\title{
New Optical Sensor Suite \\ for \\ Ultrahigh Temperature Fossil Fuel Applications
}

\section{Final Report}

DOE Award Number: $\quad$ DE-FC26-03NT41922

Reporting Start Date: 1 October 2003

Reporting Period End Date: 31 December 2007

Principal Authors: John Coggin

Tom Flynn

Jonas Ivasauskas

Daniel Kominsky

Carrie Kozikowski

Russell May

Michael Miller

Tony Peng

Gary Pickrell

Raymond Rumpf

Kelly Stinson-Bagby

Dan Thorsen

Rena Wilson

Date Report Issued: $\quad 24$ June 2008

Submitting Organization: Prime Research, LC 1750 Kraft Dr Ste 1000

Blacksburg, VA 24060 


\section{DISCLAIMER}

This report was prepared as an account of work sponsored by an agency of the United States Government. Neither the United States Government nor in the agency thereof, nor any of their employees, makes any warranty, express or implied, or assumes any legal liability or responsibility for the accuracy, completeness, or usefulness of the information, apparatus, product, or process disclosed, or represents that its use would not infringe privately owned rights. Reference herein to any specific commercial product, process, or service by trade name, trademark, manufacturer, or otherwise does not necessarily constitute or imply its endorsement, recommendation, or favoring by the United States Government were in the agency thereof. The views lists opinions of authors expressed herein do not necessarily state or reflect those of United States Government or any agency thereof. 


\begin{abstract}
Accomplishments of a program to develop and demonstrate photonic sensor technology for the instrumentation of advanced powerplants and solid oxide fuel cells are described. The goal of this project is the research and development of advanced, robust photonic sensors based on improved sapphire optical waveguides, and the identification and demonstration of applications of the new sensors in advanced fossil fuel power plants, where the new technology will contribute to improvements in process control and monitoring.
\end{abstract}




\section{TABLE OF CONTENTS}

\begin{tabular}{|c|c|}
\hline \multicolumn{2}{|r|}{ INTRODUCTION } \\
\hline \multicolumn{2}{|r|}{ 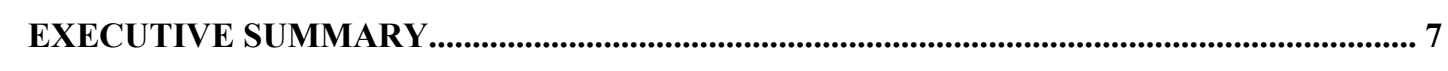 } \\
\hline 2.1 & PROGRAM OBJECTIVES.......................... \\
\hline 2.2 & ON OF SENSOI \\
\hline 2.3 & CLADDINGS FOR SAPPHIRE OPTICAL FIBERS.... \\
\hline 2.4 & DEVELOPMENT OF HigH-TEN \\
\hline \multicolumn{2}{|c|}{ 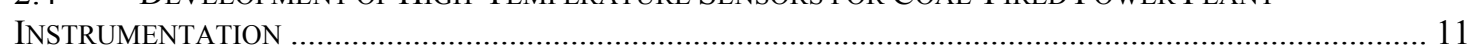 } \\
\hline 2.4 .1 & Sapphire \\
\hline 2.4 .2 & Sapphire $S$ \\
\hline 2.4 .3 & Develop \\
\hline 2.4 .4 & Dev \\
\hline 2.5 & FIELD TESTS OF HIGH- \\
\hline 2.5 .1 & Planning for Tests at Hamilton, $\mathrm{OH} P$ \\
\hline 2.5 .2 & 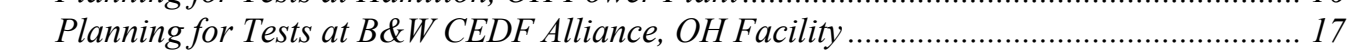 \\
\hline 2.5 .3 & 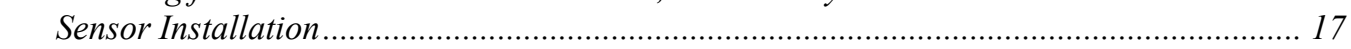 \\
\hline 2.5 .4 & Field Test Results... \\
\hline 2.6 & DEVElopment of High-Temperature Strain Gages For Solid OXide Fuel CElls. \\
\hline 2.6 & Development of Technology for Distributed Measurement of Strain in SOFCs.... \\
\hline 2.6 & evelopment of Interrogation Methods for Distributed IFPI Sensors ...... \\
\hline
\end{tabular}

3 DEVELOPMENT OF POWERPLANT SENSORS .......................................................................... 22

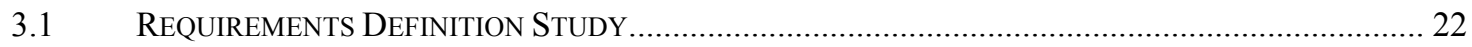

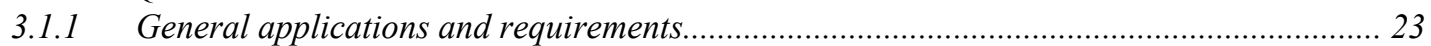

3.1.2 Requirements specific to ultrasupercritical boiler plants ................................................... 24

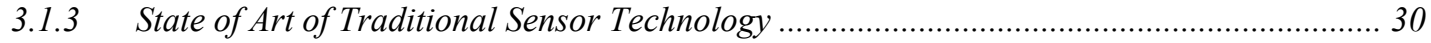

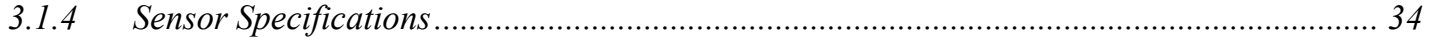

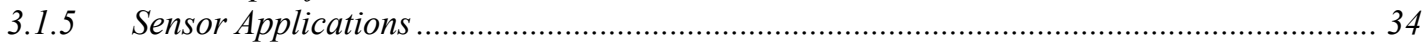

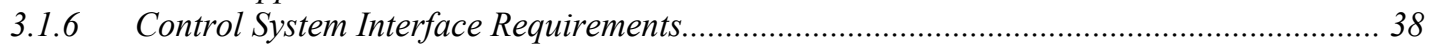

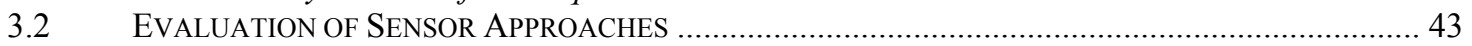

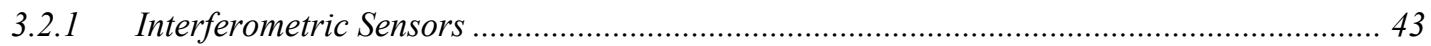

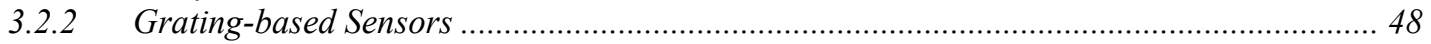

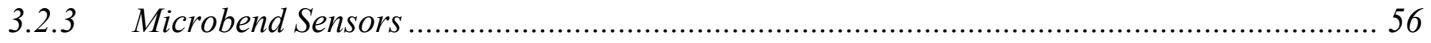

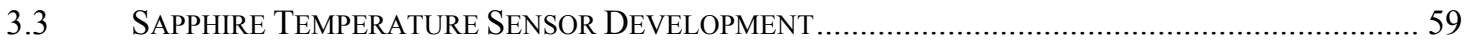

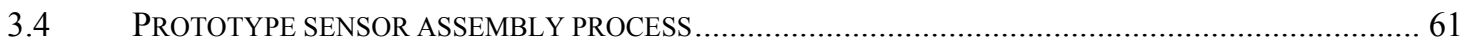

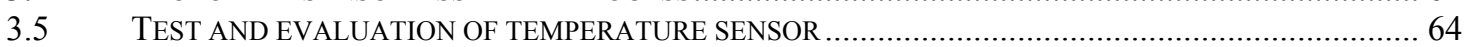

3.5.1 Room temperature testing using a clad sapphire fiber as the waveguide ............................ 64

3.5.2 Room temperature testing using an unclad sapphire fiber as the waveguide ...................... 66

3.5.3 Preliminary test results of prototype sensor at elevated temperatures ................................. 68

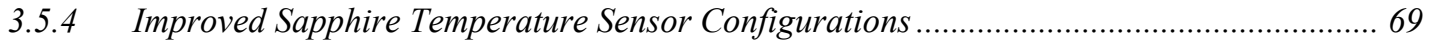

3.5.5 Sapphire Temperature Packaging Development ……...................................................... 80

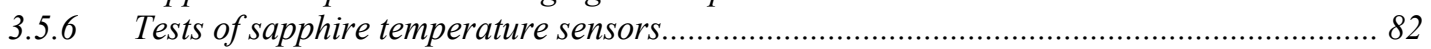

3.5.7 Fabrication of sapphire temperature sensors for field test ............................................... 83

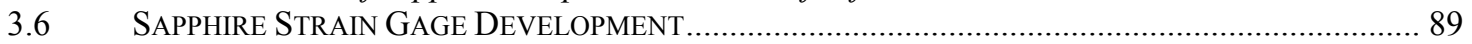

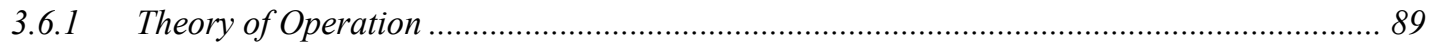

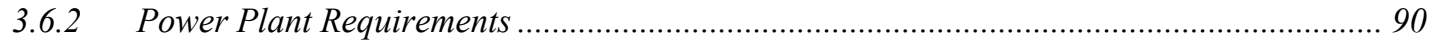

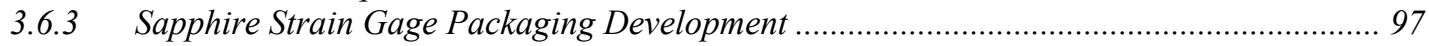

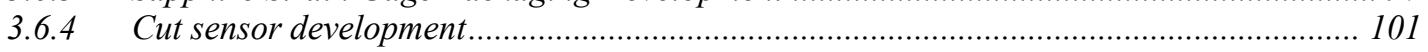

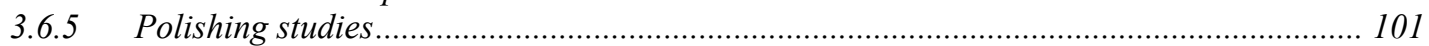

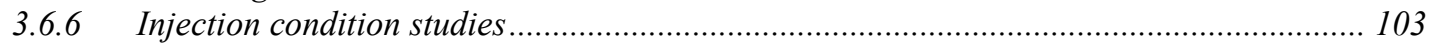

New Optical Sensor Suite for Ultrahigh Temperature Fossil Fuel Applications iv 
3.6.7 Fabrication of sapphire strain sensors for field test....................................................... 106

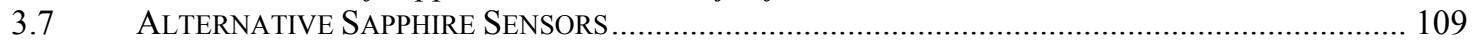

3.7.1 Sapphire Pressure Sensor Development …...................................................................... 109

3.7.2 Sapphire Sensor Development by Index Modulation …................................................... 114

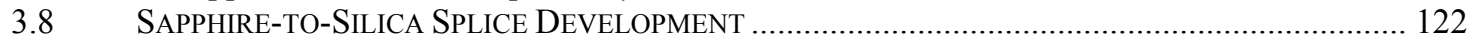

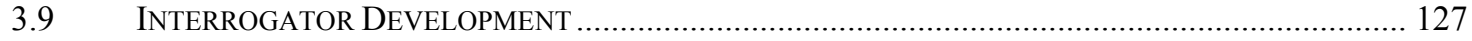

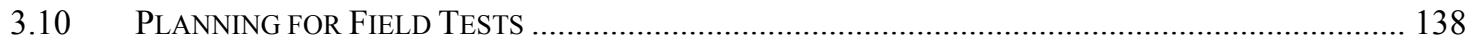

3.10.1 City of Hamilton, OH Boiler Plant ……............................................................... 138

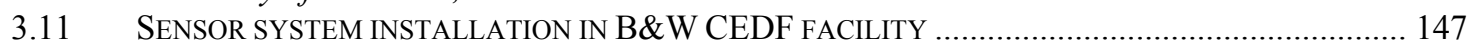

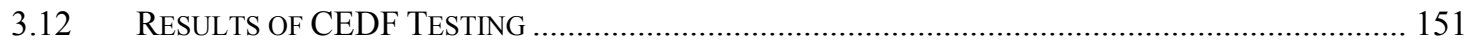

4 DEVELOPMENT OF OPTICAL CLADDING FOR SAPPHIRE FIBERS ............................. 166

4.1 InItial EXPERIMENTS Directed Towards Magnesium Aluminate Spinel CladDing .... 167

4.1.1 Preliminary Results for Spinel Cladding Development ................................................. 169

4.1.2 The high-temperature stability of the clad sapphire fiber .............................................. 173

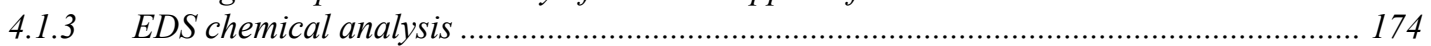

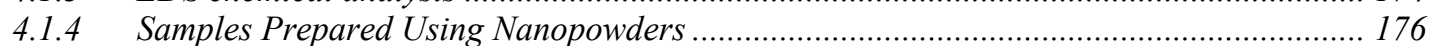

4.1.5 The use of powders with different sizes to improve packing density ................................ 187

4.1.6 The Implementation of Two Stage Milling: Dispersion Milling and Binder Milling.......... 187

4.1.7 Use of polyethylene glycol as the dispersant .............................................................. 188

4.1.8 Samples prepared using four types of powders and polyethylene glycol as dispersant...... 188

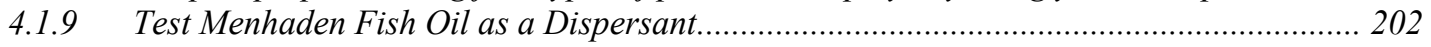

4.1.10 Study of Effectiveness of Dispersant by Particle Size Analysis................................... 206

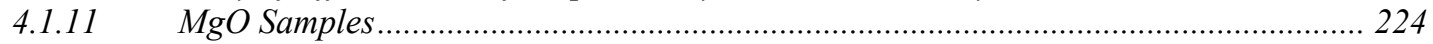

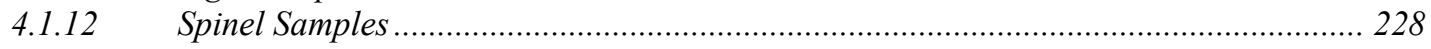

4.1.13 Measurement of Numerical Aperture and Estimate of Mode Volume........................... 229

4.1.14 Designed experiments for optimizing processing of clad sapphire fiber ......................... 235

4.1.15 Application of designed experiments to optimize sapphire cladding ........................... 240

4.1.16 High-temperature stability tests of sapphire fiber cladding ……………..................... 266

5 DEVELOPMENT OF STRAIN GAGES FOR SOLID OXIDE FUEL CELLS.......................... 272

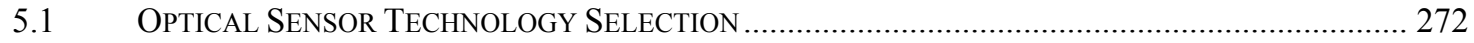

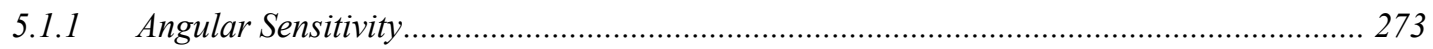

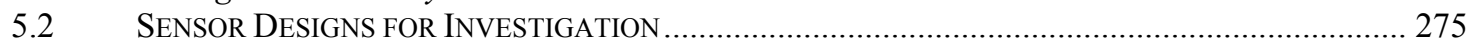

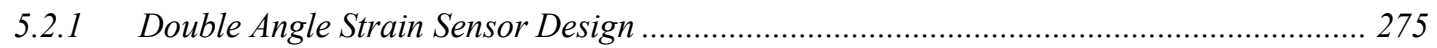

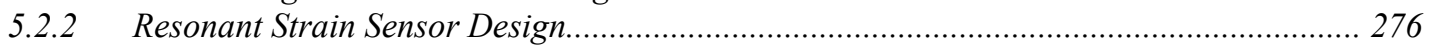

5.2.3 Miniature Fabry-Perot Strain Gages ……….......................................................... 278

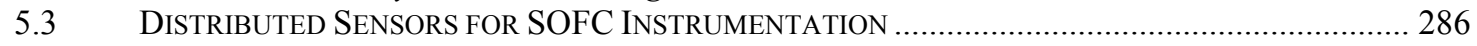

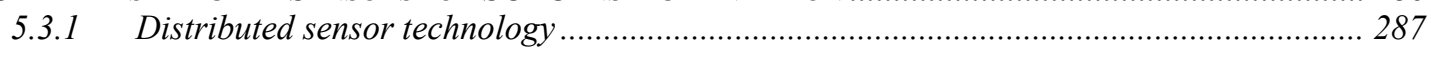

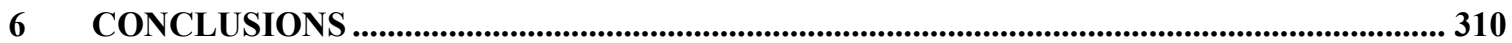

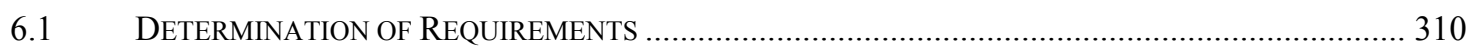

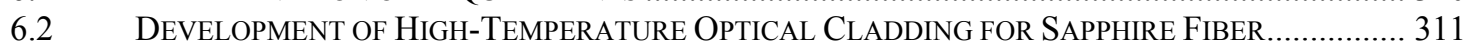

6.3 DEVELOPMENT OF SAPPHIRE SENSOR SYSTEMS FOR COAL-FIRED POWER PlanTS .................... 312

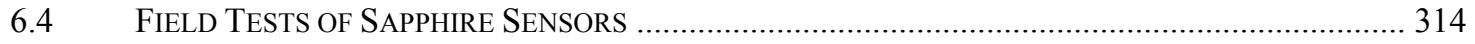

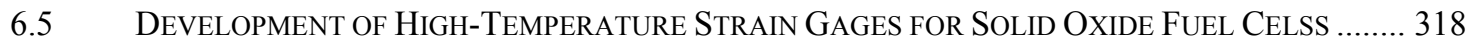

6.6 DEVELOPMENT OF TECHNOLOGY FOR DisTRIBUTED MEASUREMENT OF STRAIN IN SOFCs .... 318

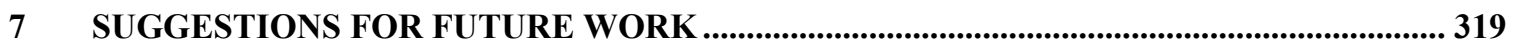

7.1 HIGH-TEMPERATURE OPTICAL CLADDING FOR SAPPHIRE FIBER .......................................... 320

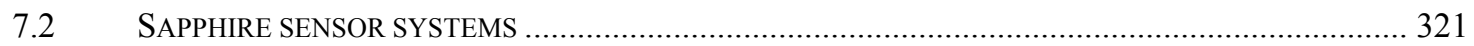

7.3 HIGH-TEMPERATURE STRAIN GAGES FOR SOLID OXIDE FUEL CELLS ……................................ 323

APPENDICES

A. B. C. D, E, F, G

New Optical Sensor Suite for Ultrahigh Temperature Fossil Fuel Applications 


\section{INTRODUCTION}

The goal of this project is the research and development of advanced, robust photonic sensors based on improved sapphire optical waveguides, and the identification and demonstration of applications of the new sensors in advanced fossil fuel power plants, where the new technology will contribute to improvements in process control and monitoring.

The advantages of fiber optic sensors over electronic sensors, particularly in environments where the electronic sensing materials can not withstand the rigors of the harsh conditions, are well known. Typically, advanced power generation systems operate at higher temperatures and pressures than traditional power plants. Traditional sensor technology for measuring temperature, pressure, flow and strain will not survive the harsh conditions anticipated in these plants. Furthermore, these plants will require more extensive process monitoring and condition assessment to maintain optimum performance and minimize maintenance costs. An effective means is required to reduce the complexity and cost of connecting the larger number of sensors to a central data acquisition platform. Therefore, one of the goals of the program is the identification of all potential applications in supercritical boiler plans where photonic sensors can be used for process control and monitoring.

For the ultrahigh-temperature environment found in current and future power generation facilities, new materials for the optical sensors will be required. Previous efforts to utilize sapphire fiber sensors that can theoretically operate above $1650^{\circ} \mathrm{C}\left(3000^{\circ} \mathrm{F}\right)$ have been limited because the fiber itself does not lend itself to incorporation in the known sensor techniques, due to its unclad and multimode construction. Another goal of this program is the development of high-temperature, ceramic claddings for sapphire fibers to improve the waveguiding properties of sapphire fibers and facilitate their use in photonic sensor systems.

Current efforts towards improvement of sapphire fibers focus on creating claddings on sapphire (aluminum oxide single crystal, $\mathrm{Al}_{2} \mathrm{O}_{3}$ ) fibers by dip-coating the fibers in a mixture of magnesium oxide $(\mathrm{MgO})$ powders and magnesium aluminate spinel $\left(\mathrm{MgAl}_{2} \mathrm{O}_{4}\right)$ powders to form a coating on the sapphire fibers. The technique involves dip coating a sapphire fiber in a suspension of $\mathrm{MgO}$ and spinel powders, drying the coating, then firing at elevated temperatures to react the $\mathrm{MgO}$ powder with the sapphire fiber, and to densify the coating. The overall reaction for the process is given by the chemical equation:

$$
\mathrm{MgO}+\mathrm{Al}_{2} \mathrm{O}_{3}=\mathrm{MgAl}_{2} \mathrm{O}_{4}
$$

which proceeds rapidly at $1750{ }^{\circ} \mathrm{C}\left(3182^{\circ} \mathrm{F}\right)$.

The coated fibers are then fired at high temperatures to facilitate the reaction between the $\mathrm{MgO}$ in the coating and the $\mathrm{Al}_{2} \mathrm{O}_{3}$ in the fibers by ion diffusion, and create a cladding consisting of $\mathrm{MgAl}_{2} \mathrm{O}_{4}$ surrounding the sapphire core. As a result, the diameters of the sapphire fibers are reduced and the number of modes propagating in the sapphire fibers is also reduced. 


\section{Executive Summary}

\subsection{Program Objectives}

The overall goal for this project was to explore the potential of optical sensors based on modified sapphire fibers for use in advanced fossil fuel power generation systems and solid oxide fuel cells. The objectives of this program were:

(1) to enable revolutionary improvements in the instrumentation of fossil fueled power plants through the development of new, robust photonic sensors based on improved hightemperature sapphire fibers,

(2) to demonstrate the application of those sensor systems to monitoring of the powerplants, and

(3) to investigate and demonstrate the use of high-temperature optical fiber sensors for characterization of the mechanical properties of developmental solid oxide fuel cells (SOFCs).

The path to achieving these research objectives involved the development of new methods to produce ultrahigh temperature clad sapphire fiber, identification of applications within advanced boiler plants for new ultrahigh-temperature photonic sensors, the development and demonstration of prototype sensors for fossil fuel power plants, the development and demonstration of high-temperature strain gages for SOFCs, and the development of technology for making distributed measurements along a single optical fiber. Specific tasks within the program included: (1) identifying applications within a next-generation fossil fuel power generation plan where ultrahigh temperature sensors are needed, (2) identifying which types of sensors are most appropriate in each location within the facility, (3) developing a repeatable process for creating modified sapphire fiber to be used in the sensors, (4) developing fiber strain gages for measurement of strain in SOFC substrates, (5) developing methods for concatenating optical sensors along a single optical fiber and reading them out individually, and (6) demonstrating success of the new sensor designs through laboratory and field measurements at high temperatures.

The project was organized into two consecutive phases. The first goal undertaken in Phase I was the identification of all potential sites within the existing and near-term boiler designs and future supercritical boiler designs where novel optical sensors could be used for process monitoring and condition assessment. After identifying the locations, the next step was the evaluation of optical sensor designs which successfully address the requirements of those applications. The final stage of Phase I was to extend the state-ofthe-art in fabrication and processing of custom single-mode and multimode sapphire fiber sensors, specifically focused on the development of new techniques to produce hightemperature optical cladding on sapphire waveguides.

The objectives for Phase II included the development of a suite of optimized sensor designs for the targeted applications, the demonstration of the feasibility and performance of the sensors, developing optical fiber strain gages for measurement of strain in SOFC substrates, and developing methods for concatenating optical sensors along a single optical fiber and reading them out individually. 


\subsection{Determination of Sensor Requirements}

Under a subcontract to Prime Research, Babcock \& Wilcox Research Center (B\&W) convened a team of engineers to consider possible applications of photonic sensors in existing and future boiler plants. In addition, a meeting was held in Alliance, $\mathrm{OH}$ between representatives of $\mathrm{B} \& \mathrm{~W}$ and Prime Research to discuss the results of the $\mathrm{B} \& \mathrm{~W}$ study, and to brainstorm other possible applications where robust sensors could be used to good advantage for controlling or monitoring power plants. Issues that were identified as potential barriers to increased use of instrumentation in advanced plants included limited availability of materials capable of surviving under typical power plant conditions (which include corrosive and reducing atmospheres), very high temperatures due to oxygen firing, a need for improved models, difficulty in interfacing with existing control systems, and resistance of customers to pay for sensors and controls.

Four classes of measurements within supercritical boiler plants were identified as the most desirable for monitoring and control of the plants. These include temperature measurements for thermal barrier coatings, in-situ strain measurements on superheater tubes, measurement of steam flow in membrane walls and tube banks, and dynamic pressure measurements for monitoring of combustion dynamics. Three specific locations were identified as primary candidates for sensor development in this program: the furnace water wall (tube temperature, heat flux, water flow), secondary superheater tubes (tube temperature, steam temperature, strain), and burners (temperature of burner components). Required sensor measurands for these locations include temperature, pressure, and strain.

Babcock \& Wilcox also investigated requirements for interfacing of fiber optic sensors to power plant control systems. Sensors were considered as an input to the plant's distributed control system, and as input to data archiving systems for trending studies and analysis. Specific interface requirements for both of these applications were derived including signal format $(0-10 \mathrm{~V}, 4-20 \mathrm{~mA}$, etc.), update rates, communications protocols, and platform architecture.

Based on the input from Babcock \& Wilcox, a matrix of potential sensing techniques and a list of requirements for high-temperature fiber and cladding were developed. The matrix compares fiber Bragg gratings (FBGs), long period gratings (LPGs), intrinsic Fabry-Perot interferometers (IFPIs), extrinsic Fabry-Perot interferometers (EFPIs), microbend sensors, optical frequency domain reflectometry (OFDR), distributed temperature sensors (DTS), polarimetric sensors, and blackbody radiation sensors according to a number of criteria, including manufacturing complexity, cost of development, suitability for multiplexing, and resolution. Based on the results of this comparative study, the requirements determined by Babcock \& Wilcox, and the experience and expertise of Prime Research engineers, sapphire sensors based on extrinsic Fabry-Perot interferometry were chosen for development as sensors for power plant applications. 


\subsection{Development of High Temperature Claddings for Sapphire Optical Fibers}

Although some success had previously been achieved by research groups in the demonstration of optical sensors based on multimode sapphire fibers, optimal function of several important classes of fiber optic sensors, including fiber Bragg gratings (FBGs) and other interferometric sensors, requires the use of single-mode waveguides. While it is possible to minimize the problems resulting from the use of multimode fibers by exciting only a small number of modes in the multimode fiber, this approach is successful only with straight, inflexible sapphire rods. Bends, splices and connectors in the fiber (which are necessary for real-world applications) cause a redistribution of power into a larger number of modes, causing intermodal interference and reducing the practicality of such multimode sensors. This limitation was the motivation for the development of hightemperature claddings in order to reduce the mode volume of the sapphire fibers.

Methods for applying magnesium aluminate spinel coatings which function as optical claddings for sapphire fibers were researched. Magnesium aluminate spinel is wellsuited for cladding of sapphire fibers due to its high melting temperature, refractive index slightly less than that of sapphire, and coefficient of thermal expansion closely matched to that of sapphire. Application of spinel coatings involves dipping the sapphire fiber in a slurry of spinel and magnesium oxide powders, firing the slurry on the fiber, and then sintering the coating to densify it on the fiber.

Research conducted during Phase I on solid-state methods for the coating of magnesium aluminate spinel claddings onto sapphire fibers demonstrated that there are several variables that influence the optical and mechanical quality of the resulting clad fibers. An investigation was undertaken in which certain parameters such as the firing profiles, the slurry dipping procedures, the atmosphere of firing, the particle sizes of the powders, and slurry compositions were systematically varied. Preliminary results indicated that a smooth interface between the sapphire core and spinel cladding, which reduces scattering and mode conversion in the fiber, can be achieved through a carefully tailored firing procedure in argon atmospheres. In addition, it was found that the core diameter of the sapphire fiber can be reduced by a specified amount through the addition of a specified proportion of magnesium oxide in the slurry.

The results indicated that the fabrication variables, which include chemical composition of the coating, processing steps for preparation of the coating slurry, and firing profiles for binder burn-out and sintering of the coatings, often interact, making determination of an optimal set of variables difficult. Therefore, a set of designed experiments was undertaken to efficiently determine the optimal set of processing variables to yield clad fibers with good optical and mechanical properties.

To guide the design of the experiments for expediency and efficiency, the Taguchi methodology was employed. First, a set of 13 desired responses for the experiments was determined. These responses fall into three general categories relating to good optical quality, good mechanical quality, and acceptable fiber geometry. Leveraging the 
extensive experience gained in the solid-state processing of spinel coatings during Phase I, a list of variables (or "factors" in Taguchi terminology) was identified for study during the designed experiments. Finally, an array of 18 experiments was derived using standard Taguchi methodology.

Experimental set-ups were designed and constructed to measure the properties of the resulting clad fibers and compare them to the desired responses. Then, 18 samples of 125 $\mu \mathrm{m}$ diameter sapphire fibers were prepared with spinel claddings, each with a unique set of factors according to the dictates of the Taguchi array. Each sample was then measured, and a numerical value was assigned for each response. Specific fabrication parameters studied included incorporation of nanopowders into the slurry, improved coating density through optimized particle packing, separation of powder milling into dispersion milling and binder milling to prevent agglomeration of powders, testing of several dispersants to control agglomeration, and determination of proper binder burnout temperature.

The measured results of the 18 experiments were statistically analyzed using Qualitek- $4^{1}$ software to determine the significance of each factor in contributing to the desired responses. The output of these statistical analyses were then used to predict the set of factors that would result in a spinel cladding with the optimal geometrical, mechanical, and optical properties.

To confirm the predictions of the Taguchi analysis, a set of four $125 \mu \mathrm{m}$ diameter sapphire fibers were clad with the magnesium aluminate spinel coating using the predicted optimal set of factors. In addition, three $75 \mu \mathrm{m}$ diameter sapphire fibers were clad using the same optimal factors. In general, the $125 \mu \mathrm{m}$ diameter clad fibers demonstrated high quality, exceeding the best results obtained during the Phase I effort. The results from the $75 \mu \mathrm{m}$ diameter clad fibers were not as good, suggesting that the optimal factors derived from designed experiments on $125 \mu \mathrm{m}$ fibers are specific for that fiber diameter.

Tests of the high-temperature stability of the clad fibers were also conducted. Four samples of clad fibers were fabricated using the optimal factors. Samples of these fibers were then heated in a furnace at temperatures of $1000^{\circ} \mathrm{C}, 1100{ }^{\circ} \mathrm{C}, 1200{ }^{\circ} \mathrm{C}, 1300{ }^{\circ} \mathrm{C}$, $1400{ }^{\circ} \mathrm{C}$, and $1500{ }^{\circ} \mathrm{C}$, for three days at each temperature. Following heat treatment at each temperature step, each fiber was polished, and its cross-section was analyzed under an optical microscope. Specifically, the fibers were examined to determine if changes in the core diameter or cladding thickness had occurred, which would indicate continued reaction of the magnesium aluminate spinel cladding with the sapphire core. The results indicated that the clad fibers were stable at temperatures up to $1300{ }^{\circ} \mathrm{C}$ for three days. At the higher temperatures, some changes in the geometry of the fibers were observed, with more dramatic changes occurring upon exposure to $1500{ }^{\circ} \mathrm{C}$ for three days, including the formation of cracks, the growth of the cladding thickness, and a reduction in the fiber core diameter. A strategy for stabilizing the cladding, involving an improved composition of the spinel slurry during coating, was proposed but not tested during this program.

\footnotetext{
${ }^{1}$ http://nutek-us.com 
In order to assess the effectiveness of the spinel cladding in reducing the number of guided modes in the sapphire fiber, an apparatus was assembled to measure the numerical aperture (NA) of a sapphire fiber before and after cladding. As the number of propagating modes in a fiber is decreased, the numerical aperture of the fiber decreases. Therefore, a change of numerical aperture can be correlated to a change in modal volume of a fiber. Using the measurement apparatus, the NA of a six-inch long unclad sapphire fiber was found to be approximately 0.59. An identical sapphire fiber was coated with a magnesium aluminate spinel cladding, and after measurement was found to have an NA of approximately 0.3 , demonstrating the efficacy of the cladding in reducing the modal volume in the sapphire fiber.

\subsection{Development of High-Temperature Sensors for Coal-Fired Power Plant Instrumentation}

A key objective for the program was the demonstration of new, ultrahigh-temperature optical sensors for specific measurements required for advanced powerplant control systems, as identified by powerplant manufacturers/integrators/operators. Towards this end, new techniques to produce sensor elements within sapphire fibers were explored and evaluated.

Tests were performed to evaluate the performance of three sensor classes: interferometric (extrinsic and intrinsic Fabry-Perot interferometer), gratings (fiber Bragg gratings and long period gratings), and microbend sensors. The sensors were configured as temperature or pressure sensors, as appropriate for the sensor method. The sensor performance was characterized in terms of sensor resolution. Of the sensor configurations evaluated, sapphire sensors based on extrinsic Fabry-Perot interferometers were determined to be the most promising approach.

Modification of sapphire fibers by doping with impurities was investigated, with a goal of creating fiber Bragg gratings or intrinsic Fabry Perot interferometer (IFPI) sensor elements in sapphire fibers. Efforts to fabricate an intrinsic Fabry-Perot cavity in a 125 $\mu \mathrm{m}$ diameter sapphire fiber by diffusing magnesium oxide into short (less than $1 \mathrm{~mm}$ ) segments of sapphire fiber were not successful. The intent was to locally reduce the refractive index of the fiber, producing two surfaces defined by the distinct change in refractive index. The Fresnel reflections generated by those surfaces would interfere, and the length of the cavity could be determined from the state of interference. Two configurations were attempted: in the first, a magnesium oxide/alumina coating was applied over a few millimeters at the end of a sapphire fiber. After the sample was heated to diffuse the magnesium oxide $(\mathrm{MgO})$ into the fiber, the end of the sapphire fiber was polished down until the section of fiber with diffused $\mathrm{MgO}$ was less than $1 \mathrm{~mm}$ in length. In this configuration, the polished end serves as one reflector in the Fabry-Perot cavity; the other is formed by the distinct transition between the doped fiber and the pure fiber. To test the sample, a swept laser spectrometer was used to inject light into the sapphire fiber and analyze the reflected spectra for interference fringes. The complex spectrum that resulted showed evidence of four cavities in the fiber: one was clearly due to the 
entire length of the sapphire fiber, and one was clearly due to the gap between the sapphire fiber and the spectrometer's silica fiber pigtail. Of the two remaining cavities, neither could be conclusively correlated with any features in the sapphire fiber.

Pressure sensors utilizing resonating mechanical structures were also investigated. Structures were fabricated that mechanically resonate when exposed to optical power that oscillates in intensity from a laser diode. The resonance, which is detected through a high-temperature fiber optic interferometric sensor and phase-locking techniques, varies in frequency with changing applied pressure, allowing for an all-optical, hightemperature pressure transducer. Laboratory tests of a prototype resonant beam pressure sensor showed that the beam's resonant frequency depended linearly on the applied pressure.

\subsubsection{Sapphire Temperature Sensors}

The design and fabrication of sapphire sensors based on Fabry Perot interferometers for measurement of temperature was researched during the program. The design investigated was based on an interferometer configuration originally developed at the Virginia Tech Center for Photonics Technology. Prime Research has licensed the intellectual property rights from Virginia Tech for this design. The specific sensor configuration uses a polished sapphire wafer as the temperature-sensitive element. The wafer is attached to a sapphire fiber (clad or unclad), and is interrogated as a Fabry-Perot cavity. Changes in the temperature of the wafer cause a change in the optical path length of the Fabry-Perot cavity, which can be related to the change in temperature through the known coefficient of thermal expansion. The high degree of parallelism of the two sides of the wafer (and thus the Fabry-Perot cavity) permits the achievement of reasonable fringe visibility, even with multimode fibers.

Methods for assembling and packaging the sensor were investigated. The use of materials that can withstand the harsh environments of boiler plants was a priority in determining the assembly process.

Several prototype sensors were fabricated and tested. A commercial optical spectrum analyzer was used to examine the resulting interference spectrum in the telecommunications C-band. Results were difficult to interpret, due to the presence of additional undesired fringes in the output spectrum. Further investigation revealed that modal noise was most likely responsible for the undesired modulation of the sensor output. If the output of a multimode fiber (such as a sapphire fiber) is restricted by an aperture of smaller spatial area than the core of the multimode fiber, the aperture will act as a spatial filter, passing a limited subset of the modes that propagate in the multimode fiber. When the number of propagating modes is filtered, intermodal interference will result. This spurious modal noise is particularly sensitive to the movement of the multimode fiber.

Further investigation indicated that the optical spectrum analyzer used for the experiments was one of the sources of the modal noise. This spectrum analyzer was 
designed specifically for use with single-mode fibers, and has an entrance pupil with a spatial extent less than that of the multimode fiber core. The influence of the modal noise can be greatly minimized, if not completely eliminated, through the use of multimode fibers exclusively in the sensor system, in addition to the use of a spectrometer designed for use with multimode fibers.

Different methods for injecting light into the sapphire optical fiber were investigated in order to determine the method that produced the least modal noise. These methods included injection of collimated light into the sapphire fiber using either a graded index rod lens or a collimator fabricated from a graded index fiber, and injection of a single mode with an expanded diameter into the sapphire fiber through the use of a fiber taper. The best and most reliable method for injection of the sapphire fiber achieved in our studies was injection using a multimode fiber with its modal volume fully filled and using standard connector ferrules for alignment.

Several prototype temperature sensors were successfully constructed using spinel-clad sapphire fibers, sapphire wafers, and supporting alumina tubes. The sensors were tested up to $900^{\circ} \mathrm{C}\left(1650^{\circ} \mathrm{F}\right)$ using a Micron Optics Component Test System as the interrogator system. Spurious oscillations in the outputs of early versions of the sapphire temperature sensors were attributed to the formation of an air gap at the sensor tip between the sapphire wafer and the polished end of clad sapphire fiber; this problem was solved by increasing the clad fiber length relative to that of the alumina supporting structure so that the clad fiber was slightly buckled, placing the interface between the fiber end and the sapphire plate in compression. The fringe stability was also improved by implementing a novel method to splice the sapphire fiber to a fused silica communications fiber, based on the use of fused silica capillary tubing to capture the two fiber ends, with borosilicate glass to bond the fiber ends. The sensors fabricated using these methods showed temperature measurement reproducibility within about 3 degree Celsius in repeated temperature tests, and the temperature sensitivities of all fabricated sensors were consistent.

Two prototype sapphire temperature sensors were fabricated and were packaged in Inconel tubes for protection to test and evaluate the final design before field tests.. Tests of the sensors were conducted at temperatures up to $1200^{\circ} \mathrm{C}\left(2192^{\circ} \mathrm{F}\right)$. One sensor performed satisfactorily and yielded a near linear relationship between applied temperature and sensor output (phase). The second sensor performed satisfactorily to $1200^{\circ} \mathrm{C}\left(2192^{\circ} \mathrm{F}\right)$, but ceased to function properly after cooling down to room temperature. Tests of the sensor indicated that the splice between the sapphire optical fiber and a glass optical fiber failed due to excessive temperature. Previous tests had shown that the splice, which is formed by using a borosilicate glass to join the sapphire and silica fibers, can fail at temperatures exceeding $450^{\circ} \mathrm{C}\left(842^{\circ} \mathrm{F}\right)$ if stress is applied to the splice. 


\subsubsection{Sapphire Strain Gages}

Several methods for fabrication of high-temperature strain sensors using sapphire fibers were investigated for applications in power generating facilities. In general, assembly of sapphire strain gages based on Fabry-Perot interferometric cavities is difficult due to the tight alignment tolerances required for multimode waveguide (sapphire fiber) components. A novel method for fabrication of Fabry-Perot cavities though the use of semiconductor manufacturing methods was evaluated for improvement of fiber alignment. In this approach, etchants were used to micromachine a V-groove in a silicon wafer. A sapphire fiber was then bonded to the $\mathrm{V}$-groove, and a diamond dicing saw was then used to cut through the silicon wafer and sapphire fiber, in a direction perpendicular to the fiber longitudinal axis. By using a dicing saw with sides that are parallel, the parallelism of the cut sapphire fiber ends is ensured. Although several attempts at constructing Fabry-Perot cavities in sapphire fibers yielded one sensor with acceptable interferometric fringes, all other attempts were not successful due to the rough surface finish on the ends of the cut fiber.

Another strain gage design investigated during the program employed V-grooves in metal coupons to hold two sapphire fibers in collinear alignment so that a gap between the fibers forms a Fabry-Perot interferometric cavity. In this method, the sapphire fibers were polished using the $\mathrm{V}$-grooves to maintain the correct polish angle. Using fibers that had been polished while bonded to V-grooves machined in metal coupons, Fabry-Perot cavities could be repeatability assembled with good interferometric fringes.

A third strain gage design employed the use of a zirconia sleeve to align the two fiber ends forming the Fabry-Perot cavity. Both sapphire fibers (input and reflector) were potted in zirconia ferrules commonly used for fiber optic connectors. A ceramic adhesive was used to pot the fibers. After curing the adhesive, the fiber and ferrule ends were polished on diamond polishing media using a polishing puck. When the polished fibers were aligned using a zirconia sleeve, acceptable interference fringes were obtained. Due to the relative simplicity of fabrication of this technique compared to the others evaluated, this fabrication method was used to fabricate the sapphire strain gages assembled for the field test.

\subsubsection{Development of Silica-to-Sapphire Fiber Splices}

Methods for joining, or splicing, the sapphire fiber to a fused silica communications fiber, were investigated and developed. Experiments showed that the most reliable splices were obtaind by using fused silica capillary tubing to capture the two fiber ends, with borosilicate glass between them to bond the fiber ends. Tests were performed to assess the maximum operating temperature for splices made in this manner. The splices were found to operate successfully to $842^{\circ} \mathrm{F}\left(450^{\circ} \mathrm{C}\right)$. Further tests were performed to determine the maximum temperature that the splice will experience after installation and during operation of the sapphire sensors in the Hamilton, Ohio power plant. By measuring the temperature profile of the sensor housing that protrudes out of the boiler 
wall, it was determined that the splice should be located no closer than 1.5 inches (38 $\mathrm{mm}$ ) from the outside of the membrane wall of the boiler plant.

\subsubsection{Development of Interrogation Methods for Sapphire Sensors}

Efforts to develop signal processing techniques to convert the optical signals from the sapphire Fabry-Perot cavities to engineering values of temperature or strain concentrated on methods to facilitate the processing of Fabry-Perot spectra, specifically concentrating on techniques to reduce the effects of intermodal interference resulting from the use of multimode sapphire fibers. Algorithms were investigated for processing the spectral responses of the sapphire Fabry-Perot sensors to yield absolute measurements (in which no data regarding the sensor history is required to obtain an accurate measurement of the environment), as opposed to relative measurements (which give only the change from a previous measurement). One method investigated was shown to achieve absolute measurements for spectra obtained from Fabry-Perot sensors made from silica fibers. Tests with Fabry-Perot sensors made from sapphire fibers, however, indicated that the complex spectra exhibited by sapphire Fabry-Perot sensors confounded the algorithm.

Therefore, development of digital spectral filters to eliminate undesired frequency components was undertaken to improve the processing of the sapphire Fabry-Perot spectra. In addition, work was directed to develop methods for uniquely determining the phase of the output signal, even when the total phase excursion of the signal exceeds $2 \pi$. The objective of those efforts is to eliminate the ambiguous output when the phase excursion exceeds $2 \pi$. Best results were realized by first calculating a coarse measure of the optical cavity length by using a measurement of the period of the spectral response of the cavity. This coarse measurement can then be used to detect and eliminate $2 \pi$ phase jumps in the calculation of cavity length from the phase of the spectral response. The phase yields a measurement of higher resolution, but is prone to modulo $2 \pi$ phase jumps if the coarse measurement of cavity length is not used to unwrap the phase jumps.

Noise in the coarse measurement contributes to noise in the fine (high resolution) measurement, so different approaches to calculate the coarse determiniation of cavity length from waveform period were evaluated. Best results were found to result from a method in which the coarse cavity size is assumed to be constant during any time in which the fine value is changing. This method then periodically re-assesses the coarse measurement at those times in which the fine measurement, and hence the measurand, is not changing. When this condition is satisfied, a weighted average of many measurements is used to establish the coarse measurement.

\subsection{Field Tests of High-Temperature Sensors for Coal-Fired Power Plants}

Prime Research worked with engineers at the Babcock \& Wilcox Research Center to identify a test site for field tests of the prototype sapphire strain and temperature sensors. 
Two facilities were considered: a municipal power plant in Hamilton, Ohio with B\&W boilers, and a combustion research facility operated by B\&W in Alliance, Ohio.

\subsubsection{Planning for Tests at Hamilton, OH Power Plant}

On April 27th, 2006, a team of engineers from PRLC met with several B\&W engineers at the pulverized coal-fired power generation plant during a scheduled outage in the city of Hamilton, Ohio to investigate potential locations for mounting and testing of sapphire temperature and sapphire strain gages.

Several locations were identified in the plant for initial testing of the sapphire sensors. A central location was identified on level 1 of the plant to place the interrogation unit and computer for collecting data during testing. A sealed cabinet with cooling capability was suggested since the testing would be over several months.

Two candidate sites in the Hamilton plant were targeted for mounting the test sensors: the first was in the secondary superheater tube bank, and the second was a crossover pipe at the top of the boiler. In the first site, superheated steam runs through the superheater tubes, high temperature steam runs through the tubes in the membrane wall, the hot gas around the superheater tubes is approximately $2000^{\circ} \mathrm{F}\left(1093^{\circ} \mathrm{C}\right)$, and a nearby soot blower make this severe location an excellent choice to test the sensor in a very aggressive environment. Two temperature sensors would be installed at this location.

The third location selected for installation of a sapphire temperature sensor in the Hamilton plant was a 15" diameter crossover pipe at the top of the plant. This location was selected due to the ease of access.

High-temperature packages were designed to protect the sapphire temperature and strain sensors during installation of the sensors in the Hamilton, $\mathrm{OH}$ coal-fired boiler plant. The sensor housings were based on Inconel, an oxidation-resistant and corrosion-resistant high-nickel alloy, and alumina, a refractory ceramic. The packages were designed to reinforce critical joints in the optical assembly and to maintain optical alignment in operation. In addition, techniques for installing the sensors were developed, and mounting adapters to attach the sensors to desired locations in the plant were designed. Three locations were to be instrumented with one temperature sensor and one strain gage in each location: on a bracket supporting the secondary superheater tube bank, in the membrane wall adjacent to the secondary superheater tube bank, and then on a crossover pipe above the boiler penthouse.

In November 2006, Prime Research learned from Babcock \& Wilcox personnel that the City of Hamilton, Ohio, which operates the coal-fired boiler plant for which the sapphire sensor packaging and installation had been designed, would no longer commit to hosting a field test of the sensors in 2007. 


\subsubsection{Planning for Tests at B\&W CEDF Alliance, OH Facility}

Following the loss of the Hamilton, $\mathrm{OH}$ plant as a field test site, $\mathrm{B} \& \mathrm{~W}$ offered an opportunity to install and test the sensors in the B\&W Clean Environment Development Facility (CEDF) in Alliance, Ohio. B\&W was up-fitting this $30 \mathrm{MW}_{\text {th }}$ demonstration facility for tests of oxy-firing combustion technologies in the summer of 2007. This plant replicates key operating conditions of steam generating systems, including burners for pulverized coal, a convection pass with simulated superheater tubes, and sootblowers for cleaning the tubes. In the CEDF plant, these tubes are not used for heat transfer and do not transport superheated steam. The tubes employ a double wall assembly. An inner steel tube carries boiling water, and is surrounded by an outer steel tube which is subjected to normal gas path temperatures. B\&W engineers suggested that the prototype sensors could be mounted to these simulated superheater tubes, and that the conditions presented to the sensors would be representative of those that would be seen in a convection pass in a conventional coal-fired supercritical boiler plant.

Prime engineers visited the CEDF facility to examine the proposed installation site, and after taking measurements of critical dimensions (tube diameter, wall thickness), began designing packaging and planning for installation of the sapphire sensors for the CEDF facility.

\subsubsection{Sensor Installation}

Two sapphire temperature sensors were assembled and packaged for installation in the Babcock \& Wilcox Clean Environment Development Facility (CEDF) combustion test facility in Alliance, $\mathrm{OH}$ for field tests. The temperature sensors and their packaging were designed for instrumentation of two locations in the $2^{\text {nd }}$ tube bank of the CEDF.

In addition, two sapphire strain gages were assembled and packaged for installation in the B\&W CEDF plant. The sensors used an extrinsic Fabry-Perot interferometer design; that is, an air gap was formed between two sapphire fibers, so that when light is injected into one of the sapphire fibers, the reflections generated by the sapphire fiber ends that form the air gap will interfere. When the two fibers are attached to a tube in the CEDF plant, then stresses applied to the tube will result in strains that will correspondingly cause a change in the sensor cavity length, which can be measured interferometrically. The sensors were packaged in Inconel housings, in order to protect the sapphire fibers from mechanical damage during handling, installation, and in service. During the final packaging of the second strain gage, the sapphire fiber was broken during insertion of the fiber into the Inconel tube, rendering that sensor unusable.

On June 21, 2007, the two sapphire temperature sensors, two thermocouples, and one sapphire strain gage were installed in the B\&W CEDF plant by Prime Research engineers. Prior to the installation date, $\mathrm{B} \& \mathrm{~W}$ personnel drilled through the CEDF wall and installed a port with a gas shield, so that the sensors could penetrate the plant wall. One sapphire temperature sensor, the sapphire strain gage, and one Type $\mathrm{K}$ thermocouple 
were attached to a CEDF simulated superheater tube using resistance welding. Due to the high temperatures anticipated by B\&W engineers (up to $2000^{\circ} \mathrm{F}\left(1093^{\circ} \mathrm{C}\right)$ ), it was not possible to mount a conventional electrical strain gage on the tube to serve as a reference for the sapphire sensor. The sapphire strain gage was mounted on a tube that was suspended from the ceiling of the superheater tube pass. Since the bottom of the tube was freely hanging, any strain resulting in the tube during the testing would result solely from the thermal expansion of the tube. Therefore, for the purpose of testing the sapphire strain gage on the CEDF tube, the measured strain was correlated with temperature.

In addition to the sapphire temperature sensor and strain gage mounted to the CEDF tube, an additional sapphire temperature sensor and thermocouple were installed. These were mounted so that the sensors sampled the temperature of the air approximately two inches from the interior wall of the CEDF.

A dustproof enclosure was used to hold two optical interrogators and a data acquisition computer. The interrogators used were a Micron Optics CTS scanning laser spectrometer (modified with a large area photodetector) for the sapphire strain gage, and an Ocean Optics USB4000 spectrometer for the two sapphire temperature sensors. The data acquisition computer was used to log the outputs from the two interrogators.

\subsubsection{Field Test Results}

Data acquisition from the sapphire strain gage, one sapphire temperature sensor, and one thermocouple began on 21 August 2007. The sapphire temperature sensor was set up to record full interference spectra every 30 seconds. Over a duration of forty-five days, a total of 130,411 spectra were recorded, ending on 5 October, 2007. On 5 October, the data collection system ceased acquisition due to a glitch in the commercial software used for recording the data. Full spectra of the output of the sapphire strain gage were recorded at one minute intervals. The sensor operated from the installation on 21 August, 2007 through 28 September, 2007. On September 28, the sensor suffered a failure at which time discernable interferometric fringes were no longer present in the captured signal. Approximately 55,000 usable spectra were logged from the strain gage.

Data obtained from the thermocouple logs indicate that the CEDF plant was not fired until 5 September 2007, so that data logged previous to that date were not used in sensor evaluation. On 18 December 2007, the sapphire sensors in the thermocouples were removed from the CEDF during a plant shutdown, for further analysis at Prime Research laboratories. At the same time, the logged data and spectra were retrieved from the data acquisition computer for further analysis.

In order to process the temperature sensor data, a series of pre-processing steps were first applied. The data were trimmed to the region of interest, and then flattened to remove the envelope of the light source used. The data were then filtered and normalized in order to yield an optimum interferogram which was then be processed in order to extract the key 
parameters which represent the measurement, specifically the frequency and phase of the signal.

An algorithm was then applied to extract data from the pre-processed spectra in order to determine the changes in the fundamental Fabry-Perot cavities. When the interference fringes acquired by the interrogator are expressed as a function of wavenumber (the reciprocal of wavelength), the frequency of the fringes is a direct measure of the FabryPerot cavity length. In the processed data from the field tests, most sections of the temperature sensor data were smoothly varying and corresponded well with the temperature changes of the system. However, other sections displayed excessive noise; it is suspected that the appearance of a second optical cavity (in addition to the sapphire wafer which serves as the measurement element and primary optical cavity) created a low-frequency interference signal in addition to the desired measurement signal. Since the frequency of the signal from the second cavity is inversely proportional to the optical length of the cavity, a small (less than one micron) cavity can generate a signal of very low frequency. This low frequency signal would heterodyne (mix) with the measurement signal, shifting the frequency of the measurement signal by an amount equal to the frequency of the second cavity. While the source of this secondary cavity has not been definitively determined, possible causes include the development of a gap between the sapphire fiber and silica fiber in the splice region, or the development of a gap between the sapphire wafer and the sapphire fiber.

In sections where high noise levels were observed, "phase jumps" resulted in offsets in the reported output, compromising the system accuracy. The phase jumps result from the periodic nature of any calculation of phase. Every $2 \pi(\sim 6.28)$ radians is a "phase jump", and one of the requirements of an interferometric sensing system is to correctly identify whether a phase jump has occurred, and unwrap it in the event that one has. In the case of excessive noise, a situation can arise in which such phase jumps are either missed, or falsely identified.

To process the data logged from the sapphire strain gage, the data was first scaled and low pass filtered to extract the highest frequency noise content. Next, the data was decimated (or downsampled) to reduce the data volume in order to maintain a reasonably short processing time. A proprietary algorithm was then used to extract the frequency, amplitude, and phase content of the signals from the decimated data.

As explained above, it was expected that the thermally induced strain in the CEDF tube would correlate well with the temperature of the tube. When the phase of the sensor output (which is proportional to the strain measured by the sensor) was plotted against the thermocouple output, a correlation coefficient of 0.7955 was obtained (with 1.0 indicating perfect correlation.)

A close inspection of the strain gage data showed that the low data sampling rate used (one sample per minute) resulted in phase jumps that reduced the accuracy of the system. When the sensor system was installed in the CEDF, it was not known definitively when the plant would begin operation. Therefore, a sampling interval of one minute was 
selected in order that the data acquisition computer's storage medium not be filled before the first firing of the plant. The actual rate at which the temperature within the environment changed was much higher than anticipated, and as a result the data collection at one minute intervals resulted in a number of phase jumps being missed. When the sensor response was successfully decoded, the slope of the phase versus temperature plot was uniform. However, when the rate of temperature change was large, the software at times failed to correctly unwrap the phase, resulting in a temporary reduction in sensitivity and a consequent reduction in accuracy. To avoid this problem in future uses of the sensor, the data acquisition rate for the strain gage interrogator should be carefully set to ensure that sufficient data is obtained to avoid phase jumps.

\subsection{Development of High-Temperature Strain Gages for Solid Oxide Fuel Cells (SOFCs)}

Strain sensors for the mechanical testing of solid oxide fuel cell (SOFC) components were developed and evaluated during the program. The initial target application was a button cell with a diameter of $1.25 \mathrm{in}$ and thickness of $137 \mu \mathrm{m}$ and operated at a temperature of approximately $800^{\circ} \mathrm{C}\left(1,472^{\circ} \mathrm{F}\right)$. Miniature Fabry-Perot strain gages based on micromachined fused silica optical fibers were evaluated. Several adhesives for bonding the optical strain gauge to the solid oxide fuel cell substrate were investigated, and a ceramic adhesive with silica filler was found to provide the best strain transfer fidelity, and to give the most stable measurements at temperatures up to $800{ }^{\circ} \mathrm{C}(1,472$ $\left.{ }^{\circ} \mathrm{F}\right)$. Development of signal processing techniques to convert the optical signals from the sapphire Fabry-Perot cavities to engineering values of temperature or strain was also addressed during the program.

Tests of the Fabry-Perot strain gage, mounted on an alumina beam next to an electrical strain gage, showed a close match between the optical and electrical strain gages when the beam was loaded in a three point configuration at room temperature. In addition, both gages showed good agreement with strains predicted by finite element analysis.

Further tests of the strain gage were performed on zirconia-based Hionic substrates at $800^{\circ} \mathrm{C}\left(1472^{\circ} \mathrm{F}\right)$. A Fabry-Perot strain gage was attached to a Hionic substrate using a ceramic adhesive, and the substrate was subjected to increasing deflection using a threepoint bend set-up. The sensor output showed changes in cavity length that were clearly correlated with the beam deflection (and hence the strain), although some hysteresis and drift was present and apparently due to slippage between the fiber sensor and the ceramic adhesive. In a final test of the sensor, the Hionic substrate was gradually loaded until failure. In this test, the increasing strain was monitored by the fiber sensor, and again some evidence of poor adhesion of the ceramic to the fiber was seen. 


\subsubsection{Development of Technology for Distributed Measurement of Strain in SOFCs}

Technologies to measure the distribution of temperature and strain across the surface of solid oxide fuel cell components were investigated and developed during the program. By concatenating multiple sensor elements along a single optical fiber, and applying optical signal processing methods to separate the responses of the individual sensors, the temperature and/or strain distribution across the fuel cell component may be mapped.

After considering various candidate sensor designs, a weakly-reflecting intrinsic FabryPerot interferometer (IFPI) was chosen as the most promising sensor type for the proposed application. Since temperatures are not expected to exceed $800^{\circ} \mathrm{C}\left(1,472{ }^{\circ} \mathrm{F}\right)$ in the solid oxide fuel cells, optical fiber sensors based on doped fused silica fiber can be applied.

A number of IFPI sensors were fabricated during this study. Techniques to write IFPI cavities in germania-doped fused silica fiber using a krypton fluoride excimer laser were developed. Efforts were made to make the fabrication process as uniform as possible, but there were significant differences observed between the particular sensors. The likely cause of this difference is the inconsistency of power and mode pattern associated with excimer lasers. Fabrication of IFPI sensors by splicing a microstructured "holey" fiber between two standard singlemode optical fibers was also evaluated, but the optical power reflected from the holey fiber was found to be too small to achieve a sufficient signal-tonoise ratio.

\subsubsection{Development of Interrogation Methods for Distributed IFPI Sensors}

Two methods for demultiplexing serially concatenated IFPIs sensors were explored: multiplexed Michelson interferometry and optical frequency domain reflectometry. A theoretical analysis was used to determine that the number of data samples required to demultiplex a $3 \mathrm{~m}$ long string of IFPI temperature sensors using optical frequency domain reflectometry (OFDR) with $1^{\circ} \mathrm{C}\left(1.8^{\circ} \mathrm{F}\right)$ resolution was prohibitively high $\left(>10^{8}\right.$ samples). An alternate approach, multiplexed Michelson interferometry (MMI), was chosen as the best alternative for further development.

The principle of operation of MMI (also called low-coherence path matching interferometry) is to use a reference cavity which is maintained within the interrogation system to sequentially match the optical cavities of each of the transducers. By accurately monitoring output signal as a function of the size of the reference cavity, one can determine the precise size of each of the transducer cavities. A prototype interrogator was assembled, and was used to successfully interrogate three concatenated Fabry-Perot sensors on a single fiber. 
The number of IFPI sensors that were multiplexed was limited by high throughput losses in the sensors, which increased the overall noise for all subsequent sensors in the chain. The overall power level which was measured returning from the sensors in the chain was approximately $2 \%$ of what could be attained with higher quality IFPIs.

A second limiting factor of the system as implemented was the optical source which was available to drive the interrogation system. The source had a much narrower spectrum than desired, resulting in a much longer coherence length. The impact of this is to make it much more difficult to localize the peaks of the resonance conditions which are indicative of having achieved the optical path match. Neither of these factors represents any limitation on the fundamental capabilities of the technology, although further development is needed to make the technology useful for practical applications.

\section{Development of Powerplant Sensors}

A key objective for the program was the demonstration of new, ultrahigh-temperature optical sensors for specific measurements required for advanced powerplant control systems, as identified by powerplant manufacturers/integrators/operators. Towards this end, requirements for high-temperature powerplant sensors were identified, new techniques to produce sensor elements within sapphire fibers were explored, prototype sapphire strain and temperature sensors were fabricated and evaluated in the laboratory, and the prototype sapphire sensors were tested in a coal-fired combustion plant.

\subsection{Requirements Definition Study}

Results of the requirements definition study to identify potential applications for process monitoring and control with advanced photonic sensors, and of the investigation and development of robust, high-temperature claddings for sapphire fibers, are described below.

On March 23, 2004, a meeting was held at Babcock \& Wilcox Research Center to discuss possible applications of advanced photonic sensors for instrumentation of current and future boiler plans. Russell May represented Prime Research as program PI. B\&W representatives present were Tom Flynn, Stan Vecci, Jeff Sarver, Bruce Young, Ralph Bailey, Greg Nakeneczny, Dennis McDonald, and Tim Fuller. In advance of the meeting, the B\&W representatives convened to discuss instrumentation needs and possible sensor applications. The objective of the meeting was to identify all potential sites within the existing/near term boiler designs and planned future supercritical boiler designs where new technology optical sensors could be used for process monitoring and condition assessment. Currently, supercritical steam boilers generally rely on indirect and calculated measurements. Advanced sensors and controls are needed to monitor 
process conditions directly to increase process efficiency, reliability, availability and detect early signs of failure. ${ }^{2}$

$\mathrm{B} \& \mathrm{~W}$ has developed designs for advanced ultrasupercritical steam boiler systems that will operate at steam conditions of $760^{\circ} \mathrm{C}\left(1400^{\circ} \mathrm{F}\right)$ and 4500 psig and cycle efficiencies up to $50 \%$. Most likely portions of the boiler will be operated at alternating oxidizing and reducing atmospheric conditions, as well as under severe slagging and fouling conditions. New alloy steels will be required to withstand the operating conditions. The long term mechanical properties of these materials under these operating conditions are not well characterized. Although traditional measurement sensors such as Type K thermocouples, pressure transducers, heat flux, flow, and strain measurements may be applied, they suffer from rapid failure rates and currently require that all the sensor leads be routed individually to the data acquisition system. Current techniques for protecting these leads are costly and expensive to install.

\subsubsection{General applications and requirements}

The following issues were identified as general barriers to efficient and cost effective process monitoring and condition assessment in advanced power plants: ${ }^{3,4}$

- Very high temperatures - Oxygen firing (increasing $\mathrm{O}_{2}$ concentration to 27-28\%) results in adiabatic flame temperatures $\left(1650{ }^{\circ} \mathrm{C}\left(3000^{\circ} \mathrm{F}\right)\right)$ higher than occur in combustion with air as the oxidant. These conditions are similar to operating temperatures $\left(1371-1650^{\circ} \mathrm{C}\left(2500-3000^{\circ} \mathrm{F}\right)\right)$ anticipated in high-temperature heat exchangers associated with the indirectly-fired cycle systems. High temperatures and ash deposition in generating banks of tubes present challenges for sensor stability and reliability.

- Models - analytical models coupled with input from robust sensor systems show promise for optimization of plant efficiency and emissions, but development of accurate models is required.

- Interfaces must be compatible with existing plant distributed control system and plant information systems.

- Materials

- Potential of failure due to harsh environmental conditions.

- High temperature thermowells for harsh conditions (erosion, corrosion, reducing atmosphere) are required.

- Cost barriers: Customers still buy on the basis of initial cost and won't pay for sensors/controls. Cost must be minimized. Current development costs for

\footnotetext{
2 “The 2002 NETL Sensor and Control Program Portfolio Review and Roadmapping Workshop: Gas, Emissions, and Process Monitoring" hosted by Department of Energy, Office of Fossil Energy, National Energy Technology Laboratory, October 15-16, 2002, Pittsburg, PA, pp17-26.

${ }^{3}$ ibid.

4 "Vision 21 Program Plan: Clean Energy Plants for the $21^{\text {st }}$ Century" Department of Energy, Office of Fossil Energy, Federal Energy Technology Center (currently NETL).
} 
advanced sensors are prohibitive without government support, especially given the price customers are willing to pay to implement the technology.

At the 2002 NETL Sensor and Control Program Roadmapping Workshop, workshop participants acknowledged that many of the sensors and control system issues stemmed from a lack of suitable sensor materials. ${ }^{2}$ Besides development of robust sensors, the DOE workshop identified the need for advancements in control systems and system integration. Participants observed that sensor technology is currently limiting rather than control system or system integration technology.

The demand for advanced sensors and control systems is usually driven by more stringent regulation than by gains in efficiency. In general, if a specific sensor need is identified, then the sensor could be developed and the control system integrated. Problems arise from the lack of knowledge of the end users' needs and the inability to convey the benefits of better measurement and monitoring systems. The extreme conditions anticipated in B\&W's ultrasupercritical boiler concepts will drive the need for new sensor technology, because it is well known that existing sensors will not be able to withstand the harsh conditions.

\subsubsection{Requirements specific to ultrasupercritical boiler plants}

A process schematic for an ultrasupercritical boiler system concept is illustrated in Figure 3-1below. ${ }^{5}$

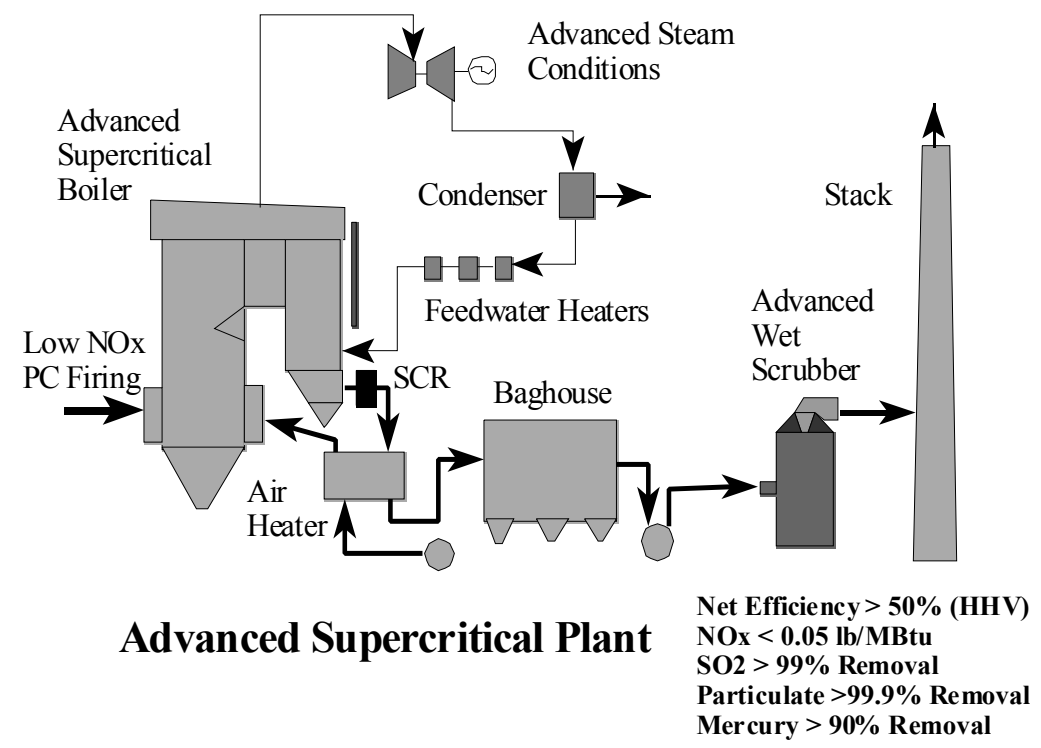

Figure 3-1. B\&W Vision for Advanced Supercritical PC Rankine Cycle

5 “B\&W's Vision for PC Boilers,” D. K. McDonald, DOE Meeting, February 18, 1999. 
Four classes of measurements within supercritical boiler plants were identified as desirable for monitoring and control of the plant. These classes are:

- $\quad$ Thermal barrier coating temperature measurement - B\&W may require thermal barriers on materials for oxygen-firing burner designs due to high temperatures.

- Strain - in-situ strain measurements on ultrasupercritical steam generation secondary superheater tubes.

- Flow measurement -steam flow in membrane walls and tube banks.

- Combustion stability - dynamic pressure measurements at high temperature, for sensing combustion dynamics. B\&W uses Flame Doctor ${ }^{\mathrm{TM}}$ to monitor conventional pulverized coal combustion flames, but will require advanced sensor technology to monitor and control the next generation of smart burners (i.e., burners that auto-adjust to coal quality changes and load changes).

In measuring water-cooled or steam-cooled furnace wall temperatures, high temperature burner components, and superheater tube temperatures (Figure 3-2), special protection must be provided because of the destructive high temperature furnace atmosphere and, in some cases, the accumulation and shedding of ash and slag deposits. For example, special provisions must be made to protect the leads between the point of measurement and the exit from the boiler setting for superheater tubes. Traditionally, this is done by containing the sheathed thermocouple in stainless steel tubing that is welded to the superheater tube. This maintains the sheath and protection tubing at a temperature approximately equal to the temperature of the superheater tube. Additional cooling of the stainless steel protection tube is frequently required. An approach to both simplify this installation and reduce the cost is required to allow for more extensive and more reliable monitoring of superheater surfaces.

Advanced once-through supercritical boiler designs are more sensitive to inaccuracies in predictions for heat flux. Further, performance of these units is more sensitive to variations in the furnace heat flux during operation. Therefore, accurate measurement of furnace heat flux is essential for the design and operation of advanced steam cycle boilers. Measurement of heat flux at more locations in the boiler will also likely be required to obtain the spatial resolution required to design the boiler and operate the boiler close to optimal conditions. Also, staging combustion to achieve low $\mathrm{NO}_{\mathrm{x}}$ emissions tends to move the more intense combustion zone upward in the furnace, which will influence furnace tube material selection.

B\&W currently uses chordal thermocouples (chord-drilled holes) or heat flux buttons to measure surface temperatures and heat flux on heat absorption surfaces such as membrane walls or generating banks. ${ }^{6}$ Measuring the temperature gradient through a tube wall is a means of determining the heat flow rate through the wall and of detecting the accumulation of certain types of external or internal deposits. Deposits on the internal surface of the tube can cause a significant increase in the tube temperature, possibly causing thermal stress beyond the yield strength of the tube material. A

\footnotetext{
${ }^{6}$ Steam: Its Generation and Use. Published by The Babcock \& Wilcox Company, Barberton, OH, U.S.A. Edited by S. C. Stultz and J. B. Kitto. 1992.
} 
temperature differential between the external and internal surface of $38^{\circ} \mathrm{C}\left(100^{\circ} \mathrm{F}\right)$ above the baseline difference is sufficient to justify chemical cleaning. Chordal thermocouples and heat flux buttons are expensive to install and maintain, so their use is often limited in large utility boilers. The chordal thermocouple requires a pressure part weld, and must be fabricated in the shop to precisely determine the location of the thermocouple location at the surface and depth within the tube wall.

Protection of pressure parts from excessive thermal stresses or overheating is also critical. Most periodic maintenance of pressure parts is performed during scheduled outages. Steam-cooled, thick wall headers such as those found on superheaters and reheaters are subject to cyclic thermal stresses as well as creep fatigue. Strain and temperature measurements are needed to calculate creep fatigue and predict remaining life.

Advanced low mass flux, supercritical vertical tube boiler designs require good measurement of steam temperature and flow through membrane walls to direct redistribution of flow to hot spots. This is a potential opportunity to combine advanced sensors and smart materials. For example, orifices that open and close at the discharge end of the tube to compensate for changes in steam conditions could be installed. The restriction orifices would open when steam conditions are hot relative to other legs, thereby preferentially increasing the water flow through the tubes that experience higher heat fluxes.

Customers are considering burning natural gas and coal in the same unit to address the restrictions in the Ozone Transport Rules (OTR). Existing low- $\mathrm{NO}_{\mathrm{x}}$ coal burners cannot achieve the $\mathrm{NO}_{\mathrm{x}}$ reduction that is possible with natural gas. An advanced dual-fuel burner capable of burning coal and natural gas in combination or individually is needed. Advanced control techniques may make it possible to achieve low $\mathrm{NO}_{\mathrm{x}}$ emissions and fuel flexibility in a single burner. 


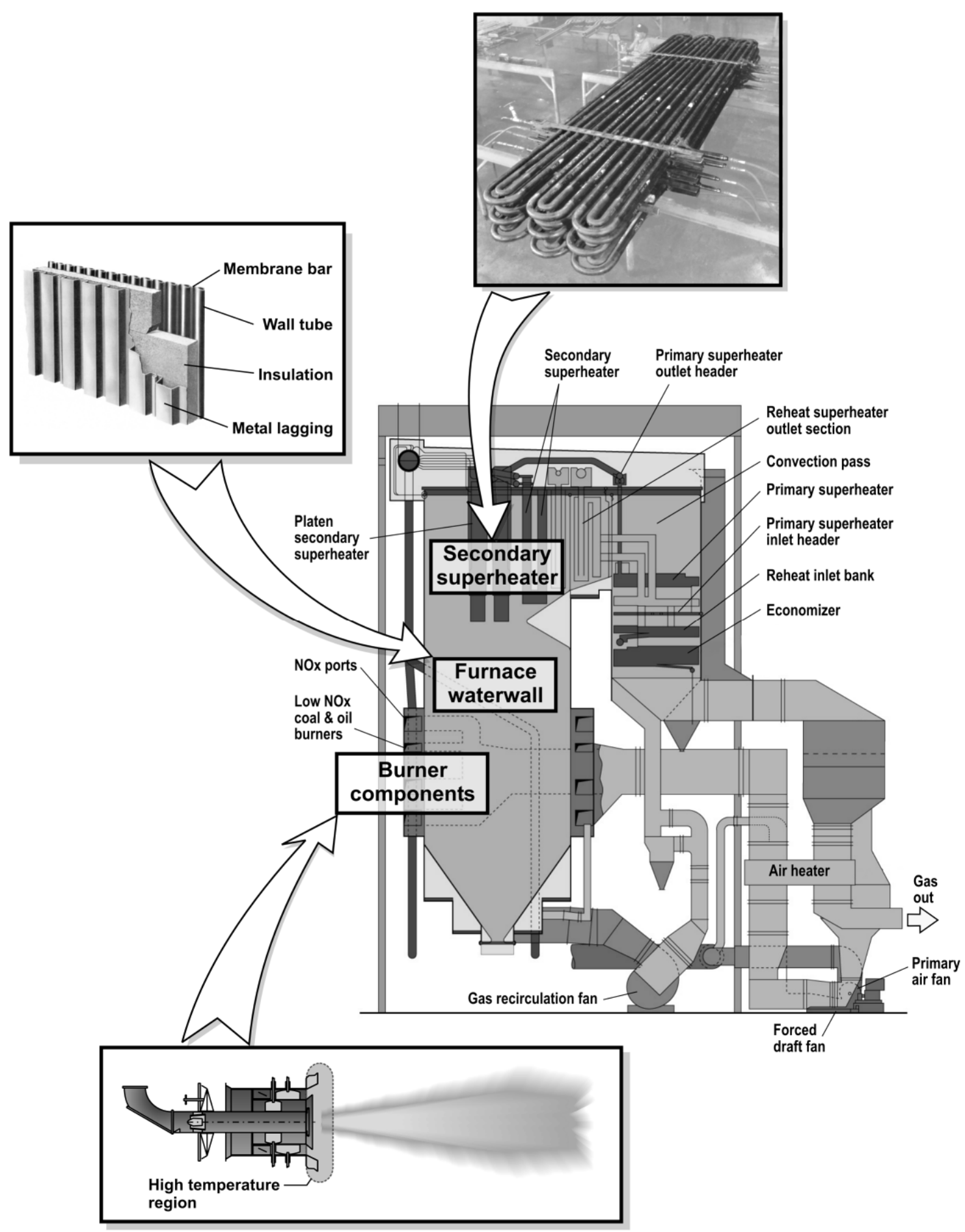

Figure 3-2. Typical Monitoring Points on a Boiler 
The following applications for high temperature optical sensors within the advanced $\mathrm{B} \& \mathrm{~W}$ plants were identified.

\section{Temperature}

- Thermal wells for pulverized coal combustors.

- Thermal wells for coal gasifier $\left(1010-1150{ }^{\circ} \mathrm{C}\left(1850-2100{ }^{\circ} \mathrm{F}\right)\right)$ suitable for corrosive reducing atmosphere (350 psi-900 psi).

- Primary stage for slagging gasifier $1427^{\circ} \mathrm{C}\left(2600^{\circ} \mathrm{F}\right)$.

- Burner components (oxygen firing) employing staged combustion $1371-1650{ }^{\circ} \mathrm{C}\left(2500-3000{ }^{\circ} \mathrm{F}\right)$, reducing conditions, oscillating reducing/oxidizing conditions.

\section{Pyrometer Measuring and Monitoring}

- Pyrometry enables both surface temperature measurements and integrity monitoring in real time.

- Combustor tiles and liners such as those on cyclone boilers. The cyclone barrels operate under slagging combustion conditions (1538$\left.1650{ }^{\circ} \mathrm{C}\left(2800-3000^{\circ} \mathrm{F}\right)\right)$, atmospheric pressure, and oscillating oxidizing/reducing atmosphere.

- Boiler burner vanes and superheater tubes can reach temperatures up to $760{ }^{\circ} \mathrm{C}\left(1400^{\circ} \mathrm{F}\right)$ at atmospheric pressure and oxidizing conditions.

- Boiler tube cladding is used for corrosion, erosion and oscillating oxidizing/reducing atmospheric conditions. Gas temperatures up to $1650{ }^{\circ} \mathrm{C}\left(3000^{\circ} \mathrm{F}\right)$ may be experienced.

\section{Strain}

- Strain measurements on ultrasupercritical steam generation secondary superheater tubes may be required. Steam conditions inside the tubes in advance cycle boilers may reach $760^{\circ} \mathrm{C}\left(1400^{\circ} \mathrm{F}\right)$ and $4500 \mathrm{psi}$. Strain measurements in the range $0.1-1.0 \%$ may be required for process monitoring and condition assessment. The team would couple pressure, temperature and strain measurements to perform condition assessment of leading secondary superheater tubes.

\section{Heat Flux}

- $\mathrm{B} \& \mathrm{~W}$ uses heat flux sensors to measure heat flux in the furnace and confirm model predictions and assess fouling on boiler wall. Water or steam blowers can be operated based on the measured heat flux. The current approach (i.e. chordal thermocouples) limits how many of these sensors can be installed, due to the cost of running all the wires back to the control room for data acquisition. 
Flow

- Steam flow in ultrasupercritical steam generating banks of tubes could be measured. The steam flow could be adjusted to optimize steam conditions for most efficient steam turbine/generator power production.

\section{Flame Monitoring and Character Method}

- Advanced burner diagnostic techniques may require nonlinear signal processing of optical sensor signal to monitor flame stability and characterize performance. Nonlinear signal processing of optical, temperature, acoustic, microwave, etc. could be coupled with chaosbased perturbation control to enhance combustion performance. B\&W may be developing a "smart burner" as part of its "smart boiler". The smart burner would be characterized by fuel flexibility (wide range of coal ranks, natural gas and oil), adaptive control system, and ultra-low $\mathrm{NO}_{\mathrm{x}}$ emissions achieved. The burner may include partial oxygen firing and internally-staged combustion.

From this range of possible applications, three locations within the next generation ultrasupercritical plant were identified as primary candidates for sensor development. The sensor applications and operating conditions are summarized below in Table 3-1.

Table 3-1. Summary of Typical Applications and Operating Conditions for Advanced Sensors

\begin{tabular}{|c|c|c|c|c|c|c|c|c|c|c|}
\hline Location & Measurement & Fluid/Material & $\begin{array}{l}\text { Gas Temp. } \\
{ }^{\circ} \mathrm{C}\left({ }^{\circ} \mathrm{F}\right)\end{array}$ & $\begin{array}{c}\text { Metal } \\
\text { Temp. } \\
{ }^{\circ} \mathrm{F}\end{array}$ & $\begin{array}{l}\text { Steam } \\
\text { Temp. } \\
\quad{ }^{\circ} \mathbf{F}\end{array}$ & $\begin{array}{c}\text { Steam } \\
\text { Pressure } \\
\text { psi }\end{array}$ & $\begin{array}{l}\text { Steam } \\
\text { Flow } \\
10^{3} \\
\mathrm{lb} / \mathrm{h}-\mathrm{ft}^{2}\end{array}$ & $\underset{\%}{\text { Strain }}$ & $\begin{array}{c}\text { Heat Flux } \\
10^{6} \\
\text { Btu/h-ft }\end{array}$ & Atmosphere \\
\hline $\begin{array}{l}\text { Furnace } \\
\text { Waterwall }\end{array}$ & $\begin{array}{l}\text { Tube } \\
\text { temperature, } \\
\text { heat flux, water } \\
\text { flow }\end{array}$ & T23 or T92 & $\begin{array}{l}1371-1650 \\
(2500-3000)\end{array}$ & $\begin{array}{l}\text { Up to } \\
510 \\
\text { (950) }\end{array}$ & $\begin{array}{l}\text { Up to } \\
482 \\
(900)\end{array}$ & $5000+$ & $\begin{array}{l}400- \\
1900\end{array}$ & $\begin{array}{l}0.1- \\
1.0\end{array}$ & $0.08-0.15$ & $\begin{array}{l}\text { Oxidizing/ } \\
\text { Reducing }\end{array}$ \\
\hline $\begin{array}{l}\text { Secondary } \\
\text { Superheater } \\
\text { Tubes }\end{array}$ & $\begin{array}{l}\text { Tube } \\
\text { temperature, } \\
\text { steam } \\
\text { temperature, } \\
\text { strain }\end{array}$ & $\begin{array}{l}\text { Austenitic } \\
\text { Stainless Steel } \\
\text { Croloy (Cr/Mo) } \\
\text { or Haynes } \\
\text { (Fe/Ni/Cr/Co) } \\
\text { Alloys }\end{array}$ & $\begin{array}{l}1093-1371 \\
(2000-2500)\end{array}$ & $\begin{array}{l}871 \\
(1600)\end{array}$ & $\begin{array}{l}760 \\
(1400)\end{array}$ & $5000+$ & $\begin{array}{l}400- \\
1500\end{array}$ & $\begin{array}{l}0.1- \\
1.0\end{array}$ & $0.02-0.04$ & Oxidizing \\
\hline Burners & $\begin{array}{l}\text { Metal } \\
\text { temperature of } \\
\text { burner } \\
\text { components - } \\
\text { vanes, coal } \\
\text { pipe, mixers }\end{array}$ & $\begin{array}{l}\text { Carbon Steel, } \\
\text { possibly } \\
\text { stainless steel } \\
\text { alloys with } \\
\text { oxygen firing. }\end{array}$ & $\begin{array}{l}1371-1650 \\
(2500-3000)\end{array}$ & $\begin{array}{l}815 \\
(1500)\end{array}$ & $\mathrm{N} / \mathrm{A}$ & $N / A$ & $\mathrm{~N} / \mathrm{A}$ & $\begin{array}{l}0.1- \\
1.0\end{array}$ & N/A & Oxidizing \\
\hline
\end{tabular}




\subsubsection{State of Art of Traditional Sensor Technology}

In this section, the limitations of existing sensor technology are discussed. The advantages and disadvantages as well as limitations of thermocouples, pressure transducers and strain gages are discussed. The matrix of sensor type, location and specification is presented. The population of existing ultrasupercritical boilers that would serve as the initial market entry point for newly developed optical sensors is provided.

\section{Thermocouples}

The objective of the optical sensor development is to provide a suitable replacement for Type K or B high temperature thermocouples that would be more robust in the high temperature gas atmosphere of a utility boiler. In general, we are not looking for a replacement of the resistance-temperature-detectors (RTDs), thermistors or integrated circuit sensor. The accuracy provided by these types of thermocouples is not required for the specific applications that are identified in the matrix.

Traditional Type K or B thermocouples offer the following advantages: ${ }^{7}$

- Self-powered

- Simple

- Rugged

- Inexpensive

- Wide variety

- Wide range of temperatures

Type C, D, G Tungsten/Rhenium are capable of measuring temperatures up to $2760^{\circ} \mathrm{C} / 5000^{\circ} \mathrm{F}$ but suffer from poor oxidation resistance and should be used in vacuum, hydrogen, or inert atmosphere. The poor oxidation resistance makes them impractical for fossil fuel applications.

The disadvantages of these Type $\mathrm{K}$ and $\mathrm{B}$ thermocouples are as follows:

- Non-linear

- Low voltage

- Reference required

- Least stable

- Least sensitive

Type $\mathrm{K}$ and $\mathrm{B}$ thermocouples also suffer from the limitation that they cannot survive the corrosive conditions of alternating oxidizing and reducing atmosphere found in the lower furnace of a large utility boiler that employs staged combustion for $\mathrm{NO}_{\mathrm{x}}$ control.

\footnotetext{
${ }^{7}$ Omega Complete Temperature Measurement Handbook and Encyclopedia Vol. 27, Appendix Z. copyright 1989.
} 
In addition, thermocouples that are used to monitor heat flux on the furnace walls and tubes are embedded directly in the furnace membrane or in the wall of the tube. These thermocouples cannot be protected with heavy metal sheath; and therefore, prone to mechanical failure. The optical temperature sensor being developed by Prime Photonics can use high-temperature, corrosion-resistant materials and therefore can address these disadvantages of Type $\mathrm{K}$ thermocouples.

The readings of Type $\mathrm{K}$ or B thermocouples are influenced by the following sources of noise:

- Power line (120V or 220V AC)

- Magnetically induced

- Stray capacitance in sensing and signal lines

- Ground loops

Traditional noise rejection techniques for typical thermocouples:

- tree switching - filters out noise due to stray capacitance in individual channels

- normal mode filtering - Analog filter removes broadband noise

- integration-averages noise over a full line cycle thereby eliminating power line related noise and its harmonics.

- guarding - filters out noise due to common mode interference such as 220V AC supply line.

- Twisted pairs extension wires.

Photonic sensors have a reduced sensitivity to electromagnetic interference due to the use of all-dielectric transducers and a dielectric signal transmission medium.

Traditional sources of measurement errors include:

- Poor junction connection

- Decalibration of thermocouple wire

- Shunt impedance and galvanic action

- Thermal shunting

- Noise and leakage currents

- Thermocouple specifications

- Documentation

Photonic sensors can be designed to be self-referencing, and therefore, are (with the appropriate design) less susceptible to some of the traditional sources of error associated with poor junction connections, decalibration of thermocouple wiring, etc. The photonic sensors offer the additional advantage that the electronics can be located remotely from the measurement point, thereby avoiding corruption or loss of the signal due to overheating of the electronics. 


\section{Pressure Sensors}

Traditionally, pressure transducers consist of a pressure sensors (diaphragm and strain gage) coupled with compensation network. Under this project Prime Photonics is developing both components of the pressure transducer. The optical sensor technology provides the means to mount the sensor in a previously inaccessible location due to the rugged design.

Desirable features of pressure sensor include: ${ }^{8}$

- Interchangeable

- Accurate over a wide temperature range

- Operable at temperature extremes

- Durable within the external environment

- Compatible with various measurands (gases, liquids, slurries, etc.)

- Isolated from the measurand

Traditional pressure sensors consist of one of the following types of construction.:

- Piezoresistive (Silicon) Diaphragms - semiconductor strain gages bonded to a silicon diaphragm

- Semiconductor strain gages bonded to a stainless steel diaphragm

- Foil grid strain gages bonded to a stainless steel diaphragm.

As indicated above for the temperature sensors, the optical sensor offers advantages over the existing sensor technology, particularly with respect to robustness at elevated temperatures.

\section{Strain Gages}

Primary application is static strain measurements rather than dynamic. The types of measurements typically made are:

- Static Loads

$\circ$ Bending

- Axial

○ Shear

- Torsional

- Derived Quantities

○ Moment -Yes

- Pressure - Yes

- Acceleration - No

○ Displacement - Yes

○ Vibration - No

\footnotetext{
${ }^{8}$ Complete Pressure, Strain and Force Measurement Handbook and Encyclopedia, Vol. 29 Appendix Z Omega Engineering, Inc. copyright 1995.
} 
Traditional types of strain gages include:

- Piezoresistive or semi-conductor gage

- Carbon-resistive gage

- Bonded metallic wire

- Foil resistance gages

Advantages of these strain gages include:

- low cost

- short gage length

- only moderately affected by temperature

- small physical size

- low mass

- high sensitivity to strain

The factors typically considered for gage selection are:

- operating temperature

- state of strain (gradient, magnitude, time dependence)

- stability

There exist several potential sources of error for electrical strain gages, including:

- Damaged sensor

- Electrical noise and interference

- Thermally induced voltages

- Magnetically induced voltages

- Temperature effects

Most strain gages are inherently unstable due to degradation of the bond, as well as temperature sensitivity and hysteresis caused by the thermoelastic strain. The optical sensors offer a superior approach to ensuring the integrity of the bond between the sensor and base material due to the high temperature materials of construction.

On utility boilers, sootblowers are used to keep the heat absorption surface in the boiler and convection pass clean so that steam can be produced with the minimum amount of fuel. Currently, the B\&W PowerClean Intelligent Sootblower system uses gas temperatures and steam flows to monitor the heat absorption in the boiler. As heat absorption surface fouls with ash and slag, the PowerClean system directs operation of the sootblowers to clean the surface. It would be very desirable to have temperature measurements on the surface of boiler tubes. To date this has not been possible because of the difficulty to get thermocouples to survive in the boiler and tube bank. Peakimpact-pressure (PIP) is a measure of the energy delivered to the deposit at a given distance from the sootblower nozzle. ${ }^{9}$ The peak-impact-pressure varies as a function of sootblower spacing, media pressure and tube spacing. For utility boilers, the high end of sootblower nozzle pressures would be around $350 \mathrm{psig}$. Using a nozzle pressure of 350 psig, Diamond Power's high performance nozzle jets will produce up to 180-200 inches Hg Peak Impact Pressure (PIP) measured at a distance of 20" from the jet exit. The

\footnotetext{
${ }^{9}$ Steam: its generation and use. $40^{\text {th }}$ ed. Babcock \& Wilcox copyright 1992 Pg 23-2. 
rugged sapphire sensors with appropriate cladding can be made to withstand the shock from the sootblower media jet impacting on the tube.

The sapphire and silica also offer superior corrosion resistance. This is especially important for monitoring burner component temperatures. Vanes and mixing devices on coal burners can reach temperatures in excess of $980^{\circ} \mathrm{C}\left(1800^{\circ} \mathrm{F}\right)$ if recirculating gases are impinging on the burner front. In addition, when the burners are staged the recirculating gases can be alternately oxidizing and reducing. This cycling of gas chemistry can cause accelerated wastage of metal components. This has made it difficult to get thermocouples or strain gages to survive in this environment. Excessive temperatures $\left(>650^{\circ} \mathrm{C} / 1200^{\circ} \mathrm{F}\right)$ can cause premature failure of the burner components that require expensive repairs during plant outages.

\subsubsection{Sensor Specifications}

Preliminary specifications are established for each of three types of sensors: temperature, pressure and strain, and are shown below in Table 3-2, Table 3-3, and Table 3-4. The list of specifications is intended to address two objectives. First, for those cases where specifications are similar between traditional sensors and the optical sensors, establish quantitative values. Second, for those cases where specifications are unique to optical sensors, identify the specifications and establish preliminary quantitative values for the specifications. In many cases, the quantitative value for the specification cannot be established until more is know about the potential application. For example, due to the future availability of the optical sensors it may be possible to make measurements not currently possible with existing sensor technology.

\subsubsection{Sensor Applications}

Numerous optical techniques can be used in the design of fiber optic sensors. In addition, different techniques are required for different measurands and multiplexing requirements. The optical electronics, signal conditioning, and acquisition equipment required for the various sensing techniques vary greatly. In some cases, the techniques do not lend themselves to implementation and a deliverable system. All sensor types must be evaluated to determine which concepts offer the greatest promise for development and application for power plant monitoring.

Table 3-5 presents a matrix evaluating nine common optical sensing techniques for several evaluation criteria. The evaluation process was based upon the experience of engineers at Prime Research, as well as that of other investigators as reported in the literature. 
Table 3-2. Draft Requirements for Temperature Sensors

\begin{tabular}{|c|c|c|c|}
\hline \multicolumn{4}{|l|}{ Temperature } \\
\hline Specification Description & Units & Value & Comments \\
\hline Range & ${ }^{\circ} \mathrm{C} /{ }^{\circ} \mathrm{F}$ & $1700 / 3000$ & $\begin{array}{l}\text { Basis Type B Thermocouple } \\
\text { (Platinum/Rhodium); Suitable } \\
\text { for oxidizing or inert } \\
\text { atmosphere but not } \\
\text { reducing, cannot be inserted } \\
\text { into metal tubes so poor for } \\
\text { measuring heat flux, } \\
\text { Contaminated by hydrogen, } \\
\text { carbon and many metal } \\
\text { vapors (Na, K), Measure } \\
\text { temperatures up to } \\
1800^{\circ} \mathrm{C} / 3270^{\circ} \mathrm{F} \text {. }\end{array}$ \\
\hline Gas & ${ }^{\circ} \mathrm{C} /{ }^{\circ} \mathrm{F}$ & $1700 / 3000$ & Flue Gas \\
\hline Metal-Burner & ${ }^{\circ} \mathrm{C} /{ }^{\circ} \mathrm{F}$ & $1100 / 2000$ & Burner parts \\
\hline Metal-Steam Tubes & ${ }^{\circ} \mathrm{C} /{ }^{\circ} \mathrm{F}$ & $870 / 1600$ & $\begin{array}{l}\text { Reheater and Secondary } \\
\text { Superheater }\end{array}$ \\
\hline Output & microvolts $/{ }^{\circ} \mathrm{C}$ & $\begin{array}{l}\text { A desirable output } \\
\text { would be between } \\
0.1 \text { and } 1 \mathrm{mv} /{ }^{\circ} \mathrm{F}\end{array}$ & $\begin{array}{l}\text { Equivalent Seebeck } \\
\text { coefficient; Type } \mathrm{S} \\
\text { thermocouple is } 7 \mu \mathrm{V} /{ }^{\circ} \mathrm{C} \text { at } \\
20^{\circ} \mathrm{C} \text {; larger number is } \\
\text { better. Type } \mathrm{K}=40 \text {. }\end{array}$ \\
\hline \multicolumn{4}{|l|}{ Performance } \\
\hline Accuracy & $\%$ & $0.50 \%$ & $\begin{array}{l}\text { ANSI Limits of Error } \\
\text { Time required to reach }\end{array}$ \\
\hline Response Time (Time Constant) & $\mathrm{ms}$ & 1 & $\begin{array}{l}63.2 \% \text { of an instantaneous } \\
\text { temperature change }\end{array}$ \\
\hline DVM Sensitivity & microvolts for $0.1^{\circ} \mathrm{C}$ & $\begin{array}{l}\text { between } \\
0.1 \text { and } 1 \mathrm{mv} /{ }^{\circ} \mathrm{C}\end{array}$ & Larger number is better. \\
\hline Linearity & $\%$ full scale output & TBD & \\
\hline Noise & & TBD & \\
\hline Stability over 1 year & $\%$ full scale output & TBD & \\
\hline Media Compatibility & & & $\begin{array}{l}\text { Most TC's are encased in a } \\
\text { sheath and compatibility } \\
\text { depends on the sheath } \\
\text { material }\end{array}$ \\
\hline \multicolumn{4}{|l|}{ Atmosphere: } \\
\hline Oxidizing & & & $\begin{array}{l}\text { Oxidizing }\left(\mathrm{CO}, \mathrm{SO}_{2}, \mathrm{O}_{2}\right. \\
\mathrm{CO}_{2}, \mathrm{NO}_{x}, \mathrm{~N}_{2}, \mathrm{HCl}, \mathrm{NaO}, \\
\mathrm{CaO}, \mathrm{SiO}) ; \\
\text { Reducing }\left(\mathrm{H}_{2} \mathrm{~S}, \mathrm{CO}, \mathrm{SO}_{2},\right.\end{array}$ \\
\hline Reducing & & & $\begin{array}{l}\mathrm{O}_{2}, \mathrm{CO}_{2}, \mathrm{NO}_{x}, \mathrm{COS}, \mathrm{HCN}, \\
\left.\mathrm{N}_{2}, \mathrm{HCl}\right)\end{array}$ \\
\hline \multicolumn{4}{|l|}{ Liquid } \\
\hline Water & & & $\mathrm{pH} 9-11,3500$ psi \\
\hline Steam & & & $3500 \mathrm{psi}, 1050^{\circ} \mathrm{F}$ \\
\hline
\end{tabular}


Table 3-3. Draft Requirements for Pressure Sensors

\begin{tabular}{|c|c|c|c|}
\hline \multicolumn{4}{|l|}{ Pressure Sensors } \\
\hline Specification Description & Units & Sensor Value & Comments \\
\hline Specifications $^{10}$ & & & Transducer Value \\
\hline Excitation & $V \mathrm{dc}$ & 12 to $40 \mathrm{VDC}$ & \\
\hline Rangeability or turndown & & $\sim 10: 1$ & can vary from $2: 1$ to $100: 1$ \\
\hline Upper Range Limit (URL) & psi & $0-5000$ & $\begin{array}{l}\text { Ranges can extend from } \\
\text { about } 0 \text { to } 30 \mathrm{in} \text {. Hg to } 0 \text { to } \\
5000 \text { psi. }\end{array}$ \\
\hline Output & $\mathrm{mV}$ & $4-20 \mathrm{ma}$ & Typical analog output \\
\hline \multicolumn{4}{|l|}{ Performance } \\
\hline Accuracy & $\begin{array}{l}\% \text { full scale } \\
\text { output }\end{array}$ & $\pm 0.25 \%$ of range & $\begin{array}{l}\text { typical, depends somewhat } \\
\text { on the span turndown }\end{array}$ \\
\hline Linearity & $\begin{array}{l}\% \text { full scale } \\
\text { output }\end{array}$ & TBD & included in accuracy spec \\
\hline Hysteresis & $\begin{array}{l}\% \text { full scale } \\
\text { output }\end{array}$ & TBD & included in accuracy spec \\
\hline Stability & $\begin{array}{l}\% \text { full scale } \\
\text { output }\end{array}$ & $\pm 0.25 \%$ of URL & over a 6 month time period \\
\hline Vibration & & $\begin{array}{c}0.1 \% \text { per g, } 0 \text { to } 200 \\
\mathrm{~Hz}\end{array}$ & \\
\hline Humidity Limits & $(\%)$ & 0 to $100 \%$ R.H. & \\
\hline Operating Temperature Range & ${ }^{\circ} \mathrm{C}$ & -40 to 85 & \\
\hline Compensated Temperature Range & ${ }^{\circ} \mathrm{C}$ & -30 to 85 & \\
\hline Thermal Zero Effect & $\%$ & $0.5 \%$ per $100 \mathrm{~F}$ & \\
\hline Thermal Span Effect & $\%$ & $0.5 \%$ per $100 \mathrm{~F}$ & \\
\hline Burst Pressure & & 10,000 psi & \\
\hline $\begin{array}{l}\text { Response time (Dynamic } \\
\text { Response) }\end{array}$ & $\mathrm{ms}(\mathrm{Hz})$ & $1 \mathrm{~ms} /(1000 \mathrm{~Hz})$ & \\
\hline Frequency response & $\mathrm{dB}$ at $\mathrm{kHz}$ & & \\
\hline Media Compatibility & & $\begin{array}{l}\text { Wetted parts typically } \\
316 \text { SS or Hastelloy C } \\
\text { with Viton O-rings }\end{array}$ & \\
\hline \multicolumn{4}{|l|}{ Atmosphere } \\
\hline Oxidizing & & & $\begin{array}{l}\mathrm{CO}, \mathrm{SO}_{2}, \mathrm{O}_{2}, \mathrm{CO}_{2}, \mathrm{NO}_{x}, \mathrm{~N}_{2} \\
\mathrm{HCl}, \mathrm{NaO}, \mathrm{CaO}, \mathrm{SiO})\end{array}$ \\
\hline Reducing & & & $\begin{array}{l}\mathrm{H}_{2} \mathrm{~S}, \mathrm{CO}, \mathrm{SO}_{2}, \mathrm{O}_{2}, \mathrm{CO}_{2}, \mathrm{NO}_{\mathrm{x}} \\
\left.\mathrm{COS}, \mathrm{HCN}, \mathrm{N}_{2}, \mathrm{HCl}\right)\end{array}$ \\
\hline \multicolumn{4}{|l|}{ Liquid } \\
\hline Water & & & pH 9-11, 3500 psi \\
\hline Steam & & & 3500 psi, 1050 F \\
\hline
\end{tabular}

${ }^{10}$ Rosemount Comprehensive Product Catalog 2002-2003 edition Emerson Process Management 
Table 3-4. Draft Requirements for Strain Gages

\begin{tabular}{|c|c|c|c|}
\hline $\begin{array}{l}\text { Strain Gages } \\
\text { Specification Description } \\
\text { Specifications }\end{array}$ & Units & Sensor Value & Comments \\
\hline Range & $\%$ & $0.1-1.0$ & \\
\hline Output & $\begin{array}{l}\mu \varepsilon @ 10 \mathrm{~V} \\
\text { Excitation }\end{array}$ & 20 & \\
\hline Sensitivity & $\mathrm{mV} / \mathrm{N} @ 1000 \mu \varepsilon$ & 2 & \\
\hline Gage factor (strain sensitivity, k) & & 2.0 & \\
\hline Gage factor tolerance & & $1.00 \%$ & \\
\hline Gage factor temperature coefficient & $\% /{ }^{\circ} \mathrm{C}$ & TBD & \\
\hline Reference temperature & ${ }^{\circ} \mathrm{C}$ & 23 & $\begin{array}{l}\text { Calibration at room } \\
\text { temperature }\end{array}$ \\
\hline $\begin{array}{c}\text { Performance } \\
\text { Service temperature range }\end{array}$ & & & \\
\hline Static & & TBD & \\
\hline Dynamic & & TBD & \\
\hline $\begin{array}{l}\text { Dynamic Response } \\
\text { Mechanical Properties }\end{array}$ & $\mathrm{kHz}$ & TBD & \\
\hline Maximum Strain & $\mu \varepsilon$ & 10,000 & $\begin{array}{l}\text { This is based on the } 1 \% \\
\text { range needed }\end{array}$ \\
\hline Hysteresis & & Negligible & \\
\hline Fatigue (at $\pm 1500 \mu \varepsilon)$ ) & cycles & $10,000,000$ & \\
\hline Smallest bend radius & & TBD & \\
\hline Media Compatibility & & & \\
\hline Atmosphere & & & \\
\hline Oxidizing & & & $\begin{array}{l}\mathrm{CO}, \mathrm{SO}_{2}, \mathrm{O}_{2}, \mathrm{CO}_{2}, \\
\mathrm{NO}_{x}, \mathrm{~N}_{2}, \mathrm{HCl}, \mathrm{NaO}, \mathrm{CaO}, \\
\mathrm{SiO}\end{array}$ \\
\hline Reducing & & & $\begin{array}{l}\mathrm{H}_{2} \mathrm{~S}, \mathrm{CO}, \mathrm{SO}_{2}, \mathrm{O}_{2}, \mathrm{CO}_{2}, \\
\mathrm{NO}_{x}, \mathrm{COS}, \mathrm{HCN}, \mathrm{N}_{2}, \mathrm{HCl}\end{array}$ \\
\hline Liquid & & & \\
\hline Steam & & & pH 9-11, 3500 psi \\
\hline Water & & & $3500 \mathrm{psi}, 1050^{\circ} \mathrm{F}$ \\
\hline
\end{tabular}




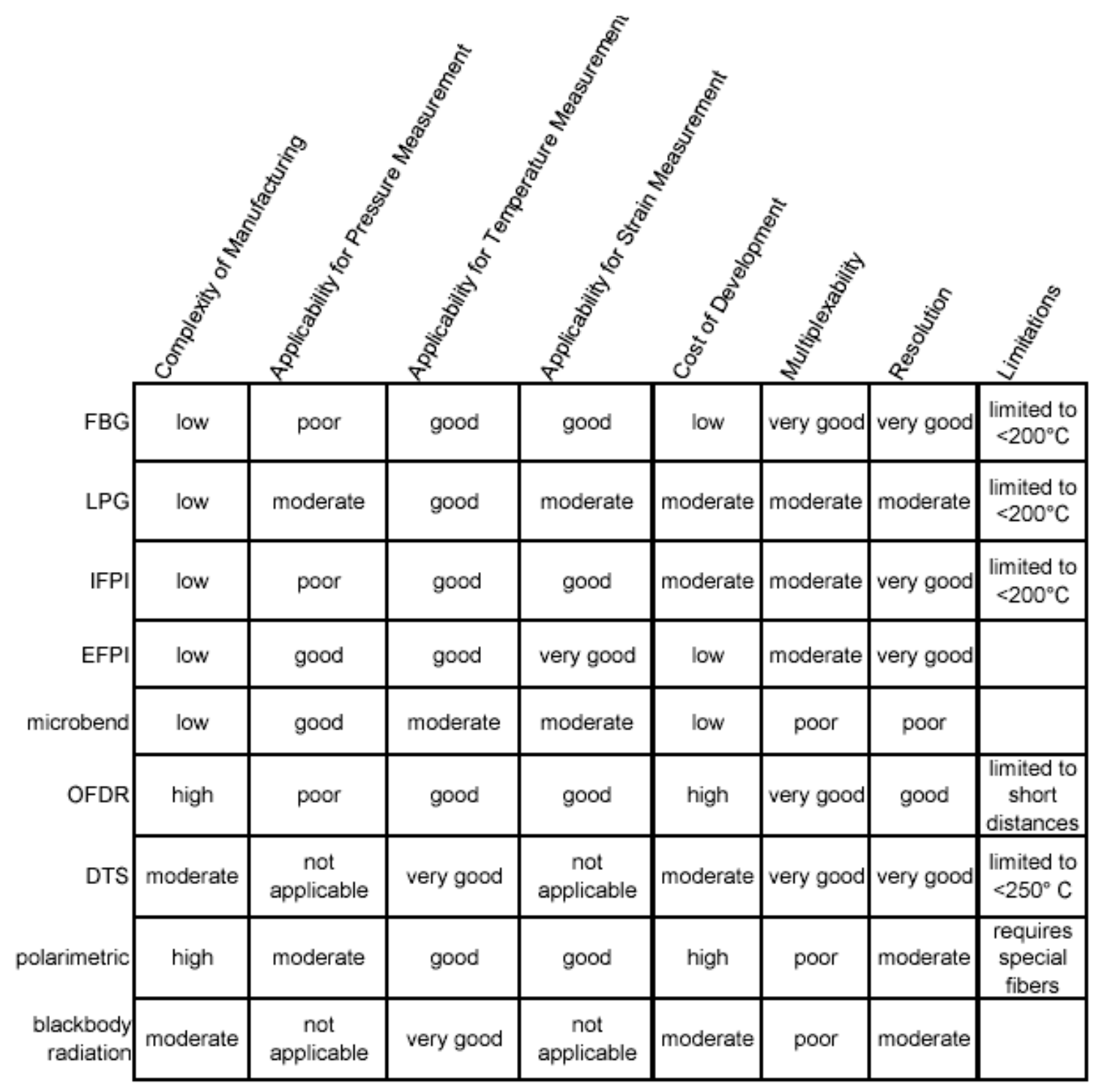

$\mathrm{PPE}=$ periodic photoelasticity sensor

IFPI = intrinsic Fabry-Perot interferometer

FBG = fiber Bragg grating

OFDR = optical frequency domain reflectometer

DTS = distributed temperature sensor

EFPI = extrinsic Fabry-Perot interferometer

Table 3-5. Sensor Evaluation Table

\subsubsection{Control System Interface Requirements}

Requirements for interface of the high-temperature optical sensors with power plant control systems were investigated by Babcock \& Wilcox engineers; results of their studies are reported in this section. 


\subsubsection{Control System Applications}

Power plant control systems typically consist of two components - a distributed control system (DCS) which is used to control the operation of the system, and a data archiving system which is used to store operating data in a database for trending and analysis.

Conceivably, the Prime Photonics sensors can be incorporated into the plant instrumentation in two ways. First, the sensors can serve as input to the DCS for control purposes. Second, the sensors can serve as input to the data archiving system for monitoring and performance assessment. [B\&W recommends that the sensors be used primarily for the second purpose. For high-temperature processes the primary concern will be mechanical integrity of components.]

Sophisticated power generation systems may employ neural net control. Multiple inputs from the DCS or data archiving systems will be sent to the neural net processor. The neural net will then attempt to optimize boiler control in either an advisory mode or a closed loop mode.

\subsection{Sootblower and Waterwall Blower Control}

The operation of individual subsystems on a power plant can be optimized by using better sensing techniques. For example, the sootblower system can be optimized by monitoring heat absorption on individual tubes and steam production throughout heat zone of the convection bank. Monitoring and control systems like B\&W's Powerclean ${ }^{\mathrm{TM}}$ acquire temperature data and using proprietary convection bank performance codes, determine optimum sootblowing sequences. This has the effect of maximizing steam production while minimizing loss of steam through the sootblowers.

The heat flux in the furnace can be monitored with surface thermocouples on the membrane walls. Variations in heat flux from optimal can be used to control waterwall blowers or to adjust the operation of the combustion system (i.e., burners and over-fire air ports).

\subsection{Burner and Overfire Air Control}

Conceivably, an array of temperature sensors mounted at the inlet to the pendent section of the convection bank can be used to guide adjustments to the combustion system i.e., burners and overfire air ports. A non-uniform temperature distribution at the furnace exit can be a sign that coal and air flow to the burners or air flow to the overfire air ports is not optimal. The individual temperature measurements could be processed by a neural net. The neural net could be used to optimize coal bias and air bias to the mills or air flow to each over fire air port. 


\subsection{Burner Air Bias and Vane Adjustment}

Temperature sensors mounted on burner mixing devices or vanes could be used to monitor metal temperatures to avoid overheating the burner elements. Overheating can cause warping and premature failure of these components. Warping can cause vanes to seize which could prevent the vanes from being adjusted properly. The vane position needs to be optimized to minimize $\mathrm{NO}_{\mathrm{x}}, \mathrm{CO}$ and unburned carbon emissions. When high temperatures are observed the operator can adjust air bias to the burner to cool the burner elements. High temperatures may also be an indication of deposits on the burner which are disrupting the flow profile out of the burner. This can cause hot recirculation gases to impinge on burner elements.

\subsubsection{Interface Requirements}

\subsection{Distributed Control System (DCS)}

If the sensor is to be connected to the plant DCS as an input to the control system, then the output of the sensor needs to be in a form compatible with existing control systems, such as 1-5 Volts, 4-20 ma, $0-10 \mathrm{~V}$ etc. Temperature output could be in $\mathrm{mV}$ similar to existing thermocouple technology. The update rate of the sensor output must be fast enough to serve an input to a control system PID loop. The use of the Prime Photonics sensors for this application is probably somewhat limited since existing instrumentation is adequate for most control applications in a boiler. However, it is possible that due to the availability of a high temperature sensor, new control strategies could be conceived that previously were not considered due to the lack of a suitable sensor that could withstand the conditions where it was installed.

\subsection{Data Archiving}

For data archiving, the same approach as the DCS application could be followed or the Prime Research sensor scheme could be packaged as a standalone system that communicates process values via an OPC protocol. This would provide flexibility to set up the system independent of the specific installation. OPC protocol is a well-established standard. With Prime Photonics multiplex architecture the OPC protocol may provide the best interfacing option. B\&W has applied this approach successfully to its Flame Doctor $^{\circledR}$ and Powerclean ${ }^{\mathrm{TM}}$ products.

\subsection{Communication Architecture}

In the event that Prime Photonics were to supply a stand alone system with its sensor processing unit acquiring the signals from the individual sensors via the multiplexed acquisition system and generating outputs for each sensor, a protocol for communicating the information to the plant DCS or data archiving system would be required. It is 
recommended that the commonly used OLE for Process Control (OPC) protocol be used. An OPC server enables communication between the OPC Client (Prime Photonics sensor processing unit) and the plant DCS or data archiving system. The OPC protocol defines the format of data that is passed between the sensor processing unit and the plant's data acquisition and control systems. B\&W has successfully employed this communication interface for its Powerclean ${ }^{\mathrm{TM}}$ and Flame Doctor ${ }^{\circledR}$ burner diagnostics products.

\subsection{Platform Architecture}

The move in instrumentation and control is clearly from analog to digital technology. Standards are being developed to connect analog devices to digital networks. Smart instrumentation provides not only the process value being sensed (primary variable), but also secondary variables such as device identification, device diagnostics, and status information.

Fieldbus, Profibus-PA or Highway Addressable Remote Transducer (HART) technology interfaces are available. These platforms provide easy access to information and control function. Fieldbus is a digital, two-way communications link among intelligent fieldlevel instruments and control devices using a multi-drop network topology. HART supports two-way digital communications for process measurement and control devices implementing a point-to-point arrangement. ${ }^{11}$.

The fieldbus protocol when fully implemented would replace the 4-20 ma standard. The fieldbus is $100 \%$ digital. Several advantages are realized when the control at a field device level can be achieved with the fieldbus technology. The two-way communication means that a value (such as process variable) can not only be read from the end device, but it would also be possible to write to this device (such as calibration parameters). Currently the transmission speed of the information on the fieldbus platform is 31.25 $\mathrm{kb} / \mathrm{s}$, which is fast enough to enable closed loop control. Standards for high-speed Ethernet communication have been developed that will allow transmission speeds up to $100 \mathrm{Mb} / \mathrm{s}$. The multi-drop capability will result in a significant cost savings since it allows multiple sensors in the same general area to be connected to the control system with a single cable rather than running individual cables for each device. Wiring cost savings of $80-90 \%$ over conventional installations have been realized. This multiplexing of signals is consistent with Prime Photonics multiplexed sensor processing technology. Problems with individual sensors or control devices can be diagnosed remotely. Moving control functions from a central control room out to the end device results in more reliable control as well as a simpler central control system.

Fieldbus was developed using Instrument Society of America (ISA) and International Electrotechnical Commission (IEC) standards. The fieldbus devices and protocols are designed to meet the stringent requirements of the process industry. Foundation ${ }^{\mathrm{TM}}$

\footnotetext{
11 *D'Aquino, R and Greene, R, "Forays in Smart Instrumentation" Chemical Engineering Progress Magazine, May 2003.
} 
fieldbus is the most comprehensive of all fieldbus technologies currently available. Foundation fieldbus has developed standard function blocks, such as analog input, digital input, PID control, analog output and digital output that provide universal interoperability between different manufacturer's devices. Unfortunately, due to the large population of analog devices currently in service, Foundation fieldbus technology will be implemented slowly over time. The best immediate application would be at a new plant.

Profibus-PA is widely used in process automation applications. Profibus-PA is an adaptation of Profibus-DP which is used for factory automation. Profibus-PA shares the same communication method as Foundation fieldbus. Profibus-PA can be configured to interface with both analog and digital devices.

The HART protocol is a hybrid that bridges the gap between analog and digital. In the HART system, the traditional 4-20 mA signal is used to represent the process variable or control output signal to or from an end device. A digital value is superimposed on top of the analog value for exchanging additional information with the device. Therefore the HART protocol is backward compatible with the installed base of instrumentation. This provides the possibility of a broader application since much of the existing instrumentation in utility power plants is still analog. FF is more readily implemented in a new installation. The HART system runs at $1200 \mathrm{~b} / \mathrm{s}$. The transmitter or control device is interrogated with a stand alone communicator device. HART-based technology is more widespread.

In addition to the HART protocol a new IEEE standard entitled "Standard for a Smart Transducer Interface for Sensors and Actuators-Mixed mode Communication Protocols and Transducer Electronic Sheet (TEDS) Formats" (Standard 1451.4) will provide a way to enable analog sensors to connect with digital protocols.

DCS/PLC system suppliers such as Rockwell Automation, Honeywell, Siemens and ABB have all developed control system technology that can interface with HART technology. Device suppliers such as Emerson Process Management and Yokogawa have developed I/O system enhancements to allow their smart sensors to interface with the control systems. HART-enabled instrumentation is available to monitor and control temperature, pressure, level and flow.

In conclusion, Prime Photonics should develop both analog and digital outputs for the sensors being developed under this project. The analog output will be compatible with many existing installation and if necessary can be converted to a digital format. The digital output will be more compatible with future platforms that are currently being developed. Smart instrumentation provides the potential to move from corrective maintenance to preventative maintenance which will reduce down time and outage costs. 


\subsection{Evaluation of Sensor Approaches}

Tests were performed to evaluate the performance of three classes of sensor: interferometric (extrinsic and intrinsic Fabry-Perot interferometer), gratings (fiber Bragg gratings and long period gratings), and microbend sensors. The sensors were configured as temperature or pressure sensors, as appropriate for the sensor method. The sensor performance was characterized in terms of sensor resolution.

\subsubsection{Interferometric Sensors}

In the Fabry-Perot fiber sensor, two reflecting surfaces are set up within the sensor fiber itself, producing local interference, the magnitude of which is related to the optical distance between the two reflectors. The two reflectors are often made from the flat ends of optical fibers, using the normal Fresnel reflections from the surfaces, as illustrated in Figure 3-3 (a). This is called an 'extrinsic' Fabry-Perot interferometer, or EFPI. The optical interference between the two reflected light waves sets up interference at the detector, which varies sinusoidally with the distance between the reflectors. These sinusoidal 'fringes' can be decoded to very accurately measure the distance between the fiber ends (the 'gap'). By attaching the two fibers to a structure, any movement in the structure can be used to indirectly calculate the strain, or related parameters such as acoustic pressure.

Alternately, a Fabry-Perot cavity can be formed an optical fiber by modifying the refractive index of the core over some short length of the fiber $(<100 \mu \mathrm{m})$. If the transition between the core's natural refractive index and the modified refractive index is distinct over a plane perpendicular to the axis of the fiber, then Fresnel reflections will be generated from the two transition zones as shown in Figure 3-3 (b), and interference can occur. This configuration of interferometer is termed an intrinsic Fabry-Perot interferometer (IFPI).

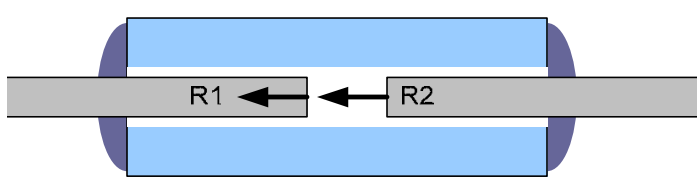

(a)

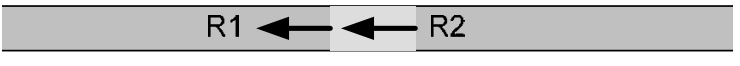

(b)

Figure 3-3. Extrinsic (a) and intrinsic (b) Fabry-Perot interferometric sensors. 


\subsubsection{Extrinsic Fabry-Perot Interferometer (EFPI)}

An alternate fiber optic sensor design incorporates an air gap between the end of an optical fiber and an extra reflector; the fiber end and the reflector form a Fabry-Perot interferometric cavity, which is generally termed an extrinsic Fabry-Perot interferometer EFPI). If some measurand induces a change in the separation between the fiber in and the reflector, the interference signal between the two reflections is modulated, and can be related to the applied measurand. For the test performed here, a variation of the EFPS sensor called the Epoxy-free Fabry-Perot Sensor (EFPS) was used. The EFPS sensor uses the same components as an EFPI to create a Fabry-Perot cavity; however, the attachment method of the fiber and diaphragm is much different as shown in Figure 3-4. The diaphragm is made of fused silica and is laser welded to the fused silica tube around the circumference of the diaphragm. In addition, the fiber is laser welded to the inside wall of the tube. Thus, the entire assembly is made of fused silica.

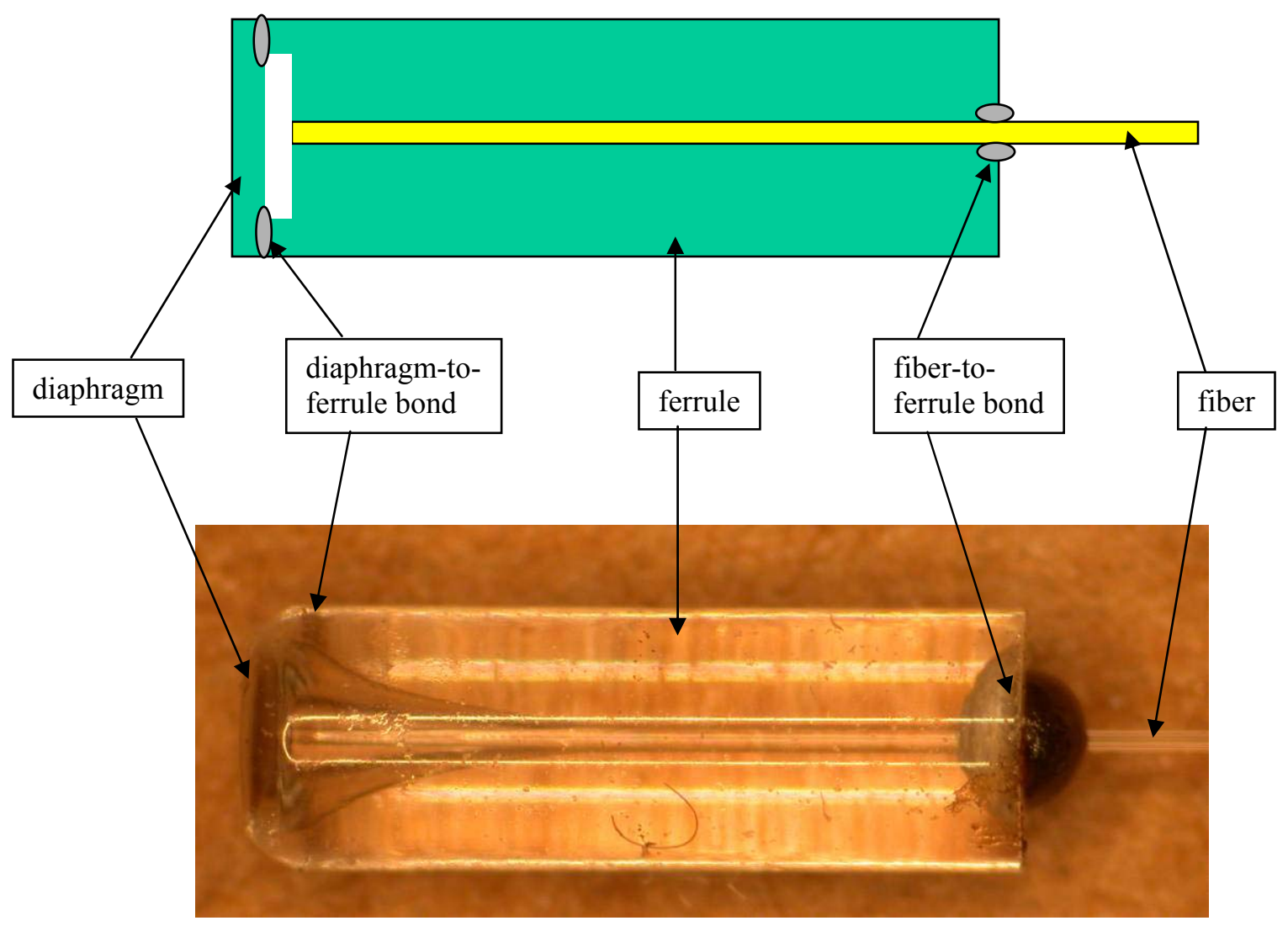

Figure 3-4. Schematic and photomicrograph of EFPS sensor.

An EFPS sensor constructed according to the schematic above was tested as a temperature sensor. The test utilized a Micron Optics si720 Component Test System 
(CTS) tunable laser spectrometer for interrogation. By measuring the spectral (wavelength) response of the Fabry-Perot cavity by the spectrometer, the cavity length of the sensor can be determined; more exactly, by monitoring the spectral response of the sensor, a change in the phase of the interferometric signal resulting from an applied measurand can be measured.

For this test, the EFPS sensor was placed in a furnace, and the temperature was taken from $50^{\circ} \mathrm{C}\left(122^{\circ} \mathrm{F}\right)$ to $200^{\circ} \mathrm{C}\left(392^{\circ} \mathrm{F}\right)$ in steps of $50^{\circ} \mathrm{C}\left(122^{\circ} \mathrm{F}\right)$, then allowed to cool back to $50^{\circ} \mathrm{C}\left(122{ }^{\circ} \mathrm{F}\right)$ and held at this temperature until conclusion of the test. It can be seen that the sensor responded to the $150^{\circ} \mathrm{C}\left(302^{\circ} \mathrm{F}\right)$ temperature increase with a change in phase of 1.2 radians. The noise amplitude is seen to be approximately 0.02 radians. The stability of the system can be observed for the portion of the test where the temperature was maintained at $50^{\circ} \mathrm{C}\left(122^{\circ} \mathrm{F}\right)$, shown in the figure. During this constant temperature the phase does not have any noticeable drift. These results indicate a resolution of approximately $2.5^{\circ} \mathrm{C}\left(4.5^{\circ} \mathrm{F}\right)$.

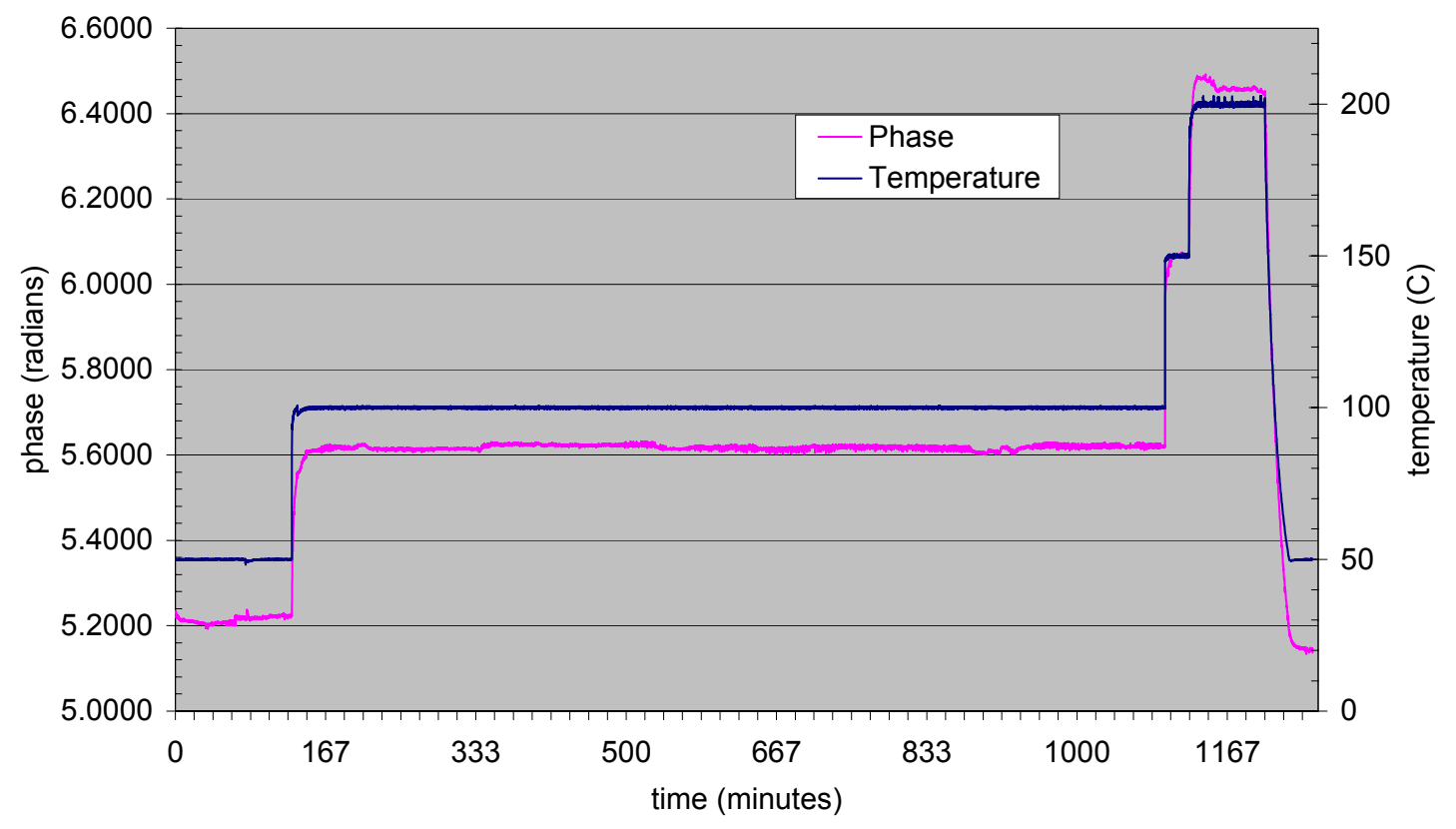

Figure 3-5. CTS interrogation of an EFPS temperature sensor. The plot shows the sensor response (phase) and the oven temperature as a function of time.

\subsubsection{Intrinsic Fabry-Perot Interferometer (IFPI)}

The sensor element is an intrinsic Fabry-Perot interferometer (IFPI) cavity in an optical fiber, as shown in Figure 3-3(b). In general, Fabry-Perot interferometers are formed by placing two parallel partially reflecting planar surfaces into the light path such that they are normal to the direction of light travel. While partially reflective surfaces can be constructed in a number of ways, the Center for Photonics Technology at Virginia Tech 
invented a unique way to place these surfaces within the existing fiber structure (hence, "intrinsic") such that there are no parts to assemble. The two planar surfaces are formed by exposing a section of photo-sensitive fiber to ultraviolet laser light. As the section of fiber is exposed to the laser light, the index of the fiber core changes such that it is different than that of the unexposed fiber. This index change creates partially reflecting surfaces at the two ends of the exposed section of fiber. This is very similar to the process used to create Fiber Bragg Gratings (FBG) in fiber; however, instead of creating a periodic pattern (many closely spaced small cavities) in the fiber as is done with FBGs, only one cavity, albeit longer in length, is written in the fiber.

To test the use of an IFPI cavity as a temperature sensor, the sensor was fabricated in Nufern GF1 single-mode fiber. This fiber is a commercially available single-mode silica fiber, with a high germanium content in the core to enhance photosensitivity. The output of a GSI Lumonics krypton fluoride excimer laser was collimated using a beam expander, and the fiber was positioned so that the laser beam was incident on the fiber perpendicular to the axis of the fiber. Approximately one inch of the polymer jacket was stripped from the fiber to expose the glass to the beam. Two metal plates were set up in front of the fiber in order to mask off the laser output. One plate was mounted to a micropositioners, so that the gap between the plates could be adjusted. This gap defines the cavity length after exposure of the fiber.

One IFPI cavity fabricated in this fashion was tested in a small furnace as a temperature sensor. Since the color centers responsible for the refractive index modulation can be erased to high temperature annealing, the test was limited to temperatures below $300{ }^{\circ} \mathrm{C}$ $\left(572^{\circ} \mathrm{F}\right)$. During fabrication, the gap between the mask plates was set to approximately $0.5 \mathrm{~mm}$. After exposing the fiber to the excimer laser output, the fiber was connected to a Micron Optics si720 CTS spectrometer. The output spectrum in Figure 3-6 was obtained, and from these fringes, it was calculated that the physical cavity length of the IFPI was $387 \mu \mathrm{m}$.

The fiber was then placed in a small furnace, with any Type $\mathrm{K}$ thermocouple to monitor the temperature. A laboratory data acquisition system was used to log the output of the CTS spectrometer during the test. The furnace was heated and after the temperature stabilized at $300{ }^{\circ} \mathrm{C}\left(572{ }^{\circ} \mathrm{F}\right)$, data logging commenced. After soaking at $300{ }^{\circ} \mathrm{C}(572$ $\left.{ }^{\circ} \mathrm{F}\right)$ for 21 hours, the temperature was decreased to $250^{\circ} \mathrm{C}\left(572^{\circ} \mathrm{F}\right)$ for approximately four hours, after which it was increased to $300^{\circ} \mathrm{C}\left(572^{\circ} \mathrm{F}\right)$ for the remainder of the test. The output phase of the sensor and the applied temperatures are shown in Figure 3-7.

The temperature and phase variations over a six-hour portion of the test, over which time the temperature was held steady at $300^{\circ} \mathrm{C}\left(572{ }^{\circ} \mathrm{F}\right)$, is reproduced in Figure $3-8$. From these data, the temperature resolution of the sensor, after subtracting the variations in applied temperature, was calculated to be $1.1^{\circ} \mathrm{C}\left(2.0^{\circ} \mathrm{F}\right)$, based on a $2 \sigma$ definition for resolution. 


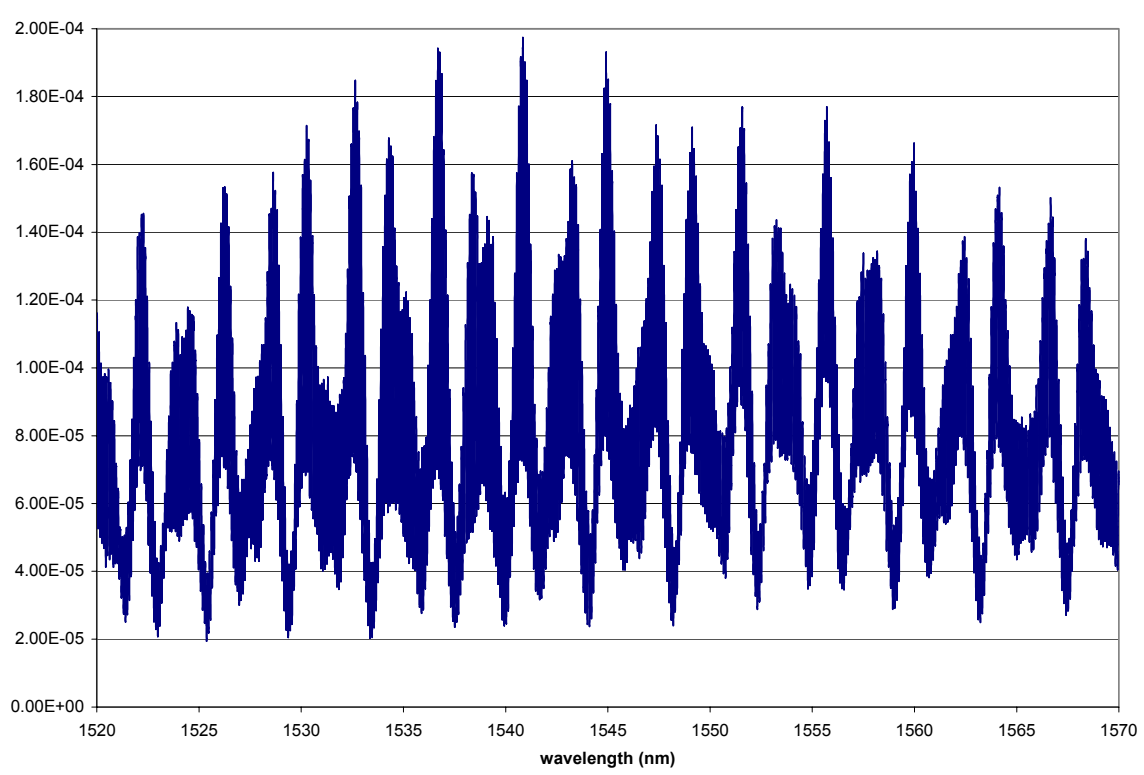

Figure 3-6. Output spectrum of IFPI sensor as measured on Micron Optics si720 CTS spectrometer.

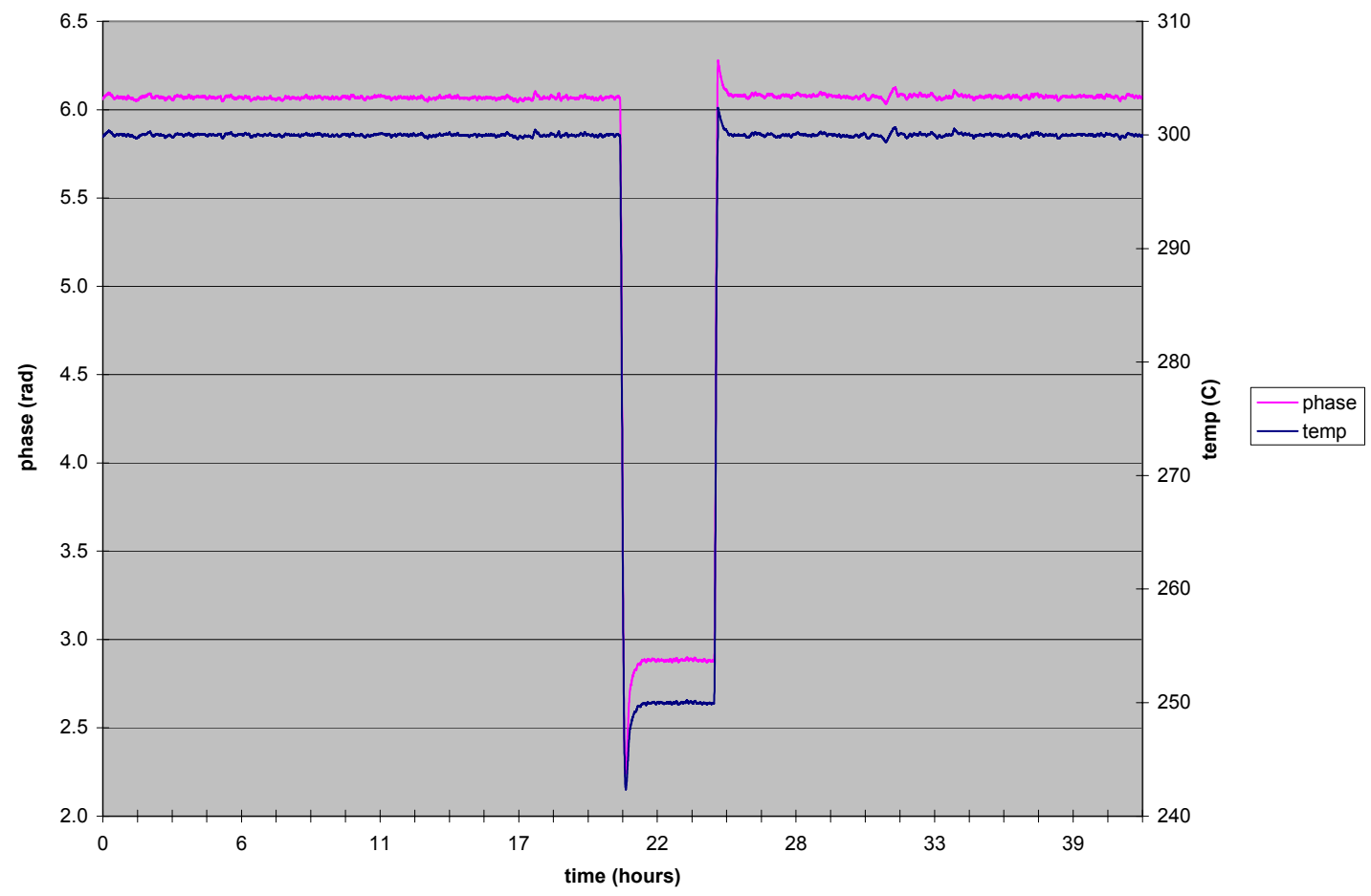

Figure 3-7. Output phase of IFPI sensor as temperature is switched from $298^{\circ} \mathrm{C}\left(568{ }^{\circ} \mathrm{F}\right)$ to $250{ }^{\circ} \mathrm{C}$ $\left(482{ }^{\circ} \mathrm{F}\right)$ and back. 


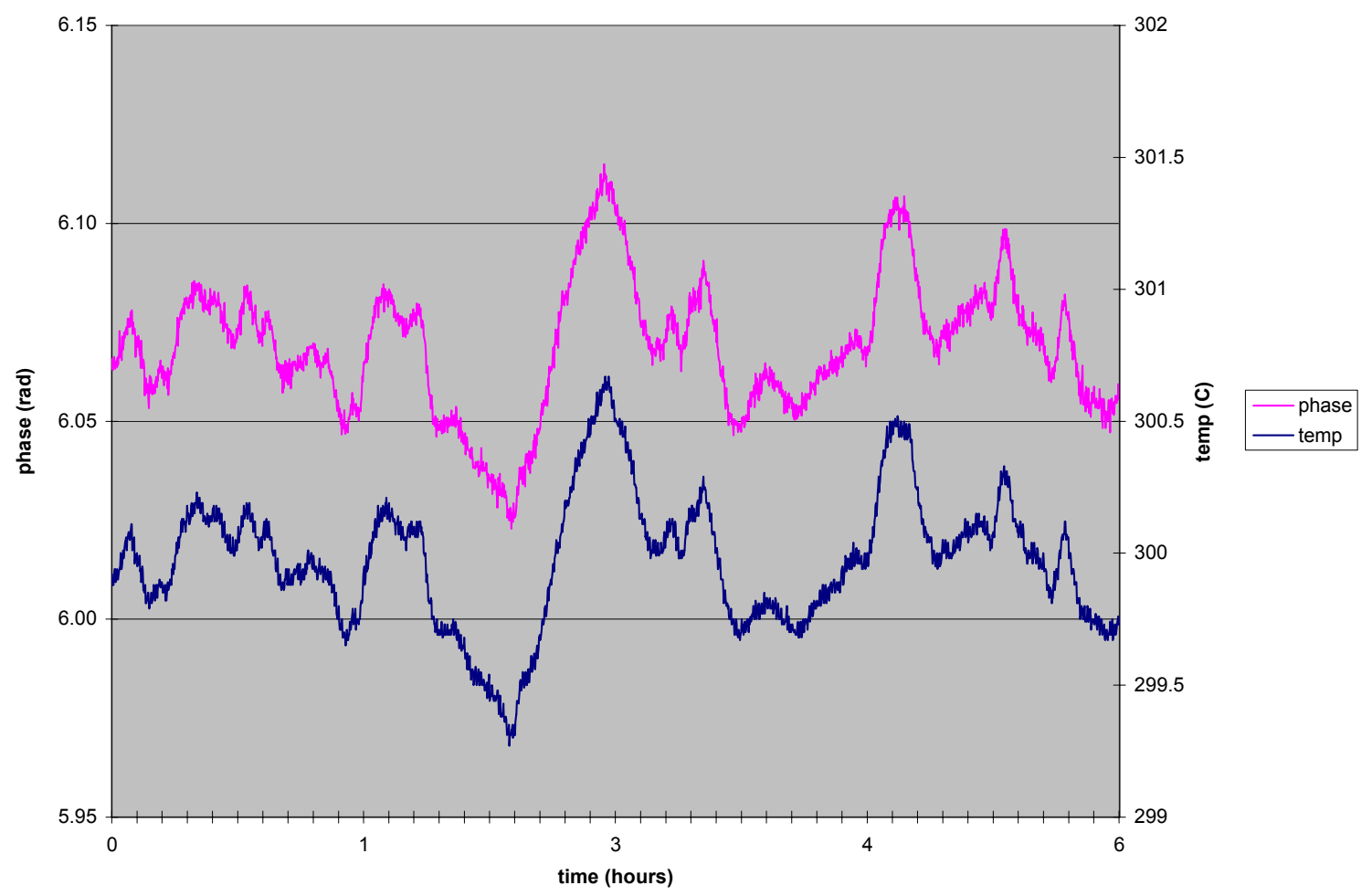

Figure 3-8. IFPI phase output and applied temperature variations over six-hour test.

\subsubsection{Grating-based Sensors}

Fiber gratings are formed when a periodic modulation of the refractive index is applied to the core of an optical fiber. If the spatial period of the modulation approximately equals half of the wavelength of the light propagating in the fiber, then the grating is classified as a fiber Bragg grating (FBG). For that structure, the FBG will reflect light with a wavelength exactly equal to the product of one half of the wavelength and the refractive index of the fiber core. In terms of fiber propagation analysis, a forward propagating LP01 mode is coupled (phase matched) by the FBG to a reverse propagating LP01 mode. Since the wavelength of the reflected light depends upon the spatial period of the grating, changes in that period due to longitudinal strain in the fiber results in a wavelength shift of the reflected light. Therefore, by designing a sensor so that the measurand induces a strain in a fiber with an FBG in the core, the magnitude of the measurand may be monitored by measuring the spectral shift of the reflected light.

If the spatial period of the refractive index modulation is on the order of 50 to $500 \mu \mathrm{m}$, then the grating is classified as a long period grating. In that case, the forward propagating LP01 mode is coupled by the LPG to modes that are guided by the fiber cladding. Since the evanescent electromagnetic field of the cladding modes extend slightly outside the fiber cladding, those modes interact with the media outside the fiber; in particular, the conditions for coupling from core modes to cladding modes depends upon the refractive index of the medium surrounding the fiber in the vicinity of the LPG. 
If the refractive index of the surrounding medium changes, then the conditions for coupling also change, and the wavelength of the light coupled into the cladding shifts. Therefore, LPGs are very effective for monitoring the refractive index of a liquid into which they are immersed. Similarly, the sensor can be engineered to monitor other measurands that can be made to result in a refractive index change of the surrounding medium. For example, LPG-based temperature sensor could be designed by surrounding the fiber with a medium whose refractive index is a known function of temperature.

In this program, FBG and LPG sensors were evaluated in laboratory tests. The LPG was a variant in which the refractive index modulation is created through application of pressure at equally spaced points along the fiber.

\subsubsection{Fiber Bragg Grating Sensors}

A Fiber Bragg Grating (FBG), written in silica fiber, was tested in order to determine its temperature resolution. The FBG was obtained from Lambda Instruments. At room temperature it demonstrates a moderate strength reflection with the center wavelength at approximately $1545 \mathrm{~nm}$, with a small side lobe on the short wavelength side. The fabricators indicated the grating to have a length of approximately $1 \mathrm{~mm}$, localized within a three inch region of the fiber.

The fiber containing the FBG was fusion spliced to a standard SMF-28 pigtail and interrogated with a Micron Optics si720 Component Test System (CTS) system. The baseline mechanism of interrogation is to acquire a full spectrum $(20,000$ points resolution over the wavelength span of 1520 to $1570 \mathrm{~nm}$ ) for post experimental analysis. The fiber was inserted into a furnace, taking care that no portion of the fiber within the furnace still possessed any polymeric coating. Once a room temperature baseline was acquired at $22^{\circ} \mathrm{C}\left(72^{\circ} \mathrm{F}\right)$, the furnace was heated. Over a period of approximately 3.5 hours the furnace was heated $495^{\circ} \mathrm{C}\left(923^{\circ} \mathrm{F}\right.$, the upper limit of this particular furnace). Scans were taken regularly ( 24 in total) during this temperature increase.

The data was analyzed in MATLAB ${ }^{\mathrm{TM}}$. After importing the data, the data was lightly smoothed to ensure accurate peak detection. A plot of the raw data (over a reduced portion of the spectrum, to highlight the salient characteristics) can be seen in Figure 3-9. The red circles indicated the locations of the peaks. In Figure 3-10, one can see the wavelength shift as a function of temperature applied to the grating. In Figure 3-11, the inverse data is illustrated, representing the temperature as a function of wavelength shift. Given the VL200 has a wavelength resolution of 1 picometer (pm) the temperature resolution of such a system is given by the slope of this curve. In this case, a temperature resolution of $0.17^{\circ} \mathrm{C}\left(0.30^{\circ} \mathrm{F}\right)$ is shown. This is likely to be improved by use of a higher quality (narrower bandwidth) FBG. 


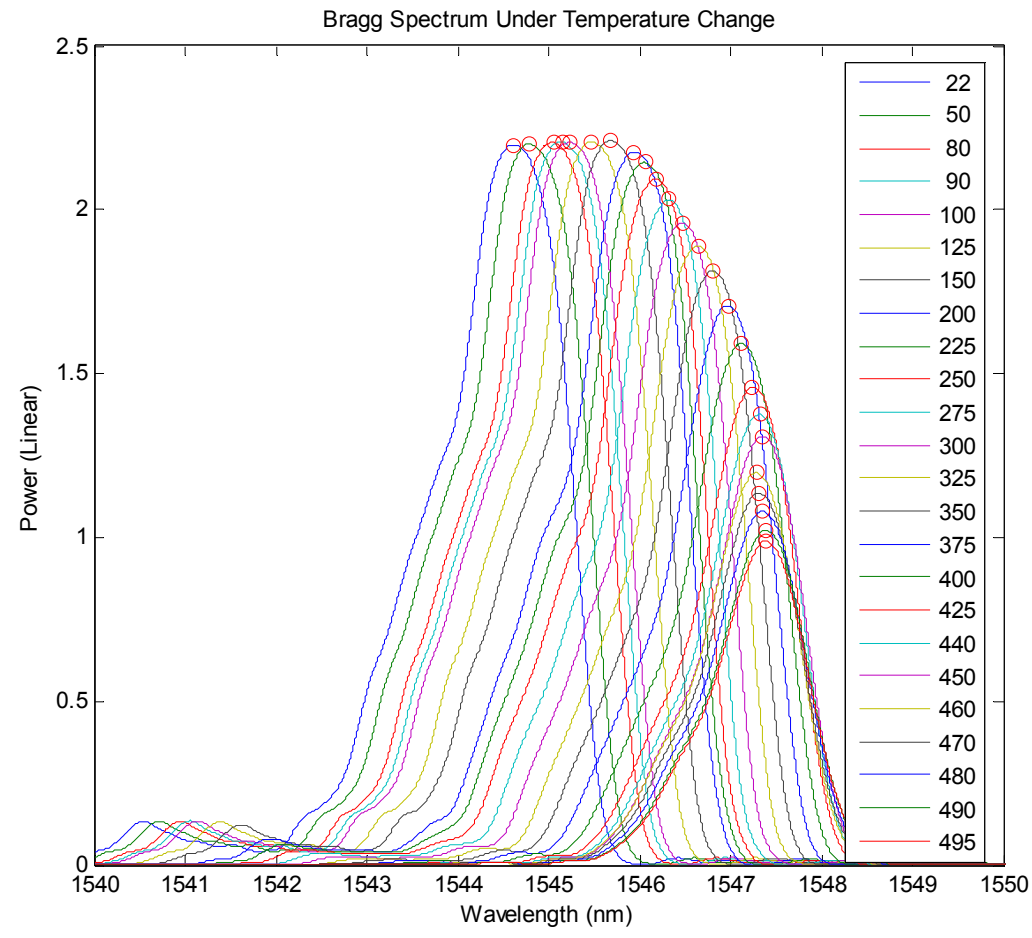

Figure 3-9. Spectral response of Bragg Grating

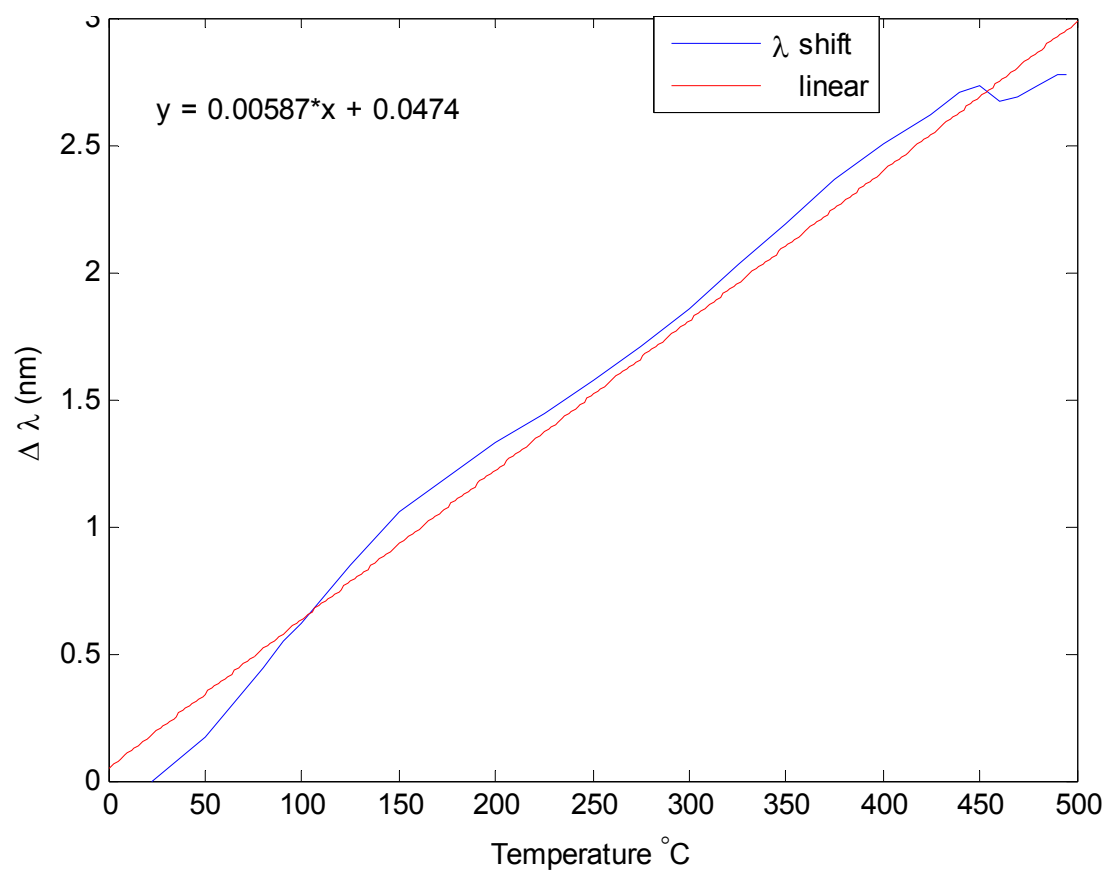

Figure 3-10: Wavelength shift as a function of temperature 
Temperature as a function of peak $\lambda$ shift

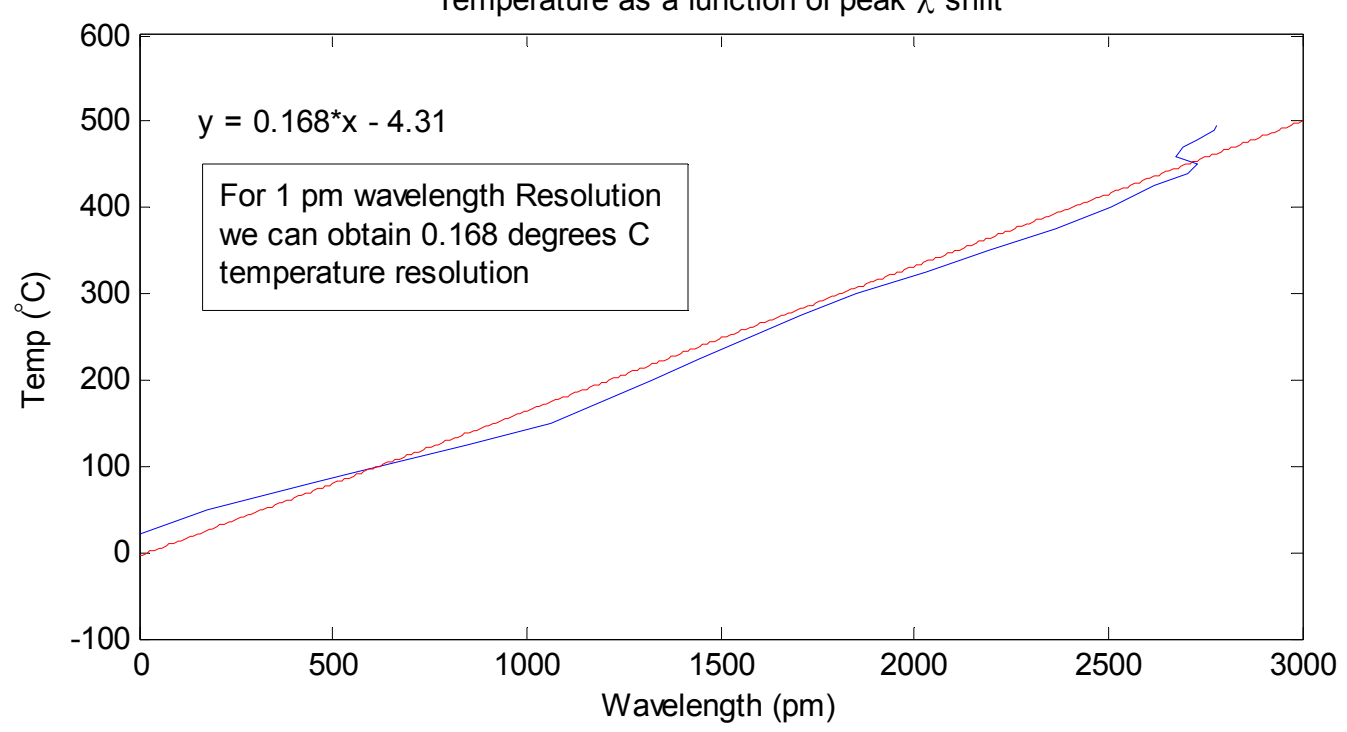

Figure 3-11: Temperature resolved as a function of wavelength

\subsubsection{Long Period Grating tests}

Most commonly, long period grating sensors are used to measure the change in refractive index of the medium surrounding the fiber. This is most useful for sensing chemical or biological elements in the immediate environment of the grating, but the coatings needed to accomplish this will generally not withstand the harsh conditions of the steam boiler or other power plant applications. However, a variation of the LPG sensor can be used to measure hydrostatic pressure. In this approach, periodic perturbations of the refractive index of the core of an optical fiber, induced by applied hydrostatic pressure, promotes coupling of guided electromagnetic modes to lossy cladding modes. As Figure 3-12 shows, a single mode fiber is positioned to rest on top of the plate with machined periodic grooves. A pressure plate is used to press the fiber against the groove plate. Through photoelasticity, the refractive index of the fiber will increase slightly in those sections were the fiber contacts the groove plate. The fiber refractive index is unchanged where the fiber rests above a groove in the bottom plate. By monitoring the transmission spectra of the fiber, losses in certain spectral bands can be correlated with the pressure amplitude. Here, the center wavelengths of the loss bands are dictated by the period of the machined grooves, and the amplitude of the loss peaks are related to the magnitude of the pressure applied to the plates.

The emphasis of the research presented here has been the demonstration of that correlation between pressure and loss. 


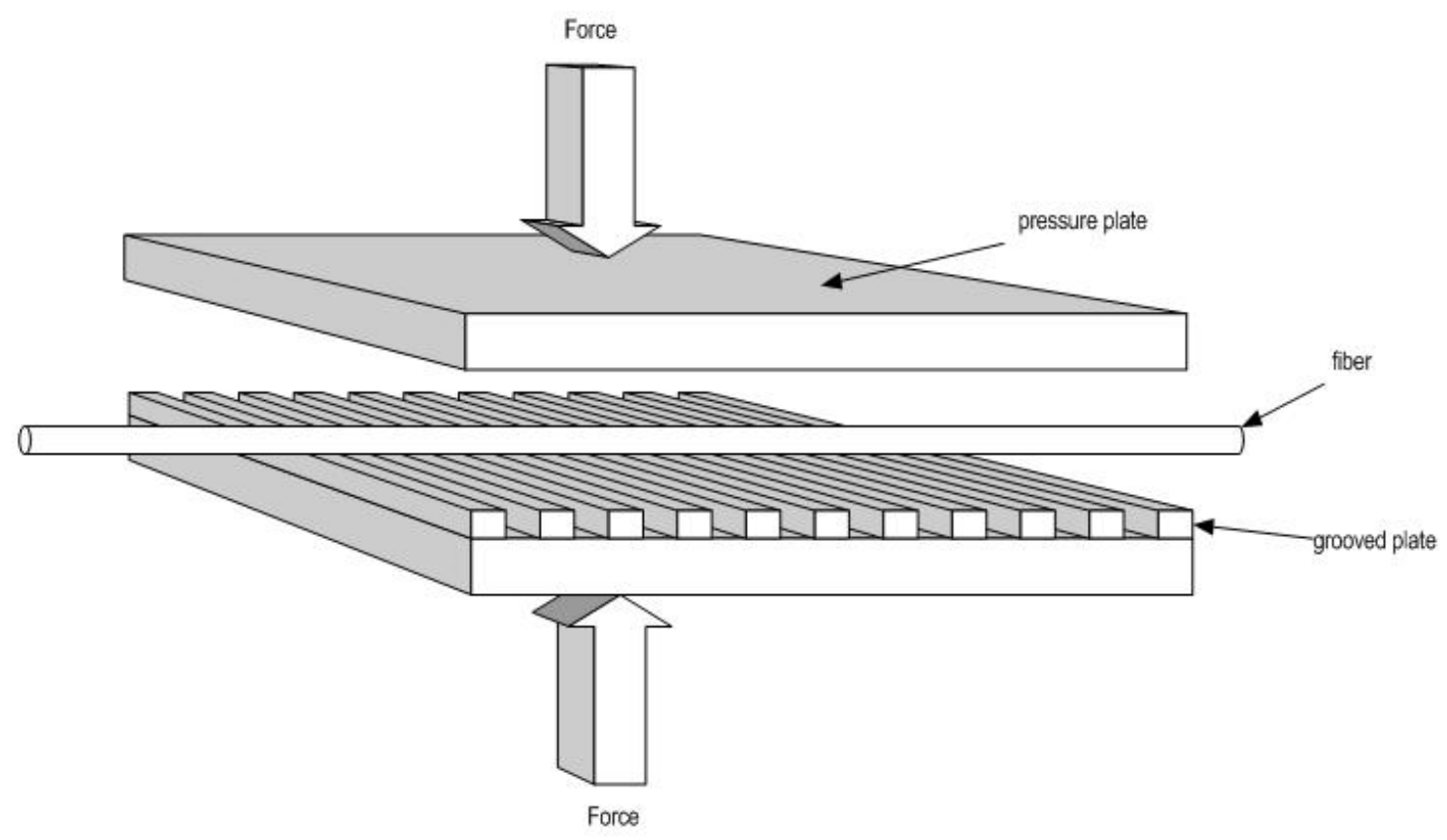

Figure 3-12. LPG sensor for measurement of pressure through periodic photoelasticity.

To demonstrate the correlation, a sensor was constructed using a 'sandwich' structure, in which a length of gold-coated optical fiber was sandwiched between two metal strips. One of the metal strips has periodic teeth machined in the surface, as shown in Figure 3-12. Metal strips with varying periodicities were tested. Room-temperature curing epoxy was used to fix the fiber to both ends of the metal strips. The whole sandwiched structure was covered tightly by a soft elastomer, with the ends sealed by epoxy.

The sensor was installed inside a pressure chamber with fiber optic pressure feedthroughs used to permit access of the fibers to the outside. A Pressure Systems Inc. pressure calibrator was used to apply hydrostatic pressure to the sensor. The working fluid was purified gaseous nitrogen. White light was injected into one end of the optical fiber, and an Anritsu optical spectrum analyzer was used to measure the transmission spectrum of the optical fiber (Figure 3-13).

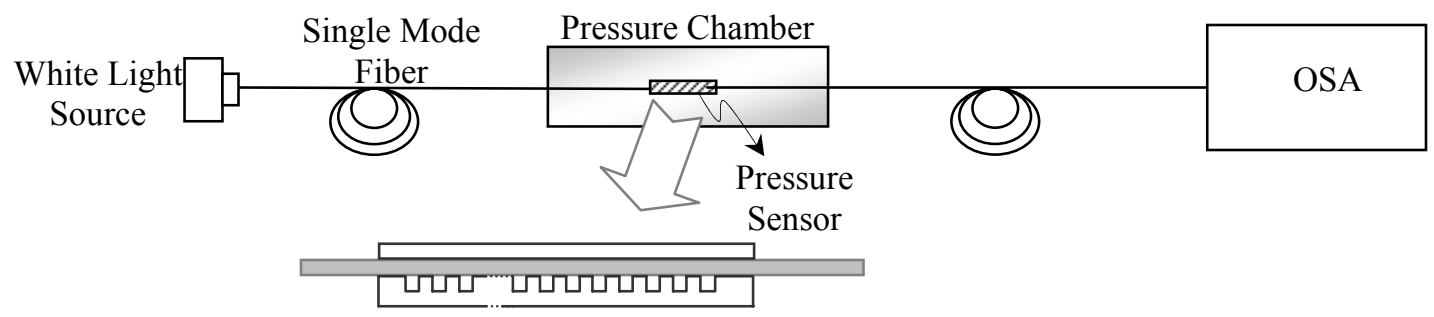

Figure 3-13. Experimental set-up to characterize pressure-induced LPG. 
In the first experiment, pressure was applied to the sensor at discrete values of 40, 50, 60, 70 and 80 psi. The corresponding spectra are shown in Figure 3-14.

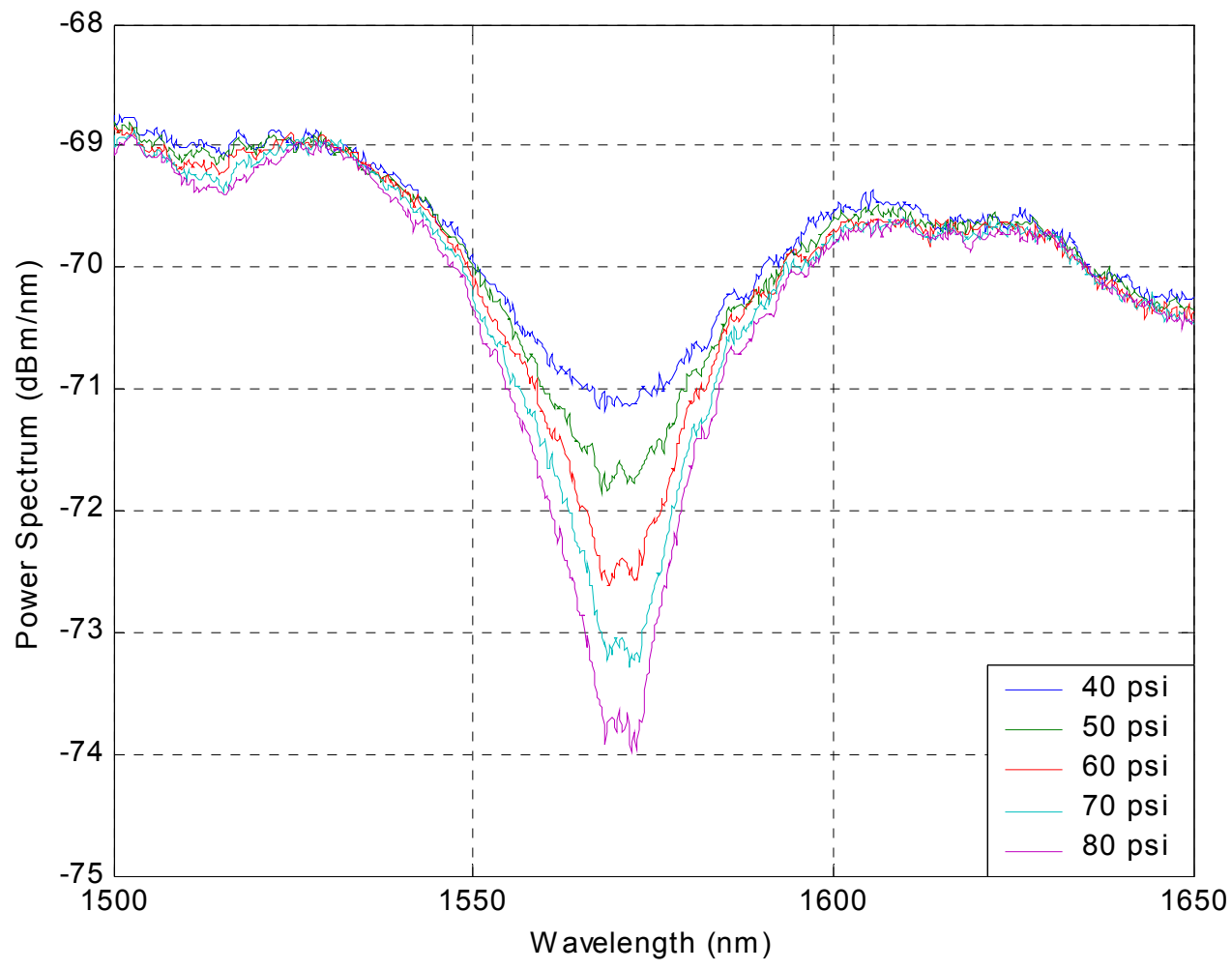

Figure 3-14. Transmission spectra of pressure-induced LPG sensor for discrete applied pressures.

In order to convert the change in transmission spectra to a single-valued parameter that could be correlated with pressure, the peak loss of the loss band in the transmission spectrum was calculated. Since the spectra are not smooth at the peaks, the average transmitted spectrum power $P_{l}(\mathrm{dBm})$ over the wavelength range from $1560 \mathrm{~nm}$ to $1580 \mathrm{~nm}$ was numerically calculated. As a baseline to determine the loss caused by mode coupling, the average power $P_{2}(\mathrm{dBm})$ over the wavelength range from $1515 \mathrm{~nm}$ to $1535 \mathrm{~nm}$, which is far removed from the peak wavelength, was calculated. Then the loss can be determined as

$$
\text { Loss }(\mathrm{dB})=P_{2}(\mathrm{dBm})-P_{1}(\mathrm{dBm})
$$

Equation 1 
The results are shown in Figure 3-15, which uses a logarithmic plot to express the result, and Figure 3-16, which employs a linear scale. Alternate approaches to determine peak loss in the spectra could employ fitting polynomials to the data, and determining the local maximum of the polynomial.

The stated loss accuracy of the Anritsu spectrometer is $\pm 0.1 \mathrm{~dB}$. From the slope of Figure 3-16, this implies a minimum resolvable pressure of $4.4 \mathrm{psi}$ for this sensor, using the Anritsu OSA to interrogate the sensor.

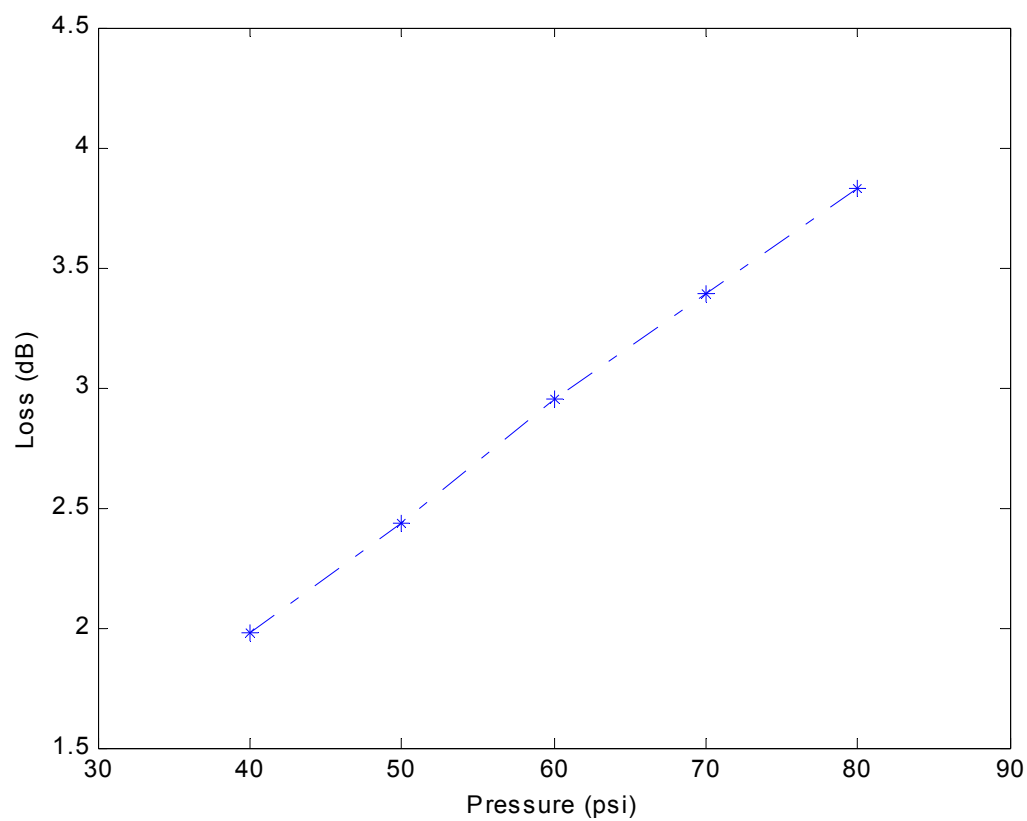

Figure 3-15. Loss vs. pressure (logarithmic scale)

The repeatability of the sensor was tested by measuring the transmission spectrum at 80 psi three times. The results are plotted in Figure 3-17. The tests show a decrease in the peak loss for each successive test. The reason for this behavior is not clear at this time, but may be related to viscoelastic (time-dependent) relaxation in the epoxy used to fix the fiber to the plates. The results of the limited experiments presented here confirm that periodic variations of the refractive index of an optical fiber core induced by photoelasticity can be used to relate changes in the transmission spectrum of the optical fiber to pressure applied to the structure encapsulating the fiber. 


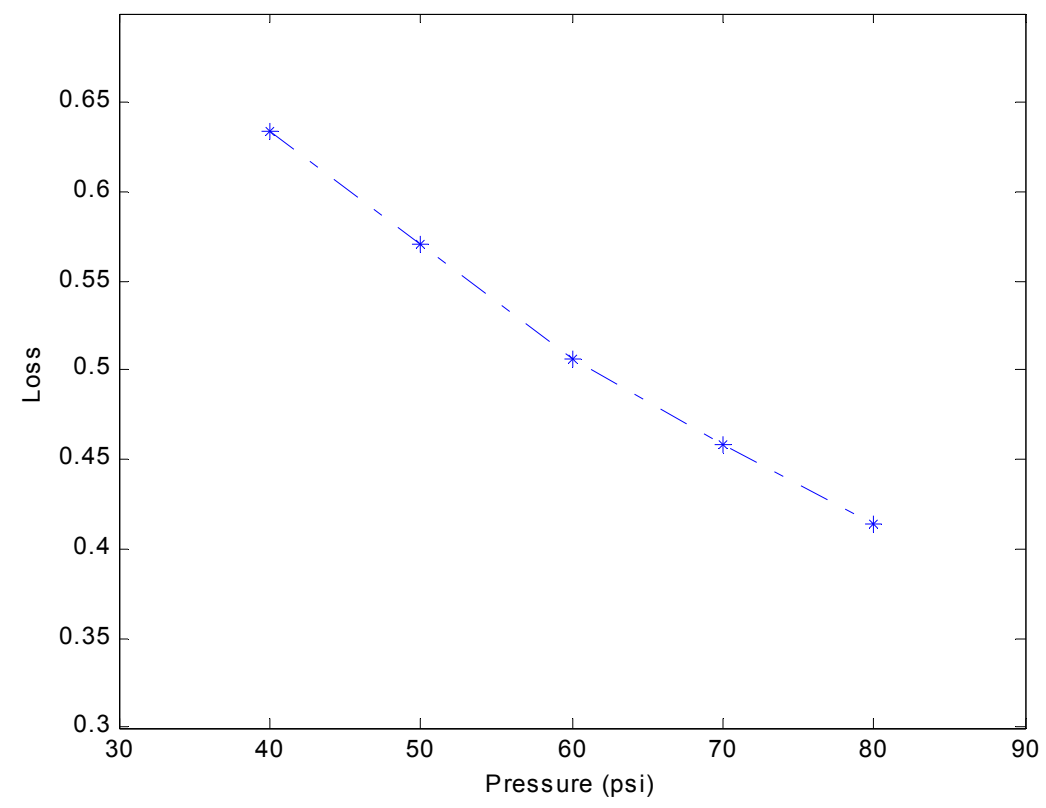

Figure 3-16. Pressure vs. normalized transmitted power (linear scale)

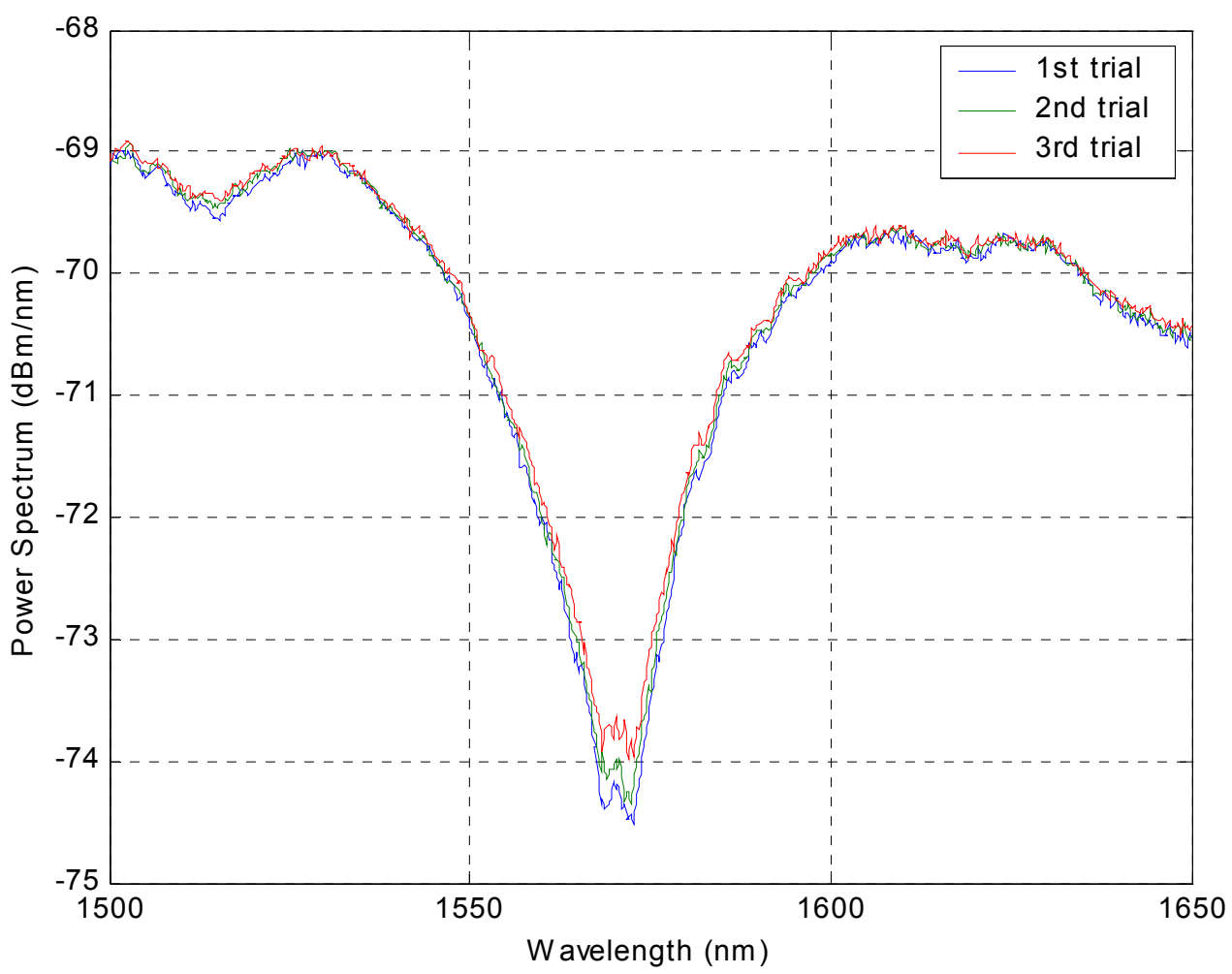

Figure 3-17. Transmission spectrum for 80 psi repeated three times. 


\subsubsection{Microbend Sensors}

Microbend displacement sensors are based on the optical loss in an optical fiber induced by small bends in the fiber by some measurand. When a fiber is subjected to microbends (small periodic deformations), light rays propagating in the fiber can exceed the critical angle required for total internal reflection; in that case, the array is no longer guided and is coupled into the cladding, word is usually absorbed by the lossy polymer jacket. Intensity based sensors generally have lower resolution and dynamic range than interferometric based sensors due to their sensitivity to noise. However, they do not require complex signal processing techniques and are much less expensive. Microbend sensors have been configured for the measurement of many different parameters including strain, temperature and pressure.

Under this Phase I program, tests were conducted to estimate the resolution of a microbend displacement sensor based on single mode fiber (core size 9.5um).

\subsubsection{The sensor configuration}

One type of microbend sensor uses multimode fibers with core size of 60 um or larger, which support the propagation of several thousand electromagnetic modes of light. In the process of microbending, the number of guided modes and the power carried by them changes. In our approach, we used the single mode fiber as the sensor element and a distributed feedback (DFB) laser diode as the source of light.

The general configuration of the sensor system is presented in Figure 3-18. The output from a laser diode with peak wavelength of $1545 \mathrm{~nm}$ is coupled into a single mode fiber. An attenuated was used to reduce the intensity of the laser output, so that the photodetector is not saturated. After attenuation, the laser light propagates through the fiber under a mechanical deformer. The deformer, used to microbend the optical fiber, consists of a pair of metal plates, each with periodic flat ridges on one side (see Figure $3-19)$. The period of the ridges is $1.6 \mathrm{~mm}$, and the total length of the deformer is $100 \mathrm{~mm}$.

The output of the fiber (after the deformer) is coupled to a photodetector, which converts the optical signal to electrical signal. A PCI-DAS6070 data acquisition card, with the digitization rate of $1000 \mathrm{kSamples} / \mathrm{s}$, was used to digitize the electrical signal. Graphical interface software written in Borland $\mathrm{C}++$ allowed us to observe the signal and calculate its standard deviation in real time.

As shown in Figure 3-19, the fiber, in its plastic jacket, passes between the two deformer plates. A static displacement of the upper plate was induced by manually adjusting a screw contacting the top of the plate; this produced quasi-square distortions of the fiber. The lower plate of the deformer was fixed in all tests. 


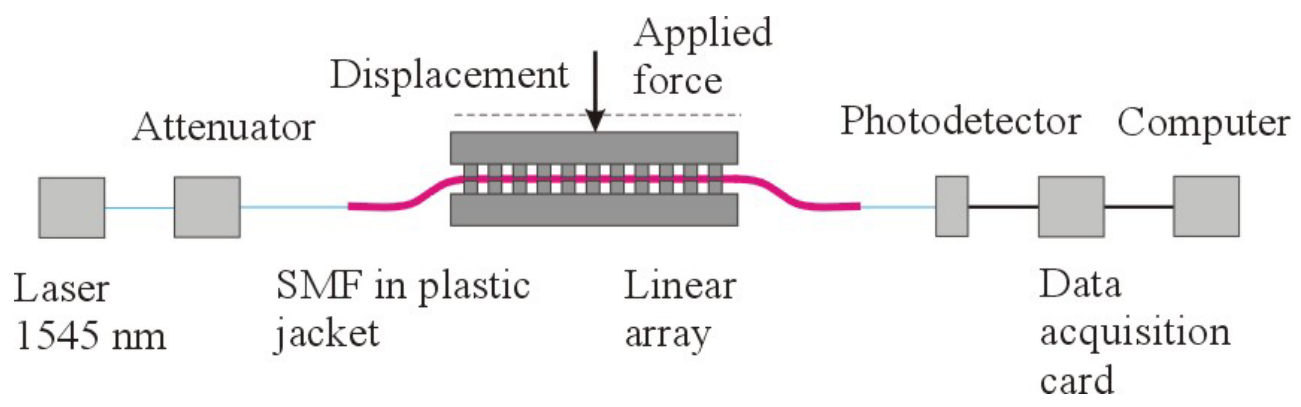

Figure 3-18. Schematic for the microbend sensor tests

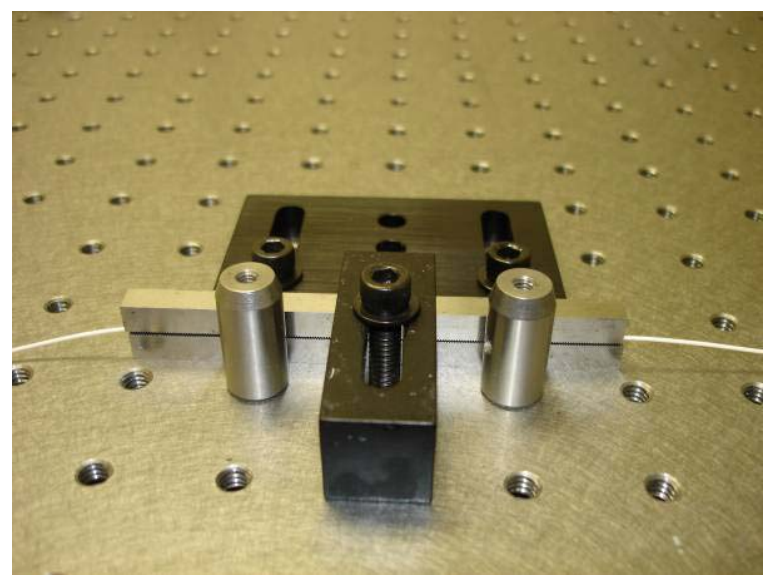

(a)

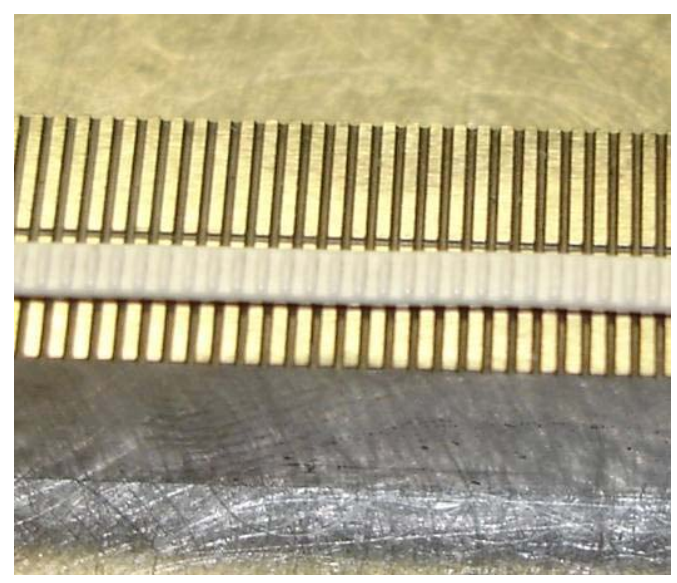

(b)

Figure 3-19. Microbend sensor design (a- mechanical deformer, b- linear deformer and optical fiber in plastic jacket)

The zero displacement position was defined as the upper plate position at which output light power started to change. Due to the viscoelastic mechanical behavior of the plastic coating, some drift was observed at the beginning of the test. However, the repeatability of the sensor was significantly improved after periodically applying high and low pressure to fiber with the objective of reducing the plastic jacket deformation memory.

\subsubsection{Microbend sensor dynamic range and resolution}

The microbend sensor dynamic range was limited by the following reasons: for small fiber deformations, the optical losses were small and the sensor had low sensitivity. Large deformations saturated the attenuation in the fiber. In the test, the deformer displacement was increased in steps of $16.1 \mathrm{um}$. For each displacement step, the data acquisition system collected 250 samples from the photodetector in a time interval of $10 \mathrm{~s}$. Then a mean value of the signal was calculated according to the formula 


$$
<x>=\frac{1}{N} \sum_{i=1}^{N} x_{i}
$$

where $x_{i}$ is the $\mathrm{i}^{\text {th }}$ measurements of $x$, the photodetector output.

The standard deviation $\sigma$ was calculated according to

$$
\sigma=\sqrt{\frac{1}{N-1} \sum_{i=1}^{N}\left(x_{i}-<x>\right)^{2}} .
$$

Equation 3

The mean value of the optical power transmitted through fiber as a function of deformer displacement is shown in Figure 3-20. In general, the curve is nonlinear for the range of displacement from $0 \mu \mathrm{m}$ to $145 \mu \mathrm{m}$. The linear part of the curve is limited from $70 \mu \mathrm{m}$ to $95 \mu \mathrm{m}$.

The sensor displacement resolution $\eta$ was calculated by formula

$$
\eta=\Delta_{\text {displacement }}\left(\frac{2 \sigma}{\Delta_{\text {power }}}\right),
$$

\section{Equation 4}

the $\Delta_{\text {displacement }}=16.1 \mathrm{um}$ is the change in displacement, and $\Delta_{\text {power }}=P_{1}-P_{2}$ is the difference in optical power corresponding to the displacement.

Figure 3-21 shows the dependence of the sensor resolution as a function of the deformer displacement. According to the curve, the sensor resolution is variable and depends upon displacement. The minimum resolution of $\sim 0.05$ um corresponds to the interval of displacement from 70um to 95um. On Figure 3-20, the same interval corresponded to the linear part of the curve. Beyond the linear range of the curve, the sensor resolution increases rapidly and reaches a value $0.6 \mathrm{um}$. 


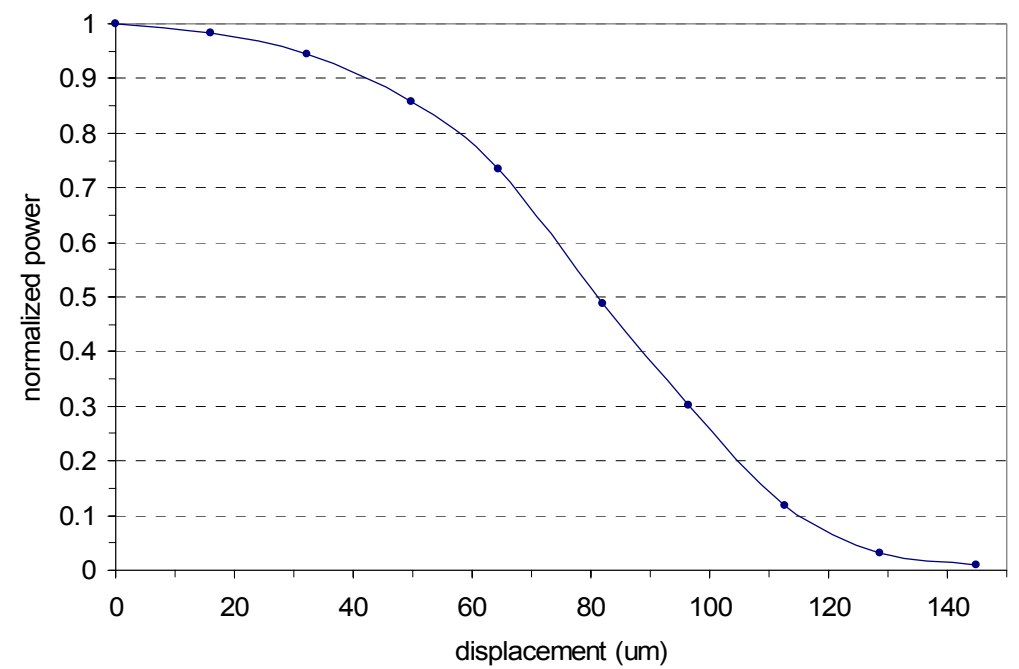

Figure 3-20. Measured attenuation of the optical power as a function of the displacement.

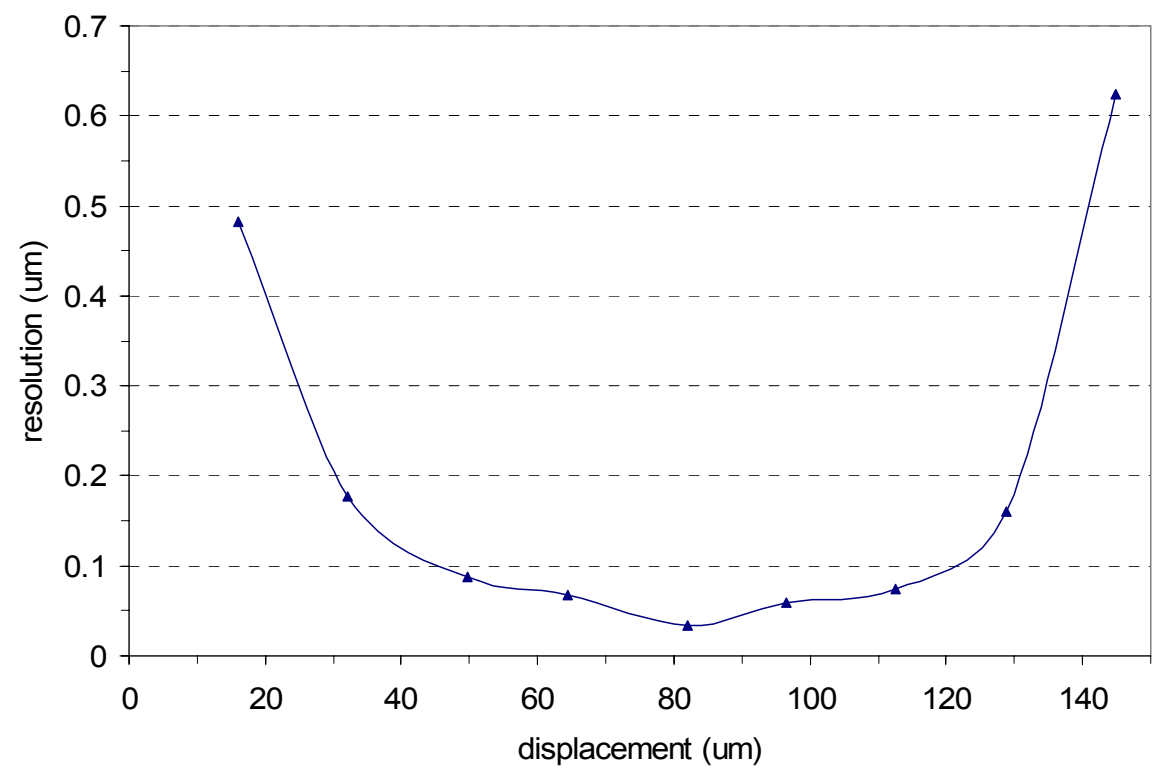

Figure 3-21. Displacement resolution of the microbend sensor

\subsection{Sapphire Temperature Sensor Development}

During the program, the design, fabrication, and testing of a sapphire sensor for measurement of temperature was researched. The design investigated was based on a Fabry-Perot interferometer originally developed at the Virginia Tech Center for Photonics Technology. Prime Research has licensed the intellectual property rights for this design from Virginia Tech. The specific sensor configuration uses a polished 
sapphire wafer as the temperature-sensitive element. The wafer is attached to a sapphire fiber (clad or unclad), and interrogated as a Fabry-Perot sensor. Changes in the temperature of the wafer cause a change in the optical path length of the Fabry-Perot cavity, which can be related to the change in temperature through the known coefficient of thermal expansion. The high degree of parallelism of the two sides of the wafer (and thus the Fabry-Perot cavity) permits the achievement of reasonable fringe visibility, even with multimode fibers.

Due to their large diameters and large numerical apertures, unclad single-crystal sapphire fibers are highly multimoded. The fringe visibility of Fabry-Perot (FP) interferometers constructed using multimode fibers is generally poor. While fringe visibility can be rigorously defined, in essence it expresses the depth of intensity modulation of the interferometer output relative to the average intensity output. The fringe visibility is highly sensitive to the flatness, the smoothness, and especially the parallelism of the two surfaces forming the FP cavity. It was reported that the fringe visibility can be significantly reduced by an angle of the order of $10^{-2}$ degree between the two faces forming the FP cavity. ${ }^{12}$ This strong dependence of fringe visibility in a multimode fiber on alignment angle has also been analyzed by Han, et al. ${ }^{13}$ The very stringent requirement of end-face alignment poses a great challenge in the sensor design of FP interferometers based on multimode fibers, including sapphire fibers.

In most of the high-temperature interferometric sensors described in published literature, an air gap between two polished sapphire fiber ends, or between a polished fiber end and some kind of reflector, is constructed as the FP cavity. As mentioned in the previous paragraph, the precise parallel alignment between the two reflective surfaces is very critical in achieving fringes with acceptable visibility. On the other hand, this kind of precise alignment is most often very difficult to achieve. Therefore, a novel approach using a sapphire wafer instead of an air gap as the FP cavity was adopted. The sensor configuration is shown in Figure 3-22 below. $^{14}$

A sapphire wafer is fixed in front of an alumina tube with a very small inner diameter by a high-temperature alumina adhesive. A sapphire fiber is inserted into the tube until its polished end reaches the wafer. In this configuration, the parallelism requirement is automatically satisfied by the very high degree of parallelism between the two surfaces of sapphire wafer, which can be readily achieved by the current workmanship of the wafer lapping-polishing industry. This approach to sapphire interferometer fabrication was originally demonstrated by the Center of Photonic Technology (CPT) at Virginia Tech. Negotiations to license this technology are currently underway.

\footnotetext{
${ }^{12}$ F. Perennes, P.C. Beard, and T.N. Mills, Appl. Opt. 38, 7026 (1999).

${ }^{13}$ Ming Han and Anbo Wang, Applied Optics, 43, 4659 (2004).

${ }^{14}$ Yizheng Zhu, Zhengyu Huang, Ming Han, Fabin Shen, Gary Pickrell and Anbo Wang, Proc. of SPIE vol. 5590, p. 19 .
} 


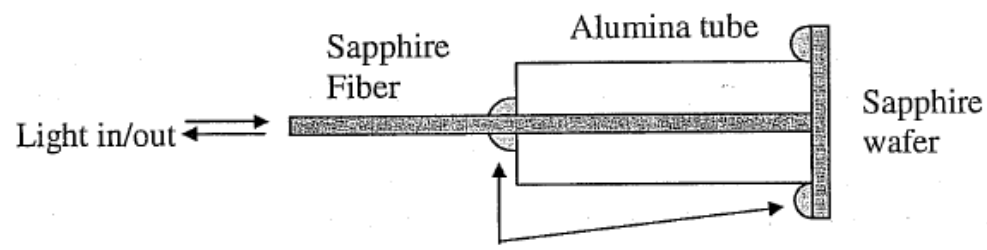

Alumina adhesive

Figure 3-22: Sensor configuration using a sapphire wafer as the FP cavity [2]

\subsection{Prototype sensor assembly process}

The prototype high temperature sapphire sensors were fabricated using the following steps. Other options to couple the silica fiber to the sapphire fiber, such as fusion splicing, are currently under review. The current sensor head configuration and the technique used to affix the sapphire wafer to the end of the ceramic tube are also under review. The procedures described here use an unclad $75-\mu \mathrm{m}$ sapphire fiber as the high temperature waveguide.

1. The first step was to prepare a polished silica fiber end to be coupled to the sapphire fiber. The end of a silica fiber was inserted into a ceramic ferrule and 5minute epoxy was used to fix the fiber end inside the ferrule. Then the ferrule with the fiber inside was polished using polishing papers with abrasive sizes down to $0.5 \mu \mathrm{m}$. After completing this polishing step, the ferrule was fixed inside a ceramic tube using 5-mninute epoxy. The configuration of the silica fiber end after preparation is shown in Figure 3-23 below. (It is to be noted that the 5minute epoxy at the ferrule tip shown in this Figure must be removed by polishing before coupling to the sapphire fiber).

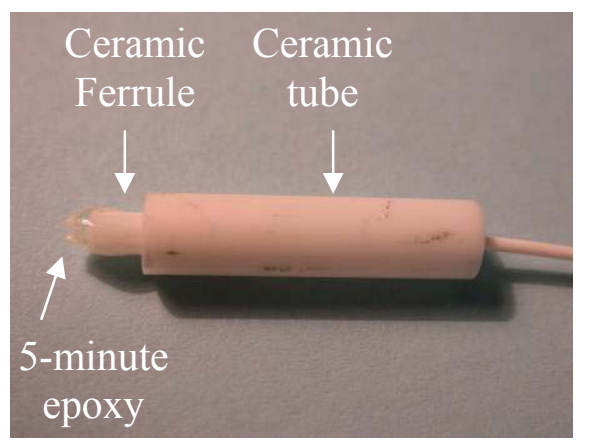

Figure 3-23: The preparation of the silica fiber for the coupling to sapphire fiber.

2. This step involves the preparation of the sapphire fiber end to be coupled to the silica fiber end. A 75- $\mu \mathrm{m}$ sapphire fiber (Micromaterials, Inc.) of about $10.8 \mathrm{~cm}$ long is inserted into a $2.5 \mathrm{~cm}$ long double-bore ceramic tube (McDaniel Advanced Ceramic, $0.13 \mathrm{~mm}$ bore diameter, and $0.79 \mathrm{~mm}$ outer tube diameter) until the end of sapphire fiber protrudes out of one end of the ceramic tube by about $1 \mathrm{~mm}$. Next, 5-minute epoxy is applied at the protruded sapphire fiber at the end of 
ceramic tube. After the epoxy is cured, the end is polished using polishing papers with abrasive sizes down to $0.5 \mu \mathrm{m}$.

3. The polished end of the double-bore ceramic tube prepared in Step 2 above and the silica fiber end prepared in Step 1 are coupled together using two micropositioners stages as shown in Figure 3-24 below. A power meter located at the other end of sapphire (not shown in this figure) fiber was used to monitor the power level and maximize power throughput during the coupling procedures. A very small drop of index matching gel was applied at the coupling junction. The silica fiber end could also be tilted to facilitate the coupling. At the moment the power throughput is maximized, UV-curable optical adhesive was applied surrounding the junction connecting the sapphire fiber end and the silica fiber end to fix the connection in place and it was cured using a UV lamp.

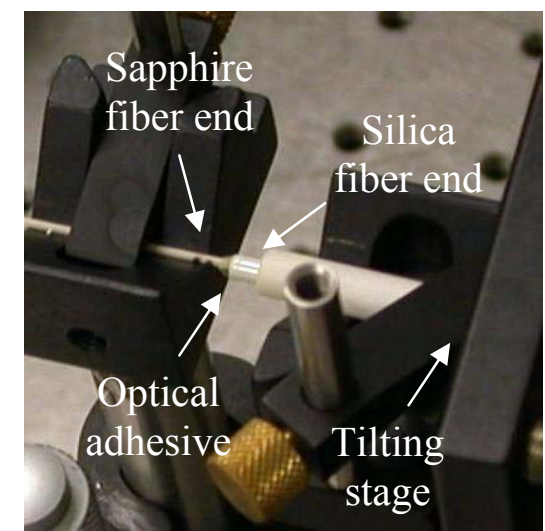

Figure 3-24: the coupling between silica and sapphire fibers.

4. At this stage of fabrication, we have a piece of $10.8 \mathrm{~cm}$ long sapphire fiber coupled to the silica fiber through a section of double-bore ceramic tube approximately $2.5 \mathrm{~cm}$ long. There is about $8.3 \mathrm{~cm}$ of bare sapphire fiber extending out from the $2.5 \mathrm{~cm}$ long double-bore ceramic tube. The sapphire fiber is then inserted into another piece of double-bore ceramic tube about $7.2 \mathrm{com}$ long, as shown in Figure 3-25 below, until the polished end of the sapphire fiber is about $1 \mathrm{~cm}$ away from the end of the double-bore tube. Now it is ready to affix the sapphire wafer to the end of the double-bore ceramic tube.

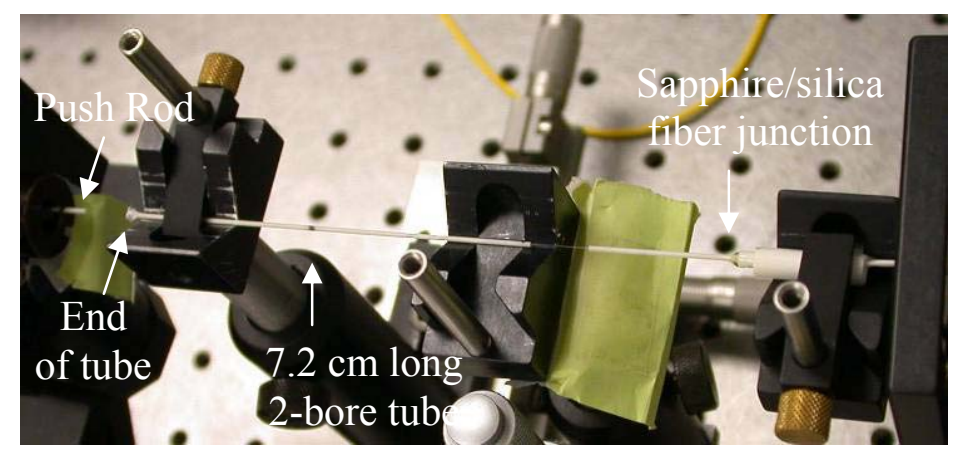

Figure 3-25: sapphire wafer assembly configuration 
5. A small piece of sapphire wafer is pushed against the end of the double-bore ceramic tube using a push rod. High-temperature alumina adhesive is applied surrounding the area where the wafer and the double-bore tube are in contact. The wafer configuration after the push rod is removed is shown in Figure 3-26 below.

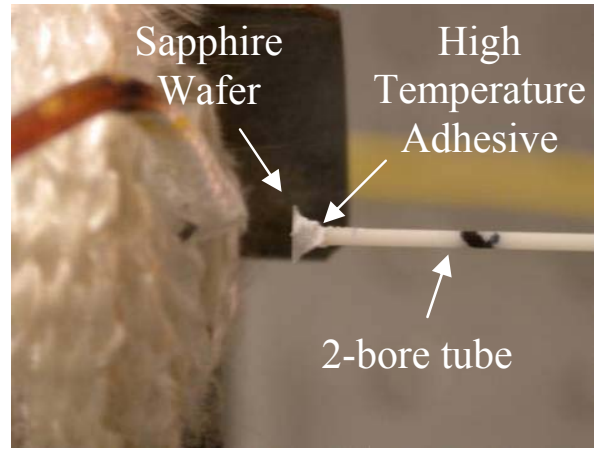

Figure 3-26: Sensor tip configuration

6. After the sapphire wafer is fixed to the end of the double-bore tube, the polished end of sapphire fiber, which is currently located about $1 \mathrm{~cm}$ away from the wafer, is pushed forward until it reaches the wafer. At this moment, there should be interference fringes, produced by the wafer, present on the optical spectrum analyzer.

7. Then the sapphire fiber inside the $7.2 \mathrm{~cm}$ long double-bore ceramic tube is fixed at the end of the ceramic tube opposite to the wafer, as shown in Figure 3-27, by a small drop of alumina adhesive. The wafer end of the double-bore ceramic rod is placed inside a coil heater. It is ready for temperature test now.

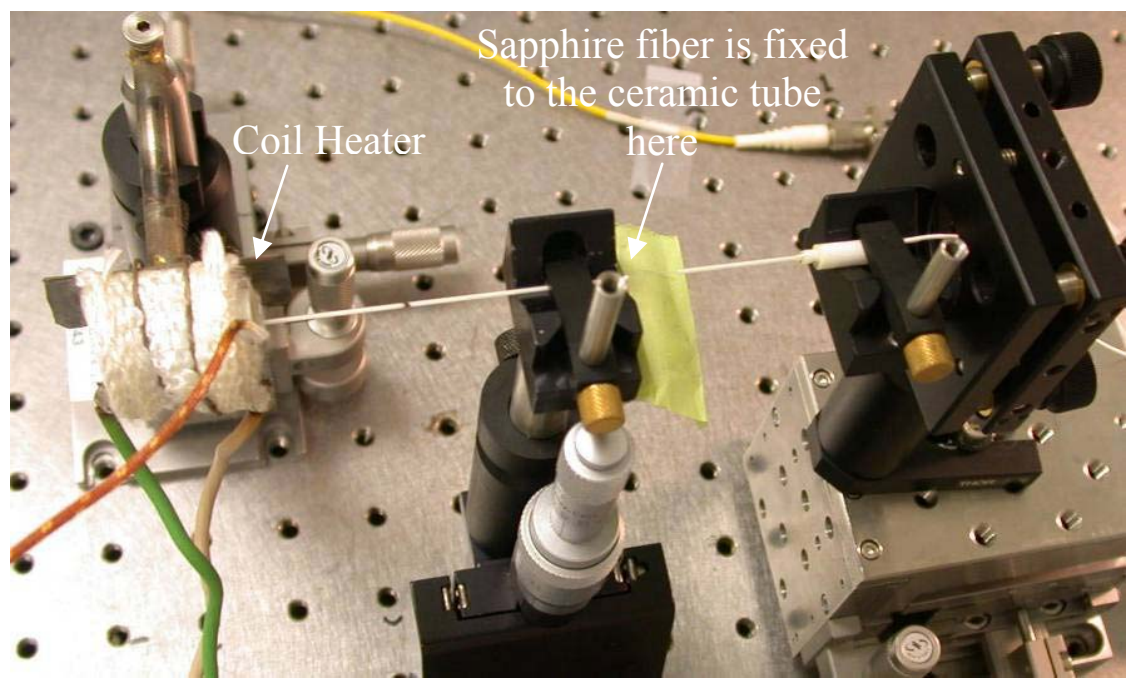

Figure 3-27: Sensor setup ready for temperature test. 


\subsection{Test and evaluation of temperature sensor}

The experimental setup for the testing is shown in Figure 3-28 below. The broadband light at $1550 \mathrm{~nm}$ from a Denselight superluminescent light emitting diode (SLED) with a single-mode fiber pigtail is fed into a single-mode $3 \mathrm{~dB} 2 \times 2$ coupler. One of the two arms of the coupler opposite to the light source end is immersed in index matching gel to minimize back reflection. The other arm of the coupler is connected to a multimode patch cord before connecting to the sensor. The interference fringes are monitored using an Anritsu optical spectrum analyzer (OSA). This OSA is designed to be used specifically with a single mode fiber as the input. It was observed that with a multimode fiber input the power level was greatly reduced.

According to the original design, the ideal testing system should be an all multimode system consisting of a light source with multimode fiber output, a 2 by 2 coupler made of multimode fibers, a spectrometer or monitoring system compatible with multimode fiber input, and a multimode fiber patch cord. The construction of an all multimode testing system was completed later in the program. The major advantage of using an all multimode system is the removal of modal noises originating from the implementation of both singlemode and multimode fibers in the experimental setup.

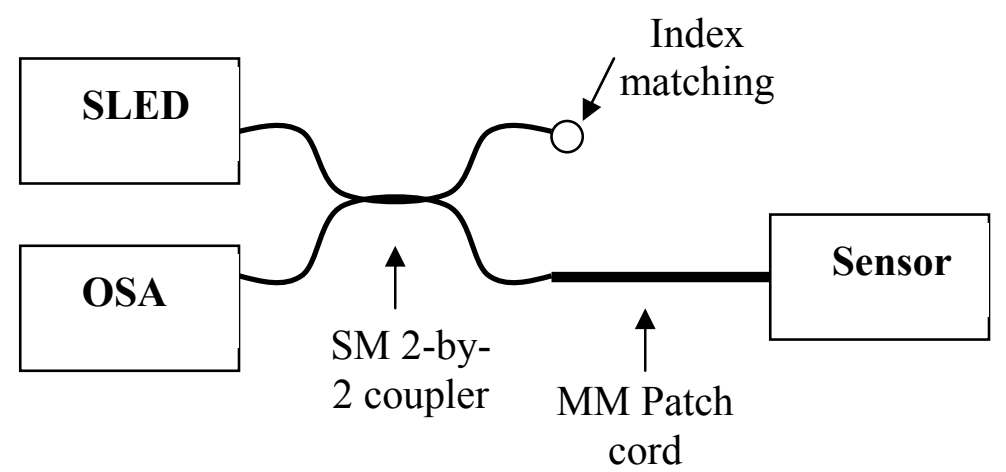

Figure 3-28: Experimental setup for evaluating temperature sensors

\subsubsection{Room temperature testing using a clad sapphire fiber as the waveguide}

This section describes the evaluation of a Fabry-Perot interferometric sensor using a clad sapphire fiber as the waveguide with a 0.005 inch thick (nominal thickness) sapphire wafer as the Fabry-Perot cavity at room temperature. The wafer had a measured thickness of $132 \mu \mathrm{m}$, and has a random crystallographic orientation (Meller Optics, Providence, RI). The clad fiber was fabricated using the procedures recommended by the Taguchi experimentation described in Section 4.1.15. A five inch long clad fiber was packaged in a ceramic tube with stainless steel ferrules at both ends. Both ends of the fiber package were polished using polishing papers with abrasive sizes down to $0.5 \mu \mathrm{m}$.

The optical bench setup for the testing is shown in Figure 3-29 (a) below. The clad fiber in the package was coupled to the silica fiber using positioning stages while a hand-held power meter (not shown in the figure) was used to maximize the power throughput at the 
other end of the clad sapphire fiber package. No index matching gel was applied at the coupling junction in this case. The power meter was then replaced with a push rod in order to affix the wafer to the end of clad fiber package as shown in Figure 3-29 (b). The interference patterns after the wafer was attached are shown in Figure 3-30 (a) and (b) below. The background with clad sapphire fiber in place but without the sapphire wafer is shown as the spectrum " $A$ " in Figure 3-30 (a). It was observed that the shape of the background spectrum " $A$ " could be easily altered by just moving the multimode fiber patch cord (see Figure 3-29 (a)) connected to the clad fiber. As the testing set-up consisted of both singlemode and multimode fibers, this change in output spectrum upon movement of the multimode fiber was attributed to modal noise, since the background spectrum " $A$ " was sensitive to the shape of the multimode fiber patch cord. Therefore, steps were taken to ensure that the multimode fiber patch cord was not disturbed at any times during the experiments, in order to achieve a stable and constant background. The spectrum after the attachment of the sapphire wafer is shown as spectrum " $\mathrm{B}$ " in Figure 3-30 (a). The net spectrum obtained by subtracting spectrum " $A$ " from spectrum " $B$ " in Figure 3-30 (a) is shown in Figure 3-30 (b). The wafer thickness, $d$, can be calculated using the relation

$$
n d=\frac{\lambda_{1} \times \lambda_{2}}{2\left(\lambda_{1}-\lambda_{2}\right)}
$$

Equation 5

where $n$ is the index of refraction of sapphire, which is 1.76 , and $\lambda_{1}$ and $\lambda_{2}$ are two consecutive peak wavelengths as indicated by the two arrows shown in Figure 3-30 (b). This expression, the product $n d$ is the optical path length attributed to the sapphire wafer thickness. The physical thickness of the wafer $d$ calculated using the above equation and the two peaks in Figure 3-30 (b), i.e., $1521.8 \mathrm{~nm}$ and $1526.8 \mathrm{~nm}$, is $132.0 \mu \mathrm{m}$, exactly the same as that of the measured wafer thickness.

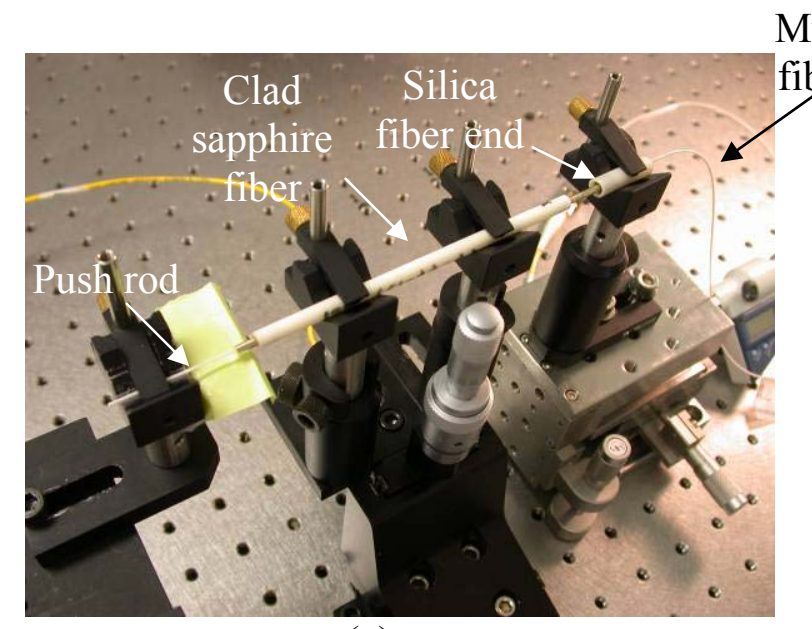

(a)

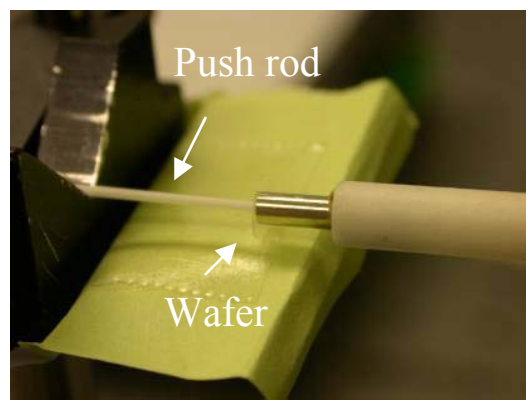

(b)

Figure 3-29: (a) Optical bench setup for the testing a clad sapphire fiber, and (b) the close up at the sapphire wafer (the wafer is sandwiched between the push rod and a stainless steel ferrule in this photo). 


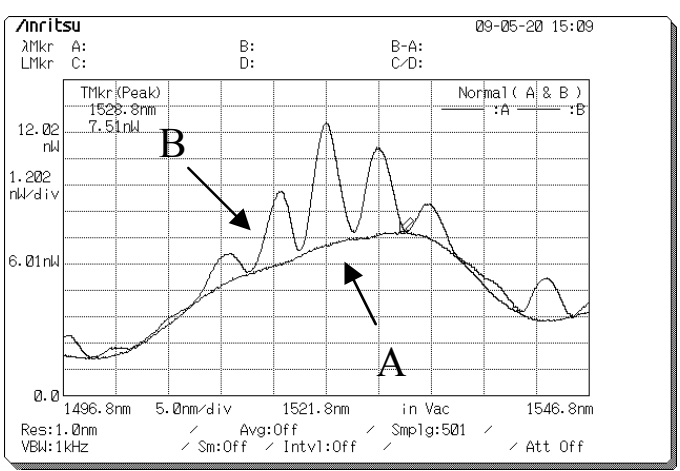

(a)

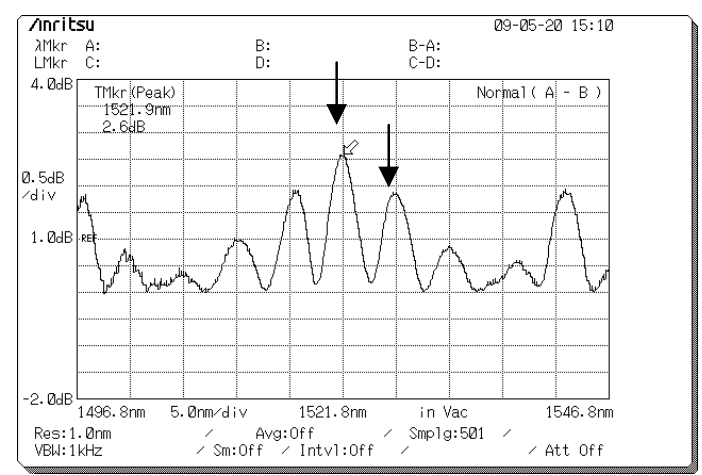

(b)

Figure 3-30: (a) The background before the attachment of the wafer (A), and the spectrum after the joining of wafer (B), (b) Spectrum B minus spectrum A. The sample is a $5 \mathrm{in}$. long clad sapphire fiber.

\subsubsection{Room temperature testing using an unclad sapphire fiber as the waveguide}

In this test, the clad sapphire fiber was replaced with an unclad sapphire fiber of $75 \mu \mathrm{m}$ diameter and 6 inch long. All other experimental details of the set-up were identical to those described in the previous section unless otherwise stated. In this case, the sapphire fiber was inserted into a 6-inch long double-bore ceramic tube (McDaniel Advanced Ceramic, $0.13 \mathrm{~mm}$ bore diameter, and $0.79 \mathrm{~mm}$ outer tube diameter), and the sapphire fiber extended out of both ends of the tube for about $1 \mathrm{~mm}$. A small quantity of 5minute epoxy was applied to either end of the ceramic tube to fix the sapphire fiber. After the epoxy is cured, both ends of the tube were polished using polishing papers with abrasive sizes down to $0.5 \mu \mathrm{m}$.

The sapphire-fiber/silica-fiber coupling and the sapphire wafer affix procedures were the same as those described in the previous section, with the exception here that an index mating adhesive was applied at the sapphire-fiber/silica-fiber junction. The interference patterns are shown in Figure 3-31 (a) and (b) below. Spectrum "A" in Figure 3-31 (a) represents the background before the attachment of sapphire wafer. It is apparent from this figure that the background here shows more signs of interference fringes than the background with a clad sapphire fiber as the waveguide as shown in the spectrum " $\mathrm{A}$ " in Figure 3-30 (a).

We believe that these interference effects present in the background spectrum are caused by modal noise, which in turn results from the combined use of multimode and singlemode fibers. The different electromagnetic modes propagating in a multimode fiber may interfere, if requirements for coherence are satisfied. However, if the active area of the photodetector used to monitor the multimode fiber output is large enough that all of the electromagnetic modes (and consequently there interference effects) are sampled simultaneously, then the interference effects are averaged out, and are not visible. However, if the photodetector active area is much smaller than the core diameter of the multimode fiber, or if a singlemode fiber is used to sample the multimode fiber output, then only the interference between a limited set of modes may be sampled, and the 
interference will not be averaged out. The smaller the photodetector (or core area of the sampling fiber), the stronger the observed interference effects will be. In the experimental set-up illustrated in Figure 3-28, the connection between the multimode fiber and the single-mode fiber of the $2 \times 2$ coupler is likely to be the source of the observed modal noise.

The net spectrum obtained by subtracting spectrum "A" from spectrum "B" in Figure 3-31 (a) is shown in Figure 3-31 (b). As can be seen in this Figure, the spacing between adjacent peaks varies a lot. For example, the spacing between peak 1 and 2 in Figure 3-31 (b) is only about $3.0 \mathrm{~nm}$, while the spacing between peak 3 and 4 is about $6.7 \mathrm{~nm}$. After taking into account the peak spacing of more adjacent peaks, the average peak spacing here is quite close to that in the case of clad fiber (see Figure 3-30 (b)), which is $5 \mathrm{~nm}$. It is believed that this variation in spacing is caused by the modulation of the desired Fabry-Perot cavity fringes by the undesired modal noise. The interference patterns (see Figure 3-30 (b)) obtained using a clad fiber as the waveguide seem to have more even peak spacing than the patterns obtained using an unclad sapphire fiber. This seems to indicate that the use of clad fiber, with its reduced modal volume, reduces the vulnerability to modal noise even in a system mixed with both SM and MM fibers.

For multimode interferometers, modal noise may be minimized by eliminating the use of single-mode fibers in the system, ensuring that connections or splices between fibers are well aligned, and ensuring that the active areas of photodetectors or the entrance pupils of spectrometers are larger than the multimode fiber core area. Therefore, for further testing, the experimental set-up of Figure 3-28 was modified by replacing the singlemode $2 \times 2$ coupler with a multimode $2 \times 2$ fiber, constructed using fiber with a $62.5 \mu \mathrm{m}$ core diameter. In addition, a multimode fiber with a $62.5 \mu \mathrm{m}$ core was used as the patch cord. However, modal noise was still observed in the background. Some investigation revealed that the Anritsu optical spectrum analyzer (OSA) used in the experiment was designed for use with single-mode fiber only, and had an entrance pupil small enough to pass only a limited set of mode from the multimode fiber, generating the observed modal noise. A spectrometer designed for use with multimode fibers has been ordered, and will be used for future interrogation of sapphire interferometric sensors.

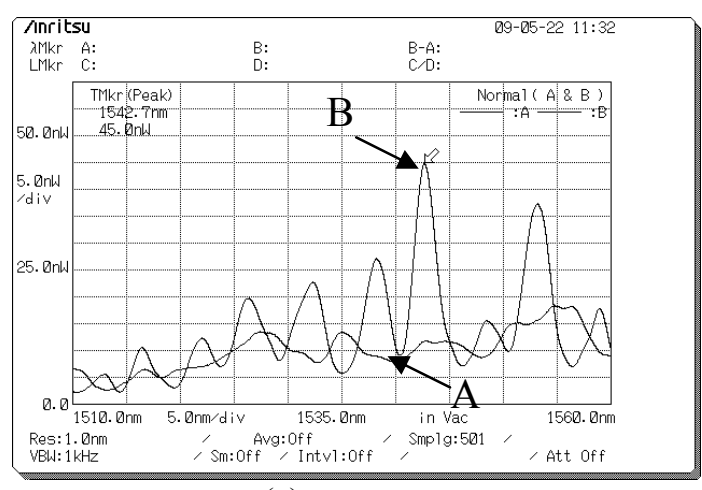

(a)

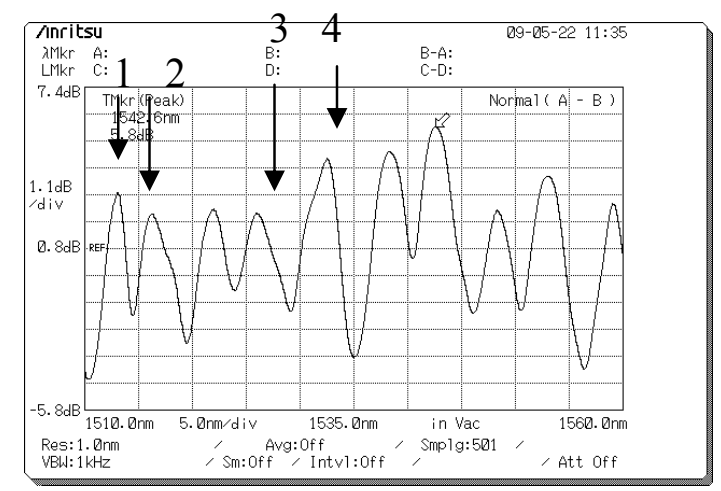

(b)

Figure 3-31: (a) The background spectrum before the attachment of wafer (A), and the spectrum after the attachment of wafer (B), (b) Spectrum B minus spectrum A. 


\subsubsection{Preliminary test results of prototype sensor at elevated temperatures}

A prototype sensor was constructed following the procedures described in Section 3.4 using an unclad 75- $\mu \mathrm{m}$ sapphire fiber as the high temperature waveguide. The sensor configuration and experimental setup are exactly the same as those illustrated in Figure 3-26 and Figure 3-27, respectively, in Section 3.4. The sapphire wafer used here has a measured thickness of $136 \mu \mathrm{m}$ with a c-axis orientation (Meller Optics, Providence, RI). The interference patterns at room temperature are shown in Figure 3-32 below. The output was similar to the patterns discussed in the previous section, as the spacing between the adjacent peaks was not even. For example, the spacing between peak 1 and peak 2 in Figure 3-32 was $4.4 \mathrm{~nm}$, and those between peak 2 and peak 3 and peak 3 and peak 4 were $4.9 \mathrm{~nm}$ and $5.7 \mathrm{~nm}$, respectively. The average spacing is $5.0 \mathrm{~nm}$. The variation in peak spacing was likely due to the modulation of the Fabry-Perot fringes by the modal noise resulting from the use of the Anritsu spectrometer optimized for use with single-mode fibers.

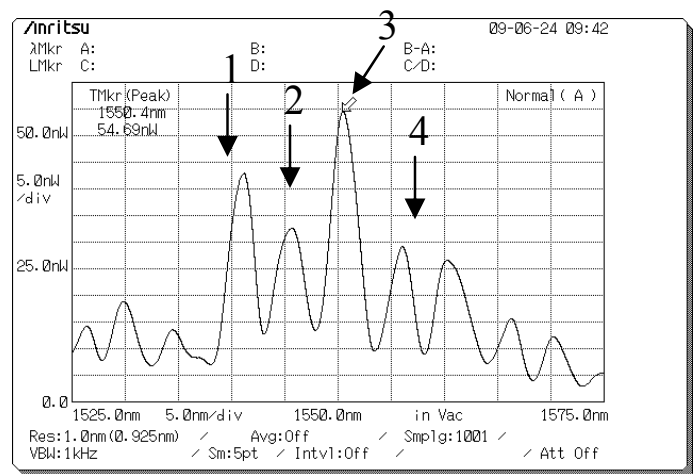

Figure 3-32: The fringe patterns of a sensor made of $75-\mu \mathrm{m}$ sapphire fiber as the waveguide and a $136-\mu \mathrm{m}$ thick sapphire wafer at room temperature.

The sensor was heated to $100^{\circ} \mathrm{C}\left(212^{\circ} \mathrm{F}\right)$ and was kept at this temperature for 20 minutes; the resulting interference patterns are shown in Figure 3-33. During the heating process, it was observed that the relative intensity of each peak varies quite significantly. That is, some peaks may increase in intensity and other peaks decrease intensity. The degree of variation of peak intensity decreased when the temperature was stable at $100{ }^{\circ} \mathrm{C}$ $\left(212^{\circ} \mathrm{F}\right)$.

As shown in Figure 3-33, the relative intensity among peak 1 to 4 changed relative to that shown in Figure 3-32. Furthermore, the peak spacing between two adjacent peaks also changed. Here the spacing between peak 1 and peak 2 is $5.0 \mathrm{~nm}$, and those between peak 2 and peak 3 and peak 3 and peak 4 are $4.6 \mathrm{~nm}$ and $5.4 \mathrm{~nm}$, respectively. As a result of the temperature increase, the spacing between peak 1 and peak 2 increases $0.6 \mathrm{~nm}$, while the spacing between peak 2 and peak 3 decreases $0.3 \mathrm{~nm}$ and the peak distance between peak 3 and peak 4 also decreases $0.3 \mathrm{~nm}$. The average peak spacing is still 5.0 $\mathrm{nm}$, the same as the case at room temperature. In an ideal system where no modal noise exists, the spacing between each pair of adjacent peaks should either all increase or all 
decrease in response to temperature change. The current testing system with modal noise creates difficulty in using the peak spacing to interpret the spectrum response.

On the other hand, another possible method to interpret the response is by the amount of peak shift as a result of temperature change. Here all four peaks shift to the right when the temperature is increased to $100^{\circ} \mathrm{C}\left(212^{\circ} \mathrm{F}\right)$. By comparing the peak locations in Figure 3-32 and Figure 3-33, peak 1 shifts to the right for $0.6 \mathrm{~nm}$, peak 2 for $1.2 \mathrm{~nm}$, peak 3 for $0.9 \mathrm{~nm}$, and peak 4 for $0.6 \mathrm{~nm}$. Therefore, the amount of shift towards longer wavelength varies from $0.6 \mathrm{~nm}$ to $0.9 \mathrm{~nm}$ among the 4 peaks. This is also most likely caused by the modulation of peak locations by modal noise. This also makes it difficult to interpret the interference pattern by tracking the amount of peak shift. An all multimode fiber system is current under fabrication to address the modal noise problem.

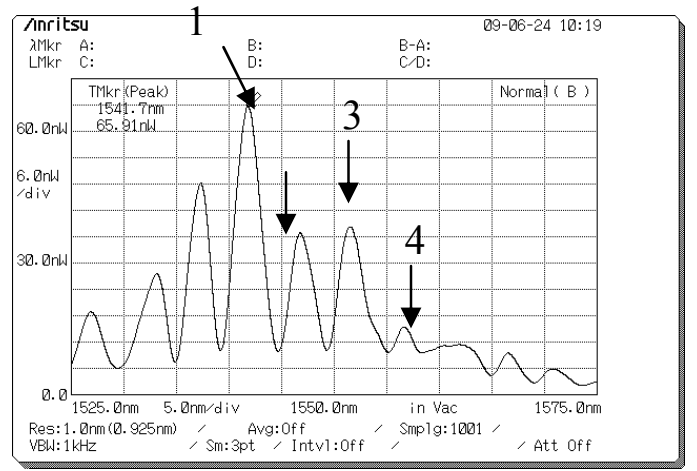

Figure 3-33: The fringe patterns of a sensor made of $75-\mu \mathrm{m}$ sapphire fiber as the waveguide and a $136-\mu \mathrm{m}$ thick sapphire wafer at $100^{\circ} \mathrm{C}\left(212^{\circ} \mathrm{F}\right)$.

\subsubsection{Improved Sapphire Temperature Sensor Configurations}

The configuration of the earlier version of temperature sensor (sensor C4) is shown in Figure 3-34(a) to (d). In this version of the sensor, the clad sapphire fiber and the multimode silica fiber are mechanically spliced together using a UV-curing adhesive (Norland optical adhesive \#68). The appearance of the splice is shown in Figure 3-34 (b). As shown in this figure, the silica fiber is protected within a zirconia ferrule located to the left of the glue joint, and the clad sapphire fiber is protected within an alumina tube located to the right of the glue joint. Due to the nature of the UV-curing adhesive, the maximum temperature of operation of the splice is estimated to be about $100{ }^{\circ} \mathrm{C}\left(212{ }^{\circ} \mathrm{F}\right)$. The clad fiber extends from this splice junction all the way to the tip of sensor where a sapphire wafer is affixed to the end of an alumina tube using ceramic adhesive. The tip of sensor is shown in Figure 3-34 (d). The sapphire wafer has a C-plane orientation and was measured to be $135 \mu \mathrm{m}$ thick using a caliper. The polished end of clad fiber contacted the sapphire wafer intimately, and there was no visible air gap existing between the clad fiber end and the wafer as fabricated. Figure 3-34 (c) shows the middle section of sensor where a part of the clad fiber was exposed. The sensor structural integrity is maintained by gluing a short piece of "support rod" to the two ceramic tubes containing the clad fiber. It is to be noted that in the sensor depicted in Figure 3-34 (c), the clad fiber was not affixed 


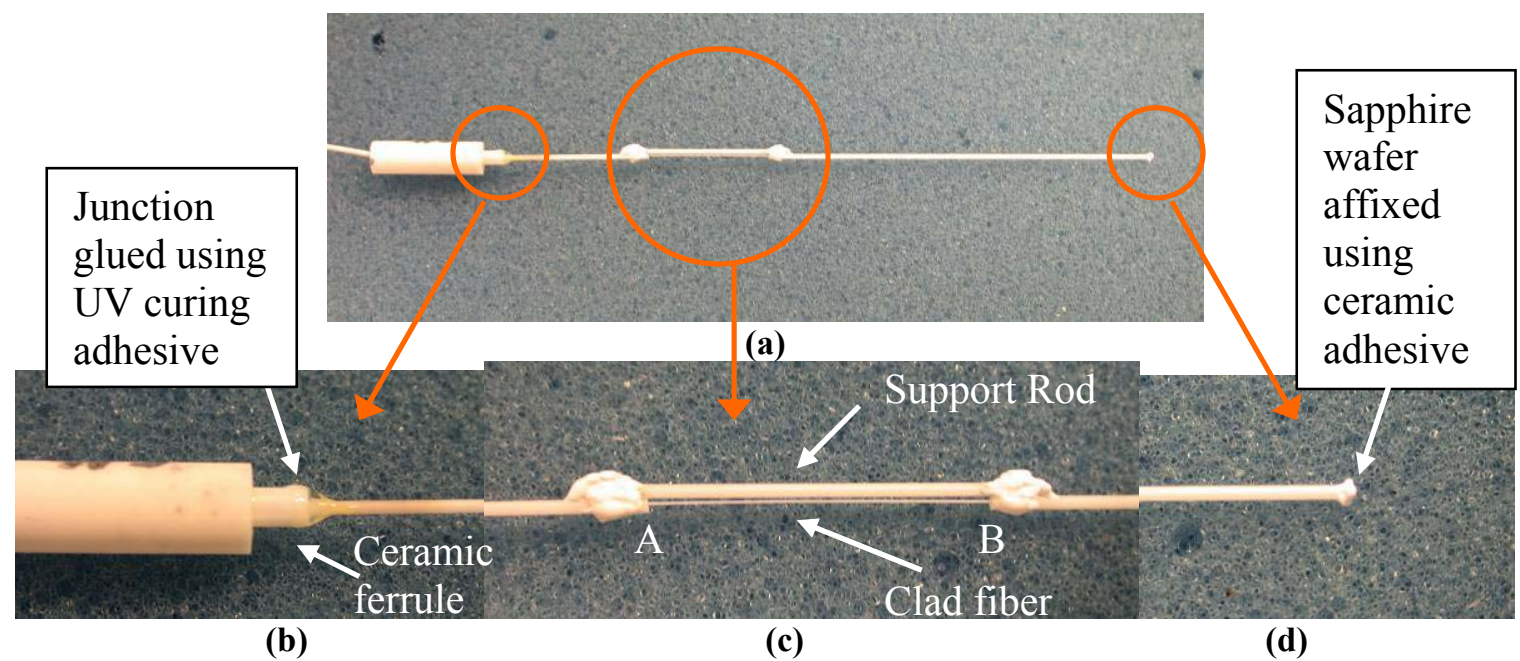

Figure 3-34. The configuration of the earlier version of temperature sensor is shown from (a) to (d). The sensor shown in this figure is Sensor C4.

at the locations labeled " $\mathrm{A}$ " and "B" to the alumina tubes using ceramic adhesive, and was free to move within the tubes. This exposed section of clad fiber is critical in avoiding sensor performance abnormality due to the formation of an air gap at the tip of sensor between the wafer and the polished clad fiber. More details will be discussed in the next section.

In order to better protect the exposed section of clad fiber, a new sensor package design incorporating an alumina support tube, as shown in Figure 3-35, was used to provide structural integrity instead of the support rod shown in Figure 3-34 (c). Another major improvement in this newer version of sensor is the implementation of a silica-to-sapphire splice based on borosilicate glass and capillary tubing that can survive at higher temperatures, as explained in 3.8. Furthermore, the possibility of using gold-coated silica fibers at the silica-to-sapphire splice junctions has the potential of further increasing the operating temperature of the splice.

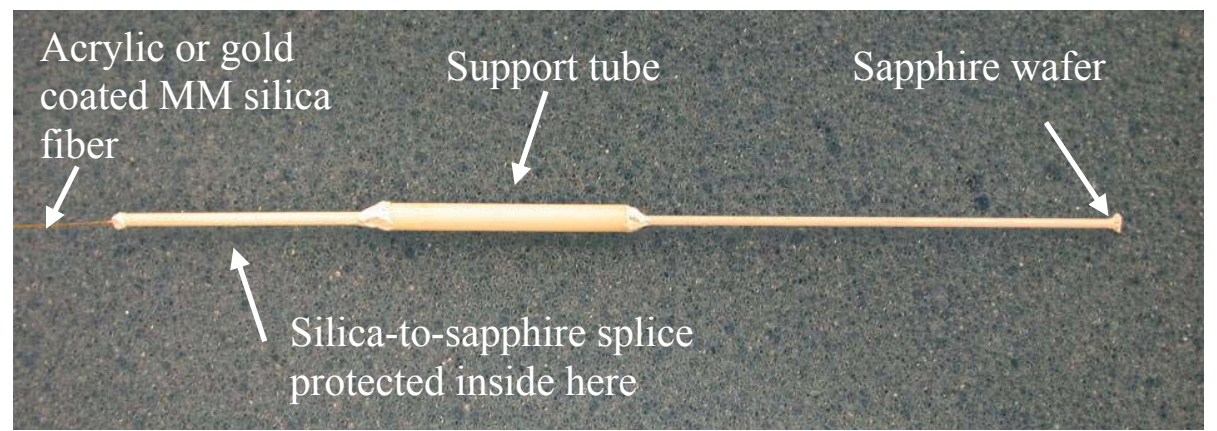

Figure 3-35. Newer version of temperature sensor with innovative silica-to-sapphire splices and support tube structures

\subsubsection{Sensor Performance}

In this section, the performance of three temperature sensors, labeled $\mathrm{C} 2, \mathrm{C} 4$, and $\mathrm{C} 5$, will be discussed. The first two sensors, $\mathrm{C} 2$ and $\mathrm{C} 4$, were made using the older, 
mechanical type of silica-to-sapphire splices, in which UV-curing adhesive is used to join the fibers, together with a simple fiber support structure, as shown in Figure 3-34 (c). Sensor C5 was fabricated using a new silica-to-sapphire splice technique based on a capillary tube and a support tube structure very similar to those depicted in Figure 3-35. The phase shift of the output of sensor C2 with increasing temperature possessed an abnormal oscillating behavior which was detrimental to the temperature data interpretation. It was discovered that this oscillating behavior was caused by the formation of an air gap between the sapphire wafer and the end of polished clad fiber at the sensor tip after temperature testing cycles. An illustration of the location of this air gap is shown in Figure 3-36.

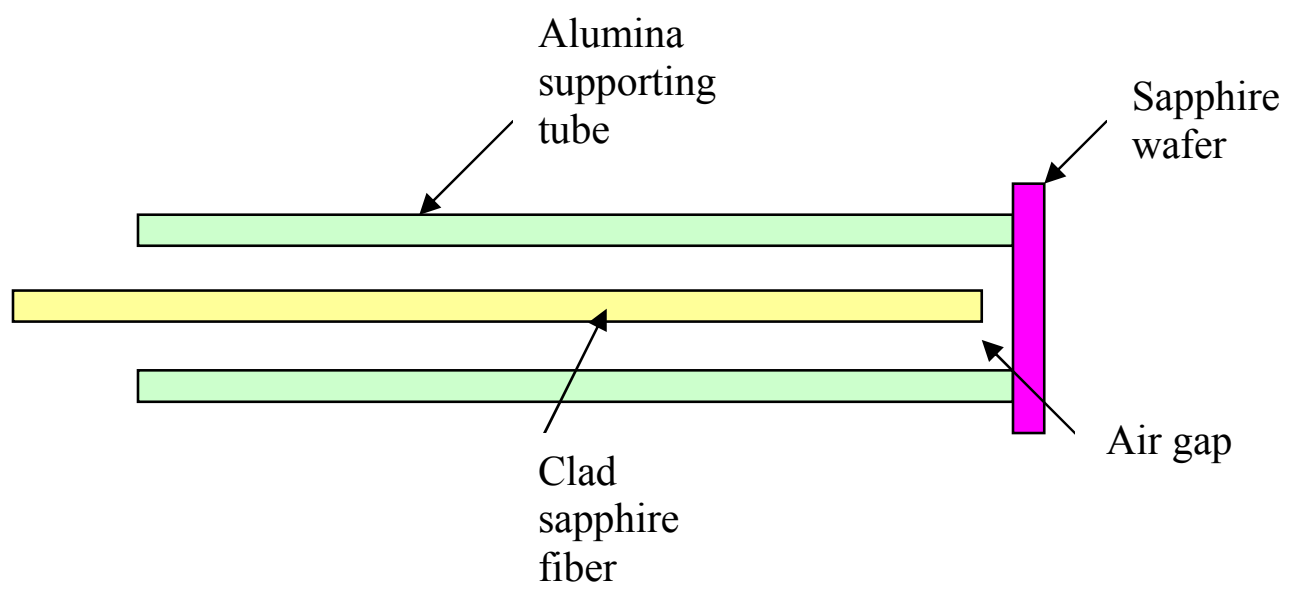

Figure 3-36. An illustration of the air gap formed between the sapphire wafer and the clad fiber end at sensor tip

To solve this problem, in sensor $\mathrm{C} 4$, a slightly longer section of clad fiber, relative to the overall length of the supporting alumina tubing structure, was integrated into the sensor through the formation of a bulge in the exposed section of clad fiber (see Figure 3-46 for the illustration of this bulge section in sensor C5). This modified fabrication approach prevented the undesirable formation of an air gap at the sensor tip. This modification was also used in the fabrication of sensor C5. Also in sensor C5, the improved silica-tosapphire splicing and the support tube structure were implemented. The methods of fabricating the exposed section of clad fiber in sensor C5 were also modified to reduce the large noise level observed in the phase shift of sensor $\mathrm{C} 4$.

\subsection{Configurations and Performance of Sensor C2}

The configuration of sensor C2 is very similar to sensor C4 shown in Figure 3-34 except that in sensor $\mathrm{C} 2$ the exposed section of clad fiber, instead of being free to move in the supporting alumina tubes at locations " $A$ " and "B" as shown in Figure 3-34 (c), is glued to the two supporting tubes using ceramic adhesive at location " $A$ " and " $B$ " (see Figure $3-37)$. The clad sapphire fiber for sensor $\mathrm{C} 2$ has a length of about $11 \mathrm{~cm}(4.3 \mathrm{inch})$. 


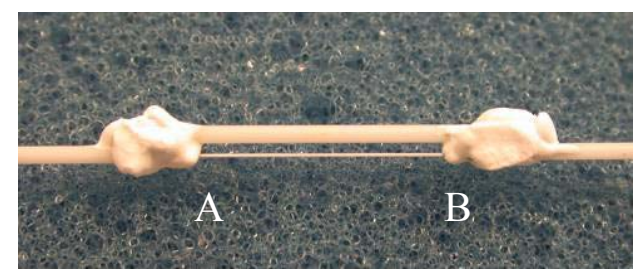

Figure 3-37. The exposed section of clad sapphire fiber of sensor C2

A multimode fiber with $62.5 \mu \mathrm{m}$ core diameter and $125 \mu \mathrm{m}$ cladding diameter is mechanically spliced to the clad sapphire fiber. The combined forward optical loss of the mechanical splice and the 4.3-inch long clad sapphire fiber was $2.2 \mathrm{~dB}$. The loss was measured using a $1550 \mathrm{~nm}$ superluminescent LED light source with a single mode fiber patch cord. The cross section of the clad sapphire fiber in sensor C2 is shown in Figure 3-38. The clad fiber has a diameter of about $135 \mu \mathrm{m}$ and a sapphire core diameter of about $98 \mu \mathrm{m}$.

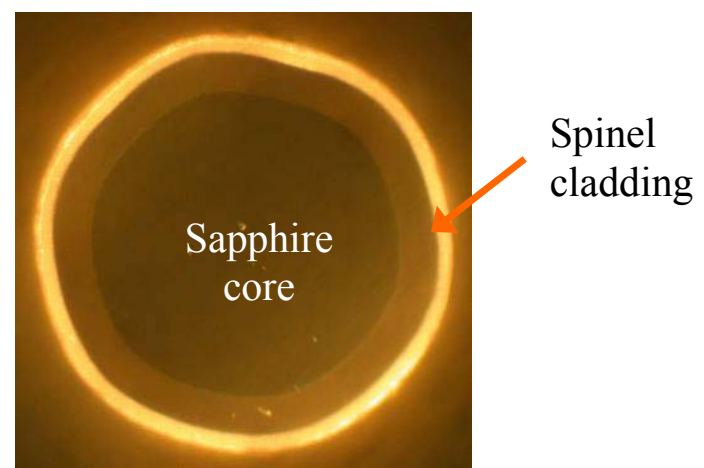

Figure 3-38. The cross section of clad sapphire fiber of sensor C2

The sensor was tested using the experimental system depicted in Figure 3-39. The light source inside the Micron Optics CTS scanning laser system is a tunable fiber ring laser with a spectral range from $1520 \mathrm{~nm}$ to $1570 \mathrm{~nm}$. The light source was connected to a multimode $(62.5 \mu \mathrm{m}$ core $/ 125 \mu \mathrm{m}$ clad) $2 \times 2$ coupler through a singlemode fiber patch cord. The laser light was transmitted to the sensor from the coupler through a multimode $(62.5 / 125)$ patch cord. Reflected light from the sensor was collected at the detector port at the CTS unit through another multimode $(62.5 / 125)$ patch cord. The CTS unit monitored the phase shift of the interference fringes as a function of temperature. The phase shift originated from the thermal expansion of the sapphire wafer due to temperature change. The phase data were recorded every 5 seconds.

Figure 3-40 shows the top of the high temperature furnace used for temperature testing. The sensor was inserted through a hole in the top of the furnace into the hot zone. A ceramic feedthrough consisting of an alumina tube sealed at one end was used to enclose the sensor in the oven to prevent possible contamination of the sapphire wafer in the oven. During testing, the opening of the feedthrough was covered by pieces of woven fiberglass sheets (not shown here in Figure 3-40) for heat insulation. A K-type thermocouple was placed close to the sensor tip to measure the sapphire wafer temperature. The furnace temperature was increased from room temperature to $900{ }^{\circ} \mathrm{C}$ $\left(1652{ }^{\circ} \mathrm{F}\right)$ in intervals of about $100^{\circ} \mathrm{C}\left(212^{\circ} \mathrm{F}\right)$ with a dwell period of 30 to 60 minutes at 
each temperature level. At the end of each dwell period, the temperature setting was increased manually to the next level.

The interference fringes of sensor $\mathrm{C} 2$ at $23^{\circ} \mathrm{C}\left(73^{\circ} \mathrm{F}\right)$ are shown in Figure 3-41. From these fringes, the calculated optical path length of the sapphire wafer was $235.7 \mu \mathrm{m}$. The physical wafer thickness can be calculated by dividing the optical path length $(235.7 \mu \mathrm{m})$ by the refractive index of sapphire (1.76), which gives a wafer thickness of $134 \mu \mathrm{m}$. This calculated thickness is almost the same as the measured thickness of $135 \mu \mathrm{m}$ using a caliper. When the wafer expands as a result of rising temperature, the fringes shown in Figure 3-41 move to the right with a positive phase shift. For example, when the temperature increased from $23{ }^{\circ} \mathrm{C}\left(73{ }^{\circ} \mathrm{F}\right)$ to $200^{\circ} \mathrm{C}\left(392^{\circ} \mathrm{F}\right)$, it was observed that peak "A" in Figure 3-41 slowly moved to the right and ended up at a new location as indicated in Figure 3-42, which shows the fringes of sensor $\mathrm{C} 2$ at $200{ }^{\circ} \mathrm{C}\left(392^{\circ} \mathrm{F}\right)$. The phase difference between the peak " $A$ " in Figure 3-41 and the peak " $A$ " in Figure 3-42 is 5.20 radians, a little less than $2 \pi(6.28)$ radians. It is to be noted that the phase difference between peaks " $A$ " and " $B$ " in Figure $3-41$ is exactly $2 \pi(6.28)$ radians.

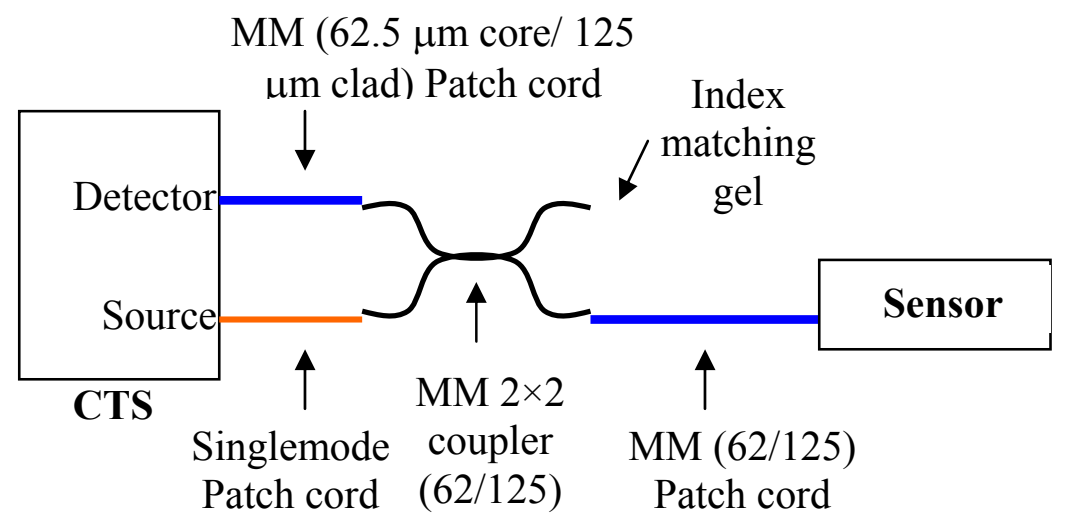

Figure 3-39. Sensor testing system

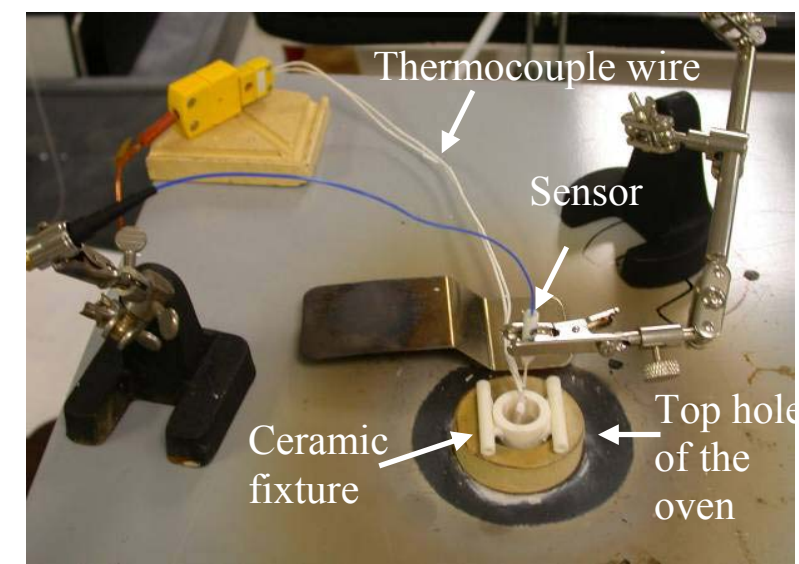

Figure 3-40. Temperature test setup of clad sapphire fiber sensor 


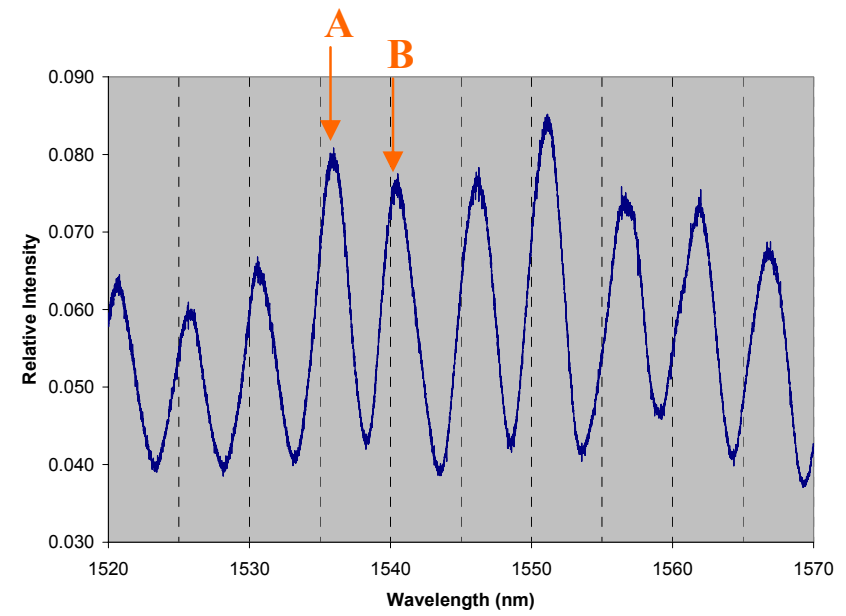

Figure 3-41. The interference fringes of sensor $\mathrm{C} 2$ at $23^{\circ} \mathrm{C}\left(73^{\circ} \mathrm{F}\right)$

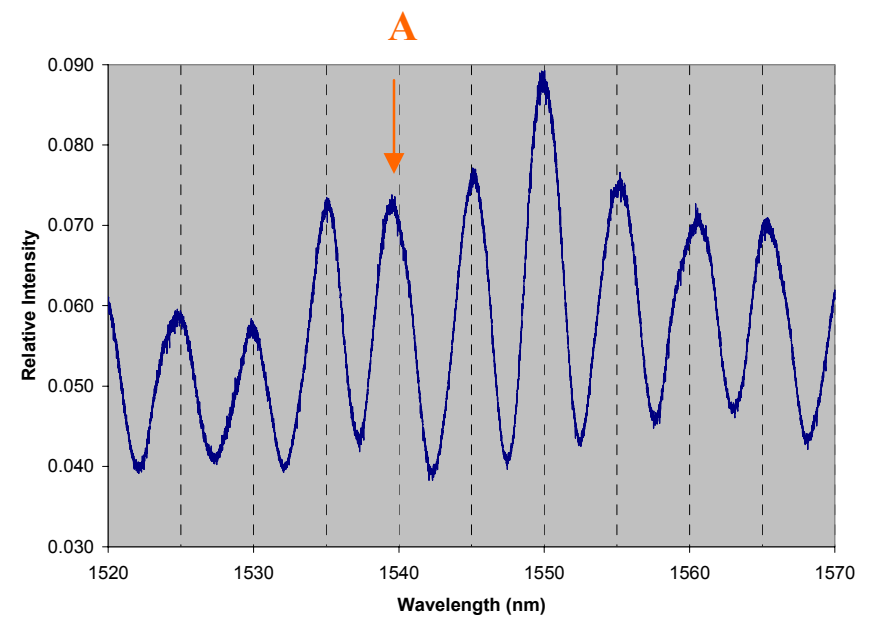

Figure 3-42. The interference fringes of sensor $\mathrm{C} 2$ at $200^{\circ} \mathrm{C}\left(392{ }^{\circ} \mathrm{F}\right)$

The phase shift of sensor $\mathrm{C} 2$ as a function of temperature from $23{ }^{\circ} \mathrm{C}\left(73{ }^{\circ} \mathrm{F}\right)$ to $899{ }^{\circ} \mathrm{C}$ $\left(1650^{\circ} \mathrm{F}\right)$ is shown in Figure 3-43. Sensor $\mathrm{C} 2$ had been undergone two similar temperature cycles before the test whose results are shown in Figure 3-43. At room temperature, the furnace controller was manually set to a temperature corresponding to a measured temperature at the sensor tip of $201{ }^{\circ} \mathrm{C}\left(394{ }^{\circ} \mathrm{F}\right)$. As shown in Figure 3-43, the phase rapidly increased as the temperature rose from room temperature toward $201{ }^{\circ} \mathrm{C}$ $\left(394^{\circ} \mathrm{F}\right)$. The phase endured a discontinuous jump to 0 when it passes $2 \pi(6.28)$ radians. The phase change at each temperature interval can be calculated from the trace in Figure 3-43. The temperature sensitivity, i.e., the average phase change per degree Celsius (Fahrenheit), at two temperature ranges from $22^{\circ} \mathrm{C}\left(72{ }^{\circ} \mathrm{F}\right)$ to $402{ }^{\circ} \mathrm{C}\left(756^{\circ} \mathrm{F}\right)$ and from $402{ }^{\circ} \mathrm{C}\left(756^{\circ} \mathrm{F}\right)$ to $800{ }^{\circ} \mathrm{C}\left(1472^{\circ} \mathrm{F}\right)$ were calculated and tabulated in Table 3-6. It was observed that the temperature sensitivity was greater at the higher temperature range. For example, as indicated in Table 3-6, the temperature sensitivity in the temperature range from $402^{\circ} \mathrm{C}\left(756^{\circ} \mathrm{F}\right)$ to $800{ }^{\circ} \mathrm{C}\left(1472{ }^{\circ} \mathrm{F}\right)$ was about $3.7 \times 10^{-2}$ radians $/{ }^{\circ} \mathrm{C}$. This average temperature sensitivity is $28 \%$ larger than that in the temperature range from $22{ }^{\circ} \mathrm{C}(72$ $\left.{ }^{\circ} \mathrm{F}\right)$ to $402{ }^{\circ} \mathrm{C}\left(756{ }^{\circ} \mathrm{F}\right)$, which was $2.9 \times 10^{-2}$ radians $/{ }^{\circ} \mathrm{F}$. 
In some locations as indicated by the orange-color arrows in Figure 3-43, the phase trace shows an oscillatory behavior which is detrimental to the interpretation of testing temperatures. To determine the mechanisms causing this oscillating behavior, the sensor tip was closely examined under an optical microscope after testing. It was observed that a $35 \mu \mathrm{m}$ thick air gap had been formed between the sapphire wafer and the polished end of clad sapphire fiber. It is believed that fringes from the air gap may have interfered with the correct interpretation of phase information by the CTS unit. The factors contributing to the formation of this air gap may include the shrinkage of ceramic adhesive after temperature cycles, and the difference in the coefficients of thermal expansion (CTE) between alumina tubes and clad sapphire fibers. The sensor fabrication procedures were modified for sensor $\mathrm{C} 4$ to prevent the formation of an air gap at the sensor tip.

There was another abnormal behavior associated with sensor $\mathrm{C} 2$ at temperatures above about $600{ }^{\circ} \mathrm{C}\left(1112^{\circ} \mathrm{F}\right)$. At and above this temperature the interference fringes (see Figure 3-41 and Figure 3-42) started to shift up and down as a whole; that is, the dc offset of the fringes appeared to change randomly with time, while the peak-to-peak separation of the fringe maxima and minima appeared to stay constant. The variation in the dc offset appeared to increase as the testing temperature approaching $899^{\circ} \mathrm{C}\left(1650{ }^{\circ} \mathrm{F}\right)$. We suspect that at these higher temperatures the mechanical splice made using UV curing adhesive started to lose its mechanical integrity.

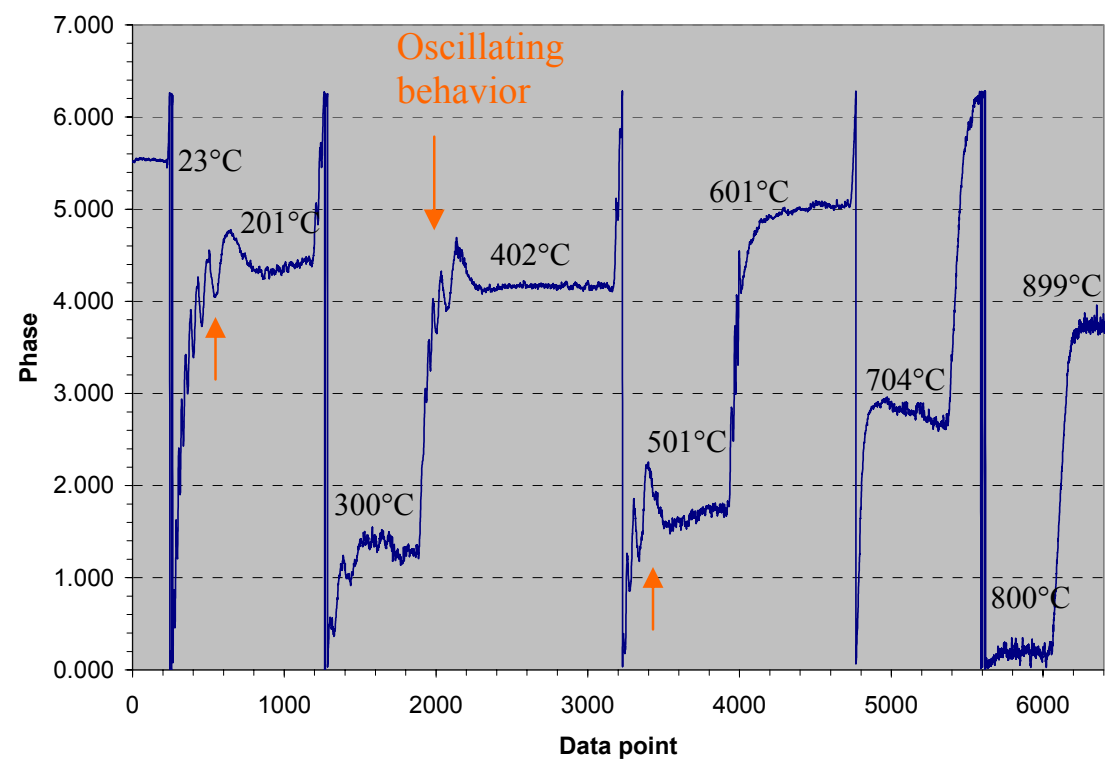

Figure 3-43. Temperature test results of sensor $\mathrm{C} 2$ from room temperature to $900^{\circ} \mathrm{C}\left(1652^{\circ} \mathrm{F}\right)$

Table 3-6. Temperature sensitivity of sensor C2

\begin{tabular}{|c|c|c|c|}
\hline Temp. Range & $\begin{array}{c}\text { Phase Change } \\
(\Delta \varphi)\end{array}$ & $\begin{array}{c}\text { Temp. Change } \\
(\Delta \mathrm{T})\end{array}$ & $\Delta \varphi / \Delta \mathrm{T}$ \\
\hline $22^{\circ} \mathrm{C}$ to $402^{\circ} \mathrm{C}$ & 11.20 & $380^{\circ} \mathrm{C}$ & $2.9 \times 10^{-2} /{ }^{\circ} \mathrm{C}$ \\
$\left(72{ }^{\circ} \mathrm{F}\right.$ to $\left.756{ }^{\circ} \mathrm{F}\right)$ & & $\left(684^{\circ} \mathrm{F}\right)$ & $\left(1.6 \times 10^{-2} /{ }^{\circ} \mathrm{F}\right)$ \\
\hline $402^{\circ} \mathrm{C}$ to $800^{\circ} \mathrm{C}$ & 14.87 & $398^{\circ} \mathrm{C}$ & $3.7 \times 10^{-2} /{ }^{\circ} \mathrm{C}$ \\
$\left(756^{\circ} \mathrm{F}\right.$ to $\left.1472{ }^{\circ} \mathrm{F}\right)$ & & $\left(712^{\circ} \mathrm{F}\right)$ & $\left(2.1 \times 10^{-2} /{ }^{\circ} \mathrm{F}\right)$ \\
\hline
\end{tabular}




\subsection{Configurations and Performance of Sensor C4}

As discussed in previous sections, the configurations of sensor $\mathrm{C} 4$ are exactly the same as those shown in Figure 3-34 (a) to (d). The critical differences between sensor C4 and sensor $\mathrm{C} 2$ are the methods by which the exposed section of clad fiber is fabricated. In sensor $\mathrm{C} 4$, efforts were made to make the exposed section of clad fiber longer by forming a bulge in the exposed section of clad fiber. Furthermore, in sensor $\mathrm{C} 4$, the exposed section of clad fiber was not glued at its ends to the supporting alumina tubes and was free to move in the tubes. After fabrication, there was no observable air gap at the tip of sensor $\mathrm{C} 4$ under careful examination using an optical microscope.

Sensor C4 was tested using the same sensor testing system as shown in Figure 3-39 and the high temperature furnace setup as shown in Figure 3-40. The phase shift results of sensor $\mathrm{C} 4$ as a function of temperature from $22^{\circ} \mathrm{C}\left(72{ }^{\circ} \mathrm{F}\right)$ to $799^{\circ} \mathrm{C}\left(1470{ }^{\circ} \mathrm{F}\right)$ in intervals of about $200^{\circ} \mathrm{C}\left(392^{\circ} \mathrm{F}\right)$ are shown in Figure 3-44. As shown in this figure, the detrimental oscillating behavior in the phase trace of sensor C2 (see Figure 3-43) is absent here. After several temperature cycles, the sensor tip was examined again under an optical microscope. There was no observable air gap found at the tip of sensor C4. However, as indicated by the orange-color arrows in Figure 3-44, there existed a relatively larger noise floor level at and above about $600^{\circ} \mathrm{C}\left(1112^{\circ} \mathrm{F}\right)$ compared to the noise floors of sensor C2 (see Figure 3-43). This larger noise level may be caused by the fact that the exposed section of clad fiber in sensor $\mathrm{C} 4$ was not glued to the two supporting alumina tubes and was free to move within the tubes. Similar to sensor C2, the dc level of the interference fringes of sensor $\mathrm{C} 4$ also shifted erratically at and above about $600{ }^{\circ} \mathrm{C}\left(1112^{\circ} \mathrm{F}\right)$.

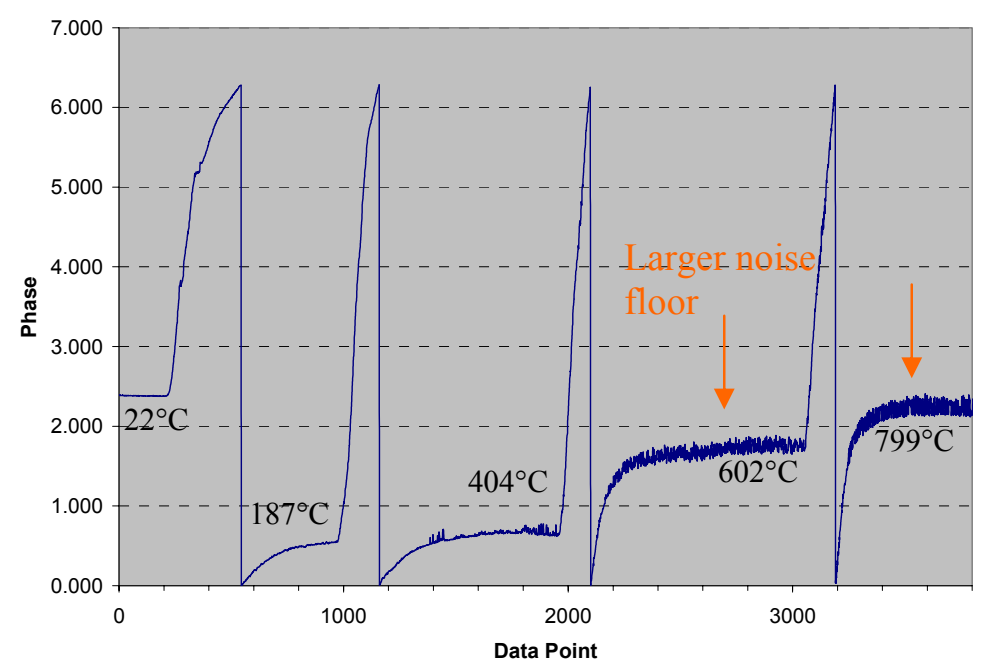

Figure 3-44. Temperature test results of sensor $\mathrm{C} 4$ from $22^{\circ} \mathrm{C}\left(72^{\circ} \mathrm{F}\right)$ to $799^{\circ} \mathrm{C}\left(1470{ }^{\circ} \mathrm{F}\right)$

Sensor $\mathrm{C} 4$ was tested two more times to determine the repeatability of its performance. In these two tests sensor $\mathrm{C} 4$ was heated to $800^{\circ} \mathrm{C}\left(1472^{\circ} \mathrm{F}\right)$ in intervals of about $400^{\circ} \mathrm{C}$ 
$\left(752^{\circ} \mathrm{F}\right)$. The phase shift data of these repeatability tests are shown in Figure 3-45 (a) and (b). Similar to the sensor behaviors in the first test (see Figure 3-44), there was no undesirable oscillating behavior associated with the phase data in both repeatability tests. Also, the noise floors at $800^{\circ} \mathrm{C}\left(1472^{\circ} \mathrm{F}\right)$ in these repeatability tests are consistent with the noise floor in the fist test as shown in Figure 3-44. The sensor tip was again carefully examined using an optical microscope after the last temperature test cycle. There was no visible air gap found from this examination.

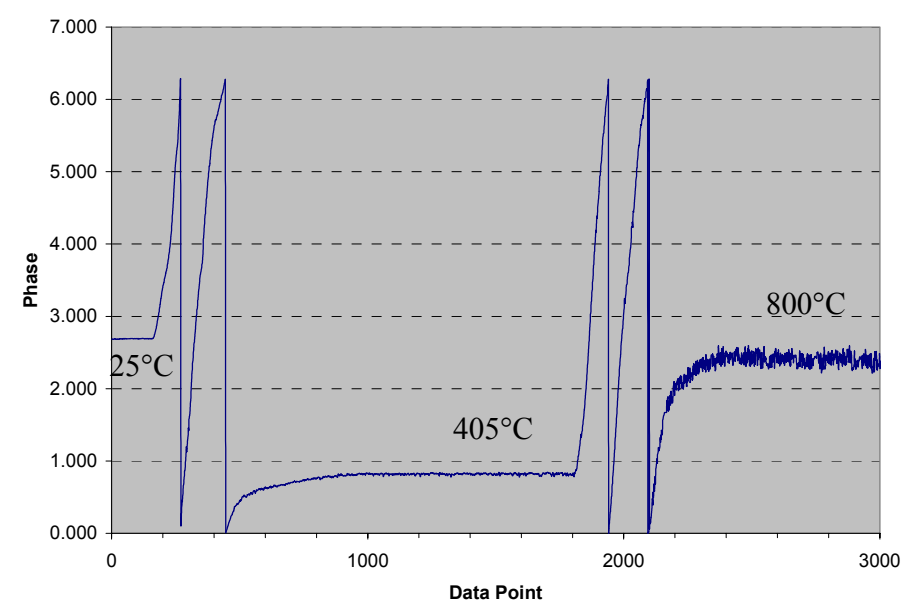

(a)

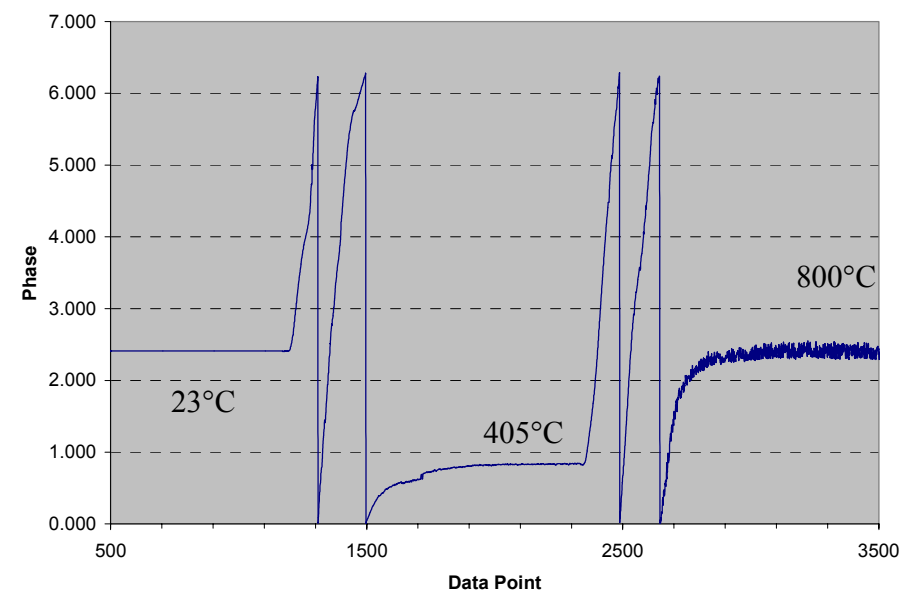

(b)

Figure 3-45. Phase shift data of the first (a) and second (b) repeatability tests of sensor C4.

The temperature sensitivity in terms of the average phase change per degree Celsius for all three tests in two temperature ranges are listed in Table 3-7. The average phase change per degree Celsius $(\Delta \varphi / \Delta \mathrm{T})$ at corresponding temperature ranges are basically the same for all three tests. Similar to the case of sensor $\mathrm{C} 2$ as illustrated by the data in Table 3-6, sensor $\mathrm{C} 4$ also had a greater temperature sensitivity of $3.6 \times 10^{-2}$ radians $/{ }^{\circ} \mathrm{C}\left(2.0 \times 10^{-2}\right.$ radians $\left./{ }^{\circ} \mathrm{F}\right)$ at the temperatures from about $400{ }^{\circ} \mathrm{C}\left(752^{\circ} \mathrm{F}\right)$ to $800{ }^{\circ} \mathrm{C}\left(1472{ }^{\circ} \mathrm{F}\right)$ compared to that of $2.8 \times 10^{-2}$ radians $/{ }^{\circ} \mathrm{C}\left(1.6 \times 10^{-2}\right.$ radians $\left./{ }^{\circ} \mathrm{F}\right)$ at the temperatures from room temperature to about $400^{\circ} \mathrm{C}\left(752^{\circ} \mathrm{F}\right)$. It is to be noted that the $\Delta \varphi / \Delta \mathrm{T}$ values for sensor $\mathrm{C} 4$ here are almost the same as those for sensor $\mathrm{C} 2$. 
The exact phase value and the noise floor peak-to-peak magnitude, i.e., the width of noise level in terms of phase, of sensor $\mathrm{C} 4$ for all three tests at three difference temperatures (room temperature, $400{ }^{\circ} \mathrm{C}\left(752^{\circ} \mathrm{F}\right), 800^{\circ} \mathrm{C}\left(1472{ }^{\circ} \mathrm{F}\right)$ ) are listed in Table $3-8$. At $800{ }^{\circ} \mathrm{C}$ $\left(1472{ }^{\circ} \mathrm{F}\right)$, the phase values reported in Table 3-8 are the average phase values within the noise floor bands. As can be seen from this Table, the temperature measurement is reproducible to within about 0 to 3 degree Celsius. For example, the phase values at

Table 3-7. Temperature sensitivity of sensor $\mathbf{C 4}$

\begin{tabular}{|c|c|c|c|c|}
\hline \multirow{5}{*}{ Test 1 } & Temp. Range & $\begin{array}{c}\text { Phase } \\
\text { Change }(\Delta \varphi)\end{array}$ & $\begin{array}{c}\text { Temp. Change } \\
(\Delta \mathrm{T})\end{array}$ & $\Delta \varphi / \Delta \mathrm{T}$ \\
& $\begin{array}{c}22^{\circ} \mathrm{C} \text { to } 404^{\circ} \mathrm{C} \\
\left(72^{\circ} \mathrm{F} \text { to } 759^{\circ} \mathrm{F}\right)\end{array}$ & 10.8 & $\begin{array}{c}382^{\circ} \mathrm{C} \\
\left(720^{\circ} \mathrm{F}\right)\end{array}$ & $\begin{array}{c}2.8 \times 10^{-2} /{ }^{\circ} \mathrm{C} \\
\left(1.6 \times 10^{-2} /{ }^{\circ} \mathrm{F}\right)\end{array}$ \\
\cline { 2 - 5 } & $404^{\circ} \mathrm{C}$ to $799^{\circ} \mathrm{C}$ & 14.17 & $395^{\circ} \mathrm{C}$ & $3.6 \times 10^{-2} /{ }^{\circ} \mathrm{C}$ \\
$\left(759^{\circ} \mathrm{F}\right.$ to $\left.1470^{\circ} \mathrm{F}\right)$ & & $\left(743^{\circ} \mathrm{F}\right)$ & $\left(2.0 \times 10^{-2} /{ }^{\circ} \mathrm{F}\right)$ \\
\hline \multirow{5}{*}{ Test 2 } & $25^{\circ} \mathrm{C}$ to $405^{\circ} \mathrm{C}$ & 10.7 & $380^{\circ} \mathrm{C}$ & $2.8 \times 10^{-2} /{ }^{\circ} \mathrm{C}$ \\
& $\left(77^{\circ} \mathrm{F}\right.$ to $\left.761^{\circ} \mathrm{F}\right)$ & & $\left(716^{\circ} \mathrm{F}\right)$ & $\left(1.6 \times 10^{-2} /{ }^{\circ} \mathrm{F}\right)$ \\
\cline { 2 - 5 } & $405^{\circ} \mathrm{C}$ to $800^{\circ} \mathrm{C}$ & 14.15 & $395^{\circ} \mathrm{C}$ & $3.6 \times 10^{-2} /{ }^{\circ} \mathrm{C}$ \\
& $\left(761^{\circ} \mathrm{F}\right.$ to $\left.1472^{\circ} \mathrm{F}\right)$ & & $\left(743^{\circ} \mathrm{F}\right)$ & $\left(2.0 \times 10^{-2} /{ }^{\circ} \mathrm{F}\right)$ \\
\hline \multirow{5}{*}{ Test 3 } & $23^{\circ} \mathrm{C}$ to $405^{\circ} \mathrm{C}$ & 10.98 & $382^{\circ} \mathrm{C}$ & $2.9 \times 10^{-2} /{ }^{\circ} \mathrm{C}$ \\
& $\left(73^{\circ} \mathrm{F}\right.$ to $\left.761^{\circ} \mathrm{F}\right)$ & & $\left(720^{\circ} \mathrm{F}\right)$ & $\left(1.6 \times 10^{-2} /{ }^{\circ} \mathrm{F}\right)$ \\
\cline { 2 - 5 } & $405^{\circ} \mathrm{C}$ to $800^{\circ} \mathrm{C}$ & 14.14 & $395^{\circ} \mathrm{C}$ & $3.6 \times 10^{-2} /{ }^{\circ} \mathrm{C}$ \\
$\left(761^{\circ} \mathrm{F}\right.$ to $\left.1472^{\circ} \mathrm{F}\right)$ & & $\left(743^{\circ} \mathrm{F}\right)$ & $\left(2.0 \times 10^{-2} /{ }^{\circ} \mathrm{F}\right)$ \\
\hline
\end{tabular}

$405^{\circ} \mathrm{C}\left(761^{\circ} \mathrm{F}\right)$ for Test 2 and Test 3 were 0.82 and 0.83 respectively with a phase difference of 0.01 , which corresponds to about $0.3^{\circ} \mathrm{C}$ based on a $\Delta \varphi / \Delta \mathrm{T}$ value of 0.036 radians (see Table 3-7). On the other hand, the phase value at $404^{\circ} \mathrm{C}\left(759^{\circ} \mathrm{F}\right)$ for Test 1 was 0.66 radians. Based on an approximate $\Delta \varphi / \Delta \mathrm{T}$ value of 0.036 , the phase value at 405 ${ }^{\circ} \mathrm{C}\left(761^{\circ} \mathrm{F}\right)$ for Test 1 can be calculated to be about 0.70 . This value is about 0.12 smaller than 0.82 , the phase value at $405^{\circ} \mathrm{C}\left(761{ }^{\circ} \mathrm{F}\right)$ for Test 2 , and corresponds to a temperature approximately $3^{\circ} \mathrm{C}$ lower than $405^{\circ} \mathrm{C}\left(761^{\circ} \mathrm{F}\right)$, assuming a temperature calibration has been established based on the performance of sensor $\mathrm{C} 4$ from Test 2. Due to the existence of larger noise floors, the phase values at $800^{\circ} \mathrm{C}\left(1472^{\circ} \mathrm{F}\right)$ were a little more uncertain.

Table 3-8. The relationship between phase and temperature of sensor $\mathrm{C} 4$

\begin{tabular}{|c|c|c|c|c|c|c|c|c|c|}
\hline & \multicolumn{3}{|c|}{ Test 1 } & \multicolumn{3}{c|}{ Test 2 } & \multicolumn{3}{c|}{ Test 3} \\
\hline Temperature & $\begin{array}{c}22^{\circ} \mathrm{C} \\
\left(72^{\circ} \mathrm{F}\right)\end{array}$ & $\begin{array}{c}404^{\circ} \mathrm{C} \\
\left(759^{\circ} \mathrm{F}\right)\end{array}$ & $\begin{array}{c}799^{\circ} \mathrm{C} \\
\left(1470^{\circ} \mathrm{F}\right)\end{array}$ & $\begin{array}{c}25^{\circ} \mathrm{C} \\
\left(77^{\circ} \mathrm{F}\right)\end{array}$ & $\begin{array}{c}405^{\circ} \mathrm{C} \\
\left(761^{\circ} \mathrm{F}\right)\end{array}$ & $\begin{array}{c}800^{\circ} \mathrm{C} \\
\left(1472^{\circ} \mathrm{F}\right)\end{array}$ & $\begin{array}{c}23^{\circ} \mathrm{C} \\
\left(73{ }^{\circ} \mathrm{F}\right)\end{array}$ & $\begin{array}{c}405^{\circ} \mathrm{C} \\
\left(761^{\circ} \mathrm{F}\right)\end{array}$ & $\begin{array}{c}800^{\circ} \mathrm{C} \\
\left(1472^{\circ} \mathrm{F}\right)\end{array}$ \\
\hline $\begin{array}{c}\text { Phase } \\
\text { (radians) }\end{array}$ & 2.38 & 0.66 & 2.26 & 2.69 & 0.82 & 2.43 & 2.41 & 0.83 & 2.40 \\
\hline $\begin{array}{c}\text { Noise floor } \\
\text { (peak-to- } \\
\text { peak) }\end{array}$ & $\mathrm{n} / \mathrm{a}$ & $\mathrm{n} / \mathrm{a}$ & 0.2 & $\mathrm{n} / \mathrm{a}$ & $\mathrm{n} / \mathrm{a}$ & 0.25 & $\mathrm{n} / \mathrm{a}$ & $\mathrm{n} / \mathrm{a}$ & 0.2 \\
\hline
\end{tabular}




\subsection{Sensor Configurations and Performance of sensor C5}

The appearance of sensor C5 are almost the same as those illustrated in Figure 3-35. For sensor C5, an acrylate coated multimode fiber was spliced to the clad sapphire fiber. The clad fiber in sensor C5 was only 2.8 inch long, shorter than the clad fiber lengths of both sensor $\mathrm{C} 2$ and $\mathrm{C} 4$, which were about 4.5 inch long. A new type of silica-to-sapphire splice based on borosilicate glass and capillary tubing, explained in Section 3.8 was implemented to increase the operating temperature of the splice. The exposed section of clad fiber was fully enclosed in an alumina protecting tube. The configuration of sensor C5 is illustrated in Figure 3-46. Similar to sensor C4, a bulging section of clad fiber was fabricated to prevent the formation of air gap at the sensor tip after temperature cycles. The methods of fixing the exposed section of clad fiber to the supporting tubes, however, were different between censor $\mathrm{C} 5$ and $\mathrm{C} 4$. For sensor $\mathrm{C} 4$, the exposed section of clad fiber was not glued to any supporting tubes. On the contrary, for sensor C5, only one end of the exposed section of clad fiber was glued to the alumina tube. As shown in Figure $3-46$, the exposed clad fiber was glued to the end of Tube 2, but not to the end of Tube 1 . It is believed that by gluing only one end of the exposed clad fiber to the support tube, the clad fiber can be mechanically more stable, thus minimizing the noise floor problem, while at the same time the bulge section can still function to prevent the formation of air gap at the sensor tip after temperature cycles.

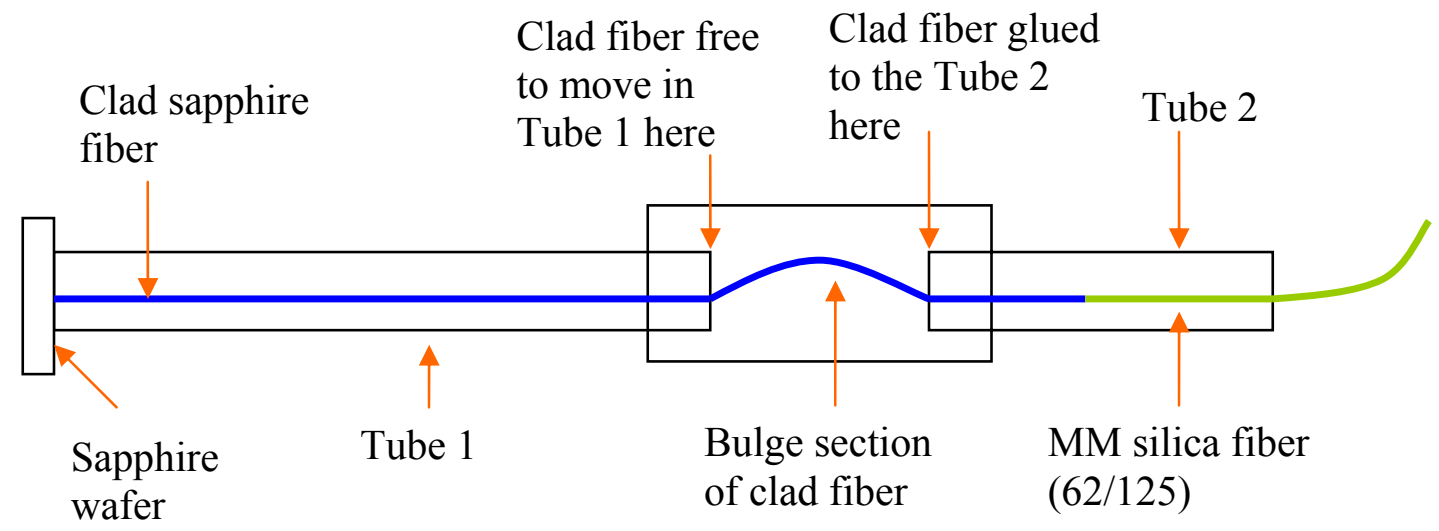

Figure 3-46. The configuration of sensor $\mathrm{C} 5$

Sensor $\mathrm{C} 5$ was tested from room temperature to close to $800^{\circ} \mathrm{C}\left(1472{ }^{\circ} \mathrm{F}\right)$. Due to the short length of sensor $\mathrm{C} 5$, the acrylate coating of the multimode silica fiber melted and smoke started to come out when the testing temperature was approaching about $783{ }^{\circ} \mathrm{C}$ $\left(1,441^{\circ} \mathrm{F}\right)$. Thus the testing was terminated prematurely at $783^{\circ} \mathrm{C}\left(1,441^{\circ} \mathrm{F}\right)$. The results of temperature test of sensor $\mathrm{C} 5$ are shown in Figure 3-47. As can be seen from this Figure, there was no undesirable oscillating behavior present, and most importantly, the noise floor at $594^{\circ} \mathrm{C}\left(1,101{ }^{\circ} \mathrm{F}\right)$ was significantly smaller than that of sensor $\mathrm{C} 4$ at 602 ${ }^{\circ} \mathrm{C}\left(1,116^{\circ} \mathrm{F}\right.$ ) (see Figure 3-44). Another promising behavior of sensor $\mathrm{C} 5$ was that the dc offset of the interference fringes was not changing at temperatures higher than $600{ }^{\circ} \mathrm{C}$ 
$\left(1,112^{\circ} \mathrm{F}\right)$. The new type of silica-to-sapphire splice that can sustain higher operating temperatures may be contributing to more stable interference fringes.

The temperature sensitivities of sensor $\mathrm{C} 5$ at two temperature ranges from $22^{\circ} \mathrm{C}\left(72{ }^{\circ} \mathrm{F}\right)$ to $392^{\circ} \mathrm{C}\left(738^{\circ} \mathrm{F}\right)$ and from $392{ }^{\circ} \mathrm{C}\left(738^{\circ} \mathrm{F}\right)$ to $783^{\circ} \mathrm{C}\left(1441^{\circ} \mathrm{F}\right)$ are listed in Table $3-9$. The two $\Delta \varphi / \Delta \mathrm{T}$ values, i.e., $2.8 \times 10^{-2}$ radians $/{ }^{\circ} \mathrm{C}\left(1.6 \times 10^{-2}\right.$ radians $\left./{ }^{\circ} \mathrm{F}\right)$ and $3.7 \times 10^{-2}$ radians $/{ }^{\circ} \mathrm{C}\left(2.1 \times 10^{-2}\right.$ radians $\left./{ }^{\circ} \mathrm{F}\right)$, are almost the same as those of both sensor $\mathrm{C} 2$ and $\mathrm{C} 4$.

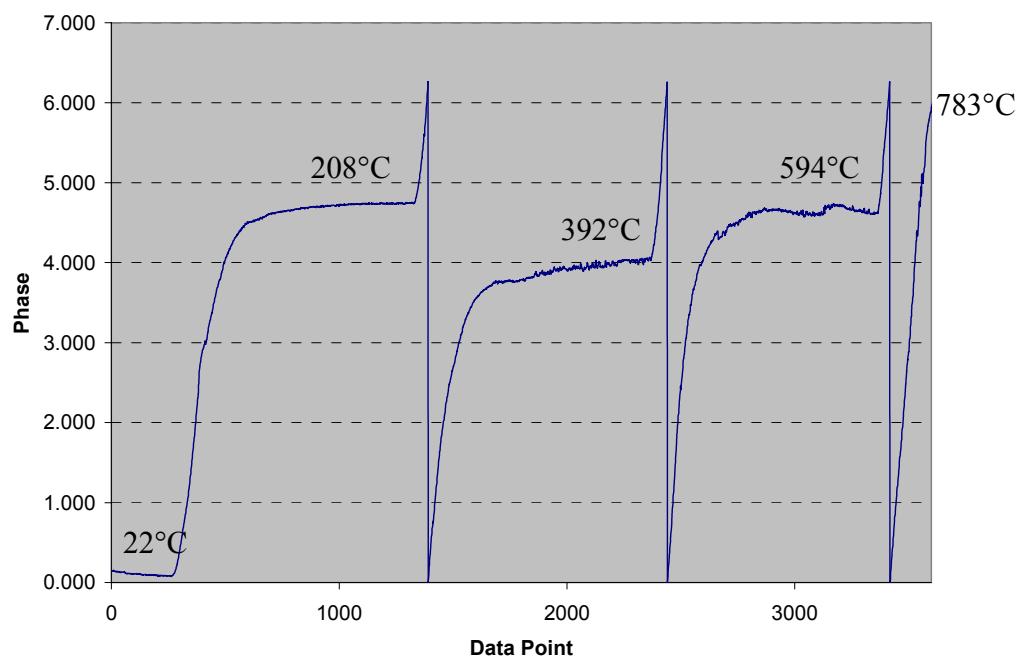

Figure 3-47. Temperature test results of sensor C5

Table 3-9. Temperature sensitivity of sensor C5

\begin{tabular}{|c|c|c|c|}
\hline Temp. Range & $\begin{array}{c}\text { Phase Change } \\
(\Delta \varphi)\end{array}$ & $\begin{array}{c}\text { Temp. Change } \\
(\Delta \mathrm{T})\end{array}$ & $\Delta \varphi / \Delta \mathrm{T}$ \\
\hline $22^{\circ} \mathrm{C}$ to $392^{\circ} \mathrm{C}$ & 10.20 & $370^{\circ} \mathrm{C}$ & $2.8 \times 10^{-2} /{ }^{\circ} \mathrm{C}$ \\
$\left(72{ }^{\circ} \mathrm{F}\right.$ to $\left.738^{\circ} \mathrm{F}\right)$ & & $\left(698^{\circ} \mathrm{F}\right)$ & $\left(1.6 \times 10^{-2} /{ }^{\circ} \mathrm{F}\right)$ \\
\hline $392^{\circ} \mathrm{C}$ to $783^{\circ} \mathrm{C}$ & 14.46 & $391^{\circ} \mathrm{C}$ & $3.7 \times 10^{-2} /{ }^{\circ} \mathrm{C}$ \\
$\left(738^{\circ} \mathrm{F}\right.$ to $\left.1441^{\circ} \mathrm{F}\right)$ & & $\left(736^{\circ} \mathrm{F}\right)$ & $\left(2.1 \times 10^{-2} /{ }^{\circ} \mathrm{F}\right)$ \\
\hline
\end{tabular}

\subsubsection{Sapphire Temperature Packaging Development}

A package was designed to facilitate the sapphire temperature sensor installation and protect the sensor in use. The package for the temperature sensor was designed to withstand the high temperatures and corrosive atmosphere of the furnace, conduct heat efficiently, and protect the sapphire sensing element from dust, shock, and vibration.

The housing and tip illustrated in Figure 3-48 are to be fabricated of inconel, a high nickel alloy with properties making it well suited for service in this extreme environment. Inconel is very resistant to oxidation and corrosion. When heated, inconel forms a stable 
passivating oxide layer protecting it from further attack. Inconel retains its strength at the high temperatures experienced in this environment.

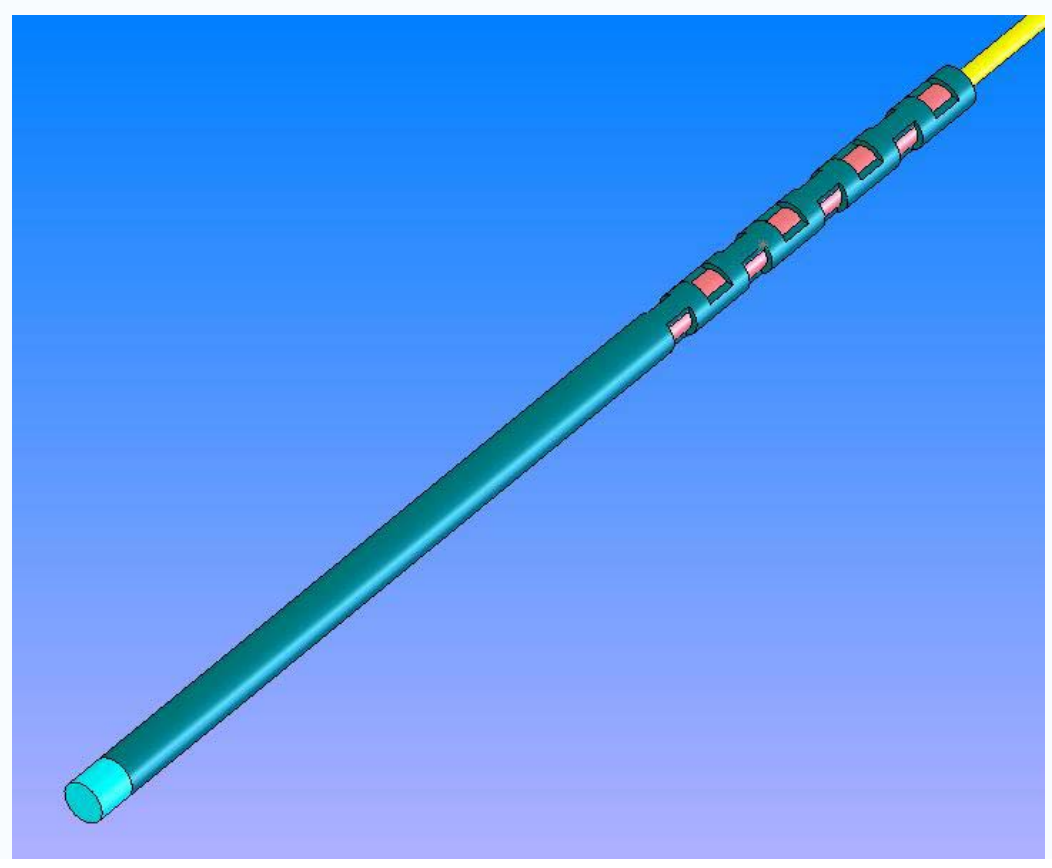

Figure 3-48. Temperature Sensor, Isometric view

The housing is fabricated from inconel tube stock. The diameter was chosen by determining the size of the components it is to contain and choosing the appropriate standard diameter. The length is determined by the sapphire transducer length, location of the splice between the sapphire fiber and the silica fiber, and additional length necessary to effect an adequate strain relief. The cavity surrounding the sapphire sensing element is kept to a minimum in order to minimize the air space and thus the response time of the sensor. Except for the reflecting element and the first millimeter of sapphire, the entire sapphire assembly is encapsulated in a high-temperature cement, as shown in Figure 3-49. The encapsulant reinforces the adhesive joints in the transducer fabrication process, eliminates movement within the housing and reduces the effects of shock and vibration on the assembly. The encapsulant material fills the tube to cover the sapphire / silica splice and to retain the Kevlar fibers of the furcation tubing, providing an anchor for the furcation tubing and allowing the fibers to "float" in the central tube (of the Furcation tubing) from the sapphire to the optical connector. The housing tube is slotted towards its distal end in order to reduce conduction through the Inconel from the hot tip end and maximize cooling for the encapsulant (Figure 3-49). The solid inconel tip is resistance welded to the housing. This is the primary structural element that will be used to attach the sensor to the bracket on the support bars and also to the crossover pipe. 


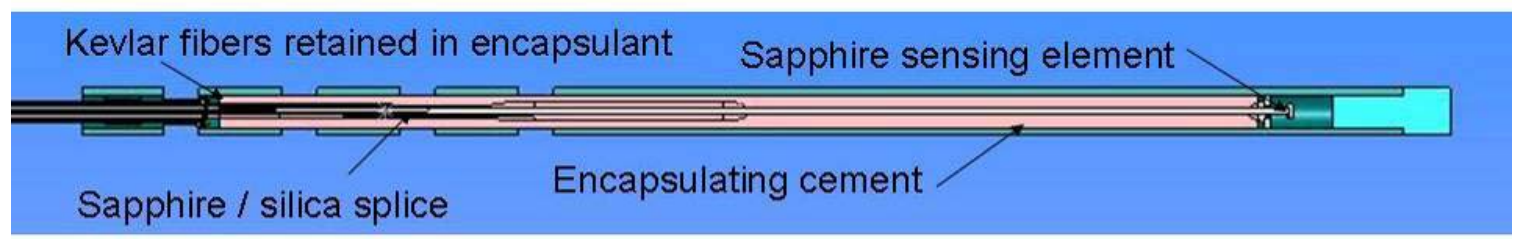

Figure 3-49. Temperature Sensor, Cross section

The sapphire components, inconel tubing, and encapsulant are all capable of withstanding temperatures in excess of $2000^{\circ} \mathrm{F}$. Through testing, it was determined that the sapphire / silica splice can withstand temperatures up to $\sim 850{ }^{\circ} \mathrm{F}\left(450{ }^{\circ} \mathrm{C}\right)$. A high temperature laboratory furnace was set to $2000^{\circ} \mathrm{F}\left(1,093{ }^{\circ} \mathrm{C}\right)$. A 4-bore ceramic insulator, equipped with a thermocouple, was installed in an inconel tube with in order to develop a temperature profile, and thus determine a "safe" location for the temperature critical splice. The mock housing was installed through the access hole on top of the furnace. The mock housing was allowed to soak at temperature for an hour before readings were taken. The housing was inserted in $1 / 4$ " steps into the furnace. Only when the end of the stainless steel housing was within $1 / 4$ " of the top of the fitting on top of the furnace (and the thermocouple was within $1 \frac{1 / 4}{4}$ ) did the thermocouple temperature reading excede $850^{\circ} \mathrm{F}\left(\sim 450^{\circ} \mathrm{C}\right)$. The housing was pulled back $1 / 4$ " and the reading decreased to $815^{\circ} \mathrm{F}$ $\left(435^{\circ} \mathrm{C}\right)$.

\subsubsection{Tests of sapphire temperature sensors}

Two sapphire temperature sensors, G4 and G9, were fully packaged in ceramic/inconel housings. Details of the packaging were provided in the last semiannual progress report; the final packaged form of sensor G4 is shown in Figure 3-50. The waveform for sensor G9 is shown in Figure 3-51 below. The plot shows the waveform at three different temperatures; this plot demonstrates that the waveform shifts to increasing wavelengths as temperature increases.

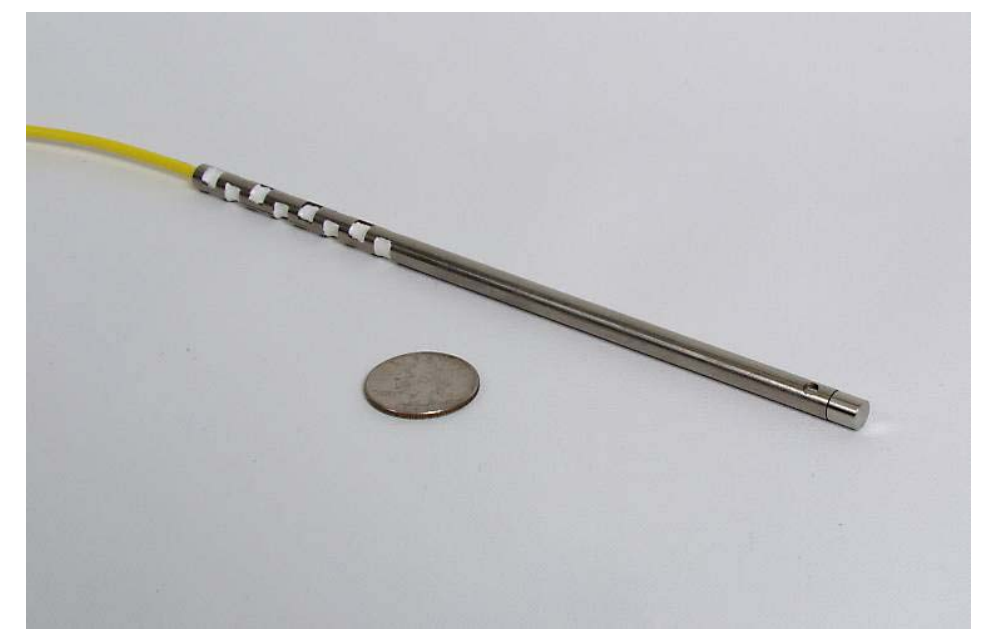

Figure 3-50. Photograph of sapphire temperature sensor G4 before temperature testing. 
Figure 3-55 shows a plot of the Fast Fourier Transform (FFT) for the same sensor. By taking the FFT of the spectrum, the relative strength of the different frequency components can be examined. In Figure 3-55 below, the FFT plot is presented as a function of cavity length in microns (in vacuum); since the fringe frequency is a linear function of the cavity length, the conversion to cavity length has been performed to aid in the interpretation of the FFT data. The ordinance is presented as phase in radians. In this plot, jumps in the output that occur when the phase equals $n 2 \pi$, where $n$ is an integer, have been removed (unwrapped). In a perfect sinusoid, a shift of $2 \pi$ radians results in an exact replication of the original signal.

The figure shows that the strongest frequency component is due to a cavity of approximately $234 \mu \mathrm{m}(0.009 \mathrm{in})$, corresponding to the optical path length of the sapphire wafer. The sensor was heated to $1200^{\circ} \mathrm{C}\left(2192^{\circ} \mathrm{F}\right)$ using the experimental temperature profile shown in Figure 3-56. The phase of the optical signal tracks the temperature very closely. The plot shown in Figure 3-57 shows the response of the sensor, which is fairly linear.

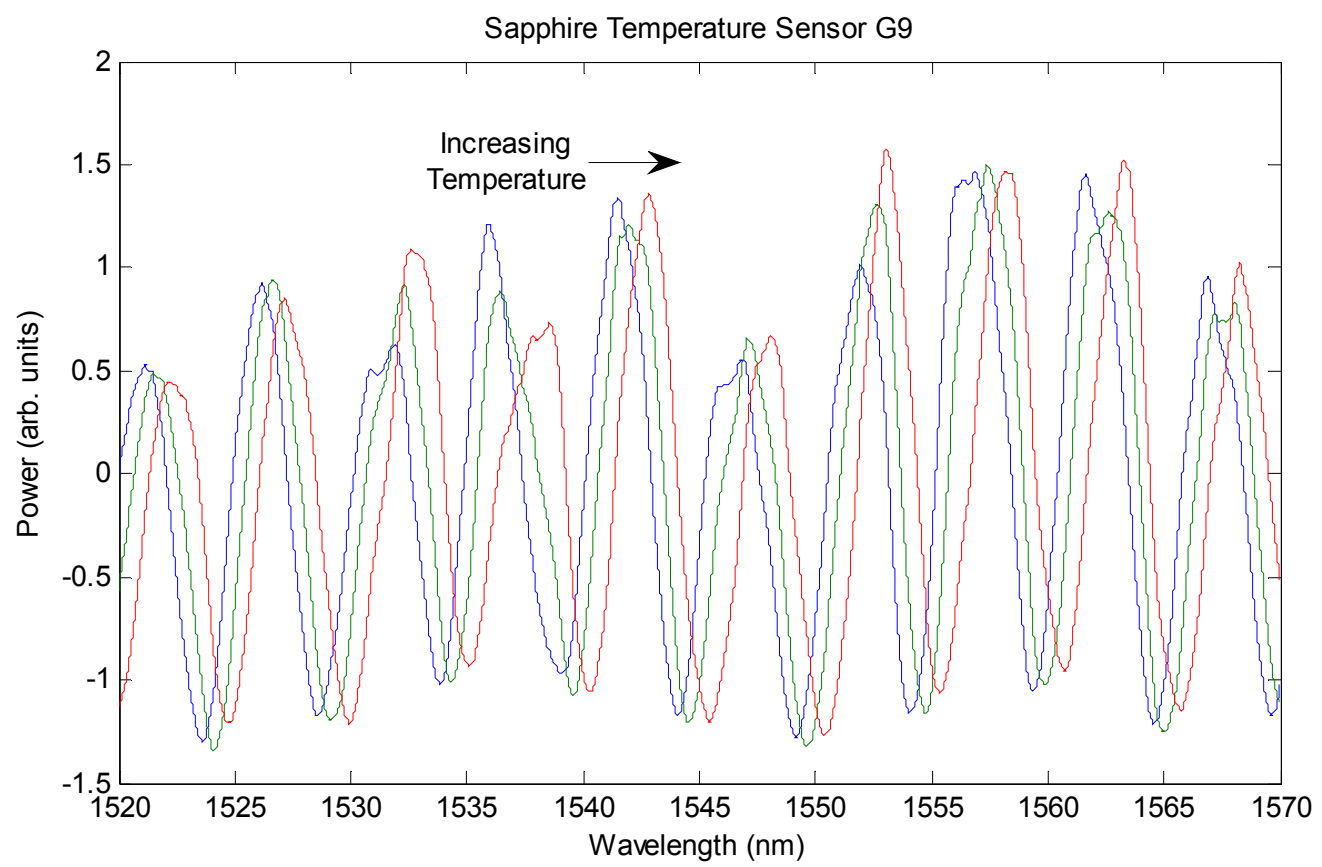

Figure 3-51. Output of sapphire temperature sensor G9, using a VectorLight 200 to convert the optical signal to an electronic output.

\subsubsection{Fabrication of sapphire temperature sensors for field test}

Two sapphire temperature sensors were assembled and packaged for installation in the B\&W CEDF combustion test facility for field tests. In the design used, a sapphire wafer with the c-plane oriented with the wafer thickness was bonded to the end of a c-plane 
sapphire fiber. In this assembly, the sapphire wafer functions as a Fabry-Perot cavity, and the optical path length of the cavity changes with varying temperature due to thermal expansion of the sapphire. Figure 3-52 illustrates the assembly schematically.

The temperature sensors and their packaging were designed for instrumentation of two separate locations in the $2^{\text {nd }}$ tube bank of the CEDF. As explained in 2.5.2, the CEDF replicates operating conditions of steam generating power plants, including burners for pulverized coal and a convection pass with simulated superheater tubes. In the CEDF plant, these tubes are not used for heat transfer and do not transport superheated steam. The tubes employ a double wall assembly, as illustrated in Figure 3-53. An inner steel tube carries boiling water, and is surrounded by an outer steel tube which is subjected to normal gas path temperatures.

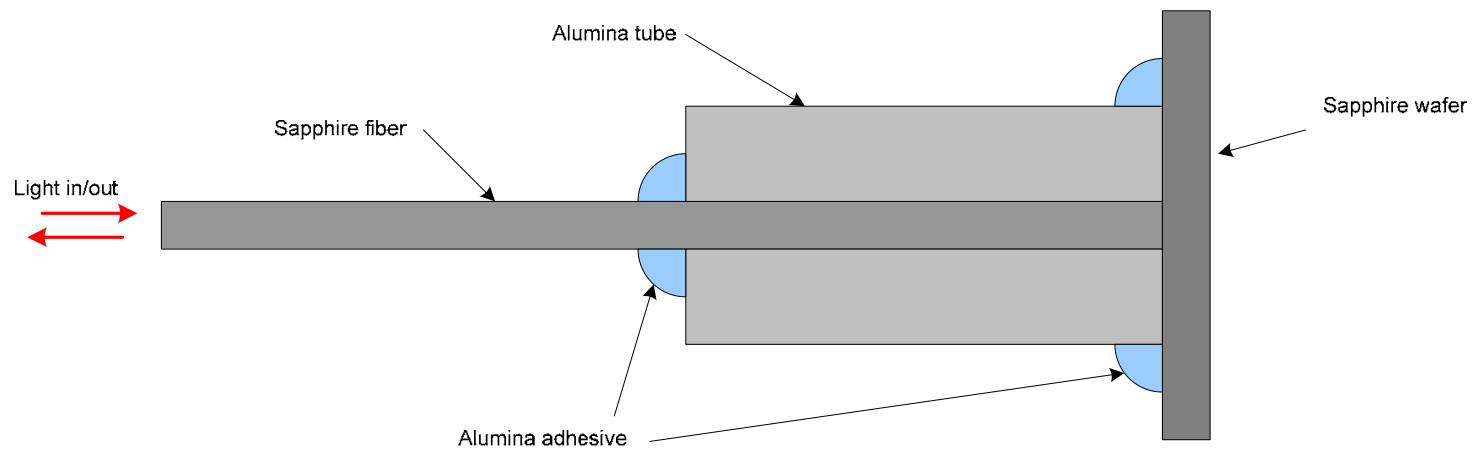

Figure 3-52. Schematic of sapphire temperature sensor optical assembly.

The first temperature sensor was designed to be mounted with the sensor tip (the end of the package containing the sapphire wafer) onto a simulated superheater tube adjacent to the boiler wall and along the trailing row of tubes in tube bank \#2. This tube is in close proximity to a soot blower and to a manway for access to the tubes. Gas temperatures in the vicinity of tube bank $\# 2$ are expected to be approximately $982^{\circ} \mathrm{C}\left(1800^{\circ} \mathrm{F}\right)$ during peak power production.

In the temperature sensor assembly, a splice is used to join the sapphire fiber with a longer silica fiber. In order to locate this splice outside the boiler wall in order to keep the splice temperature below $450^{\circ} \mathrm{C}\left(842^{\circ} \mathrm{F}\right)$, the length of sapphire fiber was set to $69 \mathrm{~cm}$ (27 in). This permitted the splice location to extend approximately $10 \mathrm{~cm}$ (4 in) outside the outer surface of the boiler wall. Previous laboratory tests have demonstrated that the sapphire-to-silica fiber splice can survive temperatures up to $450{ }^{\circ} \mathrm{C}\left(842^{\circ} \mathrm{F}\right)$.

The second temperature sensor was designed to just penetrate the boiler wall and measure the gas temperature along the inner wall of the boiler. In order to locate the splice $10 \mathrm{~cm}$ (4 in) outside the boiler wall, this required that the sapphire fiber be $56 \mathrm{~cm}$ (22 in) long. 


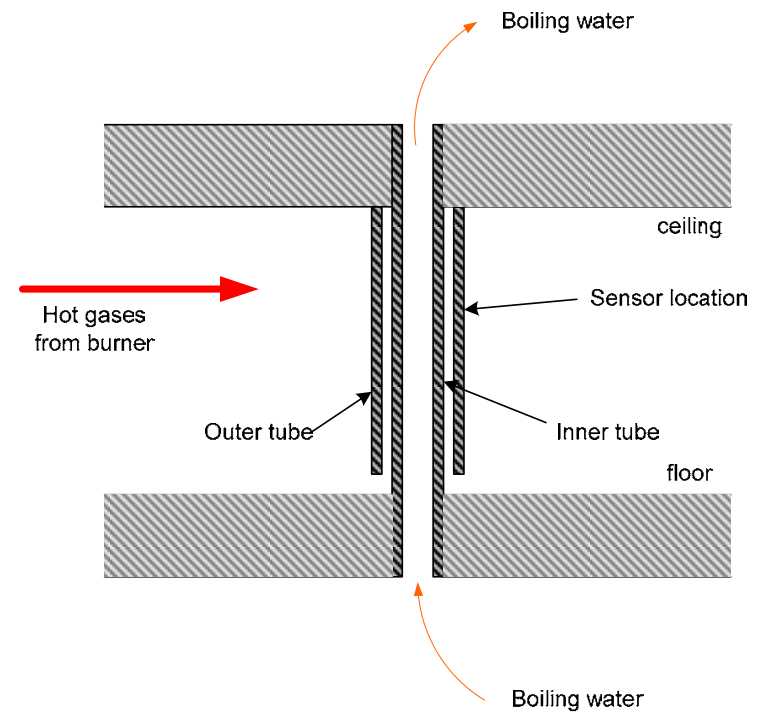

Figure 3-53. Cross-section schematic of CEDF simulated superheater tube, showing planned sensor location.

The sapphire fibers used in the fabrication of the field test sensors were not clad, due to the lengths required for the fibers. Currently, the maximum length for clad fibers prepared by Prime Research's spinel dipping process is approximately $15 \mathrm{~cm}(6 \mathrm{in})$, limited by the size of the furnace used for sintering the cladding.

The temperature sensors' optical assemblies (sapphire wafer, sapphire fiber, and splice) were packaged inside inconel tubes, which were sealed on the wafer end with inconel plugs. Standard telecommunications cable jacketing of the loose tube design was used to protect the silica fiber. The jacketing had an outer PVC sheath with an inner tubing of polypropylene, with a Kevlar aramid yarn between them. The Kevlar strength member was attached to the inconel tube using an epoxy. A polyolefin heat-shrink tube was used to protect this joint between the tube and the jacket and to provide strain relief in the event of bending.

To facilitate mounting of the long temperature sensor onto the CEDF tubes, 1 "x2"x0.025" straps were cut from 310 stainless steel sheet stock and bent to the same curvature as the CEDF tube. One strap was resistance welded to the end of the inconel tube of the long temperature sensor. A Dremel tool with a grinder attachment was used to thin the 310 SS straps at eight points on the strap to a thickness of approximately 0.010 ", to improve resistance welds at those points. During installation, the strap will be resistance welded to the CEDF tube, holding the temperature sensor against the CEDF tube. A photograph of the finished assemblies before addition of the strap to the long temperature sensor is shown in Figure 3-54. 


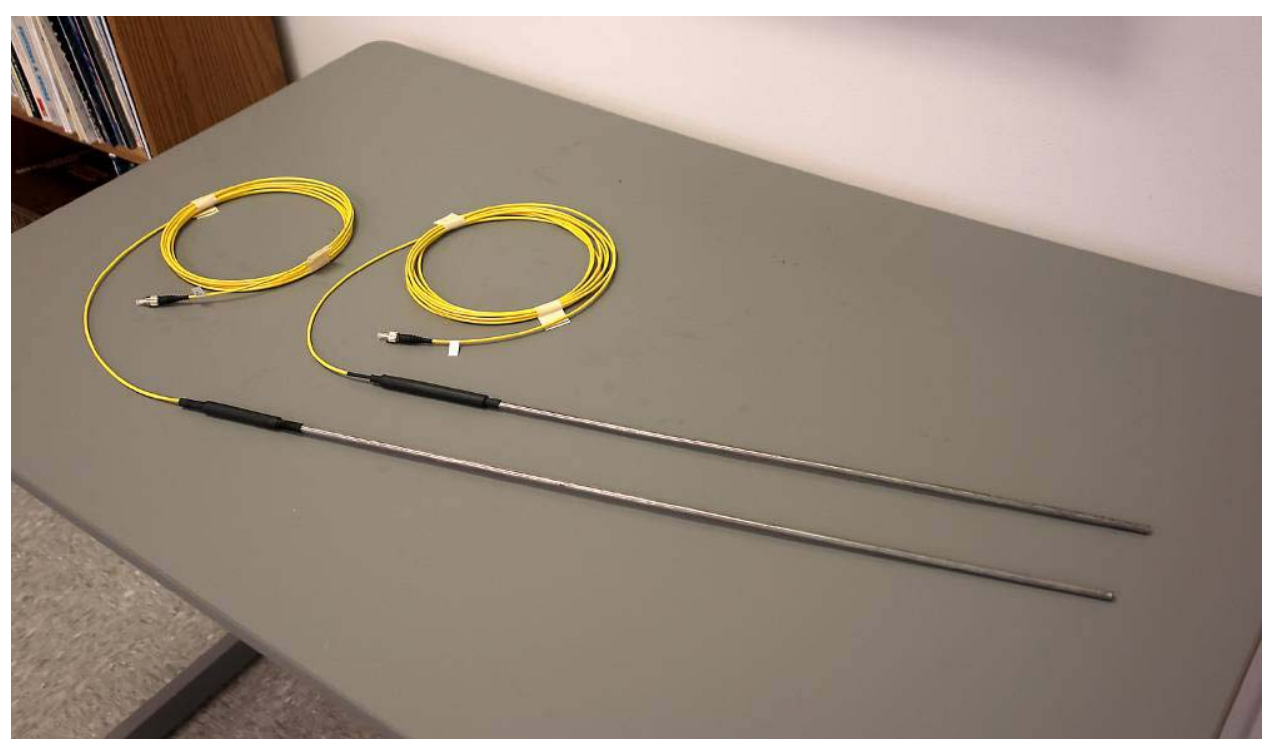

Figure 3-54. Packaged sapphire temperature sensors, before addition of 310SS mounting straps.

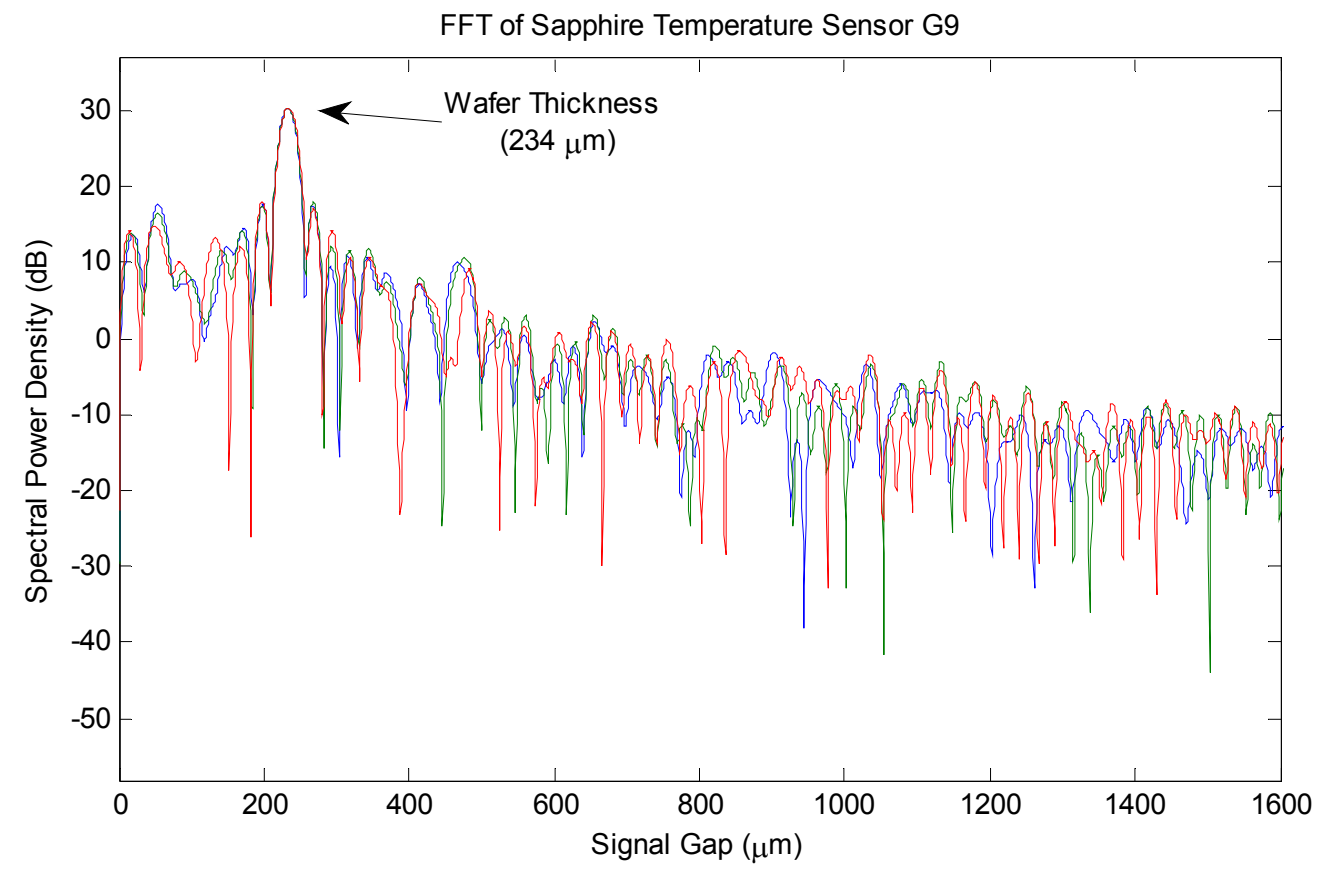

Figure 3-55. An FFT of the spectrum shown in Figure 3-51. The $x$-axis values represent the cavity length (in microns) that would produce the frequency observed, and the $y$-axis values represent the signal strength in $\mathrm{dB}$ observed for the frequency generated by the cavity length indicated. 


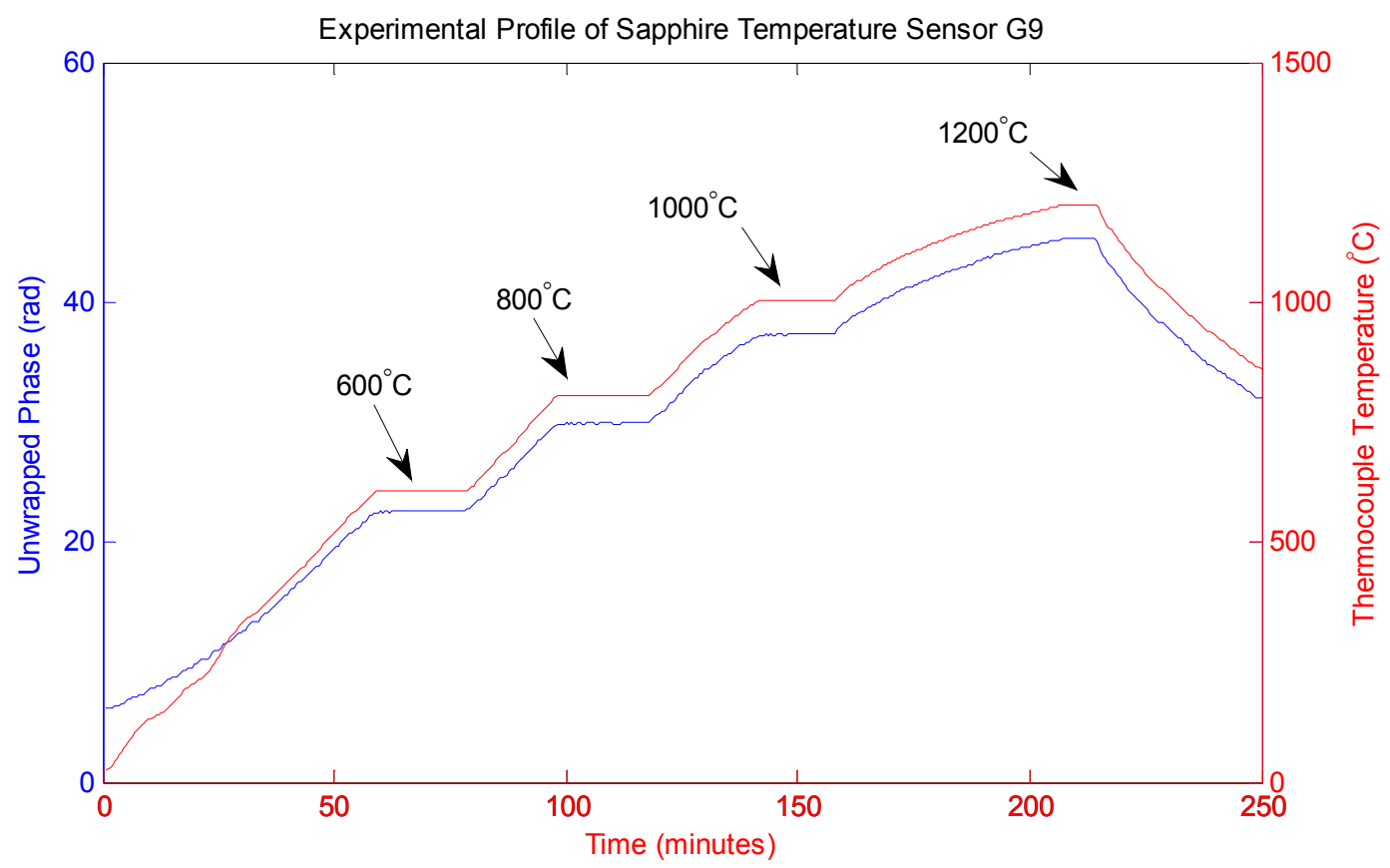

Figure 3-56. Time-temperature profile of furnace used to test sensor G9 (in red), and output of optical temperature sensor (expressed as phase, in blue).

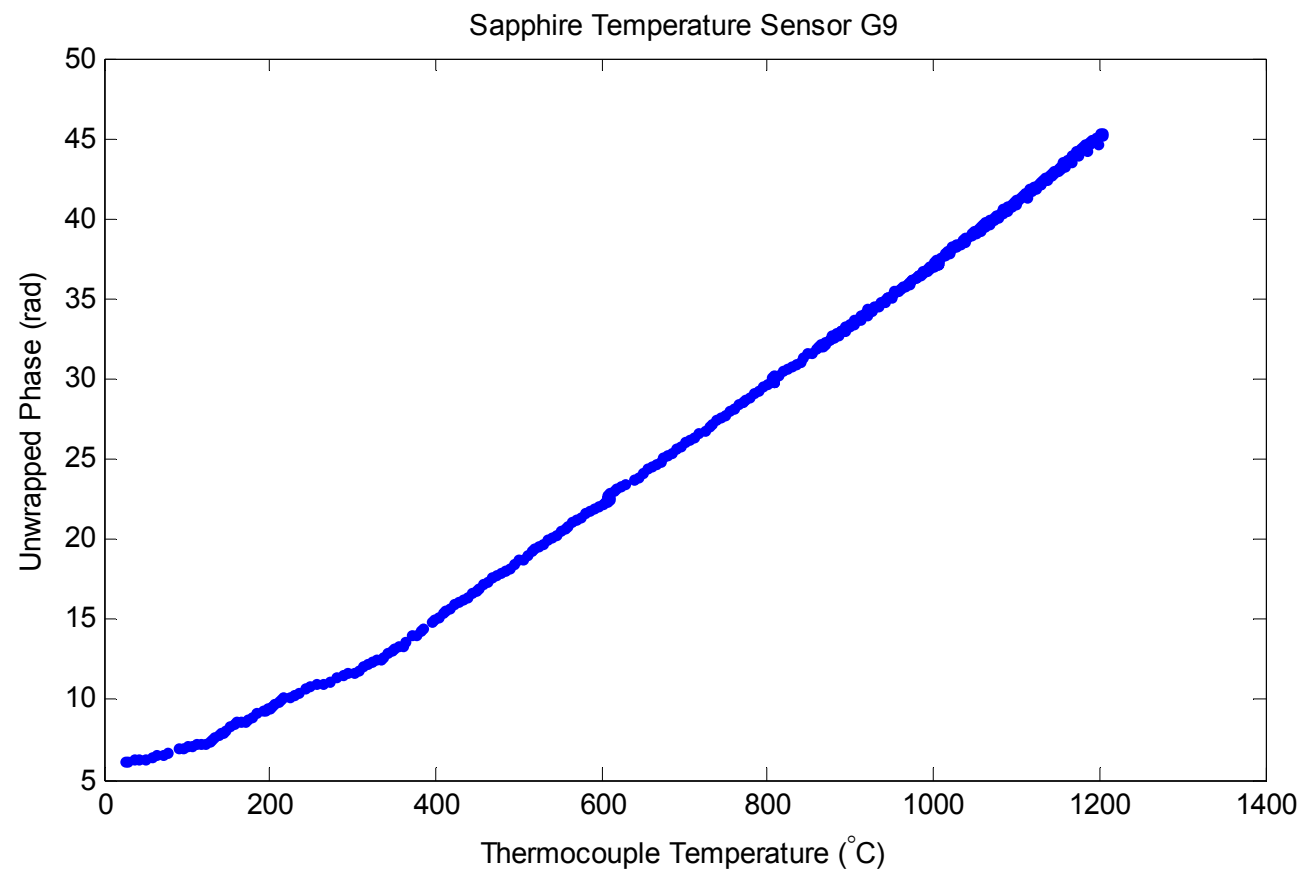

Figure 3-57. Phase output from Figure 3-56 expressed as a function of furnace temperature. 
Sensor G4 was then fabricated and packaged using the same in methods and designs as sensor G9. To test the sensor, it was heated to $1200^{\circ} \mathrm{C}\left(2192{ }^{\circ} \mathrm{F}\right)$ using the experimental temperature profile shown in Figure 3-58. The phase of the optical signal tracks the temperature very closely. The plot shown in Figure 3-59 shows the response of the sensor, which is fairly linear.

After completion of this test, interference fringes could no longer be obtained with Sensor G4. Examination of the sensor indicated that the probable failure was in the splice between the sapphire fiber and the silica fiber. Previous tests had shown that when the splice is subjected to temperatures exceeding $450^{\circ} \mathrm{C}\left(842^{\circ} \mathrm{F}\right)$, the glass joining the fibers can soften, and if stress is applied to the fiber is, they can separate. In subsequent tests of the prototype sensors, the area around the splice was instrumented with a thermocouple to monitor the temperature there.

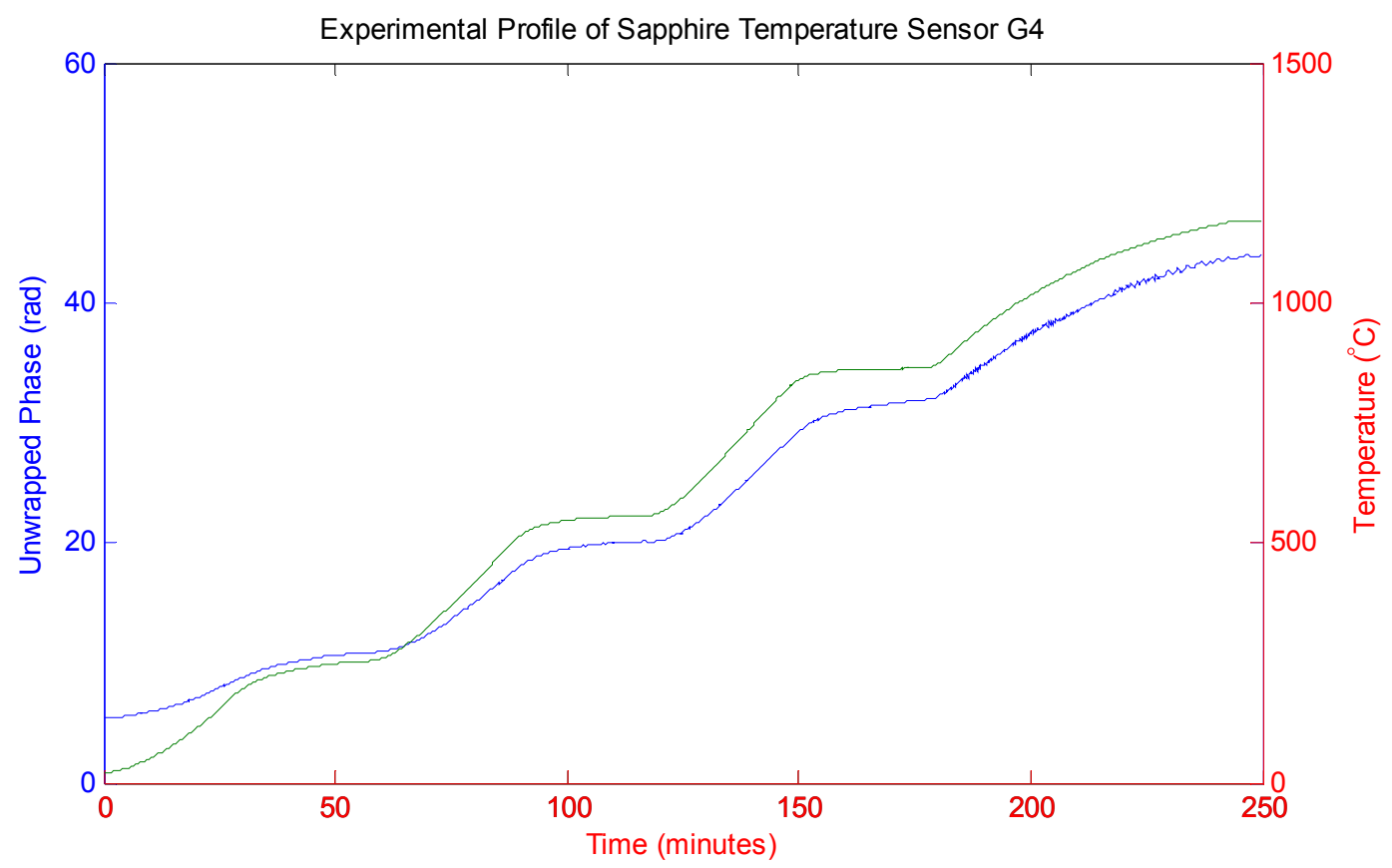

Figure 3-58. Time-temperature profile of furnace used to teach and test sensor G4 (in green), and output of optical temperature sensor (expressed as unwrapped phase, in blue). 


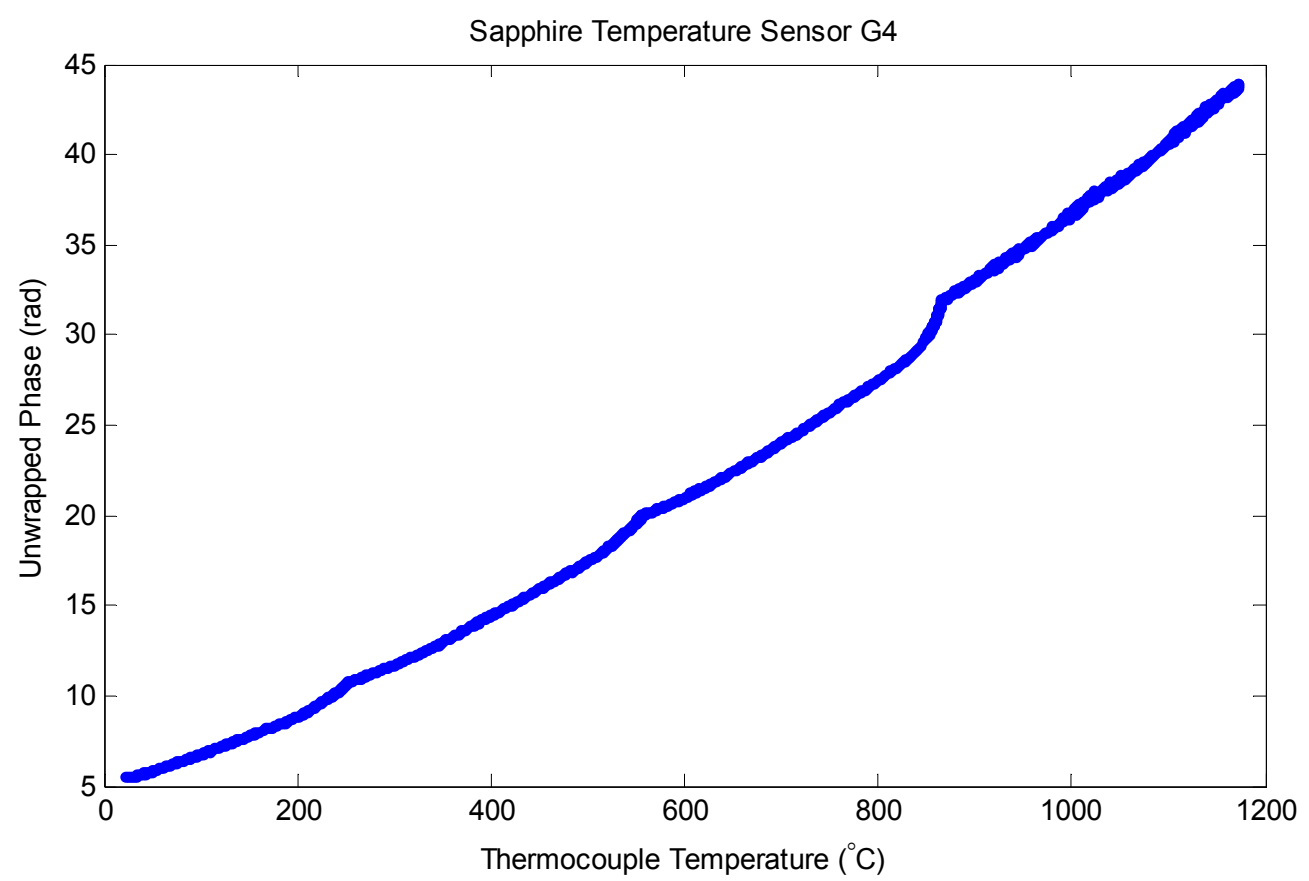

Figure 3-59. Phase output from Figure 3-58 expressed as a function of furnace temperature.

\subsection{Sapphire Strain Gage Development}

Among the many fiber-optic sensing schemes, Fabry-Perot interferometric sensors have long demonstrated several advantages such as high sensitivity and small size. During this program, efforts were directed to the development of methods for fabrication of sapphire Fabry-Perot sensors for measurement of mechanical strain in coal-fired boiler plants.

\subsubsection{Theory of Operation}

The schematic in Figure 3-60 of a sapphire fiber Fabry-Perot strain gage system shows a light source such as a light emitting diode (LED) coupled to a multimode silica glass fiber $(62.5 / 125 \mu \mathrm{m})$. A 50/50 fused coupler is used to bring the light into and away from the Fabry-Perot sensor. The light propagating from the broadband LED light source to the lead-in sapphire fiber is partially reflected at R1 (7\%) at the first sapphire to air endface. The transmitted light travels through the air-gap and is also partially reflected at R2 (7\%) at the endface of the target sapphire fiber. An interference signal is then generated by these two reflected waves, with their phase difference as a function of the length of the air-gap. To prevent further reflection, the target sapphire fiber exit end is polished to a 45 degree angle. At the receiving end, a computer controlled interrogator (spectrometer or swept laser) acquires the interference signal in the wavelength domain so that the absolute cavity length of the air-gap can be measured. Note that the lead-in sapphire fiber only needs to be long enough to get away from the hot zone, at which point the silica multimode fiber can be used for the rest of the length of fiber back to the coupler and light source and interrogator. 


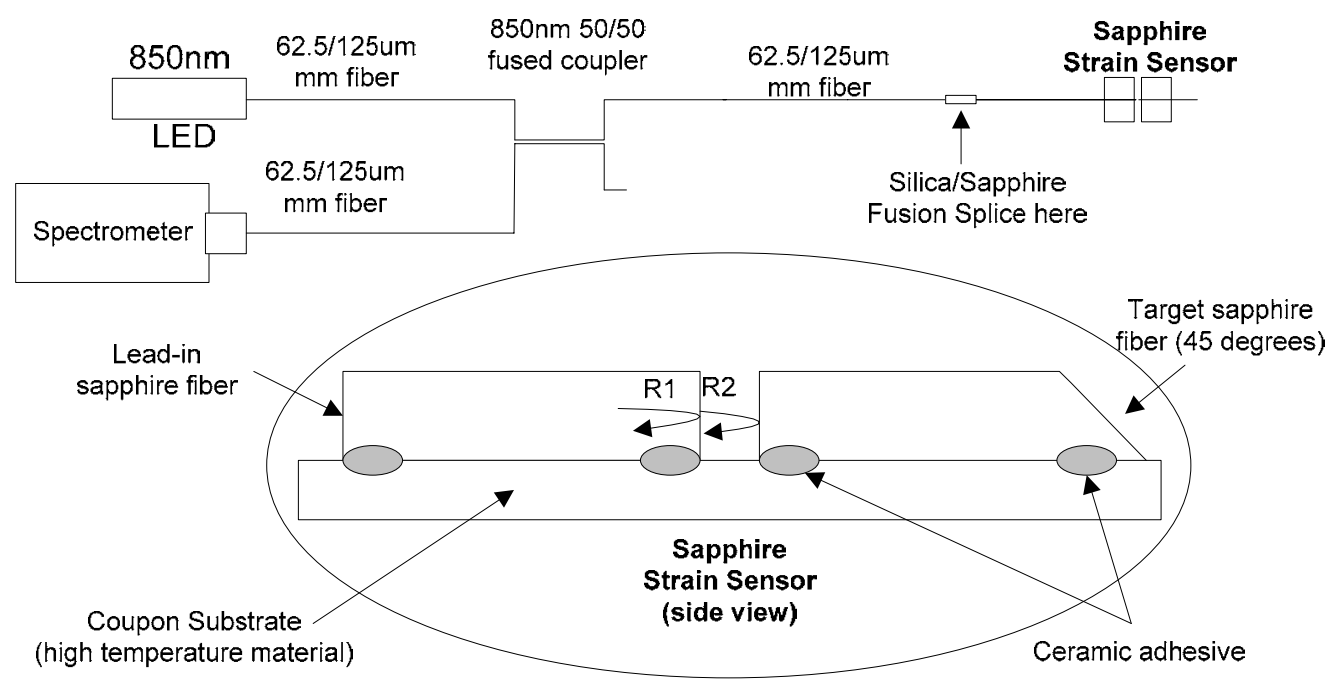

Figure 3-60. Schematic of sapphire fiber strain gage system

\subsubsection{Power Plant Requirements}

The study of sapphire fiber strain gages began through discussions with Babcock and Wilcox $(B \& W)$ regarding requirements for supercritical coal-fired power plants. These requirements included materials to be monitored for strain, testing environments, and locations within the plant. B\&W reports as well as meetings between Prime Research and $\mathrm{B} \& \mathrm{~W}$ provided specifications for the sensor development listed in Table 3-10. Construction of the supercritical power plants includes various materials including those identified in Figure 3-62, which shows the maximum allowable stresses at different temperatures for those materials. These specifications and materials will guide Prime Research to meet the goals of this project to include strain measurements in a supercritical power plant facility.

Table 3-10. Specifications provided by $B \& W$ for strain gages

\begin{tabular}{|l|l|}
\hline \multicolumn{1}{|c|}{ Specification } & \multicolumn{1}{c|}{ Description } \\
\hline Reference temperature & $23^{\circ} \mathrm{C}\left(73.4^{\circ} \mathrm{F}\right)$ \\
\hline Maximum Strain & $1,000-10,000 \mu \varepsilon$ \\
\hline Environment & \\
\hline Oxidizing & $\mathrm{CO}, \mathrm{SO}_{2}, \mathrm{O}_{2}, \mathrm{CO}_{2}, \mathrm{NO}_{\times}, \mathrm{N}_{2}, \mathrm{HCl}, \mathrm{NaO}, \mathrm{CaO}, \mathrm{SiO}$ \\
\hline Reducing & $\mathrm{H}_{2} \mathrm{~S}, \mathrm{CO}_{2} \mathrm{SO}_{2}, \mathrm{O}_{2}, \mathrm{CO}_{2}, \mathrm{NO}_{\times}, \mathrm{COS}, \mathrm{HCN}, \mathrm{N}_{2}, \mathrm{HCl}$ \\
\hline Steam & $\mathrm{pH} 9-11,3500 \mathrm{psi}$ \\
\hline Water & $3500 \mathrm{psi}, 565.6^{\circ} \mathrm{C}\left(1050^{\circ} \mathrm{F}\right)$ \\
\hline
\end{tabular}




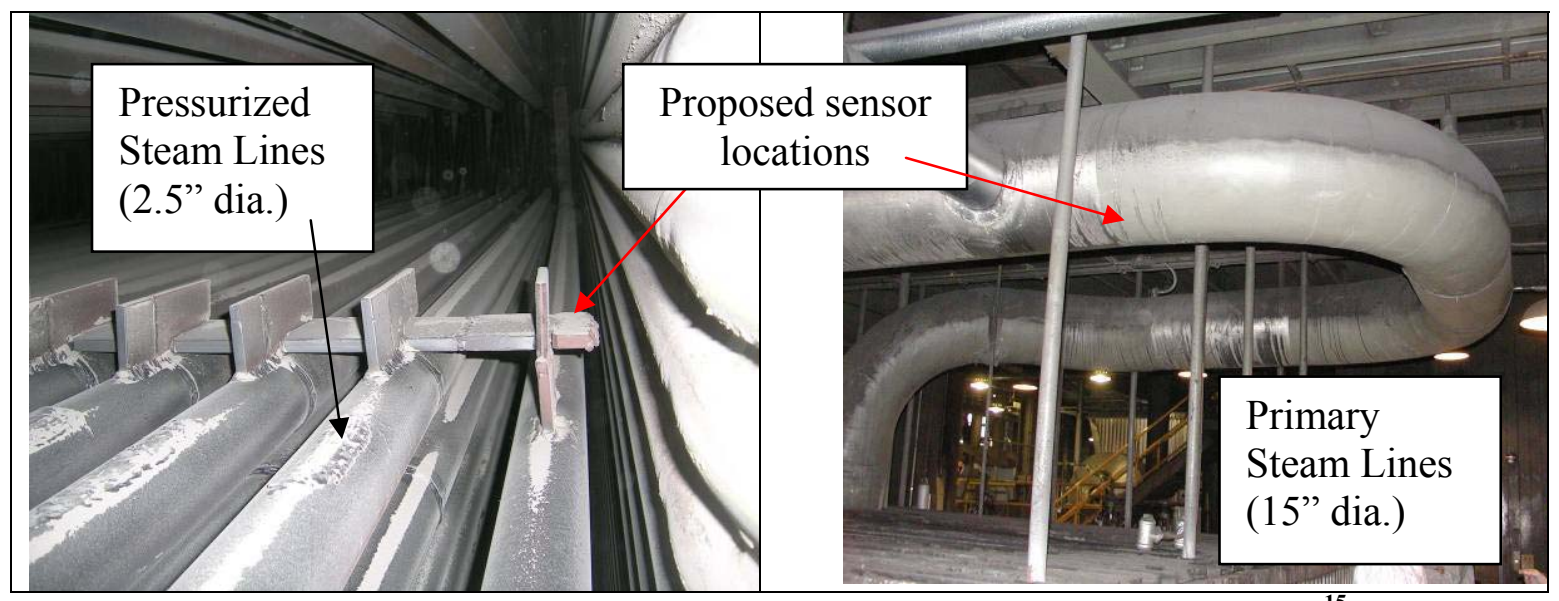

Figure 3-61. Proposed sensor locations at the Power Plant in Hamilton, $\mathrm{OH}^{15}$

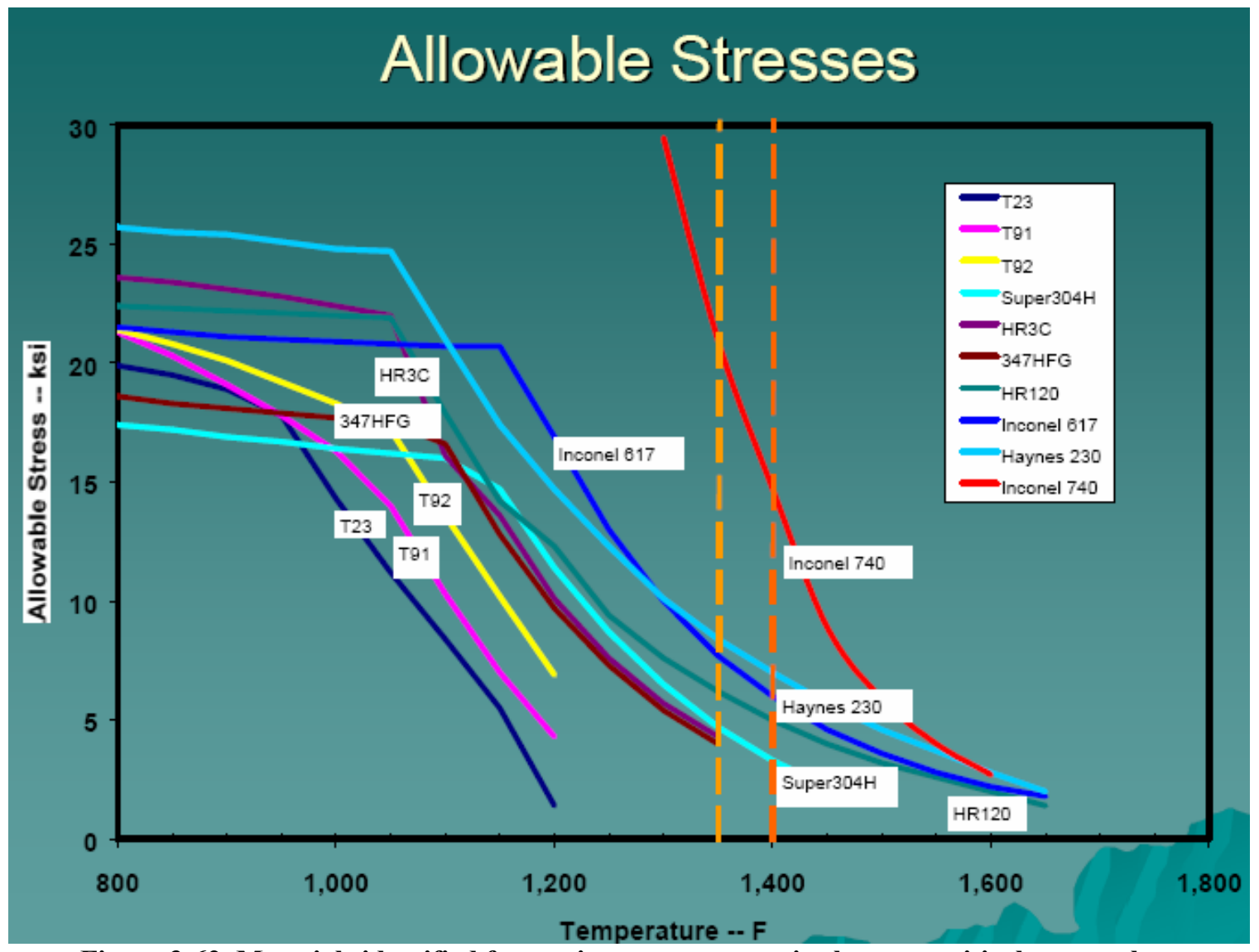

Figure 3-62. Materials identified for strain measurements in the supercritical power plant application ${ }^{15}$

\footnotetext{
${ }^{15}$ Mohn, Walt (Babcock \& Wilcox Company Barberton, OH). Presentation: Overview of the Ultrasupercritical Boiler Materials Development Program. Project duration Oct 01 - Sept 06.
} 


\subsubsection{Development of sapphire strain gages for power plant instrumentation}

Early attempts at fabrication of sapphire strain gages in this program employed a threeaxis flexure-type micropositioner to align the two sapphire fibers, as shown in Figure 3-63. Due to the very small tolerance permissible on angular alignment required to get good fringes from the air gap of the strain gage, fabrication of strain gages using this method proved difficult.

Therefore, a new approach was investigated which allowed the sapphire fiber to be bonded first to a v-groove, with the air gap subsequently created by using a special blade on a dicing saw to cut a cavity in the sapphire fiber. A single fiber is installed on the coupon and a blade is used to cut the fiber. For proof of concept experiments, a silicon wafer was used for the coupon; a concept drawing illustrating a curved metal coupon for installation of the strain gage on a boiler superheater tube is shown in Figure 3-64. Since the fiber is already in place and stabilized on the coupon, the fiber will not move out of alignment during the cutting process. After the cutting is complete, the fibers will maintain parallelism and the blade will create two perpendicular faces separated by an air gap.

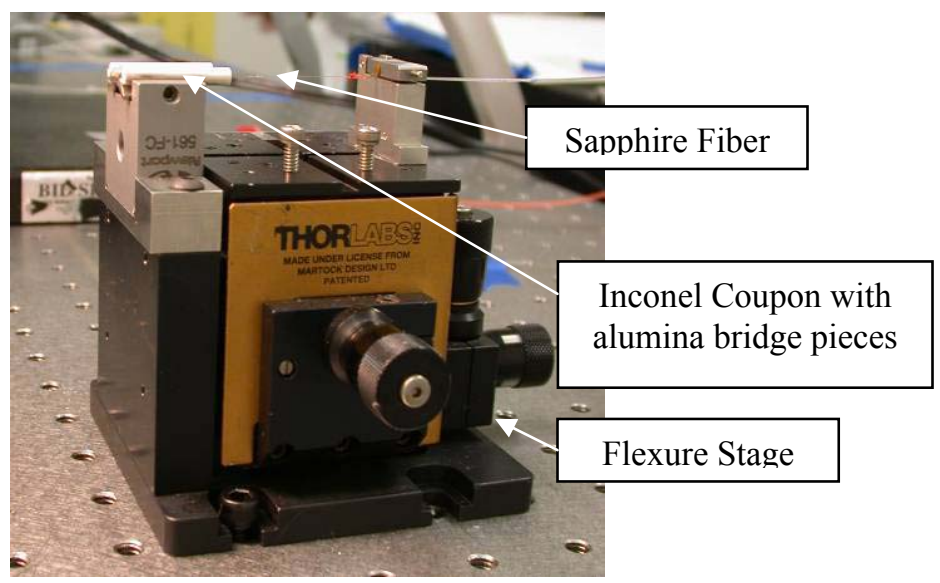

Figure 3-63: Fiber alignment on flexure

stage

An initial test was conducted on four $125 \mu \mathrm{m}$ diameter silica optical fibers bonded into grooves cut in a silicon wafer using several dicing blades, each with a different diamond grit size. Perpendicular grooves were cut to accommodate the blade depth and width so that the blade would only come in contact with the fibers. Variations in the groove widths and depths were used to understand the test set up. Additionally, various blade rotational speeds and travel speeds were tested. 


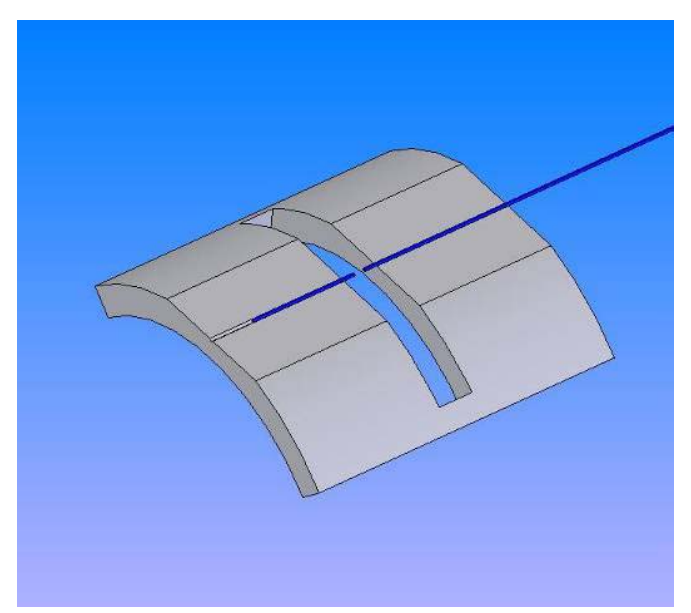

Figure 3-64. Strain gage coupon design

Observations of the results were made under an optical microscope, shown in Figure 3-65. The blade produced a perpendicular fiber end face and maintained parallel alignment of the now two fibers. However, the surface finishes of the fiber end faces were not optically polished for optimum transmission. Hence, when the fiber arrangement was analyzed as a sensor, a low power reading could be detected, but an interference pattern could not be obtained. This was most likely due to the surface finish of the fiber end faces. Additional variables such as blade speeds, grit sizes, and fiber bonding methods have been tested. By systematically varying the combination of these variables, the proof-of-concept data shown above in Figure 3-66 through Figure 3-69 were obtained.

Figure 3-66 shows the sensor test setup for a cut-away strain gage and the initial air gap at room temperature. The spectra for this test were captured using a Micron Optics CTS swept laser interrogator. Note that the sensor shown and tested was a prototype, and not constructed with the final coupon design that would be used for installation in a power plant.

As the temperature increased in the aluminum block, the air gap length increased due to thermal expansion of the block, causing the fringes shown in Figure 3-67 to move to the right. By taking the FFT of the same set of fringes, the fractional frequency can be seen in Figure 3-68 moving to the right as expected, since the increase in air gap causes more fringes to appear in the $50 \mathrm{~nm}$ range of the CTS interrogator. The fractional frequency is a measure of the number of fringes (sinusoidal periods) displayed in the CTS output The CTS records 20,000 data samples for each output spectrum, so that each sample represents an interval of $0.0025 \mathrm{~nm}$. As an example, the period of the light blue trace in Figure 3-67 $\left(51^{\circ} \mathrm{C}\right.$, or $\left.124^{\circ} \mathrm{F}\right)$ is approximately $3.8 \mathrm{~nm}$. Since the CTS output is $50 \mathrm{~nm}$ wide, there are $50 \mathrm{~nm} / 3.8 \mathrm{~nm}$ or 13.2 fringes in the full $50 \mathrm{~nm}$ wide output of the CTS. By normalizing the number of fringes to the number of data samples $(20,000)$ in the full output of the CTS, we derive a fractional frequency of $13.2 / 20000=6.6 \times 10^{-4}$. Therefore, as the air gap increases due to heating of the aluminum block, the period of the fringes decreases, so that the fractional frequency increases, and the peak associated with the fringe shifts to the right in Figure 3-68. 


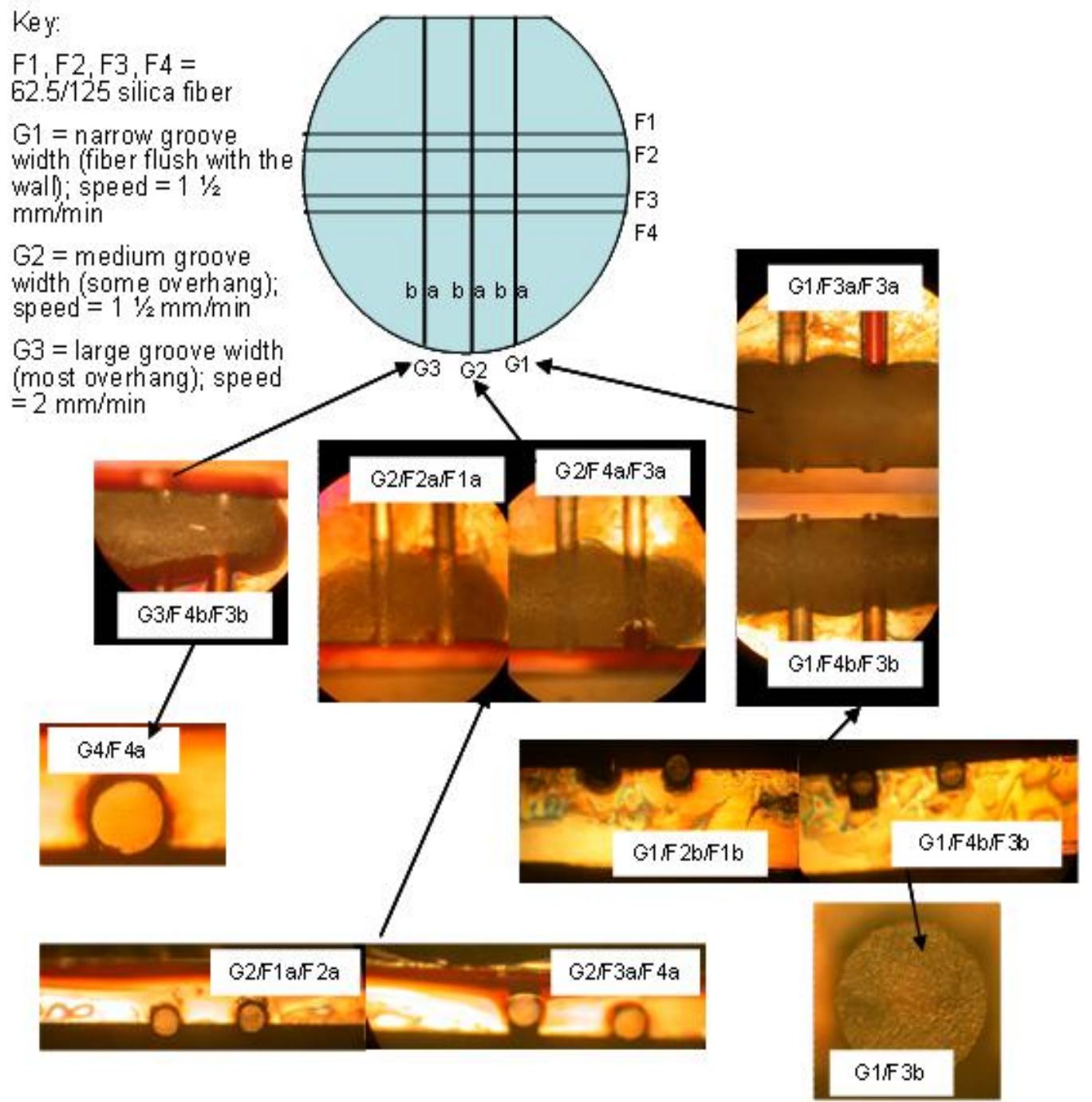

Figure 3-65. Initial cut fiber test set up with dicing equipment

To show that the relative gap changes predictably with temperature, a calculation of the CTE effects of the aluminum substrate was conducted based on the model shown in Figure 3-70. Table 3-11 shows the results of this calculation. Referencing Figure 3-66, the fibers were assumed to be attached at the edge of the coupon and the gap was determined to be $150 \mu \mathrm{m}$. The changes in the length of the gap calculated with the model were realistic values. The actual gap change could not be measured during this feasibility test; hence, further testing will be preformed. 


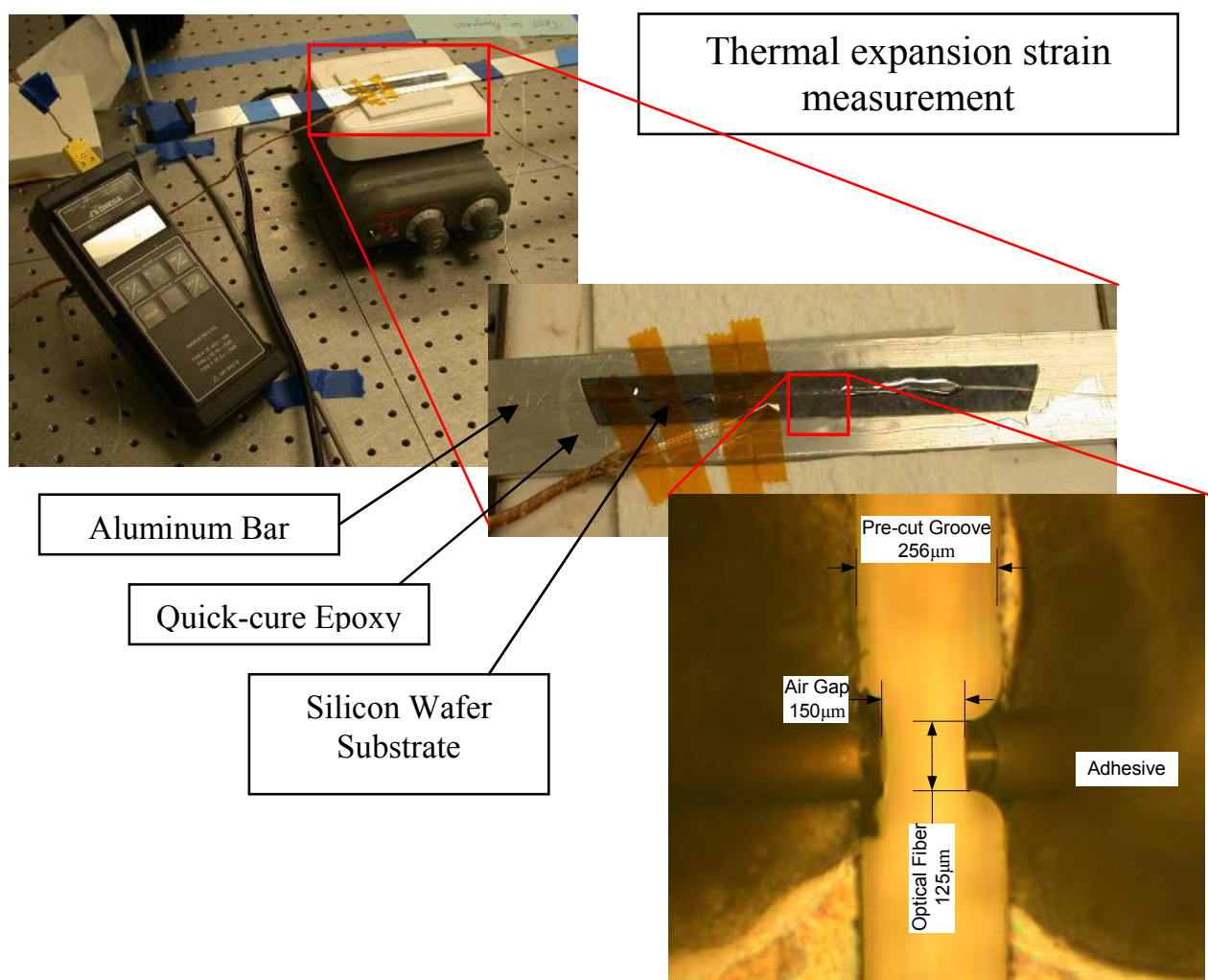

Figure 3-66. Cut Away strain gage

\section{Cut away strain gage over temperature}

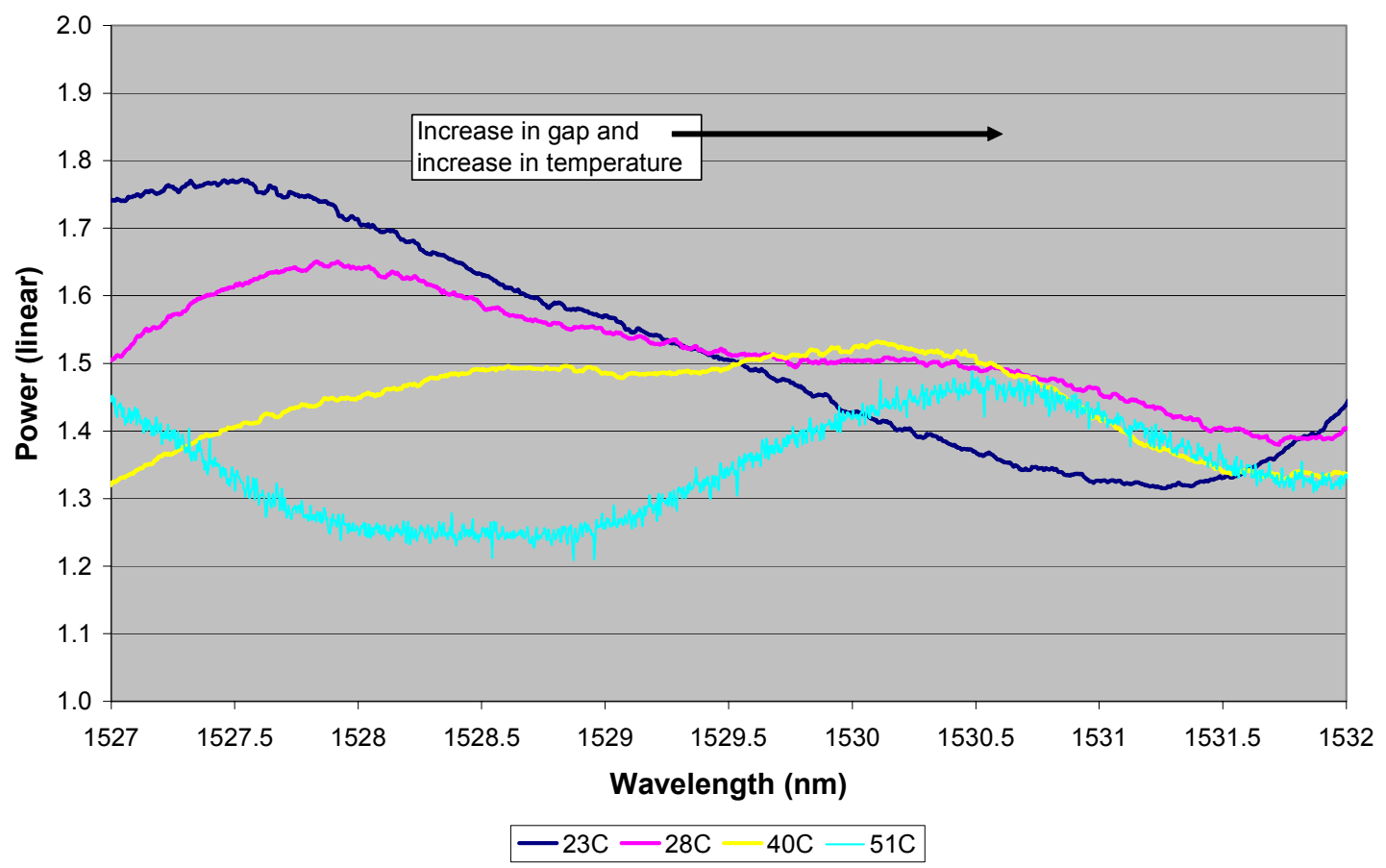

Figure 3-67. Spectra of cut away strain gage with increase in temperature 


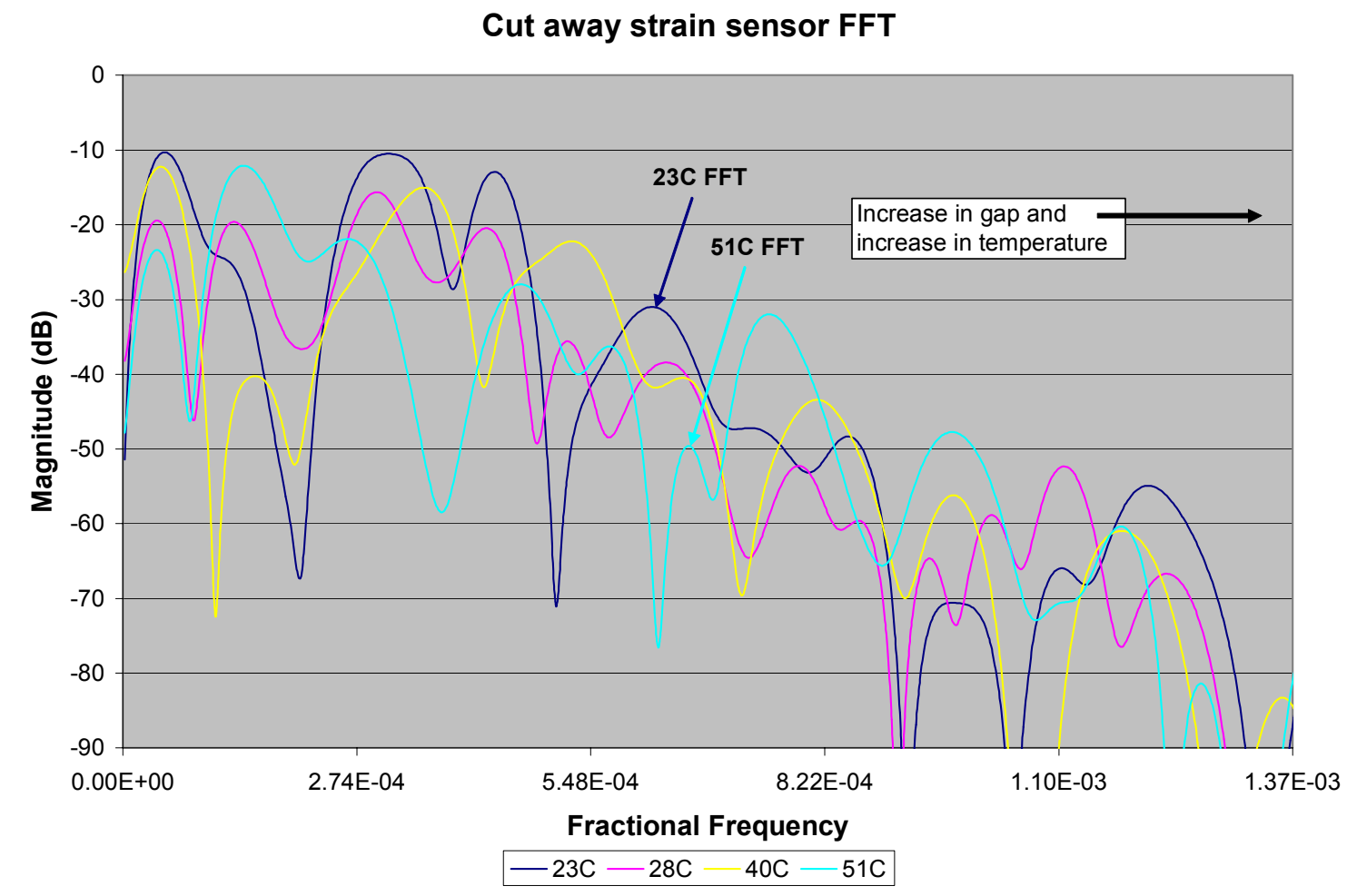

Figure 3-68. FFTs of cut away strain sensor with increase in temperature

FFT peak change with temperature

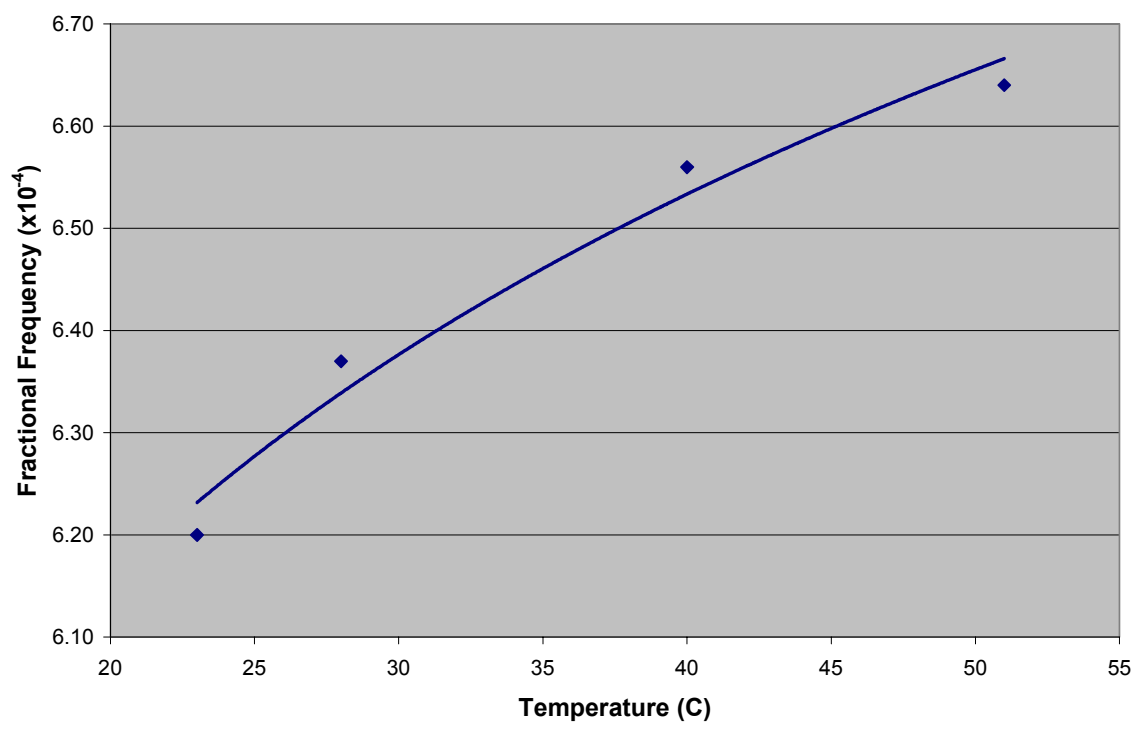

Figure 3-69. Trend with temperature for peak changes in Figure 3-68 


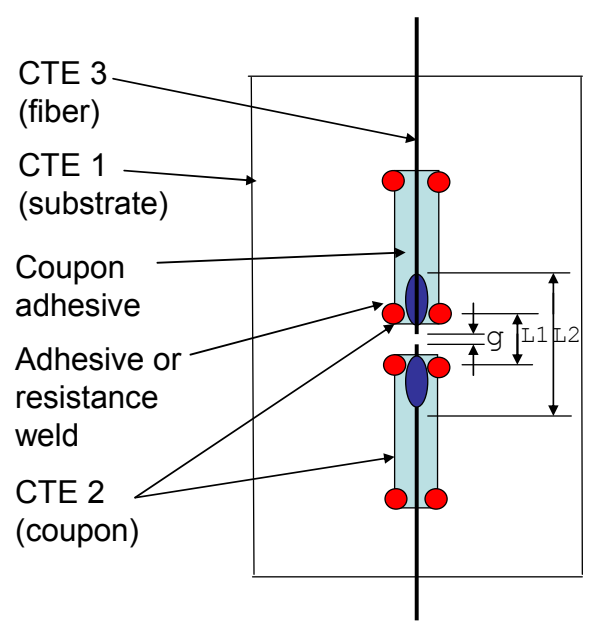

Figure 3-70. Geometrical factors used to determine temperature sensitivity of strain gage.

Table 3-11. Calculation of the gap change relative to temperature for the cut away sensor

\begin{tabular}{|c|c|c|c|c|c|c|c|}
\hline$\Delta \mathrm{g}$ & CTE 1 & CTE 2 & CTE 3 & L1 & L2 & g & $\Delta \mathrm{T}$ \\
\hline$(\mathrm{nm})$ & $\left(10^{\wedge}-6 \mathrm{~m} /{ }^{\circ} \mathrm{C}\right)$ & $\left(10^{\wedge}-6 \mathrm{~m} /{ }^{\circ} \mathrm{C}\right)$ & $\left(10^{\wedge}-6 \mathrm{~m} /{ }^{\circ} \mathrm{C}\right)$ & $(\mathrm{mm})$ & $(\mathrm{mm})$ & $(\mathrm{mm})$ & $\left({ }^{\circ} \mathrm{C}\right)$ \\
\hline & aluminum & silicon & Silica & & & & \\
\hline 30.43 & 24 & 2.49 & 0.55 & 0.256 & 0.256 & 0.15 & 5 \\
\hline 103.46 & 24 & 2.49 & 0.55 & 0.256 & 0.256 & 0.15 & 17 \\
\hline 170.40 & 24 & 2.49 & 0.55 & 0.256 & 0.256 & 0.15 & 28 \\
\hline
\end{tabular}

\subsubsection{Sapphire Strain Gage Packaging Development}

The package for the sapphire strain gage was also designed to withstand the high temperatures and corrosive atmosphere of the furnace. The base pad is fabricated of 304 Stainless Steel, the same alloy as the L bracket shown in Figure 3-139, and also the same as the pipe support brackets and pipes that make up the power plant. The base pad has recesses on the far face that are sized for nuts which will be used to temporarily attach the strain sensor assembly (Figure 3-71), and also slots which will accept a custom spot welder electrode, which will effect the permanent attachment of the sensor assembly. The base pad, with nuts installed, will be flame welded to the L bracket by certified plant personnel (Figure 3-72).

The strain sensor substrate (Figure 3-73) is fabricated from 3/32" thick Inconel plate stock. Two grooves are cut into the plate to accept the sapphire sensor fibers. While only one sensor fiber is needed, the second is redundant in case of failure of the first. Failure may occur during the fabrication or mounting process, or during use. The relative ease of adding a second sensor fiber was instrumental in making this decision. A pair of slots, perpendicular to the fiber grooves, provides the channels necessary for a saw blade to cut the sapphire fibers and make the Fabry-Perot cavity. Again the second slot is redundant, and will only be used if a second attempt is needed to make a clean dicing saw cut through the fiber. 
A $1 / 4$ " outside diameter (OD) inconel tube is attached to the strain sensor substrate (Figure 3-75). The bottom edge of the tube is tangent to the bottom face of the strain sensor substrate. An inconel block with specific geometry will be the intermediary component providing welding locations for the tube and the strain sensor substrate. A four hole ceramic rod will be installed into the inconel tube. The lower two holes will align with the two grooves in the strain sensor substrate.

A length of clad sapphire fiber is fusion spliced to gold-coated multimode fiber. The multimode fiber is terminated on the other end with an optical connector. This assembly is mounted onto a carrier assembly (Figure 3-74). A suitable length of the clad sapphire is coated with a suitable metal coating. The coating bonds to the clad surface of the sapphire and provides a conductive surface for the nickel electroplate.

The next step is to prepare the assembly for nickel electroplating. The nickel plating attaches the fibers to the inconel substrate. Nickel bonds to both the inconel substrate and the fibers and is capable of withstanding the high temperature environment of the furnace. The coefficient of thermal expansion of the inconel, a high nickel content alloy, and the nickel plating are almost identical. A sample inconel substrate with coated clad sapphire fibers installed was nickel plated. The adhesion of the nickel plating and fibers to the inconel substrate was successfully tested to $1,093{ }^{\circ} \mathrm{C}\left(2,000{ }^{\circ} \mathrm{F}\right)$.

The inconel plate assembly with the electroplated fibers is then mounted to film adhesive and hoop for cutting the sapphire fiber in the dicing saw. The plate assembly is attached to the vacuum chuck in the dicing saw, and the chuck is adjusted for tracking the blade centrally through the distal slot in the inconel plate. A cut is made dry (no fluid lubrication) through both fibers. While the sapphire assembly is still attached and aligned in the vacuum chuck, the connectors are attached to the interrogation system and signal quality is checked. If both sensors show good signal quality the unit is removed from the saw. If both are bad, then a second cut will be made through the fibers in the proximal slot. If one is good a decision will be made based on the quality of the one good signal.

Furcation tubing is installed over the ends of the fibers, and a new connector is installed on each. The end of the furcation tubing in the inconel tube is attached to the inconel tube with high alumina cement. This completes the sapphire strain sensor assembly.

The sapphire strain sensor assembly is then attached to the base pad previously welded to the L bracket. The procedure will be as follows. From the interior of the furnace, the fibers with connectors are fed out through the slot in the web and the hole in the sliding seal. The sensor assembly is attached to the base pad with two screws. With the custom jaws in the spot welder the sensor assembly is welded to the base pad in four places (Figure 3-81, Figure 3-82). Upon completion of welding, the two screws are removed (Figure 3-83).

An additional sapphire strain sensor (Figure 3-84) will be wall mounted near the support bar mounted unit. A hole will be drilled in the web between wall tubes. An L bracket will be installed on the wall in the furnace interior. The sapphire strain sensor can be attached to the bracket prior to assembly in the furnace. 


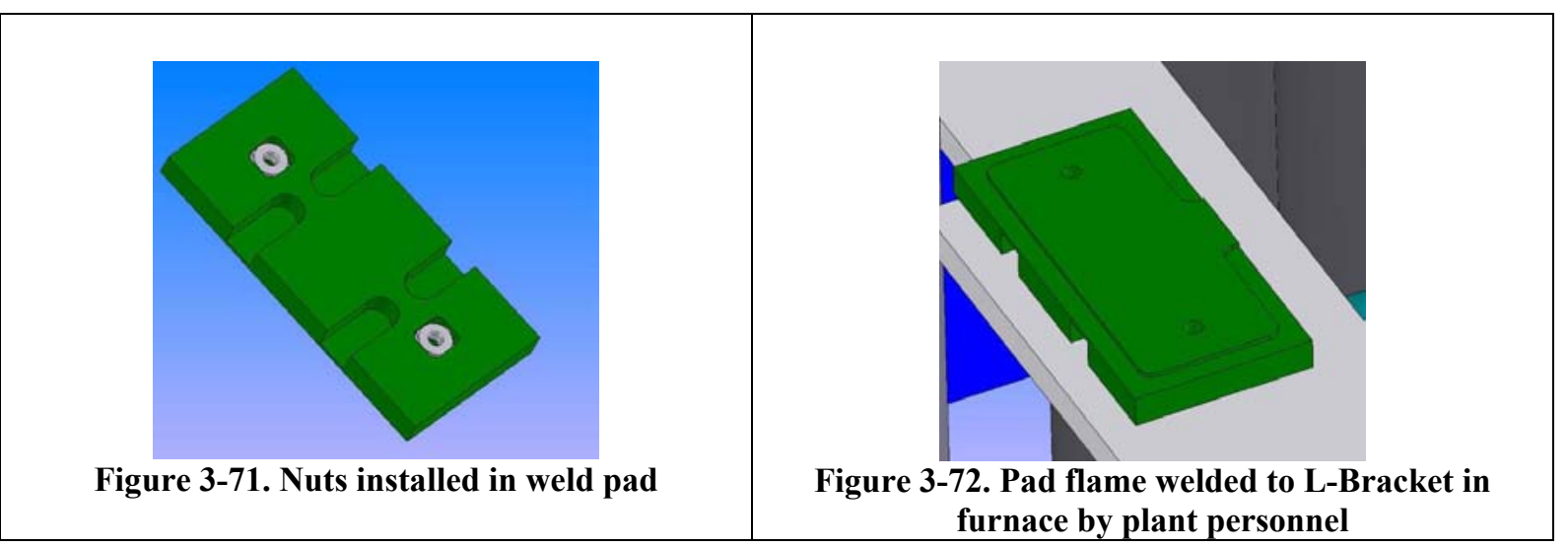

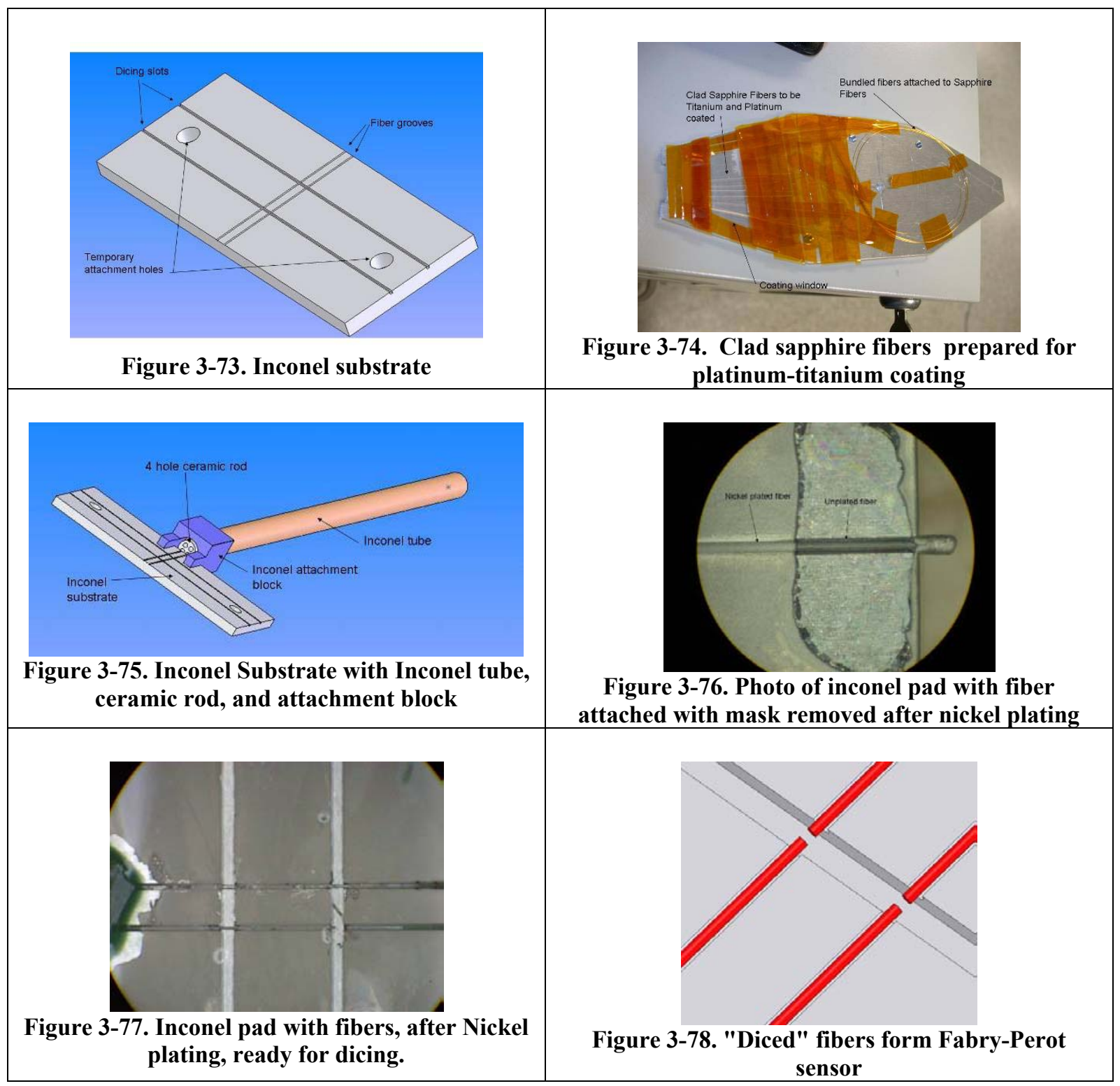




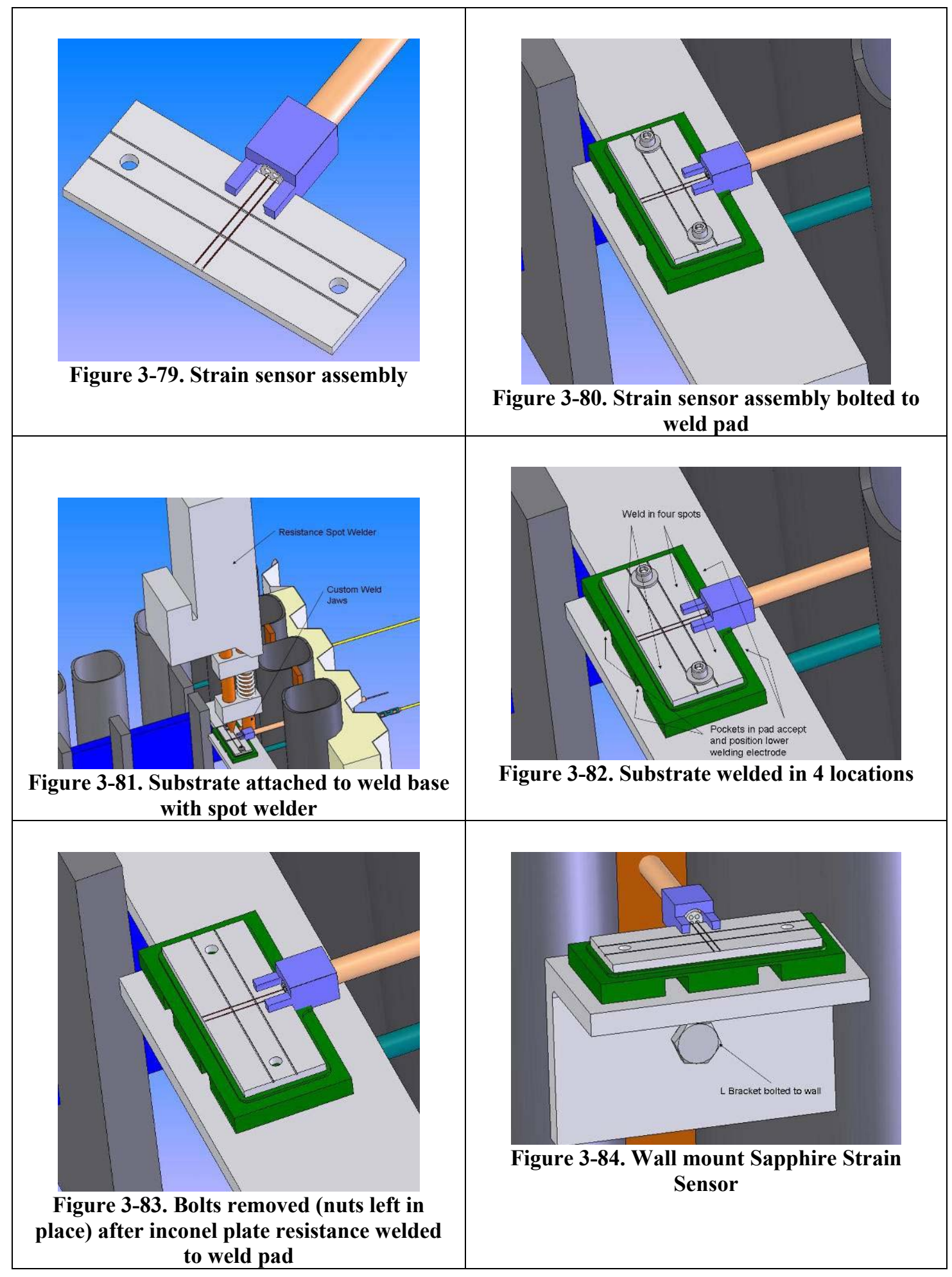




\subsubsection{Cut sensor development}

To test the design principle, one sensor was successfully made using the saw cut through a sapphire fiber bonded to a silicon wafer instead of an inconel plate. Fabrication proved difficult; seven saw cuts were required until suitable interferometric fringes were obtained. Next, a sapphire fiber bonded to an inconel plate, and several attempts were made to cut the fiber using a diamond dicing saw. While the cuts were made without

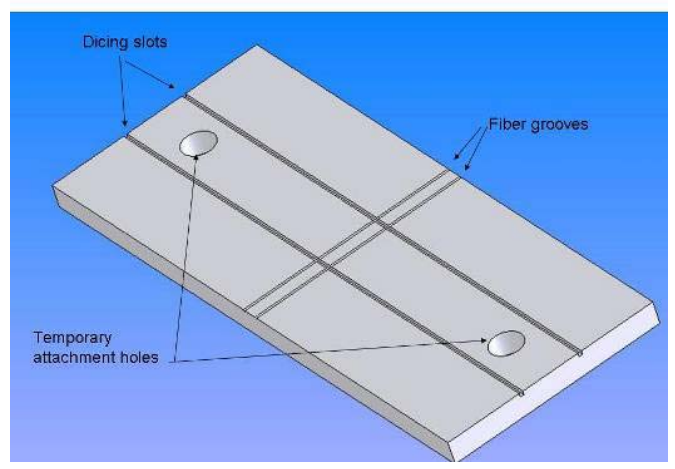

(a)

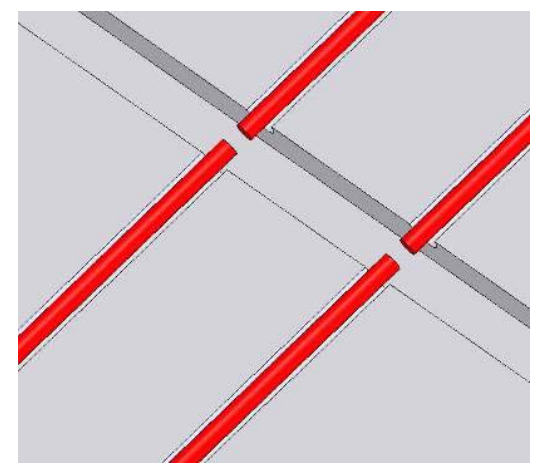

(b)

Figure 3-85. (a) Inconel used for sapphire strain gage coupon. (b) Close-up of slot for dicing of fibers, showing cut fibers (in red).

damage to the fibers, it was not possible to obtain fringes when the sapphire fiber was connected to an optical spectrum analyzer. The end faces of the saw cut sapphire were examined under a microscope, and kerf marks were observed in on the fiber end. Since optically smooth, parallel surfaces on the fiber ends are required to achieve usable fringes, it was concluded that the roughness of the fiber ends, as demonstrated by the kerf marks, was inhibiting formation of fringes. For this reason, further development concentrated on methods to form the Fabry-Perot cavity using polished sapphire fibers.

\subsubsection{Polishing studies}

The objective of the saw cut technique described in the previous section was to ensure that the two sapphire end faces in the Fabry-Perot strain sensor were parallel to each other within a tolerance of $0.1^{\circ}$. Angles of as much as $0.2^{\circ}$ will severely degrade the interference signal fringe pattern. ${ }^{16}$ Previously, the sapphire end faces were polished on an UltraTec polishing wheel with an adjustable arm that could be used to vary the angle of the polished end face with respect to the fiber axis. Yields were poor due to the difficulty of ensuring that the sapphire fiber was perfectly perpendicular to the polishing wheel when using the UltraTec polisher.

\footnotetext{
${ }^{16}$ M. Han \& A. Wang, "Exact analysis of low-finesse multimode extrinsic Fabry-Perot interferometers," Applied Optics, Vol 43, No. 24, 20 Aug 2004, pp. 4659 - 4666.
} 
In contrast, polishing the sapphire ends using techniques for polishing standard connector ferrules with a commercially available polishing puck gave excellent results. Strong interference signals could be obtained with short pieces of sapphire fiber. Fringes obtained with longer sapphire fibers were difficult to interpret due to modal noise problems which will be discussed in a later section in this report. Sapphire polished end faces were inspected in a Norland Cleave-Chek interferometer. Sapphire pieces polished using the adjustable arm had end face angles of up to one degree. Puck polished end faces were far superior.

A concept for fabricating the sapphire strain gauge by polishing fiber ends in the strain gage coupons was evaluated. As shown in Figure 3-86, two metal coupons are fabricated, with V-grooves machined across the tops. The sapphire fibers would be held in place in the $\mathrm{V}$-grooves by a high-temperature adhesive or through an electroplated metal. An adapter was designed to mount the coupons to an UltraTec polishing wheel, so that the end of the sapphire fiber (and the end of the metal coupon) could be polished. By controlling the tolerances with which the coupons are machined, the fiber ends can be polished to be perpendicular to the fiber axis within an angular tolerance approaching $0.1^{\circ}$. A slot is also machined at an acute angle to the V-groove on the coupon for the reflector fiber (Figure 3-86b). This permits the use of a diamond saw to cut the distal end of the reflector fiber at an acute angle to the fiber axis; a cut at such an acute angle will reduce undesirable reflections from the end of the reflector fiber.

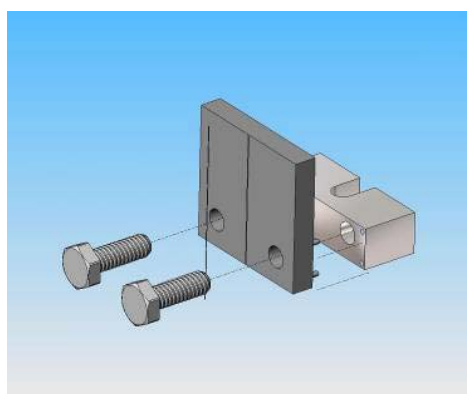

(a)

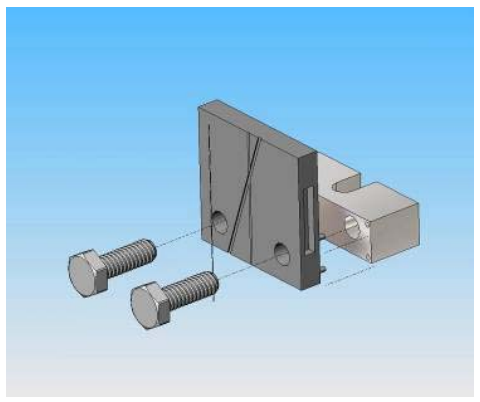

(b)

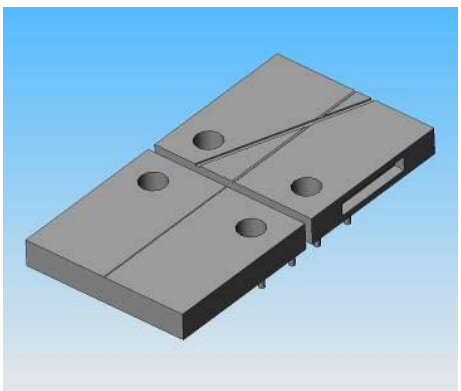

(c)

Figure 3-86. Concept design for sapphire strain gauge, employing V-grooves to align the fibers. (a) coupon to hold source fiber, with adapter (light silver) to mount coupon to polishing wheel (b) coupon to hold it reflector fiber, with adapter (light silver) to mount coupon to polishing wheel (c) assembled strain gauge.

To test this approach, a V-groove was machined in a stainless steel plate, and then the plate was cut into two pieces, with the cut perpendicular to the V-groove. Two $125 \mu \mathrm{m}$ diameter sapphire fibers were fixed to the grooves using Crystalbond adhesive. One fiber was one meter long, and served as the injection fiber, and the other was approximately 2 inches long, and served as the reflector fiber. After the fibers were attached to the steel plates, the ends on the fibers were polished using diamond media on an UltraTec polishing wheel. The two plates were then aligned, and a VectorLight 200 interrogator 
was used to check for the presence of interference. The resulting interference fringes are shown in Figure 3-87. Using the relation

$$
\text { gap }=\frac{1}{2}\left(\frac{\lambda_{1} \lambda_{2}}{\lambda_{1}-\lambda_{2}}\right)
$$

for Fabry-Perot cavities, where $\lambda_{1}$ and $\lambda_{2}$ are the wavelengths for two adjacent peaks in the spectrum, it can be determined that the gap for the cavity was $228 \mu \mathrm{m}(0.009 \mathrm{in})$. Based on the results obtained, use of sapphire fibers with polished ends for fabrication of Fabry-Perot strain gauges appears to be the most promising approach.

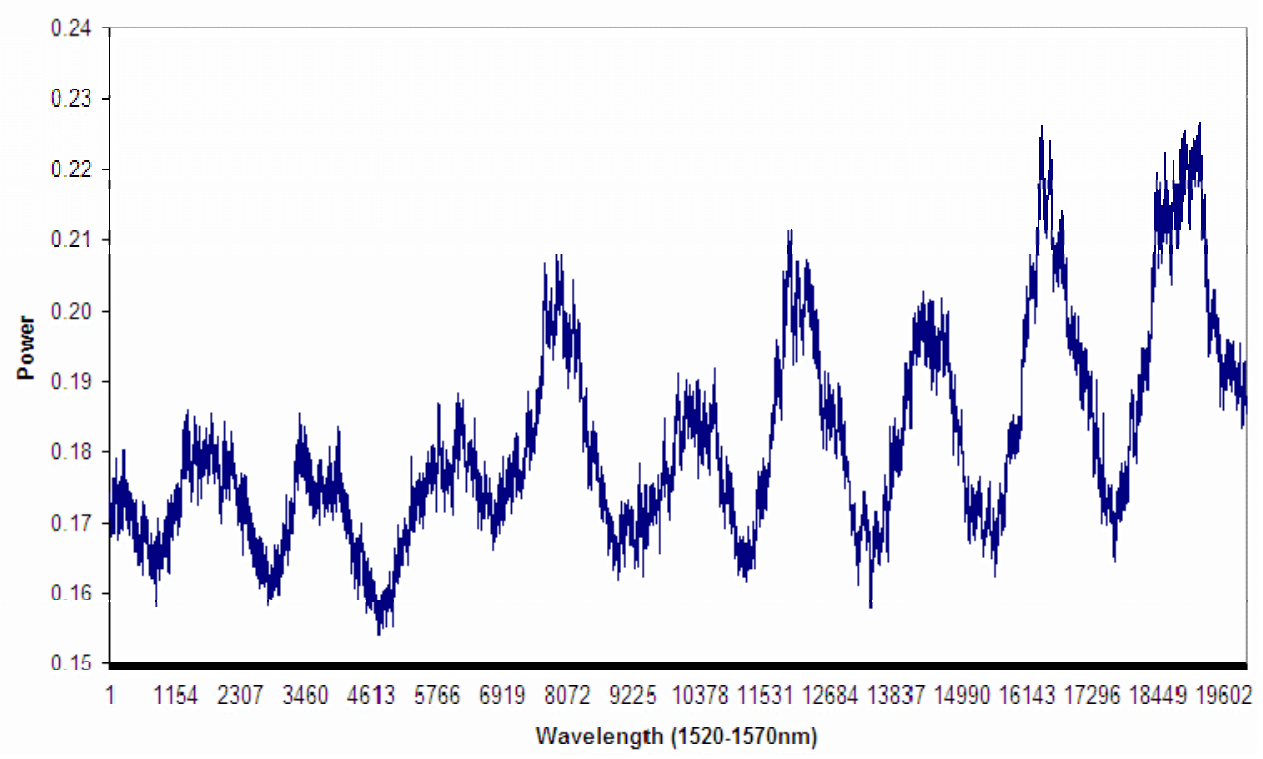

Figure 3-87. Fringes obtained from a sapphire strain gage using collinear V-grooves for alignment of one-meter long $125 \mu \mathrm{m}$ diameter sapphire fiber with a $125 \mu \mathrm{m}$ diameter sapphire fiber reflector.

\subsubsection{Injection condition studies}

Since the sapphire fiber is used in the construction of the sensors for the CEDF field test are unclad, the fibers can support propagation of many modes, which can lead to intermodal interference, which may swamp the interference signal generated by the sapphire wafer (in the case of the temperature sensor) or the air gap (in the case of the strain gage). Intermodal interference can be reduced by controlling the conditions by which light is launched into the sapphire fiber, in order that only a few modes are stimulated in the fiber (a so-called limited or low-order launch condition). However, even with only a few modes present at the input of the fiber, scattering of light by fiber imperfections or bends can increase the number of modes propagating the fiber by mode coupling. 
Several injection conditions were investigated in order to choose the method that produced the least intermodal interference. These conditions included injection of collimated light using either a graded index rod lens or a collimator fabricated from a graded index fiber, and injection of a single mode with an expanded diameter through the use of a fiber taper.

\subsubsection{Injection using collimators}

Collimators were made by splicing short lengths of $62.5 / 125 \mu \mathrm{m}$ (core diameter/cladding diameter) graded index multimode fiber onto $8.4 / 125 \mu \mathrm{m}$ bend-insensitive singlemode optical fiber. A Thorlabs BP109-IR beam profiler was used to measure the far field of each sample to determine the optimum length of multimode fiber needed to achieve collimation; proper collimation is achieved when beam spread in the far field is minimized. These far field measurements determined that collimation occurs at a length of $280 \mu \mathrm{m}$ for the particular $62.5 / 125 \mu \mathrm{m}$ graded index of fiber used for this study.

A collimator was made using this method and the output end of the collimator was then aligned to one end of a 4 in long $125 \mu \mathrm{m}$ diameter sapphire fiber using an xyz-positioner. The other end of the sapphire fiber had a $100 \mu m$ thick sapphire chip attached in order to create interference fringes. A Micron Optics Component Test System (CTS) scanning laser spectrometer was used to interrogate the signal. No signal fringes were obtained in the CTS output, regardless of the alignment of the collimator to the sapphire fiber; this indicates that the desired signal fringes were swamped by intermodal interference.

A quarter-pitch, $1.8 \mathrm{~mm}$ diameter graded index rod lens (GRIN) collimator was then purchased from Thorlabs, Inc. and tested with the beam profiling equipment. It was found to have far superior collimation compared to the 62.5/125 collimator and was therefore tested with the 4" sapphire fiber using the same method described in the previous paragraph. Good interference fringes due to the sapphire wafer were obtained. Reasonable signal fringes were also obtained when a 12 in long, $125 \mu \mathrm{m}$ diameter sapphire fiber was substituted, and weak fringes were obtained when a $1 \mathrm{~m}$ long $125 \mu \mathrm{m}$ diameter sapphire fiber was substituted.

\subsubsection{Singlemode Taper}

The diameter of a propagating electromagnetic mode guided by the core of a single-mode fiber depends on several parameters, which include the diameter of the fiber core. If the propagating mode encounters a section of fiber where the core diameter is gradually decreased, than the mode field diameter (MFD) of the propagating mode gradually increases. The MFD, defined as the diameter at which the power in the mode decreases to $\mathrm{e}^{-1}$ of its maximum, is inversely proportional to the fiber core diameter. Therefore, an effective way to enlarge the MFD of the light in an optical fiber is to reduce the fiber core diameter by making a taper in the optical fiber. This may be done by heating the fiber until a tensile force on the fiber causes it to elongate, creating the desired taper. 
If the diameter of the output end of the single-mode fiber is reduced by tapering before it is spliced to a sapphire fiber, then the expanded mode that is injected into the sapphire fiber should reduce the number of modes stimulated in the sapphire fiber, and in turn reduce the intermodal interference. However, the taper must be gradual, or radiative losses will result in the taper.

Tapers were made using a Fujikura FSM-40F arc fusion splicer, then cleaved at the narrowest point. OFS 8.4/125 bend-insensitive singlemode fiber was used and tapered to various minimum diameters. The throughput loss of each taper was measured at $1500 \mathrm{~nm}$ before cleaving. The tapers were also coated with glycerin to index match and strip light out of the fiber cladding if the signal had coupled into the cladding from the core.

Coupling of light from the core to the cladding is undesired, since it results from radiative losses in the core, and indicates that the taper is too abrupt.

Tapers with a minimum diameter of $32 \mu \mathrm{m}$ were used to inject one end of a 4 in long 125 $\mu m$ sapphire fiber. The other end of the sapphire fiber had a $100 \mu m$ thick sapphire chip attached to create interference fringes. A Micron Optics CTS was used to interrogate the signal. No signal fringes were obtained, indicating that intermodal interference was still present.

The above taper experiment was repeated using 4/125 singlemode fiber which would guide the $1550 \mathrm{~nm}$ light more loosely; due to the reduced core diameter, the propagating mode in the untapered fiber has a larger MFD than in the 8.4/125 bend-insensitive fiber This fiber was spliced to a standard $9 \mu \mathrm{m}$ core singlemode pigtail and connector. The splice loss measured at $663 \mathrm{~nm}$ was about $6 \mathrm{~dB}$ at $1550 \mathrm{~nm}$. However, it was found that the fiber was extremely sensitive to bends at $1550 \mathrm{~nm}$ and that the fiber had to be extremely straight to keep bend losses to acceptable levels. Therefore, the length of the 4 $\mu m$ singlemode fiber was changed from $1 \mathrm{~m}$ to less than $1 \mathrm{~mm}$.

A diagram of the singlemode taper is shown below in Figure 3-88. The loss through the taper and splice at $1550 \mathrm{~nm}$ was $2.5 \mathrm{~dB}$. The loss though the sapphire fiber when injected with the taper was an additional $1.0 \mathrm{~dB}$. When the $100 \mu \mathrm{m}$ thick sapphire chip was attached to the 4 in long sapphire fiber, no signal fringes were obtained. However, when a drop of glycerin was added to the taper-sapphire fiber interface, fringes were obtained, indicating that some coupling from core modes to cladding modes had occurred in the taper. The fringes that were obtained with the taper represented an improvement over those obtained using a standard singlemode fiber for injection, but were not as good as the fringes that were obtained using multimode fiber to inject the sapphire fiber. Figure 3-89 is a graph of the output signal obtained from the sapphire sensor when injected by the singlemode fiber taper, and for comparison, Figure 3-90 shows the output signal obtained with the sensor is injected with a $62.5 / 125$ multimode fiber. 


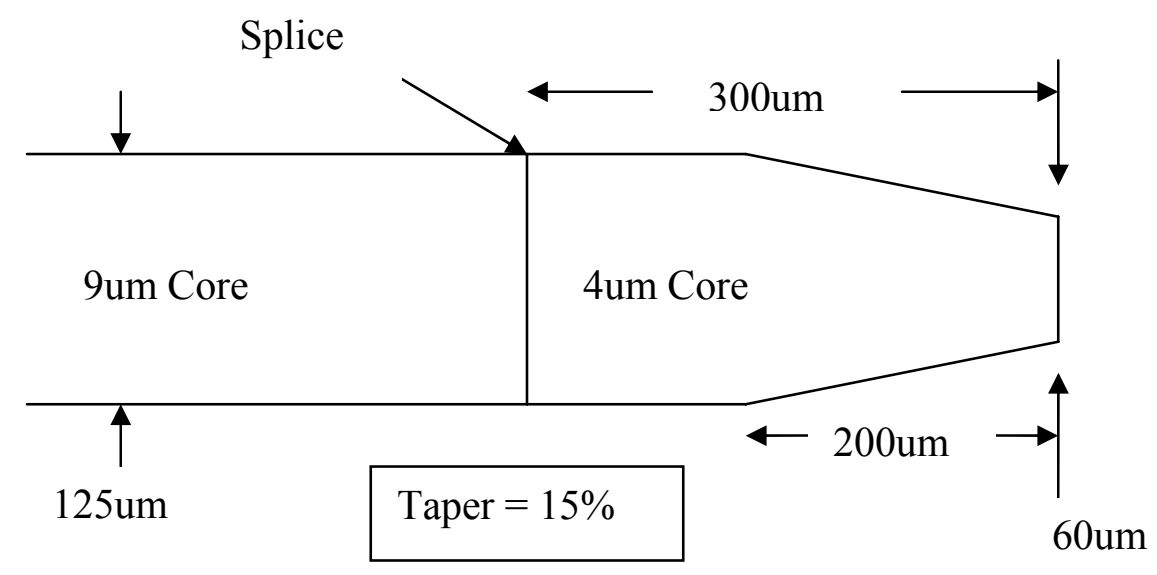

Figure 3-88. Schematic illustrating geometry of fiber taper.

\subsubsection{Conclusions from Injection Condition Study}

Extensive tests of the use of singlemode fiber tapers for injection of sapphire fiber sensors were performed with various taper geometries and singlemode fiber types, and the results were generally poor. Injecting the sapphire with a collimator on the end of a singlemode fiber resulted in much better signals than with the singlemode tapers, but alignment was extremely sensitive. Good signals could be achieved, but any movement in the sapphire fiber would cause the signal to disappear. The best and most reliable method for injection of the sapphire fiber achieved in our studies is the use of multimode fiber with standard connector ferrules for alignment. This multimode injection method was used in the fabrication of sensors for CEDF testing.

\subsubsection{Fabrication of sapphire strain sensors for field test}

Two sapphire strain gages were assembled for installation in the Babcock \& Wilcox CEDF plant. The sensors used an extrinsic Fabry-Perot interferometer design; that is, an air gap was formed between two sapphire fibers, so that when light is injected into one of the sapphire fibers, the reflections generated by the sapphire fiber ends that form the air gap will interfere. Using well-known principles from white light interferometry, the length of the Fabry-Perot cavity (the air gap) can be determined uniquely. If the two fibers are attached to a test article, then stresses applied to the test article or result in strains that will correspondingly cause a change in the sensor cavity length. By knowing in advance the distance separating the points at which the strain gage is attached to the test article, the strain in the test article can be quantitatively determined by dividing the elongation of the cavity length by the separation of the attachment points (the gage length).

To fabricate the optical assembly for each strain gauge, one end of a one $m$ long, $125 \mu \mathrm{m}$ diameter c-axis sapphire fiber was installed in a zirconia ferrule with a $140 \mu \mathrm{m}$ inner diameter and $1.8 \mathrm{~mm}$ outer diameter. An alumina ceramic adhesive was used to fix the 
fiber end inside the ferrule, and then the fiber end was polished using diamond polishing media to an optically smooth finish. Similarly, a 2 in long, $125 \mu \mathrm{m}$ sapphire fiber was fixed in a second $1.8 \mathrm{~mm}$ diameter zirconia ferrule using a ceramic adhesive. A zirconia tube was then used to align the two ferrules so that a gap of approximately $100 \mu \mathrm{m}$ length separated the two polished sapphire fiber ends.

Next, the free end of the $1 \mathrm{~m}$ long sapphire fiber was spliced to a $3 \mathrm{~m}$ long multimode glass fiber, using a mechanical splice. The two fibers were polished, butted together, and fitted in place using mechanical fixturing. The sapphire fiber was then threaded into a 1 $m$ long inconel tube, and the zirconia ferrule was fixed to the tube end using a ceramic adhesive. Also, the 2 in long sapphire fiber was fixed in a 2 in long inconel tube with the ceramic adhesive. Standard telecommunications cable jacketing of the loose tube design was used to protect the silica fiber. The jacketing had an outer PVC sheath with an inner tubing of polypropylene, with a Kevlar aramid yarn between them. The Kevlar strength member was attached to the inconel tube using an epoxy. A polyolefin heat-shrink tube was used to protect this joint between the tube and the jacket and to provide strain relief in the event of bending.

The two sapphire fibers were then joined using a zirconia tube, and proper optical alignment of the Fabry-Perot cavity was checked by using a Micron Optics Component Test System (CTS) scanning laser interrogator. This CTS system had been modified for use with multimode optical fibers by substituting the stock photodiode with a large area photodiode. The alignment check was performed by ensuring that interferometric fringes with acceptable fringe contrast were obtained. Since fringe contrast depends strongly on proper alignment of the fiber end faces, achieving good fringes ensures that the alignment is correct.

Sapphire, 125um, 4" Injected with 60 um Diameter 830 SM Taper

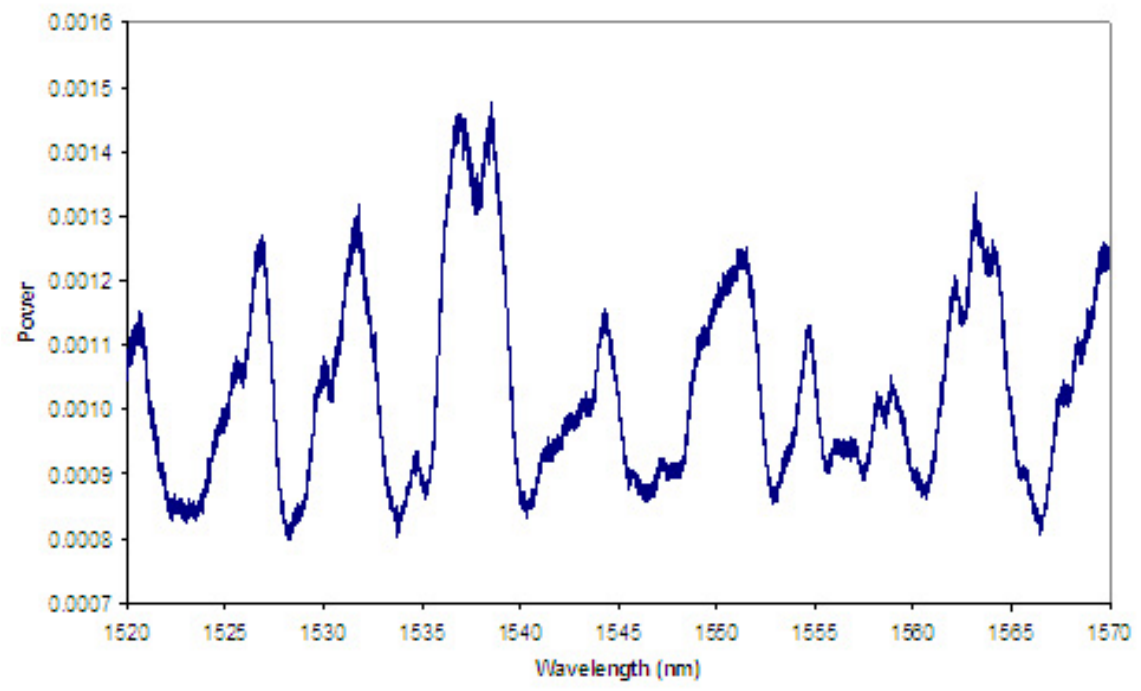

Figure 3-89. Interference fringes obtained from sapphire temperature sensor injected by a singlemode fiber taper. 


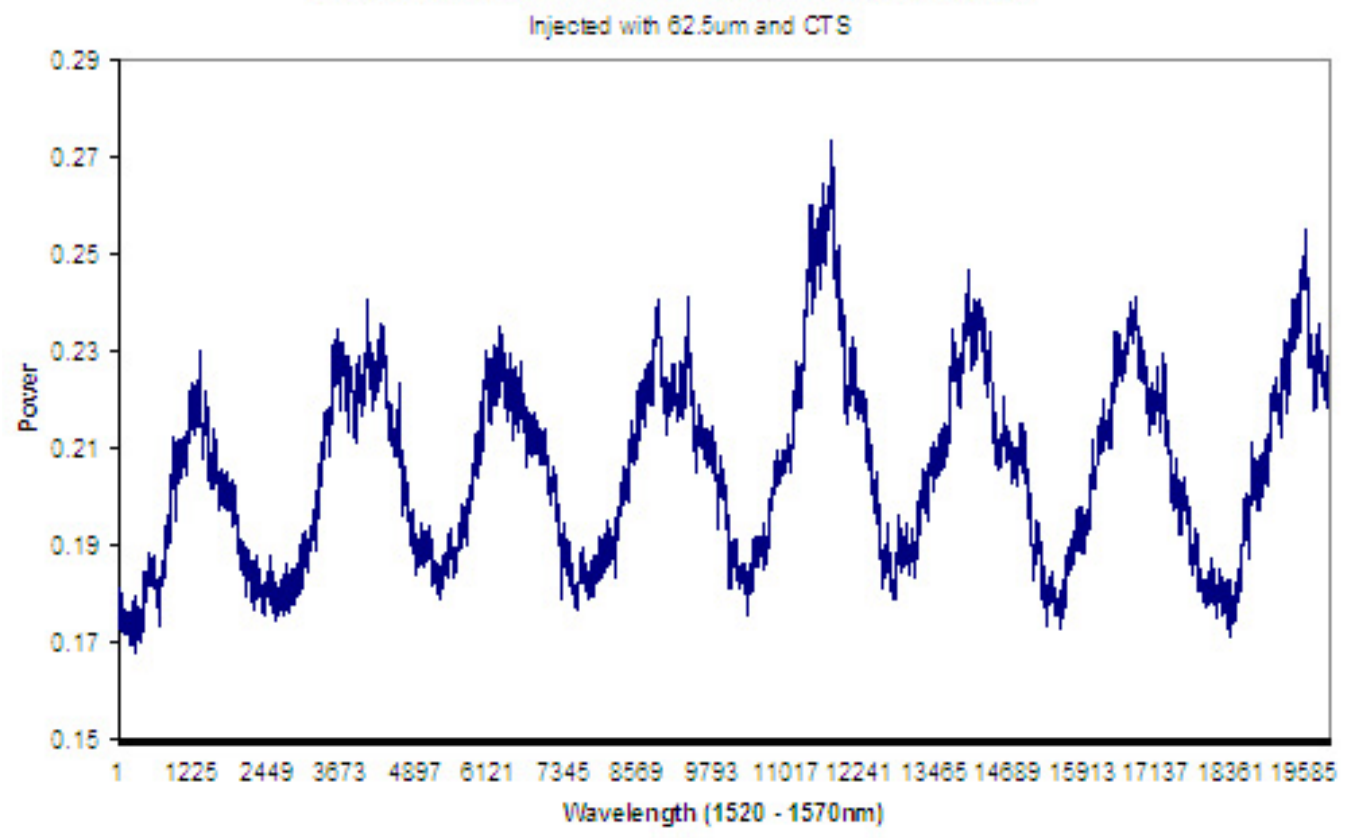

Figure 3-90. Interference fringes obtained from sapphire temperature sensor injected by a 62.5/125 multimode fiber.

During the final packaging of the second strain gage, the sapphire fiber was broken during insertion of the fiber into the inconel tube, rendering the sensor unusable. Since this failure occurred shortly before the scheduled sensor installation, and since the second strain gauge was intended as a backup for the first, the broken strain gage was not replaced.

To facilitate mounting of the strain gage onto the CEDF tubes, 1"x2"x0.025" straps were cut from 310 stainless steel sheet stock and bent to the same curvature as the CEDF tube. One strap was resistance welded to the end of the long inconel tube of the input sapphire fiber. A second strap was resistance welded to the short inconel tube of the reflector sapphire fiber. A Dremel tool with a grinder attachment was used to thin the 310 SS straps at eight points on each strap to a thickness of approximately 0.010 ", to improve resistance welds at those points. During installation, the straps will be resistance welded to the CEDF tube, holding the two sapphire fibers in proper optical alignment. Thermally induced strain in the CEDF tube will cause the gap between the two sapphire fibers to increase, resulting in a change in interference fringes. 


\subsection{Alternative Sapphire Sensors}

\subsubsection{Sapphire Pressure Sensor Development}

During the program, an effort was directed towards investigating the development of resonating structures whose natural frequency depends on the magnitude of an applied pressure. Resonant sensing refers to a technique by which the resonant frequency of a transducer is made to vary with the desired measurand, in this case pressure. The particular embodiment studied here involves optical interrogation via a Fabry-Perot interferometer, and optical stimulation via sinusoidally varying laser energy. The resonant frequency of the beam is determined by operating a Phase-Locked Loop (PLL) circuit that drives the beam at its natural frequency given the feedback signal from the interferometer. The advantages of resonant sensing over traditional methods such as Fabry-Perot or amplitude-based devices are 1) mechanical stability of the Fabry-Perot gap is not required for the device to be accurate; 2) stability of the opto-electronics (lasers, filters, etc) is not required for the device to be accurate; 3 ) fiber bend losses and wavelength dependent attenuation do not degrade accuracy. For accurate sensing, the only requirement is that the relationship between applied pressure and the transducer resonant frequency be maintained.

Figure 3-91 is a graphical illustration showing the concept of a resonant pressure transducer investigated. The resonant beam shown in green (hereafter referred to as the resonator) is attached to the diaphragm (gray). The interrogation fiber is positioned at the center of the beam where maximum deflection occurs. The fiber that delivers the sinusoidally varying laser energy (referred to as the driving fiber) is positioned at the root of the beam to maximize the response of the beam to the applied energy. Absorption of the energy from the laser beam causes a localized temperature rise, accompanied by a localized thermal expansion of the beam. By varying the intensity of the laser sinusoidally with time, the resulting sinusoidal variation in thermal expansion at the root of the beam stimulates vibration of the beam.

The resonator natural frequency depends on the stress induced in the beam, just as the natural frequency of a guitar string depends on the tension in the string. Stress in the beam is proportional to the pressure applied to the diaphragm. Figure 3-92 shows the results of a finite element analysis applying NASTRAN to predict the fundamental mode shape and natural frequency of the resonator at zero applied pressure.

Additional finite element analyses were used to predict the shift in natural frequency due to the applied pressure. Geometric parameters such as the beam and diaphragm dimensions can be tailored to give the desired frequency change over a given operating pressure range and thereby adjust the mechanics for optimal monitoring of the beam resonance by a Fabry-Perot sensor. 


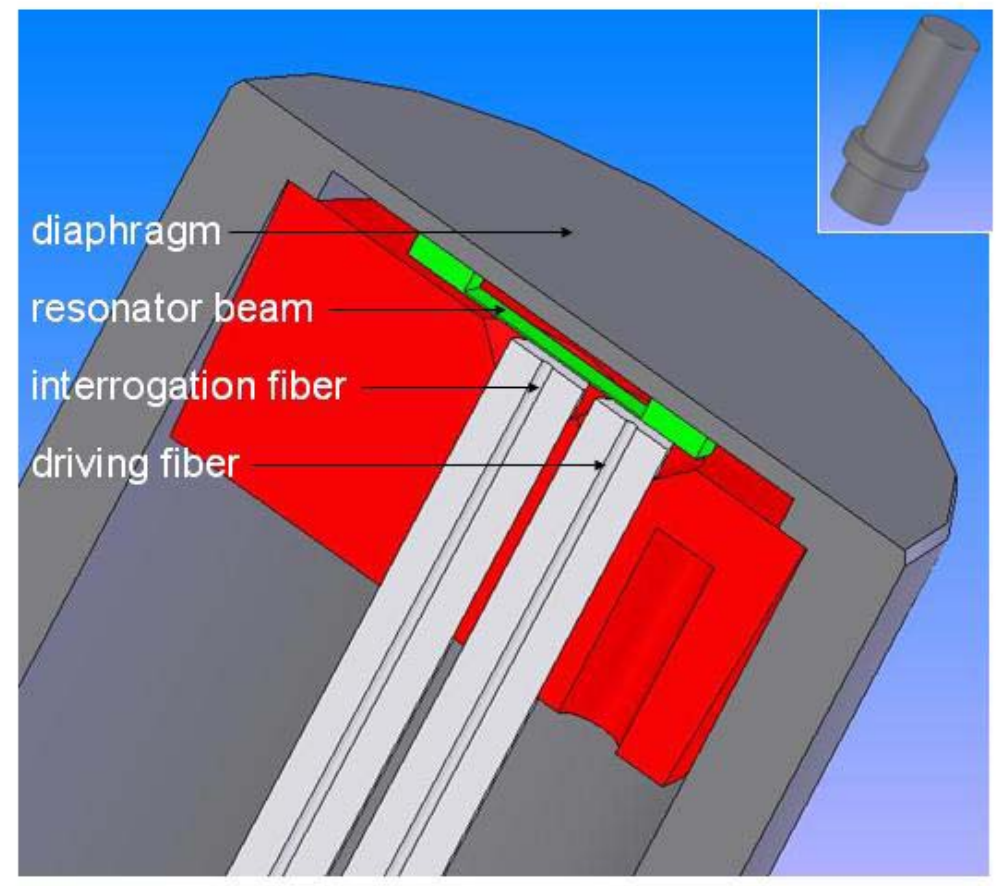

Figure 3-91. Mechanical layout of a pressure sensor based on the resonant sensing concept. Inset picture shows the exterior of the transducer, cross-sectional view details the important components of the sensor.

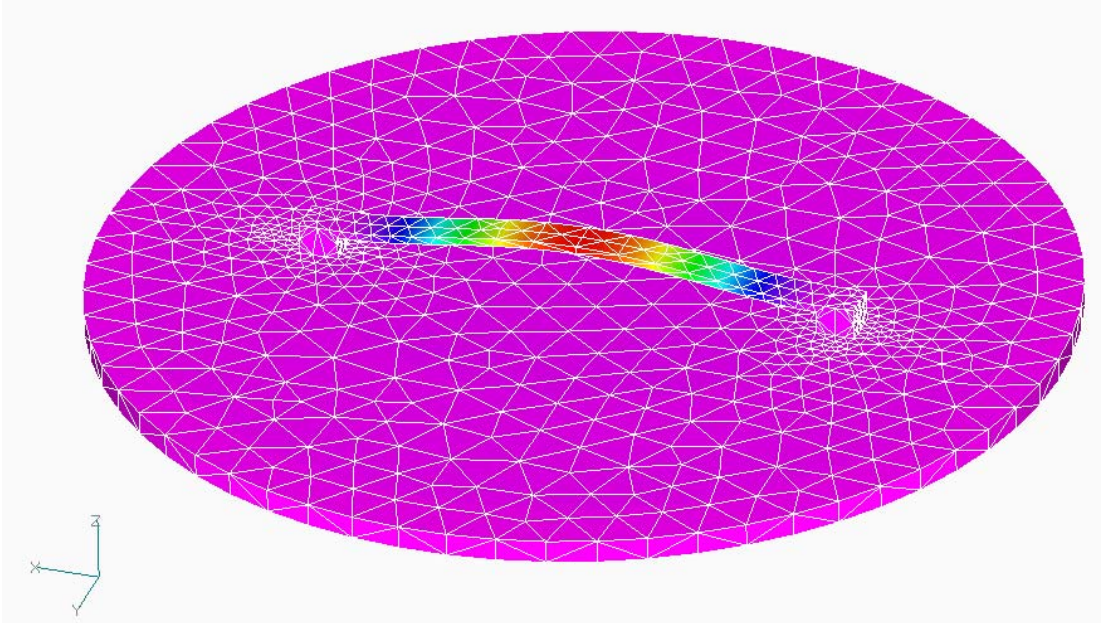

Figure 3-92. Finite element model of the pressure sensor diaphragm and beam. Magnified displacements show the structure's fundamental mode shape.

Based on the NASTRAN models, a prototype pressure sensor with a resonant beam was assembled for evaluation according to the design shown in Figure 3-91 The sensor housing, which incorporates the diaphragm on end end, is shown in Figure 3-93 after the piece was machined from 310 stainless steel. Also shown is a penny for scale, and three 
of the resonant beams are resting on the penny. The beams, shown in a close-up in Figure 3-93 (b), were machined from 310 stainless steel. One of the beams was bonded to the inside surface of the diaphgram using resistance welding.

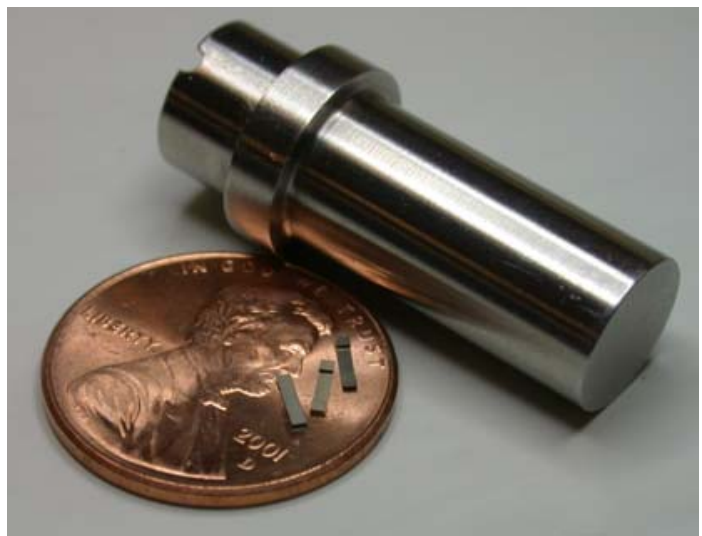

(a)

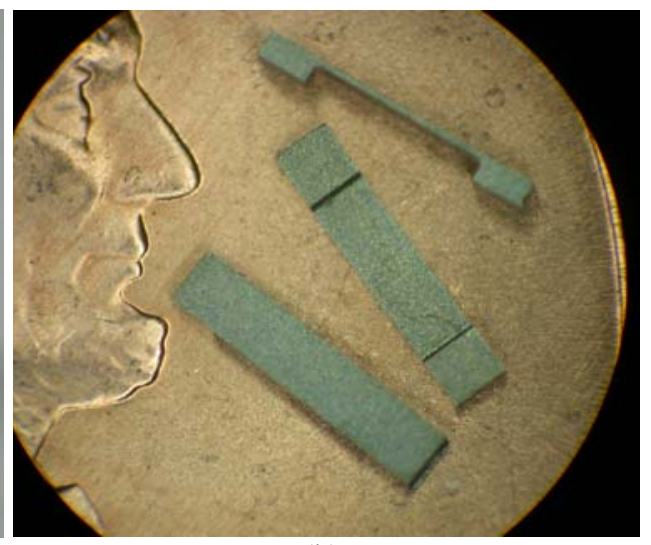

(b)

Figure 3-93. (a) housing for resonant beam pressure sensor, with diaphragm on end; (b) close up of resonant beams before bonding to diaphragm.

After mounting the interrogation fiber and driving fiber in the ferrules, which were in turn mounted in a cylindrical plug, the plug was inserted into the sensor housing. In order to maximize the optical drive of the vibrating beam and to maximize signal strength, the position of the plug was adjusted and then fixed in place.

To test the pressure sensor, it was connected by a tube to the output of a bottle of pressure regulated nitrogen gas, as depicted graphically in Figure 3-94. The pressure applied to the pressure sensor was varied by adjusting the bottled gas regulator. The frequency of the optical excitation was varied by adjusting the frequency of a signal generator connected to the drive electronics for the laser diode used to excite the beam. The resonant frequency was determined by sweeping the frequency of the signal generator over some range and watching the output of the interferometer interrogator to determine the frequency at which maximum displacement of the beam occurred. The regulated pressure output was monitored using an analog pressure gauge.

The results of the experiment are shown in Figure 3-95, where the resonant frequency was determined as the applied pressure was increased in 5 psi increments. As the red trendline fitted to the data indicates, the resonant frequency varies linearly with the pressure, although some scatter exists in the data. The scatter was within the measurement error that resulted from the low resolution of the pressure regulator's analog gauge, and the subjective assessment required of the test operator in determining the peak amplitude of the resonant beam. 


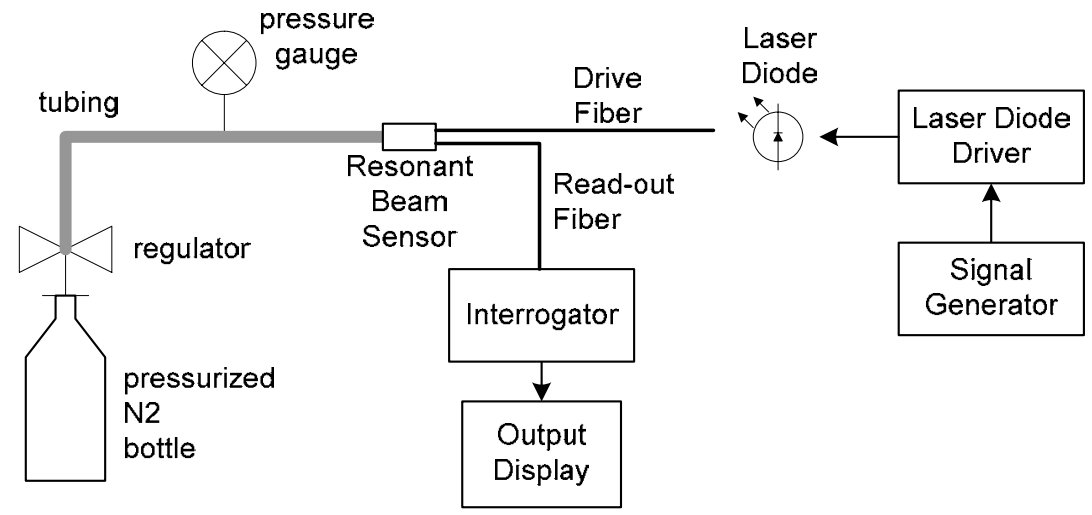

Figure 3-94. Test set-up for evaluation of prototype resonant beam pressure sensor.

To test the repeatability of the resonant beam pressure sensor, a new beam was bonded to the diaphragm, and the pressure was increased from ambient to 50 psig four times in succession. Figure 3-96 plots the results of the four successive tests.

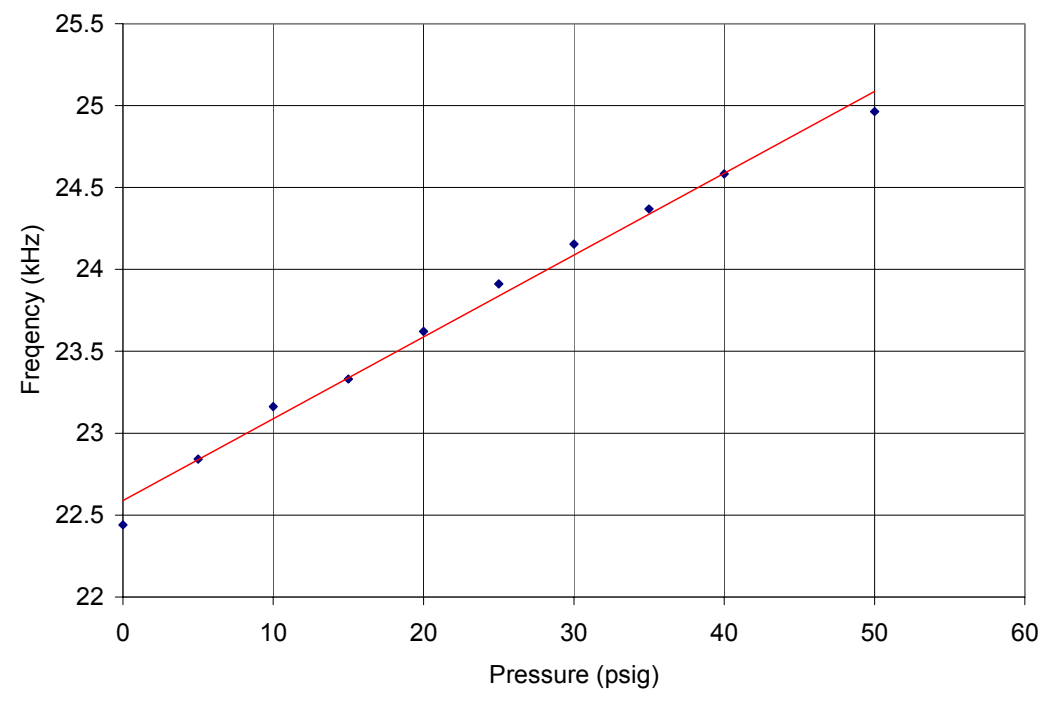

Figure 3-95. Plot of the resonant frequency of the resonant beam as the applied pressure is increased. 


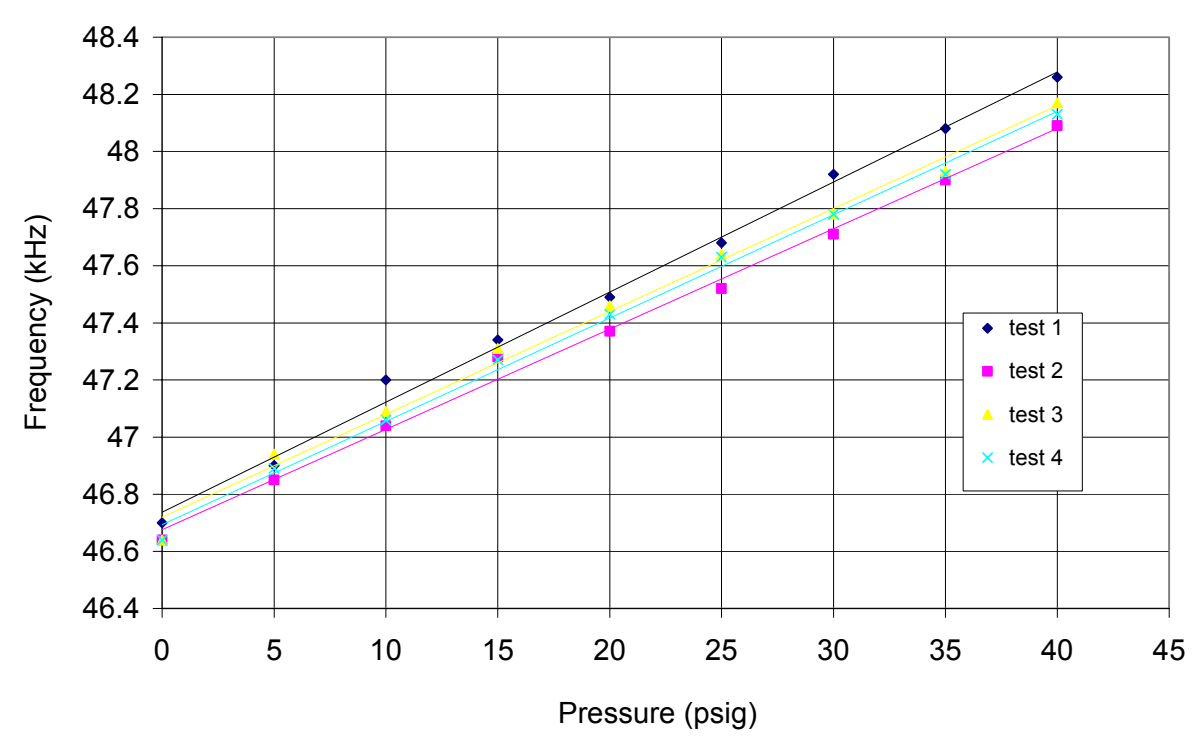

Figure 3-96. Change in resonant frequency with pressure for four tests.

To reduce the error to due determination of the frequency for which the peak beam amplitude is achieved, a phase locked loop (PLL) circuit was designed and implemented. The basic operation of the PLL is illustrated in the block diagram of Figure 3-97. The sinusoidally varying output from the interrogator in Figure 3-94 is applied to the PLL as the input signal $s_{i n}(t)$. This input signal is mixed with the sinusoidal signal $s_{V C O}(t)$ output by a voltage controlled oscillator (VCO), and the mixed signal is passed through a low pass filter. If the frequency and phase of the input signal is the same as the VCO signal, then output of the low pass filter (LPF) is the difference in the two signals, and the output $V_{\text {out }}$ of the PLL is zero. If, however, the frequency of the input signal changes, then the output of the LPF rises to a non-zero output. The output of the LPF is applied as an error signal $V_{e}$ to the $\mathrm{VCO}$, which changes the VCO frequency to match that of the input signal. In this way, the PLL output is linearly proportional to the frequency of the input signal (which is derived from the interrogator output).

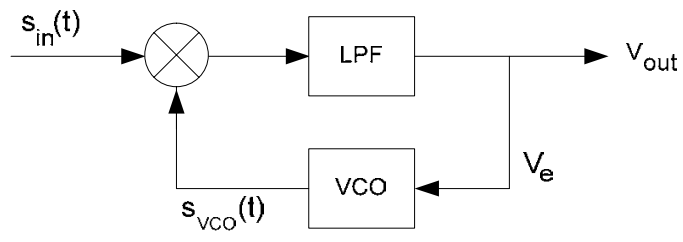

Figure 3-97. Block diagram of phase locked loop (PLL) circuit. LPF stands for Low Pass Filter, VCO stands for Voltage Controlled Oscillator, and the $\otimes$ symbol represents a mixer used as a phase detector.

Figure 3-98 shows the PLL output as a function of applied pressure when the PLL circuit was integrated into the test set-up of Figure 3-94 


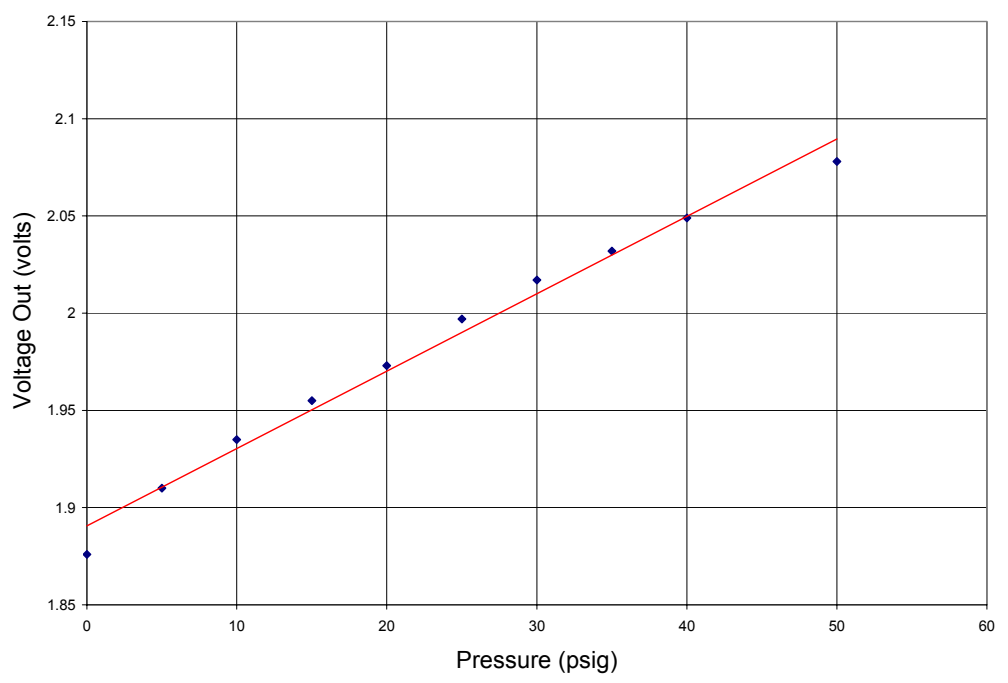

Figure 3-98. Output of the phase locked loop circuit as the pressure applied to the sensor, increasing the beam's resonant frequency.

\subsubsection{Sapphire Sensor Development by Index Modulation}

Techniques for local (on a small spatial scale) modification of the refractive index of a sapphire fiber in order to generate in-line (i.e. in the fiber) sensors were investigated. Two specific approaches were considered: periodic modification of the index to form grating structures in the fiber (both fiber Bragg gratings and long period gratings), and short $(<1 \mathrm{~mm})$ step changes in index to form intrinsic Fabry-Perot interferometers (IFPIs).

Grating-based sensors and Fabry-Perot sensors have been extensively researched in traditional glass optical waveguides such as optical fibers. Optimal function of the sensors requires single-moded waveguides; in multimode waveguides, the response of the sensor to each guided mode is different, due to differing phase constants of each guided mode when it interacts with the sensor structure. The total sensor response is a superposition of the responses of all of the modes to the sensor structure; the overlapping responses tend average out, washing out the total sensor response.

One solution to the problem is to restrict the launch conditions of the light injected into the waveguide, so that only a small number of modes are excited in the fiber. This approach has been used successfully in the past with straight, inflexible sapphire rods. ${ }^{17}$ However, since bends or splices or connectors in the fiber can cause a redistribution of

${ }^{17}$ A. Wang, S. Gollapudi, R.G. May, K.A. Murphy, and R.O. Claus, "Advances in Sapphire-Fiber-Based Intrinsic Interferometric Sensors," Optics Letters, 11/1/92, Vol. 17, No. 21. 
power into a larger number of modes, this strategy is limited in practice to applications were bends, splices, and connectors can be avoided. This limitation is the motivation for the development of high-temperature claddings for sapphire fiber in order to reduce the mode volume of the sapphire fiber.

\subsubsection{Fiber Bragg gratings sensors}

Fiber Bragg gratings (FBG) have several attributes that make them attractive for sensor systems. In particular, large numbers of FBG elements may be concatenated in order to achieve sensing along a single fiber. A number of schemes for interrogation of serially multiplexed FBGs have been developed.

Fabrication of FBGs in glass fibers relies upon the photosensitivity of germania-doped silica. The formation of FBGs is usually classified according to three distinct dynamic mechanisms. Type-I photosensitivity corresponds to a monotonic increase in the amplitude of refractive index modulation from exposure of a germania-doped silica core to intense UV radiation of the appropriate wavelength. Generation of color centers associated with $\mathrm{UV}$-induced effects in the $\mathrm{GeO}_{2}$ dopant is responsible for the refractive index modulation. Since color centers can be annealed by heat treatment, Type I FBGs are not stable at high temperatures, and can be erased at relatively low temperatures, approximately $200{ }^{\circ} \mathrm{C}$.

Protracted exposure of Type-I gratings to UV radiation will result in partial or complete erasure, followed by a large negative refractive index change. The resulting grating is classified as a Type-IIA grating (sometimes called Type III). It is assumed that the refractive index change with exposure time results from two local reactions. The first produces some defects or chemical species that lead to the positive index change; the second is slower than the first, and produces negative index change through structural reorganization. This structural reorganization is known as compaction of fiber.

Type-IIA photosensitivity can lead a refractive index change to about $1 \times 10^{-3}$ or even higher. A noticeable erasure is observed at temperature above $500{ }^{\circ} \mathrm{C}$.

Type-II gratings are distinct from other grating types. They are formed under very high, single-pulse fluences $\left(>500 \mathrm{~mJ} / \mathrm{cm}^{2}\right)$. Unlike Type-I and Type-IIA gratings, Type-II gratings can be obtained by using a high-power excimer laser. It is apparent that there is a threshold at pulse energy density of about $650 \mathrm{~mJ} / \mathrm{cm}^{2}$. When pulse energy is below the threshold, refractive index changes linearly. But refractive index change increases

dramatically above this threshold. When the pulse energy is higher than $1000 \mathrm{~mJ} / \mathrm{cm}^{2}$, the refractive index change will begin to saturate.

It is believed that Type-II photosensitivity is caused by the result of damage at the corecladding interface. Type-II photosensitivity can lead to a refractive change as high as $1 \%$. Type-II gratings show good stability to high temperature, withstanding temperatures as high as $800{ }^{\circ} \mathrm{C}$. Due to the damage at the interface of fiber core and cladding, the fiber becomes fragile at the writing point. This may be a potential problem if the sensor is not protected. 
Fiber Bragg gratings have only recently been demonstrated in sapphire fibers. ${ }^{18}$ These were achieved by the exposure of sapphire fibers with the output of an $800 \mathrm{~nm}$ femtosecond Ti: sapphire laser through a phase mask. The index modulation in this case is associated with multi-phonon ionization. Use of a femtosecond laser appears to be critical to achieve threshold fluence for ionization without heating the fiber with long pulse widths.

\subsubsection{Sapphire Intrinsic Fabry-Perot Interferometric Sensors}

In-line, or intrinsic, Fabry-Perot interferometric sensors have several features that make them attractive for measurement of physical quantities at discrete points along an optical fiber. Due to the low insertion loss of a typical IFPI sensor, many IFPI sensors can be concatenated on a single fiber without suffering significant penalty to the optical loss budget of the sensor system. Since the sensor is interferometric, high sensitivity is possible with appropriate designs, and a number of sensor interrogation methods for interferometric sensors may be applied to read out the IFPI sensors. Virginia Tech's Center for Photonics Technology has demonstrated the use of optical time domain reflectometers (OTDR) to demultiplex three IFPI sensors. Several groups, including Prime Research, have investigated the use of digital signal processing to deconvolve individual sensor responses from the spectral output of an optical fiber with multiplexed IFPI sensors.

During the Phase I research reported here, Prime Research investigated the fabrication of IFPI cavities in sapphire fibers. Since sapphire fibers do not exhibit the same sensitivity to UV radiation that germania-doped silica fibers do, a UV laser was not used in these studies. Instead, magnesium oxide was bulk diffused into unclad sapphire fibers over short $(<1 \mathrm{~mm})$ longitudinal segments of the fiber, in order to locally modify the refractive index over that length and define an intrinsic Fabry-Perot cavity.

Two configurations were attempted to the fabrication of sapphire IFPI sensors. In the first configuration, magnesium oxide (in a magnesium aluminate spinel slurry) was applied to the surface of a sapphire fiber over a short $(<1 \mathrm{~mm})$ length of the fiber. The coated fiber was then fired in a furnace in order to promote diffusion of the magnesium oxide into the sapphire fiber, in the hopes of reducing the refractive index over the short coated segment, forming an IFPI cavity.

\footnotetext{
${ }^{18}$ D. Grobnic, S.J. Mihailov, C.W. Smelser, and H. Ding, "Sapphire Fiber Brag Grading Sensor Made Using Femtosecond Laser Radiation for Ultrahigh Temperature Applications," IEEE Photonics Technology Letters, Vol. 16, No. 11, Nov. 2004, pp 2505 - 2507.
} 


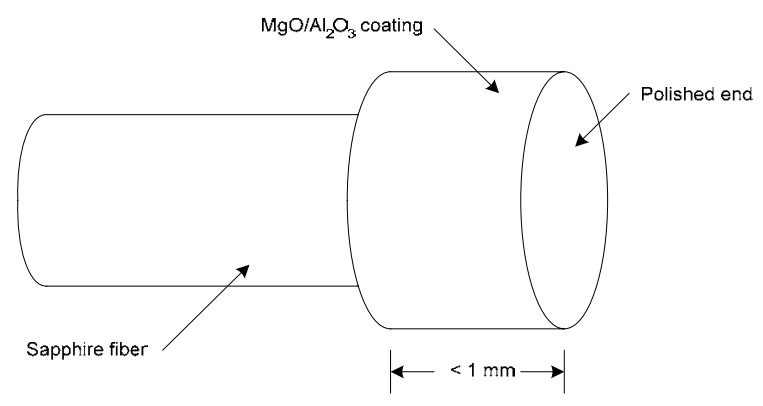

Figure 3-99. Geometry of completed sapphire IFPI sensor.

To construct a sensor of this first configuration, one end of a length of unclad $125 \mu \mathrm{m}$ diameter sapphire fiber (typically 4 inches in length) was dipped into a magnesium aluminate spinel slurry so that the coating was built up over a few millimeters of the end of the fiber. The fiber was masked with polyimide tape (a few millimeters were left uncovered at the end of the fiber), so that a distinct transition could be achieved between the coated fiber and the uncoated fiber. After a suitable number of layers of spinel had been applied to the fiber, the tape mask was carefully removed, and the partially coated fiber was fired in a furnace according to the schedule shown in Figure 4-74. The fiber was then removed from the furnace, and the coated end was polished to an optically smooth finish perpendicular to the axis of the fiber, through the use of a polishing wheel using diamond polishing media. The initial polish was conducted using coarse media until the length of the coated section of the fiber was less than $1 \mathrm{~mm}$, as shown in Figure 3-99. Upon completion of this process, the length of the sapphire fiber was measured using calipers to be $48 \mathrm{~mm}$ long, and from Figure 3-100, the coated section of the fiber was estimated to be approximately $488 \mu \mathrm{m}$ in length.

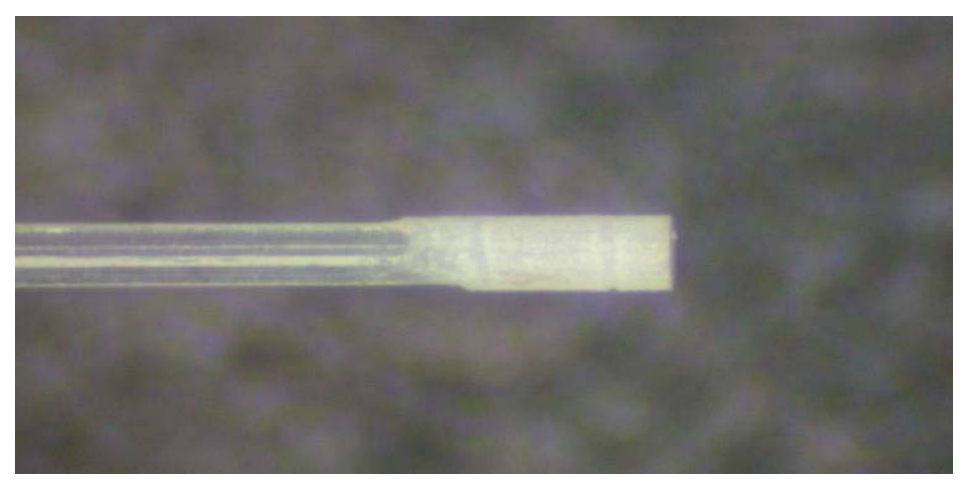

Figure 3-100. Photomicrograph of completed (fired) coating on end of sapphire fiber.

To evaluate the treated fiber and determine if an IFPI cavity was formed within the sapphire fiber, the experimental setup illustrated in Figure 3-101 was employed. The Micron Optics Component Test System (CTS) si720 is a swept laser spectrometer which was configured to analyze the spectral reflection from the sapphire fiber as its internal laser was swept from $1520 \mathrm{~nm}$ to $1570 \mathrm{~nm}$. A single-mode fiber (SMF) patch cord was 
used to connect the CTS to the sapphire fiber. The far end of the patch cord from the CTS was terminated in a FC/APC fiber optic connector polished at $8^{\circ}$ angle. The end of the sapphire fiber that was not modified to form the IFPI was also polished at $8^{\circ}$ angle.

The angled ends on the fibers are used to reduce the finesse of the Fabry-Perot cavity that may be formed between the sapphire fiber and SMF patch cord, to reduce the strength of any interferometric fringes formed in the air gap between the fibers. A three axis micropositioner was used to align the sapphire fiber input end to the fiber end in the FC/APC connector.

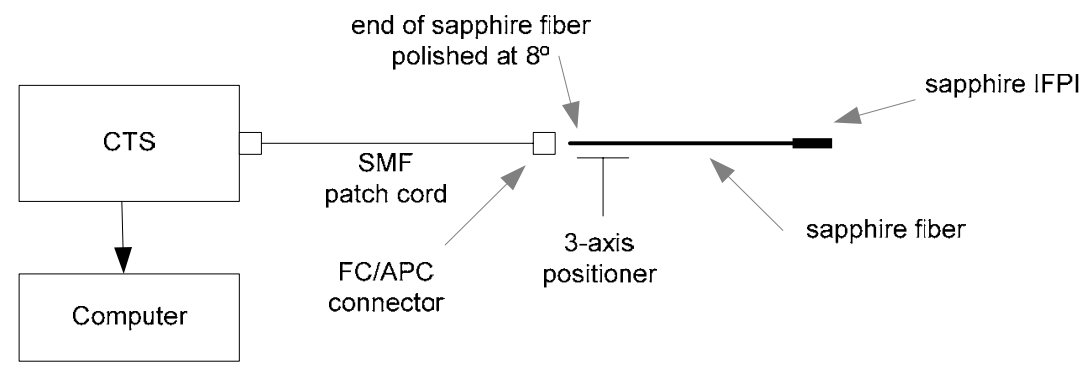

Figure 3-101. Experimental setup used to evaluate sapphire IFPI sensor. "CTS" is a Micron Optics Component Test System si720.

The spectral output obtained from the sapphire fiber is reproduced in Figure 3-102. The spectrum is a complicated signal, with several frequencies apparent. In order to facilitate interpretation of the output, the signal was Fourier transformed, with the results shown in Figure 3-104. The result shows a strong frequency content associated with a wave number of $71 \mathrm{~nm}^{-1}$, in addition to other peaks at lower frequencies. The $71 \mathrm{~nm}^{-1}$ wave number describes a spectral waveform with a wavelength of $14 \mathrm{pm}$. Using the expression relating the geometry of a Fabry-Perot cavity to its spectral output, the physical length $L$ of the cavity is given by

$$
L=\frac{\lambda^{2}}{2 \cdot n \cdot \Delta \lambda}
$$

\section{Equation 7}

where $n$ is the c-axis refractive index of sapphire, $\Delta \lambda$ is the wavelength of $14 \mathrm{pm}$, and $\lambda$ is chosen to be the wavelength at the center of the CTS scan $(1545 \mathrm{~nm})$. Upon substitution of these values into the equation, the physical length of the cavity associated with this peak is found to the $48 \mathrm{~nm}$, which agrees nicely with the measured length of the sapphire fiber. Therefore, the peak found at a wave number of $71 \mathrm{~nm}^{-1}$ is associated with fringes resulting from the Fabry-Perot cavity formed by the length of sapphire fiber. 


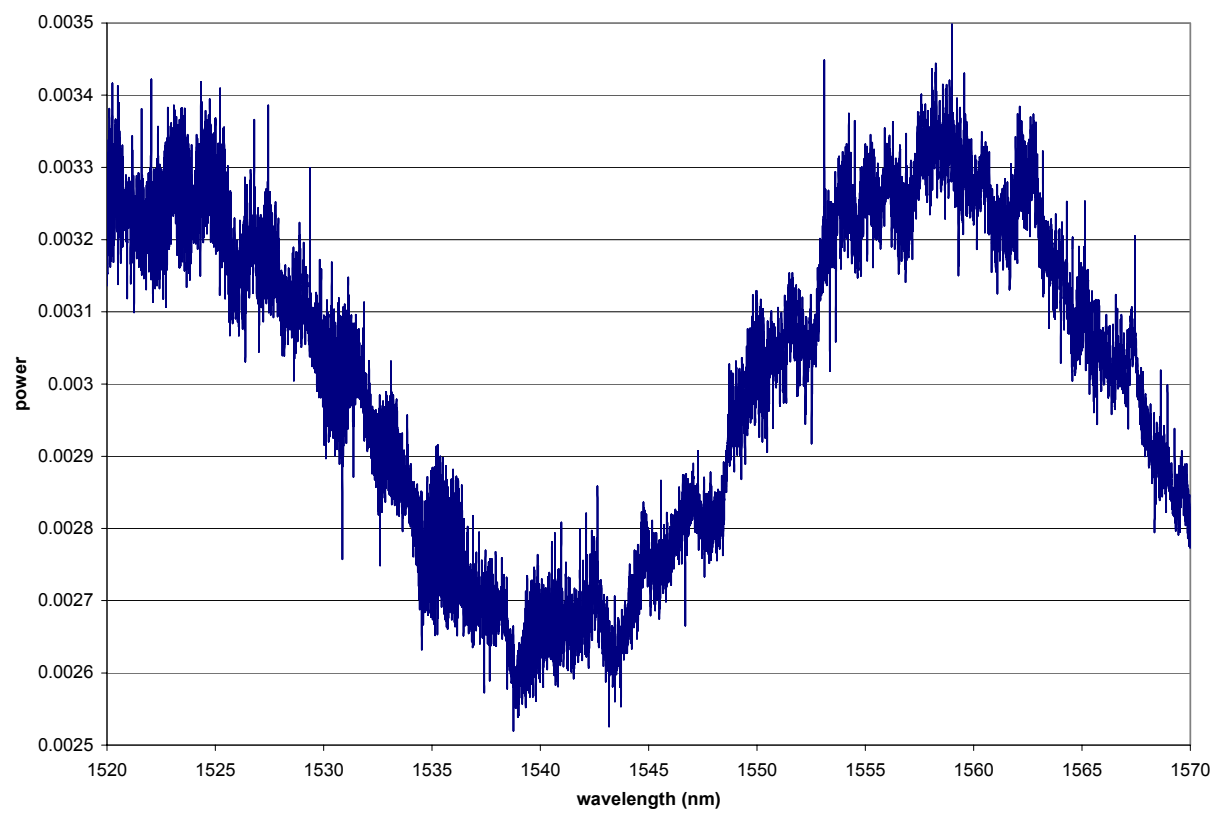

Figure 3-102. Spectral output of sapphire fiber with IFPI cavity, as measured by the CTS.

In order to analyze the lower frequency peaks, the Fourier spectrum is expanded to show the lower frequencies in Figure 3-104. Three additional peaks are seen in the spectrum from zero to $6 \mathrm{~nm}^{-1}$. The lowest of these, with a wave number of $0.02 \mathrm{~nm}^{-1}$, is associated with fringes with a period of $50 \mathrm{~nm}$, which is clearly the waveform with the largest amplitude in Figure 3-102. Using Equation again, this period is associated with a cavity of optical path length of $24 \mu \mathrm{m}$. This cavity was due to a $24 \mu \mathrm{m}$ gap between the sapphire fiber end and the fiber end in the FC/APC connector; due to an inability to rotate the fibers to align the angled fiber ends to a parallel state, the air gap was present and responsible for this peak in the Fourier spectrum.

The second peak in Figure 3-104 is associated with a fringe of period $2.17 \mathrm{~nm}$. Using the equation for the Fabry-Perot output, this fringe should be generated by a cavity with an optical path length of $550 \mu \mathrm{m}$. If we assume that this cavity is the IFPI cavity resulting from diffusion of magnesium oxide into the sapphire fiber, then the local refractive index should be 1.723 , and the resulting physical cavity length would be $550 \mu \mathrm{m} / 1.723$, or 319 $\mu \mathrm{m}$. This calculated value for the physical cavity length is approximately $150 \mu \mathrm{m}$ less than the estimate for the coated section of the fiber obtained from Figure 3-100. 


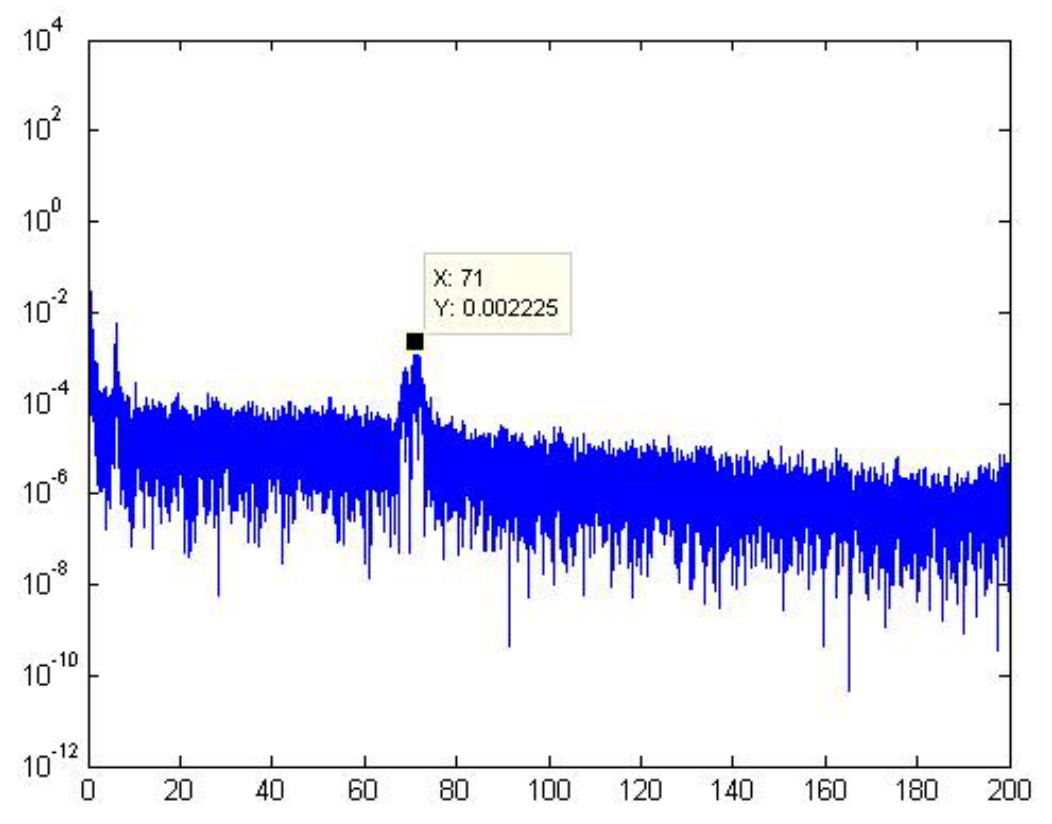

Figure 3-103. Fourier transform of data in Figure 3-102.

The origin of the third peak is as yet unclear. With a wave number of $6 \mathrm{~nm}^{-1}$, the associated fringe period is $0.16 \mathrm{~nm}$, which yields an estimated cavity optical path length of $7.45 \mathrm{~mm}$. No physical features were observed in the fiber corresponding to this length, or to a length of $4.22 \mathrm{~mm}$, which is found by dividing the optical path length by the refractive index of sapphire.

The second approach that was investigated in Phase I for fabrication of IFPI cavities in sapphire fibers is illustrated graphically in Figure 3-105. A magnesium alumina spinel coating is applied to a sapphire fiber in the usual way by dipping; however, this coating is not fired in a furnace. Instead, the fiber is fixed on a motorized rotary stage so that it can be rotated around the axis of the fiber. While the fiber rotates, the output of a $\mathrm{CO}_{2}$ laser is focused to a point on the surface of the spinel coating. As the fiber rotates, this focal point traces out a circumferential band on the coating. The intent is to heat the coating only in this circumferential band, resulting in a short band of magnesium oxide diffusion into the sapphire, to form an IFPI cavity of short length. One advantage of this approach over the previous one, in which the end of the sapphire fiber was polished to form one and of the IFPI cavity, is that IFPI cavities could be formed serially along the fiber. 


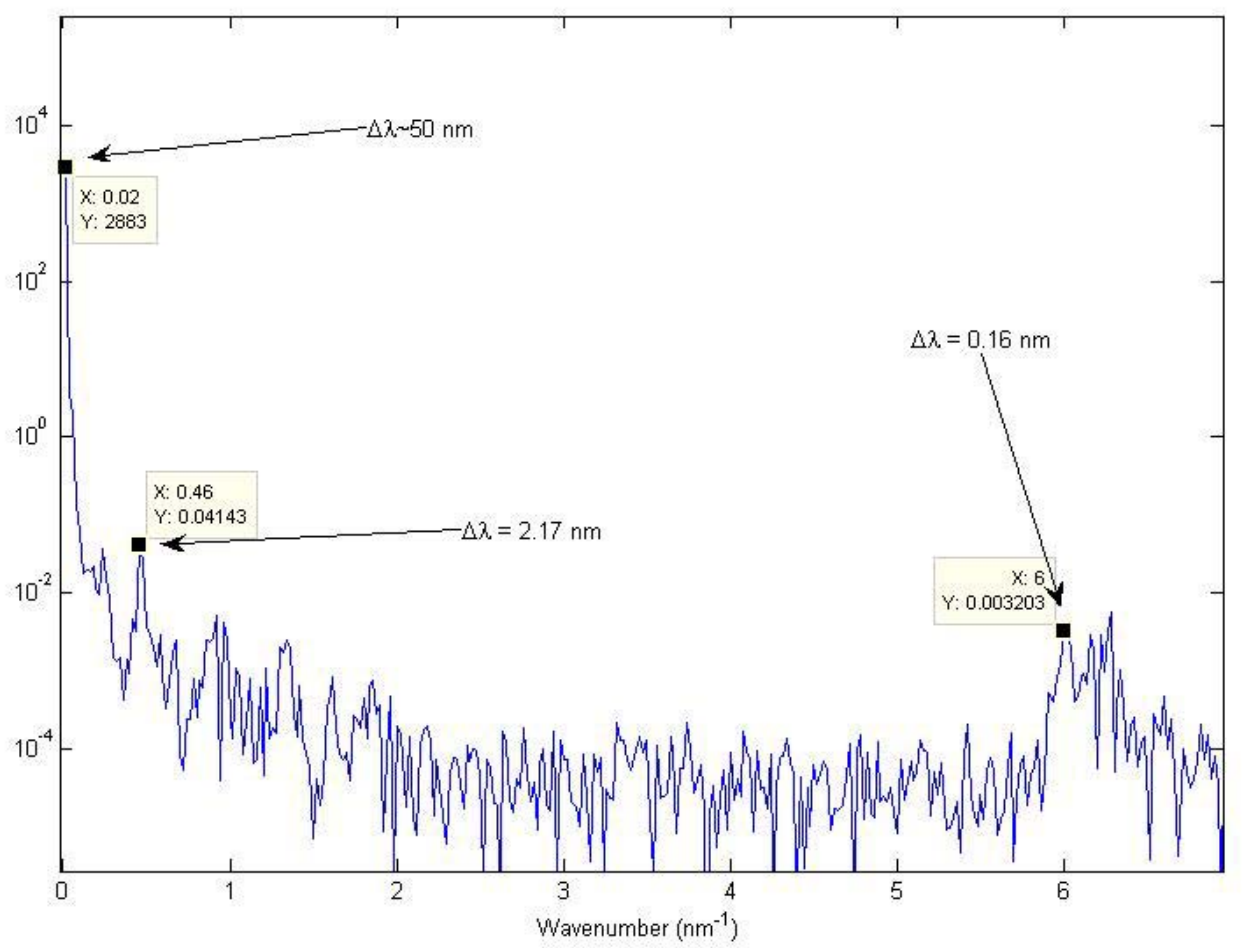

Figure 3-104. Zoomed view of the low frequency portion of Figure 3-103.

Figure 3-106 is a photograph of the apparatus set up in the laboratory to heat the coated fibers using a carbon dioxide laser. The fiber was mounted in a can place, which in turn was mounted on a step or motor shaft. In the photograph, the beam delivery optics of the $\mathrm{CO}_{2}$ laser is seen in the upper right; the fiber is seen to incandescent due to the heating in process.

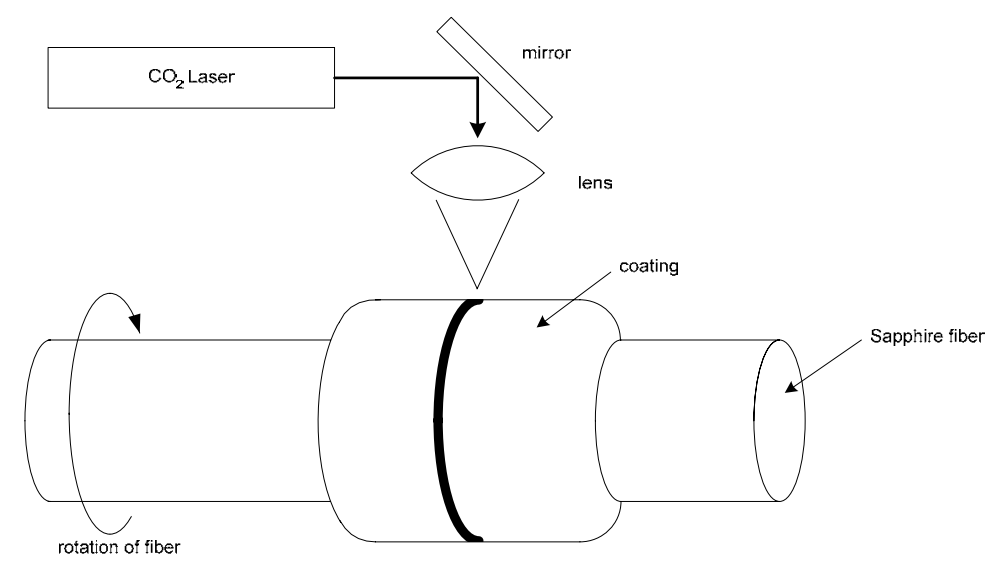

Figure 3-105. Experimental setup for selective firing of spinel coating to form IFPI in sapphire fiber. 


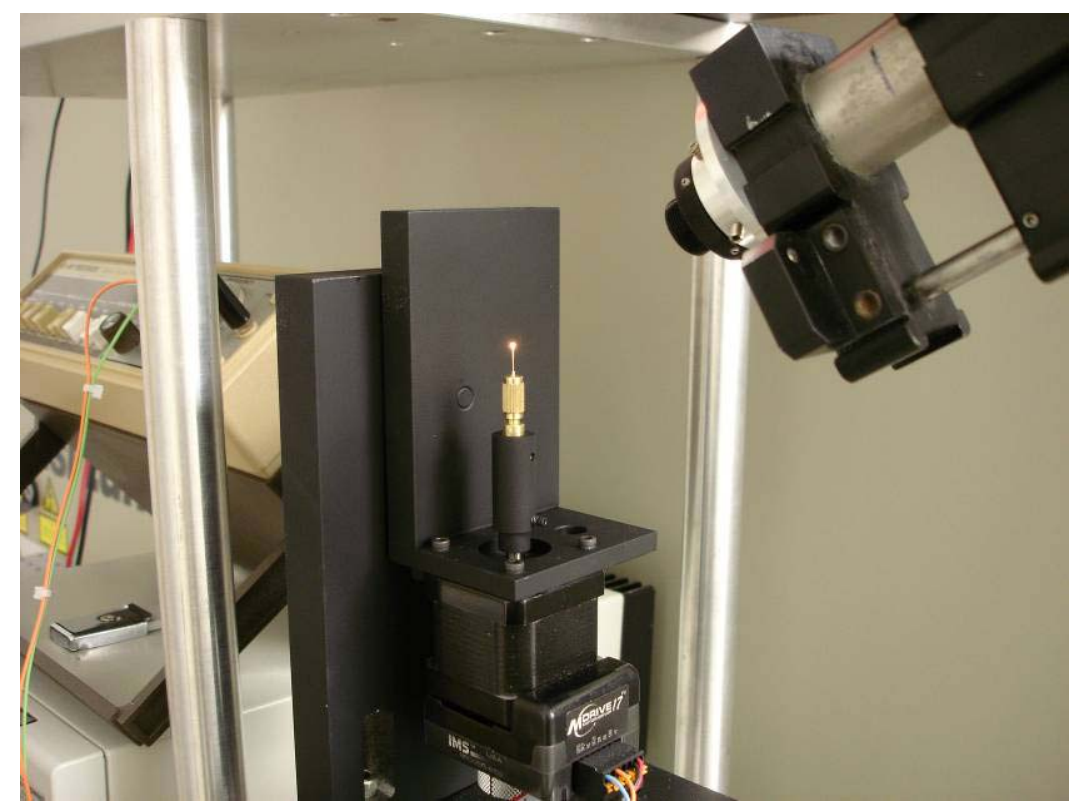

Figure 3-106. Photograph of fixture to rotate coated sapphire fiber while the radiating fiber by output of $\mathrm{CO}_{2}$ laser.

\subsection{Sapphire-to-Silica Splice Development}

In designing a sensor system using sapphire fiber, it is generally preferable to keep the length of the sapphire fiber as short as possible. Considerations driving this requirement include the high expense and attenuation of sapphire fibers relative to silica fibers. This is accomplished by using sapphire fibers only where environmental conditions (e.g. high temperatures, corrosive atmospheres) dictate, and splicing the sapphire fiber to a silica fiber once the fiber is clear of aggressive environmental conditions. For the interferometric sapphire fiber sensors being developed under this program, the sapphire is spliced to a standard 62.5 um core, $125 \mathrm{um}$ clad (62.5/125 um) multimode fiber using a configuration which can stand fairly high temperatures, but not the extreme temperatures needed for the actual sensor. The first testing of sensor prototypes was performed in the lab using splices made with epoxy and UV adhesive. The splices were actively aligned with a 5 -axis positioner. These splices could work for temperatures below $100{ }^{\circ} \mathrm{C}(212$ $\left.{ }^{\circ} \mathrm{F}\right)$ but not for the desired $800^{\circ} \mathrm{C}\left(1,472{ }^{\circ} \mathrm{F}\right)$. High temperature adhesives were considered for joining the fibers, but are not optically transparent; hence a fusion splice was considered the only reasonable approach.

Previous experience indicated that fusing the sapphire directly to the silica produced high loss splices. There is also difficulty splicing because the viscosity of fused silica is very low at temperatures approaching the melting point of sapphire. In addition, the two materials have very different coefficients of thermal expansion, causing the splice to crack during high changes in temperature. A splice design using a silica alignment tube and borosilicate glass melted between the silica fiber and sapphire fiber was tested with good results. Figure 3-107 is a diagram of the splice construction. First, an arc fusion 
splicer is used to deposit a hemispherical drop of borosilicate glass on the end of a silica fiber. The end of the silica fiber with the borosilicate drop is inserted into a short fused silica capillary tube. A sapphire fiber is then inserted into the other end of the tube until the two fibers contact. Finally, an arc fusion splicer is used to heat the fused silica until the borosilicate becomes fluid. The two fibers are pushed together until they contact, and then the heat is withdrawn. As the borosilicate cools and solidifies, it captures the silica and sapphire fibers in the tube.

A total of 15 splices were made using this technique. Forward direction splice losses (from source toward sensor only) ranged from $1.5 \mathrm{~dB}$ to $7 \mathrm{~dB}$. Splices with higher throughput losses resulted in poor sensor signal fringe contrasts when sensors were fabricated using the slices. Overheating of the borosilicate glass would produce bubbles in the optical path, and too much borosilicate would cause small cracks. The alignment tube had an ID of $150 \mathrm{um}$, resulting in loose fits for the $135 \mu \mathrm{m}$ clad sapphire and the $125 \mu \mathrm{m}$ clad silica. Active fiber alignment was difficult because it had to be done while the borosilicate was fluid which lasted only about a second during the arc fusion. Figure 3-108 and Figure 3-109 show photos of the two splices later used in sensor testing.

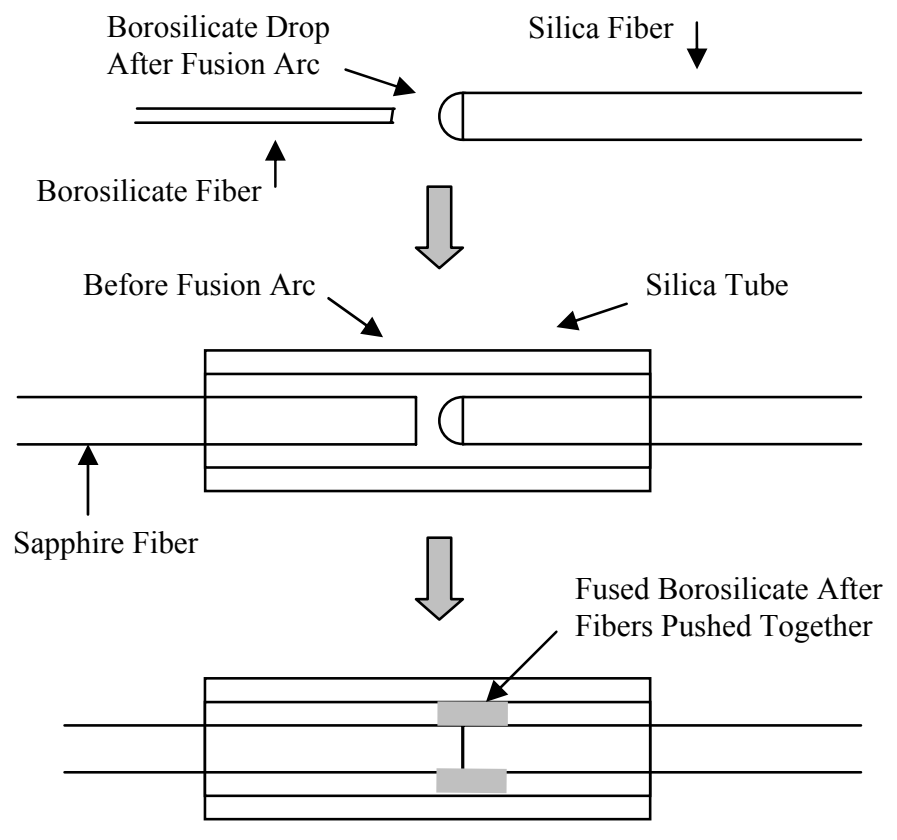

Figure 3-107. Splice Construction

Probably the most difficult problem encountered was the unpredictability of the splice signal quality, due to the intentional under-injecting of the modes in the multimode fiber from the singlemode fiber. Figure 3-110 shows diagrams of the measurement setups in which a singlemode (10 $\mu \mathrm{m}$ core, $125 \mu \mathrm{m}$ clad) fiber was used to inject light into a multimode $(62.5 \mu \mathrm{m}$ core $/ 125 \mu \mathrm{m}$ clad $)$ silica fiber; use of a single mode fiber for injection preferentially stimulates low order modes in the multimode fiber. The splice loss was calculated by comparing the splice throughput power to the reference power indicated in the figure. When the multimode sapphire fiber ( $90 \mu \mathrm{m}$ core, $135 \mu \mathrm{m}$ clad) was modally fully injected using only multimode fiber $(62.5 \mu \mathrm{m}$ core, $125 \mu \mathrm{m}$ clad $)$ the 
losses were much higher (over $10 \mathrm{~dB}$ ). This high loss was probably due to the low numerical aperture of the clad sapphire fiber. When using these injection conditions (multimode fiber only), no sensor interference signal could be seen. Singlemode injection solved the problem, but an increase in modal noise could contribute to instability in the signal.

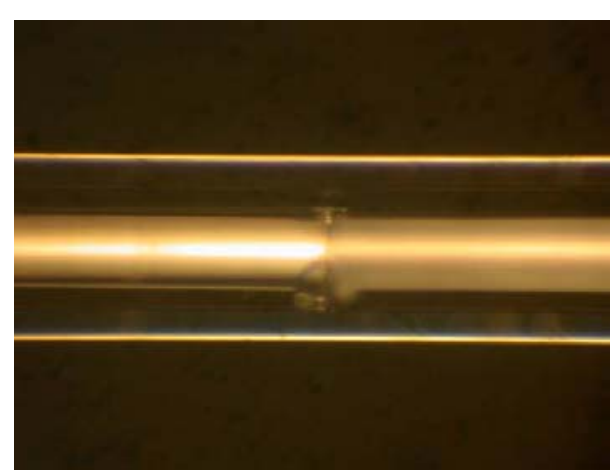

Figure 3-108. Splice \#8

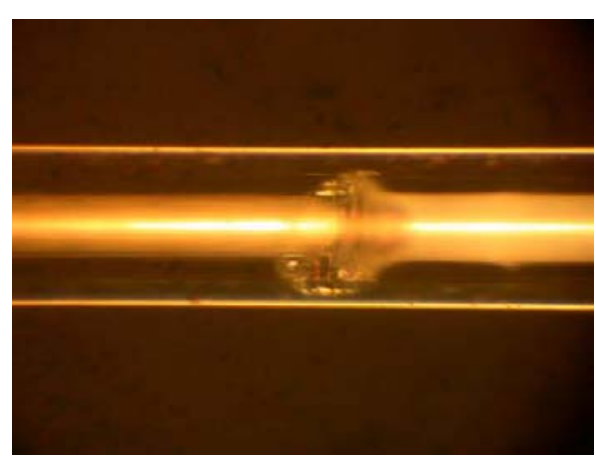

Figure 3-109. Splice \#15

Figure 3-111 shows the spectra reflected back through the splice with and without the sensor signal when a CTS interrogator was used as the interrogator. Splice \#6 had a very noisy background signal without the sensor. When the sapphire fiber tip was built into a sensor, the interference pattern from the sensor still had the uneven splice background shape. Splice \#8 and splice \#15 had much flatter splice background signals and cleaner sensor signals; however, the reason for this is still unknown. Splice \# 8 was later used in sensor C5 and splice \#15 was used in sensor G1 (Section 3.5.4.1.3).
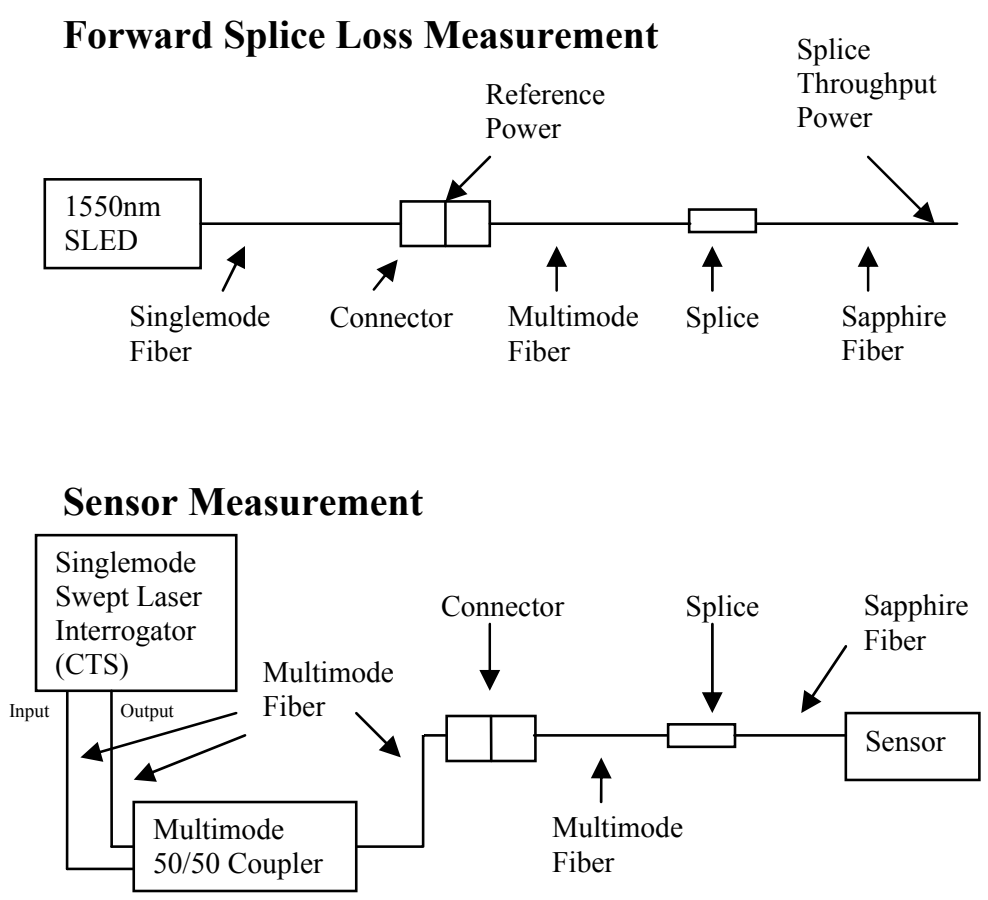

Figure 3-110. Measure Setups for Splice Loss and Quality 
In order to determine the practical maximum temperature for the splice assembly, and experiment was conducted in which the splice was exposed to a range of temperatures as the sensor output was monitored. Figure 3-112 depicts the temperature sensor in its ceramic packaging. The ceramic is the initial package before the rugged outside housing and final cabling is installed as described in Section 3.5.5. The splice is located in the last section of ceramic tubing (as labeled above).

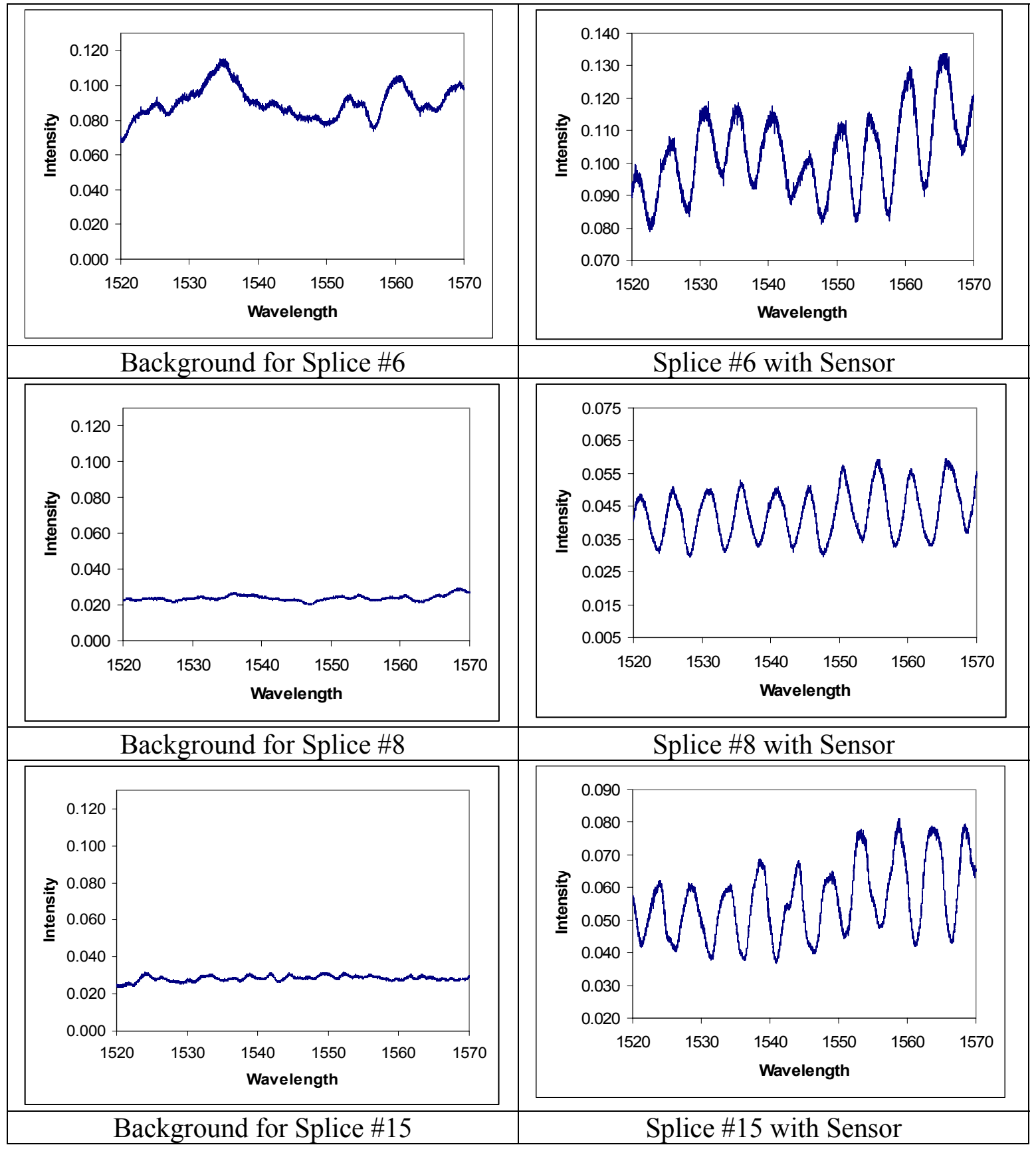

Figure 3-111. Splice background signals and sensor signals using the splices. (Background refers to the signal obtained from the interrogator when the sensor is disconnected from the $50 / 50$ coupler shown in Figure 3-110). 


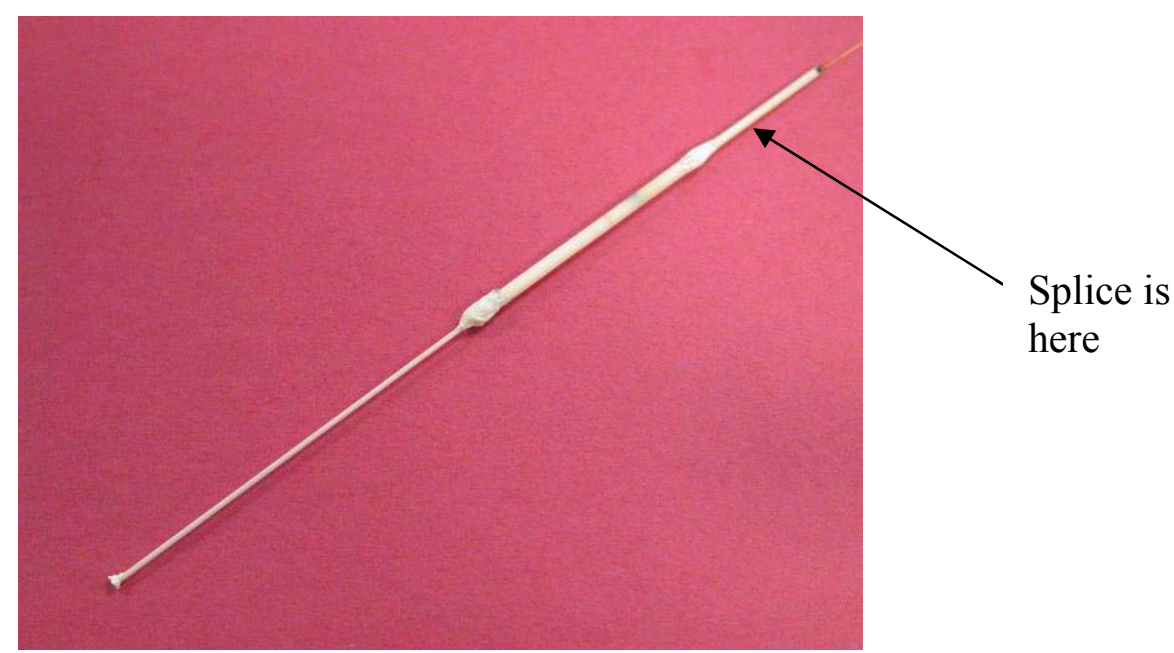

Figure 3-112. Sapphire temperature sensor in ceramic packaging, showing the location of the silica/sapphire splice.

The first test was assembled according to the photo (Figure 3-113). The temperature sensor, in its completed ceramic housing, was inserted into the heating coil positioning the splice directly in the center of the heat coil. The coil was controlled by an Omega CNi3244 temperature controller and a variac with an omega thermocouple inserted into the center of the coil. The sensor was connected to a VectorLight 200 interrogator to monitor the sensor output as the temperature was manually increased.

The first temperature was $150^{\circ} \mathrm{C}\left(302^{\circ} \mathrm{F}\right)$ for 30 minutes to check the fringes. The signal was strong, so the temperature was increased in $50^{\circ} \mathrm{C}\left(90^{\circ} \mathrm{F}\right)$ steps, allowed to stabilize, and then held for 10 minutes at each temperature. The signal remained strong at each temperature range. Upon achieving $400{ }^{\circ} \mathrm{C}\left(752{ }^{\circ} \mathrm{F}\right)$, the temperature was then stepped through more slowly, in steps of $15^{\circ} \mathrm{C}\left(27^{\circ} \mathrm{F}\right)$ and allowed to stabilize for 15 minutes after each temperature was achieved. At $450^{\circ} \mathrm{C}\left(842^{\circ} \mathrm{F}\right)$, a slight drop in the signal was observed, but it still remained a useable signal. The temperature was then increased to $475^{\circ} \mathrm{C}\left(887^{\circ} \mathrm{F}\right)$. At this temperature, the signal became unstable and within a minute, the signal was completely lost.

The sensor was cooled and successfully unpackaged. After removing the sensor and viewing the splice under a microscope, the adhesive appeared brownish in color. While under the scope, the splice fell apart. The gold fiber cleave was inspected and appeared to be a smooth cleave, therefore confirming the fact that the splice could not handle more than $450^{\circ} \mathrm{C}\left(842^{\circ} \mathrm{F}\right)$ without failing.

A second test was performed on the temperature sensor splice, but this time without being packaged in the ceramic housing. The bare sensor was inserted in the heating coil with the splice in the center of the coil. The test fixturing and procedure followed was exactly as previous test. The splice failed at $460^{\circ} \mathrm{C}\left(860^{\circ} \mathrm{F}\right)$, confirming earlier results. 


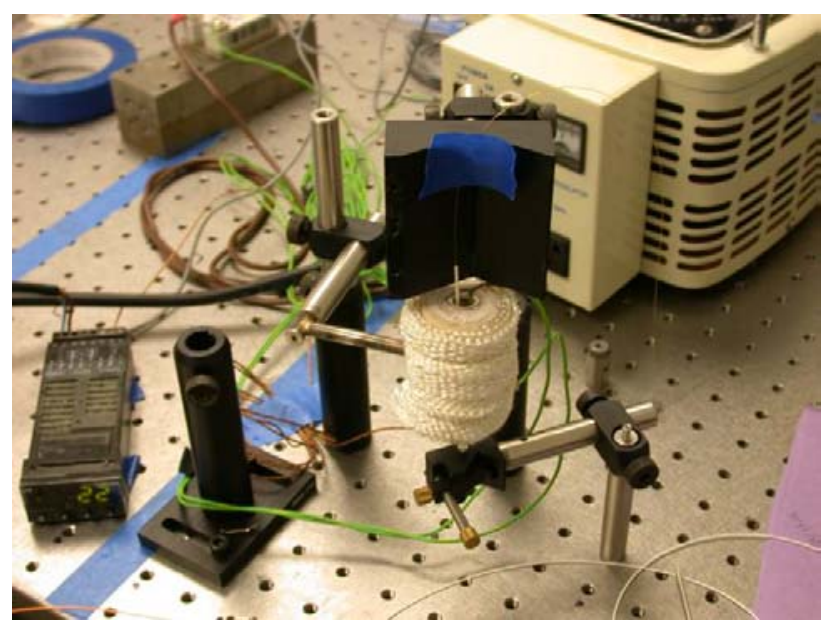

Figure 3-113. Test set-up to determine maximum operating temperature of the silica-sapphire splice.

As described in Section 2.5.2, the Babcock \& Wilcox Clean Environment Development Facility (CEDF) was ultimately selected for testing the temperature and strain sensors. The first design of the temperature sensor intended for use in the City of Hamilton, Ohio power plant had the splice about $114 \mathrm{~mm}(4.5 \mathrm{in})$ from the tip. The CEDF plant requires that the splice be at least $560 \mathrm{~mm}$ (22 in) from the sensor tip. Since the current process for applying spinel cladding to the sapphire fiber is limited by our laboratory equipment to lengths of approximately $152 \mathrm{~mm}(6 \mathrm{in})$, unclad sapphire fibers must be used to instrument the CEDF. Since longer sapphire fibers may then be used, there is no reason to have the splice so close to the heat zone. Therefore, a standard FC/PC connector can be used with standard epoxy to connect an unclad sapphire fiber to a silica fiber. Both the silica fiber and the sapphire fiber can be connectorized using standard industry procedures.

\subsection{Interrogator Development}

Several methods for interrogating the high-temperature interferometric sensors were evaluated. Two key choices are required in the area of sensor decoding. These two are the question of the interrogation (hardware) methodology, and signal processing (or algorithmic) selection. Three candidates for the interrogation system were evaluated for use in the field demonstration that took place in B\&W's CEDF facility. Two of these systems were modified versions of existing Prime Research, LC (PRLC) products, the VectorLight ${ }^{\mathrm{TM}} 200$ and VectorLight ${ }^{\mathrm{TM}}$ 300. These systems are both based on sensor interrogation with a scanning laser illumination. The required modification to these instruments is to make them compatible with the multimode nature of sapphire fiber.

The third system considered was an experimental approach based on broad band illumination with an incoherent light source (a light emitting diode, or LED), referred to as the Experimental Incoherent Interrogator (EII). All three interrogation systems rely on obtaining a spectral response from the transducer. A summary of some basic characteristics of each of these interrogation systems is shown in Table 3-12 below. 
Table 3-12: Interrogator properties

\begin{tabular}{|c|c|c|c|}
\hline & VectorLight ${ }^{\mathrm{TM}} 200$ & VectorLight ${ }^{\mathrm{TM}} 300$ & EII \\
\hline $\begin{array}{l}\text { \# of simultaneous } \\
\text { measurements }\end{array}$ & 1 & 4 & 1 \\
\hline Update rate & $1 \mathrm{~Hz}$ & $1 \mathrm{~Hz}$ & $4 \mathrm{~Hz}$ \\
\hline $\begin{array}{l}\text { Wavelength } \\
\text { Resolution }\end{array}$ & $2.5 \mathrm{pm}$ & $5 \mathrm{pm}$ & $\sim 50 \mathrm{pm}$ \\
\hline
\end{tabular}

In the area of signal processing, there are multiple measures that can be considered in comparison of algorithm suitability. The primary criteria are the algorithm's accuracy, resolution, and whether the algorithm results in an absolute measurement or a relative one. To facilitate clarity, the following definitions are used:

- An absolute measurement is one in which no data regarding the sensor history is required to obtain an accurate measurement of the environment. The sensor may still require a fixed calibration, which would be accomplished upon fabrication of the sensor.

- A relative measurement is one in which the output of the algorithm indicates the change in measurand between the previous measurement and the current one (e.g. the temperature is now $0.5^{\circ} \mathrm{C}\left(0.9^{\circ} \mathrm{F}\right)$ higher than it was one the previous cycle).

To obtain an absolute measurement from a relative measurement requires continuous tracking of the value from a known reference point.

Due to the need to instrument multiple locations in the planned field test, absolute measurements were chosen for development in this program. The use of absolute measurements allows for switching between multiple sensors without having to maintain a dedicated interrogator for each measurement. Emphasis was placed on working with the modified VectorLight ${ }^{\mathrm{TM}} 200$, due to its higher accuracy than the VectorLight ${ }^{\mathrm{TM}} 300$. The data shown and discussed below was all gathered from a modified VectorLight ${ }^{\mathrm{TM}}$ 200.

The sensors which were used in this program are in the category of low finesse FabryPerot Interferometers. The characteristic spectral response of a low finesse system is a near sinusoidal variation of reflected intensity as a function of wavenumber for a pair of reflective surfaces. Wavenumber $k$ is defined by $k=1 / \lambda$, where $\lambda$ is the wavelength of the light being reflected. As additional surfaces are introduced into the sensor, the spectral response grows increasingly complex; the response is the superposition of individual Fabry-Perot cavities formed by all of the surfaces. Theoretical models of the spectra from two and three surface systems can be seen below in Figure 3-114 and Figure $3-115$, respectively. It can be seen that the addition of an extra reflector results in a more complex spectrum. 


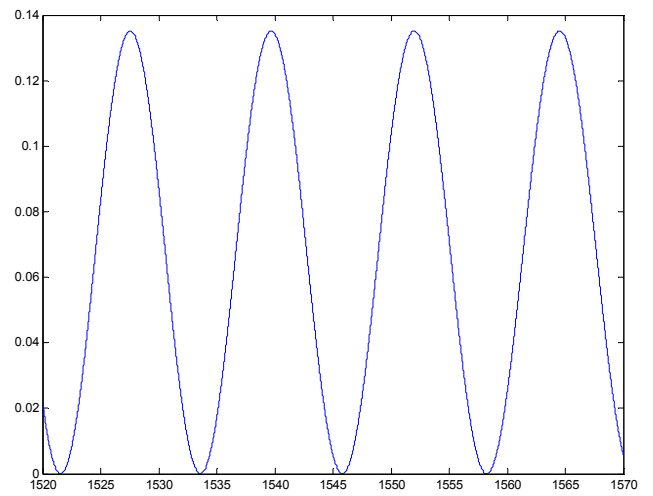

Figure 3-114: Theoretical simulation of the spectral response from a low finesse two surface interferometer

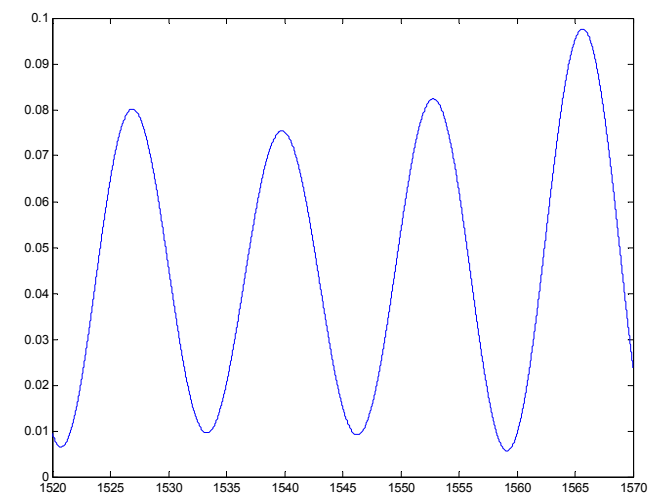

Figure 3-115: Theoretical simulation of the spectral response from a low finesse three surface interferometer

The digital signal processing used to condition the output of the Prime Photonics, LC sapphire sensors is composed of a collection of signal processing modules. The raw data provided by the interrogation system represents a large number (greater than ten thousand for the swept laser interrogator, and 3646 for the EEI) of samples of the reflected power intensity over a given range of wavelengths (nominally the C-band, from 1520 to 1570 $n m)$.

The basic components of the processing sequence are:

- Collection of raw spectral data from the reflected signal (Figure 3-116)

- Spectral flattening to account for losses that are linear in wavelength (if necessary)

- Filtering any high frequency noise from the data (Figure 3-117)

- Conversion of sampled waveform from uniform spacing in wavelength to uniform spacing in wavenumber

- Appropriate decimation, or downsampling, of the resulting data

- A module whose function is to determine the approximate size of the cavity (coarse measure)

- A module which acts to determine a precise response of the cavity (fine measure)

- Conversion from coarse and fine cavity measurements to sensor parameter (cavity length)

While all of these aspects of the algorithm were already in existence, in the course of this program, efforts were made to enhance the spectral filtering, decimation, and the coarse measurement functions of the algorithm. 


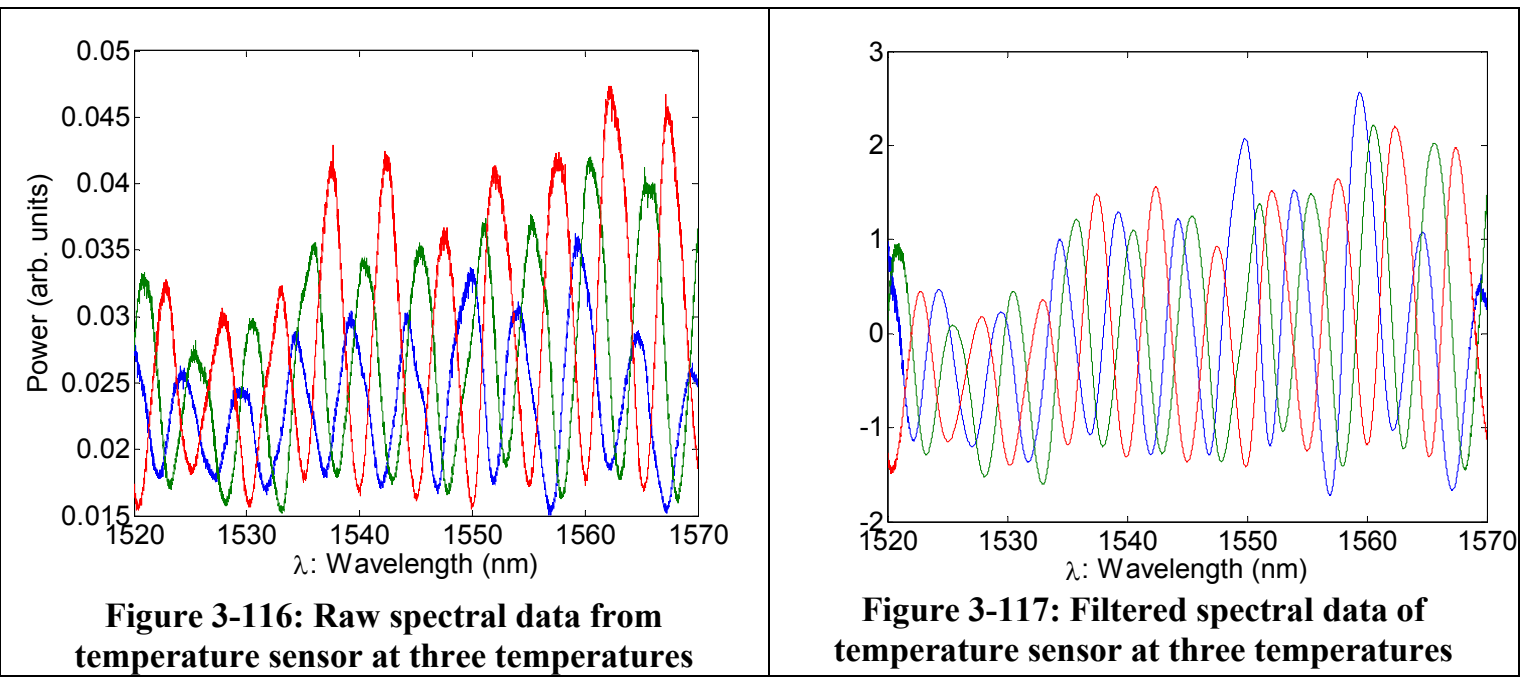

Several new filtering methods were implemented which can be utilized based on the precise characteristics of the sensor in question. One of these methods is based on the use of a baseband shift in order to achieve an extremely narrow bandpass filter, which improves the resistance to noise caused by extra reflective surfaces. Multi-rate filtering was also attempted in an attempt to accelerate the processing.

The two modules which are responsible for measuring cavity response are based on approximation of the decimated data with a superposition of mathematical sinusoids. The oscillatory response of a single Fabry-Perot interferometer is described by

$$
I(\lambda, L) \propto \cos \left(\frac{4 \pi n L}{\lambda}\right)=\cos \left(\frac{4 \pi n L v}{c}\right) .
$$

For a given cavity size $L$, the phase of the sinusoid in the intensity pattern varies inversely as a function of wavelength $\lambda$ (or linearly as a function of optical frequency, $v$ ). Because Fabry-Perot sensors transduce the physical measurand by changing the cavity length $L$, an accurate measure of this length is the key to decoding a sensor's response.

There are two primary characteristics of these spectra which change as the sensor responds to the measurand (Figure 3-118). The first is the phase shift which is shown by the marking A. This is a highly sensitive response to changes in the sensor. A problem from which it suffers, however, is that due to the periodic nature of sinusoids, when the measurand traverses a large enough range, it will return to the same value of phase. The second spectral response is a shift or change in the period (or spacing) between successive peaks in the spectrum (Figure 3-118-marked B). This is a much less sensitive measure than the change in phase. As a result, it is substantially more susceptible to noise in the original measurement. It is, however, an absolute measurement, with each value of period correlating to exactly one value of measurand. 


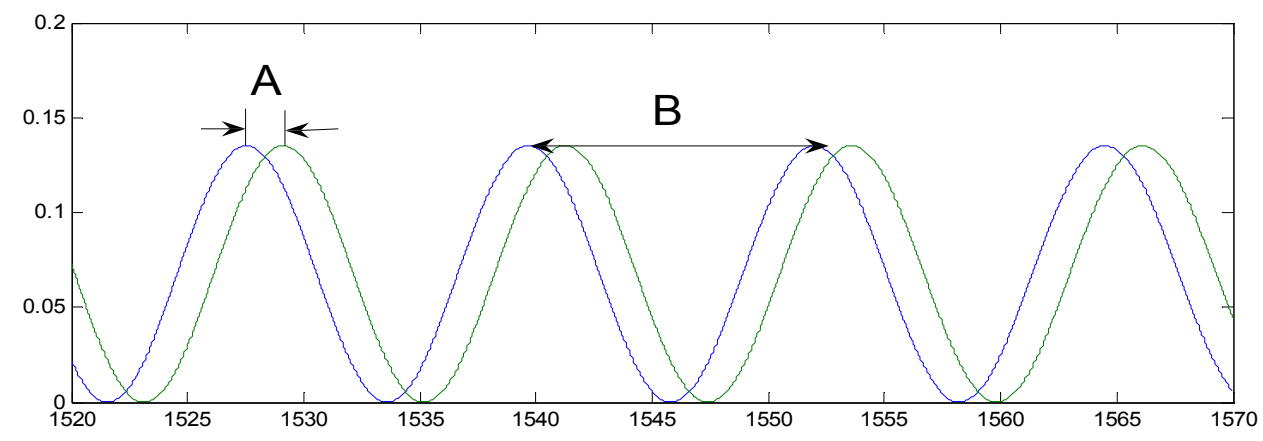

Figure 3-118: Spectral responses of sensor to measurand: (A) phase shift, and (B) change in period

The most significant algorithmic effort undertaken during the program was in the area of effectively assessing the coarse cavity size, which is derived from the spectral period (Figure 3-118-marked B). While this is a nearly trivial problem in the case of a noiseless sinusoid by the use of an FFT or similar computation, it is highly challenging when there is significant noise, or when there exists the overlap of multiple signals with similar frequencies.

Processed spectra from a silica pressure sensor and a sapphire temperature sensor can be seen in Figure 3-119 and Figure 3-120 respectively. In both cases, the data shown represent measurements taken at continuously increasing values of pressure or temperature (although the rate of increase is not constant). The green trace is the calculation of spectral phase. This data is relatively noise free but ambiguous due to the cyclic repeating of values. The blue trace is the optical path length of the sensor (the coarse measure), which is a measurement derived from the period of the spectral fringes. This data is noisier, but in the case of the silica sensor, is certainly adequate to resolve the appropriate integer multiple of $2 \pi$ to add to obtain the absolute phase.

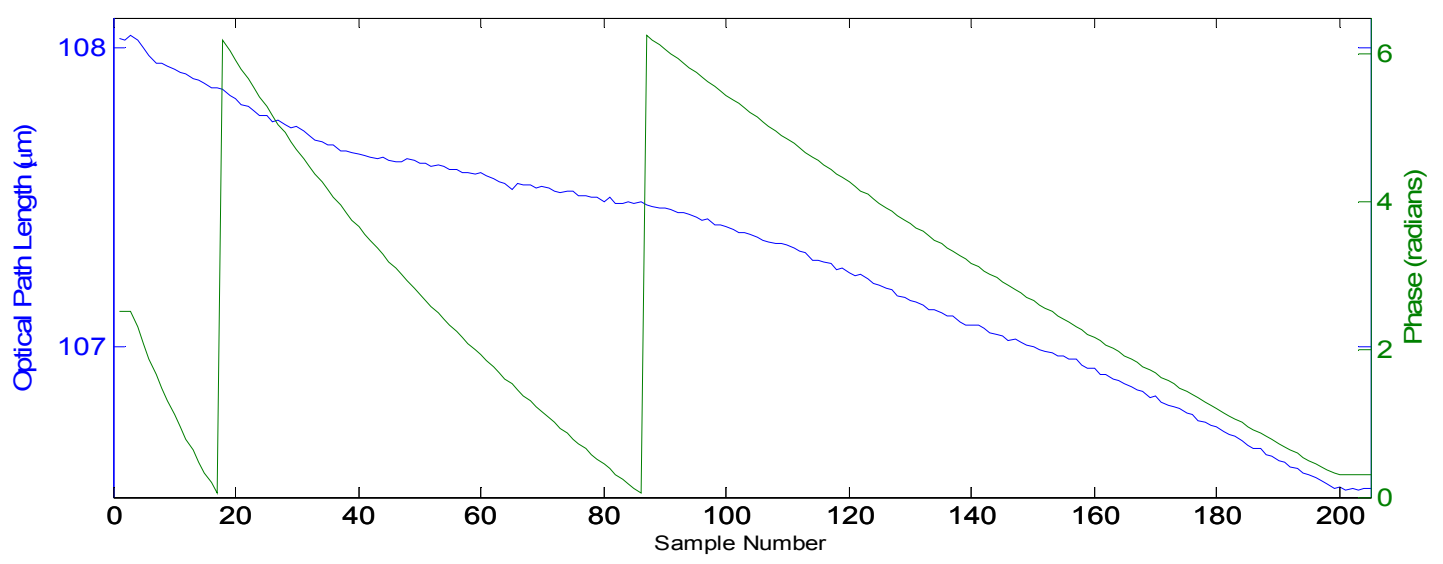

Figure 3-119: Phase and period measurements from 205 spectra taken from a silica pressure sensor

In Figure 3-120, one notes that the optical path length measurement is not currently usable for distinguishing between ambiguous values of phase, as it was in the case of the silica sensor. This is the result of errors in calculation induced by the additional noise present in gathering spectra from the sapphire sensor, most likely from modal noise 
(intermodal interference). The noise is clearly visible in the comparison of Figure 3-121 with Figure 3-122. In Figure 3-121, it is clear that there are relatively few spectral components, which allows for straightforward separation and detection of signals. The spectra from the sapphire sensor, shown in Figure 3-122, contain a much richer spectral composition, was well as more variation of that composition from sample to sample. As a result, it is much more difficult to accurately isolate the desired components for analysis.

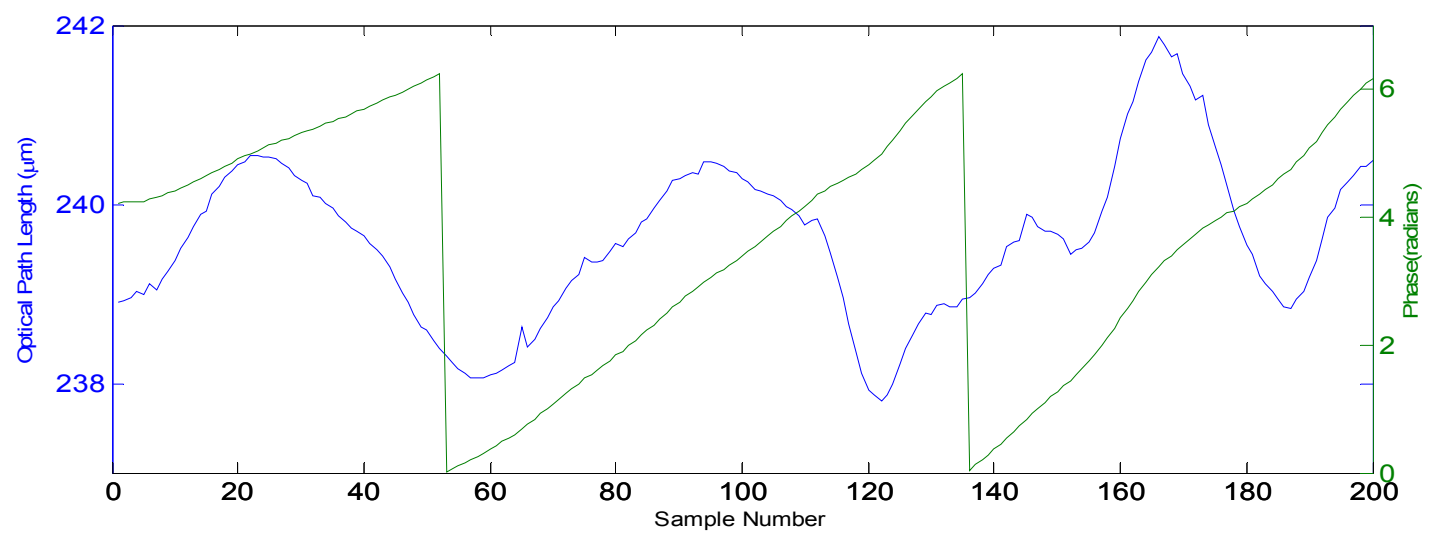

Figure 3-120: Phase and period measurements from 200 spectra taken from a sapphire temperature sensor

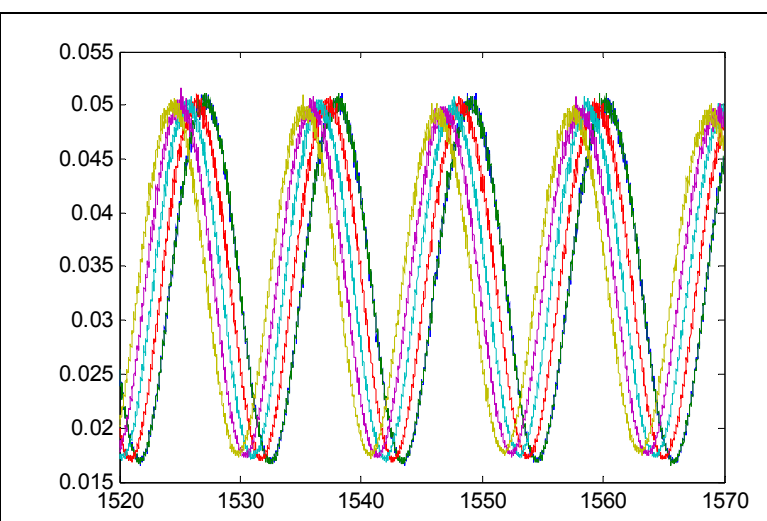

Figure 3-121: Six sequential spectra taken from silica sensor

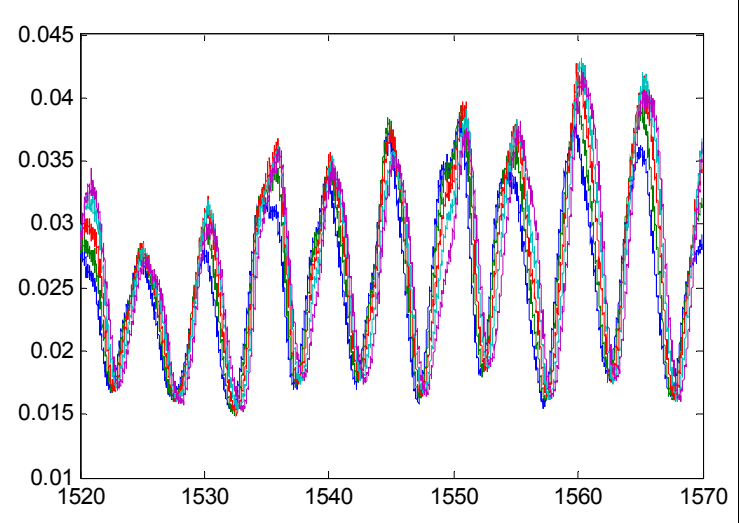

Figure 3-122: Six sequential spectra taken from sapphire sensor

A number of approaches were developed to improve processing in the presence of significant noise, or when there exists the overlap of signals from multiple reflectors. In order to compare the effectiveness of the various approaches, the processed results of the same collection of data will be presented after each of the methods has been applied. The data which is being used represents the performance of the sapphire temperature sensor, G9. The profile of the temperature to which the sensor was exposed, and the number of data samples obtained at each step, are shown in Table 3-13. 
Table 3-13: Profile of test data collected on sapphire temperature sensor G9

\begin{tabular}{|l|c|}
\hline Test Profile & Number of Samples \\
\hline Ramp to $600^{\circ} \mathrm{C}$ & 120 \\
\hline Soak at $600{ }^{\circ} \mathrm{C}$ & 35 \\
\hline Ramp to $800^{\circ} \mathrm{C}$ & 46 \\
\hline Soak at $800{ }^{\circ} \mathrm{C}$ & 35 \\
\hline Ramp to $1000^{\circ} \mathrm{C}$ & 52 \\
\hline Soak at $1000^{\circ} \mathrm{C}$ & 35 \\
\hline Ramp to $1200^{\circ} \mathrm{C}$ & 100 \\
\hline Soak at $1200^{\circ} \mathrm{C}$ & 10 \\
\hline Ramp down from $1200^{\circ} \mathrm{C}$ & 67 \\
\hline
\end{tabular}

A full, raw (unprocessed) spectrum was saved at each sample point, and later postprocessed using each of three methods described below. A sample spectrum can be seen in Figure 3-123. Every spectrum consists of 20,000 measurements of intensity equally spaced in wavelength from 1520 to $1569.9975 \mathrm{~nm}$. In every case, the same steps were applied to flatten, digitally filter, and decimate the data.

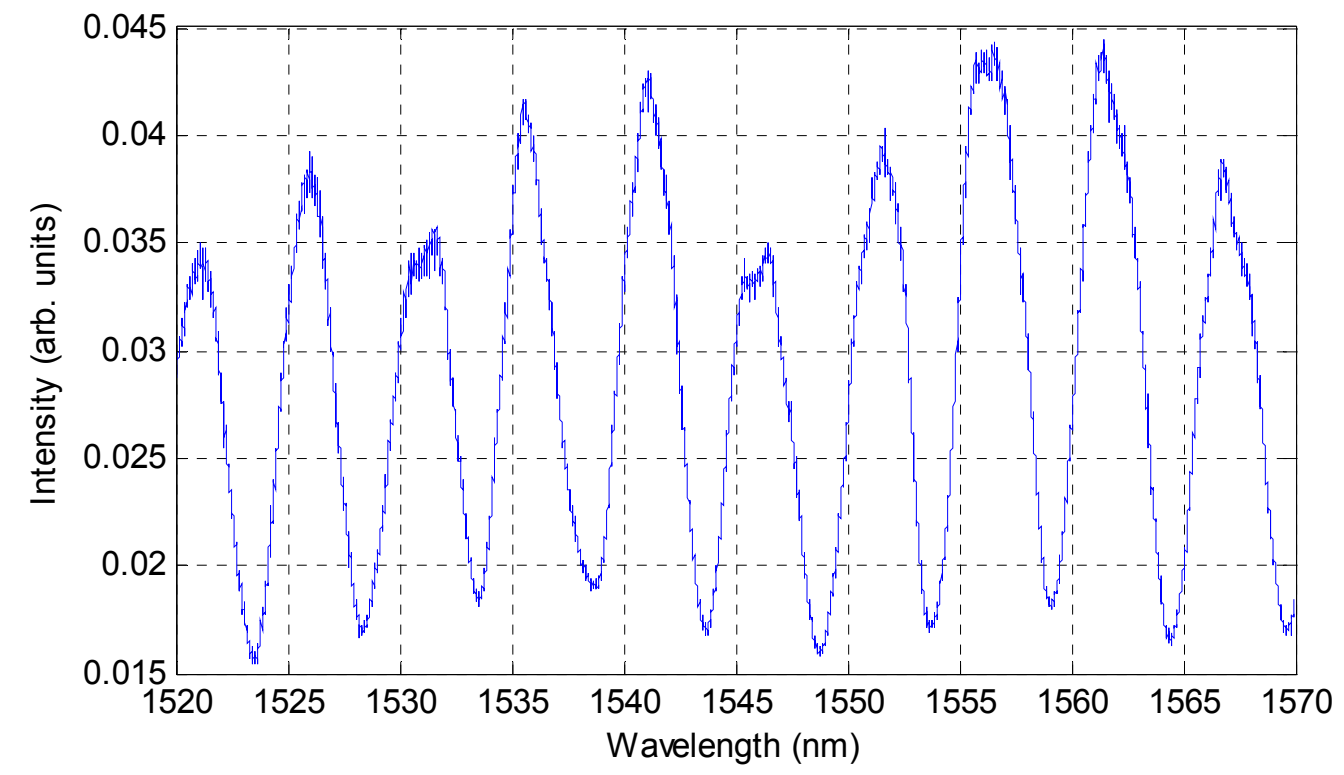

Figure 3-123: Raw data spectrum from sapphire temperature sensor G9

The initial algorithm was based upon the iterative optimization of a single variable equation. The results of this method of calculation can be seen in Figure 3-124. The time taken to perform the calculation on 500 samples was approximately 316 seconds.

The upper plot shows the result of the coarse optical path length calculation derived from the waveform period, whereas the lower represents the fine adjustment of the calculation based on phase tracking. 

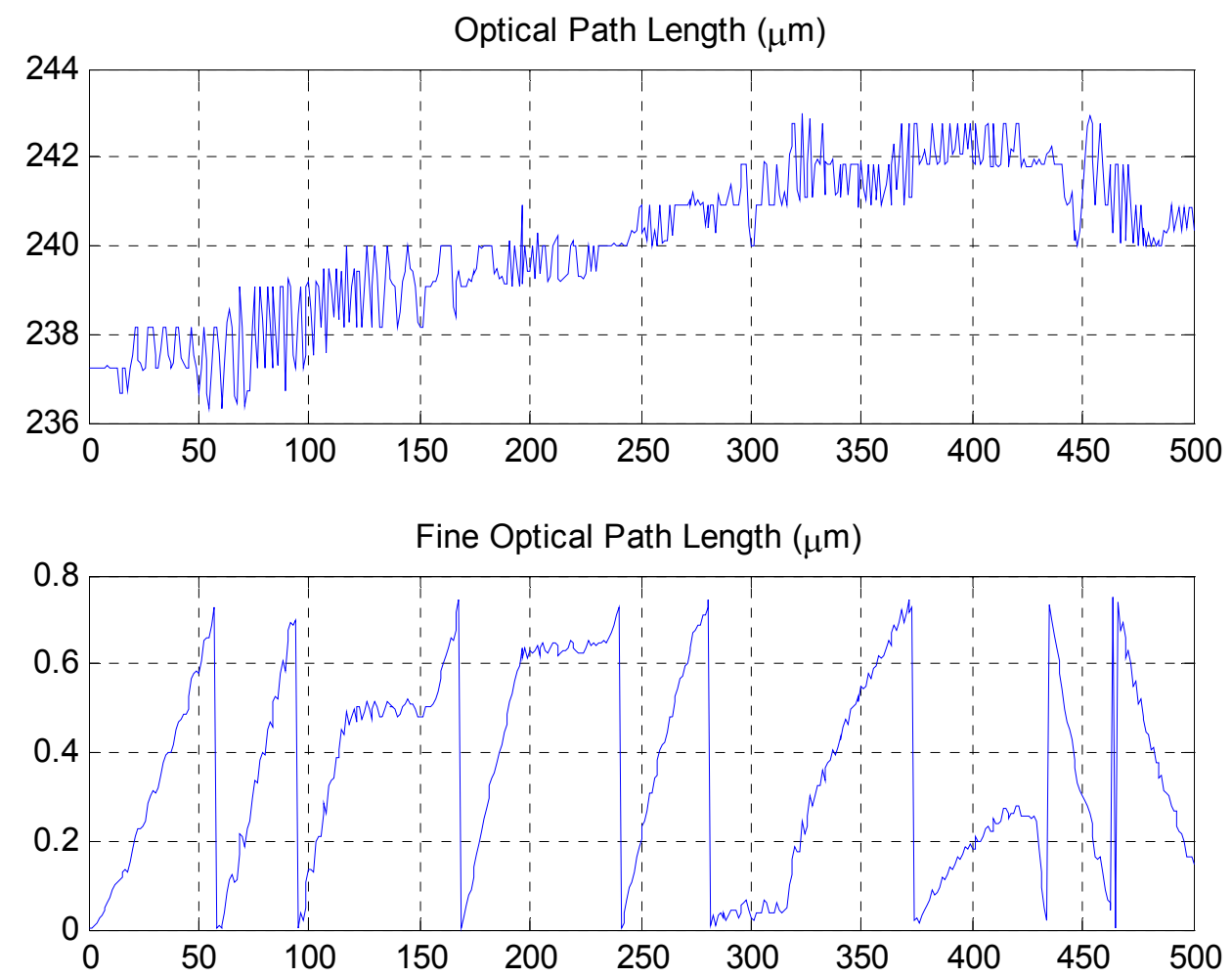

Figure 3-124: Processing of data set with single variable optimization

One of the characteristics to note in the lower plot of Figure 3-124 (which will also be evident for all other methods) is the fact that the result value wraps back around to zero on a regular basis. This is due to the periodic nature of sinusoidal signals. In a perfect sinusoid, a shift of $2 \pi$ radians results in an exact replication of the original signal. This phenomenon is what we refer to as the "phase jump." It is the primary function of the coarse measurement to allow discrimination between phase jumped values of the fine measurement. There is also a secondary phenomenon, in which error from the coarse measurement increases the error of the fine measurement. As can be seen from Figure $3-124$, the initial single variable optimization is insufficient to the task.

The first adaptation of this method was to expand the optimization to a more efficient routine, which employed multivariate optimization (in many cases extending upwards to a modeling of seven independent spectral responses). The calculations of our sample data using this method were accomplished in 94 seconds. The results shown in Figure 3-125 indicate some of the benefits and difficulties associated with this method. The degree of coarse measurement error variation is generally a little bit smaller than was the case for single variable optimization, except for the region between samples 100 and 162 . In this instance, the multivariate solver selected the incorrect value as the dominant one and fell into a false optimum. 

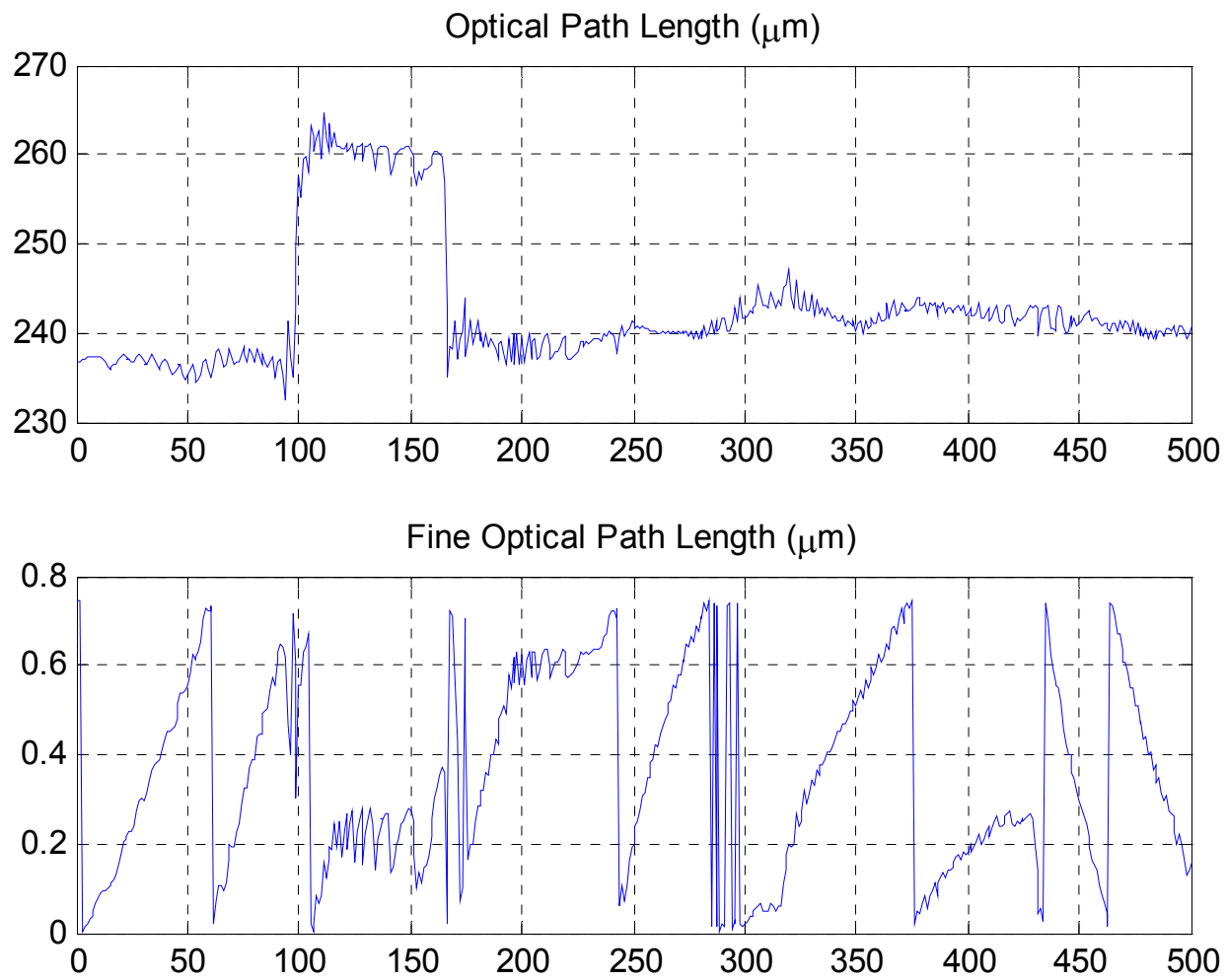

Figure 3-125: Performance of data analysis using a multivariate optimization routine

The false optimum issue which occurs with multivariate optimization routines is caused by the extremely non-linear merit function which is involved when one is modeling a sinusoidal function, combined with the tremendous number of local extremes associated with such a fitting function. A demonstration of this can be seen in Figure 3-126. An arbitrary, perfectly noiseless sinusoid was modeled. Then the amount of error associated with all fits in the immediate region of the parameter space was determined and the quality of the fitness (vertical axis) was plotted as a function of the phase and frequency mismatch (horizontal axes). Naturally, the best fit occurs when there is zero deviation in phase or frequency, but there are a very large number of false maxima surrounding this true peak, making optimization very difficult. This challenge is generally addressed by a field known as global optimization, and there is no overall method which can resolve such a problem with great assurance, if one does not have prior knowledge of at least some of the solution. 


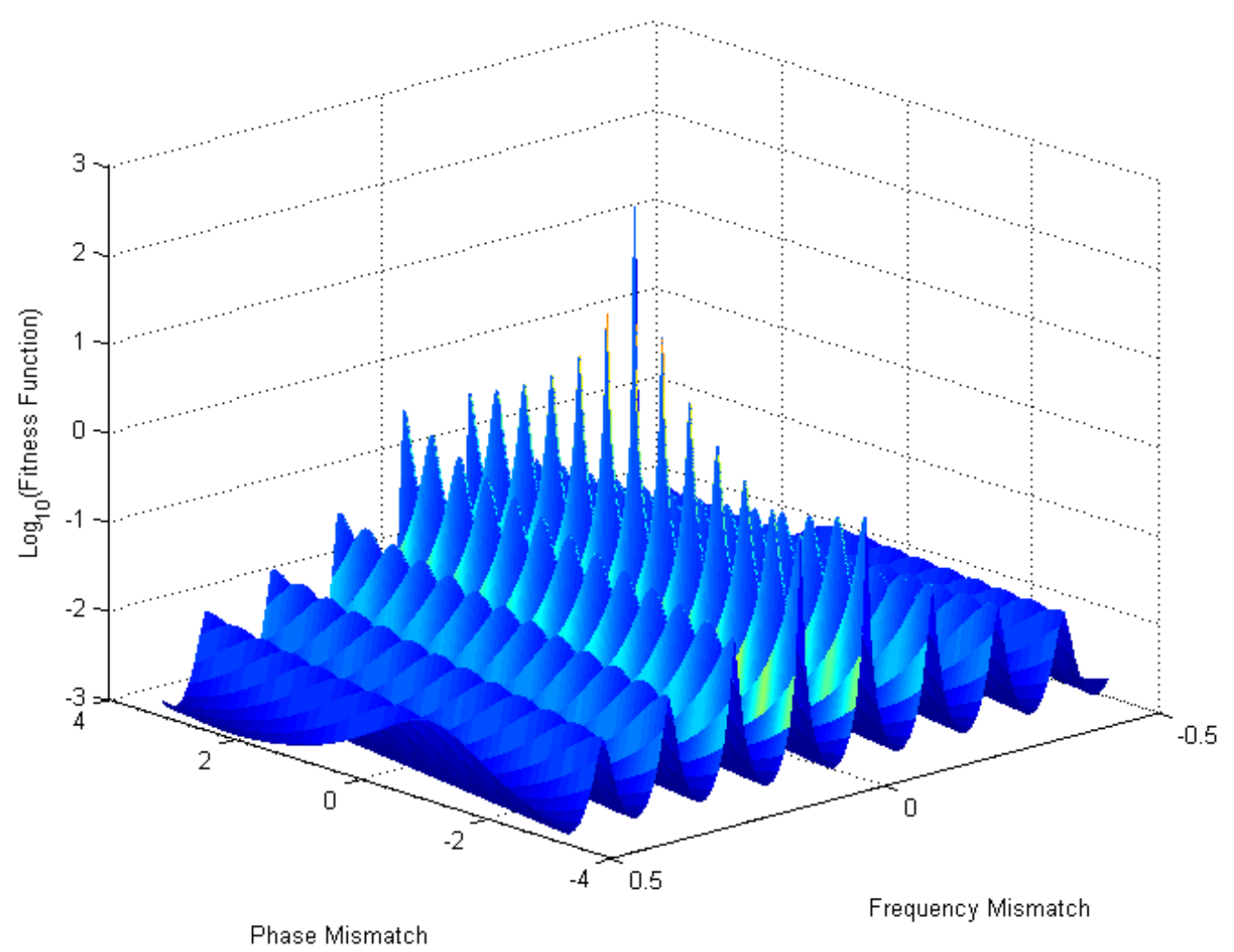

Figure 3-126: Surface plot of fitness function for fitting a single arbitrary sinusoid

An additional approach which was applied to the coarse measurement problem was the utilization of the class of solvers known as subspace algorithms. These are a class of solvers which act to break down a signal into any specified number of periodic signals, based on the periodicities which are present in the complex waveform. They require virtually no assumptions as to the underlying structure of the signal waveforms, but are very particular as to the specific details of implementation. When our sample data was processed through this method, the calculations were concluded in 34 seconds. The results of this calculation can be seen in Figure 3-127. Of all three methods discussed thus far, this one yielded the lowest noise levels for the majority of samples. The level of noise in the coarse measurement is still too large, however, to uniquely resolve the phase jump problem. Also, as discussed before, because noise in the coarse measurement adds to noise in the fine measurement, even the high resolution portion of the measurement is noisier than is desired.

To demonstrate the possible resolution of the fine measurement, a set of calculations were run where the value for the coarse measurement was taken as a fixed value, rather than calculated. This processing required only 23 seconds to complete, with results shown in Figure 3-128. The top plot of Figure 3-128 shows a horizontal line (at $236 \mu \mathrm{m}$ ), indicating the fixed value of the coarse measurement, whereas the lower plot shows the low noise results for the fine adjustment. While this calculation yields by far the cleanest fine adjustment data, it suffers the grave flaw that it does so at the total expense of a coarse measurement, allowing no method for resolving the phase jump issue. 

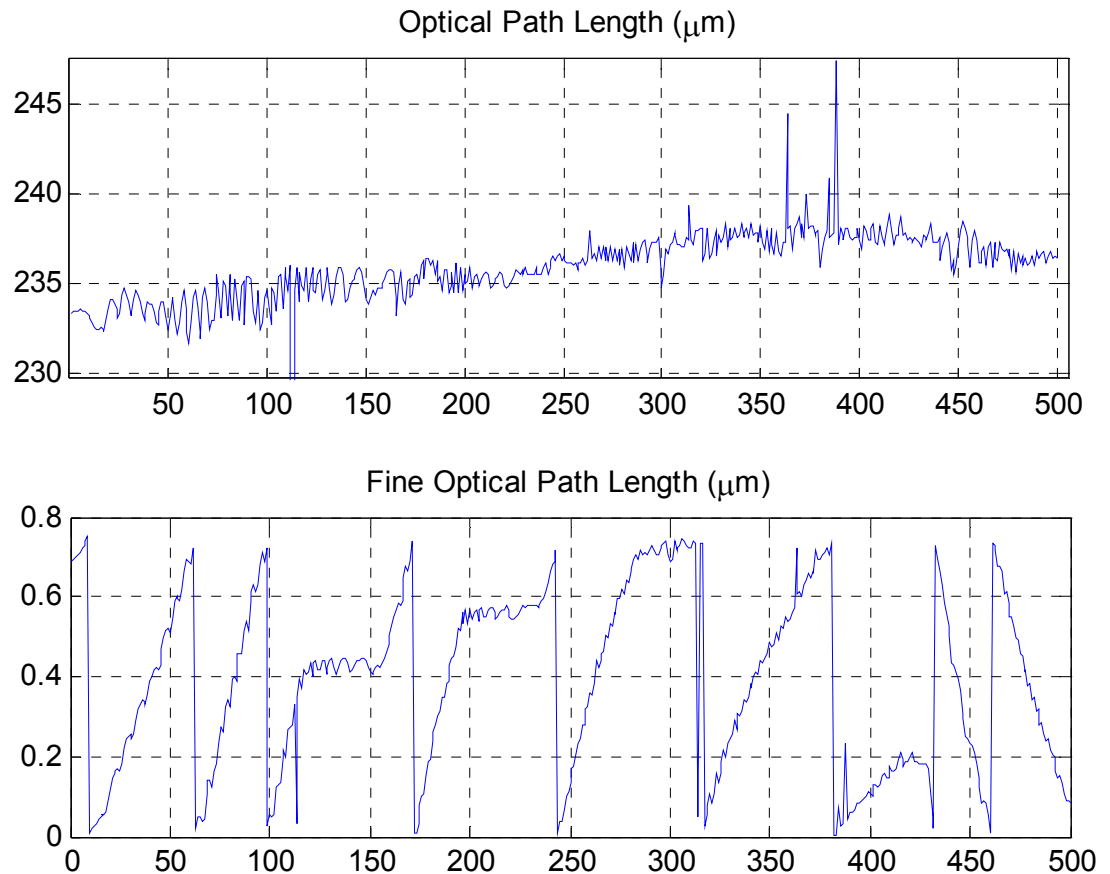

Figure 3-127: Performance of direct subspace modeling of coarse and fine optical path lengths
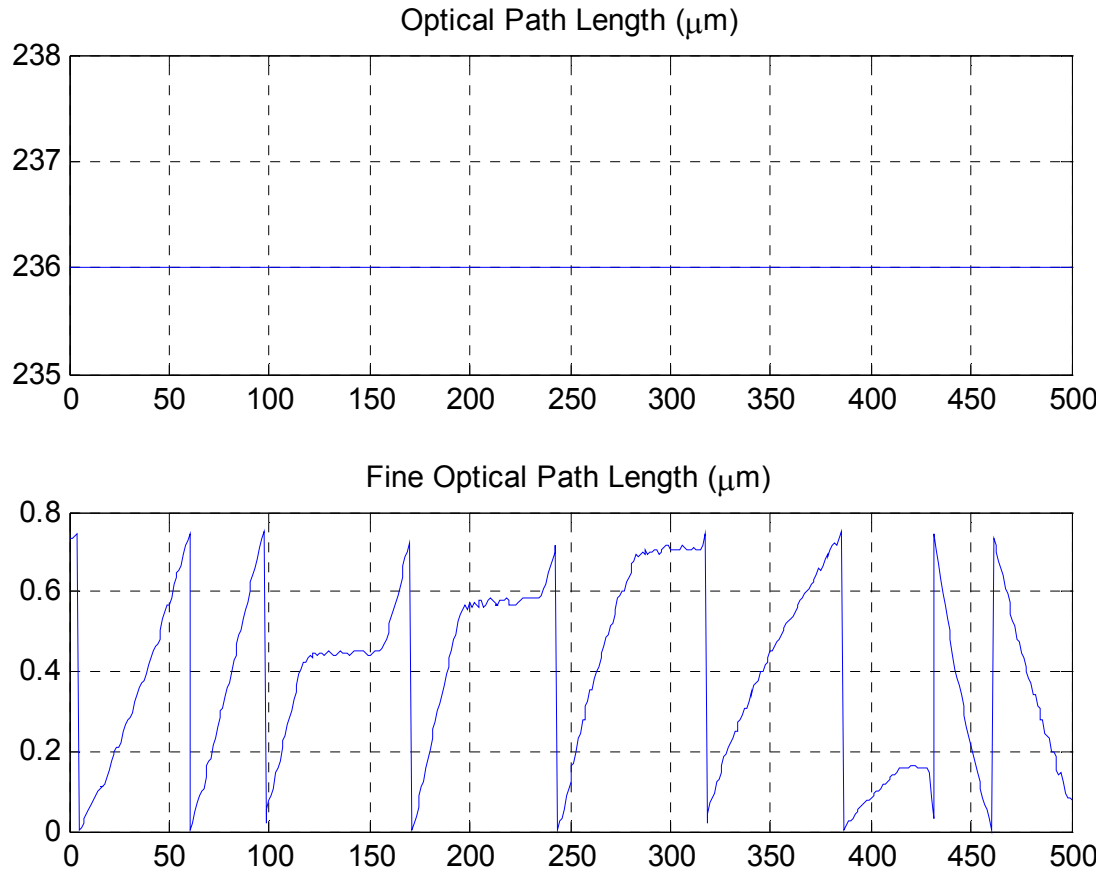

Figure 3-128: Performance of the fine measurement calculations using a fixed coarse measurement 
One potential method for resolving this and obtaining the high resolution of the fixed coarse measurement along with the phase jump discrimination is to use the fixed coarse calculation most of the time, and to periodically perform calculations of the coarse gap size only with data which is deemed highly trustworthy. This was implemented by measuring a running standard deviation of the fine adjustment value. When this value is sufficiently stable, a carefully weighted average of the spectra which contributed to the standard deviation is generated, and then processed for coarse measurement values. The results of this can be seen in Figure 3-129. When this hybrid method was used to unwrap phase jumps in the outputs from sapphire sensors, missed phase jumps and false phase jumps were greatly reduced when compared to the previous methods. The hybrid method was later used to process the data from the field test of the sapphire strain gage (as described in Section 3.12.1.1).

Also shown in the lower plot of Figure 3-129 is an unwrapped version of the fine measurement (shown in green). This is generated simply by recognizing that a sufficiently abrupt change in value of the fine measurement is an instance of a phase jump and adding (or subtracting) the appropriate constant. This is the means by which absolute values can be extracted from a relative measurement. If a system could be devised where the continual operation could be guaranteed, this alone would suffice to adequately measure the performance of the sensor at all time, simply by always referencing against a known original state. This is generally not a viable condition, which is the reason for the necessity of the coarse measurement.

\subsection{Planning for Field Tests}

\subsubsection{City of Hamilton, OH Boiler Plant}

On April 27th, 2006, a team of engineers from PRLC met with several B\&W engineers at the pulverized coal-fired power generation plant during a scheduled outage in the city of Hamilton, Ohio to investigate potential locations for mounting and testing of sapphire temperature and sapphire strain gages.

Several locations were identified in the plant for initial testing of the sapphire sensors. One goal of these first tests, to be setup during the next scheduled shutdown in the October 2006 timeframe, is to verify that the sensors will be robust enough for the harsh environment, which includes high temperatures, corrosive atmospheres, and high vibrations. A central location was identified on Level 1 of the plant to place the interrogation unit and computer for collecting data during testing. A sealed cabinet with cooling capability was suggested since the testing would be over several months. 


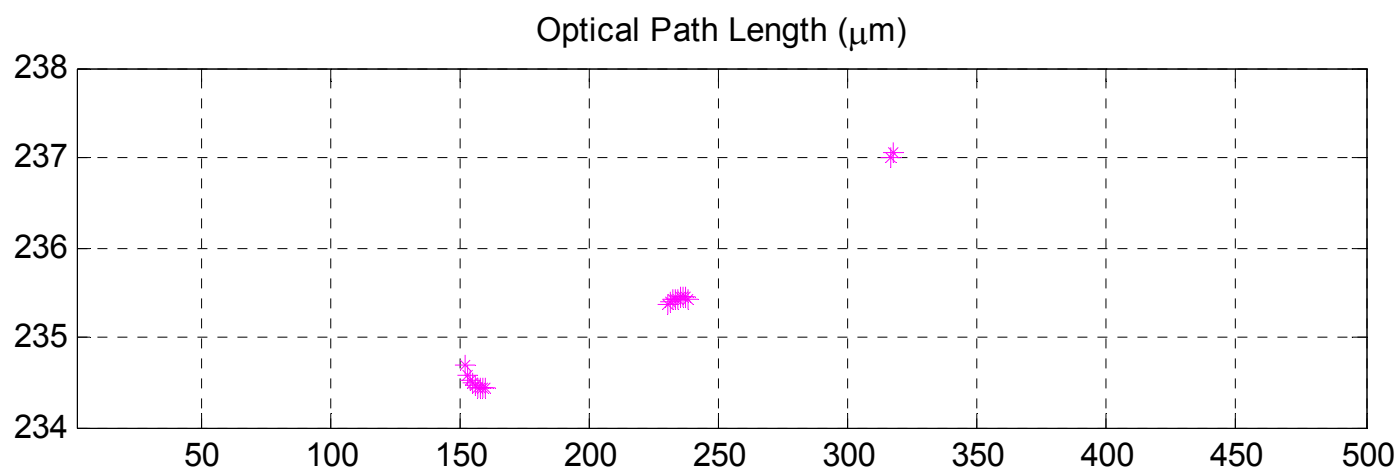

Fine Optical Path Length $(\mu \mathrm{m})$

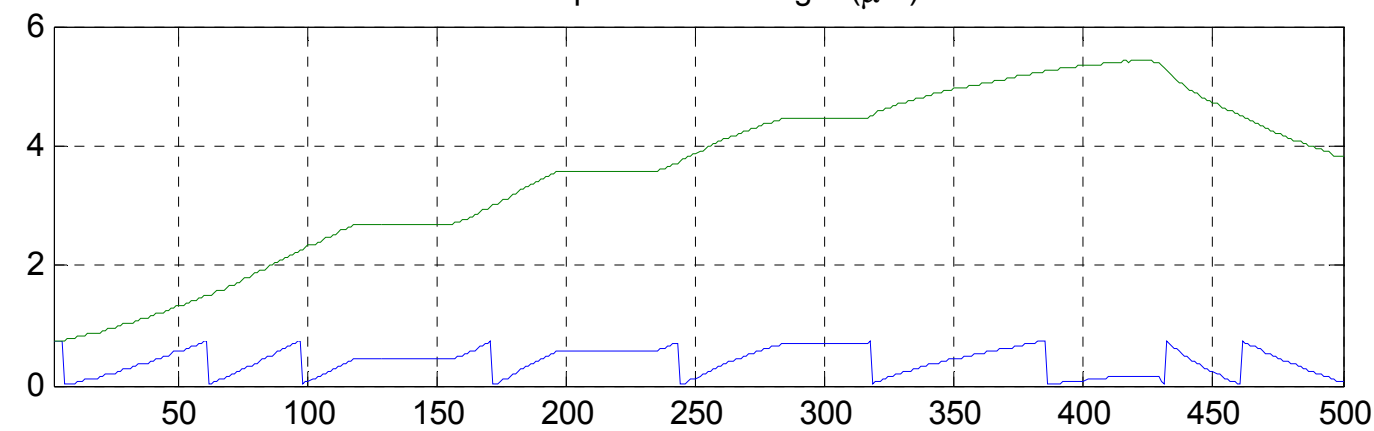

Figure 3-129: Demonstration of trust region method of determining coarse measurement

Figure 3-130 shows the circular burner on one of the lower levels of the plant which could potentially be used to locate a sapphire temperature sensor near the burner flame. Figure 3-131 shows an unused view port that could be tapped to support the entry of the temperature sensor.

Figure 3-132 and Figure 3-133 show a manway on level 3 near the 2-1/2 O.D. superheater tubes which would allow a sapphire temperature and strain gage to be mounted near the membrane wall.

Lastly, a potential location offering ease of installation and monitoring directly on the primary steam pipe on level 4 of the Hamilton plant is shown in Figure 3-134. 


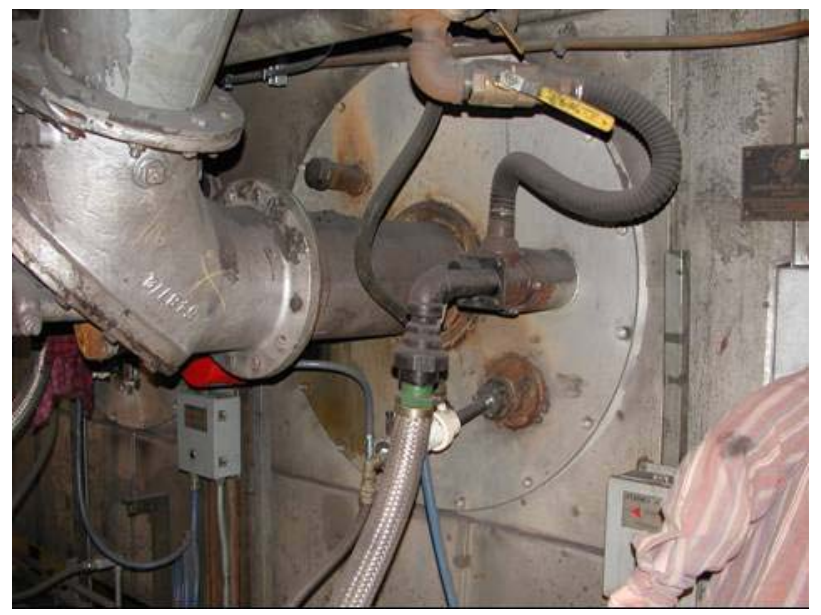

Figure 3-130 Circular burner

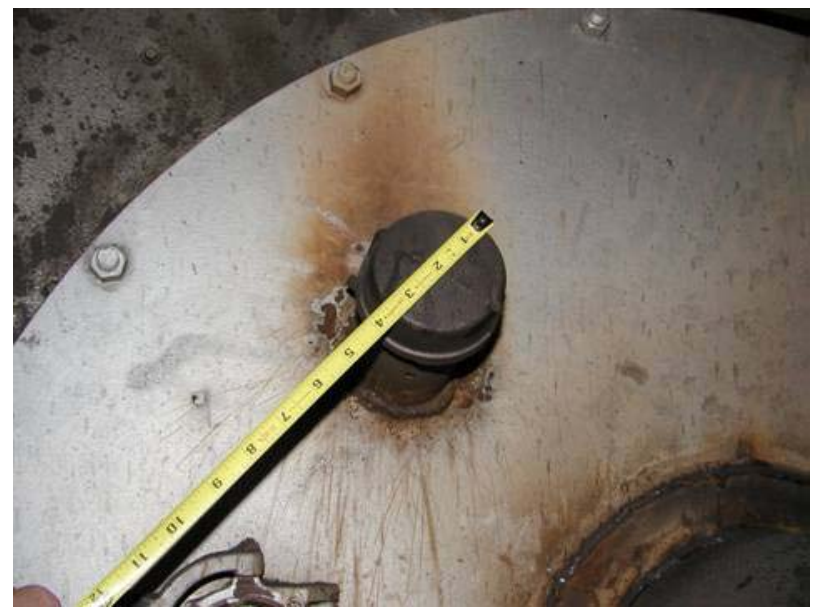

Figure 3-131 Burner view port

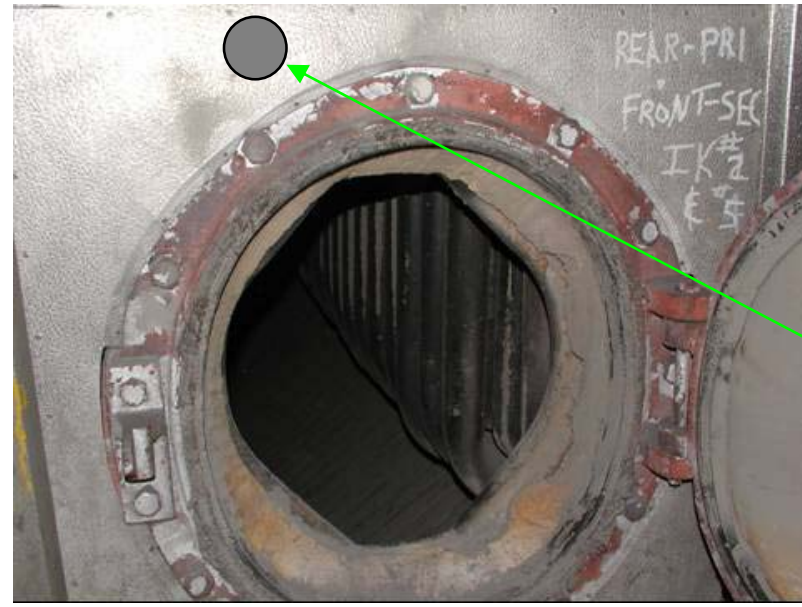

Drill hole here through membrane wall for penetration of optical cable.

Figure 3-132. Manway to super critical tubes 


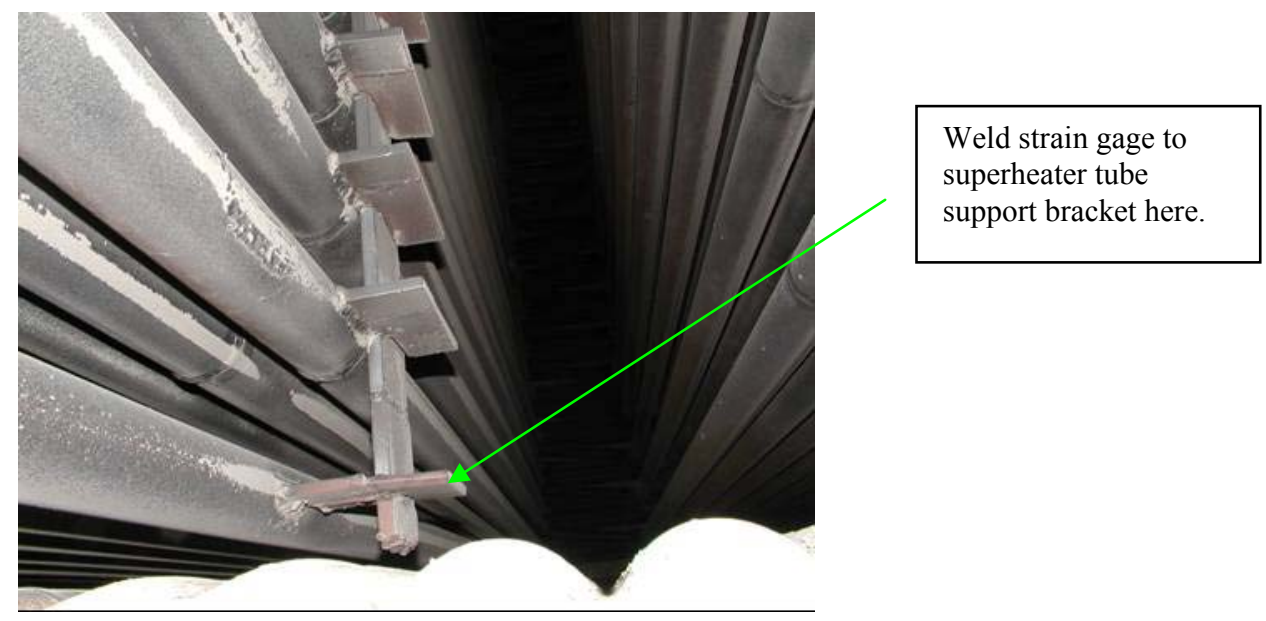

Figure 3-133. Mounting location for sensors on tube support near membrane

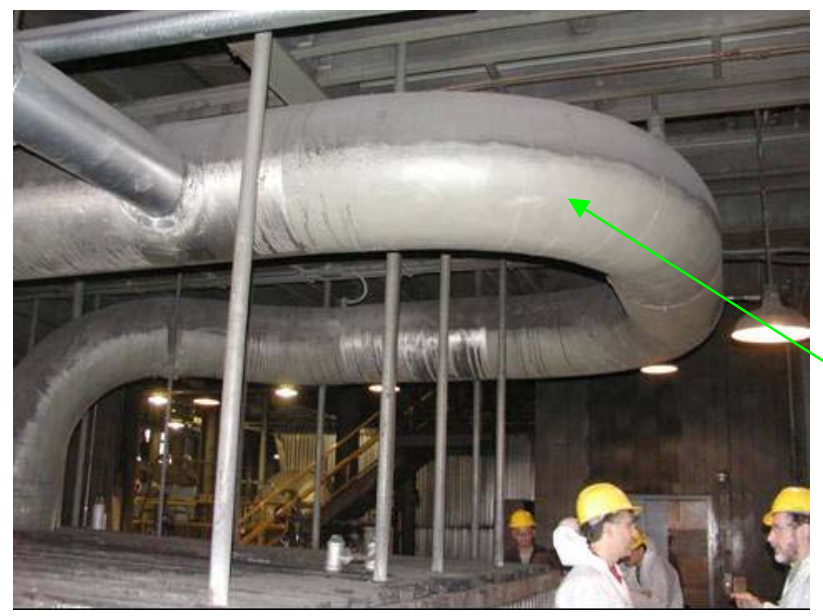

Possible location for strain gage and temperature sensor (under insulation).

Figure 3-134. Primary steam pipe

Two candidate sites in the Hamilton, Ohio boiler plant for mounting the test sensors were targeted: the first is in the secondary superheater tube bank, and the second is a crossover pipe at the top of the boiler. The first site is on the third floor level. In that location, superheated steam runs through the superheater tubes, high temperature steam runs through the tubes in the membrane wall, the hot gas around the superheater tubes is approximately $2000{ }^{\circ} \mathrm{F}\left(1093{ }^{\circ} \mathrm{C}\right)$, and a nearby soot blower make this severe location an excellent choice to test the sensor in a very aggressive environment. Two temperature sensors will be installed at this location. Steam tubes with $1 / 2$ " thick metal web form the wall. A layer of Kaowool insulation and corrugated sheet metal ( Figure 3-135) cover the wall tubes and form the exterior skin. The floor-level manway (Figure 3-136) provides convenient access to the test location. Support brackets (Figure 3-137) provide 
a location to mount sensors without the need to weld components to operation-critical steam pipes, and possibly compromise their integrity.

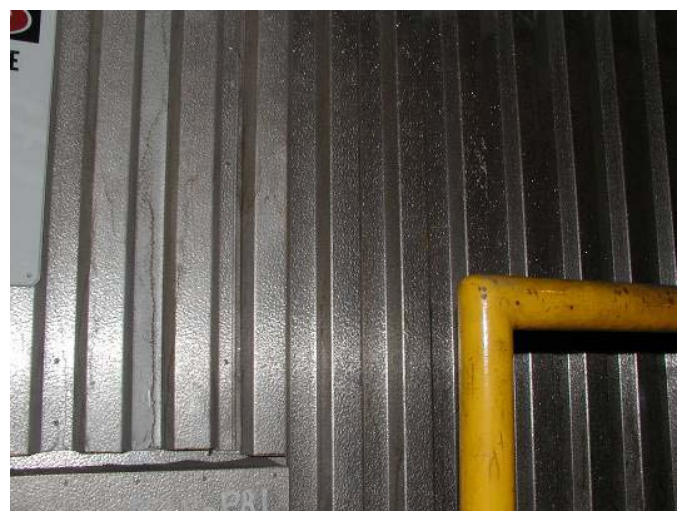

Figure 3-135. View of Exterior corrugated wall

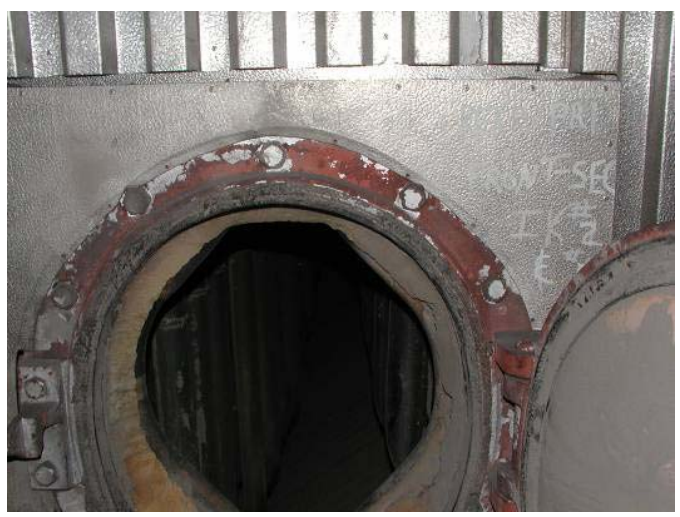

Figure 3-136. Manway

An accurate solid model of the steam tubes (Figure 3-138), supporting brackets, and wall was constructed representing the area in which the sensors would be installed for testing. The model was constructed utilizing dimensions from construction blueprints, and photos and dimensions taken at the site.

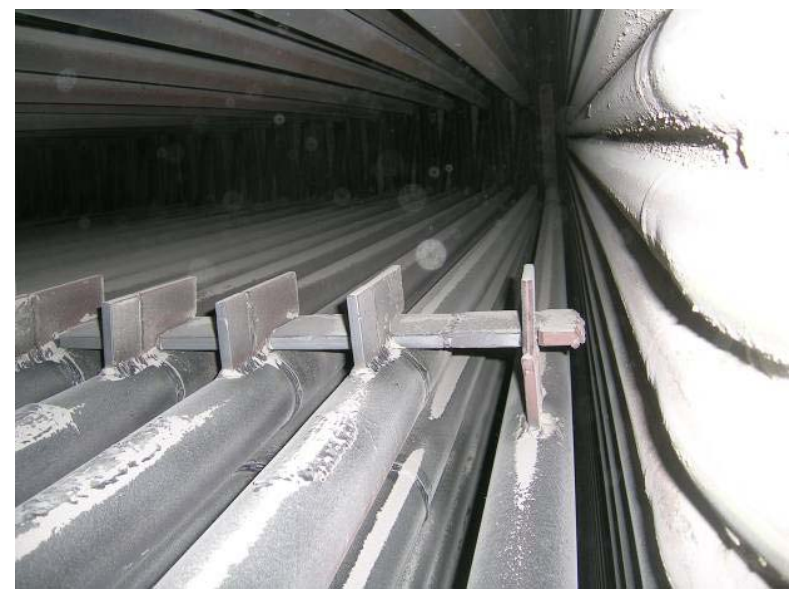

Figure 3-137. Secondary superheater tubes and supporting brackets. In this picture, the view is looking up words along the superheater tubes (lower left). The tubes on the right are part of the membrane wall.

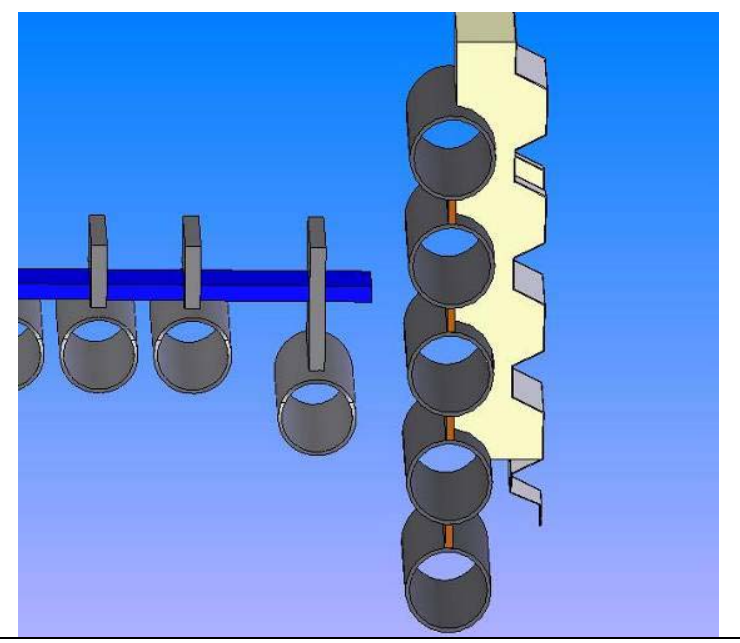

Figure 3-138. Solid model. In this picture, the membrane wall with its insulation and tubes are on the right, and the superheater tubes are on the left.

It was initially decided to mount one temperature sensor attached to the support bracket. The conduction through the steam pipes to the support brackets would provide the most accurate indication of the pipe temperature, short of mounting it directly on the pipe (which was avoided in order to minimize concerns of the Hamilton, Ohio plant engineers about compromising the reliability of the superheater tubes by welding a sensor directly to the tube). 
A 304 stainless steel bracket with multiple temperature sensor mounting holes will be welded to the support bar (Figure 3-139), and an access slot will be cut into the between pipe web by power plant personnel (Figure 3-140). The temperature sensor will be installed in this bracket (Figure 3-141), and an anti-rotation retainer will be resistance welded to this by Prime personnel (Figure 3-142).

In coordination with Babcock \& Wilcox engineers, it was calculated that up to 9" of vertical travel could result from thermal expansion of the steam tubes. A slot will be cut into the web between the pipes to accommodate this movement and a sliding baffle will be installed to minimize the opening to the outside environment.

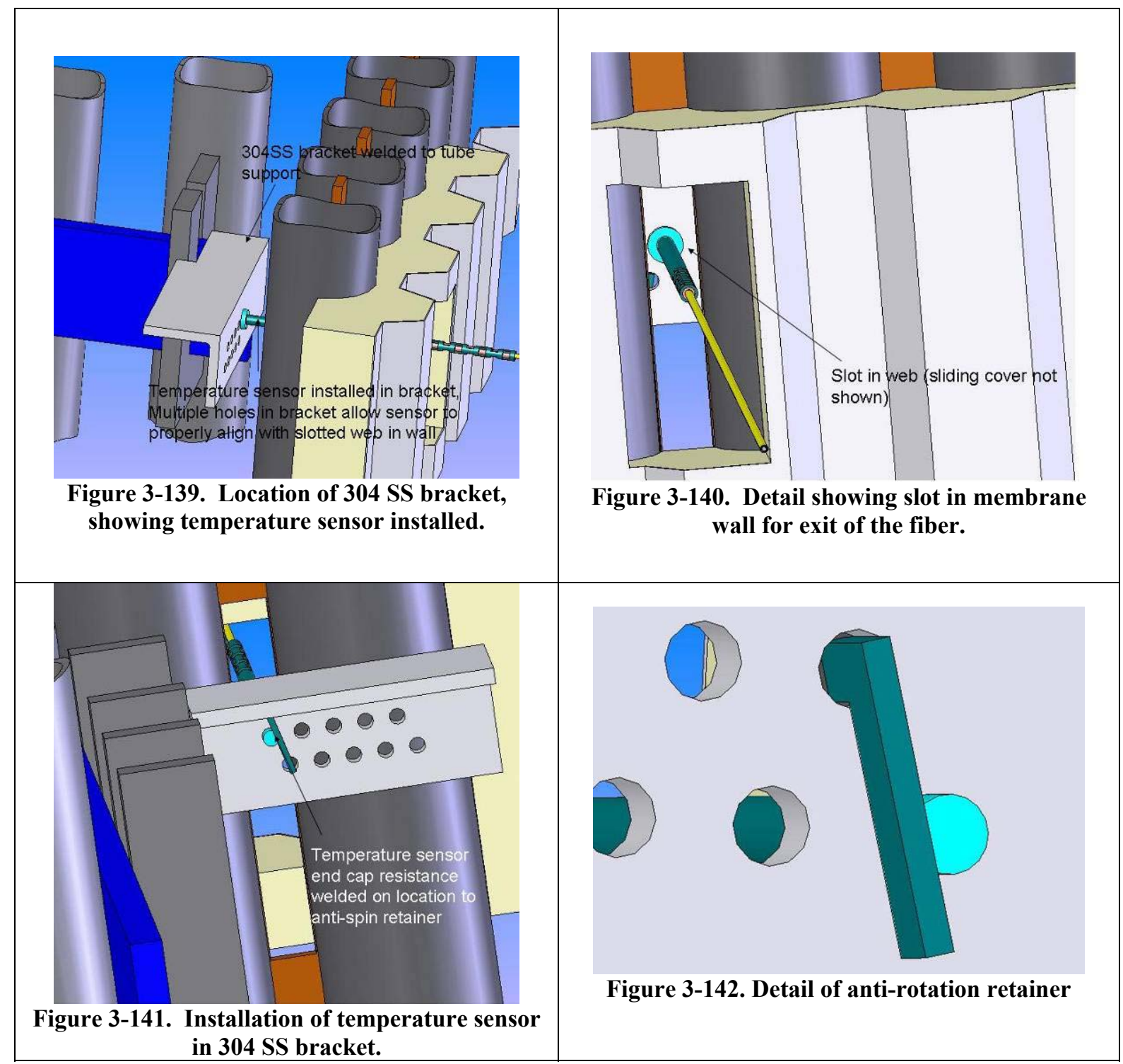


Prime Research was later informed that horizontal (lateral) travel of the secondary superheater tubes could also be encountered. Babcock \& Wilcox engineers provided a video showing the tubes swinging sideways, with an excursion estimated to be in excess of 8 inches. In discussion with the Babcock \& Wilcox engineers, it was decided that lateral motion of this magnitude was not likely during normal operation. The motion of the tubes in the video was likely caused by human excitation, since the video showed a work light and extension cord close by the tubes. The presence of the light and cord suggested that the video of the superheater tube motion was not made during plant operation, when temperatures around the light and extension cord would have been about $2000^{\circ} \mathrm{F}$. However, the video was useful in that it showed that the superheater tube mounting could not constrained significant lateral motion of the tubes. As a result, we decided to include backup sensors in a nearby location that would not be subject to damage from horizontal pipe movement.

The additional sapphire temperature sensor will be wall mounted near the support bar mounted unit. A hole will be drilled in the web between wall tubes. The split bushing will be installed from the furnace interior (Figure 3-143). The spacer will be slid over the split bushing, as shown in Figure 3-144. The split collar will be installed over the split bushing and pushed tight against the spacer, while the flanged end of the split bushing is pushed up against the furnace interior wall. The split collar will be tightened only enough to retain all components in place (Figure 3-145). The sapphire temperature sensor will then be slid into the split bushing and the split collar will be tightened to secure the unit (Figure 3-146).

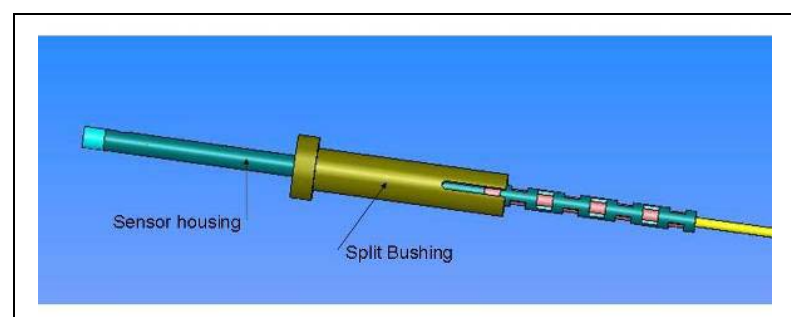

Figure 3-143. Split Bushing, Thru wall mounting

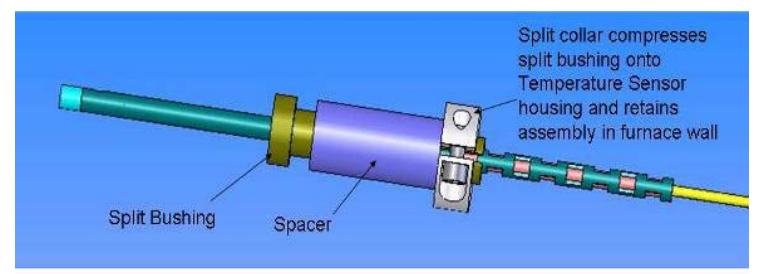

Figure 3-145. Complete assembly

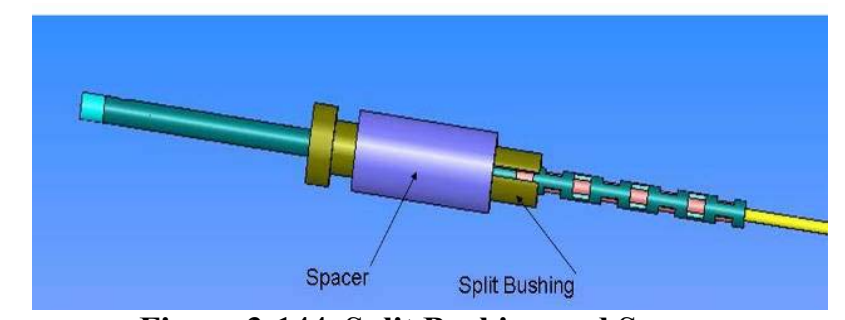

Figure 3-144. Split Bushing and Spacer

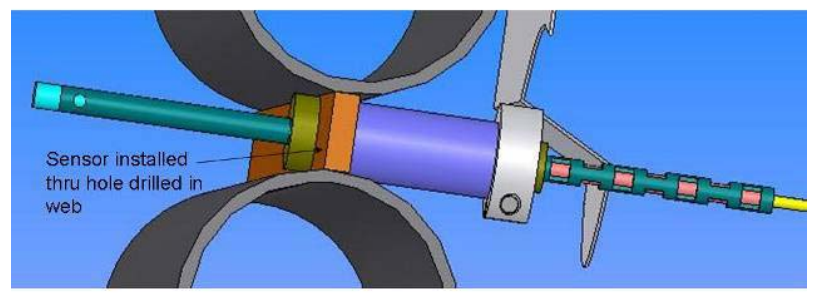

Figure 3-146. Assembly mounted in furnace

The third location for installation of a sapphire temperature sensor in the Hamilton plant will be on a 15 " diameter crossover pipe, which is shown in the photograph in Figure 3-147. This location was selected due to the ease of access. A saddle will be attached to 
the pipe and a sapphire temperature sensor will be attached to the saddle (Figure 3-149 \& Figure 3-150). No drilling or welding will be required for this application. The only preparation required will be to remove a layer of insulation from the pipe at the installation point.

The housing configuration does not change for this application except for the profile of the tip, as illustrated in Figure 3-148. The contour of the tip is specific for this application only.

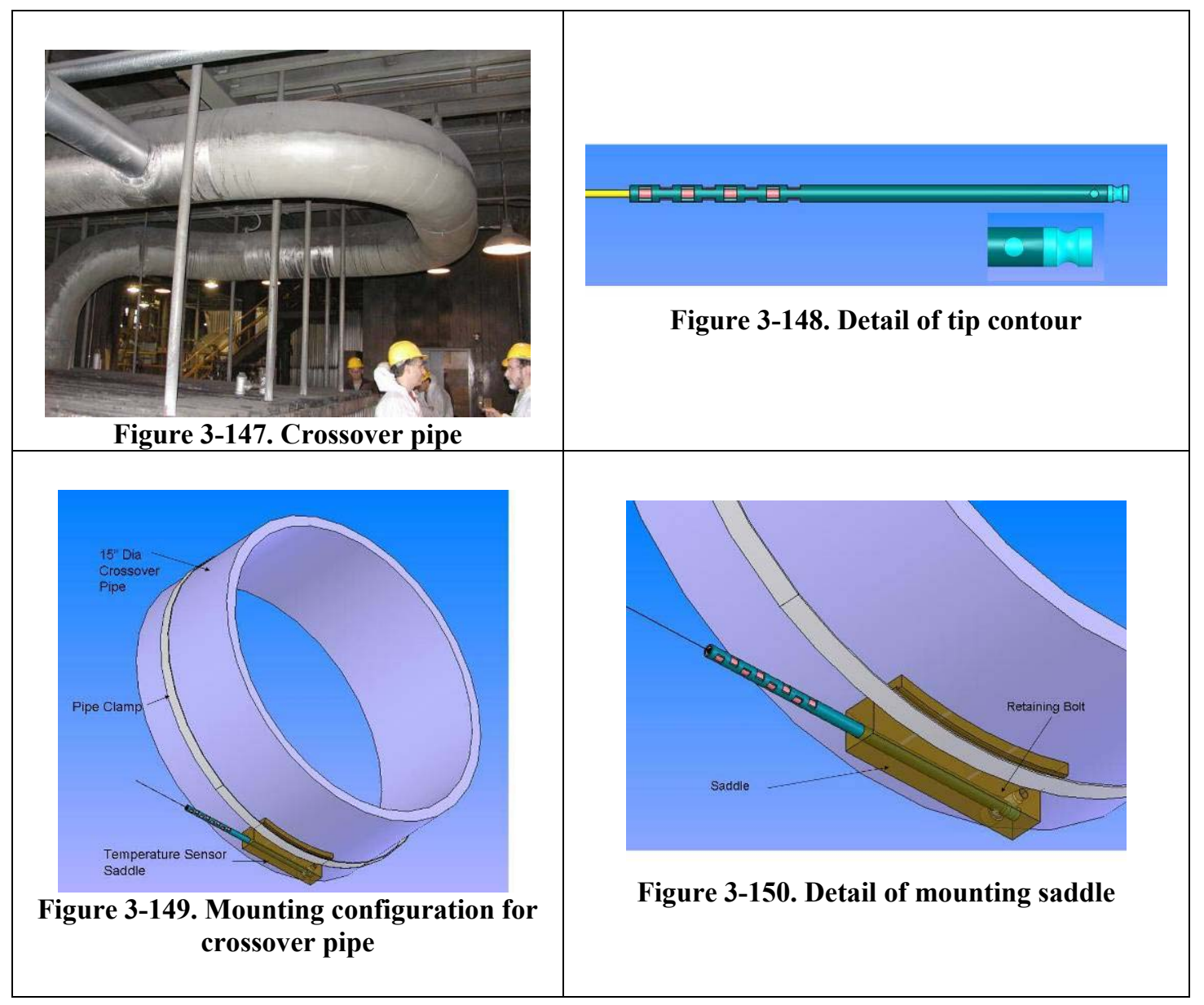

\subsubsection{Babcock \& Wilcox Clean Environment Development Facility (CEDF), Alliance, $\mathrm{OH}$}

In November 2006, Prime Research was notified by Babcock \& Wilcox (B\&W) that the Hamilton, $\mathrm{OH}$ plant would no longer be available for testing of prototype sensors. As communicated to Prime Research by B\&W, this decision was largely due to uncertainty by the City of Hamilton whether the plant would be operated in 2007. Typically, the plant is only operated in summer months. In late 2006, Hamilton was undecided whether 
to operate the plant in 2007, and therefore could no longer commit to support the planned sensor tests.

With the assistance of B\&W engineers, Prime Research began seeking alternative test sites for the field tests. In early 2007, B\&W offered an unusual opportunity. Plans were being made to use the B\&W Clean Environment Development Facility (CEDF) in Alliance, $\mathrm{OH}$ for tests of oxy-coal combustion technologies, and the facility offered a unique opportunity to conduct field tests of the sensors. The CEDF is a $30 \mathrm{MW}_{\text {th }}$ demonstration facility for testing new combustion and emissions technologies (Figure 3-151). It replicates key operating conditions of steam generating systems, including burners for pulverized coal and a convection pass with simulated superheater tubes. In the CEDF plant, these tubes are not used for heat transfer and do not transport superheated steam. The tubes employ a double wall assembly. An inner steel tube carries boiling water, and is surrounded by an outer steel tube which is subjected to normal gas path temperatures. B\&W engineers suggested that the prototype sensors could be mounted to these simulated superheater tubes, and that the conditions presented to the sensors would be representative of those that would be seen in a convection pass in a conventional coal-fired supercritical boiler plant.

In February, 2007, three Prime Research engineers visited the CEDF facility in Alliance $\mathrm{OH}$ to examine the suggested installation site and to take measurements to permit final sensor packaging to proceed. Based on this inspection, it was decided to mount sensors on the trailing side of tube bank \#2. This location was chosen due to the close proximity of a manway for access to the tubes, the convenience of a platform to support personnel performing sensor installation, and a convenient nearby site for location of the support electronics and signal conditioners. Gas temperatures in the vicinity of tube bank \#2 are expected to be approximately $982{ }^{\circ} \mathrm{C}\left(1800^{\circ} \mathrm{F}\right)$ during operation. The tubes will be cleaned by a soot blower at least twice daily during operation of the plant. 


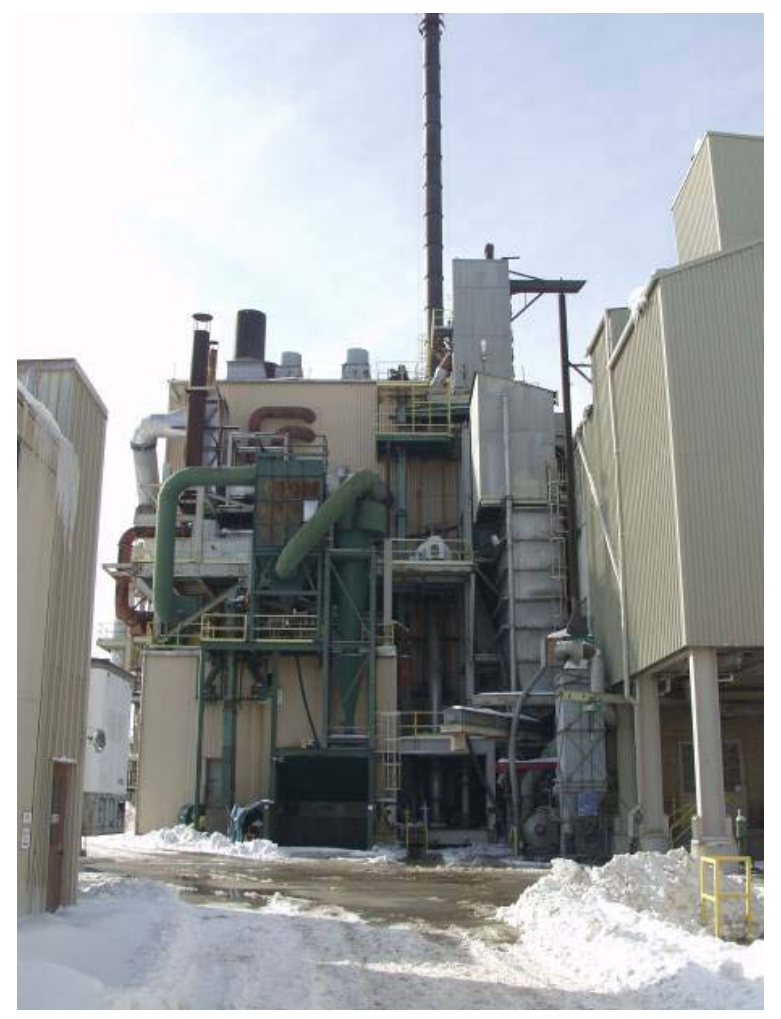

Figure 3-151. Exterior of Babcock \& Wilcox's Clean Environment Development Facility

\subsection{Sensor system installation in B\&W CEDF facility}

On June 21, 2007, the two sapphire temperature sensors, two thermocouples, and the sapphire strain gauge were installed in Babcock and Wilcox's CEDF plant in Alliance, Ohio. The installation was performed by John Coggin and Russell May from Prime Research, with assistance from Ralph Bailey and Jeff Kidwell from Babcock \& Wilcox.

Prior to the installation date, Babcock \& Wilcox personnel drilled through the CEDF wall and installed a port with a gas shield, so that the sensors could penetrate the plant wall. During installation, sensors were passed through this port, and held against the nearest tube while their location for installation was marked on the tube. Surface oxides on the tube over the sensor installation locations were then removed with an angle grinder.

The long temperature sensor was mounted first on the tube. The sensor was held in place on the tube by a $C$-clamp while resistance welder was used to tack weld the 310SS strap to the tube, thereby holding the temperature sensor in place against the tube.

Since the sapphire strain gauge comprised two parts (a long inconel tube containing the 1 $m$ sapphire fiber and a short inconel tube holding the 2 in reflector fiber), mounting the strain gauge to the CEDF tube required a two-step process. First, the inconel tube containing the $1 \mathrm{~m}$ fiber was passed through the CEDF port and its 310SS strap was 
positioned over the CEDF tube as the strap was resistance welded to the CEDF tube. Second, a zirconia alignment tube was fitted over the zirconia ferrule at the end of the 1 $m$ fiber, and the zirconia ferrule on the end of the reflector fiber was fitted into the open end of the zirconia tube. The connectorized end of the strain gauge was connected to the Micron Optics Component Test System (CTS) scanning laser spectrometer, and as the video display of the spectrometer was monitored, the reflector fiber ferrule position was adjusted until interferometric fringes with a reasonable fringe contrast were observed. Using the software in the CTS spectrometer to measure the Fabry-Perot cavity (airgap) length, the longitudinal position of the reflector fiber was adjusted until a separation of approximately $100 \mu \mathrm{m}$ was obtained. At that point, the strap attached to the inconel tube housing the reflector fiber was resistance welded to the CEDF tube.

Next, a Type K thermocouple was threaded through the CEDF port and then fixed with a 310SS strap to the CEDF tube in close proximity to the long sapphire temperature sensor. Finally, the short sapphire temperature sensor was threaded through the CEDF port so that the tip protruded about 1 in into the CEDF interior. A second thermocouple was threaded into the port and positioned to be in close proximity to the short sapphire temperature sensor. After the sensors had been successfully attached to the CEDF tube, the port in the CEDF wall was packed with refractory wool in order to seal it against the escape of hot gases from the CEDF interior. Finally, the fiber cables outside the CEDF wall were bundled and routed to the interrogators.

Figure 3-152 is a photograph of the sensors welded to the CEDF tube. After it was confirmed that all sensors were outputting acceptable signals, a stainless steel shield was mounted over the sapphire strain gauge. This shield was intended to protect the ceramic alignment tubes from the output of a nearby sootblower. The sootblower was located approximately 6 feet from the sensors, and positioned so that it would directives deemed to the side of the CEDF tube on which the sensors were mounted. Figure 3-154 and Figure 3-155 show the stainless steel shield after installation. Note that the shield is attached to the CEDF tube only below the strain gage location; in this way, the CEDF tube was not stiffened in the proximity of the strain gage.

The dustproof enclosure that was used to hold the two interrogators and the data acquisition computer is shown in Figure 3-153. The enclosure is a 22 ' $\mathrm{D}$ x 24 ' $\mathrm{W} \times 27$ ' $\mathrm{H}$ steel cabinet with a filter of cooling fan. In the photograph, the enclosure is shown with a drop cloth that was used to keep the enclosure clean despite the presence of fly ash in the plant. The interrogators used were a Micron Optics CTS scanning laser spectrometer (modified with a large area photodetector) for the sapphire strain gage, and an Ocean Optics USB2000 spectrometer for the sapphire temperature sensor. A laboratory computer was used to log the outputs from the two interrogators. 


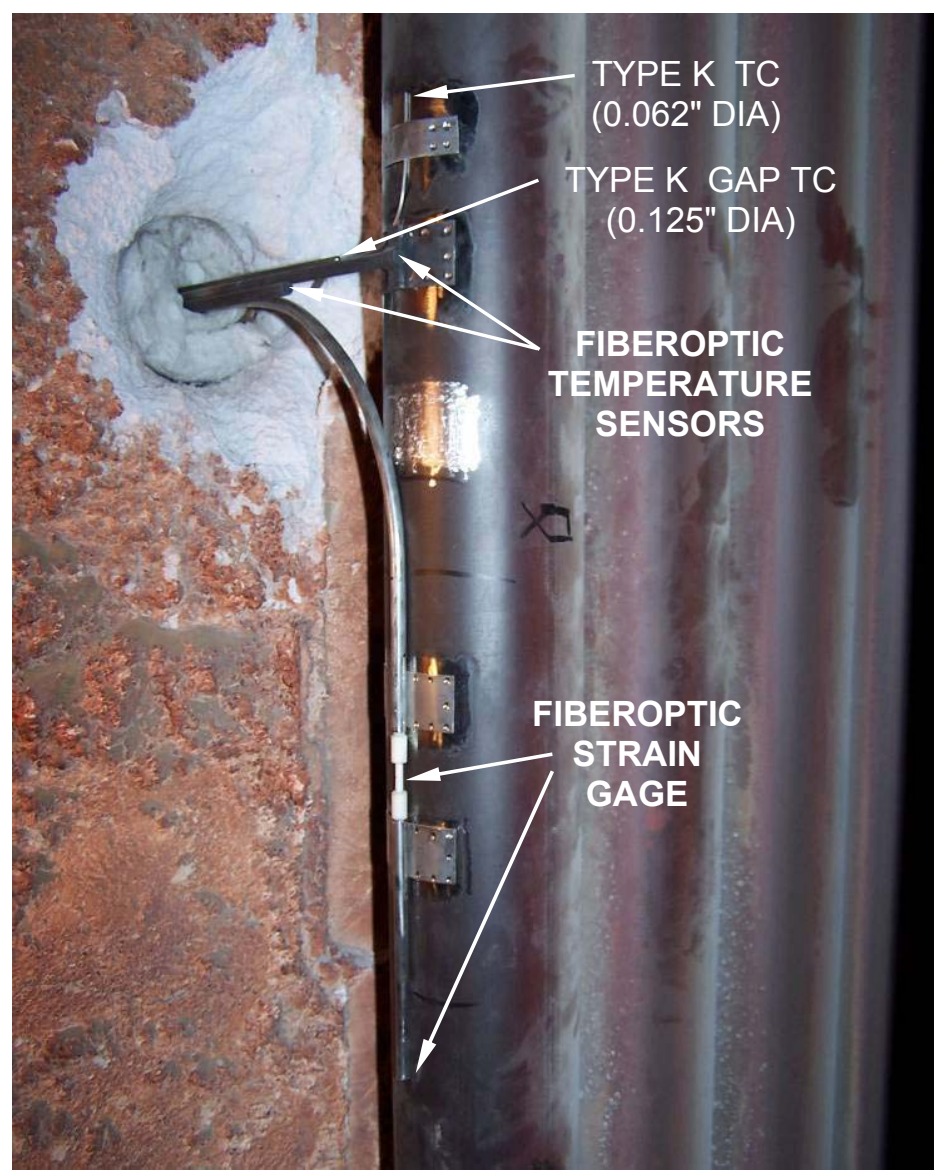

Figure 3-152. Installation of sensors for CEDF field test, showing the location of the thermocouples, sapphire temperature sensors, and sapphire strain

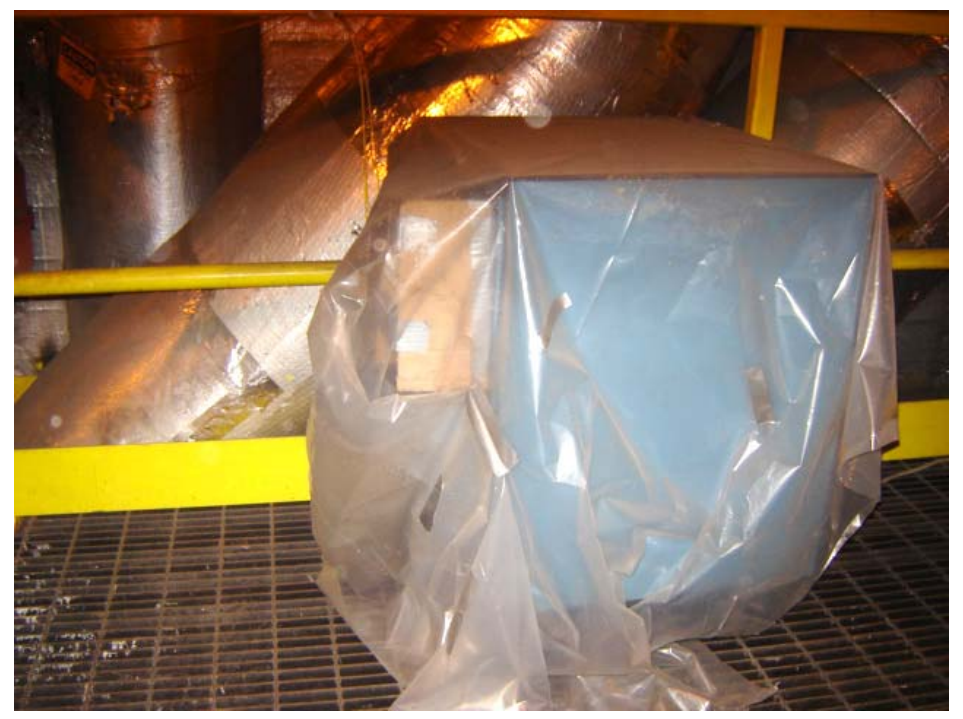

Figure 3-153. Dustproof enclosure holding two interrogators and data acquisition computer at CEDF. 


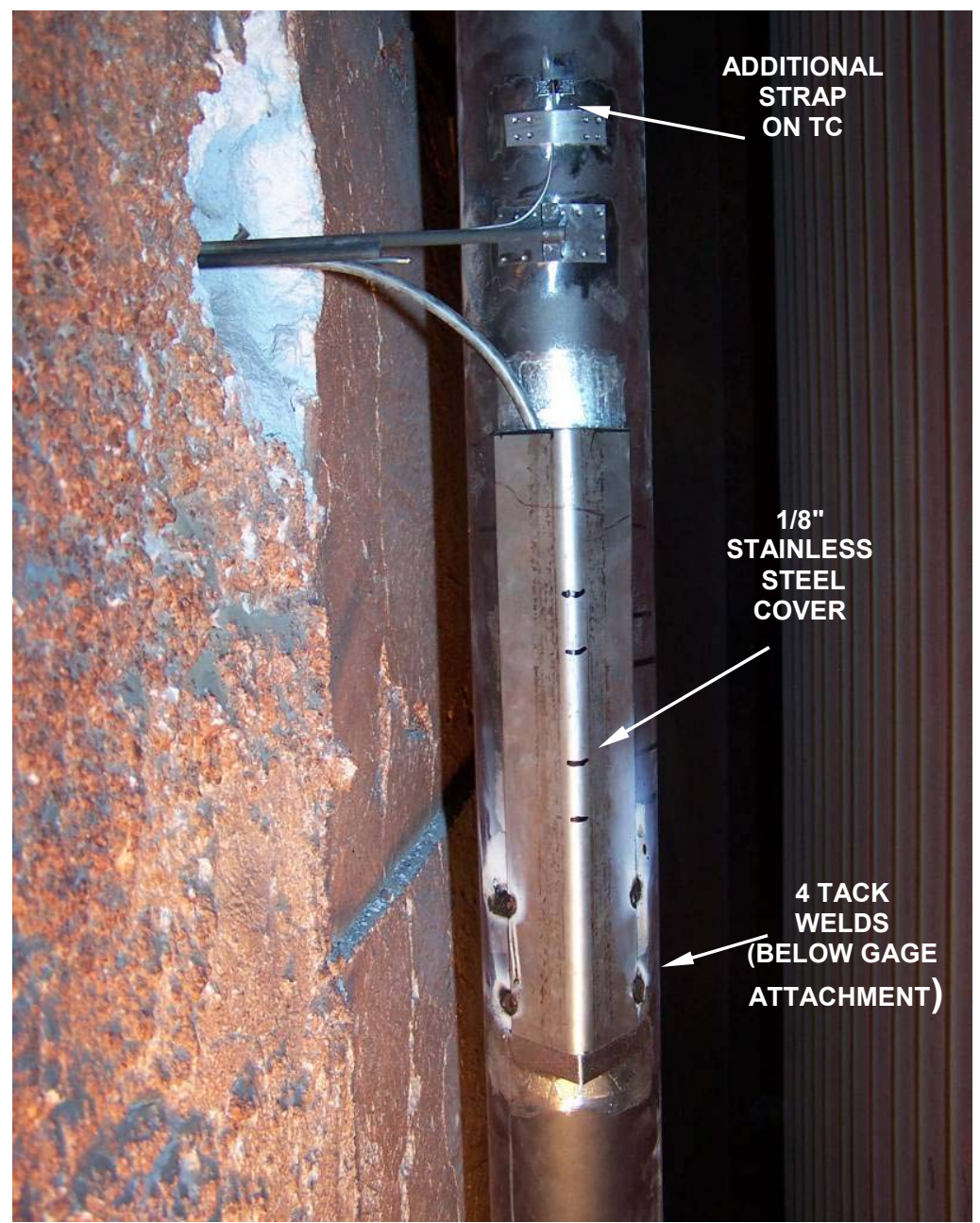

Figure 3-154. Stainless steel cover installed over strain gage.

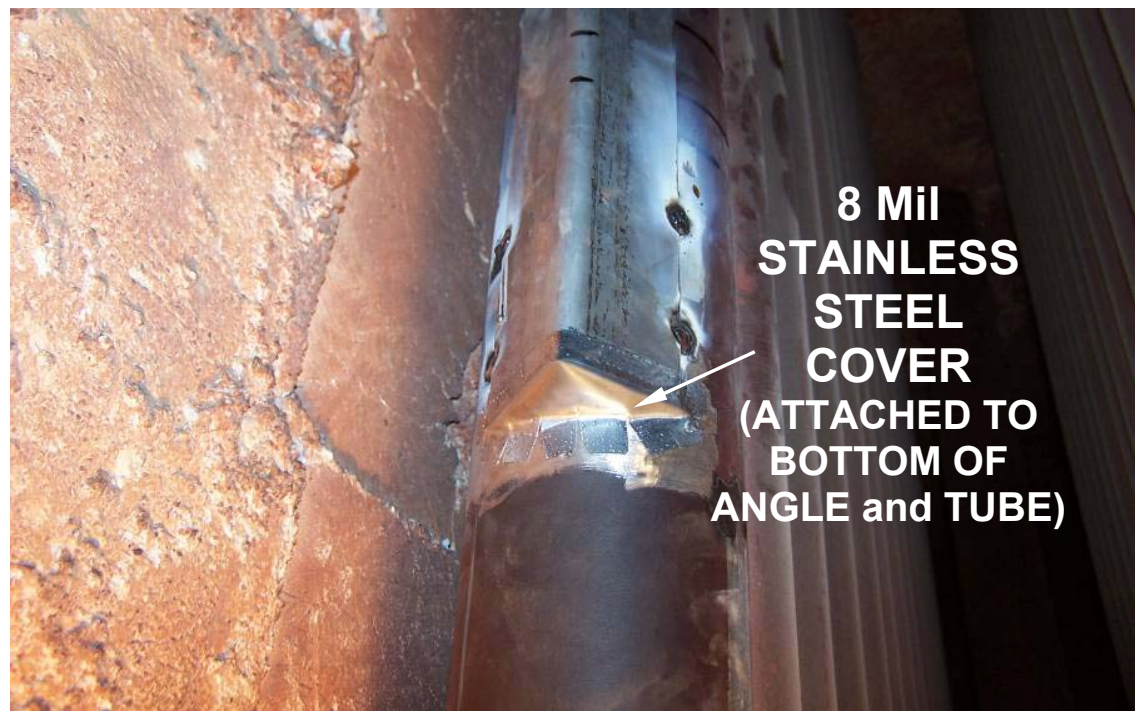

Figure 3-155. Detail of stainless steel cover at bottom of strain gage. 


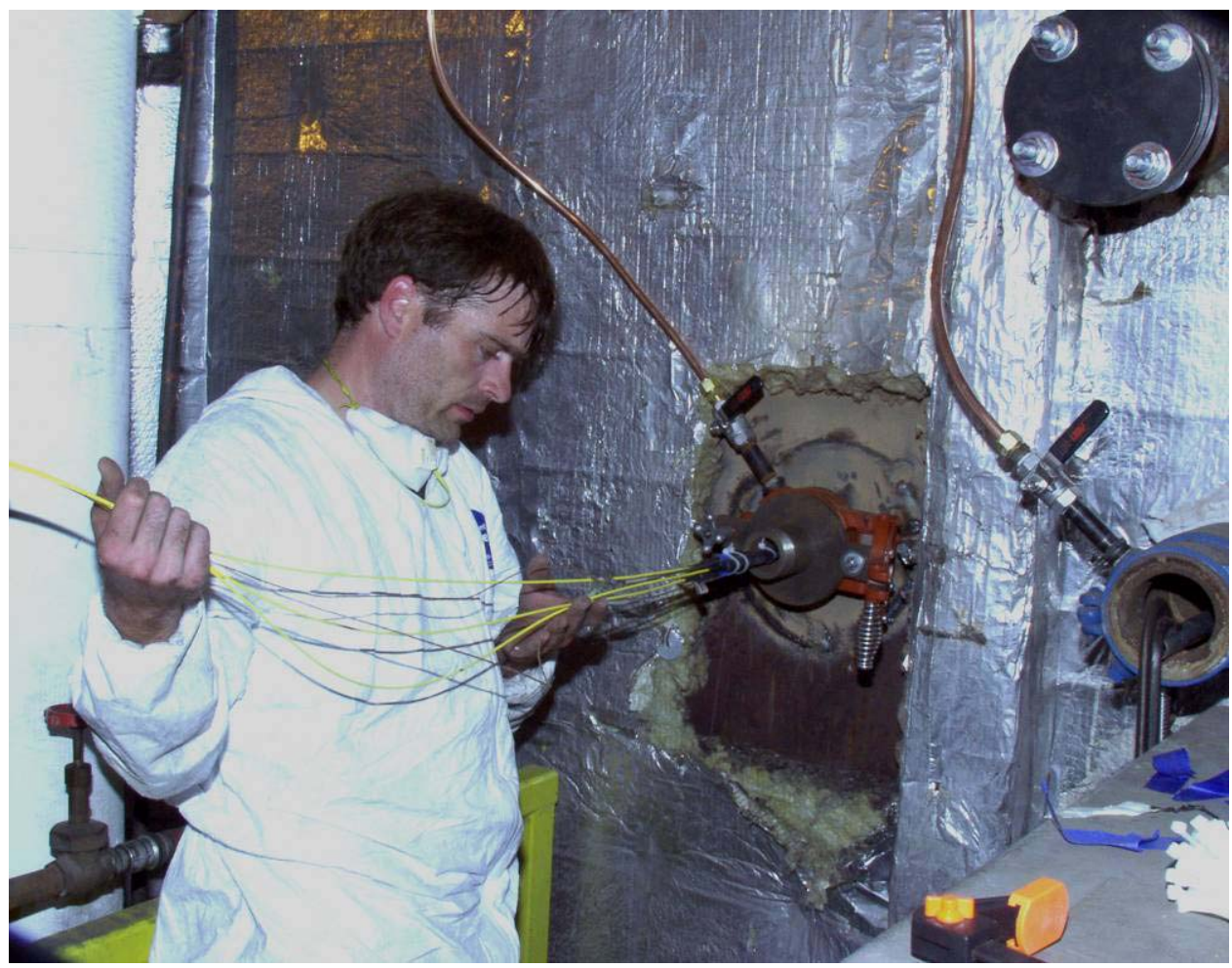

Figure 3-156. Prime Research engineer John Coggin bundles cables through passthrough in CEDF wall.

\subsection{Results of CEDF Testing}

On December 18, 2007, Prime Research engineer John Coggin removed the sapphire sensors and thermocouples from the CEDF for analysis at the Prime Research laboratories. In addition, he retrieved the test data that had been logged to the data acquisition computer during the test.

An angle grinder was used to break the weld that held the protective stainless steel cover over the sapphire strain gage. Upon removal of the cover, it was discovered that the strap that held the reflector fiber to the CEDF tube had disbonded, and fallen to the bottom of the stainless steel cover. Figure 3-157 shows the CEDF tube after the cover was removed. The input fiber can still be seen at the top of the photo. Light-colored fly ash can be seen covering the tube. A shadow can be seen in the fly ash (Figure 3-157), indicating where the reflector fiber in the strap attaching it to the two had previously been located. The observation that fly ash had not coated the shadowed area suggests that the reflector fiber strap had disbonded from the two at a time closer to the end of the field test, rather than at the beginning. 


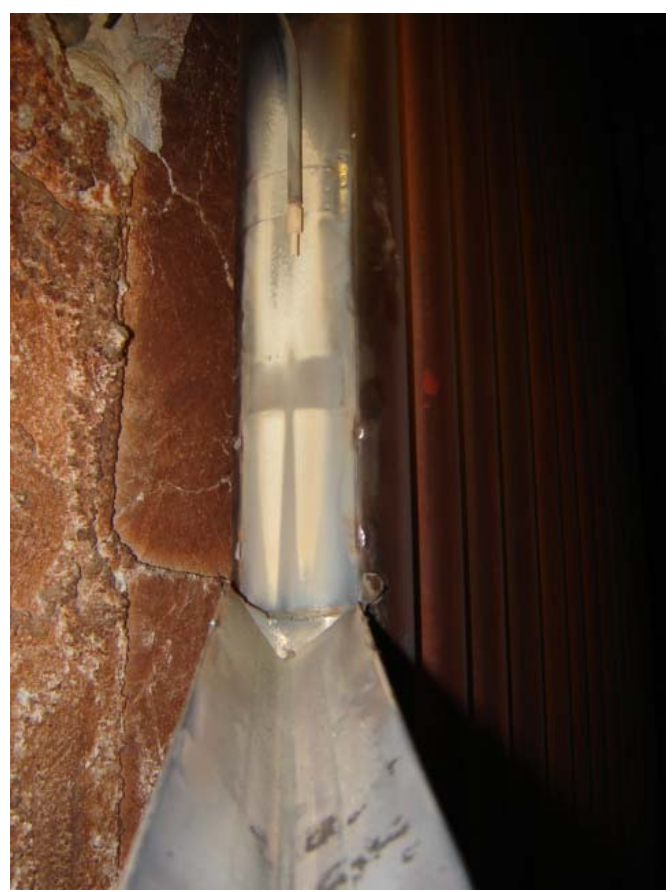

(a)

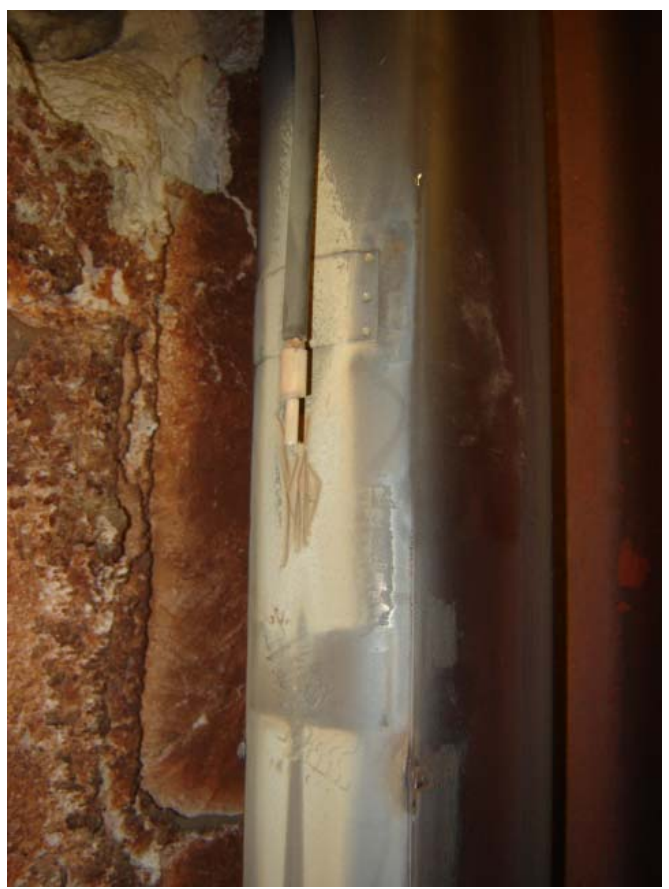

(b)

Figure 3-157. CEDF tube showing sapphire strain gage after protective shield was removed following field test.

During the removal of the sensors, it was observed that the cables had been bent where the yellow polyurethane cable jacket exits the stainless steel tubes (the junction between the tube in the polyurethane cable is under the black heat shrink tubing. Figure 3-158(a) is a close-up of the cable bundle as it exits the pass-through in the CEDF wall. A bend in one of the optical cables (in this case, the cable for the strain gauge) is highlighted in the circled area. Figure 3-159 is a photograph of the three sapphire sensor packages arrayed on a table after removal from the CEDF; the bends in the packages are clearly visible.

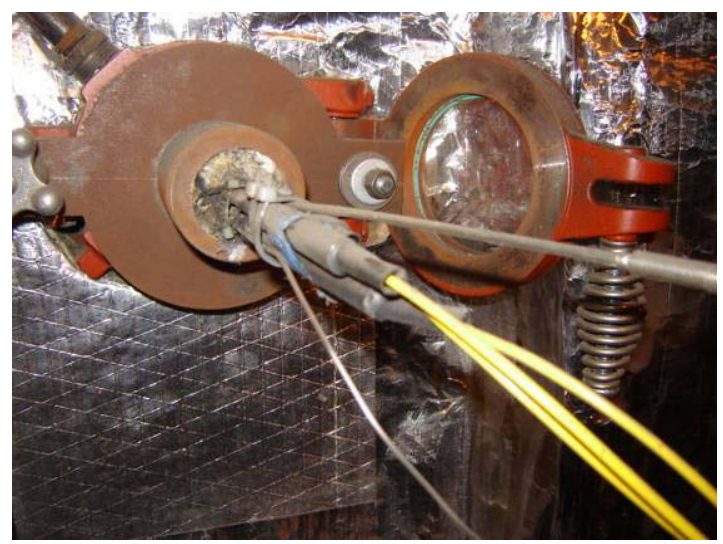

(a)

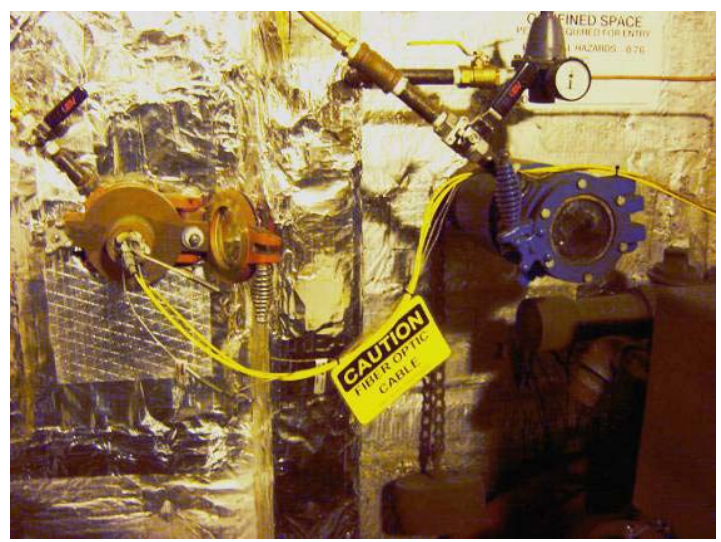

(b)

Figure 3-158. Feed-though in wall of CEDF, showing optical fiber cables and thermocouple leads following the field test. 


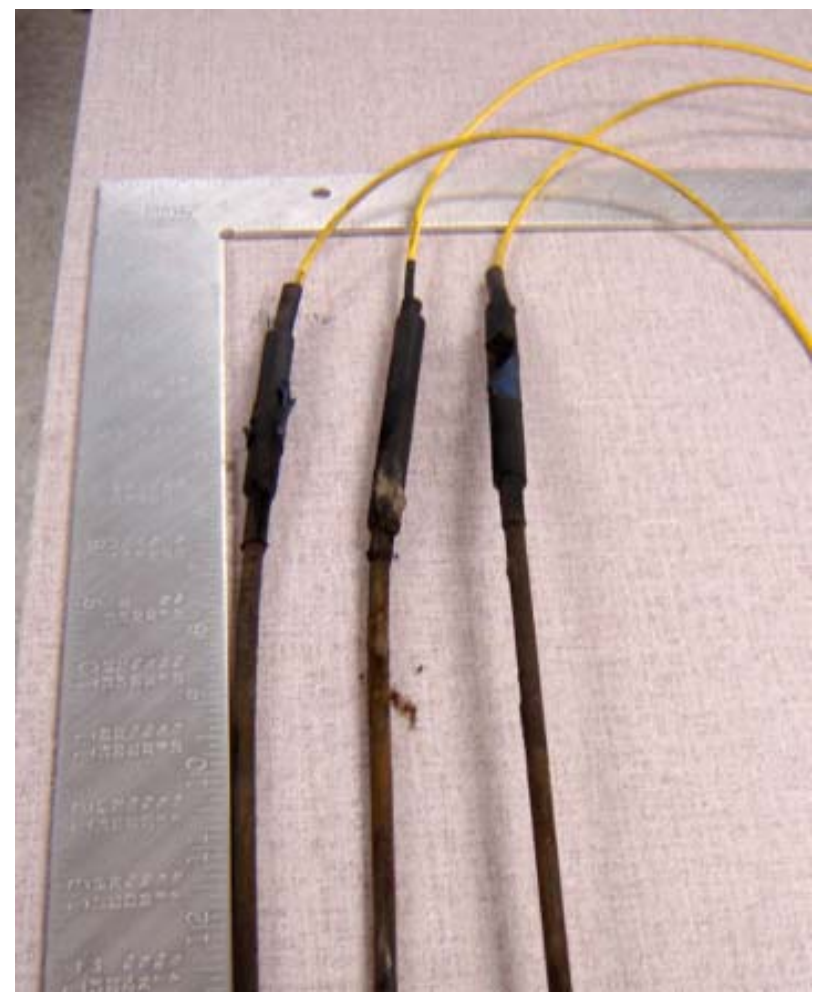

Figure 3-159. Sapphire sensors after removal from CEDF, showing bent protective Inconel tubes.

The bends in the sapphire fiber packages suggest that some force have been applied to the cables at some time after installation. Close examination of Figure 3-158, which shows the CEDF wall just before retrieval of the sensors, and comparison with Figure 3-156, which shows the CEDF wall just after sensor installation, reveals that additional insulation had been applied to the CEDF wall around the pass-through after the sensor installation. Possibly the cables were moved by workmen when the insulation patch was applied to the CEDF wall. It seems unlikely that the forces applied to the strain gauge package were responsible for the disbonded of the reflector fiber strap. Since the reflector fiber was mechanically decoupled from the input fiber, excessive forces on the strain gauge package should have resulted in a disbond of the input fiber strap before a disbond of the reflector fiber strap. A more likely scenario is that stresses resulting from differences in thermal expansion of the strap and CEDF tube caused shear stresses in the spot welds holding the strap to the tube, eventually causing the disbond.

Figure 3-160.(a) and (b) displays photomicrographs made after the retrieval the sensors; (a) shows the end of the input fiber of the sapphire strain gage. The fiber was bonded in a zirconia ferrule using an alumina cement. Fly ash (light tan in color) can be seen on the end of the fiber and ferrule. Figure 3-160.shows the end of the reflector fiber in a zirconia ferrule. 


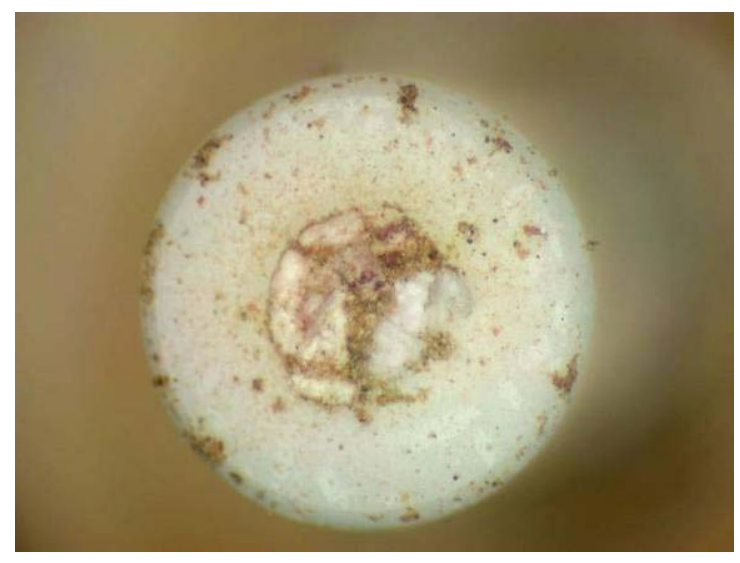

(a)

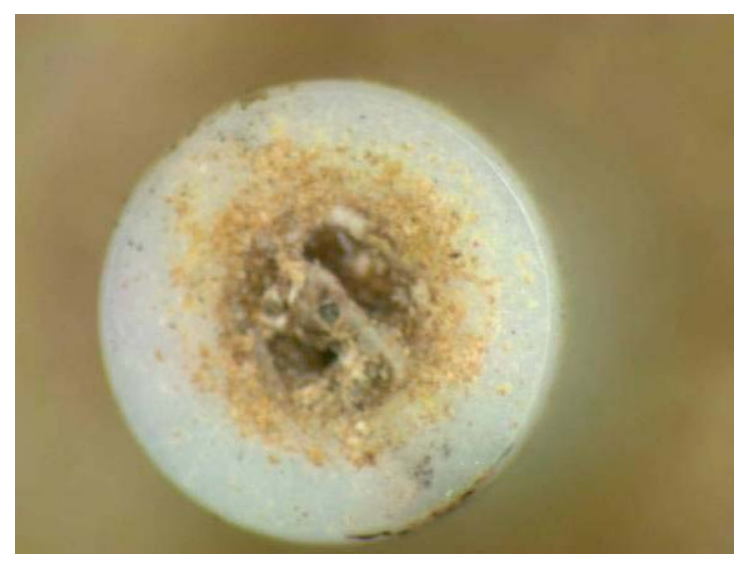

(b)

Figure 3-160. Photomicrographs taken after removal of sensors from CEDF, showing the ends of the sapphire fibers that formed the sapphire strain gage. Both fibers are mounted in zirconia ferrules. (a) shows the end of the input fiber, and (b) shows the end of the reflector fiber.

\subsubsection{Analysis of Logged Data}

During the period of testing which began on 21 August 2007, a Type K thermocouple was sampled at one minute intervals, to provide a temperature reference for the testing. This time series is plotted in Figure 3-161. There is an extended duration of room temperature at the beginning of this time period, which is the time prior to the first heating of the facility. The data required some manipulation to correct an offset in the time stamps (the thermocouple timestamps lagged the actual time by approximately 4.4 hours), as well as interpolation of 154 samples which were the result of data communications errors.

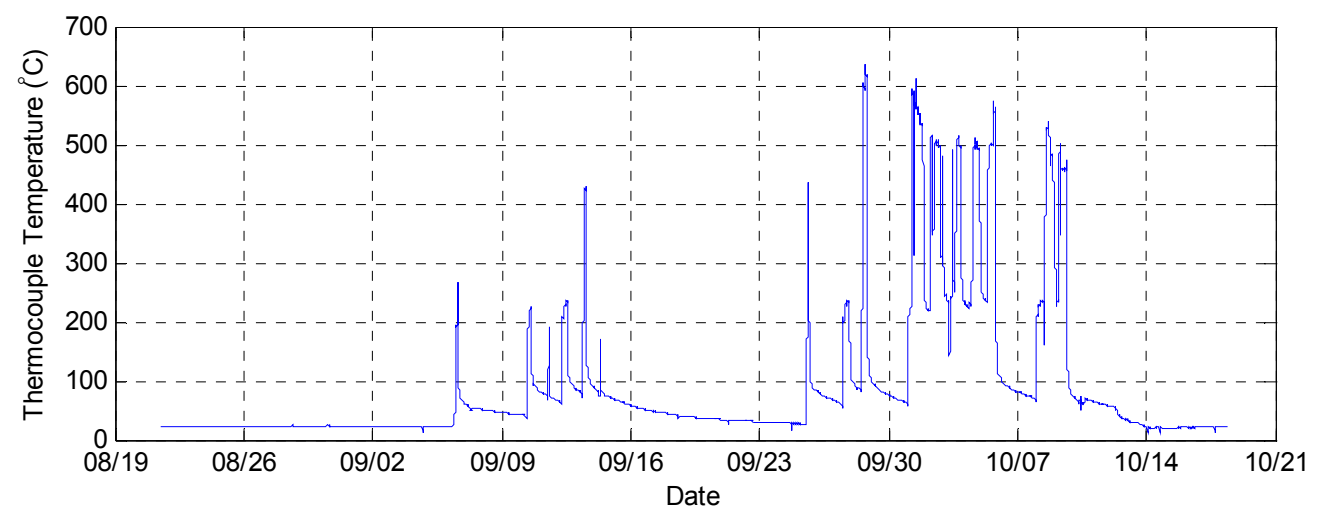

Figure 3-161: Temperature profile as reported by a type $K$ thermocouple over the period of 8/21/07 through 10/18/07 


\subsection{Sapphire Temperature Sensor (T1)}

The sapphire temperature sensor $\mathrm{T} 1$ was set up to record full interference spectra at an interval of 30 seconds starting on 21 August 2007. Over a duration of forty-five days, a total of 130,411 spectra were recorded, ending on 5 October, 2007. At approximately 19:30 on 5 October, the data collection system ceased acquisition due to a glitch in the commercial software used for recording the data. Shortly thereafter, the hard drive containing the data was removed from the PC and returned to PRLC for data processing. All of the data was recorded with the maximum possible resolution in order to ensure no loss of data. An example of a single data sample (one interference spectrum) is shown in Figure 3-162.

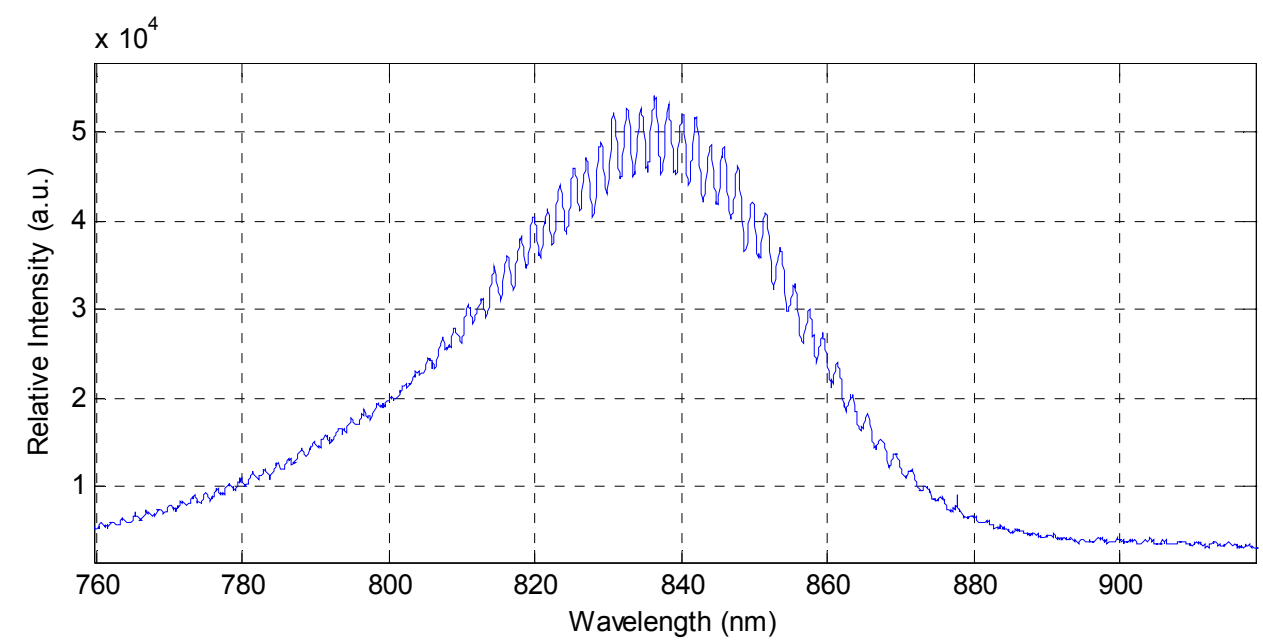

Figure 3-162: A sample of the raw data from temperature sensor T1

In order to process the data shown in Figure 3-162, a series of pre-processing steps must first be applied. Samples of the data at each of these steps are shown in Figure 3-163. In the top graph is a sample of the same raw data seen above. The data is trimmed to the region of interest, and then flattened to remove the impact of the overall shape of the return signal (which results from the envelope of the light source used). The results of this process are shown in the center graph. The data is then filtered and normalized in order to yield an optimum interferogram which can then be processed in order to extract the key parameters which represent the measurement, specifically the frequency and phase of the signal. The output of this is shown in the bottom plot. The red asterisks shown in the bottom plot of Figure 3-163 mark the downsampled data which are passed to the primary processing algorithm. 

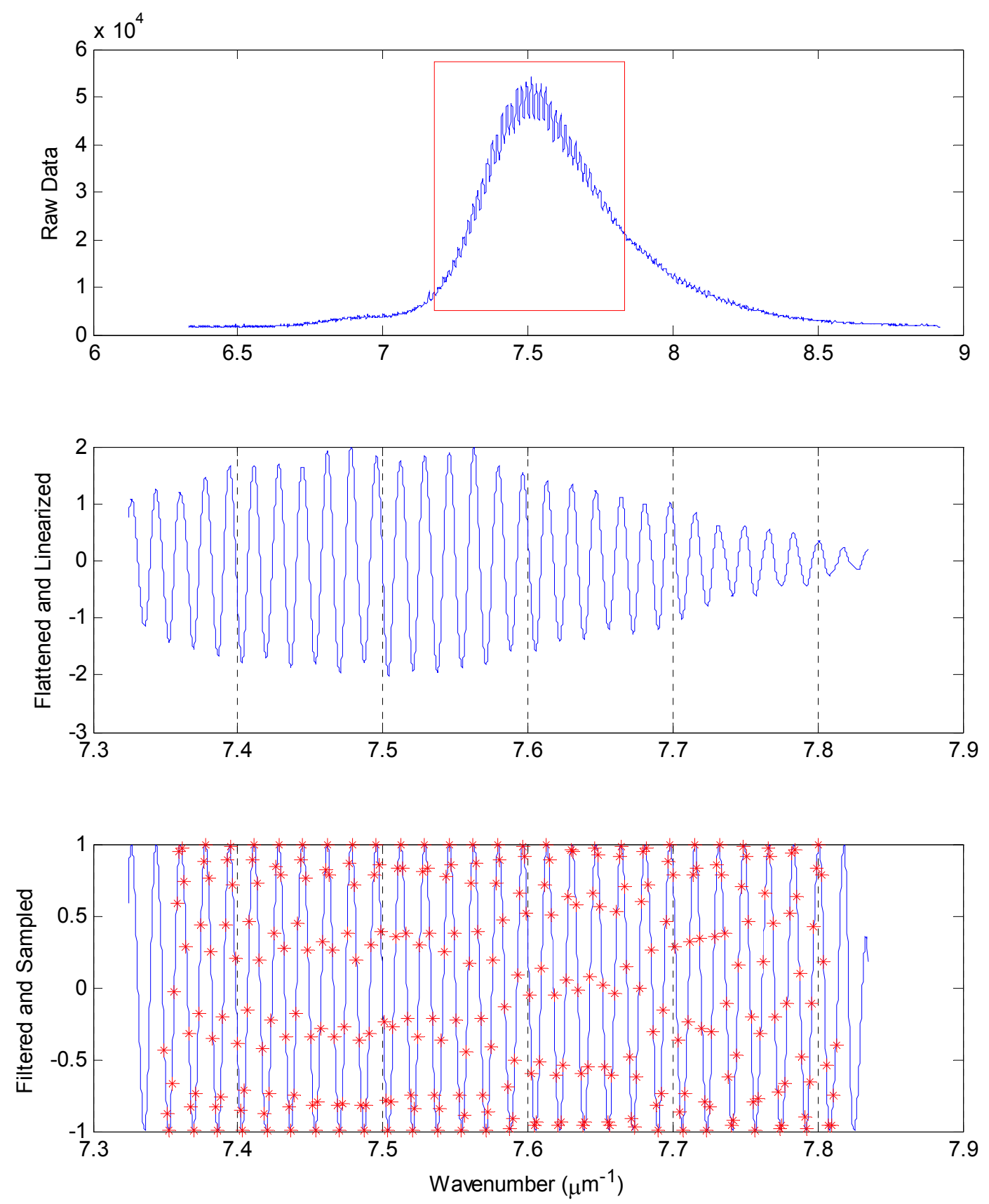

Figure 3-163: Demonstration of the data preparation for processing from temperature sensor T1. Top: Raw data with red rectangle to show region used for later steps of processing. Center: Data after it has been flattened and interpolated to uniform sample spacing. Bottom: Data after filtering and normalization, red stars show the downsampled points of the data used for content extraction.

The primary algorithm extracts data from the pre-processed spectra in order to determine the changes in the fundamental Fabry-Perot cavity. The two principal measures of the cavity change are the optical cavity size and the phase of the interferogram, which can be thought of, respectively, as a coarse and fine measure of the parameter of interest. It is worthy of note that these are not two completely independent measures of the sensor, as there exist cross-dependencies in the calculations of these two properties. 
There are several methods by which this data can be represented. Two methods for displaying the results of the coarse measure of the optical cavity are shown in Figure 3-164. In the top of the plot is a display of the dominant characteristic cavity size from the data which has been processed with the primary processing algorithm. The middle section of the figure is a spectrogram which is the graphical representation of the amount of power which is present at a given frequency over time. Each vertical slice of the data shown in the spectrogram is the frequency analysis of one of interferograms such as the one shown in the bottom of Figure 3-163. The dark red line which traverses the density plot represents the frequency of the dominant signal for that particular sample, and approximately correlates with the data (optical cavity size) shown above. The right half of the plot shows the smooth, anticipated variations of the frequency content, with minor variations which correspond well with the temperature changes of the system.

An area of difficulty is shown in the left hand side of the data where data artifacts create a highly noisy spectrogram which is largely uncorrelated with the actual temperature. The source of this noise has not been conclusively determined, although it is suspected that the appearance of a second optical cavity (in addition to the sapphire wafer which serves as the measurement element and primary optical cavity) creates a low-frequency interference signal in addition to the desired measurement signal. Since the frequency of the signal from the second cavity is inversely proportional to the optical length of the cavity, a small (less than one micron) cavity can generate a signal of very low frequency. This low frequency signal would heterodyne (mix) with the measurement signal, shifting the frequency of the measurement signal by an amount equal to the frequency of the second cavity. While the source of this secondary cavity has not been definitively determined, possible causes include the development of a gap between the sapphire fiber and silica fiber in the splice region, or the development of a gap between the sapphire wafer and the sapphire fiber. It is noted that similar gaps between the wafer and fiber were observed in early testing of the sapphire temperature sensor (see Section 3.5.4.1). Surprisingly, the signals tend to be noisiest at times of relative temperature stability, rather than during the fast temperature ramps.

Shown in Figure 3-165 is a plot of the phase extracted from each interferogram along with the thermocouple temperature, over the same period of time. In order to render the phase plot more easily discerned, it has been magnified by a factor of five. It is clear that there is a good correlation of the optical phase with the temperature, although there are regions of noise, particularly in the early half of the data, as well as a few discrete steps of offset. The overall correlation coefficient for these data sets is 0.7383 (1.0 indicating perfect correlation). The noise in the earlier half of the data shown in Figure 3-165 is highly correlated with the noise which is present in the coarse measurement, as shown in Figure 3-164. 


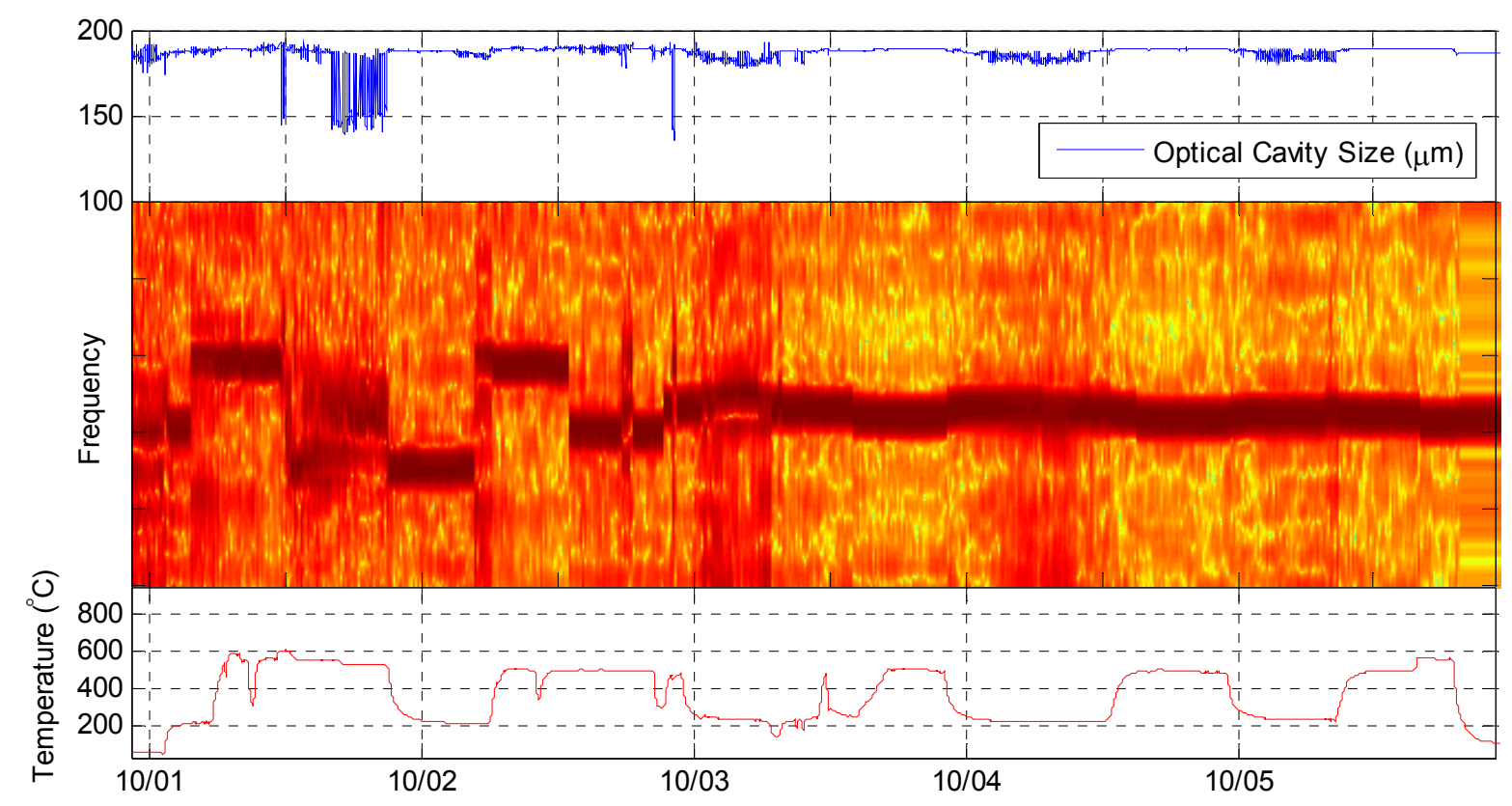

Figure 3-164: Optical cavity dimension and power spectral density of sensor T1 from 10/1/07 through 10/5/07

The step offsets shown in Figure 3-165 result from the periodic nature of any calculation of phase. Every $2 \pi(\sim 6.28)$ radians is a "phase jump", and one of the requirements of an interferometric sensing system is to correctly identify whether a phase jump has occurred, and unwrap it in the event that one has. This is highlighted in Figure 3-166, in which the data has been marked at each point where a $2 \pi$ phase jump has been corrected for. In the case of excessive noise, a situation can arise in which such phase jumps are either missed, or falsely identified, which is the source of the abrupt noise jumps shown in the data. It is worthy of note, however, that once the system noise diminishes again, the ability to track through the phase jumps is restored.

To offer a direct comparison of the relationship between sensor output and thermocouple temperature, the two sets of data have been plotted against each other in Figure 3-167. For clarity, the data has been color coded based on the time of the data sample. An ideal sensor response would be linear, with all data lying on a straight line. In this scatter plot, phase jumps that are missed by the algorithm result in horizontal lines and a horizontal shift of the data. When the data is incorrectly interpreted as containing a phase jump, a vertical line results, and the data is shifted vertically. 


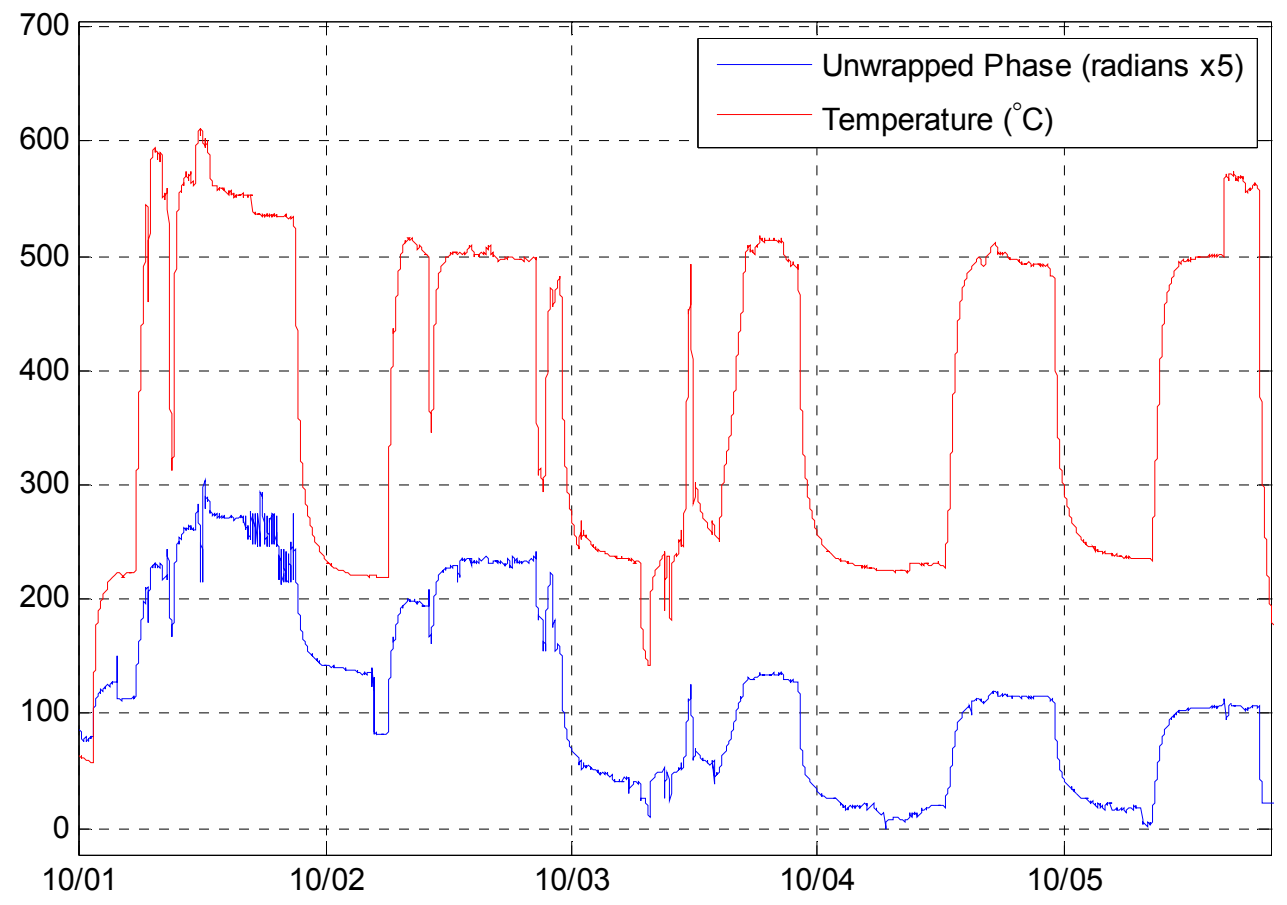

Figure 3-165: Comparison of the thermocouple temperature reading (red) with the phase (fine) optical measurement (blue).

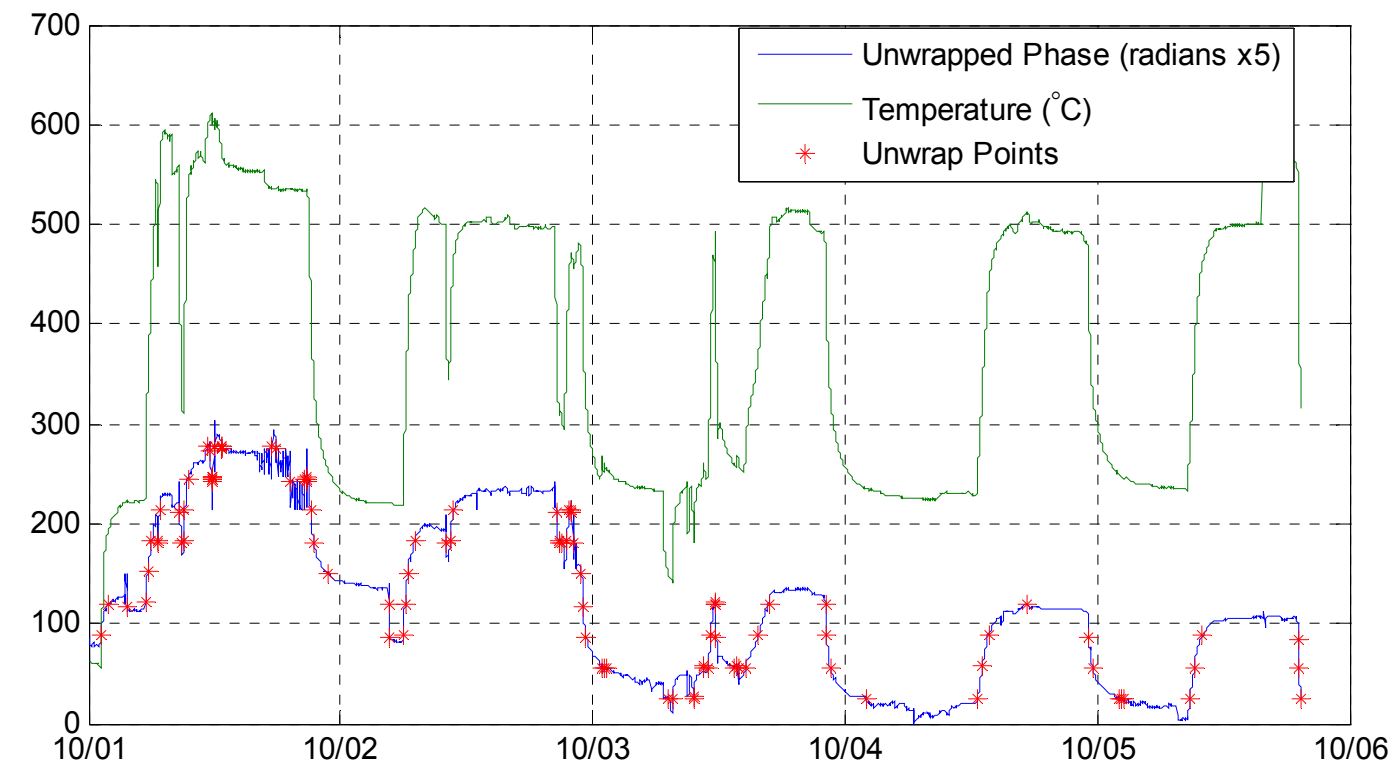

Figure 3-166: Replication of Figure 3-165 with red asterisks marking all points where the phase was unwrapped 


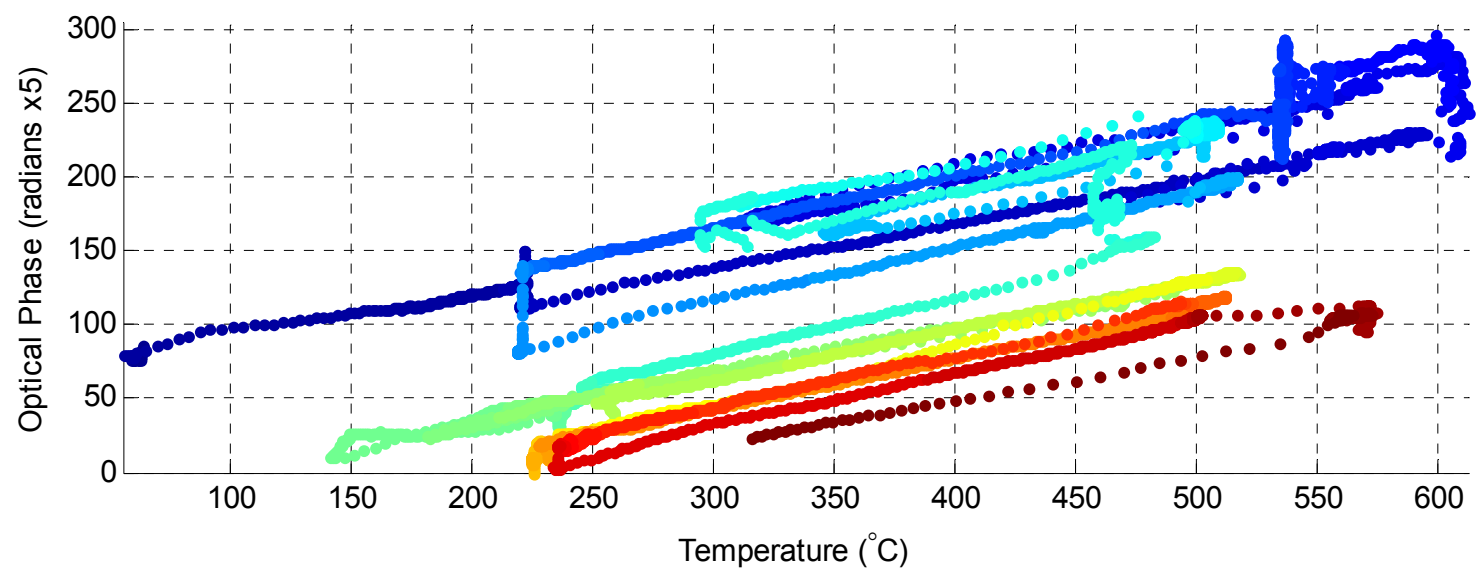

Figure 3-167: Scatter plot of optical phase vs. temperature (as reported by the thermocouple). The data is color coded based on the time of the sample, with blue as the earliest data, and red as the latest.

The shifts of data in Figure 3-167 illustrate the severe impact of noise on the sensor output. For example, if the data resulting from the first temperature cycle of the sensor (the dark blue trace) were used to calibrate the sensor, then data as 20 radians recorded near the end of the test (dark red trace) would be incorrectly interpreted as $100{ }^{\circ} \mathrm{C}$, rather than the true temperature of $550{ }^{\circ} \mathrm{C}$. Either reducing the noise in the measurement data, or reducing the algorithm's sensitivity to the noise, should be a priority for continued development of the sapphire temperature sensor.

\subsection{Strain Sensor}

In addition to the installation of the temperature sensor, a strain sensor (S1) was also installed and monitored with a modified scanning laser spectrometer (Micron Optics Component Test System, or CTS). Full spectra of the response from the sensor were recorded at one minute intervals. The sensor operated from the installation on 21 August, 2007 through 28 September, 2007. On September 28, at approximately 12:30 p.m. the sensor suffered a failure at which time discernable interferometric fringes were no longer present in the captured signal. The data presented in this analysis begins on 5 September, 2007, as all data prior to that time represents a baseline at steady state (since the CEDF burners were not fired before that date). A plot of the temperature profile during the test, as reported by the thermocouple, is shown in Figure 3-168, with a red star to indicate the point at which the strain sensor ceased to provide meaningful data. The response from S1 at times later than this are indicative of the sensor potentially becoming misaligned. As explained in Section, it was discovered when the sensors were retrieved from the CEDF during a plant shutdown that the bottom half of the sensor (containing the reflector fiber) had disbonded from the superheater tube and was found lying on the bottom of the protective shield. There was, therefore, no Fabry-Perot cavity in the strain gage after the event producing the disbond (presumably on 28 September), and any periodic structure in the spectra recorded subsequent to this date were primarily due to modal noise or spurious cavities. 


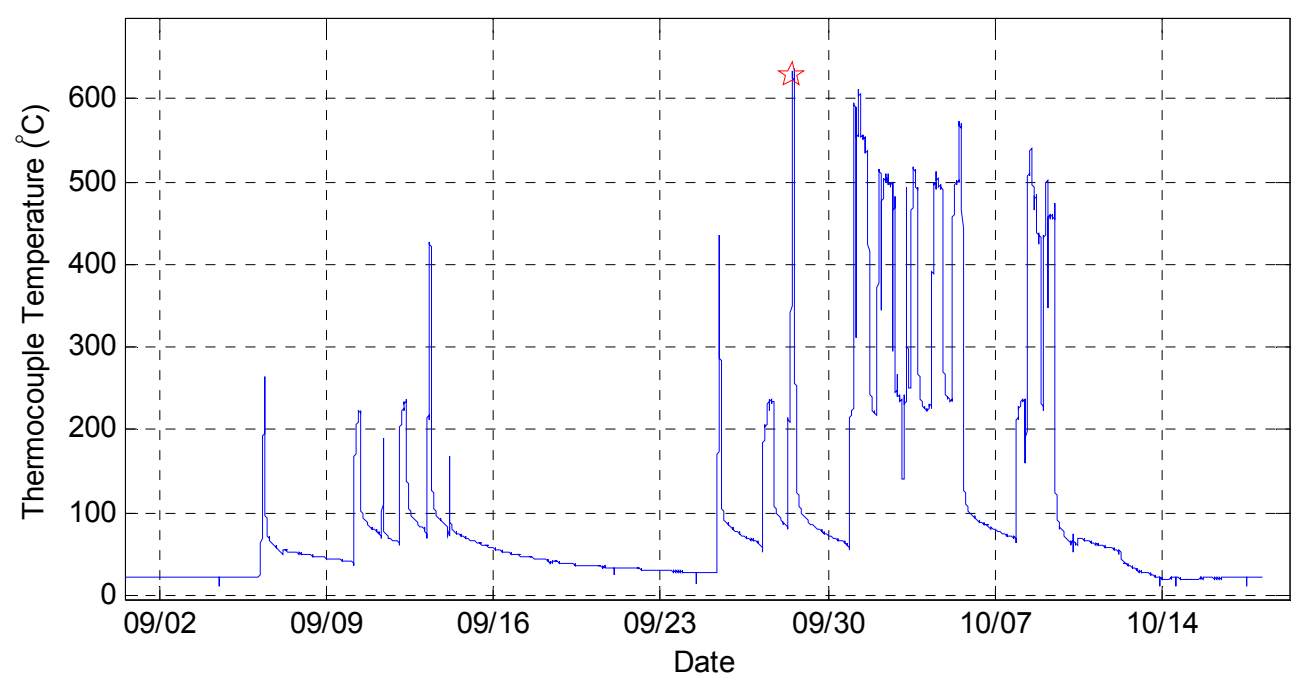

Figure 3-168: Plot of the temperature profile of the test as indicated by the thermocouple mounted next to the sapphire strain gage $S 1$. The red star indicates the time of the last sensor data point.

Figure 3-169 shows an example of a single sample from the strain sensor before the interference fringes were lost. This data is clearly visually different from the temperature sensor spectrum which was shown in Figure 3-162 (see page 155). The reason for this difference is because the two sensors were interrogated with systems which operate by very different principles. The strain sensors were interrogated by the VectorLight ${ }^{\mathrm{TM}} 200$ which operates at a longer wavelength and which utilizes a swept laser light source in order to achieve spectrally flat illumination. In addition, the VectorLight ${ }^{\mathrm{TM}} 200$ returns approximately six times as many data points as the system which was used with $\mathrm{T} 1$. The strain data is encoded in the low frequency wave which is seen in the plot (which has approximately 4.5 full cycles visible). The high frequency (rapidly oscillating) content represents noise which is a result of signals from additional cavities or intermodal interference within the fiber optic sensing system.

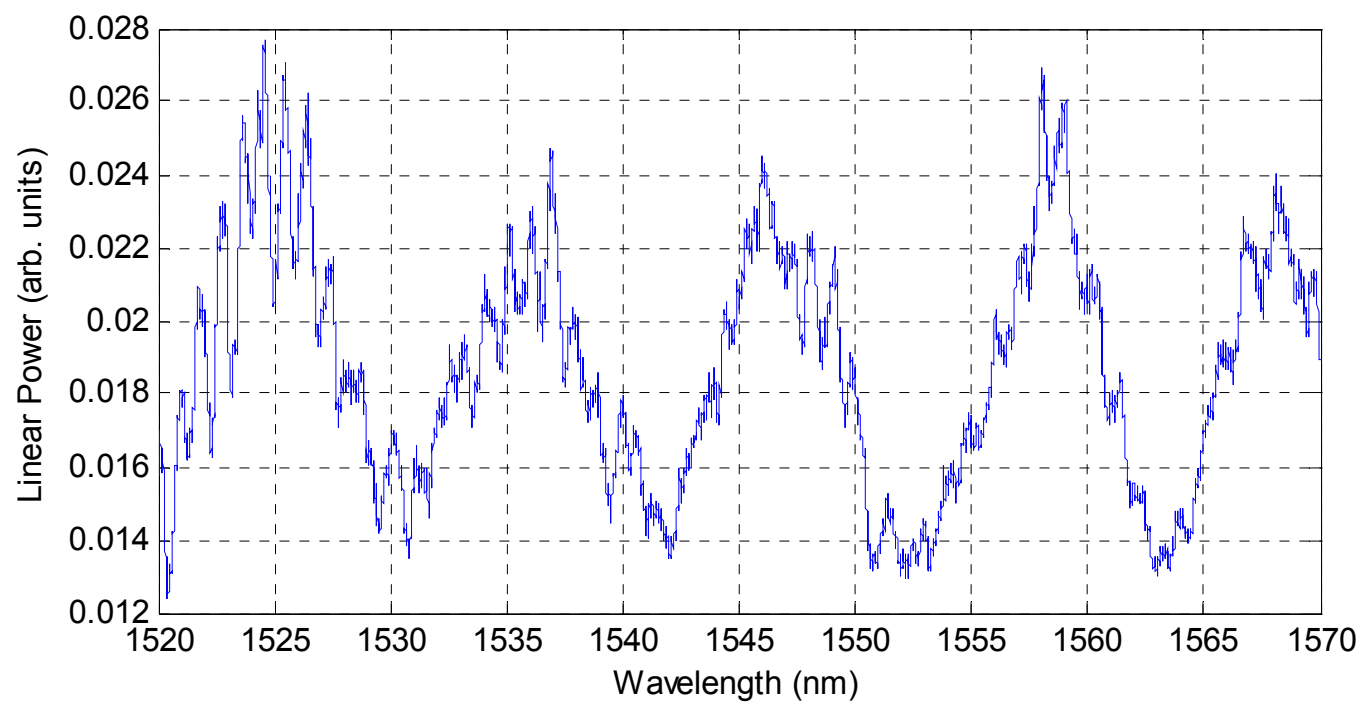

Figure 3-169: Example of raw data from strain sensor 
In Figure 3-170, the basic processing steps are shown for the decoding of the signal which is returned from the strain sensor. In the top plot is the raw data which is reported by the VectorLight ${ }^{\mathrm{TM}} 200$ hardware. The data is then scaled and low pass filtered to eliminate the highest frequency noise content. This result is shown in the middle plot, as the blue line. In order to maintain a reasonably short processing time, the data is then decimated (or downsampled) to a subset, which is shown as the red dots. This decimated data is then passed into the PRLC algorithm which extracts the frequency, amplitude, and phase content of the signals. The bottom plot shows the reconstructed signal based on those derived values (shown in blue), with the red dots carried from the preceding plot for comparison.

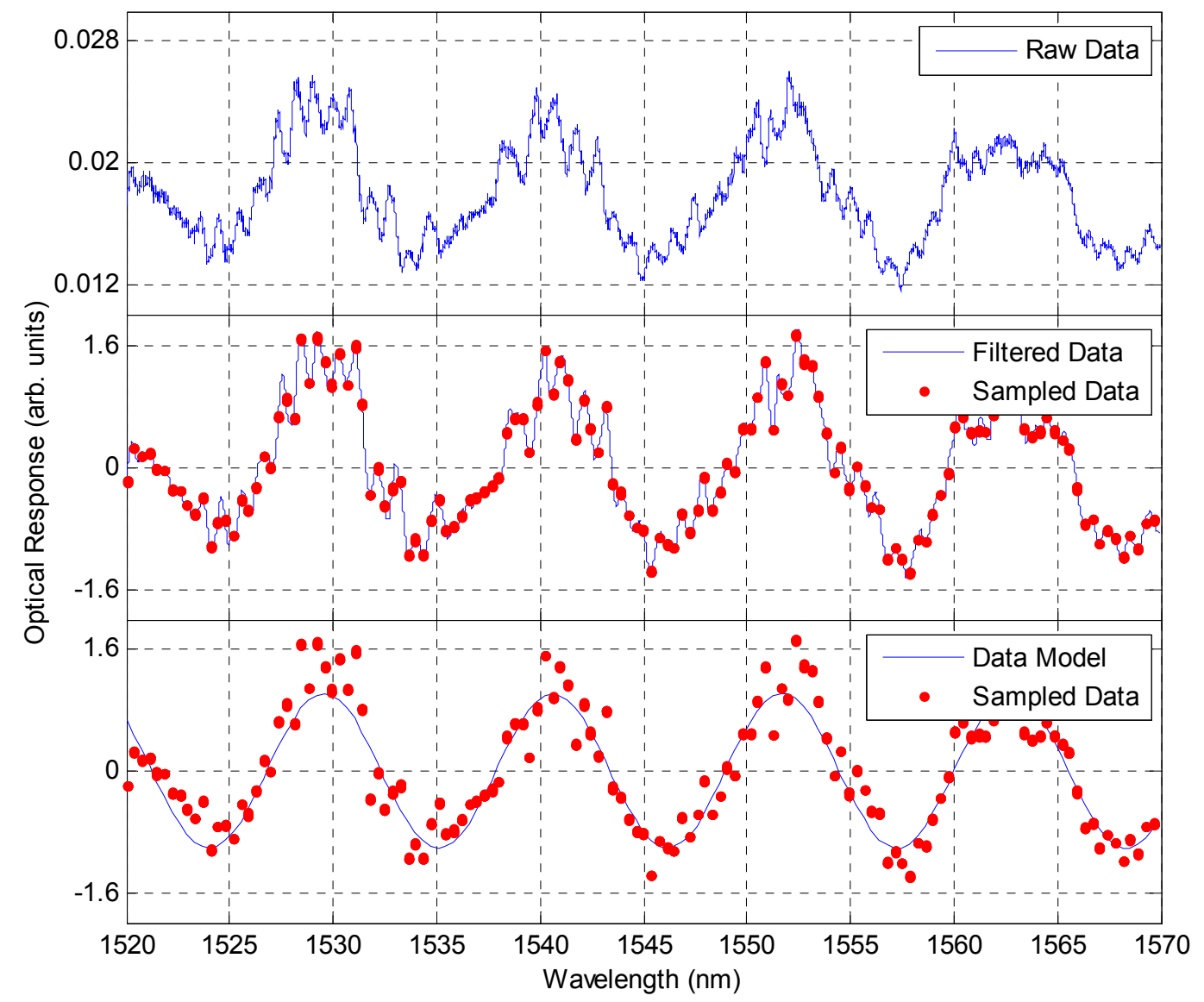

Figure 3-170: Processing steps for decoding content of strain sensor response. The top plot shows the raw data from the instrument. The middle plot shows the data which has been scaled and filtered (blue), then downsampled (red). The bottom plot shows the same downsampled data (red) and the modeled response of the system (blue).

As with the temperature sensor, there are two key measurements of the spectral data which correlate with the value of the strain detected. The coarse measurement of the cavity size is represented in the frequency of the sinusoidal signal, while the fine response is detectable as the phase of the waveform. Figure 3-171 shows the results of the coarse cavity measurements through two independent analysis methods. While the two methods yield the same overall structure, there are some fine details which may be 
seen in the top plot, which are indicative of a moderately lower noise band for method 2 (shown in green). The lower plot shows the scatter relationship between each of these two methods when plotted against the temperature, which again highlights the lower noise performance of method 2 .

As explained in Section 3.11, the sapphire strain gage was mounted to a tube in the convection pass of the CEDF facility. Due to the high temperatures anticipated by B\&W engineers (up to $2000{ }^{\circ} \mathrm{F}\left(1093^{\circ} \mathrm{C}\right.$ )), it was not possible to mount a conventional electrical strain gage on the tube to serve as a reference for the sapphire sensor. The sapphire strain gage was mounted on a tube that was fixed and suspended from the ceiling of the superheater tube pass. Since the bottom of the tube was freely hanging, any strain resulting in the tube during the testing would result solely from the thermal expansion of the tube. Therefore, for the purpose of testing the sapphire strain gage on the CEDF tube, the measured strain was correlated with temperature.

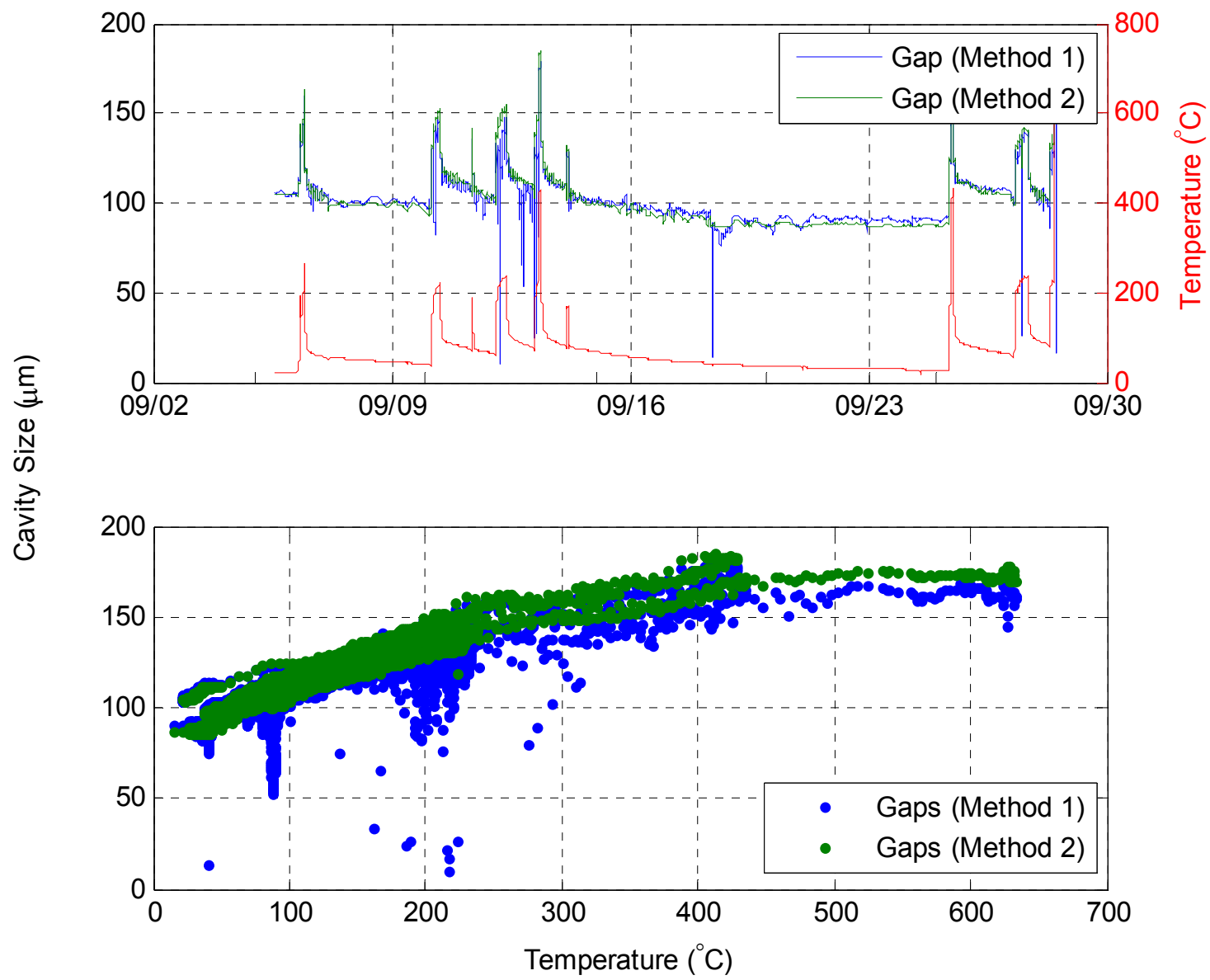

Figure 3-171(: (Top): Plot of optical cavity size as determined through two methods (blue and green) and Temperature (red) versus time. (Bottom): Plot of optical cavity size versus temperature for both methods. 
Figure 3-172 shows the spectrogram of the signals from S1 throughout the duration of the test. Although there are small noise features, the signal is highly continuous, indicating that $\mathrm{S} 1$ did not suffer from any changes in the spectral content which were not correlated with the actual measurand.

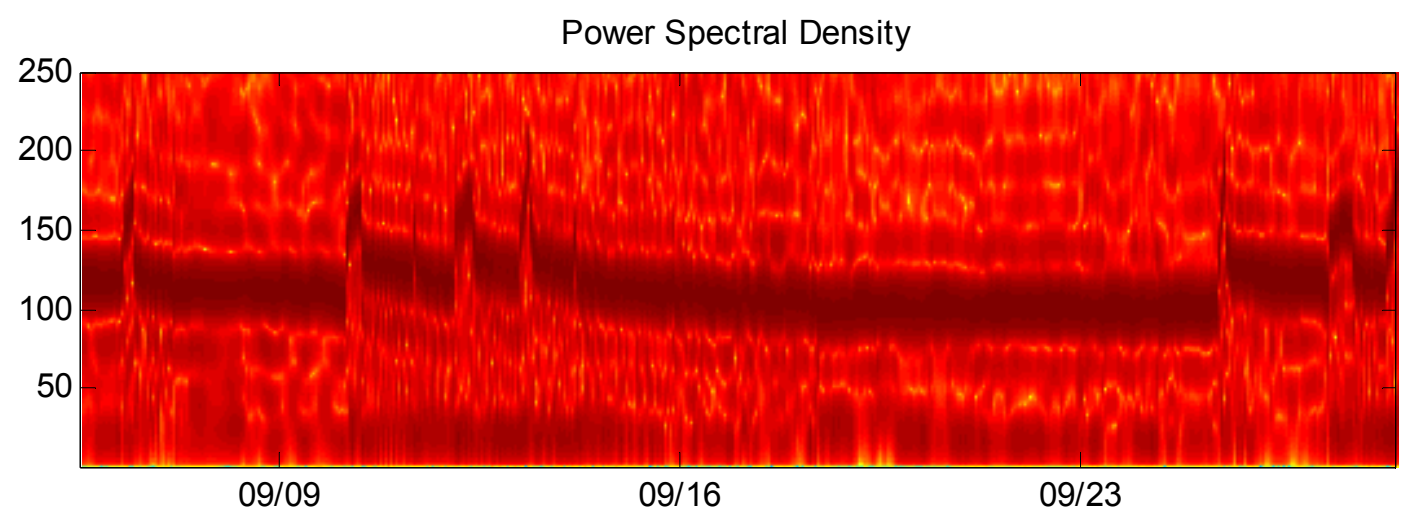

Figure 3-172: Frequency spectrum of strain sensor $S 1$ over the duration of the test.

The fine measure of strain reported from $\mathrm{S} 1$ is found in the phase of the interferograms. An unwrapped display of the phase is shown in Figure 3-173 (blue) along with the temperature (green). There is a good correlation between the phase and the temperature. The correlation coefficient for is 0.7955 (with 1.0 indicating perfect correlation.)

Results from Strain Sensor

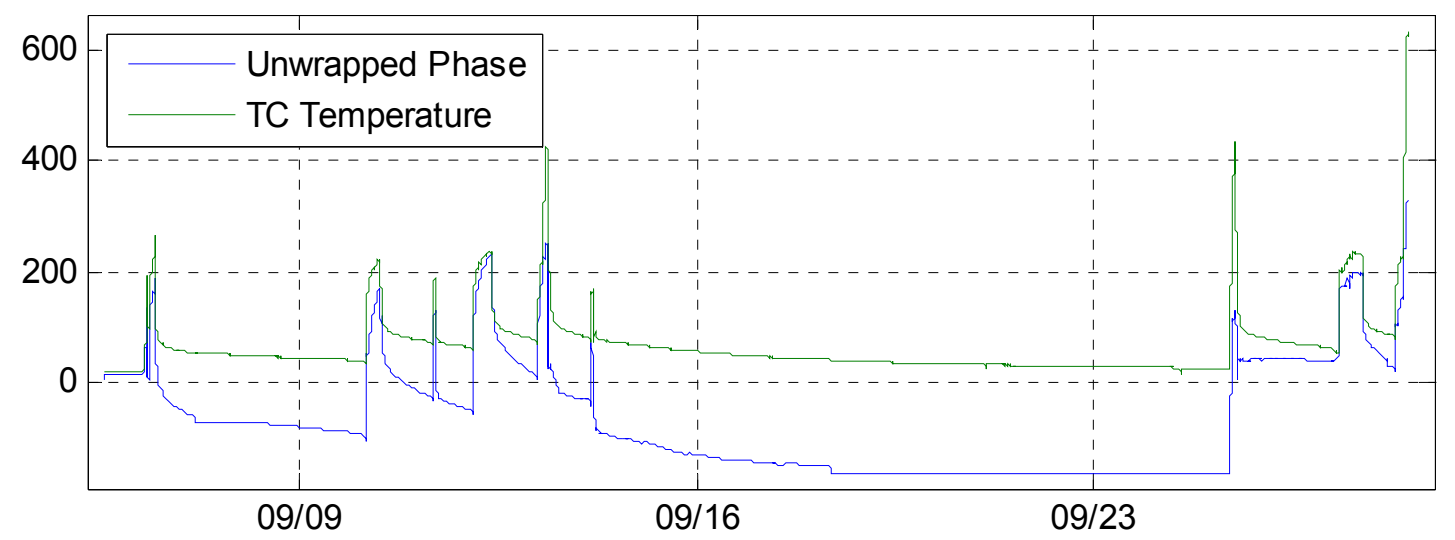

Figure 3-173: Plot of the phase (unwrapped) and temperature results from S1 over the duration of the test

There are two factors which contribute to lowering the correlation between the temperature and the phase shown in Figure 3-173. The first is the difference which arises between the measurement of air temperature and the development of strain in the substrate material (the pipe). Because the thermocouple has so much lower of a thermal mass than the pipe, it responds faster to changes in temperature than does the pipe. The second issue is the result of unidentified phase jumps in the data. Those are cases in which the phase should have been unwrapped, but wasn't. The key contributor to this was the low sampling rate of the data collection; the algorithm missed phase jumps (phase excursions of greater than $2 *$ pi radians) that occurred between samples. 
When the sensor system was installed in the CEDF, it was not known for sure when the plant would begin operation. Therefore, a sampling interval of one minute was selected in order that the data acquisition storage medium not be filled before the first firing of the plant. The actual rate at which the temperature within the environment changed was vastly higher than anticipated, and as a result the data collection at one minute intervals resulted in a number of phase jumps being missed. This phenomenon can be seen in Figure 3-174. When the sensor response is successfully decoded, the slope of the phase versus temperature plot is uniform. However, when the rate of temperature change is large (as indicated by the red or blue points), the software at times fails to correctly unwrap the phase, resulting in a very small slope (a large change in temperature without a corresponding change in phase).

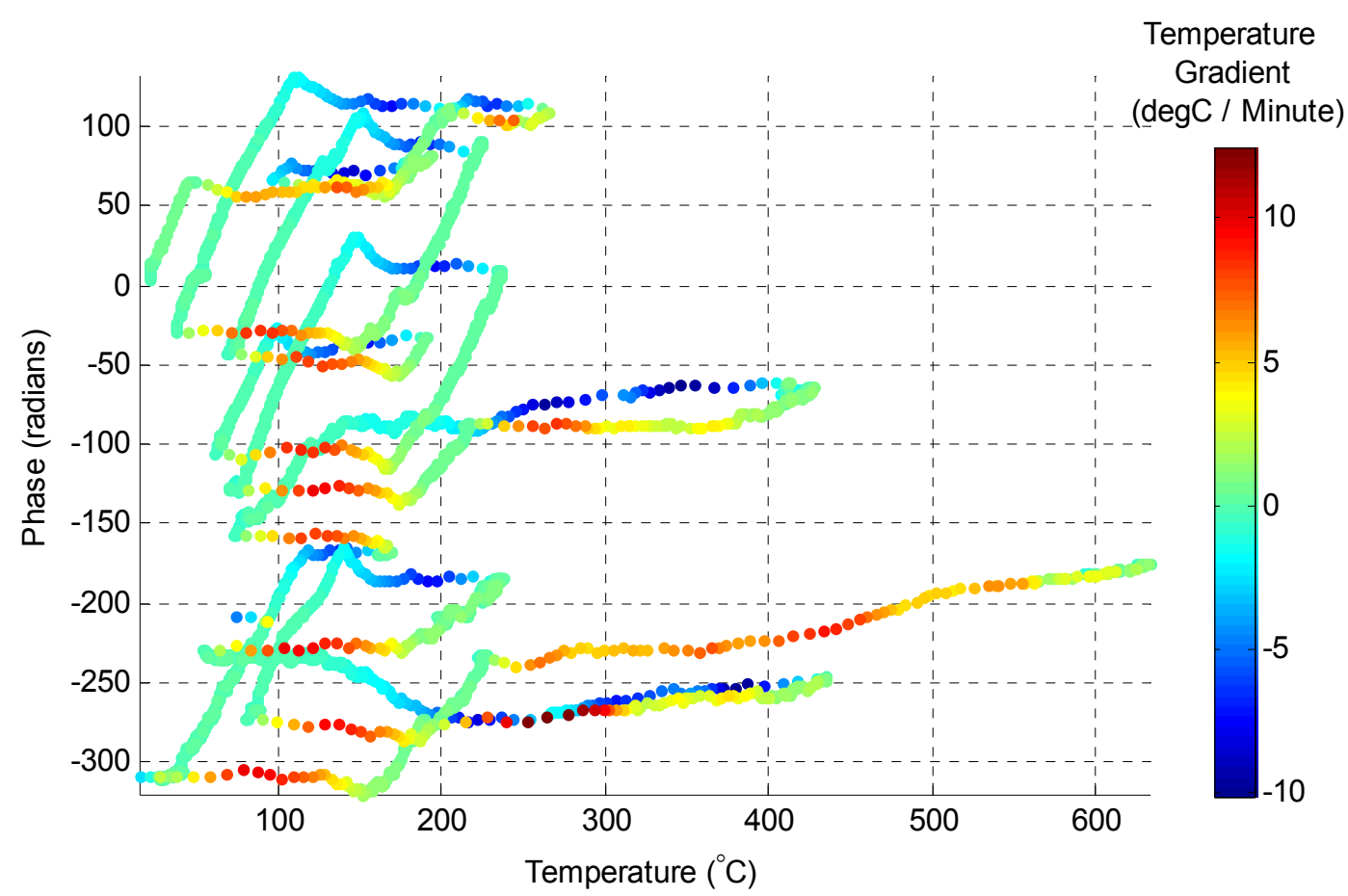

Figure 3-174: Scatter plot of the unwrapped phase versus temperature. The color of the points is indicative of the rate of temperature change in degrees Celsius per minute

In order to correct for this problem, the phase unwrapping was completed using a hybrid method which relies upon both of the methods for calculating the coarse gap. The scatter plot of this data is shown below in Figure 3-175. While the hybrid method was vastly more effective than the original approach, there were still some instances in which it was unable to keep up with the rapidly changing temperatures. The ultimate method to resolving this issue is to increase the sampling frequency of the interrogator, which prevents such dramatic changes in value between sequential samples. 


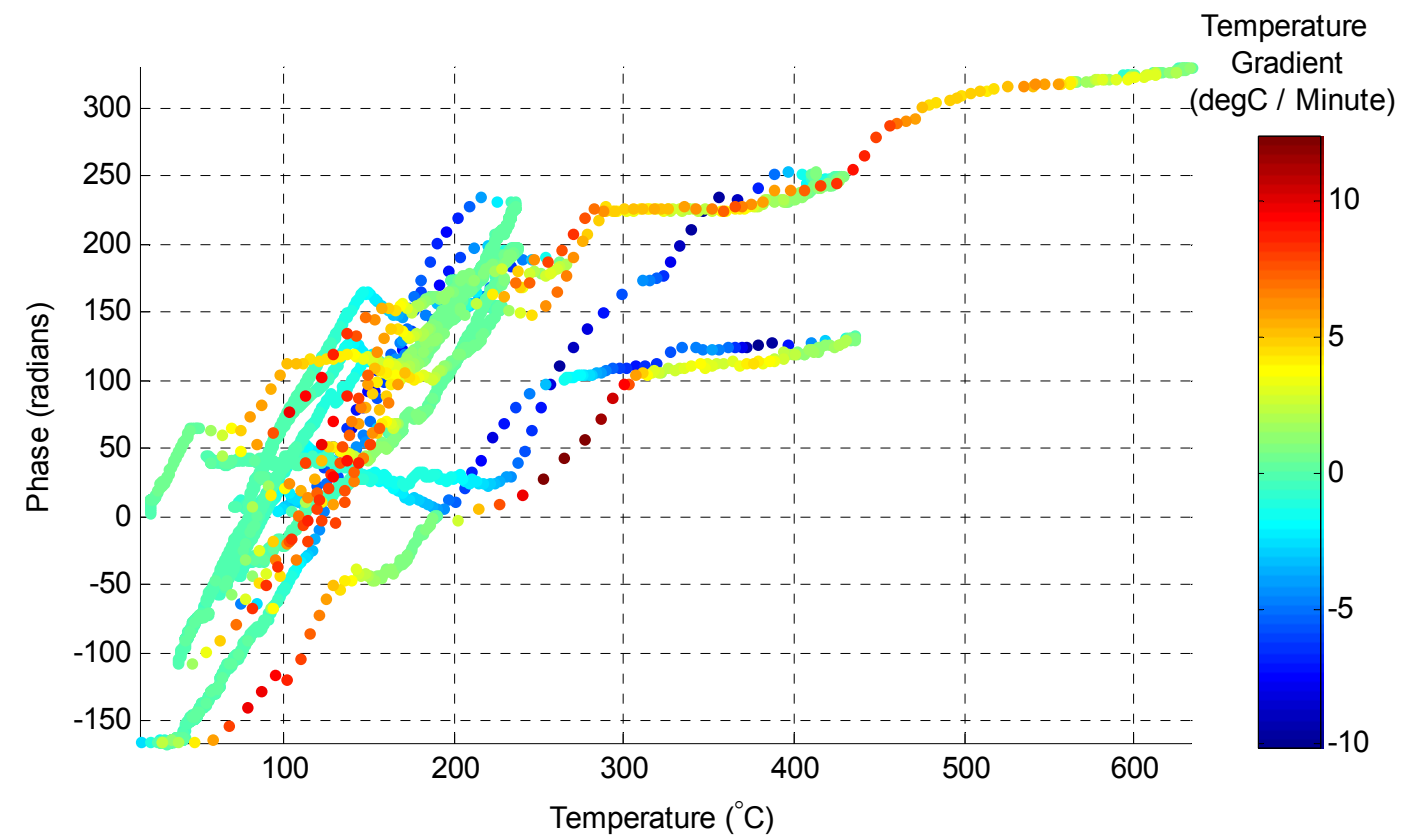

Figure 3-175: Scatter plot of the corrected phase versus temperature. The color of the points is indicative of the rate of temperature change in degrees Celsius per minute

\section{Development of Optical Cladding for Sapphire Fibers}

For the ultrahigh-temperature environment found in current and future power generation facilities, new materials for the optical sensors will be required. Previous efforts to utilize sapphire fiber sensors that can theoretically operate above $1650{ }^{\circ} \mathrm{C}\left(3000^{\circ} \mathrm{F}\right)$ have been limited because the fiber itself does not lend itself to incorporation in the known sensor techniques, due to its unclad and multimode construction. Another goal of this program is the development of high-temperature, ceramic claddings for sapphire fibers to improve the waveguiding properties of sapphire fibers and facilitate their use in photonic sensor systems.

Current efforts towards improvement of sapphire fibers focus on creating cladding in sapphire (aluminum oxide single crystal, $\mathrm{Al}_{2} \mathrm{O}_{3}$ ) fibers by dip-coating the fibers in a a mixture of magnesium oxide $(\mathrm{MgO})$ powders and magnesium aluminate spinel $\left(\mathrm{MgAl}_{2} \mathrm{O}_{4}\right)$ powders to form a coating on the sapphire fibers. The technique involves dip coating a sapphire fiber in a suspension of $\mathrm{MgO}$ and spinel powders, drying the coating, then firing at elevated temperature to react the $\mathrm{MgO}$ powder with the sapphire fiber and to densify the coating.

The overall reaction for the process is given by the chemical equation:

$$
\mathrm{MgO}+\mathrm{Al}_{2} \mathrm{O}_{3}=\mathrm{MgAl}_{2} \mathrm{O}_{4}
$$

which proceeds rapidly at $1750{ }^{\circ} \mathrm{C}\left(3182^{\circ} \mathrm{F}\right)$. 
The coated fibers are then fired at high temperatures to facilitate the reaction between the $\mathrm{MgO}$ in the coating and the $\mathrm{Al}_{2} \mathrm{O}_{3}$ in the fibers by ion diffusion, and create a cladding consisting of $\mathrm{MgAl}_{2} \mathrm{O}_{4}$ surrounding the sapphire core. As a result, the diameters of the sapphire fibers are reduced and the number of modes propagating in the sapphire fibers is also reduced.

\subsection{Initial Experiments Directed Towards Magnesium Aluminate Spinel Cladding}

The spinel slurry is prepared by dissolving a powder mixture of $\mathrm{MgO}$ powders (EMD Chemical Inc., formally EM Science, Gibbstown, NJ; average particle size $\approx 15 \mu \mathrm{m}$ ) and $\mathrm{MgAl}_{2} \mathrm{O}_{4}$ powders (Baikowski International, Rayleigh, $\mathrm{NC}$; average particle size $\approx 3 \mu \mathrm{m}$ ) in a solvent consisting of isopropyl alcohol (2-propanol, Jade Scientific, Canton, MI), propylene glycol methyl ether (1-methoxy-2-propanol from Fluka, Milwaukee, WI), polyvinylpyrrolidone (Aldrich Chemical Company, Milwaukee, WI), and polyethylene glycol (SPI supplies, West Chester, PA). To maintain its homogeneity, the slurry is constantly stirred in a beaker covered with parafilm on a hot plate using a magnetic spinning bar.

After the slurry is prepared in a beaker on a hot plate with magnetic stirring, the slurry is poured into a 250 c.c. polypropylene bottle for ball milling. The bottle is filled to about $50 \%$ in volume with alumina grinding media as shown in Figure 4-1(a). The grinding medium is in a cylindrical shape $1 / 4$ inch long and $1 / 4$ inch in diameter. For best milling efficiency, more isopropyl alcohol is added into the bottle to the level just covering the grinding media. The bottle is then placed and fastened using rubber bands in a porcelain container for ball milling as shown in Figure 4-1 (b). The ball miller is made by US Stoneware (East Palestine, $\mathrm{OH}$ ). The ball milling step is critical in achieving a slurry with fine particle sizes and uniform and homogeneous thickness (viscosity).

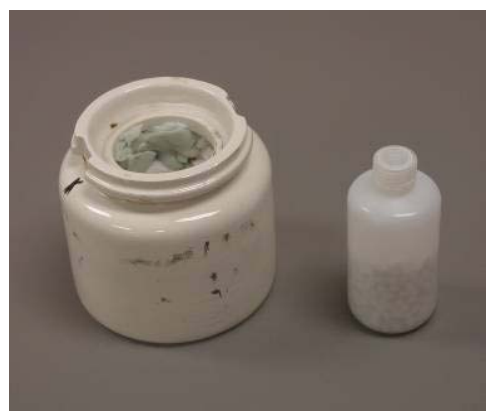

(a)

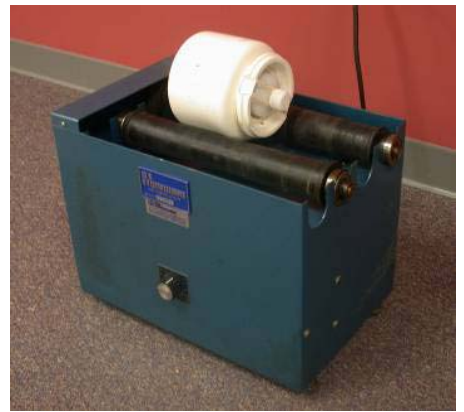

(b)

Figure 4-1. (a) The 250 c.c. polypropylene bottle half filled with grinding media (right) and the porcelain container (left) for ball milling;

(b) The container on the ball milling machine ready for milling. 
After ball milling, the slurry is poured back to a beaker without a parafilm cover and is stirred using a spinning bar on a hot plate. In this process, the additional isopropyl alcohol gradually evaporates and the slurry slowly gets thicker and thicker. When the desired slurry thickness is reached, it is ready for fiber dipping.

Before dipping, the $150 \mu \mathrm{m}$ diameter sapphire fiber (Photran LLC, Poway, CA) is cleansed with hydrochloric acid, deionized water, and finally isopropyl alcohol. The fiber dipping is performed using the experimental apparatus shown in Figure 2 (a) and (b). The fiber is clamped vertically using the stand shown in Figure 4-2 (a). The vertical orientation of the fiber is critical in creating a coating with uniform thickness. A graduated cylinder or a small glass bottle is used to contain the slurry. The cylinder or the bottle sits on a small lab jack. The dipping is achieved by raising and lowering the cylinder or the bottle using the lab jack. Several dips are needed to form a thick enough coating on the fiber, and the overall time of the dipping procedures may take from several minutes to tens of minutes. In order to maintain a constant viscosity of the slurry during dipping, the cylinder or the bottle is capped, and a small hole is drilled in the stopper of the graduated cylinder or in the cap of the bottle as shown in Figure 4-2 (b); as a result, the fiber can pass through the hole while the evaporation of the solvent, i.e., isopropyl alcohol, in the slurry is minimized. To increase the uniformity of the coating thickness along the length of the fiber, a smooth motion of the platform of the lab jack with a constant speed is required. Since maintaining a constant speed by manually adjusting the height of the lab jack using the turning knob is not easy, so only best efforts are made to achieve this goal.

The step-by-step slurry preparation and fiber dipping procedures are described in Appendix 1. The dipped fibers are finally fired in either a Thermolyne 46100 hightemperature furnace or Thermolyne 48000 furnace (Thermolyne, Dubuque, Iowa). The former is referred to as the "Large Furnace" and the latter the "Small Furnace" in the firing profiles illustrated in Appendix 2.

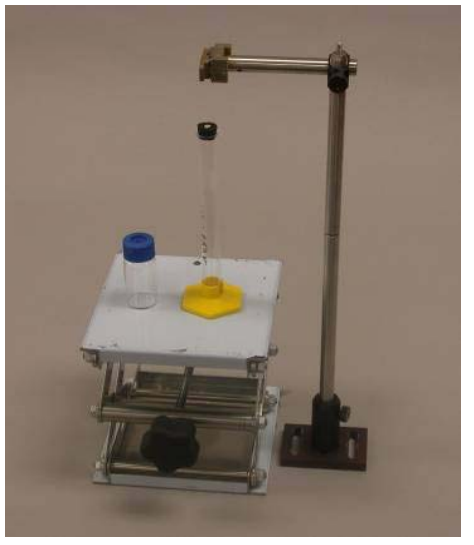

(a)

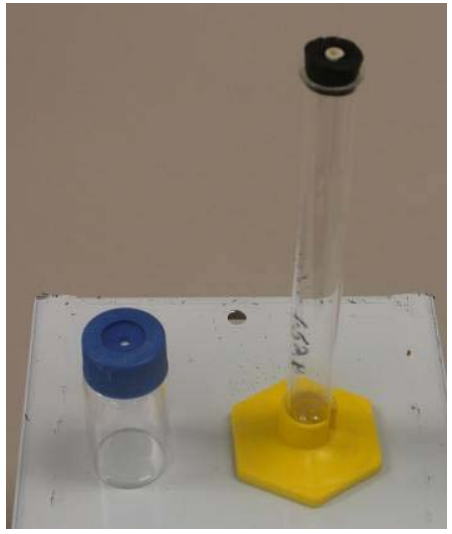

(b)

Figure 4-2. The experimental apparatus for the dipping of fibers 


\subsubsection{Preliminary Results for Spinel Cladding Development}

Samples of magnesium alumina spinel $\left(\mathrm{MgAl}_{2} \mathrm{O}_{4}\right)$ clad sapphire (aluminum oxide, $\mathrm{Al}_{2} \mathrm{O}_{3}$ ) fibers with reduced core diameters and optically smooth cladding/core interfaces capable of light guiding were successfully fabricated. The light guiding ability was qualitatively observed using an optical microscope (Nikon Optiphot-100) and was judged by the degree of smoothness of the cladding/core interface. The cross sections of the clad fiber are shown in Figure 4-3(a), (b), (c) and (d) below. The fiber was fired at $1600{ }^{\circ} \mathrm{C}$ $\left(2912^{\circ} \mathrm{F}\right)$ for 30 minutes in argon. The detailed firing profiles are illustrated in Appendix 2.

In Figure 4-3(a) and (b) the sapphire fiber is dipped for 14 times in the spinel slurry, while in (c) and (d), 17 times. The details of the slurry preparation and dipping procedures are described in Appendix 1. In the case of the fiber with 14 dips, the core diameter is reduced from $150 \mu \mathrm{m}$ to about $75 \mu \mathrm{m}$, and in the case of 17 dips, to about $50 \mu \mathrm{m}$. It is apparent in most of the figures that the core part of the fiber is significantly brighter than the cladding part, which indicates the light guiding ability of the core.

Especially in Figure 4-3 (b), a certain kind of pattern of brightness exists within the core, which possibly represents the mode patterns of light propagation in the core.

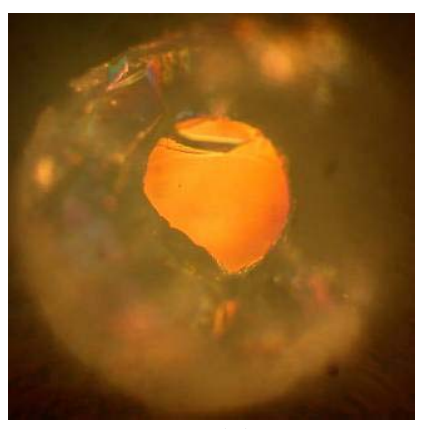

(a)

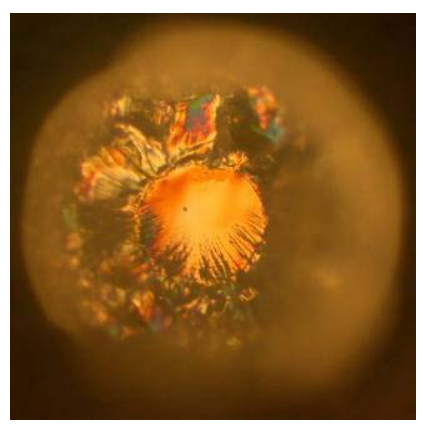

(c)

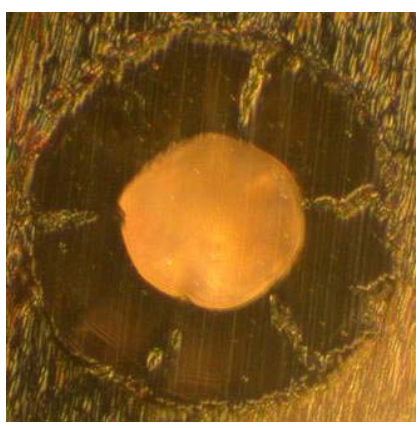

(b)

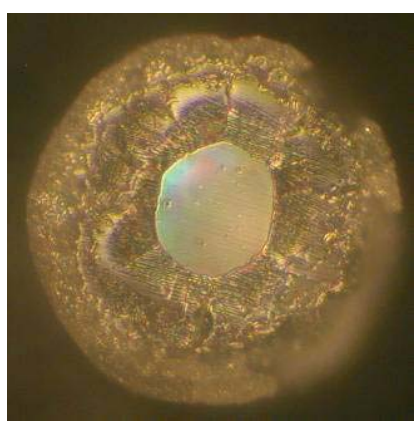

(d)

Figure 4-3. The fractured (a) and polished (b) cross sections of the clad sapphire fiber with 14 dips in the slurry, and the fractured (c) and polished (d) cross sections of the clad sapphire fiber with 17 dips in the slurry. (Both from the same sample with sample I.D. 14(17)s). 
An investigation was undertaken in which certain parameters such as the firing profiles, the slurry dipping procedures, the atmosphere of firing, the particle sizes of the powders, and the slurry compositions were systematically varied. A total of 37 samples have been prepared as of the date of this report. The names of the samples, the dates on which the samples were dipped and fired, the firing atmosphere, the firing profiles, the type of powders, and the number of dips for all samples are summarized in Appendix 3.

The preliminary results show that to achieve an optically smooth $\mathrm{MgAl}_{2} \mathrm{O}_{4}$-spinelcladding $/ \mathrm{Al}_{2} \mathrm{O}_{3}$-sapphire-core interface, a carefully tailored firing procedure in argon (whose profile depends on the type of powders and the number of dips, i.e., the thickness of the slurry) is required. The suspected key mechanism at play is the crystalline grain growth of the polycrystalline spinel at the cladding/core interface. The results supporting this assumption in terms of the effects of firing profile, the firing atmosphere, the powder particle size, and the thickness (viscosity) of the slurry will be presented in the following sections. The issue of high-temperature stability of this materials system will be explored thereafter, and followed by the presentation of some results on the chemical composition analysis of the clad fibers using EDS technique. Finally the problems and difficulties encountered and their mitigating strategies will be discussed.

\subsubsection{The effects of firing profile}

An optimal firing profile completes the reaction between the slurry coating and the sapphire fiber, while minimizing the grain growth of the polycrystalline spinel at the cladding/core interface. Over-firing causes the spinel grains at the cladding/core interface to grow, resulting in a rough interface which contributes to the scattering of light at this interface, thus diminishes the capability of light guiding in the core.

An extreme case of over-firing is shown in Figure 4-4 below. This sample was fired in air at $1650{ }^{\circ} \mathrm{C}\left(3000^{\circ} \mathrm{F}\right)$ for 16 hours. The flower-shape cladding/core interface presumably results from the growth of the $\mathrm{MgAl}_{2} \mathrm{O}_{4}$ spinel grains at the interface. In similar overfired clad samples, depth profiling of the cross sections was performed by polishing the clad fiber ends in $25 \mu \mathrm{m}$ steps. The results indicate that the cladding/core interface patterns change dramatically from one cross section to another along the lengths. Since the spinel grain size is presumably smaller than $25 \mu \mathrm{m}$, the assumption is that the interface pattern actually reveals the spinel grain structures at the cladding/core interface, which vary drastically in a $25 \mu \mathrm{m}$ interval along the length of the fibers.

\subsubsection{The effects of firing atmosphere}

From various tests, it was observed that the firing in argon under current firing profiles seems to slow down the spinel grain growth compared to samples that were fired in air, judging from the smoothness of the cladding/core interfaces of the samples. In general, the samples fired in argon possessed smoother interfaces compared to the ones fired in air. This implies that the oxygen in air must have played an important role in the spinel grain growth process. 


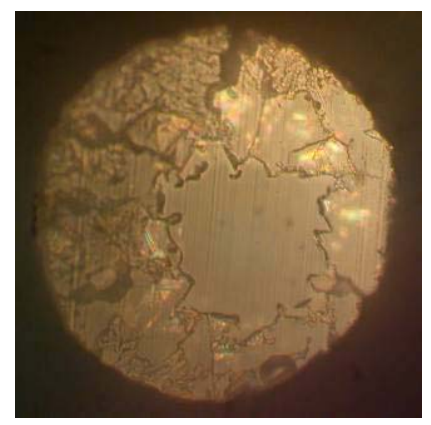

Figure 4-4. The cross section of a spinel clad sapphire fiber fired in air at $1650{ }^{\circ} \mathrm{C}\left(3000{ }^{\circ} \mathrm{F}\right)$ for 16 hours (Sample I.D. 6e).

\subsubsection{The effects of powder particle size}

The important role the particle size in the slurry coating plays in the smoothness of the cladding/core interface is a function of the dependence of the spinel grain growth on the reactivity of the powders, i.e., the finer the particle sizes of the powders in the slurry, the more reactive the powders, and the more rapid the spinel grain growth at the cladding/core interface.

Two samples were prepared using slurries with different powder particle sizes. In one sample, the slurry was prepared using fine powders; these powders were prepared by hand-grinding the original $\mathrm{MgO}$ and $\mathrm{MgAl}_{2} \mathrm{O}_{4}$ powders for 1 to 2 hours, followed by extensive ball milling for several days. In the other sample, the slurry is a mixture of the fine and the coarse powders; the coarse powders are the original powders with only about 30-minute ball milling. The exact portions of mixture between the fine and the coarse powders are not available; however, the ratio is believed to be close to 1:1. The two samples were both fired in argon at $1600^{\circ} \mathrm{C}\left(2912^{\circ} \mathrm{F}\right)$ for 1 hour. The exact firing profiles are illustrated in Appendix 4. The numbers of dips are comparable for both samples, in the range from about 20 to 30 dips. Figure 4-5 (a) and (b) show the cross sections of these samples. As shown in both figures, the cladding thickness is comparable in both cases, which implies that the amounts of powders in the coatings are comparable in both cases. Note that the cladding/core interface in Figure 4-5 (a) is much smoother than that in Figure 4-5 (b); it indicates that the firing profile is close to optimal for the sample shown in Figure 4-5 (a), which is prepared using the slurry with a mixture of coarse and fine powders. On the other hand, based on the assumption that the finer powders are more reactive, for the sample shown in Figure 4-5 (b), which is prepared using the fine powders only, the same firing profile is excessive and promotes reconfiguration of the cladding/core interface configuration caused by additional grain growth of the spinel grains. This results in an undesirable rough interface. 


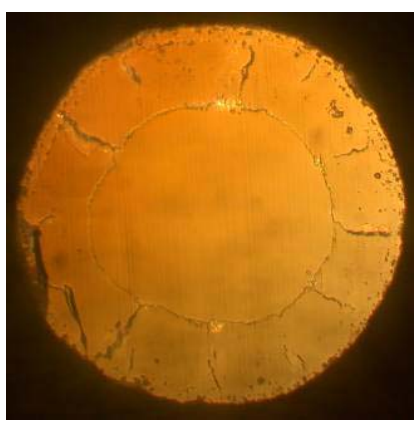

(a)

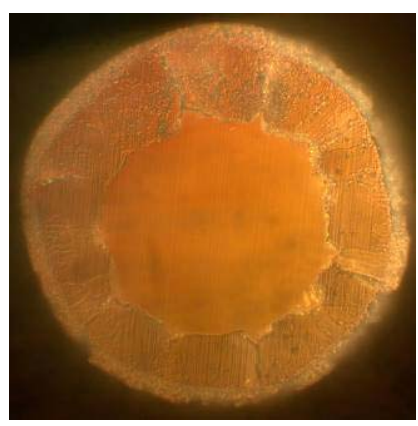

(b)

Figure 4-5 (a) The cross section of the clad fiber prepared using the slurry with a mixture of coarse and fine powders (Sample I.D. (20-35)o), and (b) the sample prepared using the slurry with the fine powders only (Sample I.D. 19(25)r). Both samples are fired in argon at $1600{ }^{\circ} \mathrm{C}\left(2912^{\circ} \mathrm{F}\right)$ for 1 hour with a $10{ }^{\circ} \mathrm{C} /$ minute heating rate and $5^{\circ} \mathrm{C} /$ minute cooling rate.

\subsubsection{The effects of the slurry thickness (viscosity)}

The preliminary results indicate that the ability to achieve a smooth core/cladding interface depends also on the slurry thickness (viscosity), which dictates the number of dips required to obtain a cladding with certain thickness. When all other experimental conditions (such as the powder particle size, firing profile, and the firing atmosphere) are the same and the cladding thickness is comparable for two samples, the thinner the slurry (i.e., the larger the number of dips required to obtain the cladding thickness), the more reactive the dipped coating surrounding the fiber, and the lesser degree of firing is needed. This assumption is best illustrated by the following results.

Efforts were made to reproduce the clad fiber (Sample I.D. 14(17)s) which had previously demonstrated promising light guiding properties, as depicted in Figure 4-3. The same batch of fine-powder slurry was used to prepare the new sample (Sample I.D. $30 \mathrm{t}$ ); however, this time the slurry was thinner than the one used to prepare sample 14(17)s, due to the additional amount of isopropyl alcohol added. The new sample was dipped 30 times while sample 14(17)s was dipped 14 times. The same firing profile of sample 14(17)s (as illustrated in Appendix 2) was used to fire the new sample, except that the argon flow rate for the new sample was greater (148 bubbles per minute, instead of the 84 bubbles per minute used for sample 14(17)s).

Figure 4-6(a) and (b) show the cross sections of sample 14(17)s and the new sample, respectively. As shown in Figure 4-6(b), the core/cladding interface of the new sample is much rougher than that of sample 14(17)s as shown in Figure 4-6(a). This implies that the firing profile optimal for achieving a smooth core/cladding interface in sample 14(17)s is excessive for the new sample, presumably due to its more reactive coating structure. 


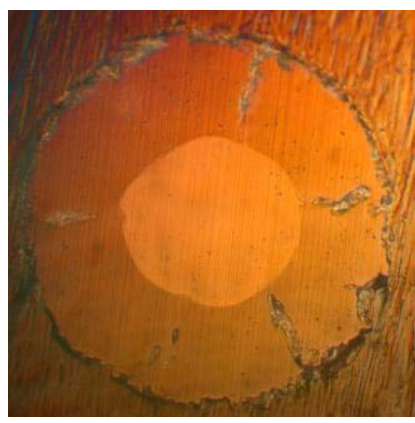

(a)

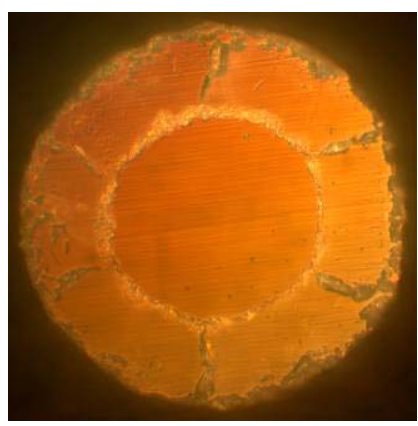

(b)

Figure 4-6 (a) the cross section of sample 14(17)s, and (b) that of the new sample (Sample I.D. 30t). Both samples are fired in argon for 30 minutes at $1600{ }^{\circ} \mathrm{C}\left(2912^{\circ} \mathrm{F}\right)$, and the heating and cooling rates are both $15^{\circ} \mathrm{C}\left(27^{\circ} \mathrm{F}\right)$ per minute. Sample $30 \mathrm{t}$ is prepared with a thinner slurry than that in the case of sample 14(17)s.

The suspected mechanism at work is that the coating structure created by the thinner slurry with a larger number of dips is denser in its packing. The powder particles move more freely in thinner slurry, and these particles can accommodate themselves more freely in successive dips to fill the voids existing on the surface of the coating, thus creating a denser coating structure, which is more reactive compared to a coating structure with a lot of voids, which is suspected to be the case when thicker slurry is used to prepare the sample.

\subsubsection{The high-temperature stability of the clad sapphire fiber}

Preliminary tests were performed to examine the high temperature stability of the fabricated fiber sample 14(17)s. The results indicate that the configuration of the core/cladding interface at the end face of the polished sample begins to change after the sample is heated to $1500{ }^{\circ} \mathrm{C}\left(2732^{\circ} \mathrm{F}\right)$ for about 1 day. This may imply a degradation of light guiding ability of the clad fiber after prolonged operation in this temperature range. Further investigation is needed to clarify whether this phenomenon is due to the surface effects at the end face or it is a bulk behavior through the whole fiber. More data is needed to establish a guideline for the operation at elevated temperatures.

The sample was first heated at $1300^{\circ} \mathrm{C}\left(2372{ }^{\circ} \mathrm{F}\right)$ for 22 hours, and the cross section of the sample was examined under an optical microscope. Then the sample was further heated to $1400{ }^{\circ} \mathrm{C}\left(2552^{\circ} \mathrm{F}\right)$ for 22 hours and to $1500{ }^{\circ} \mathrm{C}\left(2732^{\circ} \mathrm{F}\right)$ for another 22 hours, and after each heat treatment, the sample was examined again under an optical microscope. The results are shown in Figure 4-7. As shown in Figure 4-7(a), (b) and (c), there are no appreciable changes in the core/cladding interface after sequential heat treatments up to $1400^{\circ} \mathrm{C}\left(2552^{\circ} \mathrm{F}\right)$. However, as can be seen in Figure 4-7(d), significant changes occur at the interface after the heat treatment at $1500^{\circ} \mathrm{C}\left(2732{ }^{\circ} \mathrm{F}\right)$ for additional 22 hours; further investigation is needed to interpret the newly formed patterns of the interface shown in Figure 4-7(d), in particular to determine if the changes are present only on the end-face surface of the fiber or within the body of the fiber. 


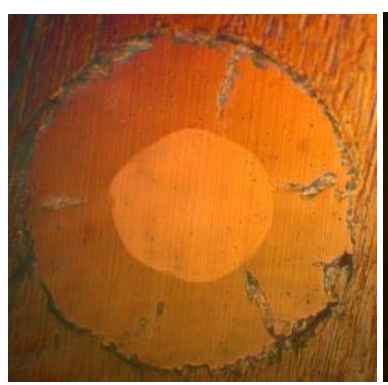

(a)

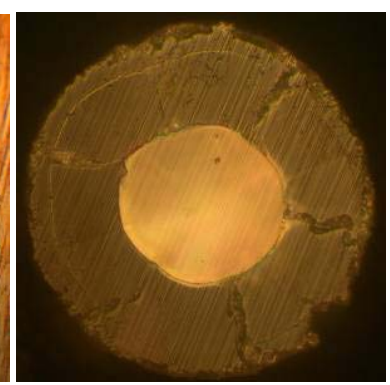

(b)

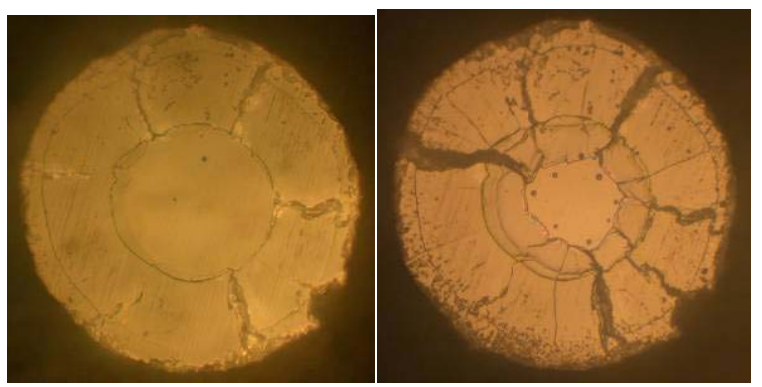

(c) (d)

Figure 4-7 (a) the original sample 14(17)s before the high temperature heat treatment, and (b) the same cross section end face after the treatment at $1300{ }^{\circ} \mathrm{C}\left(2372{ }^{\circ} \mathrm{F}\right)$ for 22 hours, and (c) after additional treatment at $1400{ }^{\circ} \mathrm{C}\left(2552{ }^{\circ} \mathrm{F}\right)$ for 22 hours, and (d) after additional treatment at $1500{ }^{\circ} \mathrm{C}$

$\left(2732^{\circ} \mathrm{F}\right)$ for 22 hours. The heating and cooling rates in all heat treatments are $10{ }^{\circ} \mathrm{C}$ per minute.

\subsubsection{EDS chemical analysis}

Some clad sapphire fiber samples were examined using energy dispersive X-ray spectroscopy (EDS) at the Chemistry Department of Virginia Tech, to determine the chemical compositions in the core and cladding of the fibers. The preliminary results (Figure 4-8) show that the atomic ratio between the magnesium and the aluminum in the cladding parts is 0.16 , which translates to a spinel composition of $0.23 \mathrm{MgO} \cdot 0.77 \mathrm{Al}_{2} \mathrm{O}_{3}$. This result in general lies within the ball park of the composition predicted from the $\mathrm{MgO}-\mathrm{Al}_{2} \mathrm{O}_{3}$ phase diagram (Figure 4-9) for the spinel formed at $1650{ }^{\circ} \mathrm{C}\left(3000{ }^{\circ} \mathrm{F}\right)$ from $\mathrm{Al}_{2} \mathrm{O}_{3}$, which is about $0.33 \mathrm{MgO} \cdot 0.67 \mathrm{Al}_{2} \mathrm{O}_{3}$. The reason for the discrepancy between the $0.23 \mathrm{MgO} \cdot 0.77 \mathrm{Al}_{2} \mathrm{O}_{3}$ in our case and the prediction $0.33 \mathrm{MgO} \cdot 0.67 \mathrm{Al}_{2} \mathrm{O}_{3}$ is not clear at this moment and is still under investigation. However, the fact that the cladding thickness ceases to increase at a certain point following prolonged firing supports the hypothesis that the $\mathrm{MgO}$ reacts with the sapphire until the spinel composition reaches the one on the phase boundary close to the point "c" in Figure 4-9, and the reaction continues until all the $\mathrm{MgO}$ in the dipped coating is depleted, and at that point the reaction ceases to proceed. ${ }^{19}$ This conclusion implies that the cladding thickness can be well controlled by the amount of the $\mathrm{MgO}$ in the dipped coating, i.e., the thickness of the dipped coating, and the firing temperature. However, this conclusion is supported by consideration of the equilibrium phase diagram of the magnesium aluminates spinel $\left(\mathrm{MgO}-\mathrm{Al}_{2} \mathrm{O}_{3}\right)$ system, which treats the system in equilibrium only. Additional testing will be required to evaluate the effect of kinetics on the high temperature stability of the spinel coatings.

One typical EDS output for the cladding section of one fiber is shown in Figure 4-8 below. The fiber analyzed in the plot is sample $2 \mathrm{~b}$. As shown in the figure, there are prominent peaks representing $\mathrm{Al}, \mathrm{Mg}, \mathrm{Au}, \mathrm{Fe}$, and $\mathrm{O}$. The first two elements and the $\mathrm{O}$ belong to the spinel; the Au comes from the Au coating evaporated on the surface of the fiber before the EDS examination; the Fe peaks occur from time to time due to the scattering of electrons from the sample holder. By comparing the areas under the $\mathrm{Al}$ and

\footnotetext{
${ }^{19}$ W. D. Kingery, H. K. Bowen and D.R. Uhlmann, “Introduction to Ceramics”, John Wiley \& Sons, 1976, p.133.
} 
$\mathrm{Mg}$ peaks and after taking into account the adjustment factors for $\mathrm{Al}$ and $\mathrm{Mg}$, the relative atomic ratio between $\mathrm{Mg}$ and $\mathrm{Al}$ is calculated to be $16 \%$. The complete data of sample $2 \mathrm{~b}$ and 8 other samples are listed in Appendix 6. For all samples, the core parts consist of almost pure alumina. A general expression of spinel composition can be written as (1$\mathrm{x}) \mathrm{MgO} \cdot \mathrm{xAl}_{2} \mathrm{O}_{3}$, and the atomic ratio between $\mathrm{Mg}$ and $\mathrm{Al}$ is $(1-\mathrm{x}) / 2 \mathrm{x}$. If the atomic ratio between $\mathrm{Mg}$ and $\mathrm{Al}$, i.e., $(1-\mathrm{x}) / 2 \mathrm{x}$, is 0.16 , then $\mathrm{x}$ is 0.76 . The $\mathrm{MgO}-\mathrm{Al}_{2} \mathrm{O}_{3}$ phase diagram is shown in Figure 4-9 below. As depicted by the vertical dotted line in the figure, the $\mathrm{x}$ value for the spinel formed from $\mathrm{Al}_{2} \mathrm{O}_{3}$ at $1650^{\circ} \mathrm{C}\left(3000^{\circ} \mathrm{F}\right)$, the firing temperature of sample $2 \mathrm{~b}$, is about 0.67 . As mentioned in the previous paragraph, the discrepancy of the value 0.67 predicted from the $\mathrm{MgO}-\mathrm{Al}_{2} \mathrm{O}_{3}$ phase diagram and the calculated value of 0.76 for sample $2 \mathrm{~b}$ is not clear at this moment.

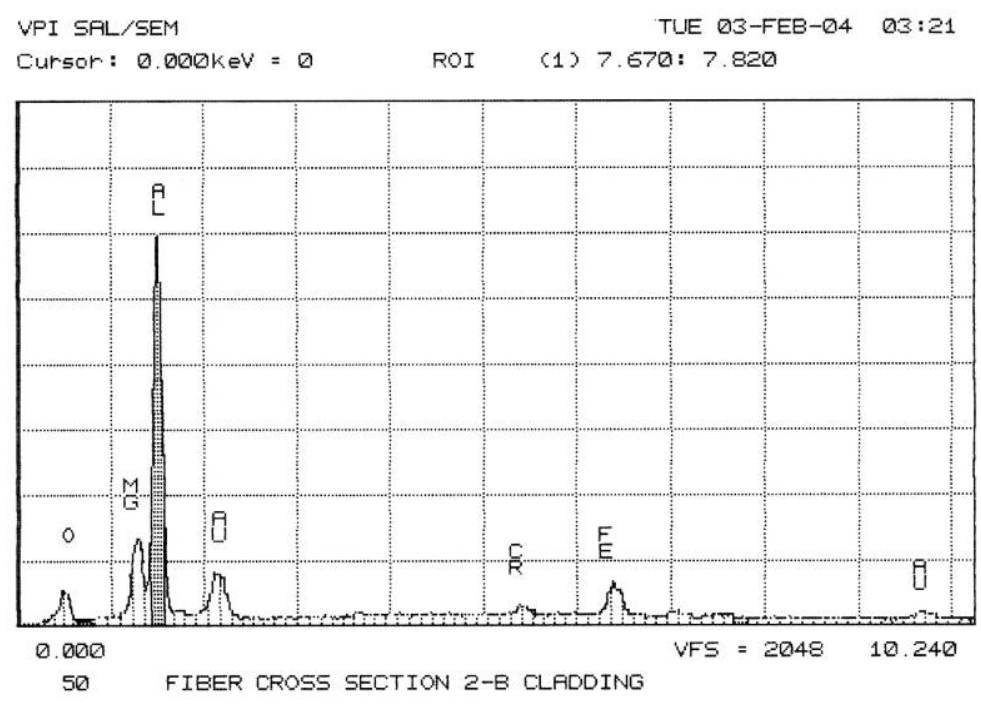

Figure 4-8. A typical EDS result at the cladding part of the fiber

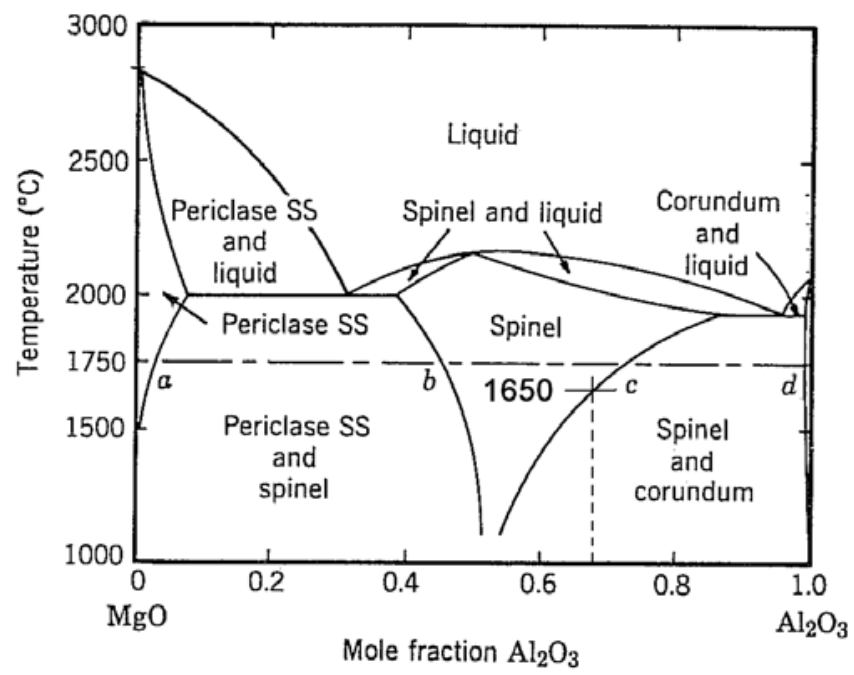

Figure 4-9. The ${\mathrm{MgO}-\mathrm{Al}_{2} \mathrm{O}_{3} \text { phase diagram }}^{20}$

${ }^{20}$ Ibid.

New Optical Sensor Suite for Ultrahigh Temperature Fossil Fuel Applications 


\subsubsection{Samples Prepared Using Nanopowders}

The goal to incorporate $\mathrm{MgO}$ nanopowders is to examine their effects on the spinel cladding properties. In general, in the sintering of ceramic body, the smaller the particle size, the larger is the driving force for sintering. This implies that powders with finer sizes can be sintered at lower temperatures and in a shorter period of time. However, the driving force for grain growth is also larger for finer grain powders. In the sintering of nanopowders, the competition between densification and grain growth greatly diminishes the chance of achieving dense ceramic body with nanometer-scale structures. ${ }^{21}$ Another goal for using nanopowders is to increase the mechanical strength of the converted spinel cladding.

In contrast to the sintering of consolidated ceramic body described above, in the current project, the slurry consisting of $\mathrm{MgO}$ and $\mathrm{MgAl}_{2} \mathrm{O}_{4}$ powders is dip-coated on the surface of a sapphire fiber. After drying and binder burnout, the ceramic powders in the coating pack less densely in comparison to, say, the packing density of a ceramic body consolidated using isostatic pressure. As the temperature increases, the $\mathrm{MgO}$ in the coating starts to diffuse into the sapphire, and reacts with $\mathrm{Al}_{2} \mathrm{O}_{3}$ by converting it to $\mathrm{MgAl}_{2} \mathrm{O}_{4}$. At the same time, the $\mathrm{Al}_{2} \mathrm{O}_{3}$ in the fiber also diffuses outwardly into the coating, although to a lesser extent. ${ }^{22}$ In addition, the sparse coating structure consolidates when the temperature rises.

\subsubsection{The First Attempt on Samples Prepared Using Nanopowders}

The first batch of nanopowder slurry was prepared using $\mathrm{MgO}$ nanopowders from Aldrich $\left(130 \mathrm{~m}^{2} / \mathrm{g}\right.$, ) and spinel powders from Alfa Aesar $\left(10 \mathrm{~m}^{2} / \mathrm{g}\right.$,). The constituents of the slurry are listed in Table 4-1. The slurry was ball milled after mixing. There was a delay of several days between the slurry preparation and the actual fiber dipping, so the weight $\%$ of 2-propanol at the moment of dipping may be smaller than the number shown in Table 4-1, due to the evaporation of 2-propanol during processing and storage. A fiber, sample $34 \mathrm{x}$, was coated using this slurry. The drying, binder burnout, and sintering profile are shown in Figure 4-10. Based on the assumption that nanopowders have a greater driving force for sintering, the dwelling time at $1600{ }^{\circ} \mathrm{C}\left(2,912{ }^{\circ} \mathrm{F}\right)$ was chosen to be much less than for sample 14(17)s. The polished cross section of sample $34 \mathrm{x}$ is shown in Figure 4-11. The fiber is still imbedded in the matrix used in polishing. It can be seen in Figure 4-11 that there is a significant amount of slurry coating surrounding the

21 I.-Wel Chen \& X.-H. Wang, "Sintering dense nanocrystalline ceramics without final-stage grain growth", Nature, Vol 404, 9 March 2000, p.168.

${ }^{22}$ Ronald C. Rossi and Richard M. Fulrath, "Epitaxial of Spinel by Reaction in the Solid Stae", Journal of the American Ceramic Society, v. 46, n. 3, March, 1963, pp.145-49. 
cladding not fully reacted. It is clear that the dwelling time at $1600^{\circ} \mathrm{C}\left(2,912^{\circ} \mathrm{F}\right)$ was not enough to fully react the entire slurry coating. The same sample was fired again at $1600^{\circ} \mathrm{C}\left(2,912^{\circ} \mathrm{F}\right)$ for a longer time to examine whether the residuals can further react and disappear. The polished cross section after the section firing is shown in Figure 4-12. Here the cladding thickness is much larger compared to that before the second firing, and the degree of residuals on the surface of cladding is greatly reduced. This indicates that during the second firing, the remaining residuals diffuse inward and reacted with the sapphire core. Furthermore, large cracks start to appear, presumably due to the huge increase in volume, i.e., $50 \%$, originating from the phase transformation between sapphire and spinel. ${ }^{23}$

The core/cladding interface here is rougher and more irregular. There are two possible causes for this interface roughness. First, the powder packing in the coating is not uniform, denser in some regions and sparser in others. A sparse and inhomogeneous powder packing results in a porous structure with large voids in the coating after consolidation. These voids can impede the local diffusion rate of molecules in the coating towards the sapphire fiber, thus reduce the reaction rate of the converted spinel cladding. After diffusion and interaction with the sapphire fiber, this tenuous structure in the coating regenerates itself at the core/cladding interface. Packing density and uniformity of the slurry coating also influence the amount of residuals not fully reacted after firing: the better the powder packing, the less are the residuals after firing. Another cause for the irregular interface might be the excessive grain growth of the spinel grains at core/cladding interface due to over-firing. In order to achieve the largest possible packing density and its uniformity in the coating, the powder sizes, their distributions, and the perfect dispersion of the powders in the slurry are critically important.

Table 4-1. Components of slurry batch Nano-1.0 (sample 34x)

\begin{tabular}{|c|c|c|c|}
\hline \multicolumn{4}{|c|}{$\begin{array}{c}\text { Sample Name: } 34 x \\
\text { Slurry Batch: Nano-1.0 }\end{array}$} \\
\hline & Component & Weight $(\mathrm{g})$ & "Weight $\%$ \\
\hline Solvent 1 & 2-propanol & 28.62 & 92.0 \\
\hline Solvent 2 & 1-ethoxy-2-peopanol & 2.04 & 6.6 \\
\hline Binder & PVP (1.3M m.w.) & 0.32 & 1.0 \\
\hline Plasticizer & Polyethylene glycol & 0.14 & 0.4 \\
\hline \multirow[t]{2}{*}{ Powders } & $\mathrm{MgO}$ (Aldrich, & 2.76 & \multirow{2}{*}{$\begin{array}{l}\text { Total: } \\
3.68\end{array}$} \\
\hline & $\begin{array}{l}\mathrm{MgAl}_{2} \mathrm{O}_{4}(\mathrm{Alfa} \\
\text { Aesar) }\end{array}$ & 0.92 & \\
\hline \multicolumn{4}{|c|}{ Solid Loading $=3.68 /(3.68+28.62)=11.4 \%$} \\
\hline
\end{tabular}

${ }^{23}$ Louis Navias, "Preparation and properties of spinel made by vapor transport and diffusion in the system $\mathrm{MgO}-\mathrm{Al}_{2} \mathrm{O}_{3}$ ", Journal of the American Ceramic Society, v. 44, n. 9, September 1961, pp.434-46. 


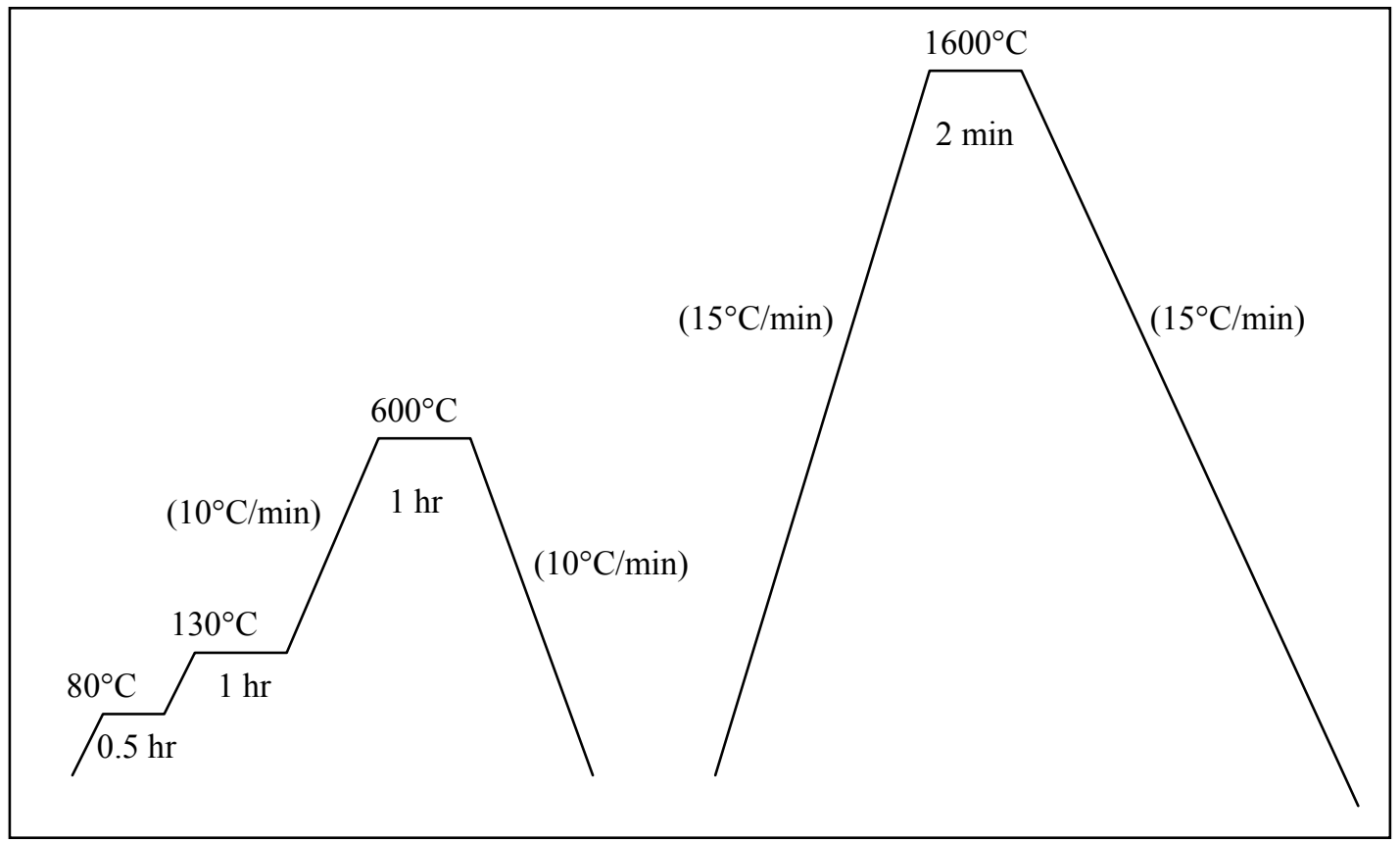

Figure 4-10. The firing profile of sample 34x

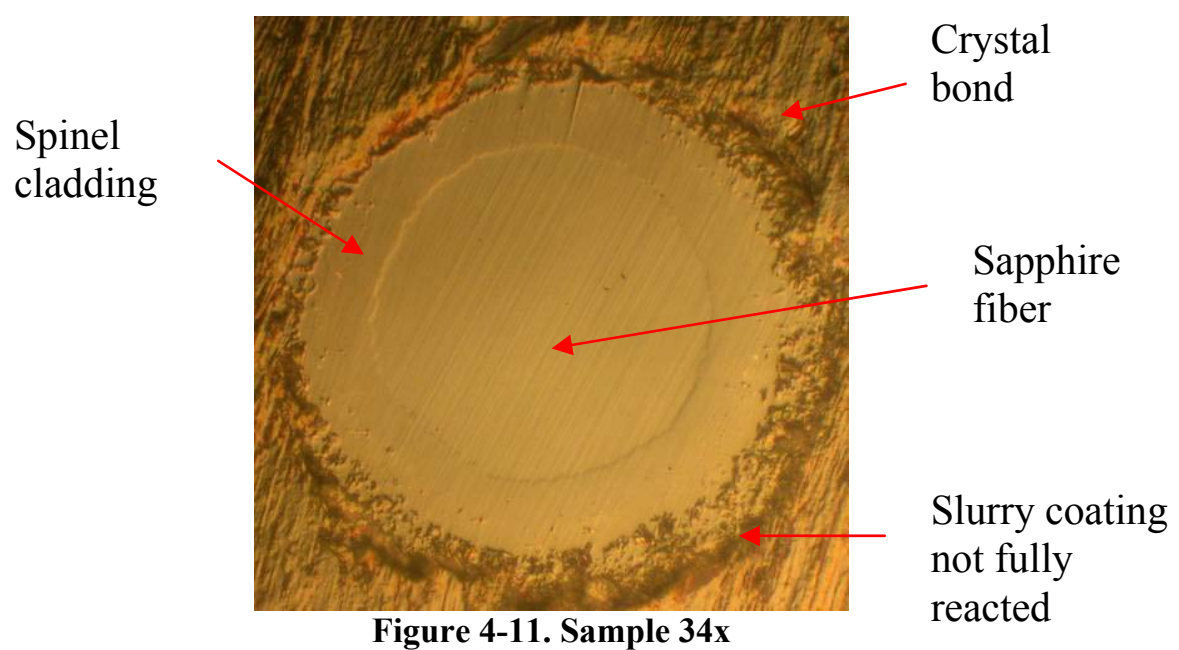




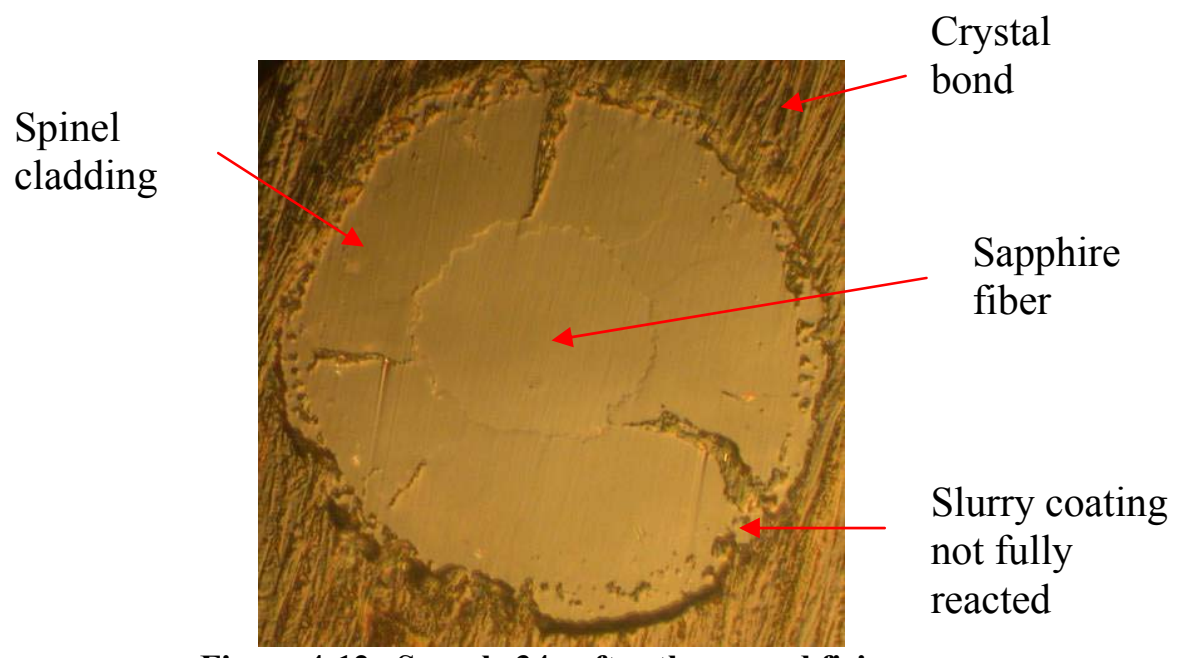

Figure 4-12. Sample 34x after the second firing

Sample 34x prepared using nanopowders has two rounds of firing at $1600^{\circ} \mathrm{C}$ for a total of 32 minutes, while the sample using $\mu \mathrm{m}$-range powders, i.e. sample 14(17)s, has one round of firing at $1600^{\circ} \mathrm{C}$ for 30 minutes. The drying and binder burnout stages are almost the same for these two samples. The firing profiles and slurry compositions of the two samples are quite similar. As shown in Figure 4-12, sample 14(17)s does not have as much residuals as in sample $34 \mathrm{x}$, which indicate that the powder packing in sample $34 \mathrm{x}$ may be less dense and lea uniform than those of sample 14(17)s. This tenuous coating structure in the coating prepared by nanopowders impedes the molecular diffusion into the fiber, thus increases the amount of residuals. It shows that the particle sizes and their distributions and the degree of powder dispersion in the nanopowder slurry are inferior. On the other hand, the core/interface of sample 14(17)s is smoother than that of sample $34 \mathrm{x}$, which points out that the single firing stage at $1600^{\circ} \mathrm{C}$ for 30 minutes is optimal to achieve a smooth interface for sample $14(17) \mathrm{s}$, while the two rounds of firing at $1600^{\circ} \mathrm{C}$ for a total of for 32 minutes is excessive for sample $34 \mathrm{x}$. It is also possible that the rough interface in sample $34 \mathrm{x}$ is a direct regeneration of its tenuous coating, as discussed the previous paragraph.

After the second firing, the slurry was ball milled again, and then it was stirred constantly in a beaker for until it was used to dip another fiber, sample 24z. Sample $24 \mathrm{z}$ was fired at $1600^{\circ} \mathrm{C}$ for 30 minutes, as illustrated in Figure 4-13. It has the same drying and binder burnout profiles as those of sample $34 \mathrm{x}$. The $30^{\circ} \mathrm{C}$ per minute rapid cooling from $1600^{\circ} \mathrm{C}$ is designed to reduce the chance of excessive grain growth at high temperatures, and the slow cooling from $1100^{\circ} \mathrm{C}$ to room temperature is for reducing the degree of cracking in the cladding. It was expected that this firing profile should creates samples similar to sample $34 \mathrm{x}$, in which there is only a negligible amount of residuals. The polished cross section of sample $24 \mathrm{z}$ after firing is shown in Figure 4-14. It shows huge amounts of residuals, much more than sample $34 \mathrm{x}$. The features in sample $34 \mathrm{x}$ can not be repeated 
using the same batch of slurry that has been stirred in a beaker for a period of 15 days. It is suspected that the degree of agglomeration of powders in the slurry may be slowly increasing, thus resulting in even poorer packing density after drying.

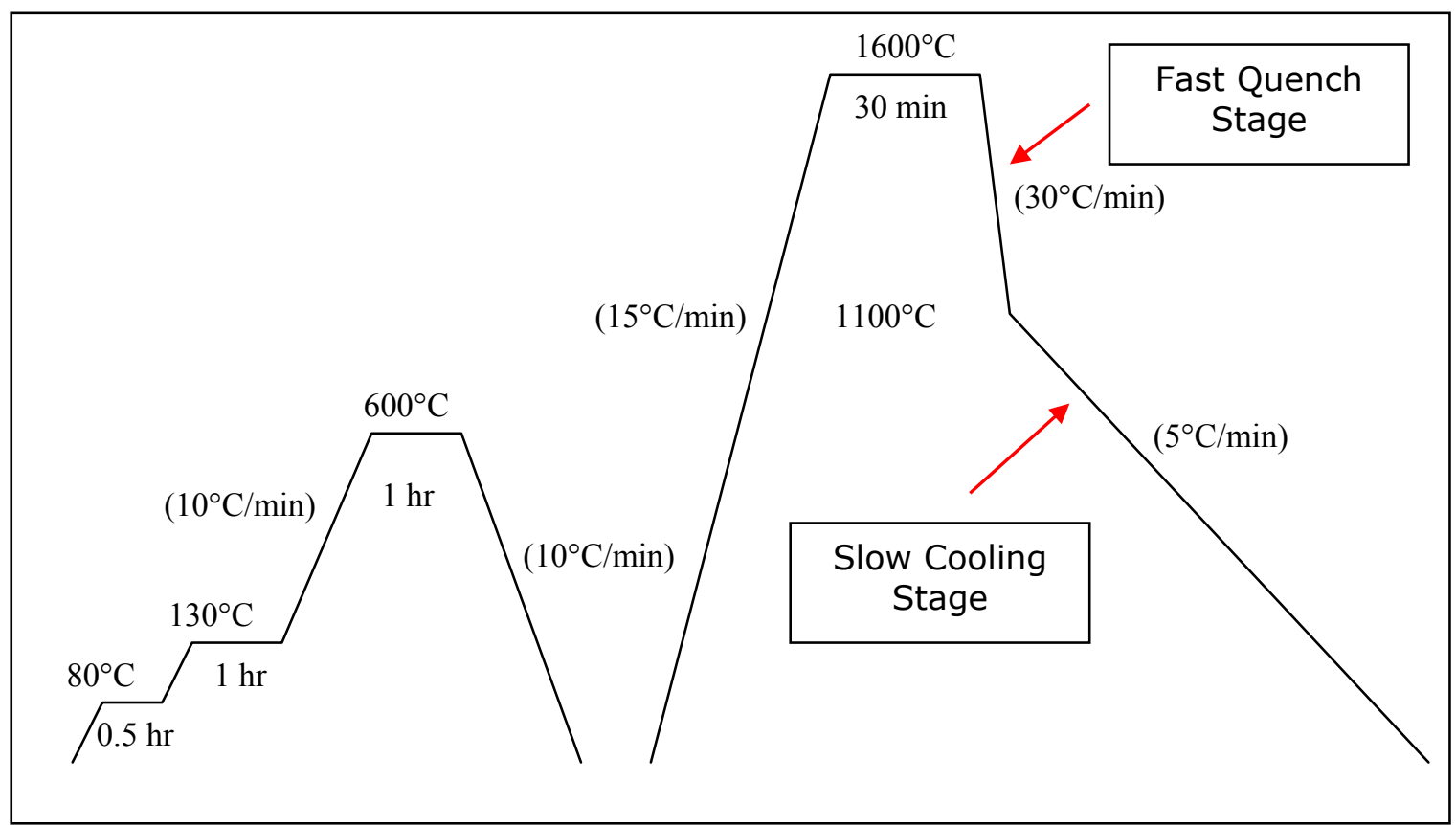

Figure 4-13. The firing profile of sample $24 \mathrm{z}$

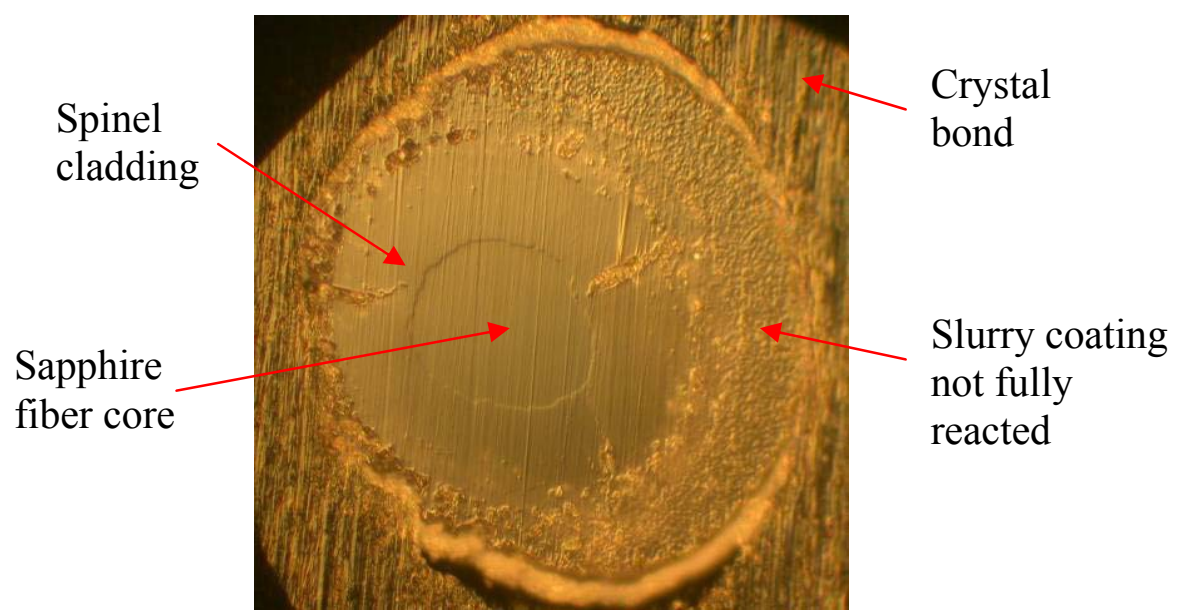

Figure 4-14. Sample 24z

To examine whether prolonged firing can further react the residuals, the same sample was fired again at $1600{ }^{\circ} \mathrm{C}\left(2,912^{\circ} \mathrm{F}\right)$ for additional 60 minutes. The heating and cooling rates are both $30^{\circ} \mathrm{C}$ per minute $\left(54^{\circ} \mathrm{F} / \mathrm{min}\right)$. The outcome of the second firing is shown in Figure 4-15. With a total of 90 minutes of sintering, the residuals left in sample $24 \mathrm{z}$ after the first firing seem mostly disappeared, but the core/cladding interface turns rougher. It is interesting to note that a detailed look at the core/cladding interface in Figure 4-15 reveals that the lower-right portion of the interface, where the cladding is the thickest and is formed most recently after the second firing, is smoother than the interface at the 
upper-left portion. This portion of the interface has the thinnest cladding and was formed earlier in the first firing, and the second firing ruins the relatively smooth interface at this portion of the interface. This evidence enforces the assumption that rough core/cladding interface is caused by over-firing.

By comparing sample 14(17)s (Figure 4-6 and Figure 4-7), 34x (Figure 4-11 and Figure 4-12), 24z (Figure 4-14 and Figure 4-15), in general, the degree of cracking in the samples prepared by nanopowders is less severe than that in the sample prepare by $\mu \mathrm{m}$ sized powders.

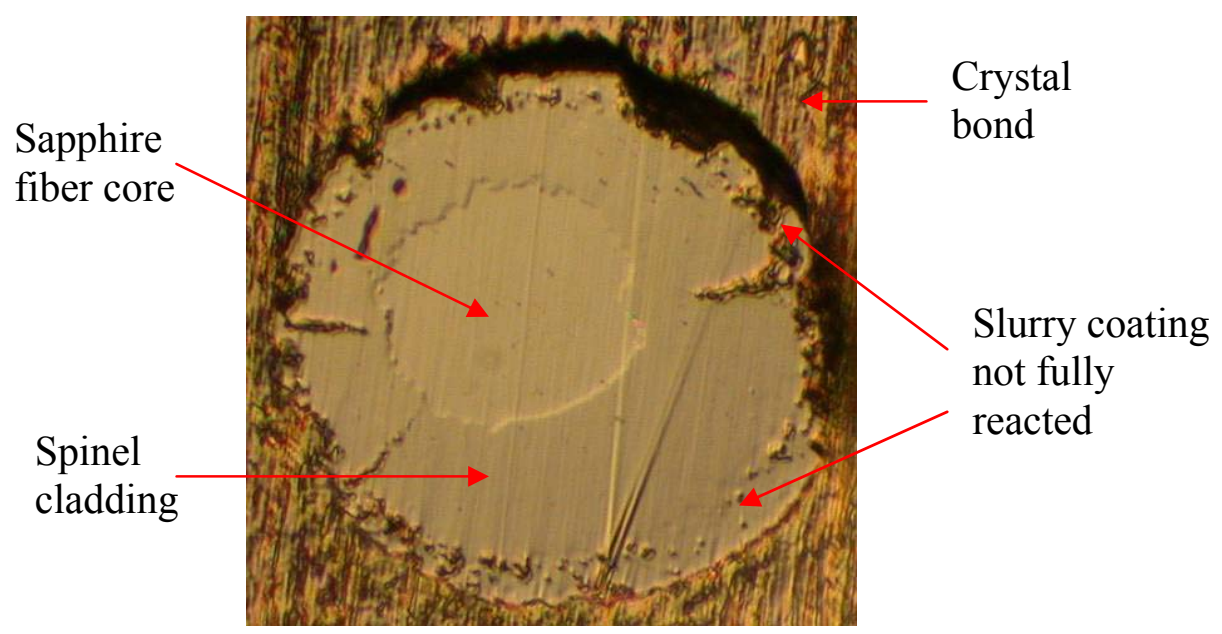

Figure 4-15. Sample $24 \mathrm{z}$ after the second firing

\subsubsection{The Second Attempt on Samples Prepared Using Nanopowders}

A new type of nanopowders was used to prepare the following samples. The powders, NanoActive MgO Plus, from NanoScale have very large surface areas of $600 \mathrm{~m}^{2} / \mathrm{g}$ and a very small crystalline size of $4 \mathrm{~nm}$. However, the mean aggregate size is large, $12 \mu \mathrm{m}$, and there are visible carbon black contaminate particles in the powders. Both the $\mathrm{MgO}$ powders from NanoScale and the spinel powders from Alfa-Aesar were hand-ground for before use. The constituents of the slurry are listed in Table 4-2. The -solvents, binder, and plasticizer contents in this slurry batch, Nano-2.0, are very similar to those of sample $34 \mathrm{x}$ (Table $4-1$ ), but the solid loading here is much larger, $19.3 \%$ verses $11.3 \%$. The slurry was ball milled before being used to dip a fiber. Figure 4-16 illustrates the firing profile of this sample.

The heating rate to $1600{ }^{\circ} \mathrm{C}\left(2,912^{\circ} \mathrm{F}\right)$ was changed from $15^{\circ} \mathrm{C}$ per minute $\left(27^{\circ} \mathrm{F} / \mathrm{min}\right)$ to $10^{\circ} \mathrm{C}$ per minute $\left(18^{\circ} \mathrm{F} / \mathrm{min}\right)$. The cross sections after fracture and after polishing and annealed are shown in Figure 4-17 and Figure 4-18 respectively. The sample was annealed at $1200^{\circ} \mathrm{C}\left(2,192^{\circ} \mathrm{F}\right)$ for 3 hours. Sample $30 \mathrm{G}$ has a relatively smooth core/cladding interface and a relatively small amount of residuals not fully reacted. This hints that the heating profile illustrated in Figure 4-16 is close to what is needed to both fully react the slurry coating and to create a smooth core/cladding interface. But the clad 
Table 4-2. Components of slurry batch Nano-2.0 (sample 30G)

\begin{tabular}{|l|l|c|c|}
\hline \multicolumn{4}{|c|}{$\begin{array}{c}\text { Sample Name: 30G } \\
\text { Slurry Batch: Nano-2.0 }\end{array}$} \\
\hline \hline & \multicolumn{1}{|c|}{ Components } & $\begin{array}{c}\text { Weight } \\
(\mathrm{g})\end{array}$ & Weight \% \\
\hline Solvent 1 & 2-propanol & 24.94 & 91.0 \\
\hline Solvent 2 & 1-ethoxy-2-peopanol & 2.02 & 7.4 \\
\hline Binder & PVP (1.3M m.w.) & 0.29 & 1.1 \\
\hline Plasticizer & Polyethylene glycol & 0.14 & 0.5 \\
\hline Powders & $\begin{array}{l}\text { MgO (NanoScale } \\
\text { crystalline) }\end{array}$ & 4.48 & Total: \\
\cline { 2 - 3 } & $\begin{array}{l}\text { MgAl } \mathrm{O}_{4}(\mathrm{Alfa} \\
\text { Aesar) }\end{array}$ & 1.46 \\
\hline \hline \multicolumn{3}{|l|}{ Solid Loading $=5.96 /(5.96+24.94)=19.3 \%$} \\
\hline
\end{tabular}

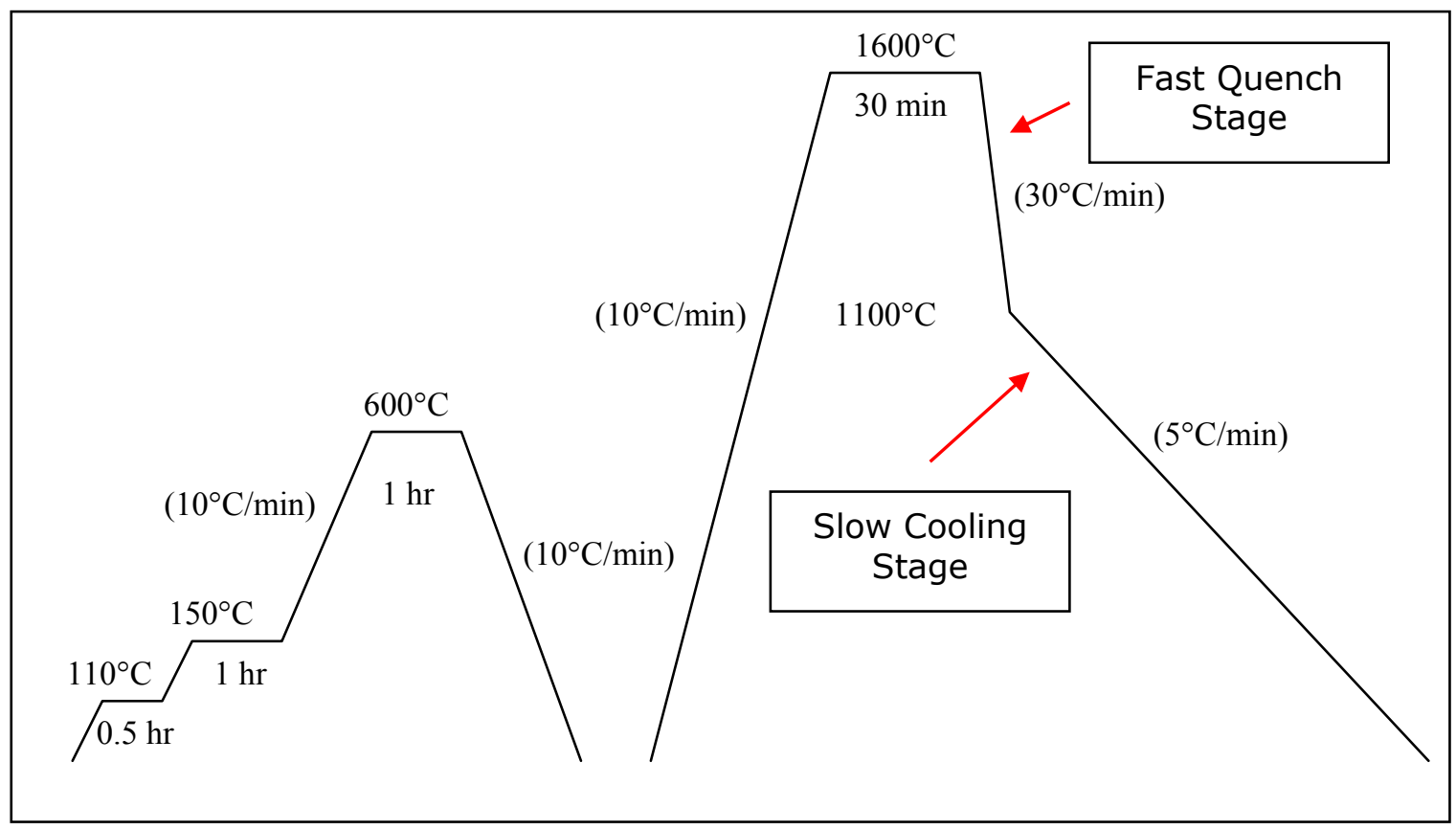

Figure 4-16. The firing profile of sample 30G

fiber is quite fragile, and its surface on its sides is rough and uneven as shown in Figure 4-19. From now on the surfaces as well as the cross sections morphologies are monitored. This roughness hints that the amounts of constituents in the slurry as tabulated in Table 4-2 are not optimized. Most likely the amount of binder is too little to hold the coating together in one uniform piece after drying. The fragility is caused by the significant amount of cracking in the cladding. 


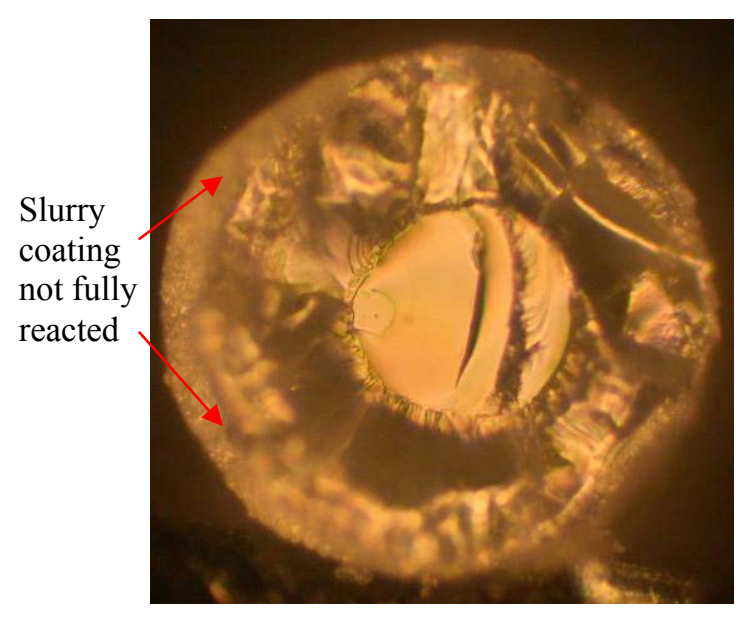

Figure 4-17. The fractured surface of cross section of sample 30G

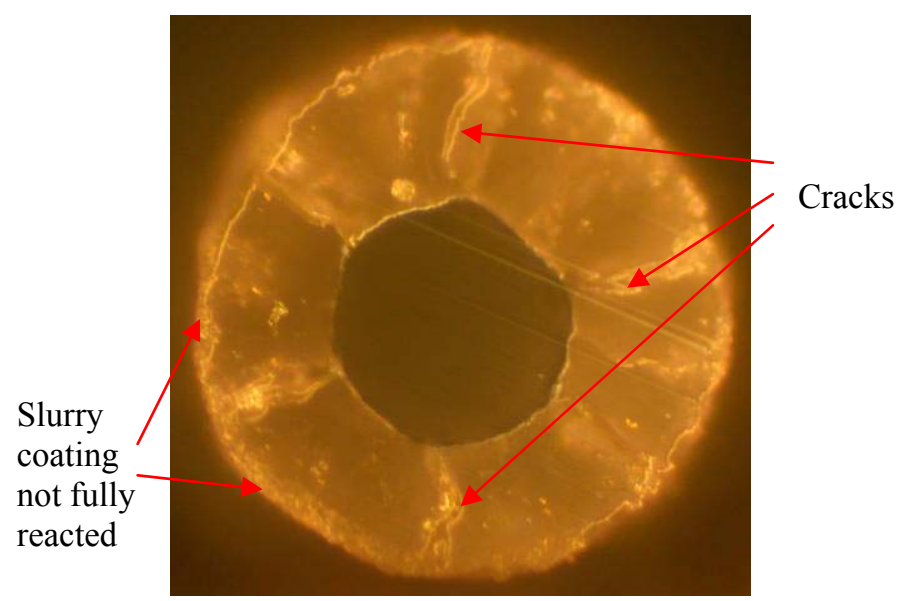

Figure 4-18. The polished and annealed sample 30G

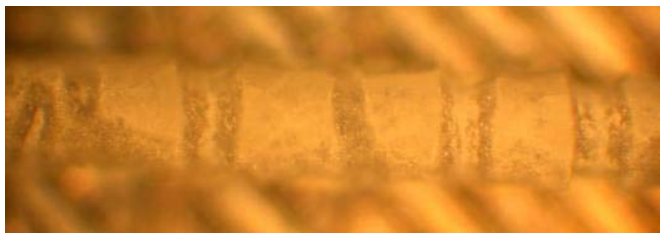

Figure 4-19. The surface on the sides of sample 30G 
Table 4-3. Components of slurry batch MgO-1.0 (sample 10H-4)

\begin{tabular}{|l|l|c|c|}
\hline \multicolumn{4}{|c|}{$\begin{array}{c}\text { Sample Name: 10H-4 } \\
\text { Slurry Batch: MgO-1.0 }\end{array}$} \\
\hline \hline Solvent 1 & \multicolumn{1}{|c|}{ Components } & $\begin{array}{c}\text { Weight } \\
(\mathrm{g})\end{array}$ & Weight \% \\
\hline Solvent 2 & 2-propanol & 12.7 & 91.1 \\
\hline Binder & 1-ethoxy-2-peopanol & 1.03 & 7.4 \\
\hline Plasticizer & PVP (1.3M m.w.) & 0.15 & 1.1 \\
\hline Powders & Polyethylene glycol & 0.06 & 0.4 \\
\hline & $\begin{array}{l}\text { MgO (NanoScale, } \\
\text { crystalline) }\end{array}$ & 2.47 & $\begin{array}{c}\text { Total: } \\
2.47\end{array}$ \\
\hline \multicolumn{4}{|l|}{ Solid Loading = 2.47/(2.47+12.7)=16.3\% } \\
\hline
\end{tabular}

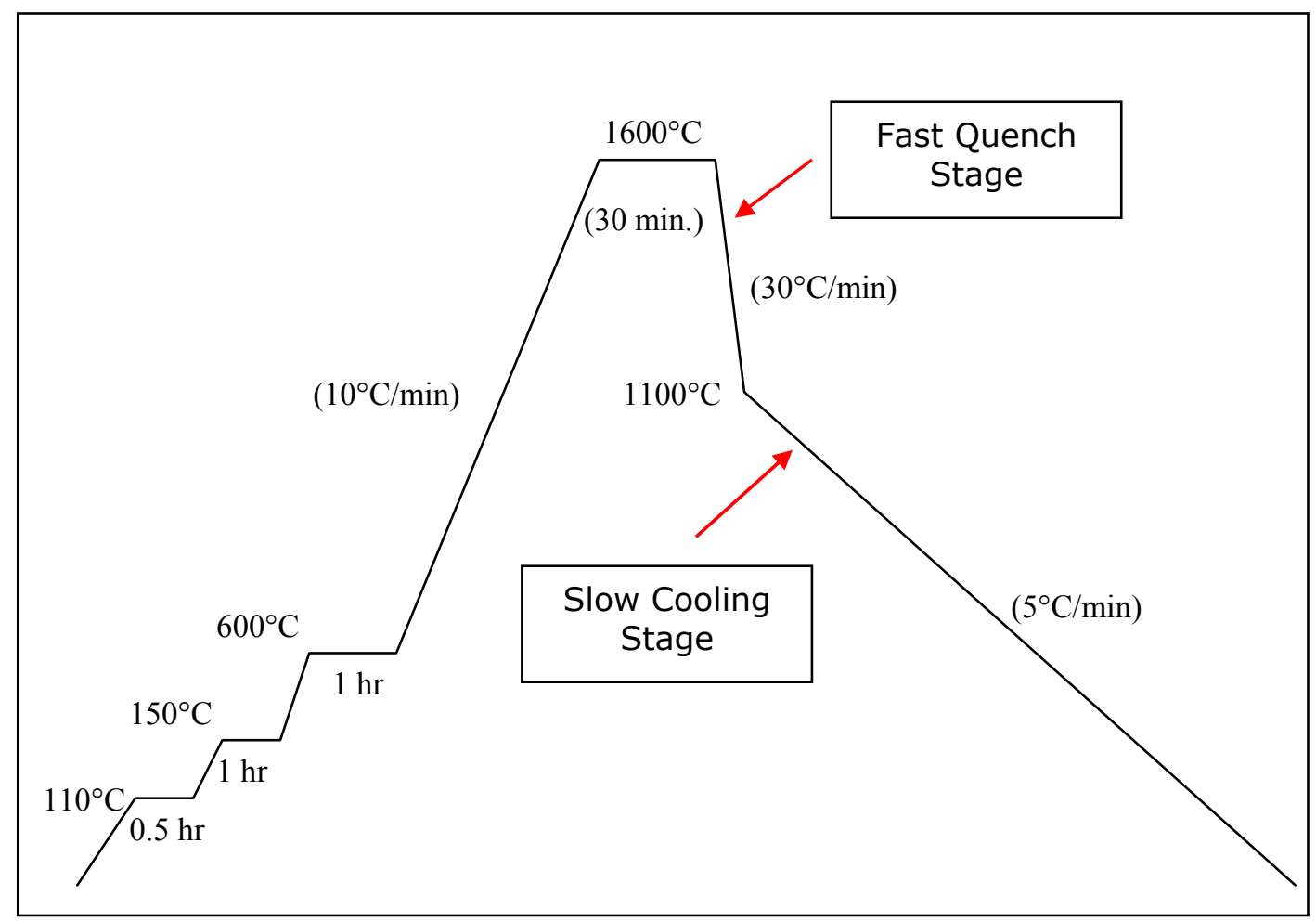

Figure 4-20. The firing profile of sample 10H-4 


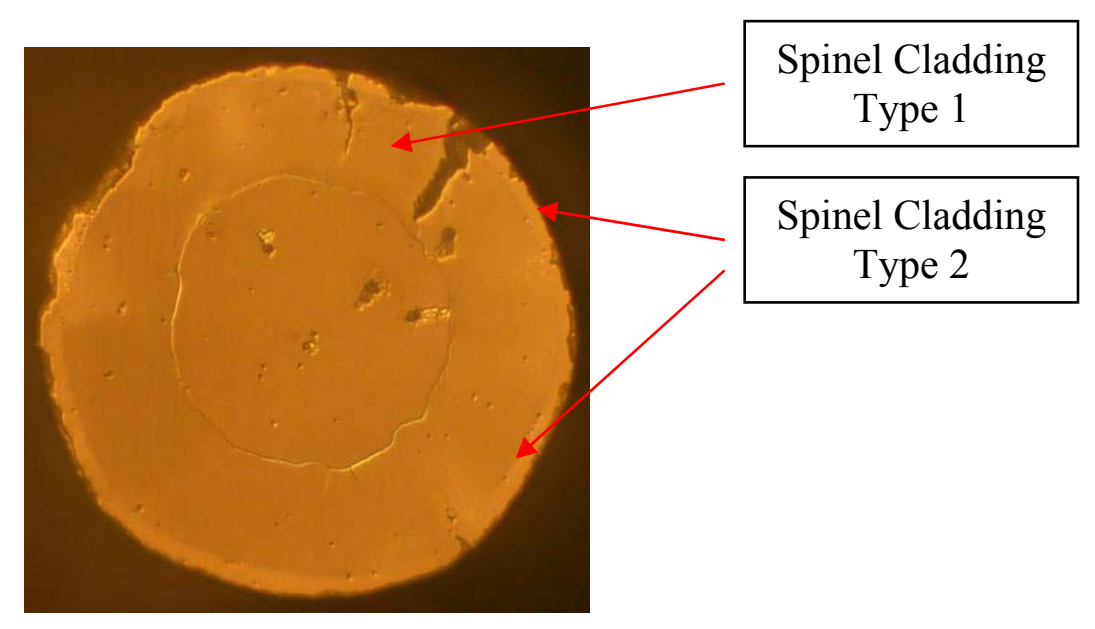

Figure 4-21. The polished and annealed cross section of sample 10H-4

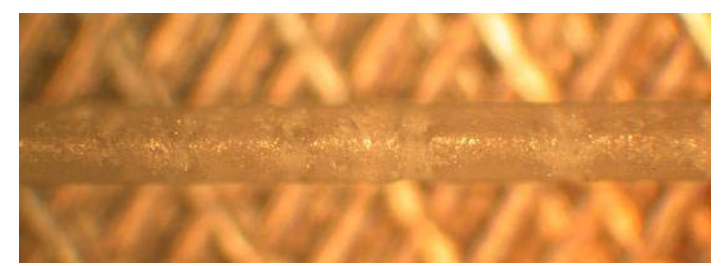

Figure 4-22. The surface on the sides of sample $10 \mathrm{H}-4$

All the slurries used to dip the fibers up till now are prepared using a weight ratio of 3 to 1 between $\mathrm{MgO}$ and spinel powders. The spinel powders serve as nucleation centers to assist the nucleation of spinel grains when $\mathrm{MgO}$ interacts with the $\mathrm{Al}_{2} \mathrm{O}_{3}$ of sapphire fibers. It is suspected that, during firing, while the $\mathrm{MgO}$ powders in the coating diffuse towards the sapphire fiber, the spinel powders in the coating do not have as large a driving force to diffuse to the sapphire fiber as do the $\mathrm{MgO}$ powders, consequently resulting in residuals on fibers after firing. If the only role of spinel powders is to serve as nucleation centers, the powders should be required only at the coating/fiber interface, not within the bulk of the coating, since as long as the spinel grains are nucleated at the core/cladding interface, they can grow towards the center of fiber like columns without assistance of spinel powders in the bulk of the coating. To verify this assumption, an experiment was designed to use two batches of slurries to dip one single fiber. The first batch is the regular slurry consisting of both $\mathrm{MgO}$ and spinel powders, and the second batch has $\mathrm{MgO}$ powders only. The fiber was dipped using the first batch first, and then followed by the second batch. Only coating close to the core/cladding interface has spinel, not in the bulk of the coating. The constituents of the $\mathrm{MgO}$ only slurry is listed in Table 4-3. The MgO powders were hand ground before mixing. The regular batch with both $\mathrm{MgO}$ and spinel powders is the same batch used to dip sample 30G, and the constituents of this batch, Nano-2.0, are listed in Table 4-2. The fiber, sample 10H-4, was dipped first with the regular slurry, and followed by the $\mathrm{MgO}$ only slurry. The firing profile is illustrated in Figure 4-20. An attempt was made to combine the drying and binder burnout stage with the high temperature sintering stage. One concern is that 
usually binder needs oxygen for its burnout, and it is not sure at this moment whether PVP, the binder used in our experiments, can be successfully burned out. The polished and annealed cross section of sample 10H-4 is shown in Figure 4-21. The surfaces of the clad fiber on its sides are shown in Figure 4-22. As revealed by Figure 4-21, first, there are almost no residuals left on the surfaces of the clad fiber, and second, the cracking in the cladding is limited to only one large crack and one small crack in the upper-right portion of the cladding, and third, although the core/cladding interface is not perfectly concentric, it is quite clean and smooth. Furthermore, there are two types of distinctive cladding structures; the first type, Type 1 shown in Figure 4-21, occupies more than 90\% of the cladding, and the second type, Type 1 in Figure 4-21, locates only at the surface of cladding, and is only $1 / 8$ of the thickness of the first type. It is believed that the Type 1 cladding is formed by the inward diffusion of $\mathrm{MgO}$ from coating into fiber, while Type 2 cladding is formed by the outward diffusion of $\mathrm{Al}_{2} \mathrm{O}_{3}$ from fiber towards coating. It seems that the incorporation of all $\mathrm{MgO}$ coating can minimize the amount of residuals and the cracking in the cladding. The non-concentricity of the core/cladding interface may be caused by the non-uniformity of the $\mathrm{MgO}$ plus spinel coating thickness around the fiber or the non-uniformity of the powder packing density in the slurry.

The surfaces of sample $10 \mathrm{H}-4$ on its sides are still rough, but they are much better than those of sample $30 \mathrm{G}$ shown in Figure 4-19. It is to be noted that one sign of an excessive amount of residuals is that the surface of the clad fiber looks white, in contrast to a fiber fully reacted that looks translucent. These results show promise of using this 2-batch dipping method to reduce residuals, cracking, and promote a smooth core/cladding interface.

\subsubsection{Summary of the results of the samples prepared using nanopowders}

1. With similar slurry compositions and firing profiles, the samples prepared by nanopowders have more residuals on the fiber after firing, and the core/cladding interfaces of these samples are rougher and more irregular than those of the samples prepared using $\mu$ m-range powders. It is suspected that the slurry composition, which is optimal for the $\mu \mathrm{m}$-range powders, is not feasible for nanopowders.

2. In order to achieve the largest possible packing density and its uniformity in the coating, the powder sizes, their distributions, and the perfect dispersion of the powders in slurry are critically important.

3. The nanopowder slurry prepared using the existing composition is not stable as a function of time, even when the slurry is stirred constantly after preparation. The samples prepared using aged slurry have a lot of residuals not fully reacted after firing. It is suspected that the degree of agglomeration of powders in the slurry may be slowly increasing, thus resulting in even poorer packing density after drying.

4. Rough core/cladding interface may be caused by either over-firing or sparse and tenuous coating structures. 
5. The degree of cracking in the samples prepared by nanopowders seems to be less severe than that in the sample prepare by $\mu \mathrm{m}$-sized powders.

6. Judging from the rough and uneven surface morphology in some of the samples, the current amount of binder may not be enough to hold the coating together in one uniform piece after drying.

7. Slurry coating consisting of an undercoat made of $\mathrm{MgO}$ and spinel to help spinel nucleation at the core/cladding interface, and of $\mathrm{MgO}$ only topcoat, shown promise of reducing residuals, cracking, and promote a smooth core/cladding interface after firing.

\subsubsection{The use of powders with different sizes to improve packing density}

When spheres of different sizes are mixed together, the packing density depends on the size distributions of the spheres and their weight fractions. The greatest possible packing density of spheres with the optimized size distributions and weight fractions can be calculated according to the model of mixed solid spheres. The results are shown in Table 4-4. With spheres of only one size, the calculated packing density is $60.5 \%$. The introduction of spheres 8.3 times smaller with a weight fraction of $27.4 \%$ can increase the packing density to $84.8 \%$. The further introduction of spheres 5.5 times smaller than the ones just introduced, with a weight fraction of $20.9 \%$, can boost the packing density further to $95.2 \%$. Attempts were made to blend 4 types of powders, $\mathrm{MgO}$ (EM Science), spinel (Baikalox), spinel (Alfa Aesar), and $\mathrm{MgO}$ (Aldrich) in the slurry to improve the clad fiber properties. Different techniques have been used to measure the particle sizes of the above powders. The results will be discussed later.

Table 4-4. Packing density of mixed spheres of different $\operatorname{sizes}^{24}$

\begin{tabular}{|c|c|c|c|c|c|c|}
\hline \multirow[b]{2}{*}{ : } & \multicolumn{4}{|c|}{$\begin{array}{c}\text { Diameter (cm) } \\
\text { (weight fraction of spheres) }\end{array}$} & \multicolumn{2}{|c|}{ Packing Density (\%) } \\
\hline & 1.28 & 0.155 & 0.028 & 0.004 & Calculated & Experimental \\
\hline & 1.000 & - & - & - & 60.5 & 58.0 \\
\hline & 0.726 & 0.274 & - & 一 & 84.8 & 80.0 \\
\hline & 0.647 & 0.244 & 0.109 & - & 95.2 & 89.8 \\
\hline & 0.607 & 0.230 & 0.102 & 0.061 & 97.5 & 95.1 \\
\hline
\end{tabular}

Source: R.K. McGeary, J. Am. Ceram. Soc. 44(10), 513-522 (1961).

Size ratio 320/39/7/1.

\subsubsection{The Implementation of Two Stage Milling: Dispersion Milling and Binder Milling}

For all the slurries prepared to this point, the solvents, binder, plasticizer, and the powders were all mixed and milled simultaneously. It was discovered from the literature

${ }^{24}$ R. K. Mcgeary, J. Am. Ceram. Soc., 44(10), 1962. p.513-522. 
that it is difficult to achieve the highest degree of powder dispersion in the slurry this way. The slurry should be prepared in two stages: dispersion milling stage and binder

milling stage. ${ }^{25}$ In dispersion milling only the solvent(s), powders, and a

dispersant/deflocculant are added, but not binder. Binder competes with dispersant in occupying the surface sites of powders, thus reducing the effectiveness of the dispersants added. Only after thorough powder dispersion after dispersion milling is binder added in order to hold the green body together. The two-step milling will be implemented in all slurry preparation from now on.

\subsubsection{Use of polyethylene glycol as the dispersant}

In all the slurries prepared so far, polyethylene glycol is identified as plasticizer, which makes the coating plastic and deformable after drying by shortening or partially dissolving the polymer chains of the binder. The current binder used in our experiments is PVP with a molecular weight of 1,300,000. From Table 4-5, polyethylene glycol can also be used as dispersant. With the introduction of dispersion milling stage, a proper dispersant is needed in our slurry system. According to Table 4-5, polyethylene glycol will be used as the dispersant in the following samples.

\begin{tabular}{|c|c|}
\hline \multicolumn{2}{|c|}{ Some Reported Dispersants/Deflocculants used for Tape Casting } \\
\hline $\begin{array}{l}\text { Polyisobutylene } 48 \\
\text { Linoleic acid } \\
\text { Oleic acid } \\
\text { Citric acid } \\
\text { Stearic acid } \\
\text { Lanolin fatty acids } 47 \\
\text { Salts of polyacrylic acids } \\
\text { Salts of methacrylic acids } \\
\text { Blown menhaden fish oil } \\
\text { Corn oil } \\
\text { Safflower oil } \\
\text { Linseed oil } \\
\text { Glycerol trioleate } \\
\text { Svnthetic waxy esters }\end{array}$ & $\begin{array}{l}\text { pH adjustments } \\
\text { Sodium silicate } \\
\text { Dibutyl amine } \\
\text { Substituted imidazolines }{ }^{47} \\
\text { Sulfanates } \\
\text { Aliphatic hydrocarbons } 47 \\
\text { 2-amino-2-methyl-1-propanol }{ }^{47} \\
\text { Polyethylene glycol }{ }^{47} \\
\text { Polyvinyl butyral } \\
\text { Sodium sulfosuccinates }^{47} \\
\text { Ethoxylate }^{47} \\
\text { Phosphate ester } \\
\text { Glycerol tristearate } \\
\text { Many proprietary chemicals }\end{array}$ \\
\hline
\end{tabular}

\subsubsection{Samples prepared using four types of powders and polyethylene glycol as dispersant}

Four types of powders were used to prepare the slurry: $\mathrm{MgO}$ (EM Science), spinel (Baikalox), spinel (Alfa Aesar), and MgO (Aldrich). All powders, except MgO from

\footnotetext{
${ }^{25}$ Richard E. Mistler and Eric R. Twiname, "Tape Casting, Theory and Practice”, The American Ceramic Society, Westerville, OH, 2000, p.27.
} 
Aldrich, were hand-ground in 2-propanol followed by ball milling. The dispersant, polyethylene glycol, was mixed with the solvent, 2-propanol to prevent solvent evaporation. The finest powder, $\mathrm{MgO}$ from Aldrich, were first added into the beaker, followed by the spinel from Alfa Aesar, the spinel from Baikalox, and finally by the $\mathrm{MgO}$ from EM Science. Between each powder addition step, the bottle is hand-shaken to disperse the powders in the slurry. The dispersant slurry was then ball milled in the bottle.

The PVP binder can have different molecular weight depending on its chain length. Besides the 1,300,000 molecular weight PVP that has been used so far, the 10,000 molecular weight PVP was also available for evaluation at this moment. After dispersion milling binders were added to the slurry in the sequence listed in Table 4-6. The viscosity of slurry was significantly smaller after $0.33 \mathrm{~g} 10 \mathrm{k}$ binder was added compared to the slurries where the $1.3 \mathrm{~m}$ binder was used. More binders were added to increase slurry viscosity. The reason for separating the addition of binders is to prevent them from sticking together as a lump in the slurry. The rationale for increasing the viscosity is to increase the coating thickness after each dipping, thus reducing the number of dips needed to obtain a certain spinel coating thickness; for slurry in the Newtonian region, the coating thickness from each dipping is proportional to both slurry viscosity and dipping speed. ${ }^{26}$

\begin{tabular}{|c|c|c|c|c|}
\hline \multirow[b]{2}{*}{$\begin{array}{l}\text { Sequence } \\
\text { of } \\
\text { addition }\end{array}$} & \multirow{2}{*}{$\begin{array}{l}\text { Table 4-6. } \\
\text { binder }\end{array}$} & \multicolumn{3}{|c|}{ Binder addition sequence for sample $80 Q$} \\
\hline & & $\begin{array}{l}\text { Molecular } \\
\text { weight }(\mathrm{g})\end{array}$ & $\begin{array}{l}\text { Weigh } \\
\text { added } \\
(\mathrm{g})\end{array}$ & $\begin{array}{l}\text { Ball milling time after } \\
\text { binder addition } \\
\text { (hour) }\end{array}$ \\
\hline 1 & PVP & $10 \mathrm{k}$ & 0.33 & 1.3 \\
\hline 2 & PVP & $10 \mathrm{k}$ & 1.84 & 1.25 \\
\hline 3 & PVP & $10 \mathrm{k}$ & 0.10 & 1.0 \\
\hline & & $1.3 \mathrm{~m}$ & 2.40 & \\
\hline
\end{tabular}

Because of the different binder burnout temperatures for binders with different molecular weights, it is common to mix these binders together to reduce the impact of binder burnout on the green body structure. The final constituents of the slurry are shown in Table 4-7. Polyethylene glycol is used here as both dispersant and plasticizer. The weight fraction of each powder was determined roughly according to the mixed sphere models illustrated in Table 4-4. The reason for not being able to determine the exact weight fractions at this moment is that the accurate particle sizes of each powder have not been independently measured. Furthermore, the weight ratio between $\mathrm{MgO}$ and spinel powders has to be 3 to 1 , and this limits the ability to exactly follow the guideline listed in Table 4-4. Even with the huge amount of binder added, $6.3 \mathrm{w} . \% 10 \mathrm{k}$ binder and $6.9 \mathrm{w} . \% 1.3 \mathrm{~m}$ binder, the viscosity was still relatively small.

\footnotetext{
${ }^{26}$ Huang, Yuhong; Zheng, Haixing; and Forsyth, Ian, "Application of Functional Ceramic and Hybrid Nanostructure Coating Fabrication via Sol-Gel Processes," Fine, Ultrafine and Nano Powders 2000, Oct. 29-31, 2000, Montreal, Canada.
} 
This small viscosity implies that two-stage milling is critically important in the thorough dispersion of powders. When powders in a solution are badly dispersed, the powders form large agglomerates with large voids among them filled with solvents. These large agglomerates are difficult to pass one another when the slurry flows, thus increasing the slurry viscosity. On the other hand, if the powders are finely dispersed, it is easier for them to pass each other in the slurry, resulting in small viscosity. Another factor influencing viscosity is solid loading: in general, the larger the solid loading, the larger is the viscosity. After taking into account the solid loading of sample $80 \mathrm{Q}, 14.2 \%$, and those of the previous samples discussed earlier, $12.1 \%$ for sample $14(17)$ s, $11.4 \%$ for sample $34 \mathrm{x}, 19.3 \%$ for sample $30 \mathrm{G}, 16.3 \%$ for sample $10 \mathrm{H}-4$, it is still very impressive that the viscosity of the slurry for sample $80 \mathrm{Q}$ is significantly smaller than those of all other samples.

A fiber, sample $80 \mathrm{Q}$, was dipped using this slurry. The firing profiles are illustrated in Figure 4-23. There are two minor adjustments on the firing profiles. First, the heating and cooling rates in the drying and binder burnout were reduced from $10^{\circ} \mathrm{C}$ per minute to $5^{\circ} \mathrm{C}$ per minute. The purpose for this change is to improve the structural integrity of the coating by decreasing the water and binder removal rates from coating. Second, the rapid quenching from $1600^{\circ} \mathrm{C}\left(2,912{ }^{\circ} \mathrm{F}\right)$ to $1100^{\circ} \mathrm{C}\left(2,012{ }^{\circ} \mathrm{F}\right)$ was changed to from $1600^{\circ} \mathrm{C}$ $\left(2,912{ }^{\circ} \mathrm{F}\right)$ to $1200^{\circ} \mathrm{C}\left(2,192^{\circ} \mathrm{F}\right)$. From the previous results stated in the first semiannual report, a prolonged annealing at $1200^{\circ} \mathrm{C}\left(2,192^{\circ} \mathrm{F}\right)$ has no detrimental effects on the core/cladding smoothness of sample 14(17)s. Therefore, it is harmless to start the slow cooling stage from $1200^{\circ} \mathrm{C}$ instead of $1100^{\circ} \mathrm{C}\left(2,012^{\circ} \mathrm{F}\right)$. The intention here is to lower the temperature drop in the fast quench stage from $500^{\circ} \mathrm{C}\left(932^{\circ} \mathrm{F}\right)$ to $400{ }^{\circ} \mathrm{C}\left(752^{\circ} \mathrm{F}\right)$, in order to minimize the thermal stresses developed due to the different thermal expansion coefficients of sapphire and spinel.

Table 4-7. Constituents of slurry batch Blend-2.0 (sample 80Q)

\begin{tabular}{|c|c|c|c|c|}
\hline \multicolumn{5}{|c|}{$\begin{array}{c}\text { Sample Name: } 80 \mathrm{Q} \\
\text { Slurry Batch: Blend-2.0 }\end{array}$} \\
\hline & Components & $\begin{array}{l}\text { Weight } \\
\text { (g) }\end{array}$ & \multicolumn{2}{|c|}{ Weight $\%$} \\
\hline Solvent 1 & 2-propanol & 27.33 & \multicolumn{2}{|c|}{78.6} \\
\hline Solvent 2 & 1-ethoxy-2-peopanol & 2.56 & \multicolumn{2}{|c|}{7.4} \\
\hline Dispersant & Polyethylene glycol & 0.12 & \multicolumn{2}{|c|}{0.3} \\
\hline \multirow[t]{2}{*}{ Binder } & PVP (10k m.w.) & 2.27 & 6.5 & \multirow{2}{*}{13.4} \\
\hline & PVP (1.3m m.w.) & 2.4 & 6.9 & \\
\hline Plasticizer & Polyethylene glycol & 0.11 & \multicolumn{2}{|c|}{0.3} \\
\hline \multirow{4}{*}{$\begin{array}{l}\text { Powders } \\
\text { (Total: } \\
4.54 \text { ) }\end{array}$} & $\mathrm{MgO}$ (EM Science) & 2.72 & \multicolumn{2}{|c|}{59.9} \\
\hline & $\mathrm{MgO}$ (Aldrich) & 0.64 & \multicolumn{2}{|c|}{14.1} \\
\hline & Spinel (Baikalox) & 1.09 & \multicolumn{2}{|c|}{24.0} \\
\hline & $\begin{array}{l}\text { Spinel (Alfa Aesar) } \\
\end{array}$ & 0.09 & \multicolumn{2}{|c|}{2.0} \\
\hline & Solid Loading $=4.54$ & $=14.2^{\circ}$ & & \\
\hline
\end{tabular}


The annealed cross section of sample 80Q is shown in Figure 4-24, and the surfaces of the sample on its sides are shown in Figure 4-25. The spinel cladding is still thin, because of the small slurry viscosity compared to those of previous slurries. The cladding thickness is relatively uniform with some cracks in it. The core/cladding interface seems to consists of small and shinny grains that thicken the interface. There is only a very thin layer of residuals on the cladding surface. The surfaces on the sides of the sample look quite smooth and uniform without steps and cracks, possible owing to the profound amounts of binders added that are capable of holding the coating together after drying.

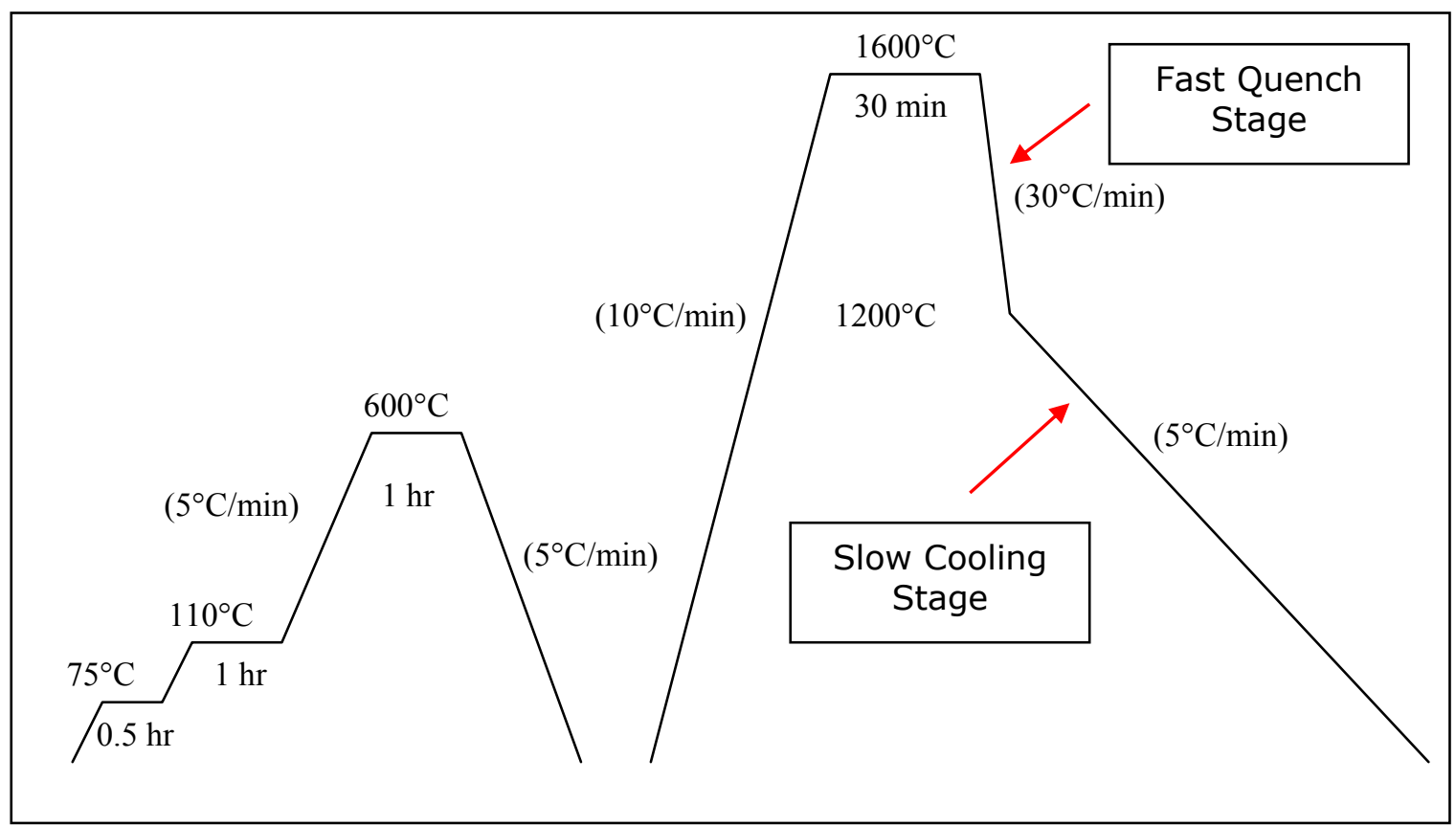

Figure 4-23. The firing Profile of sample 80Q

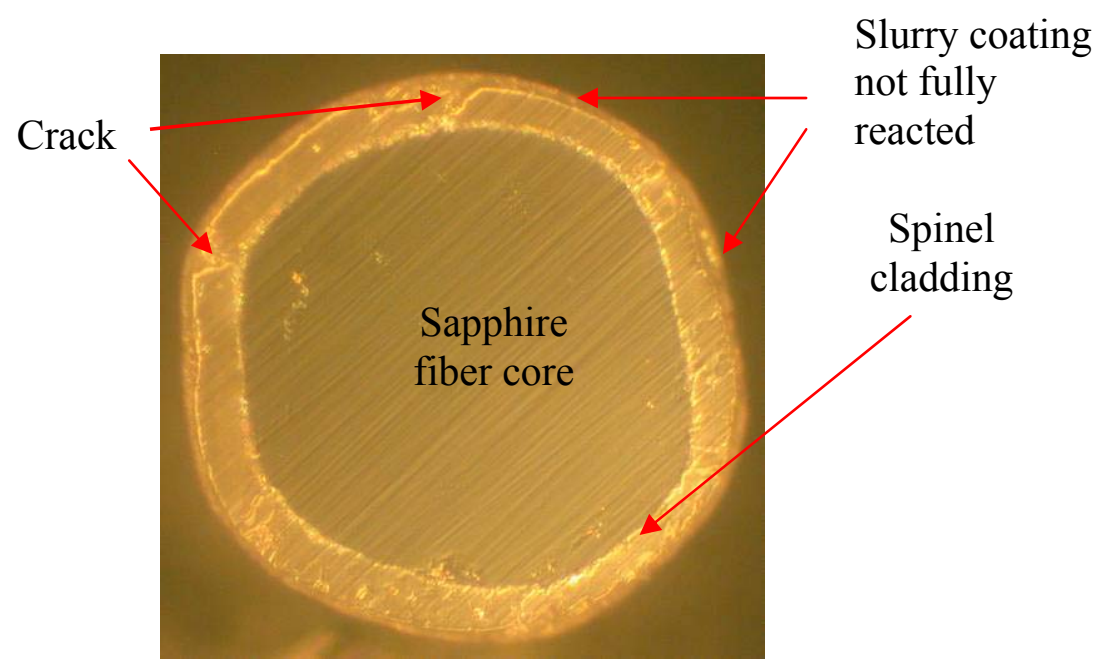

Figure 4-24. The annealed cross section of sample $80 Q$ 


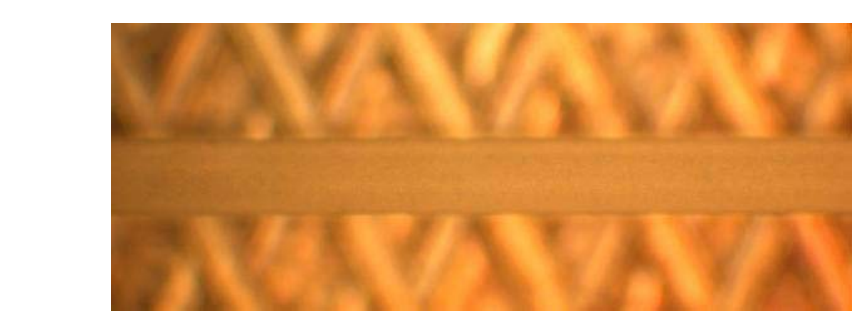

Figure 4-25. The surfaces on the sides of sample $80 Q$

To examine further the effect of binder, $0.16 \mathrm{~g}$ of $1.3 \mathrm{~m}$ PVP was added to the slurry, followed by ball milling, and then a fiber, 100R, was dipped. The dipping was performed one day after the firing of sample $80 \mathrm{Q}$. The constituents of the slurry are listed in Table 4-8. Here the amount of $1.3 \mathrm{~m}$ PVP binder is $0.4 \mathrm{w} . \%$ more than that of sample $80 \mathrm{Q}$. The firing profiles of sample 100R were exactly the same as those of sample 80Q. The annealed cross section of sample 80Q is shown in Figure 4-26, and the surfaces of the sample on its sides in Figure 4-27. As can be seen in Figure 4-26, the cladding thickness is extremely not uniform, and the interface is wavy and out of concentricity. But the amount of residuals on cladding is also small. There are some cracks in the cladding, yet their widths are very small, which indicates superior mechanical strength of the cladding. The surfaces on the sides of the fiber are not as smooth and as uniform compared to those of sample 80Q. It is suspected that the high molecular weight binder was not dissolved thoroughly and homogeneously in the slurry, thus resulting in large cladding thickness variation and roughness on the fiber surface. It is also believed that it is not a good practice to add high molecular weight binder sequentially in larger amounts, because the binders are difficult to dissolve this ways.

Table 4-8. Constituents of the slurry batch Blend-2.1 (sample 100R)

\begin{tabular}{|c|c|c|c|c|}
\hline \multicolumn{5}{|c|}{$\begin{array}{c}\text { Sample Name: 100R } \\
\text { Slurry Batch: Blend-2.1 }\end{array}$} \\
\hline & Components & $\begin{array}{l}\text { Weight } \\
\text { (g) }\end{array}$ & \multicolumn{2}{|c|}{ Weight $\%$} \\
\hline Solvent 1 & 2-propanol & 27.33 & \multicolumn{2}{|c|}{78.2} \\
\hline Solvent 2 & 1-ethoxy-2-peopanol & 2.56 & \multicolumn{2}{|c|}{7.3} \\
\hline Dispersant & Polyethylene glycol & 0.12 & \multicolumn{2}{|c|}{0.3} \\
\hline \multirow[t]{2}{*}{ Binder } & PVP (10k m.w.) & 2.27 & 6.5 & \multirow[t]{2}{*}{13.8} \\
\hline & PVP (1.3m m.w.) & 2.56 & 7.3 & \\
\hline Plasticizer & Polyethylene glycol & 0.11 & \multicolumn{2}{|c|}{0.3} \\
\hline
\end{tabular}




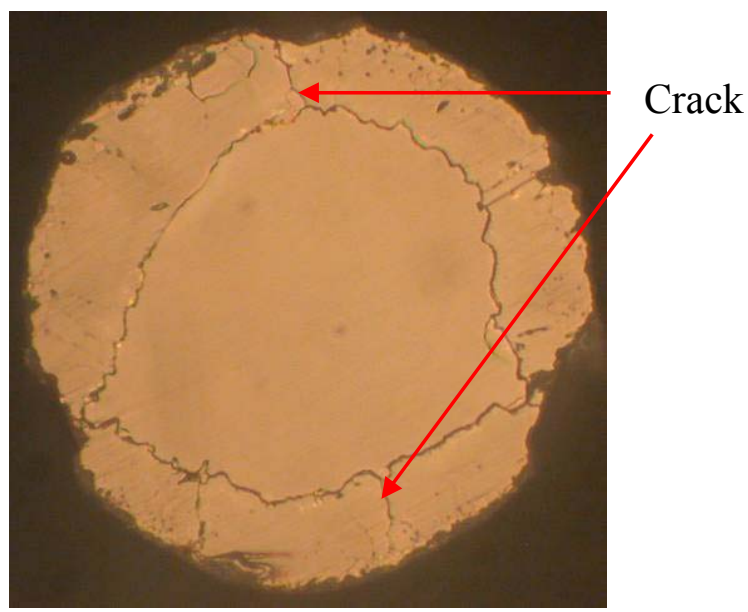

Figure 4-26. The annealed cross section of sample 100R

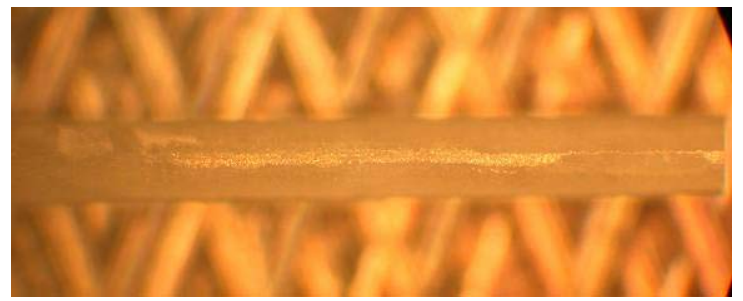

Figure 4-27. The surfaces on the sides of sample 100R

Additional $0.17 \mathrm{~g}$ of $1.3 \mathrm{~m}$ PVP binder was added to the slurry, followed by ball milling. Another fiber, sample 80S, was dipped. This was one day after the firing of sample 100R. The constituents of the slurry are listed in Table 4-9. The firing profiles of sample 80S were exactly the same as those of sample $80 \mathrm{Q}$, except that the dwelling time at $1600{ }^{\circ} \mathrm{C}$ $\left(2,912^{\circ} \mathrm{F}\right)$ was reduced from 30 minutes to 15 minutes. The goal of this change is to reduce the roughness at the core/cladding interface. The annealed cross section of sample 80Q is shown in Figure 4-28, and the surfaces of the sample on its sides in Figure 4-29. Here the results are similar to those of sample 100R: irregular cladding thickness and rough core/cladding interface, apart from that the interface is widened by shinny grains. There are little residuals on cladding surface, and there seems to be only one large crack.

Table 4-9. Constituents of the slurry batch Blend-2.2 (sample 80S)

\begin{tabular}{|c|c|c|c|c|}
\hline \multicolumn{5}{|c|}{ Sample Name: 80S; Slurry Batch: Blend-2.2 } \\
\hline & Components & $\begin{array}{c}\text { Weight } \\
(\mathrm{g})\end{array}$ & \multicolumn{2}{|c|}{ Weight $\%$} \\
\hline Solvent 1 & 2-propanol & 27.33 & \multicolumn{2}{|c|}{77.8} \\
\hline Solvent 2 & 1-ethoxy-2-peopanol & 2.56 & \multicolumn{2}{|c|}{7.3} \\
\hline Dispersant & Polyethylene glycol & 0.12 & \multicolumn{2}{|c|}{0.3} \\
\hline \multirow[t]{2}{*}{ Binder } & PVP (10k m.w.) & 2.27 & 6.5 & \multirow{2}{*}{14.3} \\
\hline & PVP (1.3m m.w.) & 2.73 & 7.8 & \\
\hline Plasticizer & Polyethylene glycol & 0.11 & \multicolumn{2}{|c|}{0.3} \\
\hline
\end{tabular}




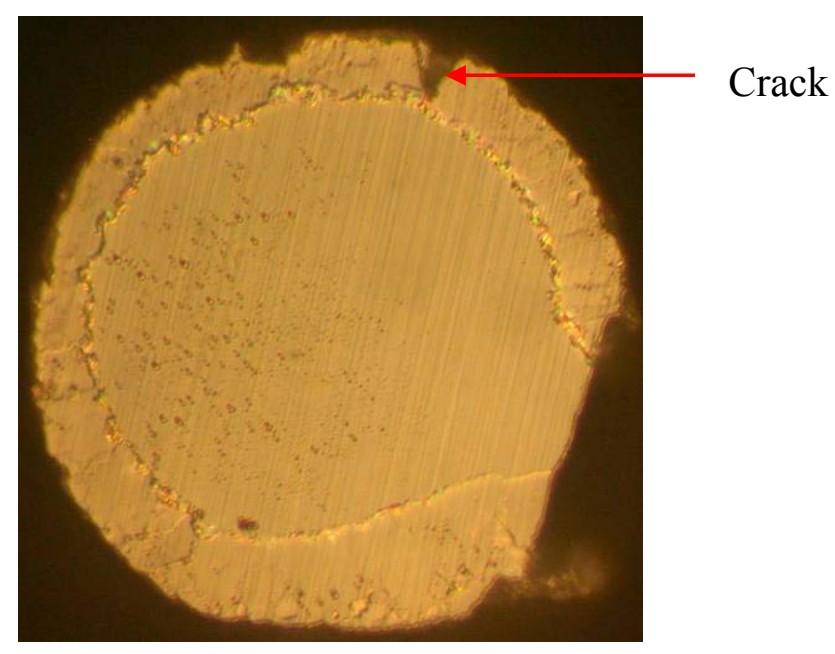

Figure 4-28. The annealed cross section of sample $80 \mathrm{~S}$

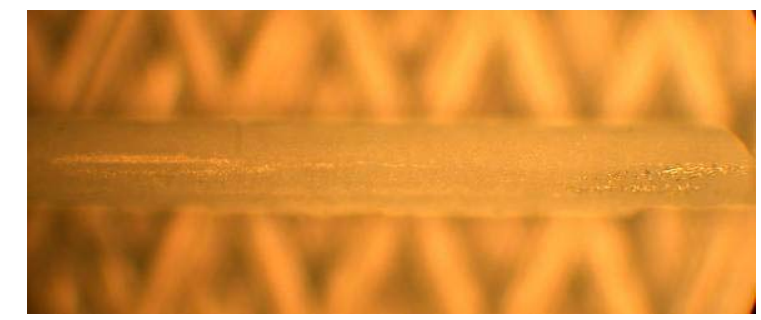

Figure 4-29. The surfaces on the sides of sample $80 \mathrm{~S}$

To examine the effect of low molecular weight PVP binder, additional $0.34 \mathrm{~g}$ of $10 \mathrm{k}$ PVP binder was added to the slurry. The slurry was ball milled before dipping. Another fiber, sample 80T, was dipped. The dipping was performed one day after the firing of sample 80S. The constituents of the slurry are listed in Table $4-10$. The w. $\%$ of $10 \mathrm{k} \mathrm{PVP}, 7.4 \%$, is again comparable to that of $1.3 \mathrm{~m}$ PVP, $7.7 \%$. The firing profiles of sample $80 \mathrm{~T}$ were exactly the same as those of sample $80 \mathrm{~S}$. The annealed cross section of sample $80 \mathrm{~T}$ is shown in Figure 4-30, and the surfaces of the sample on its sides in Figure 4-31. There are a lot of cracks in the cladding; however, the cladding thickness seems to be relatively uniform again. Although the core/cladding interface is still rough, shinny, and granular, yet some portions of the interface are significantly smooth. There are minimal amount of residuals on fiber surface. It seems that the reduction of sintering temperature from $1600{ }^{\circ} \mathrm{C}\left(2,912^{\circ} \mathrm{F}\right)$ to $1550{ }^{\circ} \mathrm{C}\left(2,822^{\circ} \mathrm{F}\right)$ increase the likelihood of a smooth core/cladding interface. It also appears that the addition of 10k PVP can slightly improve the cladding thickness uniformity and cladding surface smoothness. 
Table 4-10. Constituents of the slurry batch Blend-2.3 (sample 80T)

\begin{tabular}{|c|c|c|c|c|}
\hline & $\begin{array}{r}\text { Sample Na } \\
\text { Slurry Batch: }\end{array}$ & & & \\
\hline & Components & $\begin{array}{l}\text { Weight } \\
\text { (g) }\end{array}$ & \multicolumn{2}{|c|}{ "Weight \% } \\
\hline Solvent 1 & 2-propanol & 27.33 & \multicolumn{2}{|c|}{77.1} \\
\hline Solvent 2 & 1-ethoxy-2-peopanol & 2.56 & \multicolumn{2}{|c|}{7.2} \\
\hline Dispersant & Polyethylene glycol & 0.12 & \multicolumn{2}{|c|}{0.3} \\
\hline \multirow[t]{2}{*}{ Binder } & PVP (10k m.w.) & 2.61 & 7.4 & \multirow{2}{*}{15.1} \\
\hline & PVP (1.3m m.w.) & 2.73 & 7.7 & \\
\hline Plasticizer & Polyethylene glycol & 0.11 & \multicolumn{2}{|c|}{0.3} \\
\hline
\end{tabular}

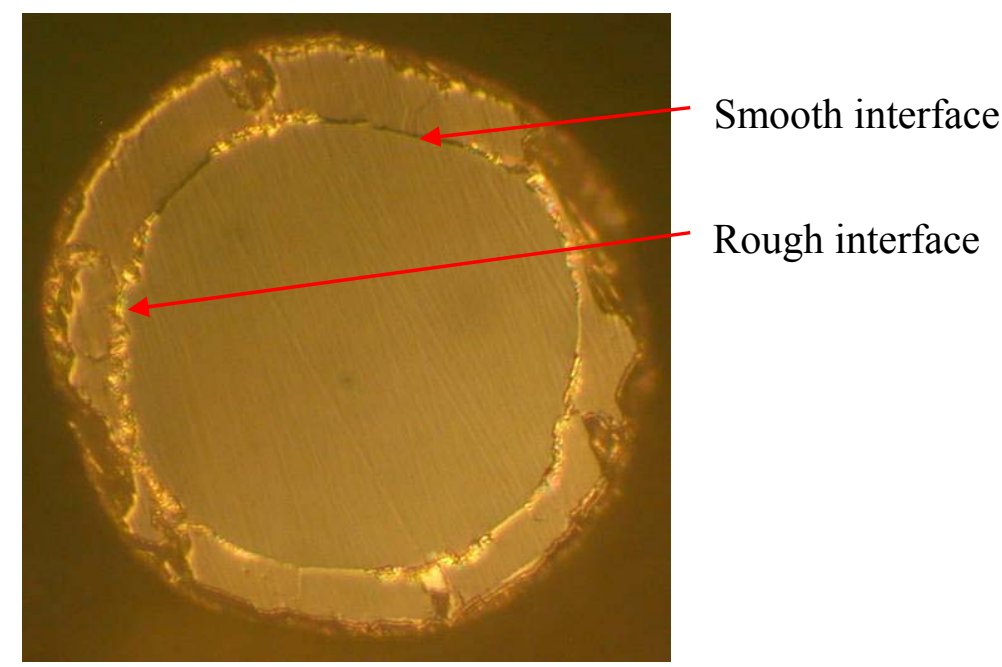

Figure 4-30. The annealed cross section of sample 100R

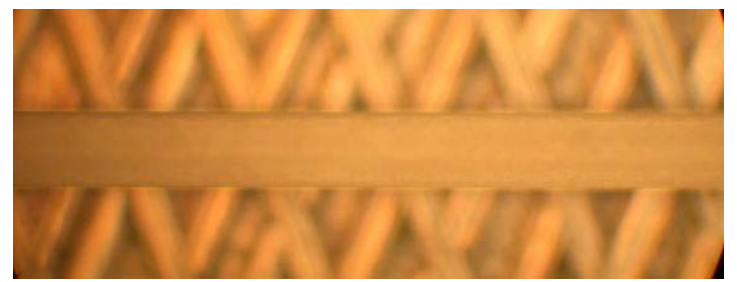

Figure 4-31. The surfaces on the sides of sample 100R

Another fresh batch of slurry blended with four types of powders and dispersed using polyethylene glycol was prepared. One new step in powder preparation was implemented: all powders were dried at $500^{\circ} \mathrm{C}\left(932^{\circ} \mathrm{F}\right)$ for at least 1 hour before adding to the slurry batch. The goal for this step is to removal the chemically absorbed water on powders, especially on the nanopowders from Aldrich. The slurry was milled with 
polyethylene glycol as the dispersant in a 4-oz polypropylene bottle half filled with grinding media. The constituents of the slurry are listed in Table 4-11. The powder weight fractions here are the same as those of sample 80Q listed in Table 4-7, but the powder loading was increased from $14.2 \%$ to $19.9 \%$, in order to increase the slurry viscosity. The major change in slurry preparation is that only 10k PVP binder was used in order to maintain slurry uniformity. There was no polyethylene glycol added in the binder milling stage as a plasticizer, since the molecular weight of 10k PVP binder is very small by itself, thus there was no need to break down the carbon chains any further.

Table 4-11. Constituents of the slurry batch Blend-3.0 (sample 80V)

\begin{tabular}{|l|l|c|c|}
\hline \hline \multicolumn{4}{|c|}{$\begin{array}{c}\text { Sample Name: 80V } \\
\text { Slurry Batch: Blend-3.0 }\end{array}$} \\
\hline \hline \multirow{2}{*}{ Components } & $\begin{array}{c}\text { Weight } \\
(\mathrm{g})\end{array}$ & Weight \% \\
\hline Solvent 1 & 2-propanol & 18.18 & 82.9 \\
\hline Solvent 2 & 1-ethoxy-2-peopanol & 1.43 & 6.5 \\
\hline Dispersant & Polyethylene glycol & 0.19 & 0.9 \\
\hline Binder & PVP (10k m.w.) & 2.13 & 9.7 \\
\hline Plasticizer & Polyethylene glycol & 0 & 0 \\
\hline \multirow{2}{*}{$\left.\begin{array}{l}\text { Powders } \\
\text { (Total: }\end{array} 4.54\right)$} & MgO (EM Science) & 2.69 & 59.4 \\
\cline { 2 - 4 } & MgO (Aldrich) & 0.67 & 14.8 \\
\cline { 2 - 4 } & Spinel (Baikalox) & 1.07 & 23.6 \\
\cline { 2 - 4 } & Spinel (Alfa Aesar) & 0.10 & 2.2 \\
\hline \hline \multicolumn{4}{|c|}{ Solid Loading $=4.53 /(18.18+4.53)=19.9 \%$} \\
\hline
\end{tabular}

The binder was added in a small amount, about $0.2 \mathrm{~g}$, at a time, followed by hand shaking the bottle to dissolve the binder. An interesting phenomenon was observed in this process. The viscosity of slurry, as a result of binder addition, instead of increasing, surprisingly decreases when the binder was added. The viscosity keeps on decreasing until a total of $0.8 \mathrm{~g}$ of binder was added, and it starts to slowly increase after that. The same phenomenon must have occurred during the slurry preparation for sample $80 \mathrm{Q}$, but it was overlooked at that time. It is believed that the low molecular weight PVP was, in fact, serves as a dispersant rather than a binder here. After each binder addition step, one drop of the slurry was let dried on a microscope glass slide, and the pattern of the dried slurry surface was observed. A network of cracks appeared after a few minutes when the slurry is dry. After a total of $2.13 \mathrm{~g}$ of binder has been added, the surface cracks ceased to appear on the dried slurry surface. The slurry was binder milled and a fiber, sample $80 \mathrm{~V}$, was dipped using this slurry. The firing profiles are shown in Figure 4-32. Here the sintering temperature was reduced to $1550{ }^{\circ} \mathrm{C}\left(2,822^{\circ} \mathrm{F}\right)$ in order to lessen the core/cladding interface roughness. The temperature drop in the fast quench stage was also reduced to $250^{\circ} \mathrm{C}\left(482^{\circ} \mathrm{F}\right)$ with a target temperature of $1300^{\circ} \mathrm{C}\left(2,372{ }^{\circ} \mathrm{F}\right)$ instead of $1200{ }^{\circ} \mathrm{C}\left(2,192^{\circ} \mathrm{F}\right)$; the purpose is to minimize the thermal stresses developed during this stage. 
The annealed cross section of sample 80T is shown in Figure 4-33, and the surfaces of the sample on its sides in Figure 4-34. The spinel cladding is still relatively thin; the diameter of sapphire fiber is reduced from $150 \mu \mathrm{m}$ to $125 \mu \mathrm{m}$. The core/cladding interface is both concentric and smooth. There are cracks in the hexagonally symmetrical directions, yet their widths are small, indicating that the degree of cracking is constrained. The residuals on cladding surface are minimal. The fiber surface as seen in Figure 4-34 is both smooth and uniform.

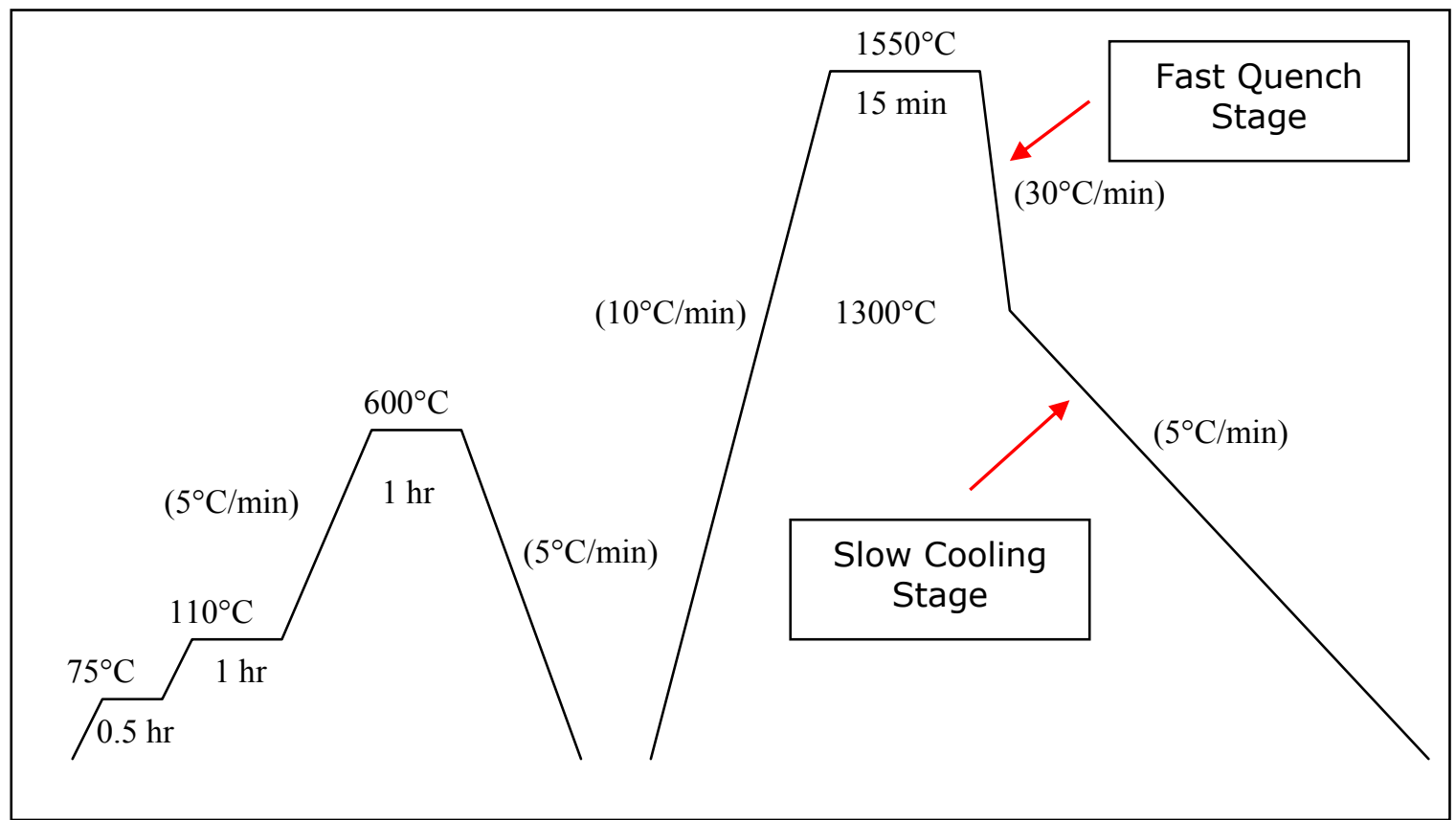

Figure 4-32. The firing profile of sample $80 \mathrm{~V}$

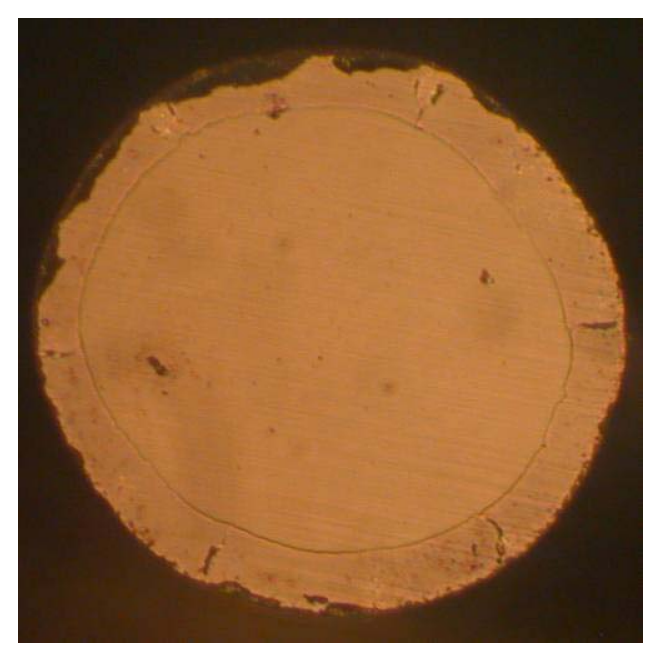

Figure 4-33. The annealed cross section of sample 80V 


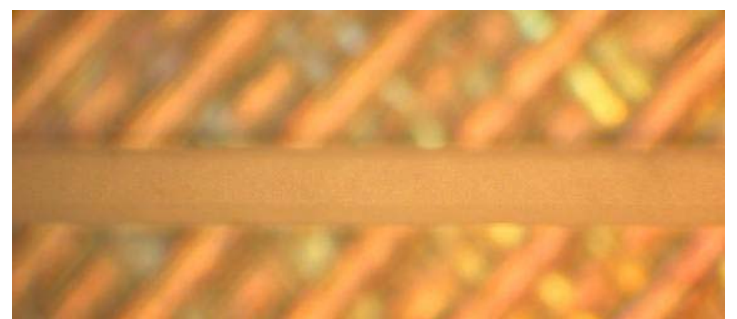

Figure 4-34. The surfaces on the sides of sample $80 \mathrm{~V}$

In order to increase the cladding thickness, $0.1 \mathrm{~g}$ of $1.3 \mathrm{~m}$ PVP binder was added to the slurry to increase its viscosity. The slurry was then ball milled. The composition of the slurry is listed in Table $4-12$. Here the $1.3 \mathrm{~m}$ PVP binder is only a very small fraction of the 10k PVP. A fiber, sample 70W, was dipped using this slurry. The dipping was performed one day after sample $80 \mathrm{~V}$. The annealed cross section of sample $70 \mathrm{~W}$ is shown in Figure 4-35, and the surfaces of the sample on its sides in Figure 4-36. Here the spinel cladding is much thicker than that of sample $80 \mathrm{~V}$. The cladding thickness is relatively uniform, and the core/cladding interface is concentric and relatively smooth. There are some fine cracks and a lot of voids in the cladding, presumably caused by the $1.3 \mathrm{~m}$ PVP binder not thoroughly and uniformly dissolved in the slurry.

Table 4-12. Constituents of the slurry batch Blend-3.1 (sample 70W)

\begin{tabular}{|c|c|c|c|c|}
\hline \multicolumn{5}{|c|}{$\begin{array}{c}\text { Sample Name: } 70 \mathrm{~W} \\
\text { Slurry Batch: Blend-3.1 }\end{array}$} \\
\hline & Components & $\begin{array}{l}\text { Weight } \\
\text { (g) }\end{array}$ & \multicolumn{2}{|c|}{ Weight $\%$} \\
\hline Solvent 1 & 2-propanol & 18.18 & \multicolumn{2}{|c|}{82.5} \\
\hline Solvent 2 & 1-ethoxy-2-peopanol & 1.43 & \multicolumn{2}{|c|}{6.5} \\
\hline Dispersant & Polyethylene glycol & 0.12 & \multicolumn{2}{|c|}{0.9} \\
\hline \multirow[t]{2}{*}{ Binder } & PVP (10k m.w.) & 2.13 & 9.7 & \multirow{2}{*}{10.2} \\
\hline & PVP (1.3m m.w.) & 0.1 & 0.5 & \\
\hline Plasticizer & Polyethylene glycol & 0 & & 0 \\
\hline
\end{tabular}

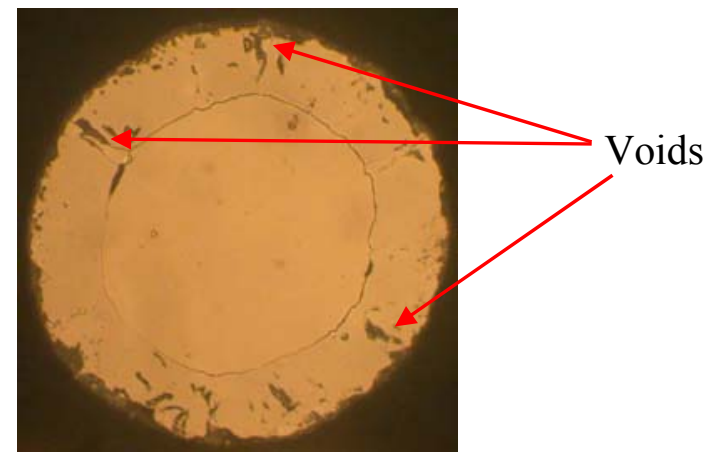

Figure 4-35. The annealed cross section of sample 70W 


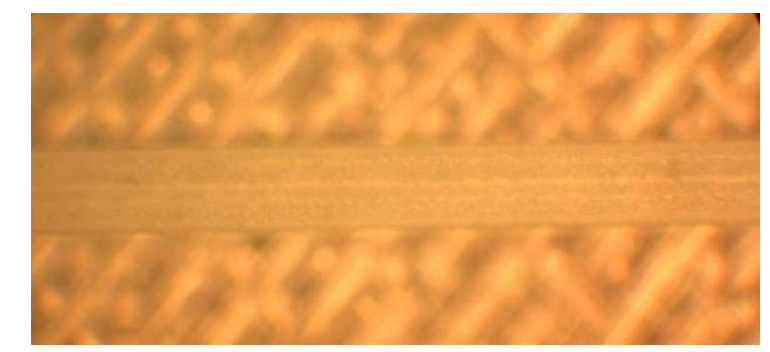

Figure 4-36. The surfaces on the sides of sample 70W

One thing worthy of noting is that the viscosity of the slurry batch Blend-3.1 increases drastically 3 days after being stored in the polypropylene bottle. The slurry looks all gelled together with the grinding media dispersed in it. This hints that the slurry with the current compositions is not stable as a function of time, which could create problems in the repeatability and consistency of the slurry behavior. A varied degree of gelation also appears in many other batches. It is suspected that the combination of polyethylene glycol as a dispersant and PVP as binder is still not suitable.

A fresh batch was prepared using a little more percentage of polyethylene glycol, $1.4 \mathrm{w}$. $\%$, a lot less both the 10k and 1.3m PVP binder, a total of $1.0 \mathrm{w} . \%$, and a lot higher solid loading, $24.3 \%$. The slurry viscosity was extremely high after dispersion milling, not suitable for dipping. It is assumed that the current slurry composition is not ideal for a relatively high solid loading of $14.3 \%$, thus resulting in very high viscosity, an indication of poor powder dispersion. It is also possible that after a certain maximum solid loading, the viscosity starts to drastically increase regardless a well designed slurry composition.

Table 4-13. Constituents of the slurry batch Blend-3.2

\begin{tabular}{|c|c|c|c|c|}
\hline \multicolumn{5}{|c|}{ Slurry Batch: Blend-3.2 } \\
\hline & Components & $\begin{array}{c}\text { Weight } \\
\text { (g) }\end{array}$ & \multicolumn{2}{|c|}{ Weight $\%$} \\
\hline Solvent 1 & 2-propanol & 28.29 & \multicolumn{2}{|c|}{93.1} \\
\hline Solvent 2 & 1-ethoxy-2-peopanol & 1.4 & \multicolumn{2}{|c|}{4.6} \\
\hline Dispersant & Polyethylene glycol & 0.44 & \multicolumn{2}{|c|}{1.4} \\
\hline \multirow[t]{2}{*}{ Binder } & PVP (10k m.w.) & 0.27 & 0.9 & \multirow{2}{*}{1.0} \\
\hline & PVP (1.3m m.w.) & 0.04 & 0.1 & \\
\hline Plasticizer & Polyethylene glycol & 0 & & 0 \\
\hline \multirow{4}{*}{$\begin{array}{l}\text { Powders } \\
\text { (Total: } \\
9.1 \text { ) }\end{array}$} & $\mathrm{MgO}(\mathrm{EM}$ Science, $15 \mu \mathrm{m})$ & 5.38 & \multicolumn{2}{|c|}{59.9} \\
\hline & $\mathrm{MgO}$ (Aldrich, $0.013 \mu \mathrm{m})$ & 1.33 & \multicolumn{2}{|c|}{14.1} \\
\hline & Spinel (Baikalox, $3 \mu \mathrm{m})$ & 2.15 & \multicolumn{2}{|c|}{24.0} \\
\hline & Spinel (Alfa Aesar, $<1 \mu \mathrm{m})$ & 0.24 & \multicolumn{2}{|c|}{2.0} \\
\hline \multicolumn{5}{|c|}{ Solid Loading $=9.1 /(28.3+9.1)=24.3 \%$} \\
\hline
\end{tabular}

Efforts were made to reproduce the sample $80 \mathrm{~V}$, but with thicker cladding. All powders were dried at $500{ }^{\circ} \mathrm{C}\left(932^{\circ} \mathrm{F}\right)$ for 3 hours. First, 2-propanol was mixed with polyethylene glycol in a $150 \mathrm{cc}$ beaker. Powders were added to the beaker in the sequence of 
increasing powder sizes: stating with the powders with the smallest sizes, $\mathrm{MgO}$ nanopowders, and ending with the powders with the largest sizes, $\mathrm{MgO}$ powders from EM Science. In between each powder addition the slurry was stirred for two minutes to disperse the powders. The slurry was then poured into a 4-oz polypropylene bottle half filed with grinding media. The bottle was then hand shaken followed by ball milling. The binder was added to the slurry in the bottle in a unit of about $0.2 \mathrm{~g}$ at a time, followed by hand-shaking the bottle for 30 seconds. The slurry after binder addition was then ball milled. The slurry composition is listed in Table 4-14. This slurry composition is almost the same as that of sample $80 \mathrm{~V}$. The firing profiles are illustrated in Figure 4-37. Except for the $500^{\circ} \mathrm{C}$ binder burnout temperature, and the $2{ }^{\circ} \mathrm{C}$ per minute $(3.6 / \mathrm{min})$ cooling rate, the profiles are exactly the same as those of sample $80 \mathrm{~V}$. A fiber, sample $200 \mathrm{hh}$, was dipped. It was realized during the fiber dipping that if ethanol, instead of 2-propanol, was used to clean the original sapphire fiber surface, the coating formed would not be uniform. The annealed cross section of sample 17ee is shown in Figure 4-38, and the surfaces of the sample on its sides in Figure 4-39. As shown in Figure 4-38, the cladding thickness is quite uniform; the core/cladding interface is both smooth and concentric. There are a few very thin cracks in the cladding, and there are also some residuals on the cladding surface. However, this clad fiber has already shown considerable improvement. The sapphire fiber core was reduced from $150 \mu \mathrm{m}$ to $105 \mu \mathrm{m}$ with light-guiding spinel cladding.

Table 4-14. Constituents of the slurry batch Blend-4.0

\begin{tabular}{|l|l|c|c|}
\hline \hline \multicolumn{4}{|c|}{$\begin{array}{c}\text { Sample Name: 200hh } \\
\text { Slurry Batch: Blend-4.0 }\end{array}$} \\
\hline \hline \multirow{2}{*}{ Components } & $\begin{array}{c}\text { Weight } \\
(\mathrm{g})\end{array}$ & Weight \% \\
\hline Solvent 1 & 2-propanol & 18.29 & 82.8 \\
\hline Solvent 2 & 1-ethoxy-2-peopanol & 1.44 & 6.5 \\
\hline Dispersant & Polyethylene glycol & 0.19 & 0.8 \\
\hline Binder & PVP (10k m.w.) & 2.18 & 9.9 \\
\hline Plasticizer & Polyethylene glycol & 0 & 0 \\
\hline \hline \multirow{2}{*}{$\left.\begin{array}{l}\text { Powders } \\
\text { (Total: }\end{array} 4.55\right)$} & MgO (EM Science) & 2.70 & 59.3 \\
\cline { 2 - 4 } & MgO (Aldrich) & 0.68 & 14.9 \\
\cline { 2 - 4 } & Spinel (Baikalox) & 1.07 & 23.5 \\
\cline { 2 - 4 } & Spinel (Alfa Aesar) & 0.10 & 2.2 \\
\hline \hline \multicolumn{4}{|l|}{ Solid Loading $=4.55 /(18.29+4.55)=19.9 \%$} \\
\hline
\end{tabular}




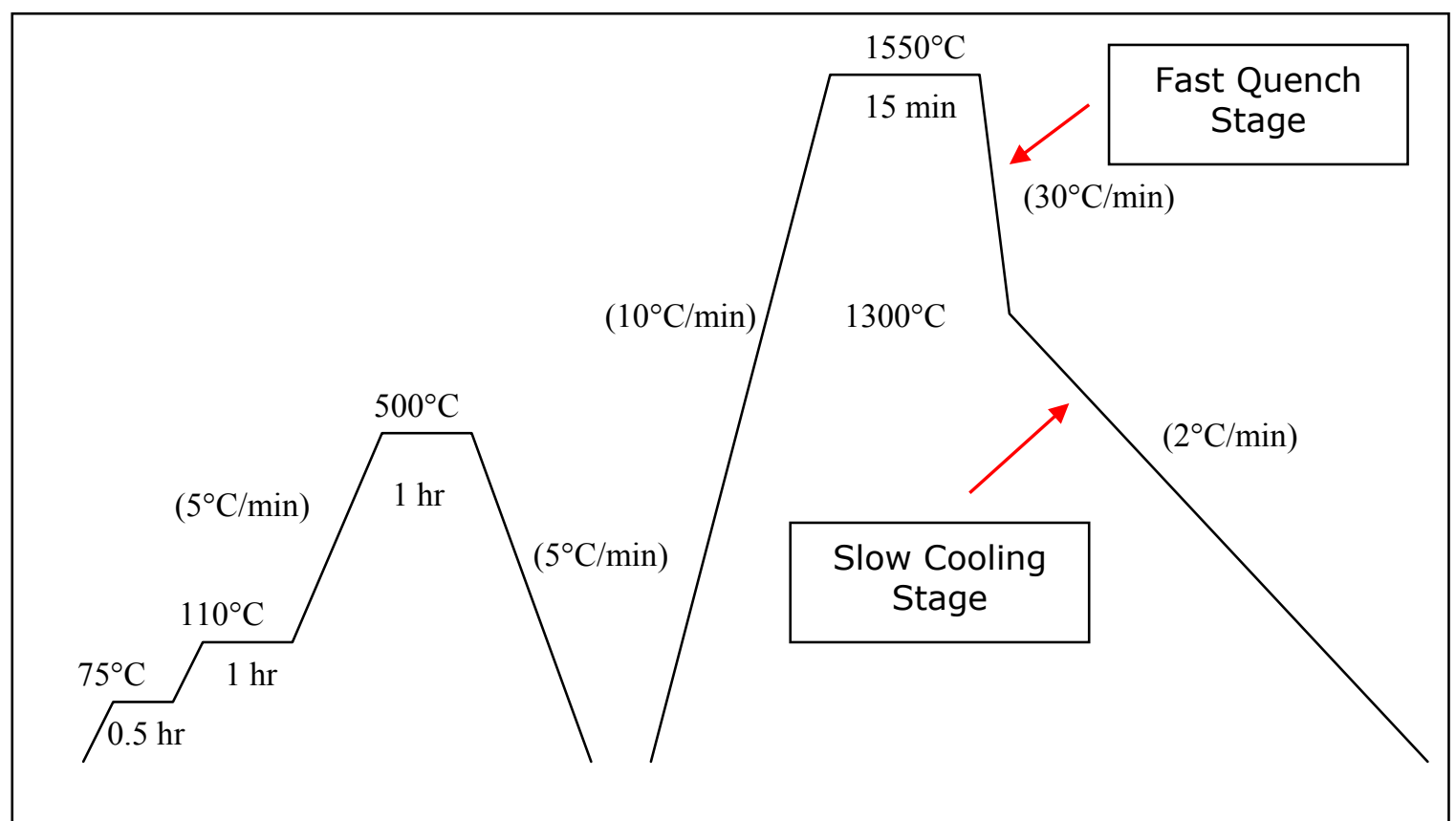

Figure 4-37. The firing profile of sample $200 \mathrm{hh}$

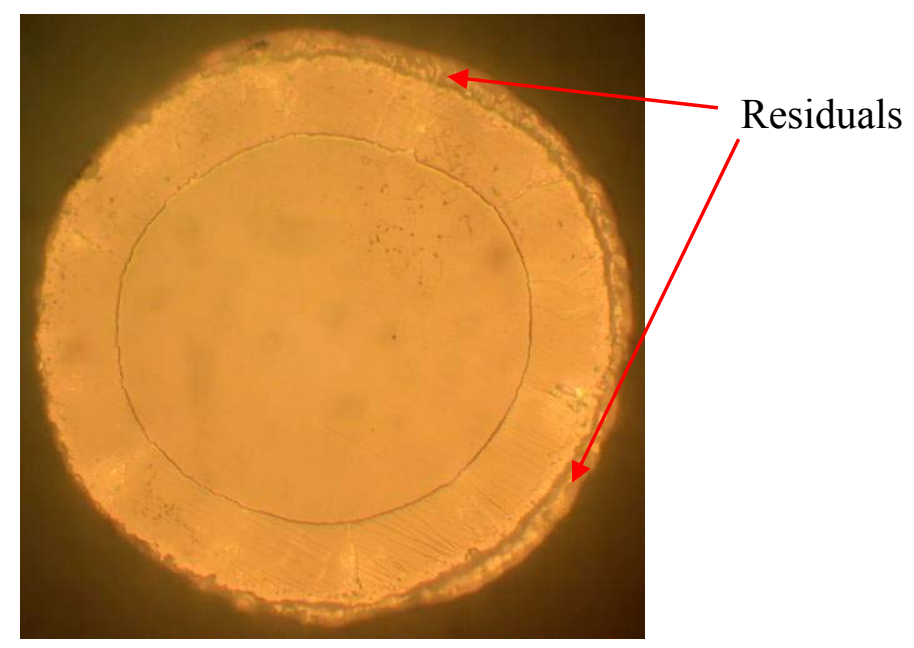

Figure 4-38. The annealed cross section of sample $200 \mathrm{hh}$

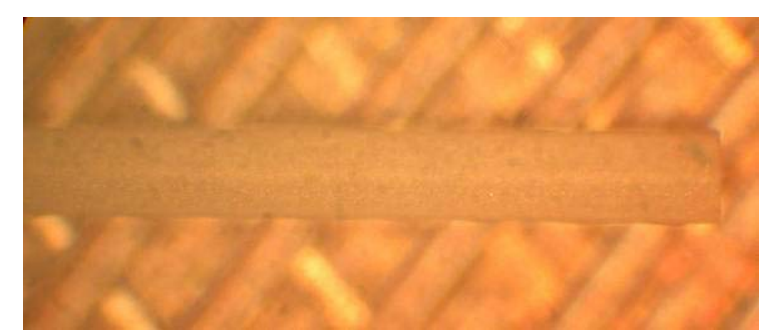

Figure 4-39. The surfaces on the sides of sample $200 \mathrm{hh}$ 


\subsubsection{Test Menhaden Fish Oil as a Dispersant}

Efforts were made to explore different types of dispersant, such as those shown in Table 4-5. Three dispersant candidates, oleic acid, stearic acid, and citric acid, were excluded first, because that after these chemicals were mixed with 2-proponal and ceramic powders, the viscosity of the slurries was very high, and the slurries even gelled together in some cases. The common aqueous dispersant sodium polyacrylate (Darvan 811 from R.T Vanderbilt Company, Inc., Norwalk, CT) was also eliminated because of the $50 \%$ water content in the dispersant. Finally, three candidates were chosen for further exploration, Menhaden fish oil (Aldrich), phosphate ester (Triton QS-44 surfactant from The Dow Chemical Company, Midland, MI), and CoatOsil 1211 coating additive from GE Silicones, Friendly, WV). Slurries prepared using these chemicals have reasonably small viscosity. The particle sizes of powders dispersed in these chemicals were measured using variety of techniques; the results will be discussed later. In this section two samples prepared using Menhaden fish oil as dispersant will be discussed.

Several batches of slurry with a range of composition were prepared to examine the effect of Menhaden fish oil as dispersant. The results of one of the batches are discussed here. In dispersant milling stage, Menhaden fish oil was first dissolved in 2-propanol, and the powders were added sequentially starting from the finest $\mathrm{MgO}$ powders from Aldrich, and ending with the coarsest powders from EM Science. All powders were dried at $400{ }^{\circ} \mathrm{C}\left(752^{\circ} \mathrm{F}\right)$ for several hours and the slurry was ball milled. Afterward, the 10k PVP binder, 1-ethoxy-2-peopanol, and polyethylene glycol were added to the slurry. The slurry composition is shown in Table 4-15. Here the PVP binder amount is significantly smaller, and that of 1-ethoxy-2-peopanol for solvent evaporation control is considerably larger. The solid loading is comparable to those of other slurry batches. The slurry was quite thick after binder milling. It is believed that the large viscosity is caused by imperfect powder dispersion in the slurry. A fiber, sample 17ee, was dipped using this slurry. The coating surface after dipping is not smooth. The firing profiles are illustrated in Figure 4-40. The annealed cross section of sample 17ee is shown in Figure 4-41, and the surfaces of the sample on its sides in Figure 4-42. Because of the large slurry viscosity, a comparatively thick cladding is obtained after only 17 dips.

Even though it is desirable to have smaller number of dips, the high slurry viscosity would likely bring about inferior cladding properties, which are clearly shown in Figure 4-41. There are six hexagonally symmetric cracks in the cladding, and their widths are relatively large. The core/cladding interface is more or less concentric, but it is not very smooth. In general, there are more residuals on the cladding surface, and the cladding surface looks whiter, compared to those samples using polyethylene glycol as dispersant. There are spotty patterns on fiber surfaces, presumably caused by binder deficiency. One thing worthy of noting is that the slurry prepared using fish oil as dispersant does NOT show signs of significant increase in viscosity as a function of time. 
Table 4-15. Constituents of the slurry batch Fish-B-1

\begin{tabular}{|l|l|c|c|}
\hline \multicolumn{4}{|c|}{$\begin{array}{c}\text { Sample Name: 17ee } \\
\text { Slurry Batch: Fish-B-1 }\end{array}$} \\
\hline \hline \multirow{2}{*}{ Components } & $\begin{array}{c}\text { Weight } \\
(\mathrm{g})\end{array}$ & Weight \% \\
\hline Solvent 1 & 2-propanol & 21.15 & 78.6 \\
\hline Solvent 2 & 1-ethoxy-2-peopanol & 5.29 & 19.6 \\
\hline Dispersant & Menhaden fish oil & 0.07 & 0.3 \\
\hline Binder & PVP (10k m.w.) & 0.2 & 0.7 \\
\hline Plasticizer & Polyethylene glycol & 0.2 & 0.7 \\
\hline \multirow{2}{*}{$\begin{array}{l}\text { Powders } \\
\text { (Total: }\end{array}$} & MgO (EM Science) & 2.74 & 59.8 \\
\cline { 2 - 4 } 4.58) & MgO (Aldrich) & 0.65 & 14.2 \\
\cline { 2 - 4 } & Spinel (Baikalox) & 1.09 & 23.8 \\
\cline { 2 - 4 } & Spinel (Alfa Aesar) & 0.10 & 2.2 \\
\hline \hline \multicolumn{4}{|c|}{ Solid Loading =4.58/(21.15+4.58)=17.8\% } \\
\hline \multicolumn{4}{|c|}{} \\
\hline
\end{tabular}

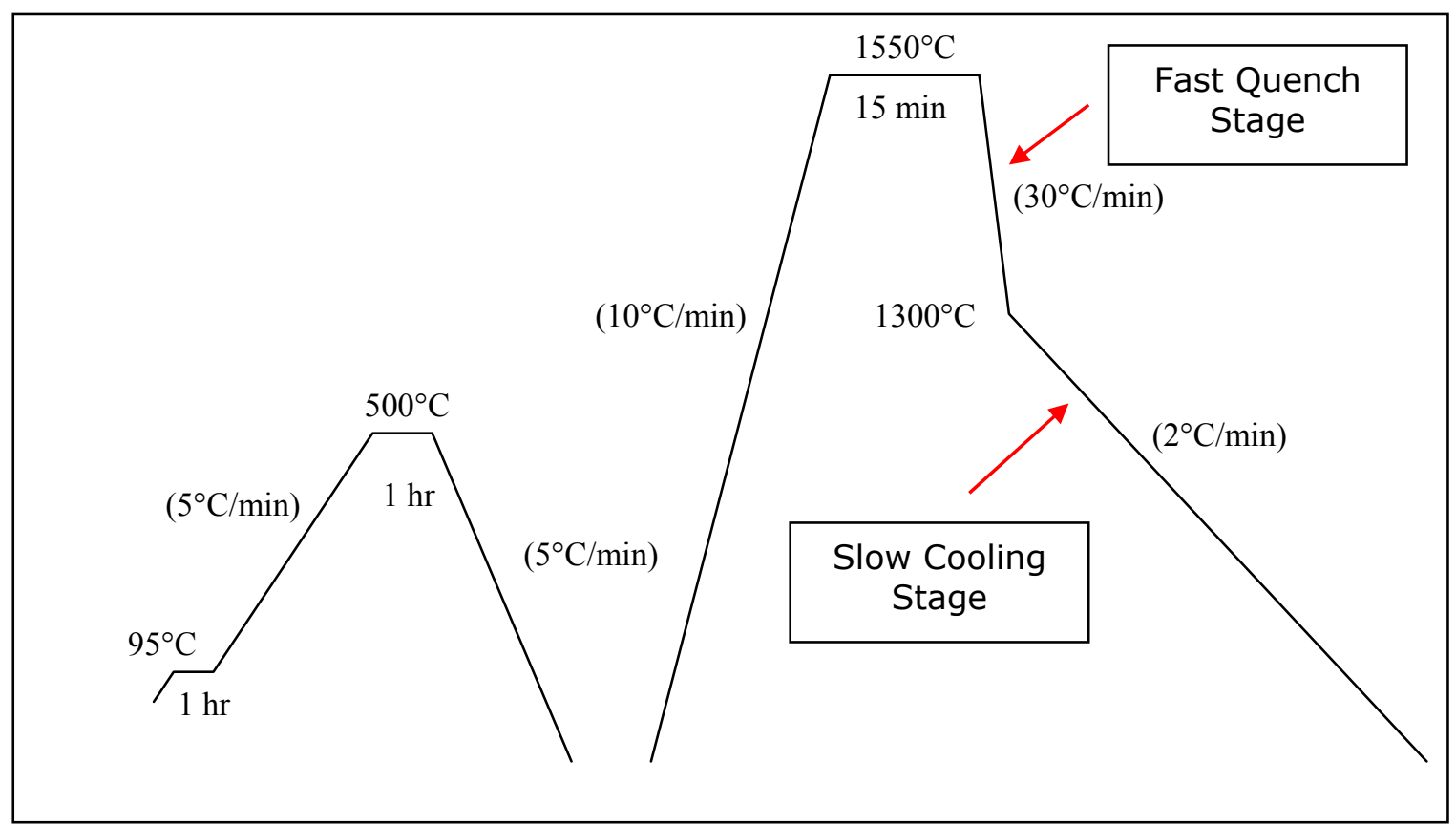

Figure 4-40. The firing profile of sample 17ee 


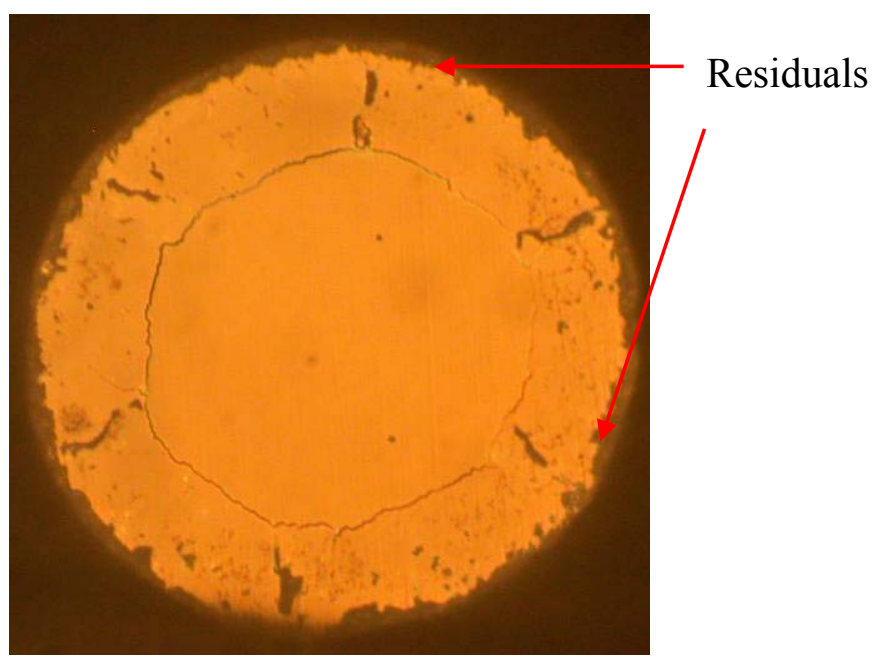

Figure 4-41. The annealed cross section of sample 17ee

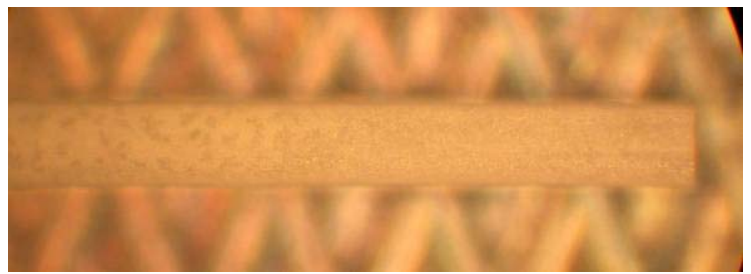

Figure 4-42. The surfaces on the sides of sample 17ee

Another sample, sample 200kk, with much improved cladding properties is presented here. The slurry composition is listed in Table 4-16. The 10k PVP binder amount is only about half of that for sample 200hh. The firing profiles are illustrated in Figure 4-43. The firing profile of sample 200kk There is major change in the drying and burnout profile, which will be discussed later. The annealed cross section of sample $200 \mathrm{kk}$ is shown in Figure 4-44, and the surfaces of the sample on its sides in Figure 4-45.

The results here are very similar to those of sample $200 \mathrm{hh}$, which is prepared using polyethylene as dispersant. The cladding thickness is quite uniform; the core/cladding interface is both smooth and concentric. There are a few very thin cracks in the cladding, and there are also some residuals on the cladding surface. This sample and sample 200hh are the best quality samples manufactured so far. 
Table 4-16. Constituents of the slurry batch Fish-B-2

\begin{tabular}{|l|l|c|c|}
\hline \multicolumn{4}{|c|}{$\begin{array}{c}\text { Sample Name: 200kk } \\
\text { Slurry Batch: Fish-B-2 }\end{array}$} \\
\hline \hline \multirow{2}{*}{ Components } & $\begin{array}{c}\text { Weight } \\
(\mathrm{g})\end{array}$ & Weight \% \\
\hline Solvent 1 & 2-propanol & 23.57 & 87.4 \\
\hline Solvent 2 & 1-ethoxy-2-peopanol & 2.04 & 7.6 \\
\hline Dispersant & Menhaden fish oil & 0.09 & 0.3 \\
\hline Binder & PVP (10k m.w.) & 1.27 & 4.7 \\
\hline Plasticizer & Polyethylene glycol & 0 & 0 \\
\hline \multirow{2}{*}{$\begin{array}{l}\text { Powders } \\
\text { (Total: }\end{array}$} & MgO (EM Science) & 2.73 & 59.3 \\
\cline { 2 - 4 } 4.60) & MgO (Aldrich) & 0.67 & 14.6 \\
\cline { 2 - 4 } & Spinel (Baikalox) & 1.10 & 23.9 \\
\cline { 2 - 4 } & Spinel (Alfa Aesar) & 0.10 & 2.2 \\
\hline \hline \multicolumn{4}{|c|}{ Solid Loading =4.60/(23.57+4.60)=16.3\% } \\
\hline \multicolumn{4}{|c|}{} \\
\hline
\end{tabular}

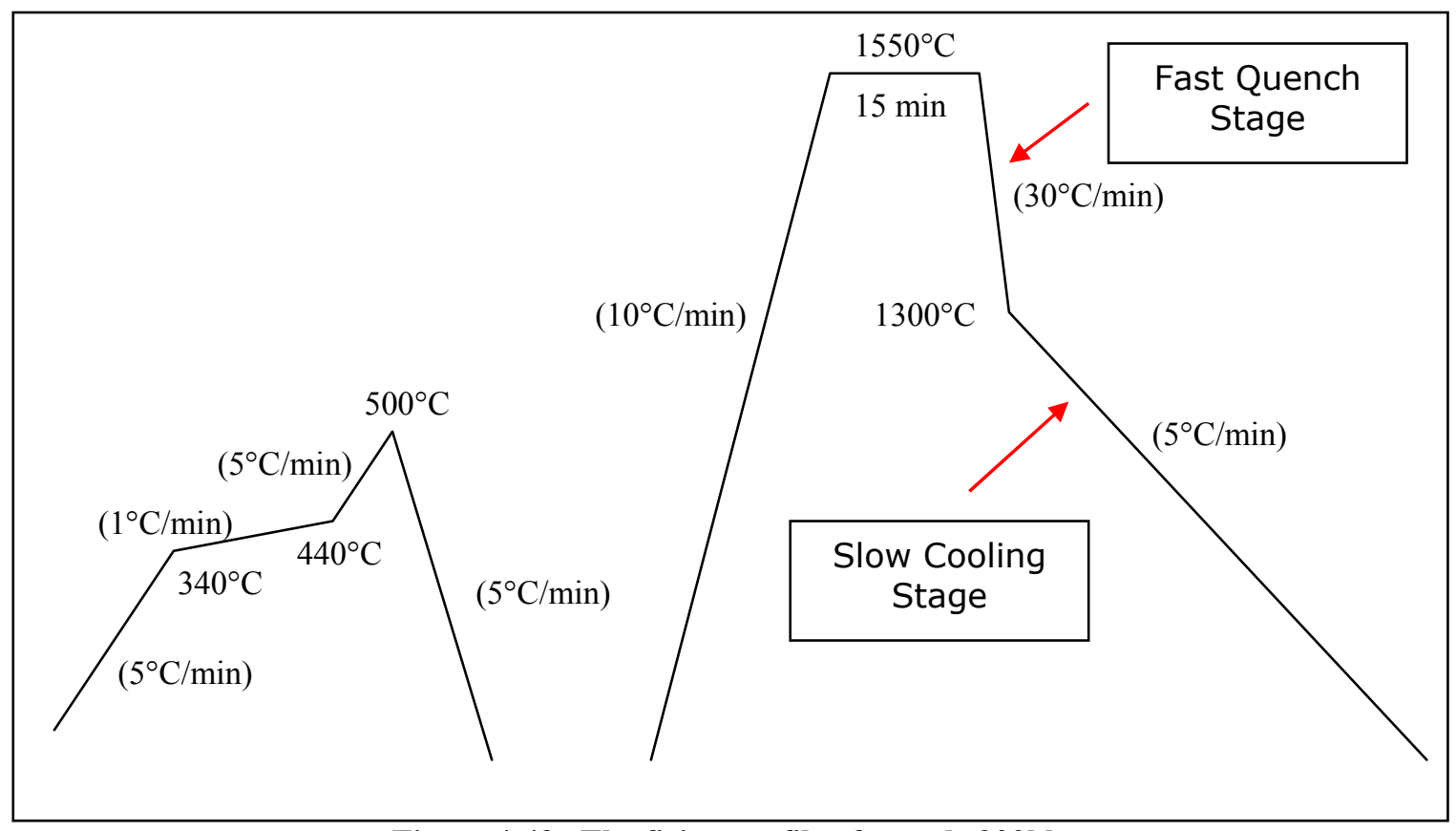

Figure 4-43. The firing profile of sample 200kk 


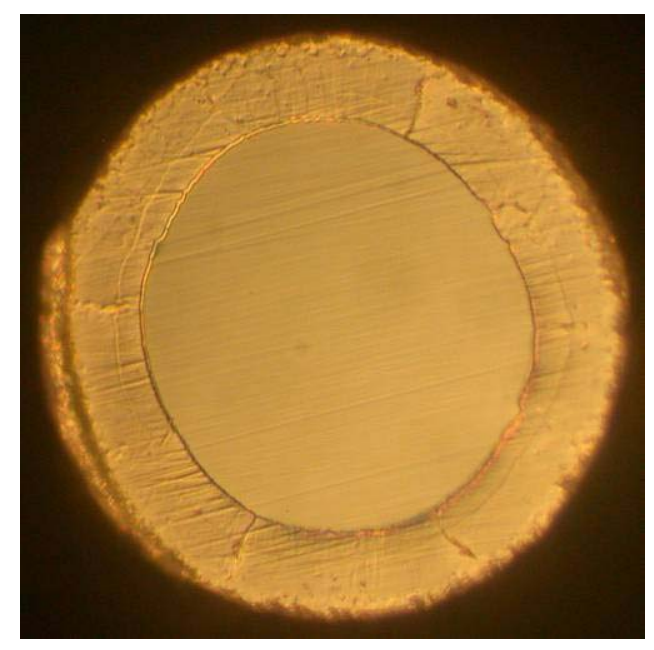

Figure 4-44. The annealed cross section of sample $200 \mathrm{kk}$

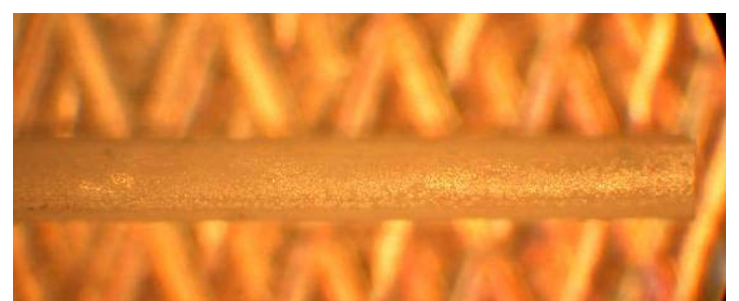

Figure 4-45. The surfaces on the sides of sample $200 \mathrm{kk}$

\subsubsection{Study of Effectiveness of Dispersant by Particle Size Analysis}

\subsubsection{Particle Size Analysis of Spinel Nanopowders from TAL Using the Zetasizer 3000HS from Malvern Instruments}

The Zetasizer 3000HS measures particle sizes based on the Brownian motion of the particles in suspension. The system configuration is shown in Figure 4-46. Laser light scattered from the sample cell is detected by a photomultiplier tube oriented at $90^{\circ}$ with respect to the laser beam. The angle of the photomultiplier tube can be varied to obtain the spatial distribution of the scattered light. If the particles in suspension were motionless, a classical stationary speckle pattern, where the speckle size and position are stationary, can be formed as shown in Figure 4-47. The bright patches in the speckle pattern are produced by the constructive interference from ALL the light scattered by ALL the particles. Similarly, the dark patches are generated by the destructive interference from all the light. If the particles undergo Brownian motion, the position and intensity of each speckle will be in constant motion. The rate of speckle fluctuation depends on the particle sizes; the smaller the sizes, the faster are the fluctuation rates. With known viscosity and index of refraction of the solvent, the particle size distribution 
can be converted from the rates of speckle fluctuation. The sample concentration is also very important in obtaining the correct size data; if the particle concentration is too high, a single laser ray could be scattered multiple times by numerous particles in its path, thus resulting in distortion of the fluctuation rate. Furthermore, the system is design to measure particles with sizes less than about $1 \mu \mathrm{m}$; for particles above this size limit, the Brownian motion may not be detectable, and the sedimentation of particles may also affect the scattered light intensity. Due to the delicate nature of the size measurement based on Brownian motion, it may require careful experimental design and repeated tests to obtain reliable size data.

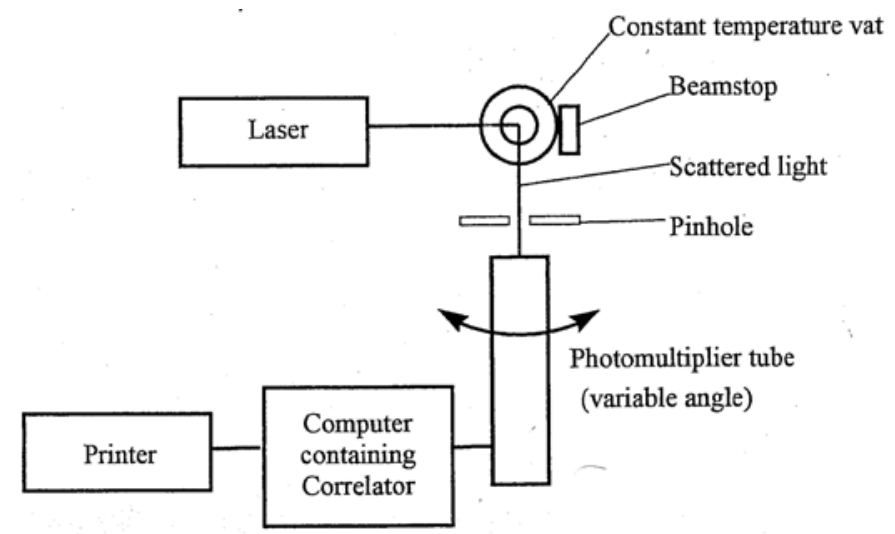

Figure 4-46. The system configuration of Zetasizer 3000HS

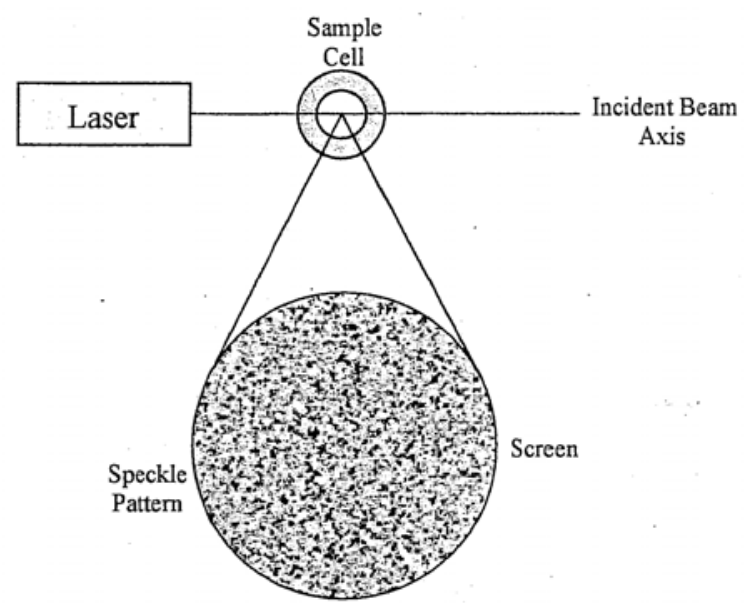

Figure 4-47. Schematic representation of a speckle pattern

\subsubsection{The Size Measurement of spinel nanopowders from TAL $(0.050 \mu \mathrm{m})$ dispersed using Menhaden fish oil, Triton QS-44, and CoatOsil 1211}

Since the $\mathrm{MgO}$ nanopowder from Aldrich is quite expensive, the lower cost nanopowders from TAL were used to evaluate the performance of Zetasizer 3000HS. The equipment is located in the Environmental department in VT. The nanopowders from TAL were first dried at $500^{\circ} \mathrm{C}$ for 2.5 hours before mixing. Three batches of samples were prepared: 0.4 
w. \% fish oil, 0.2 and $0.4 \mathrm{w}$. \% Triton QS-44 in 2-propanol. The solid loading in each batch is $10 \mathrm{w} . \%$. Since the slurry as produced is almost opaque, it needs to be diluted before measurement. $35 \mu \mathrm{L}$ of the slurry was withdrawn and mixed with 3 c.c. of 2propanol to reduce its concentration. It is to be noted that this recipe of dilution is arbitrarily chosen, and may not produce the optimal particle concentration. The index of refraction of 2-propanol, 1.377, and its viscosity, $2.3 \mathrm{cp}$, were input to the equipment as parameters. The duration of each single measurement was set to be 10 seconds. Each batch of sample was measured 30 times in a time period of about 10 minutes. The results are shown in Figure 4-48. Each data point represents the average particle size in each measurement. As can be seen in Figure 4-48, the samples dispersed using Triton QS-44 have larger particle sizes than those of the sample dispersed using fish oil. And the batch with 0.4 w. \% Triton QS-44 has smaller sizes compared to those with $0.2 \mathrm{w}$. \% Triton QS-44. It is suspected that, while the exact particle sizes obtained here may not be accurate, the relative trends among the samples may still provide some insight; fish oil seems to be superior to Triton as dispersant. As seen in Figure 4-48, the particle sizes range from $300 \mathrm{~nm}$ to $600 \mathrm{~nm}$ among all samples. For the fact that the nominal particle size provided by the manufacturer is only $50 \mathrm{~nm}$; these results indicate that the powders are forming large agglomerates in the samples. In the time period of 10 minutes, the particle sizes of all samples increase as a function of time. It is not clear whether the agglomerates are really growing in size so quickly or the results are generated by unknown experimental anomaly.

The $0.4 \mathrm{w} . \%$ fish oil sample was sonicated for 25 seconds and then measured again; the results are shown in Figure 4-49. It can be seen that the agglomerate sizes decrease for about $60 \mathrm{~nm}$ to $90 \mathrm{~nm}$ after 25 seconds of sonication. This implies that the large agglomerates are broken up by sonication. The sample was further diluted with 2propanol; the exact amount of dilution was not recorded. As shown in Figure 4-49, as a result of dilution, the agglomerate sizes increase, and there is a rapid increase of particle size as a function of time after dilution, from $400 \mathrm{~nm}$ to $900 \mathrm{~nm}$ in 10 minutes.

It was later realized that, to prevent multiple scattering of laser light, the sample has to be diluted to a level that the count rate of the photomultiplier tube falls between 10k and $500 \mathrm{k}$. In this level the sample is almost transparent. The particle size is supposed to increase as the sample is diluted, and it would reach a plateau when the count rate falls into the above level. In the current sample, before sonication and dilution (the dark blue diamond in Figure 4-49), the average count rate is $2800 \mathrm{~K}$. The subsequent sonication does not affect count rate. The count rate after dilution is $360 \mathrm{~K}$, just accidentally fall right into the required level. 
Particle Size Analysis of Spinel Nanopowders from TAL (30 measurement in 10 minutes; solvent: 2-propanol)(8-17-04)

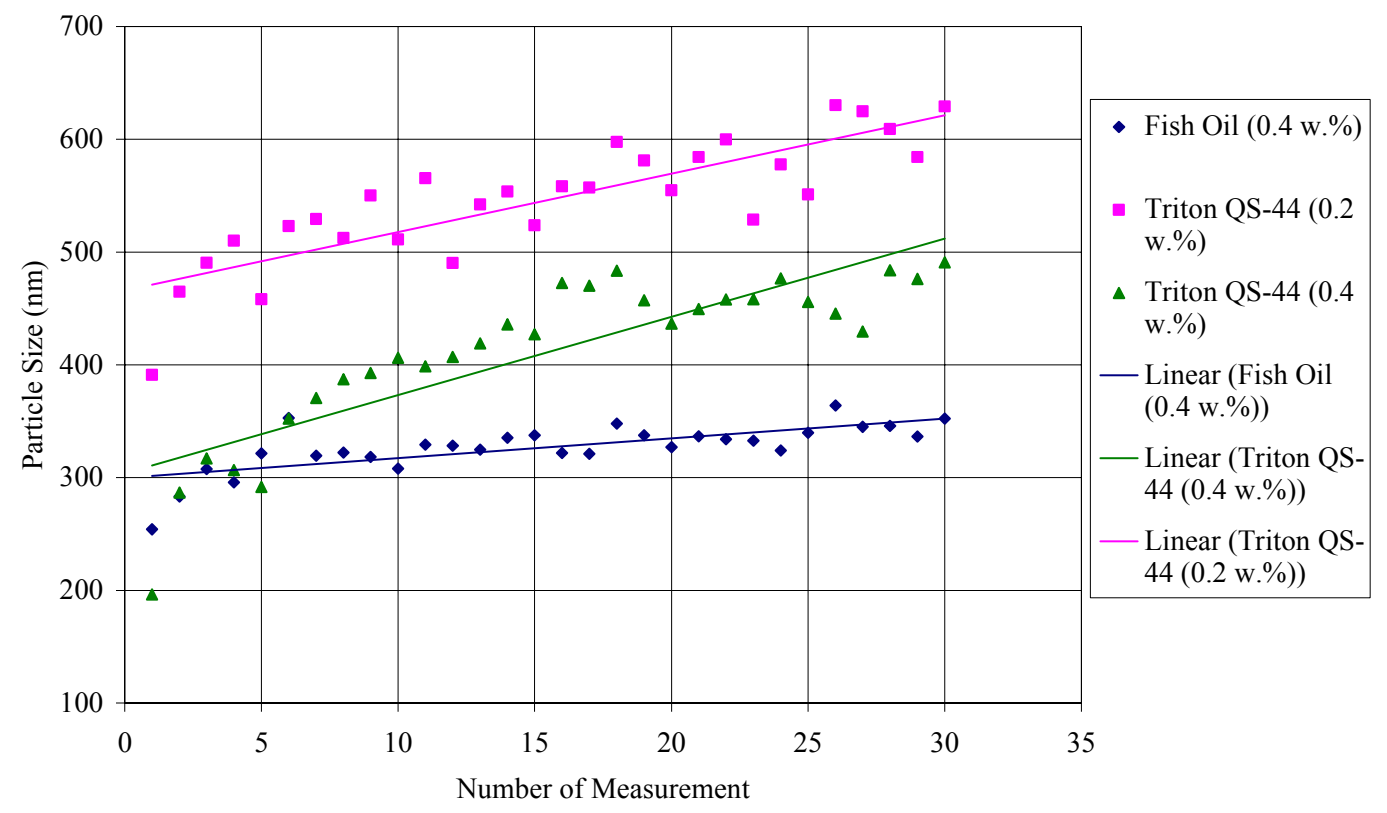

Figure 4-48. Particle size analysis of spinel nanopowders from TAL.

The diluted sample was sonicated for another 30 second and re-measured. As seen in Figure 4-49, instead of decreasing, the sizes increase with an even large rate of increasing as a function of time. These results seem confusing at this moment, and in later discussion, this abnormality is contributed to possible variation in the power of sonication.

Three new batches of samples were prepared; $0.4 \mathrm{w}$. \% fish oil, 0.3 and $0.6 \mathrm{w} . \%$ CoatOsil 1211 in 2-propanol. The $0.4 \mathrm{w} . \%$ fish oil sample has the same composition as the fish oil sample tested on August 17. No Triton samples were examined in this attempt. The solid loading in each batch is also $10 \mathrm{w} . \%$. The samples were ball milled for 27.5 hours after mixing. Similarly, $35 \mu \mathrm{L}$ of the slurry was withdrawn and mixed with 3 c.c. of 2-propanol to reduce its concentration. The results are shown in Figure 4-50. For the $0.4 \mathrm{w} . \%$ fish oil ample, the agglomerate size slowly increases from $200 \mathrm{~nm}$ to $250 \mathrm{~nm}$ in 10 minutes; these sizes here are about $100 \mathrm{~nm}$ smaller than those of the fish oil sample measured on August 17. The photomultiplier tube count rate of the current fish oil sample is $2250 \mathrm{k}$, and that of the other sample tested on August 17 is $2800 \mathrm{k}$; both of them are too large to obtain accurate size numbers. The 0.3 and $0.6 \mathrm{w} . \%$ CoatOsil samples have comparable agglomerate sizes of about $340 \mathrm{~nm}$. According to the trends here, it seems that fish oil is also superior to CoatOsil as dispersant in its ability of breaking up large agglomerates. 


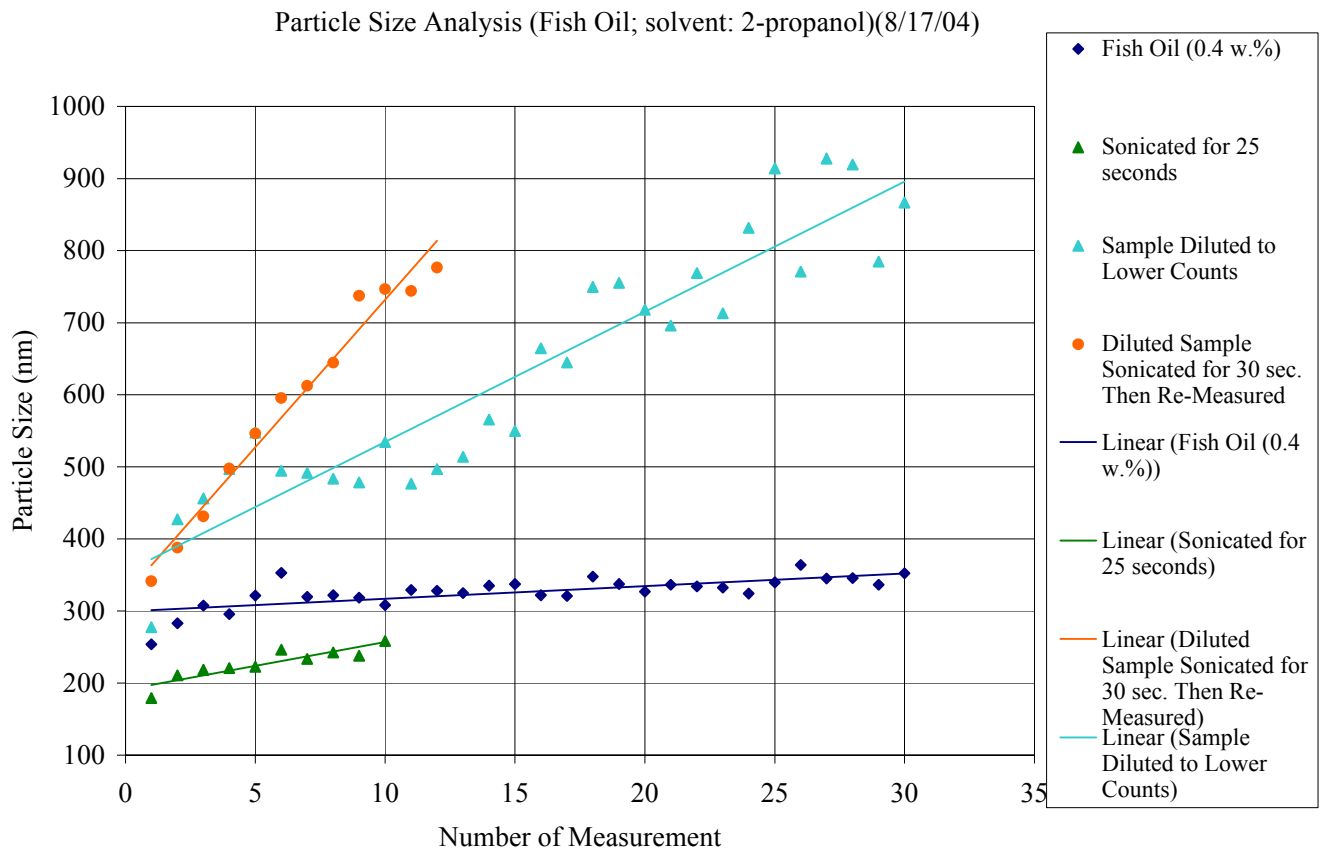

Figure 4-49. Particle size analysis of spinel nanopowders from TAL using fish oil as dispersant

Another batch of sample with $0.3 \mathrm{w} . \%$ CoatOsil was prepared by adding $35 \mu \mathrm{L}$ of the slurry into 3 c.c. of 2-propanol. This new 0.3 w. \% CoatOsil sample, together with the 0.6 w. \% CoatOsil sample and the $0.4 \mathrm{w}$. \% sample tested earlier were all sonicated for 30 seconds and re-measured. The results are shown in Figure 4-51. The agglomerate sizes of both CoatOsil samples decrease for about $70 \mathrm{~nm}$ after sonication. On the contrary, the sizes of the fish oil sample increase for about $60 \mathrm{~nm}$ after sonication. The agglomerate sizes of all samples are very close after sonication, about $260 \mathrm{~nm}$.

The behavior of the samples with fish oil after sonication is very inconsistent, sometimes the sizes increase, and at other times they increase. It is suspected that this is due to the power setting of the ultrasonic bath. It was not realized until some time later that the adjustable knob on the equipment is for power adjustment, not for time adjustment. As a result, the power of sonication may be different for different samples. It is assumed that very weak sonication, instead of breaking up large agglomerates as expected, may in fact promote agglomeration in fish oil samples.

To explore further the stability of slurry as a function of time, the samples prepared on August 17 with 0.4 w. \% Triton QS-44 and 0.4 w. \% fish oil were re-measured. Similarly, $35 \mu \mathrm{L}$ of the slurry was diluted with 3 c.c. of 2-propanol to reduce its concentration. The results are shown in Figure 4-52. For the Triton batch, the agglomerate sizes increase for an amount of $300 \mathrm{~nm}$ after 16 days of storage in a polypropylene bottle. In many of these measurements, the polydispersity index, an indication of the width of particle size distributions, is 1 . This suggests that the agglomerates in the sample are highly polydispersed with very wide size distributions. In sharp contrast, the agglomerate sizes of the fish oil sample, after 16 days, not only show no signs of increasing, they actually 
decrease by about $40 \mathrm{~nm}$. At the end of the test, the count rate of the fish oil sample is $2500 \mathrm{k}$, and that of the Triton sample is $2700 \mathrm{k}$. These results hint that the sample with fish oil is more stable than that with Triton as a function of time; the particles in the Triton sample easily form soft agglomerates that can be easily broken up by sonication. On the contrary, the sizes of the already small agglomerates in fish oil sample only reduce a little by sonication.

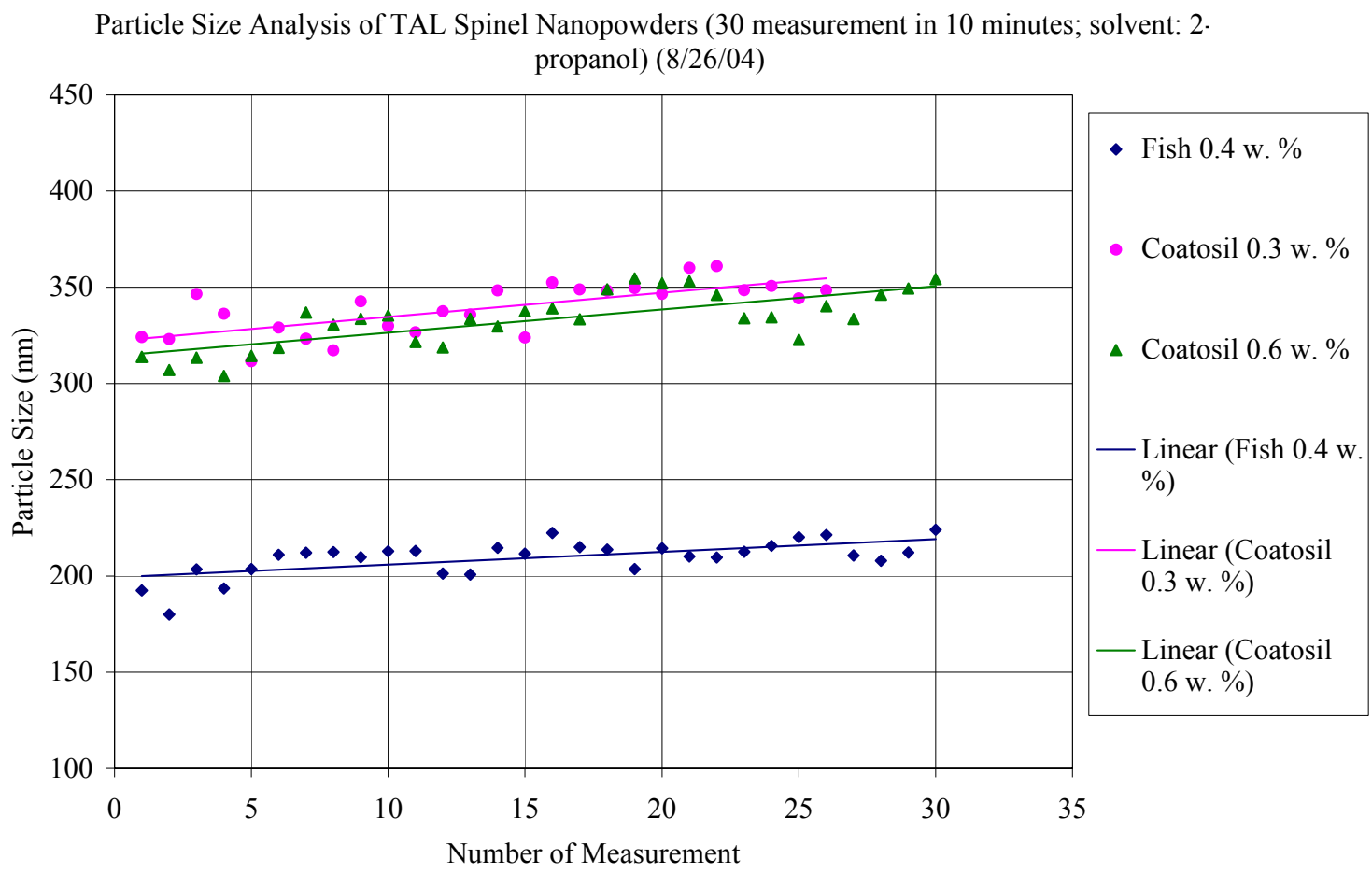

Figure 4-50. Particle size analysis of spinel nanopowders from TAL

Both sample were diluted by replacing $2.9 \mathrm{cc}$ of the suspension with 2-propanol. After dilution, the photomultiplier tube count rates dropped to $370 \mathrm{k}$ for the Triton sample, and to $136 \mathrm{k}$ for the fish oil sample. The final count rates for both samples are well within the required level. The agglomerate sizes for both samples increase for about $50 \mathrm{~nm}$ after dilution. It is believed that the current data most accurately reflect the true sizes of agglomerates in each sample. 
Particle Size Analysis of TAL Nanopowders after Sonication (30 measurement in 10 minutes; solvent: 2-propanol) 8/26/04

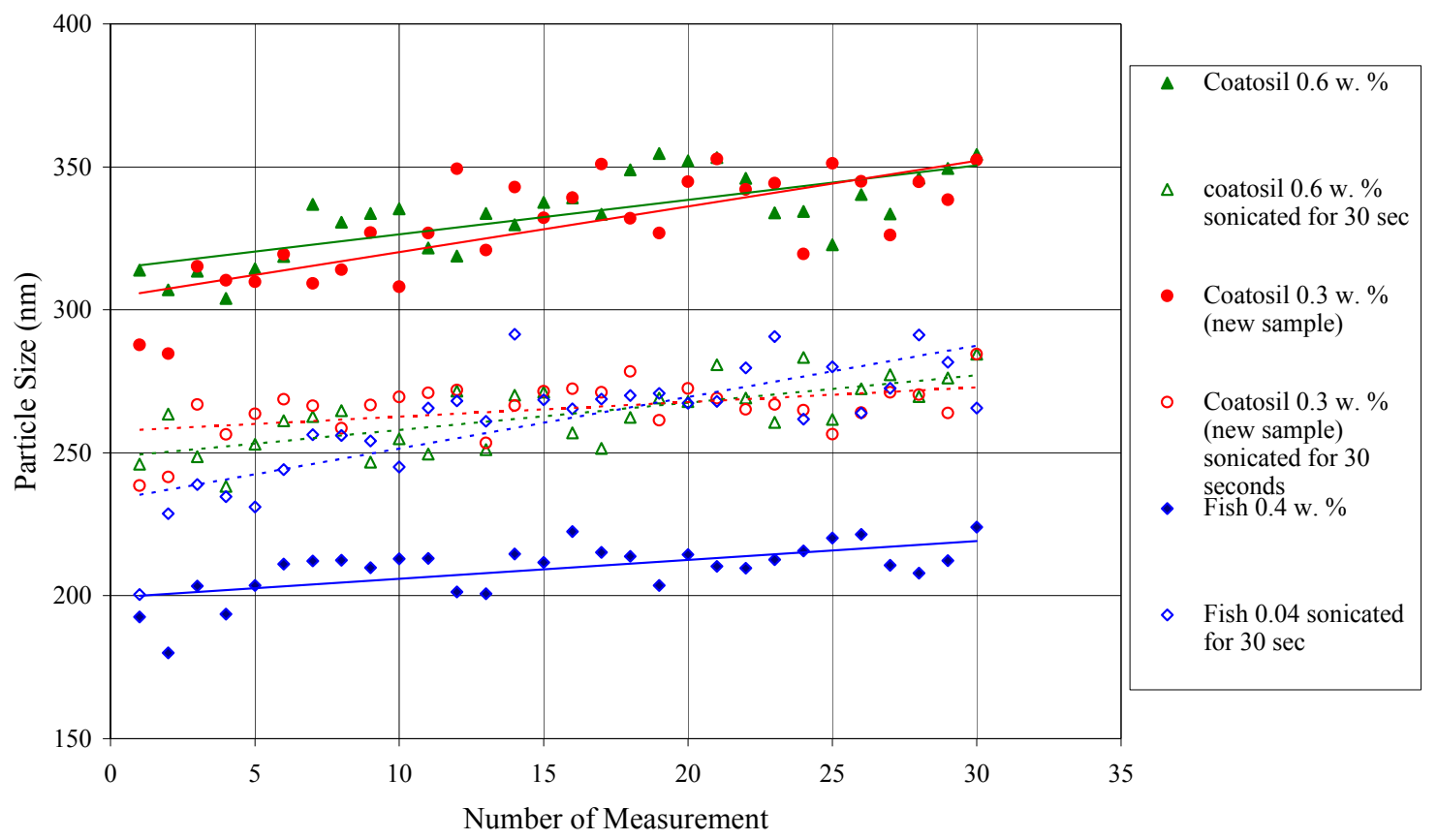

Figure 4-51. Particle size analysis of spinel nanopowders from TAL after sonication

Particle Size Analysis of TAL Spinel Nonopowders using Triton as Dispersant (9/2/04)

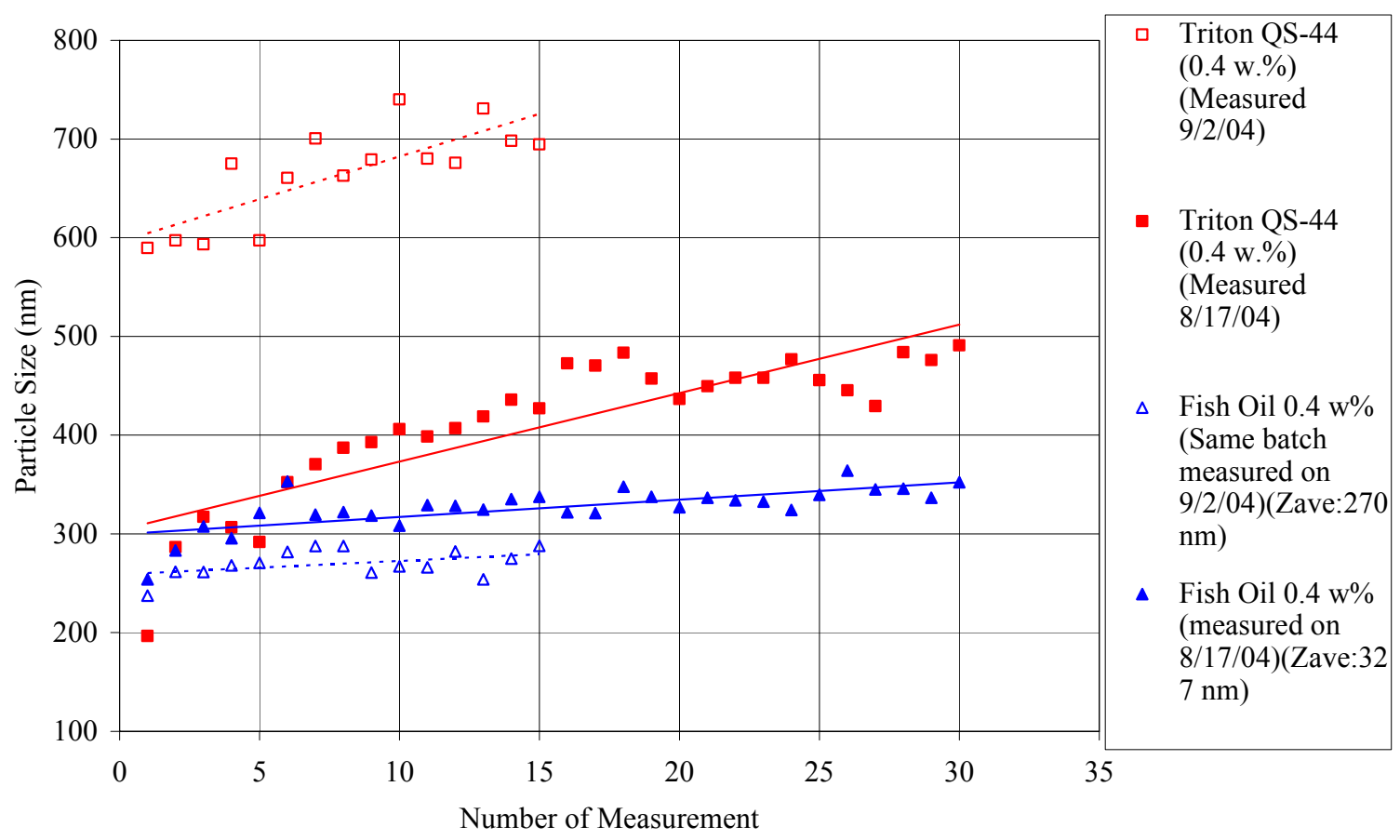

Figure 4-52. Particle size analysis of spinel nanopowders from TAL

New Optical Sensor Suite for Ultrahigh Temperature Fossil Fuel Applications 
Particle Size Analysis of TAL Spinel Nanop[owders using Triton and fish oil as dispersants

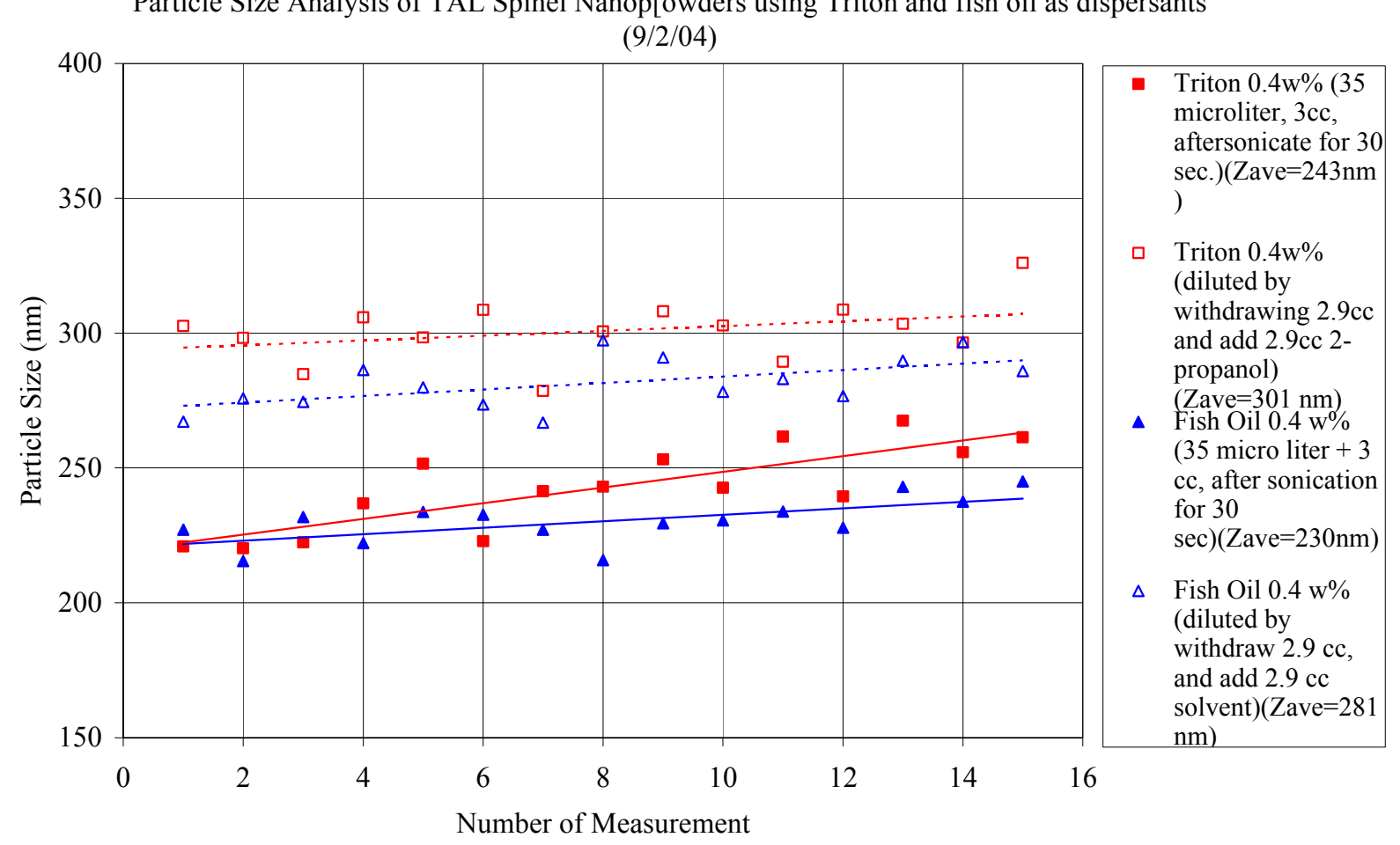

Figure 4-53. Particle size analysis of spinel nanopowders from TAL

To explore the suspension stability of CoatOsil samples, the sample prepared on August 26 with 0.3 w. $\%$ CoatOsil was re-measured. Similarly, $35 \mu \mathrm{L}$ of the slurry was diluted with $3 \mathrm{cc}$ of 2-propanol to reduce its concentration. The results are shown in Figure 4-54. Similar to the fish oil cases, the sample prepared using CoatOsil also shows no signs of size increase after 7 days of storage; in fact, the agglomerate sizes measured here are about $18 \mathrm{~nm}$ smaller than those measured initially. The sample was further diluted by replacing $2.9 \mathrm{cc}$ of the suspension with 2-propanol. After dilution, the photomultiplier tube count rates dropped from $2600 \mathrm{k}$ to $512 \mathrm{k}$, and the agglomerate sizes increase from $321 \mathrm{~nm}$ to $353 \mathrm{~nm}$. The suspension was further diluted by replacing 50\% of the suspension with 2-propanol, as a result the count rate dropped further to $283 \mathrm{k}$, and the agglomerate size is almost the same after the $50 \%$ dilution. This indicate that the particle concentration has reaches the desired level where reproducible size data can be obtained. Furthermore, in about 5 minutes, the agglomerate sizes show no sign of increasing. 


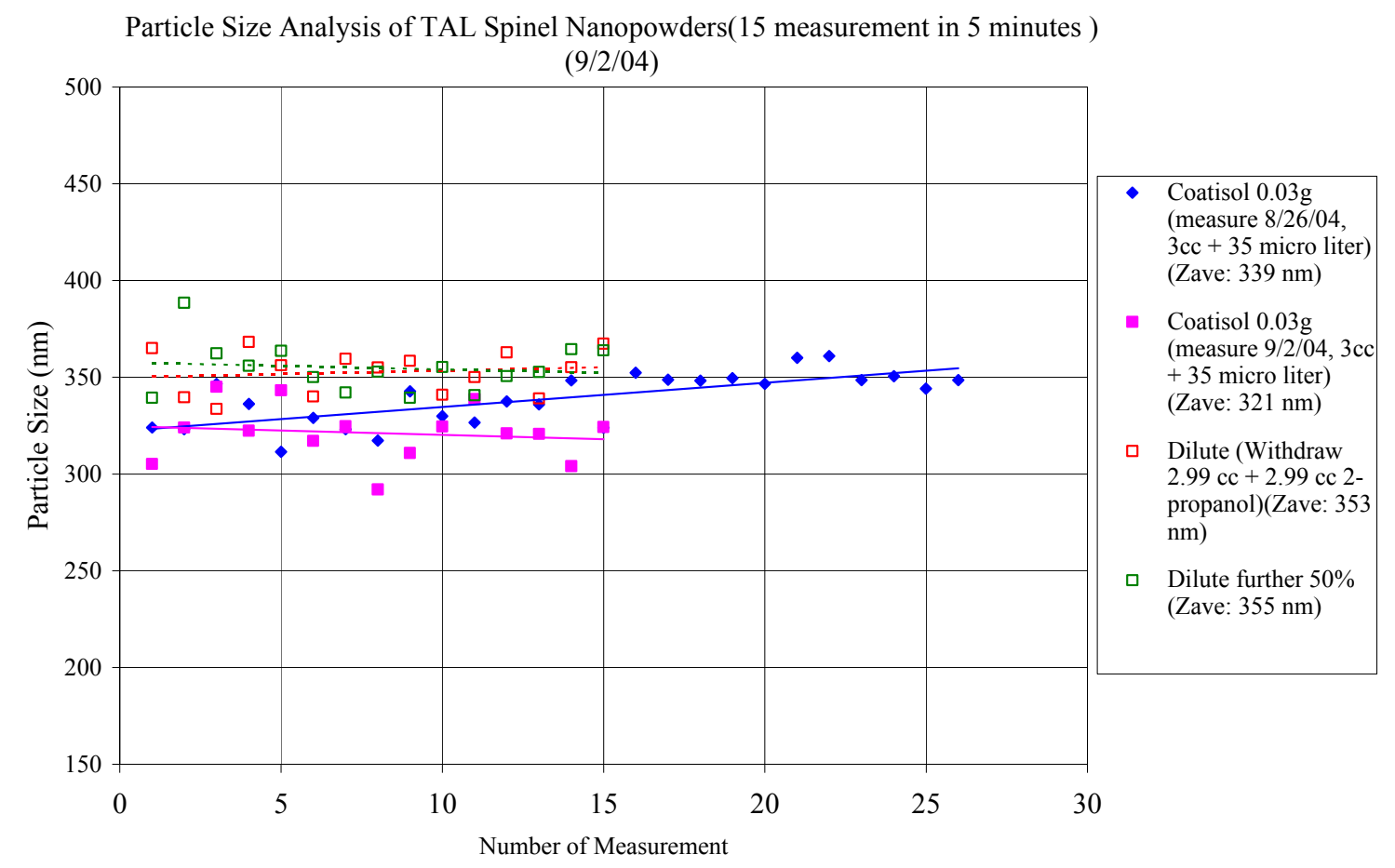

Figure 4-54. Particle size analysis of spinel nanopowders from TAL

\subsubsection{Particle Size Analysis of MgO nanopowders from Aldrich Using Zetasizer with Menhaden Fish Oil and CoatOsil as dispersant}

After the confidence on the data obtained using Zetasizer has been established using the spinel nanopowders from TAL, it is a good time now to measure the $\mathrm{MgO}$ nanopowders from Aldrich, which is one of the powder components used to prepare the clad sapphire fibers.

Two batches of samples were prepared with 0.4 w. \% CoatOsil 1211 and 0.5 w. $\%$ Menhaden fish oil as dispersant. The solid loading was 10 w. \% for both samples. The samples were ball milled for 21 hours. Before measurement, $35 \mu \mathrm{L}$ of the slurry was mixed with $3 \mathrm{cc}$ of 2-propanol, and the sample was further diluted by replacing $2.9 \mathrm{cc}$ of the suspension with 2-propanol. The sample was measured 30 times in about 10 minutes. The data gathering time for each measurement was set to be 10 seconds. The results are shown in Figure 4-55. The photomultiplier tube count rates for the two measurements are $190 \mathrm{k}$. The average agglomerate size is $412 \mathrm{~nm}$, and there is little sign of size increase in 10 minutes.

The 10-second data gathering time for each measurement was an arbitrarily chosen time to obtain preliminary size data, and it is not long enough to obtain size data with high resolution. Therefore, the sample was measured again using the parameters "Auto 
Period" and "Auto Mode" in the equipment setting. In this setting it takes about 10 minutes for each single measurement. The results of the measurement using "Auto Period" and "Auto Mode" are shown in Figure 4-56(a). Here the volume percentage of the powders as a function of agglomerate size is plotted. The photomultiplier tube count rate was 189k, and the polydispersity index was 0.299. As can be seen in Figure 4-56(a), there are three distinctive size distributions; the first one centers at $42.8 \mathrm{~nm}$ with a volume percentage of only $1 \%$, and the second one at $440 \mathrm{~nm}$ with $83 \mathrm{v} . \%$, and the last one at $707 \mathrm{~nm}$ with $16 \mathrm{v} . \%$. As mentioned earlier, the powder size provided by the manufacturer is only $13 \mathrm{~nm}$. It is believed that the nano powders form agglomerates with a variety of size distributions as illustrated in Figure 4-56(a). The average agglomerate size is $401 \mathrm{~nm}$. This size is quite close to the average sizes of $412 \mathrm{~nm}$ obtained using a 10 -second measurement period.

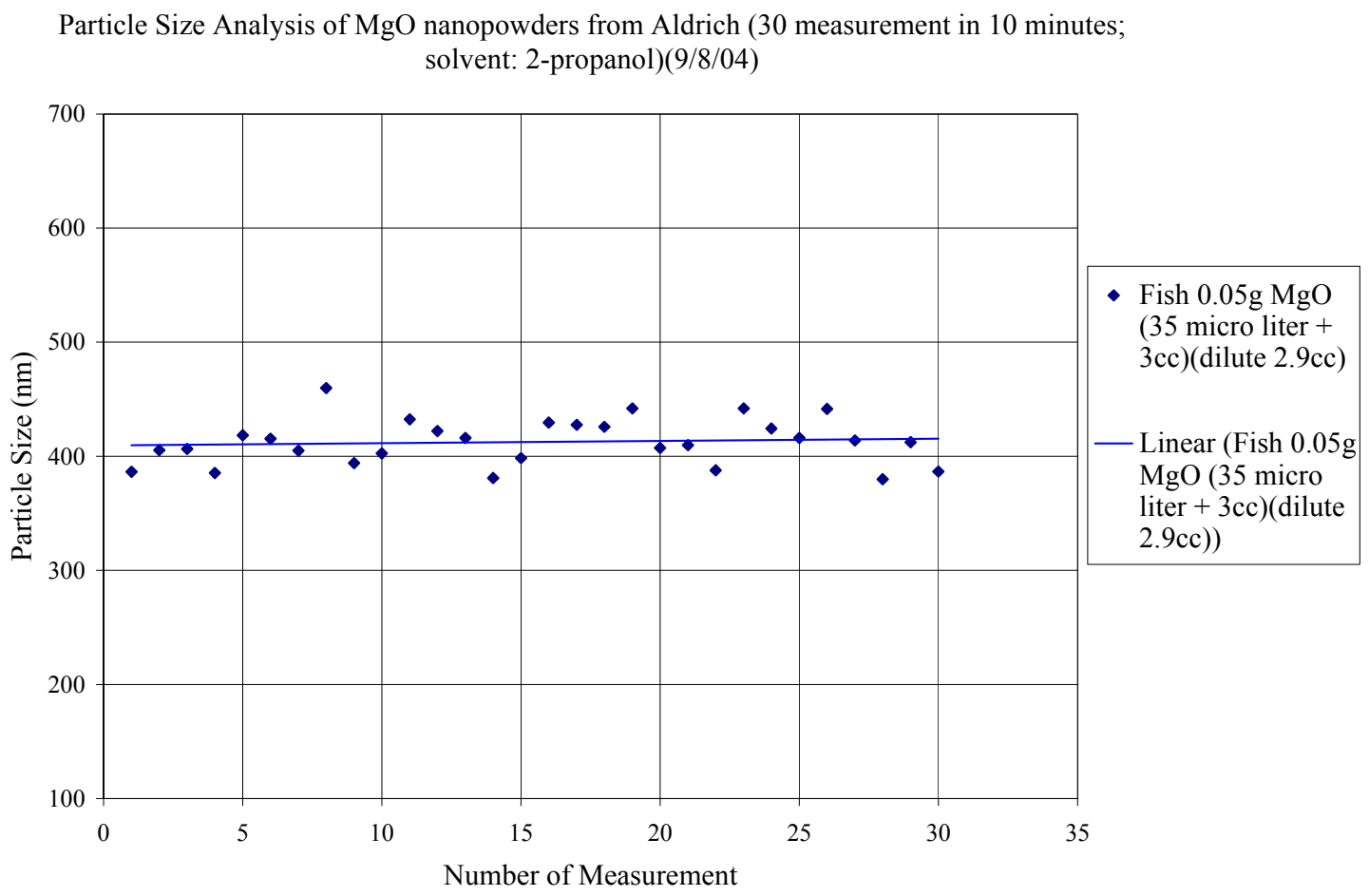

Figure 4-55. Particle size analysis of MgO nanopowders from Aldrich dispersed using fish oil.

The sample was sonicated for 30 seconds and re-measured. The count rate was $212 \mathrm{k}$, and the polydispersity index was 0.031 . The results are shown in Figure 4-56(b). In this figure there are only two size distributions, one at $271 \mathrm{~nm}$ with $81 \mathrm{v} . \%$, and the other at $405 \mathrm{~nm}$ with $19 \mathrm{v} . \%$. The average agglomerate size is $258 \mathrm{~nm}$. After sonication, the size peaks existing before sonication are shifted to smaller peak locations. It is assumed that the larger soft agglomerates are broken apart by sonication and form smaller agglomerates. The same sample was measured again one hour after sonication, and the results are shown in Figure 4-57. The count rate was 208k, and the polydispersity index was 0.245. There is only one broader size distribution with an average agglomerate size of $264 \mathrm{~nm}$. 
This distribution is quite similar to the one measured immediately after sonication, with a slight size increase of $6 \mathrm{~nm}$. It is suspected that in the time period of 1 hour there is no appreciable re-agglomeration of the powders in the suspension.

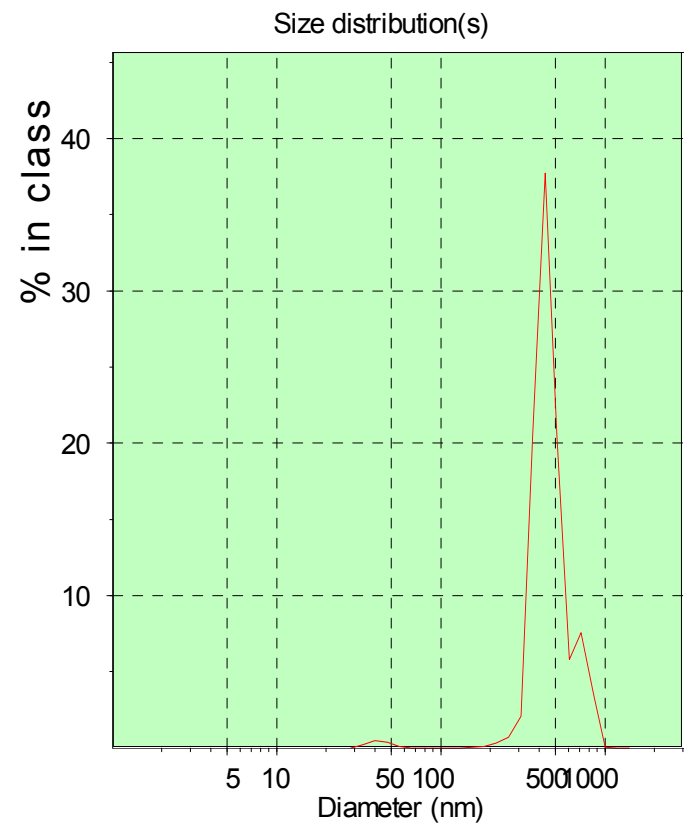

(a)

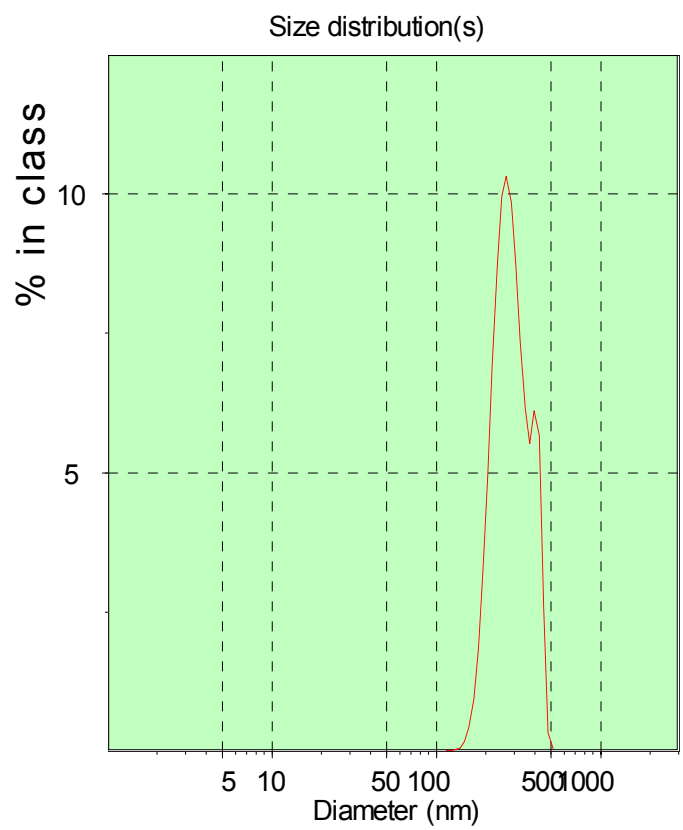

(b)

Figure 4-56. Particle size analysis of MgO nanopowders dispersed using fish oil (a) before, and (b) after 30 seconds of sonication

The results of the sample dispersed using 0.4 w. \% CoatOsil 1211 are shown in Figure 4-58. Here the sample was measured 45 times in about 20 minutes. Before measurement, $35 \mu \mathrm{L}$ of the slurry was mixed with $3 \mathrm{cc}$ of 2-propanol, and the sample was further diluted by replacing $2.9 \mathrm{cc}$ of the suspension with 2-propanol. The data gathering time for each measurement was 10 seconds, and the count rate is $385 \mathrm{k}$. The average agglomerate sizes are $357 \mathrm{~nm}$. The size slightly increases for about $17 \mathrm{~nm}$ in 20 minutes. The sample was re-measured again using the "Auto Period" and "Auto Mode", and the volume fractions of agglomerates are plotted as a function of size in Figure 4-59. As shown in Figure 4-59(a), before sonication, there is one particle size peak at $393 \mathrm{~nm}$, and the average size is $359 \mathrm{~nm}$. The polydispersity index was 0.268 . 


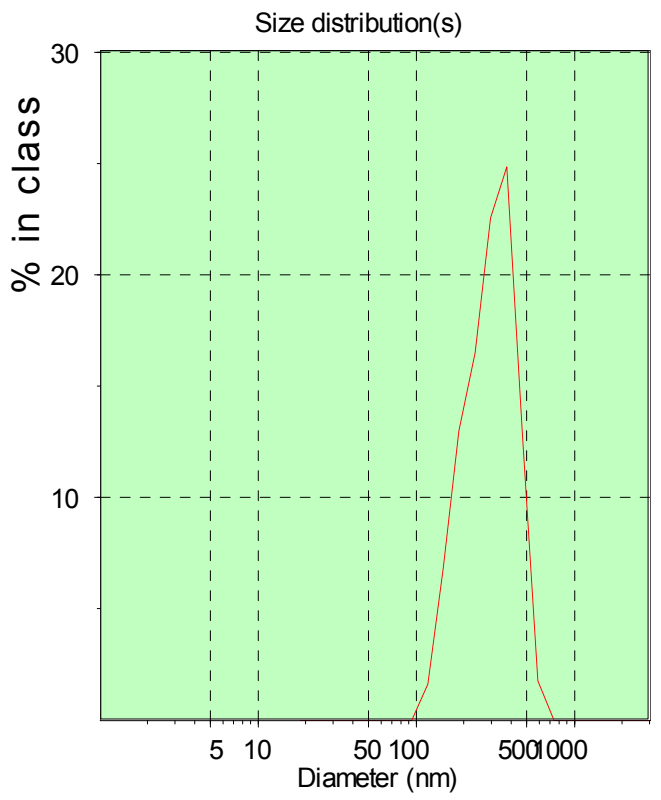

Figure 4-57. Particle size analysis of MgO nanopowders from Aldrich dispersed using fish oil, one hour after sonication.

Particle Size Analysis of MgO nanopowders from Aldrich (In 20 minutes; solvent: 2propanol $)(9 / 8 / 04)$

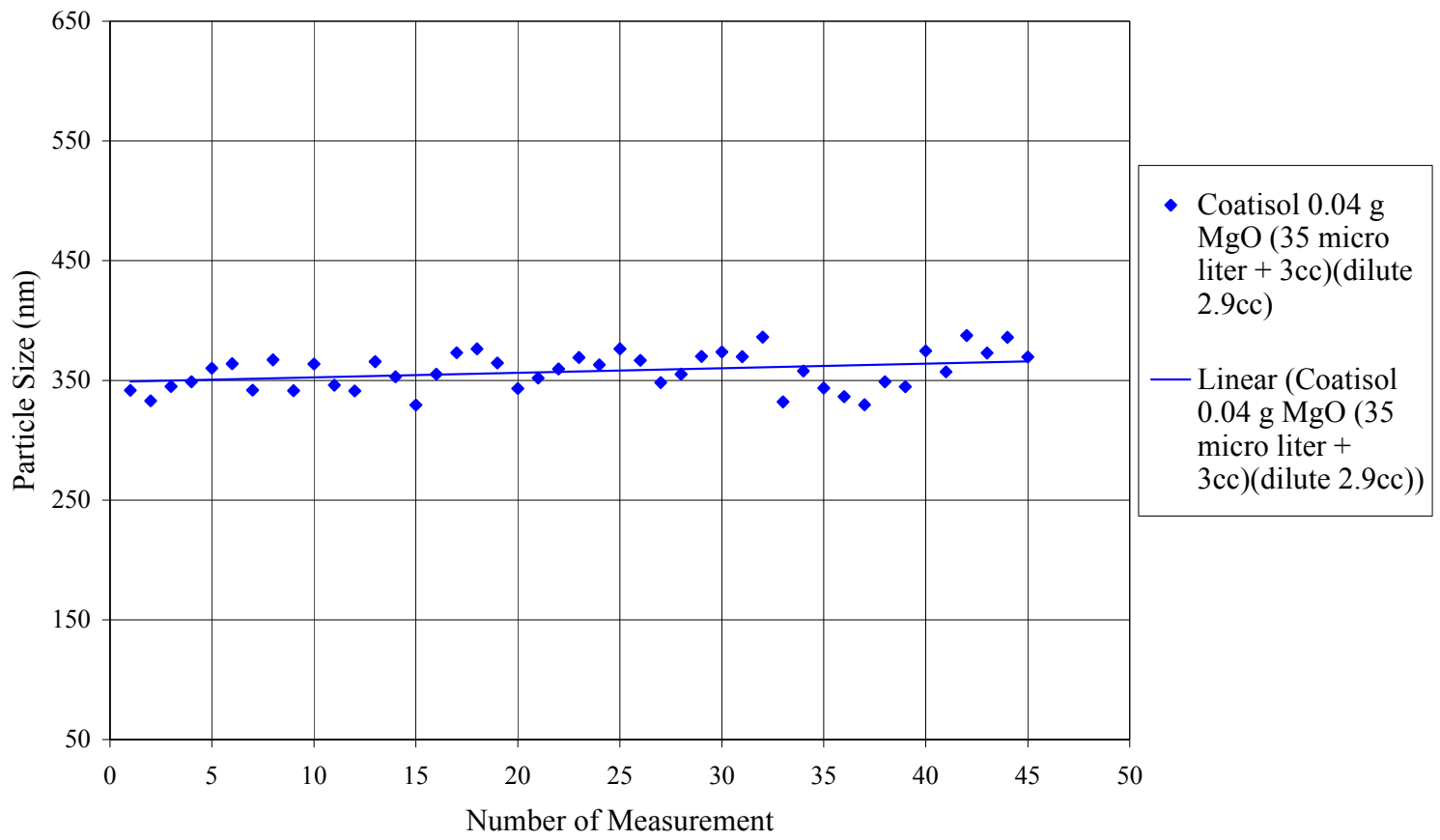

Figure 4-58. Particle size analysis of MgO nanopowders from Aldrich 

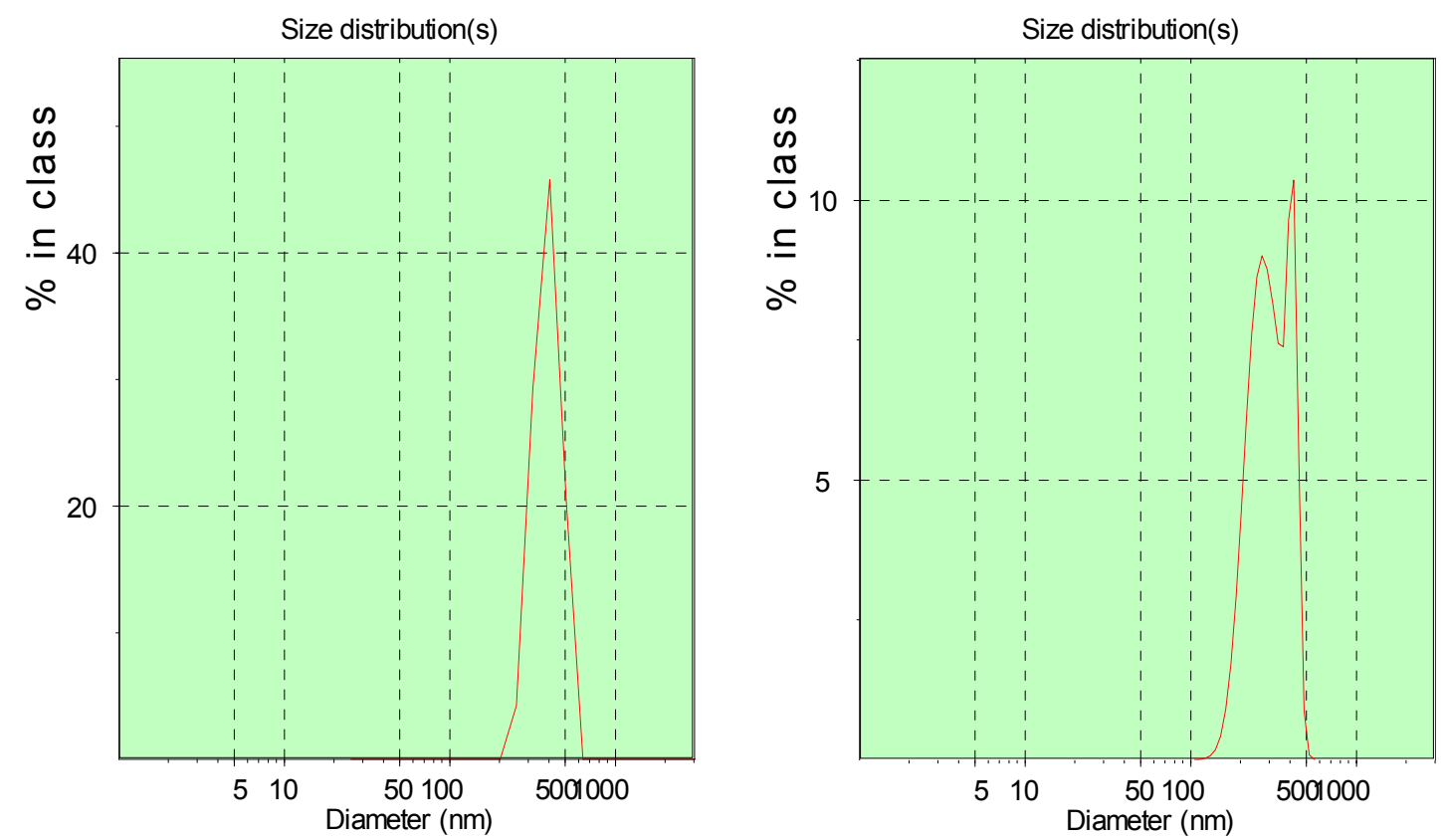

Figure 4-59. Particle size analysis of MgO nanopowders dispersed using CoatOsil 1211 (a) before, and (b) after 30 seconds of sonication

After 30 seconds of sonication, as shown in Figure 4-59(b), another peak at a smaller size, $271 \mathrm{~nm}$, develops with 69 v. \%. Another peak is located at $404 \mathrm{~nm}$, very close to the one before sonication, $393 \mathrm{~nm}$. The average size is $259 \mathrm{~nm}$. The polydispersity index is 0.038 . These results again agree with the assumption that larger agglomerates were broken up by sonication into smaller agglomerates. The sample was re-measured 20 minutes after sonication, the results show that there is one size peak with an average agglomerate size of $262 \mathrm{~nm}$, very close to the one immediately after sonication, $259 \mathrm{~nm}$.

In this attempt two $\mathrm{MgO}$ nanopowder (from Aldrich) samples were prepared with higher concentrations of CoatOsil 1211, 0.8 w. $\%$ and 1.2 w. \%. The solid loading was $10 \%$ in each sample. The samples were ball milled. Similarly, before measurement, $35 \mu \mathrm{L}$ of the slurry was diluted with $3 \mathrm{cc}$ of 2-propanol, and the concentration was further diluted by replacing $2.9 \mathrm{cc}$ of the suspension with 2-propanol. The results are shown in Figure 4-60. The $0.8 \mathrm{w} . \%$ sample was tested twice, and the $1.2 \mathrm{w} . \%$ sample was tested one. In each test fifteen measurements were take in five minutes. Also shown in Figure 4-60 are the results of the $0.4 \mathrm{w}$. \% sample measured on September 2. The average agglomerate size for the $0.8 \mathrm{w}$. $\%$ sample is $456 \mathrm{~nm}$, and that for $1.2 \mathrm{w}$. $\%$ is $425 \mathrm{~nm}$. The average agglomerate size for the $0.4 \mathrm{w}$. \% sample tested on September 2 is $359 \mathrm{~nm}$. The results indicate that the increase of CoatOsil concentration from $0.4 \mathrm{w}$. \% to 0.8 and $1.2 \mathrm{w} . \%$ does not decrease the agglomerate sizes as anticipated. And the average size of the $1.2 \mathrm{w}$. $\%$ sample is even smaller than that of the $0.8 \mathrm{w}$. \% sample. The $0.8 \mathrm{w} . \%$ sample was remeasured using the "Auto Period" and "Auto Mode". The results are shown in Figure 4-61(a). There is a highly localized peak at $451 \mathrm{~nm}$. The count rate was $305 \mathrm{k}$ and the polydispersity index was 0.164 . The results after 30 seconds of sonication are shown in Figure 4-61(b). The size drastically decreases to a highly localized peak at $246 \mathrm{~nm}$. The 
count rate was $290 \mathrm{k}$ and the polydispersity index was 0.632 . It is not clear why such a localized size distribution can have such a large polydispersity index.

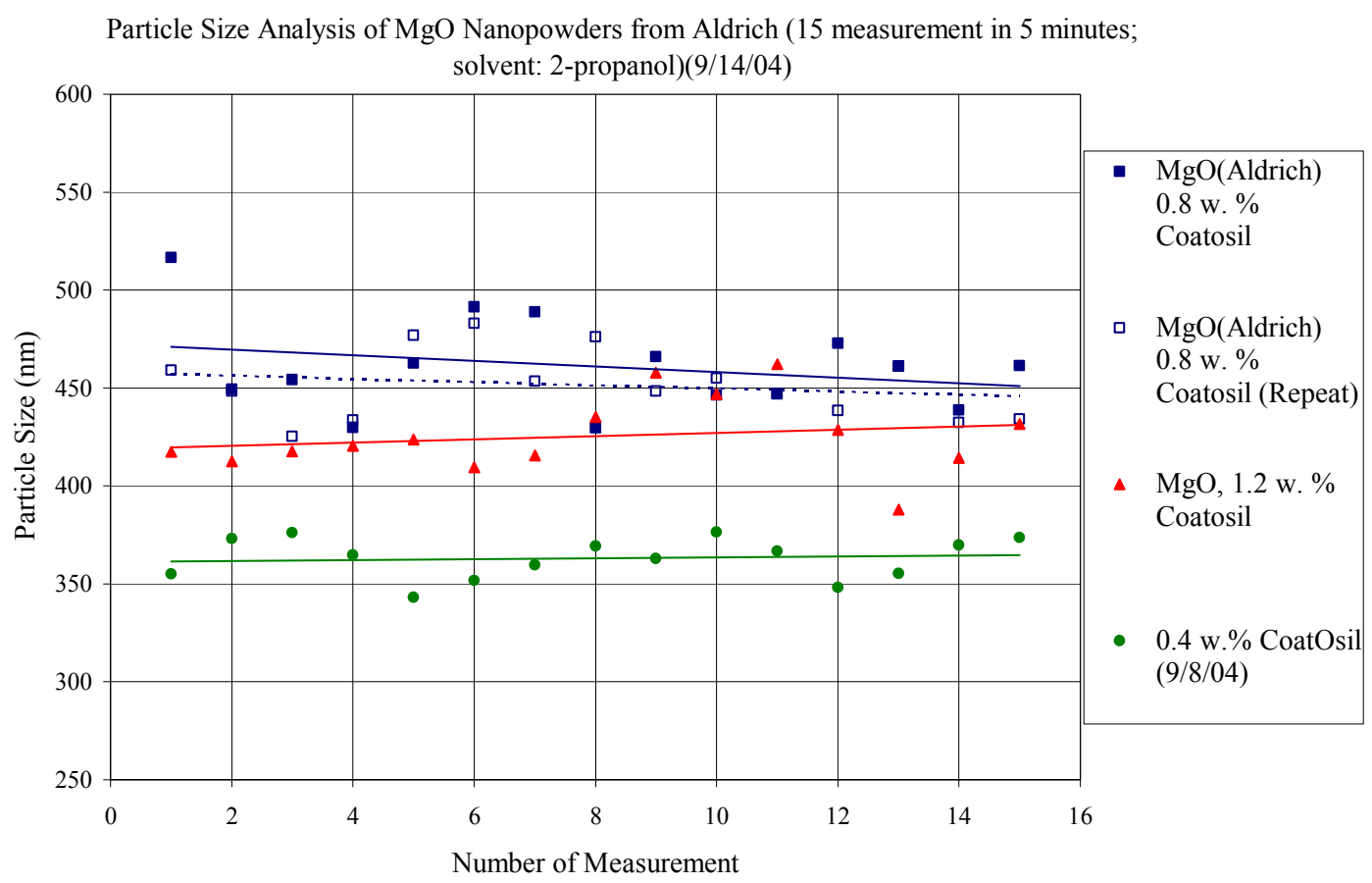

Figure 4-60. Particle size analysis of MgO nanopowders from Aldrich
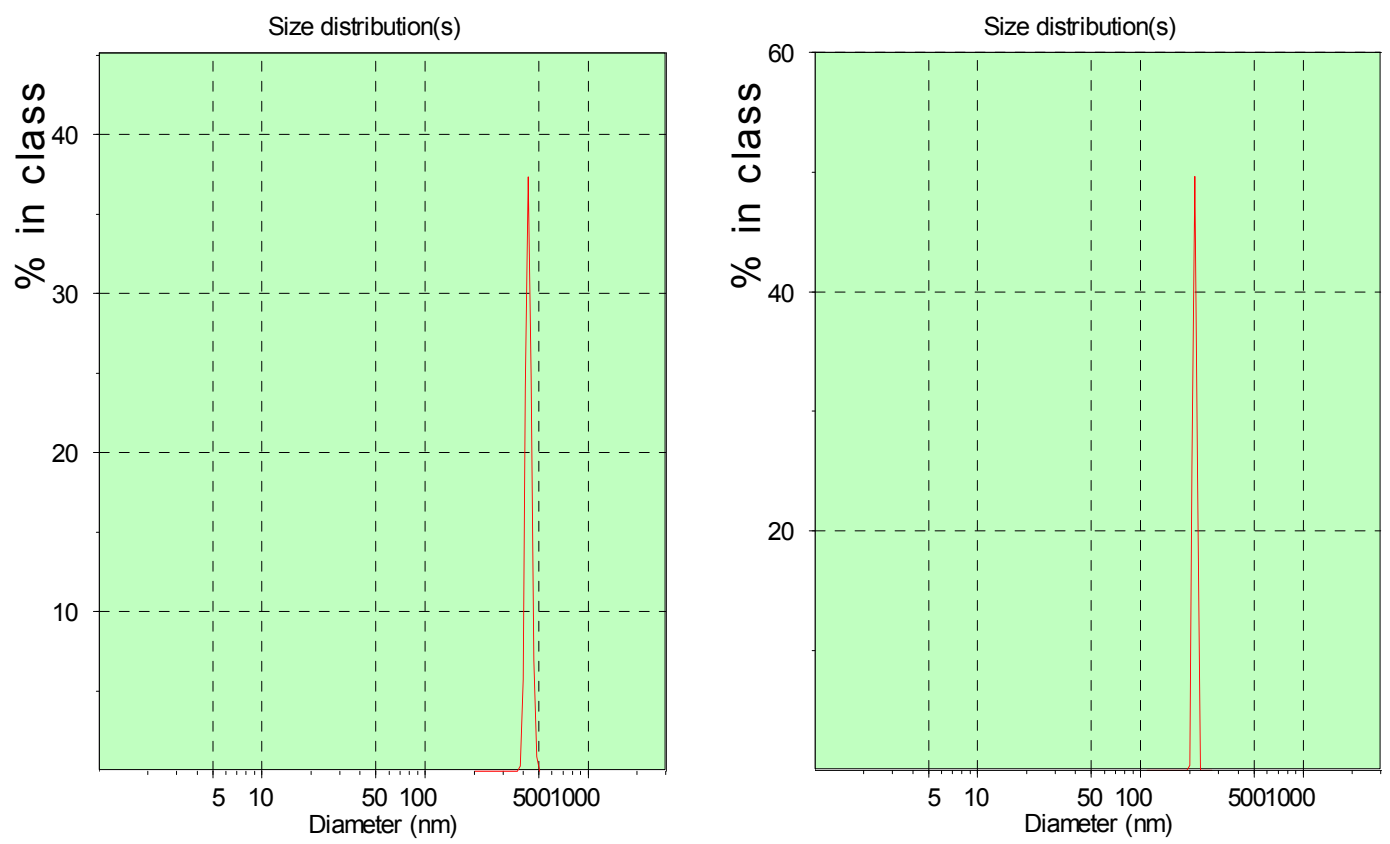

Figure 4-61. Particle size analysis of MgO nanopowders dispersed using CoatOsil 1211 (a) before, and (b) after 30 seconds of sonication 
The 1.2 w. \% sample was also re-measured using the "Auto Period" and "Auto Mode". The results are shown in Figure 4-62(a). There are two peaks, one located at $427 \mathrm{~nm}$ with $77 \mathrm{v} . \%$ and the other at $705 \mathrm{~nm}$ with $23 \mathrm{v}$. \%. The average size is $424 \mathrm{~nm}$, and the polydispersity is 0.187 . The sample was also sonicated for 30 seconds, and the results are shown in Figure 4-62(b). There are also two peaks in the size distribution. However, the peaks are located in much smaller sizes, one at $274 \mathrm{~nm}$ with $67 \mathrm{v}$. \%, and the other at $58.3 \mathrm{~nm}$ with $33 \mathrm{v} . \%$.
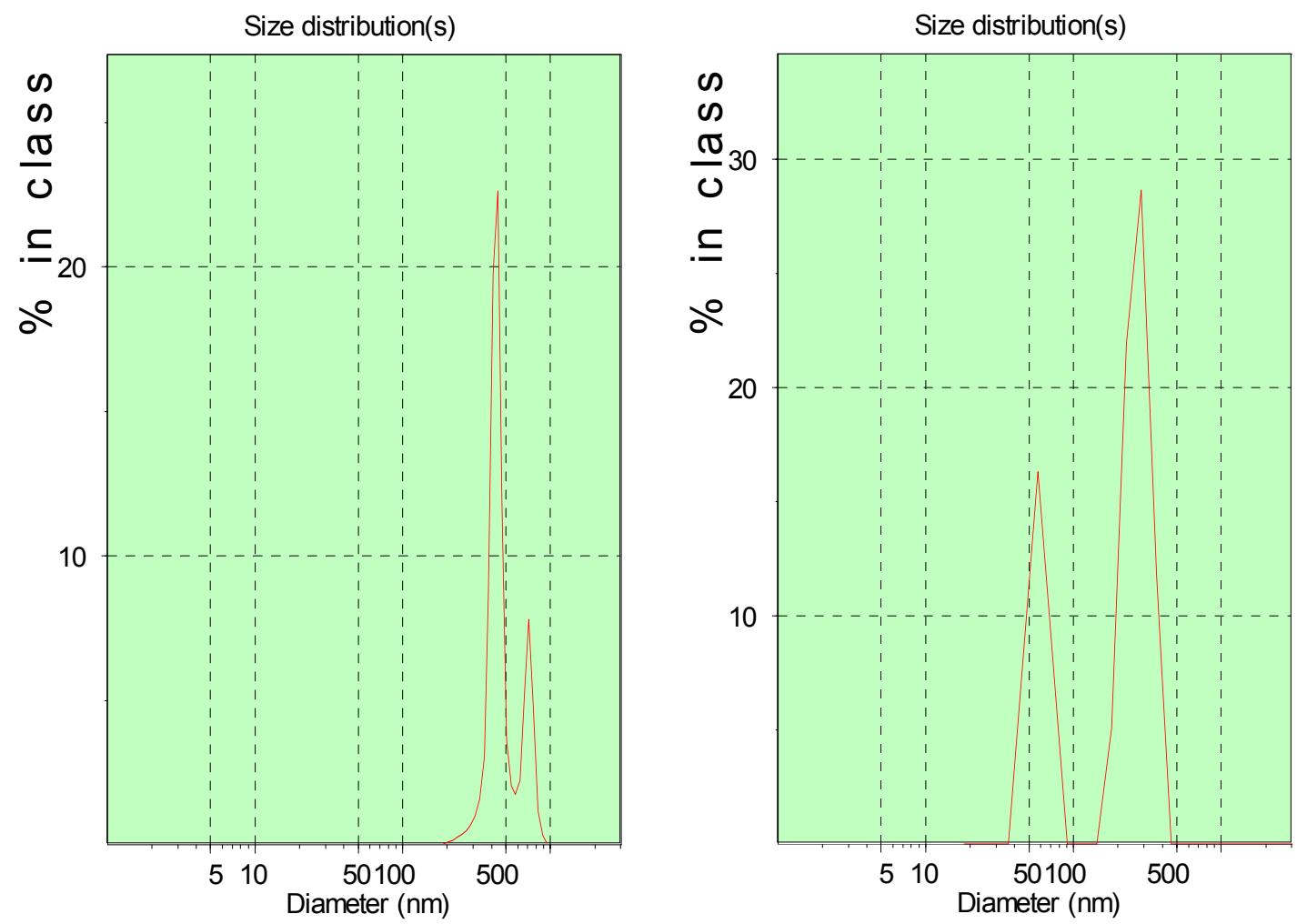

Figure 4-62. Particle size analysis of MgO nanopowders dispersed using CoatOsil 1211 (a) before, and (b) after 30 seconds of sonication

\subsubsection{Use a graticule with $10 \mu$ grid to estimate the particle sizes of the $\mathrm{MgO}$ powders from EM Science and the spinel powders from Baikalox under an optical microscope}

As mentioned earlier, the Zetasizer can only measure particles with sizes of less than $1 \mu \mathrm{m}$. Therefore, other means are needed to determine the particle sizes of the other two types of powders, $\mathrm{MgO}$ from EM Science and spinel from Baikalox. In this section the results of the particle size measurement using a graticule with scale $1 \mathrm{~mm}$ long in $0.01 \mathrm{~mm}(10 \mu \mathrm{m})$ divisions are discussed. The graticule $(01 \mathrm{~B} 21217, \mathrm{NE} 28-21 \mathrm{~mm}$, $1 \mathrm{~mm} / 100 \mathrm{Pts})$ was purchased from Pyser-Sgi Ltd. in UK. 
Two batches of $\mathrm{MgO}$ samples were prepared with 0.4 and 0.8 w. \% CoatOsil 1211 dispersant. The solid loading was $10 \mathrm{w} . \%$ for each sample. The periods of ball milling of the samples were not recorded. Four drops of the slurry were diluted with $3.6 \mathrm{~g}$ of 2propanol before measurement. The pipettes used for slurry transportation are the fine-tip disposable transfer pipettes from VWR International (Cat. \#: 14670-330). Four drops of the slurry have a volume of about $85 \mu \mathrm{L}$. One drop of slurry was dispensed on the graticule for observation under an optical microscope. Two graticule samples were prepared and observed for each batch of slurry. Several pictures were taken from one graticule sample. The representative results are shown in Figure 4-63(a) to (d). Figure 4-63(a) and (b) show the representative pictures for $0.8 \mathrm{w}$. \% CoatOsil slurry, sample 1 and sample 2, respectively. Figure 4-63(c) and (d) show the representative pictures for 0.4 w. \% CoatOsil slurry, sample 1 and sample 2, respectively. As shown in the pictures, it is clear that the two samples with $0.4 \mathrm{w} . \%$ CoatOsil form larger agglomerates on the graticule than those with $0.8 \mathrm{w}$. \% CoatOsil. Image processing was performed on Figure 4-63(a) to enhance the visibility of powder particles. The results are shown in Figure 4-64. As mentioned earlier, the total length of the scale is $1 \mathrm{~mm}$ with 100 divisions, so each division is $0.01 \mathrm{~mm}(10 \mu \mathrm{m})$ long. The yellow bars are $2.7 \mu \mathrm{m}$ wide. All the particles seem to be less than the width of the bar, so all the particles are less than $2.7 \mu \mathrm{m}$. The smallest particles are about $1 \mu \mathrm{m}$. A rough estimation puts the average particle size at about $1.7 \mu \mathrm{m}$.

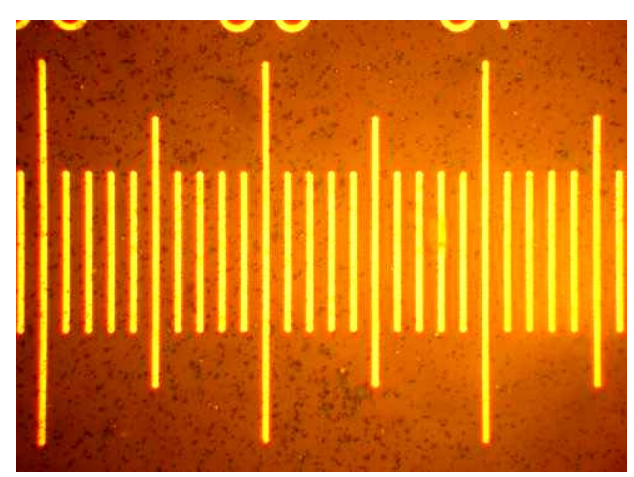

(a) 0.8 w. \% CoatOsil, sample 1

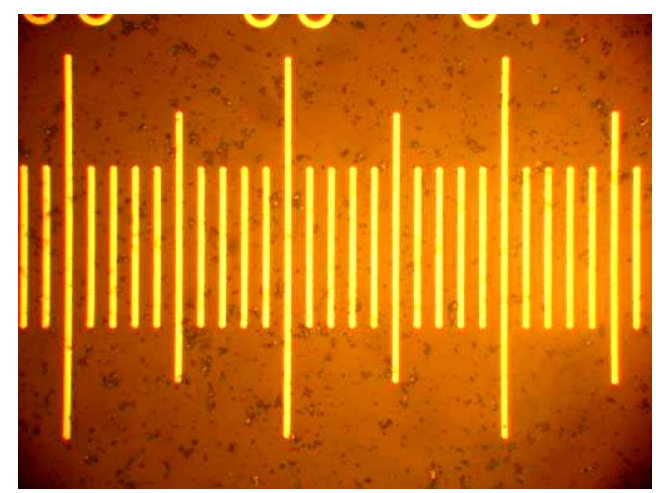

(c) 0.4 w. \% CoatOsil, sample 1

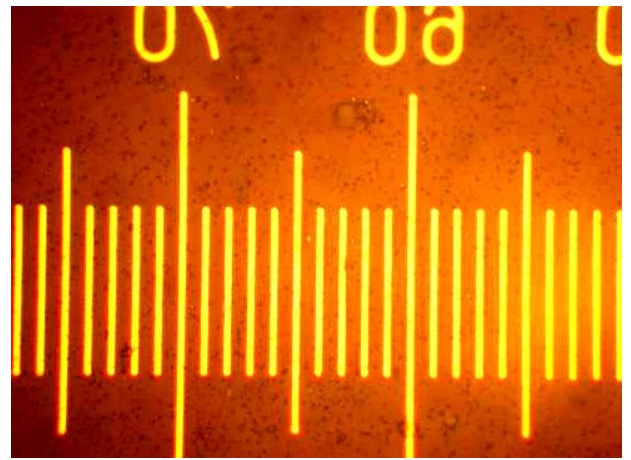

(b) 0.8 w. \% CoatOsil, sample 2

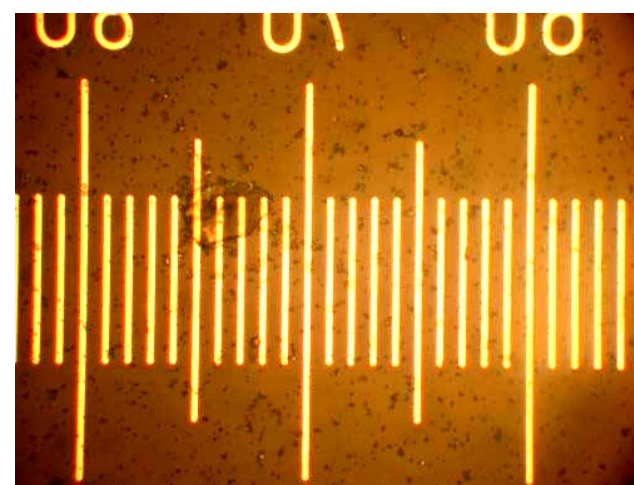

(d) 0.4 w. \% CoatOsil, sample 2

Figure 4-63. The particle analysis of MgO powders from EM Science using a graticule under an optical microscope. 


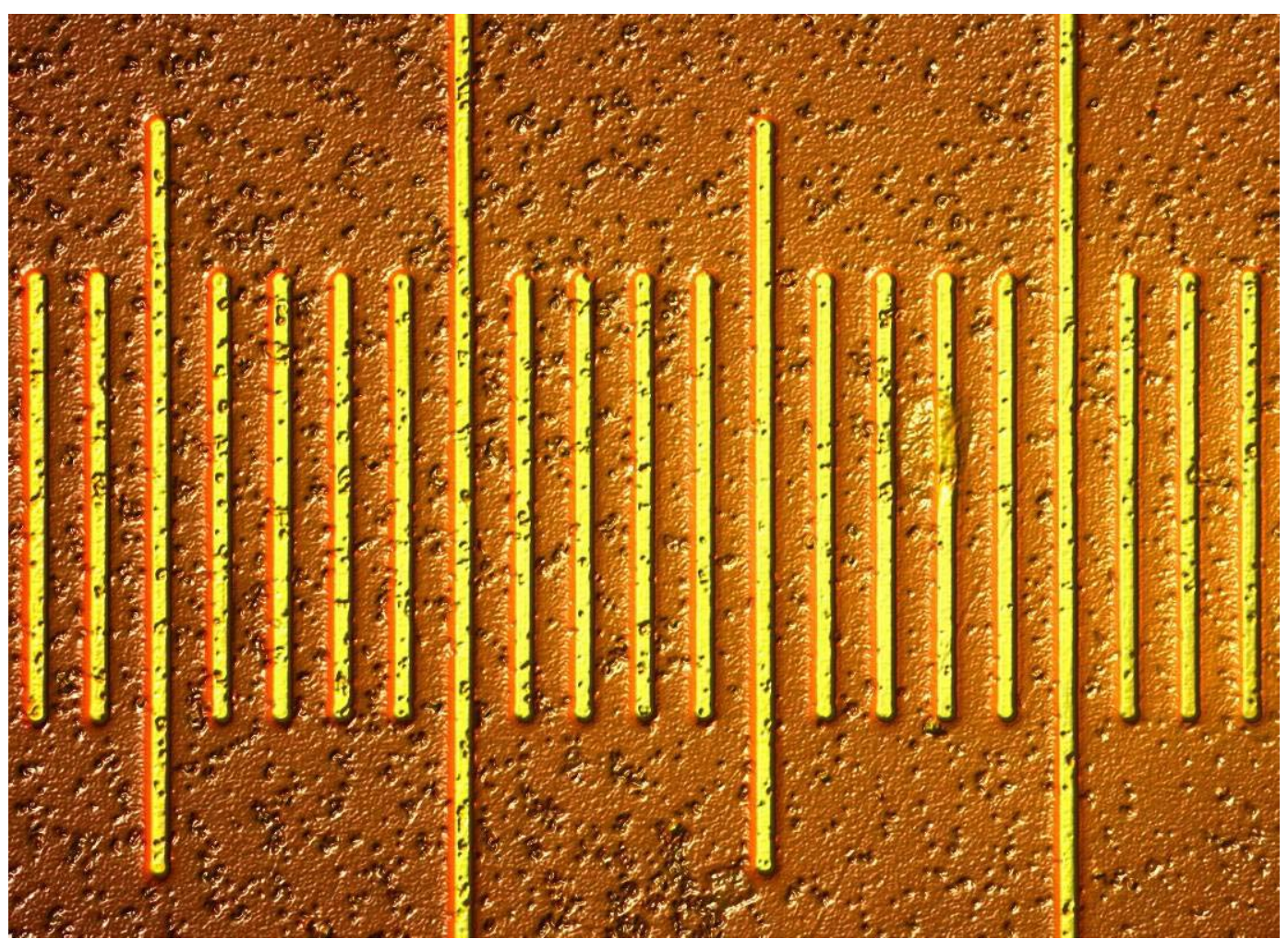

Figure 4-64. MgO powders from EM Science with 0.8 w. \% CoatOsil

Two batches of spinel samples were prepared with 0.5 and 1.0 w. \% CoatOsil 1211 dispersant. The solid loading was $10 \mathrm{w} . \%$ for each sample. The results are shown in Figure 4-65(a) to (d). Figure 4-65(a) and (b) show the representative pictures for $1.0 \mathrm{w}$. $\%$ CoatOsil slurry, sample 1 and sample 2, respectively. Figure 4-65(c) and (d) show the representative pictures for $0.5 \mathrm{w}$. \% CoatOsil slurry, sample 1 and sample 2, respectively. It is obvious from the pictures that the samples with $1.0 \mathrm{w}$. \% CoatOsil have very large agglomerates. And the samples with $0.5 \mathrm{w}$. \% CoatOsil possess relatively small agglomerates in Figure 4-65(d) and finely dispersed particles in Figure 4-65(c). It appears that, with an excessive amount of dispersant and relatively finer and lighter particles, the dispersant tends to coagulate together and drag the fine particles with it, thus resulting in powder agglomerates.

Figure 4-65 (c) is image processed to enhance the particle visibility. The results are shown Figure 4-66. By comparing the particle size with the width of the bar, which is $2.7 \mu \mathrm{m}$, all particles are about less than half of the size of the bar, which is $1.3 \mu \mathrm{m}$. Many of the particles are in the range or less than $1 / 4$ of the width of the bar, which is $0.7 \mu \mathrm{m}$. A rough estimation puts the average particle at $0.5 \mathrm{~nm}$. 


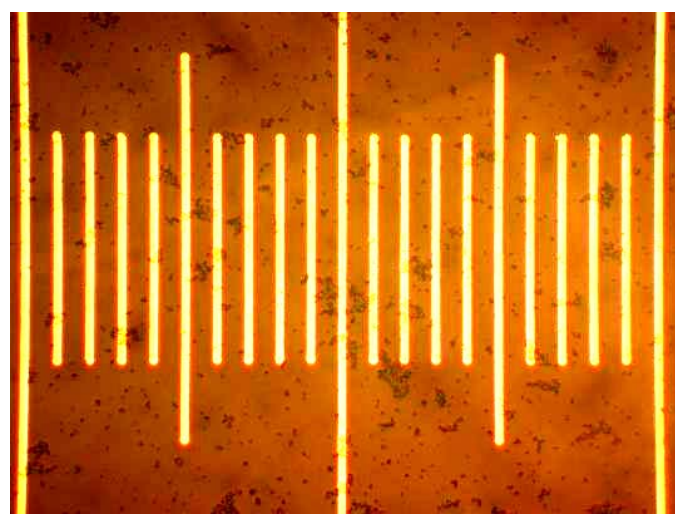

(a) 1.0 w. \% CoatOsil, sample 1

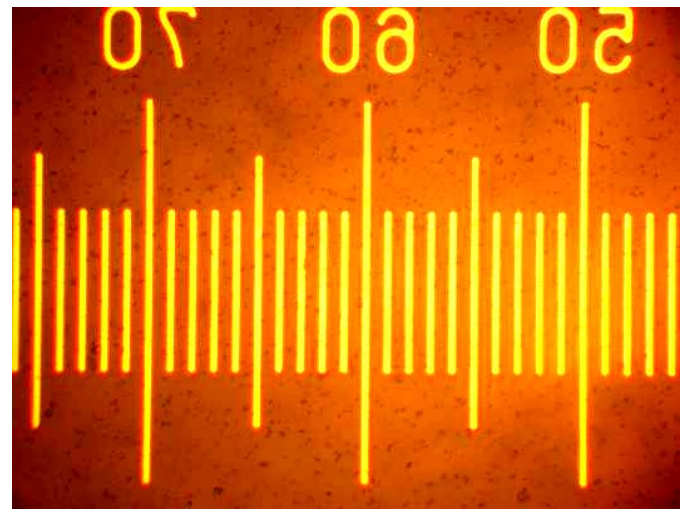

(c) 0.5 w. \% CoatOsil, sample 1

Figure 4-65. The particle analysis of spinel powders from Baikalox using a graticule under an optical

microscope

\subsubsection{Sedimentation Tests to Estimate the Optimal amount of CoatOsil 1211 as Dispersant on the MgO Powders from EM Science and the Spinel Powders from Baikalox}

If the powders in solvent are not fully dispersed, large agglomerates could form. In a sedimentation test, these large agglomerates settle relatively faster and with larger sediment volume, i.e., smaller packed-bed density. So the criterion for good powder dispersion in a sedimentation test is small sediment volume and long settling time. The $\mathrm{MgO}$ powders from EM Science and the spinel powders from Baikalox were tested to estimate the optimal amount of CoatOsil 1211 as dispersant.

Three series of settling tests were initiated. The solid loading for all samples are $10 \mathrm{w} . \%$. The first series consisted of two batches of $\mathrm{MgO}$ samples with 0.4 and $0.8 \mathrm{w}$. \% CoatOsil and two batches of spinel samples with 0.5 and $1.0 \mathrm{w} . \%$ CoatOsil. The ball milling time for the samples were not recorded. The second series consisted of two batches of $\mathrm{MgO}$ samples with 1.2 and $1.6 \mathrm{w} . \%$ CoatOsil and two batches of spinel samples with 1.5 and 2.0 w. \% CoatOsil. All samples were balled milled. The last series consist of three batches of $\mathrm{MgO}$ samples with $0,0.2$, and $0.4 \mathrm{w} . \%$ CoatOsil and one batch of spinel 
sample with $2.5 \mathrm{w}$. \% CoatOsil. All samples were ball milled. During the testing, $10 \mathrm{cc}$ of each sample was poured into a $10 \mathrm{ml}$ cylinder, whose opening was sealed with Parafilm and a rubber band to prevent the solvent from evaporation.

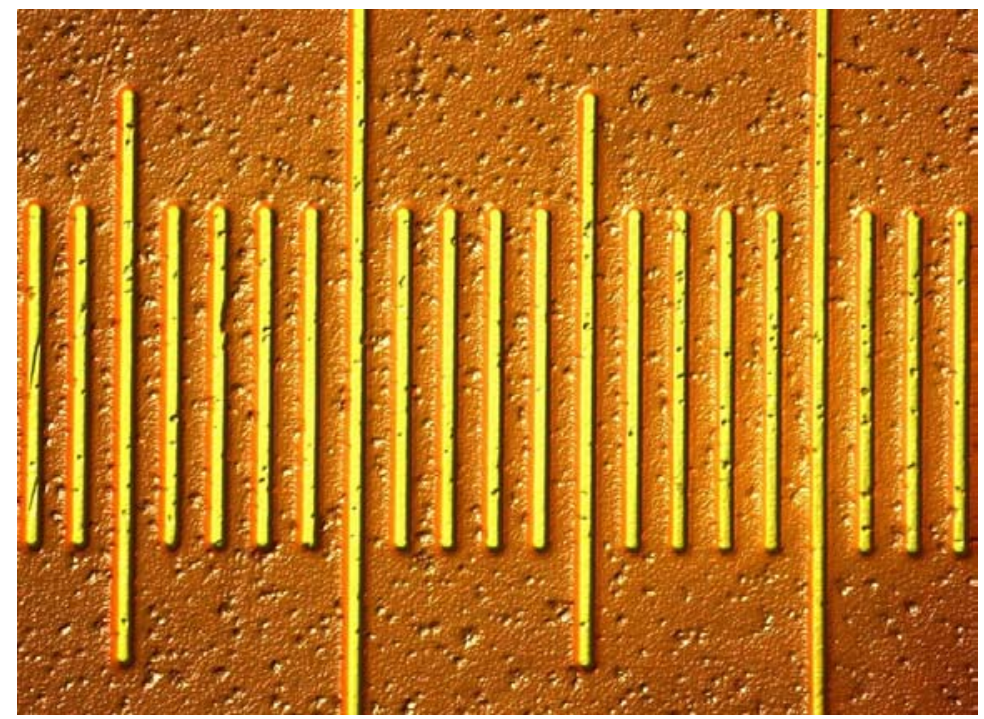

Figure 4-66. Spinel powders from Baikalox with 0.5 w. \% CoatOsil

\subsubsection{MgO Samples}

The experimental setup for four of the $\mathrm{MgO}$ batches with $0,0.2,0.4$, and $1.2 \mathrm{w} . \%$ CoatOsil are shown in Figure 4-67. Each cylinder was filled with 10c.c of the solution at the beginning of the test as marked by the blue arrows in the figure. The red arrows indicate the sediment bed-packing volumes of the samples. All samples in the figure have been tested for 22 days, except for the $1.2 \mathrm{w}$. \% sample, which has been tested for 24 days. The sediment volumes as a function of time for all seven $\mathrm{MgO}$ samples are shown in Figure 4-68. There is inconsistency in the results with the two 0.4 w. \% samples. The first sample prepared settled more rapidly than the second. The cause for this inconsistency is not clear at this moment. The sample with no CoatOsil settles very slowly compared to the sample with even a very small amount of CoatOsil, 0.2 w. $\%$. After 520 hours of testing, the sediment volumes of the samples with $0,0.2$, and 0.4 (September 24 sample) w. \% are still slowly decreasing; it is not sure whether, with long enough test time, the final sediment volumes of these samples would reach the levels of other samples with higher CoatOsil contents. The samples with relatively high CoatOsil amounts, i.e., $0.8,1.2$, and $1.6 \mathrm{w}$. \%, all settle rapidly to similar sediment volumes. 


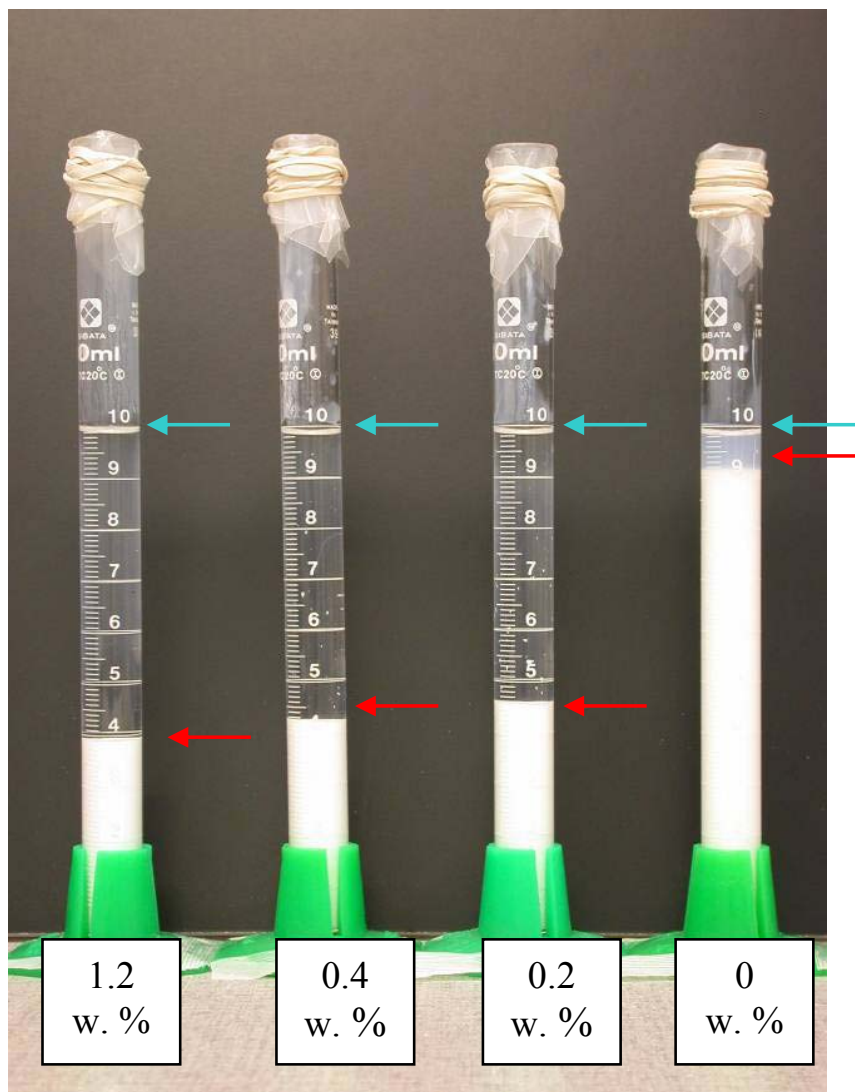

Figure 4-67. The settling test of MgO powders from EM Science using CoatOsil 1211 as dispersant. In each sample $1.0 \mathrm{~g}$ of the powders are mixed with $10.0 \mathrm{~g}$ of isopropyl alcohol. From right to left: no dispersant, $0.2 \mathrm{w} . \%$ dispersant, $0.4 \mathrm{w} . \%$ dispersant, and $1.2 \mathrm{w} . \%$ dispersant. All samples have been tested for at least 22 days, except for the $1.2 \mathrm{w}$. \% sample, which has been tested for 24 days.

From Figure 4-67, in the cylinder containing 0 w. \% CoatOsil sample, the solution above the sediment/solvent interface at $9 \mathrm{cc}$ is milky, indicating that there are some fine particles suspended in the solution. As mentioned earlier, the criterion for good dispersion is small sediment volume and longer settling time. Since the testing time here is not long enough for the $0,0.2$, and $0.4 \mathrm{w} . \%$ samples to fully settle, it is not certain which sample has the smallest sediment volume. On the other hand, judging from the settling rate of each sample, the addition of CoatOsil greatly increase the settling rates, implying that large agglomerates are formed as a result of the addition of CoatOsil. Therefore, CoatOsil 1211, instead of serving as dispersant as expected, most likely acts as flocculant in the solution. It is suspected that CoatOsil causes the aggregation of fine suspended particles, and the floc settles to the bottom of the cylinder in a much faster rate than the sample without any CoatOsil.

Based on the Stoke's Law, the agglomerate sizes can be calculated using the settling rates. For spherical particles in a viscous fluid in the Reynold's region, the constant particle settling rate resulting from the force balance between the gravitational force and the fluid dragging force can be expressed as 


$$
v_{s}=\frac{g d_{p}^{2}\left(\rho_{p}-\rho_{f}\right)}{18 \mu}
$$

where $v_{s}$ is the constant particle settling rate in $\mathrm{cm} / \mathrm{sec}, g$ the gravitational constant in $\mathrm{cm} / \mathrm{sec}^{2}\left(980 \mathrm{~cm} / \mathrm{sec}^{2}\right), d_{p}$ the particle diameter in $\mathrm{cm}, \rho_{p}$ and $\rho_{f}$ the densities of the particle and the fluid in $\mathrm{g} / \mathrm{cm}^{3}$ respectively $\left(3.6 \mathrm{~g} / \mathrm{cm}^{3}\right.$ fro both $\mathrm{MgO}$ and spinel, $0.785 \mathrm{~g} / \mathrm{cm}^{3}$ for 2 -propanol), and finally $\mu$ the fluid viscosity in poise $\left(2.3 \times 10^{-2}\right.$ poise for 2-propanol). As seen from Figure 4-68, the samples with higher CoatOsil concentrations, $0.8,1.2$, and $1.6 \mathrm{w} . \%$, have almost the same settling rate at the first 18 hours of the test; they settle for $4.43 \mathrm{~cm}$ in 18 hours as represented by the first set of data points. With proper values of all the parameters substituted, Equation (1) can be expressed as

$$
\left(\frac{4.43}{18 \times 60 \times 60}\right)\left(\frac{\mathrm{cm}}{\mathrm{sec}}\right)=\frac{980\left(\frac{\mathrm{cm}}{\mathrm{sec}^{2}}\right) d_{p}^{2}\left(\mathrm{~cm}^{2}\right)(3.6-0.785)\left(\frac{\mathrm{g}}{\mathrm{cm}^{3}}\right)}{18 \times 2.3 \times 10^{-2}\left(\frac{\mathrm{g}}{\mathrm{cm} \times \mathrm{sec}}\right)}
$$

Equation 10

The agglomerate diameter calculated from the above equation is $0.24 \mu \mathrm{m}$. This agglomerate size is much smaller than expected, since the particle size estimated from the optical microscopy shown in Figure 4-64 is $1.7 \mu \mathrm{m}$. As can be seen from Figure 4-68, the sample with no CoatOsil added settles very slowly, for about $0.93 \mathrm{~cm}$ in about 526 hours. From Equation (1) the calculated particle size is only $0.02 \mu \mathrm{m}(20 \mathrm{~nm})$. One possible cause of the size inconsistency is the non-spherical particle shapes. However, from Figure 4-64 the shape of particles seems to be not very deviated from spheres. So at this moment no explanation can be provided for this inconsistency.

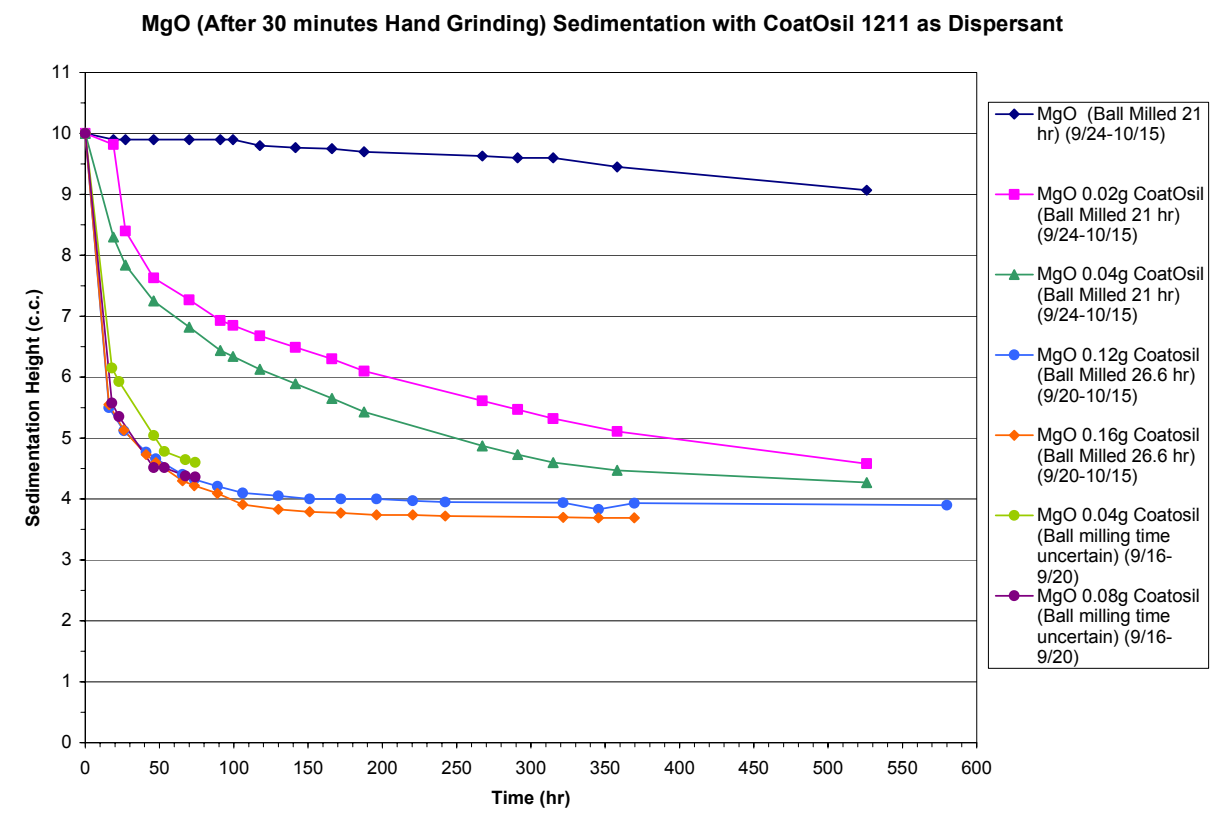

Figure 4-68. All seven MgO samples dispersed with CoatOsil 1211 


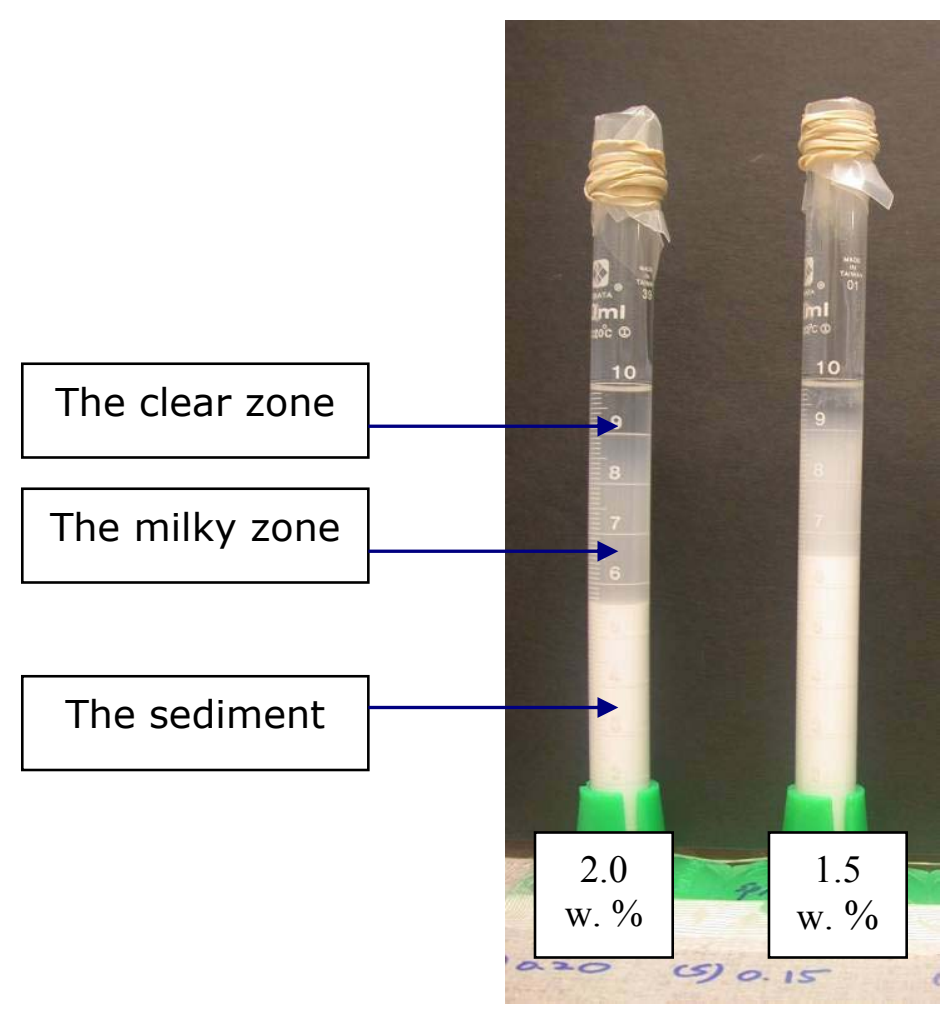

Figure 4-69. The settling test of spinel powders from Baikalox using CoatOsil 1211 as dispersant. In each sample $1.0 \mathrm{~g}$ of the powders are mixed with $10.0 \mathrm{~g}$ of isopropyl alcohol. From right to left: $1.5 \mathrm{w}$. $\%$ dispersant, and $2.0 \mathrm{w} . \%$ dispersant. The samples have been tested for 16 hours.

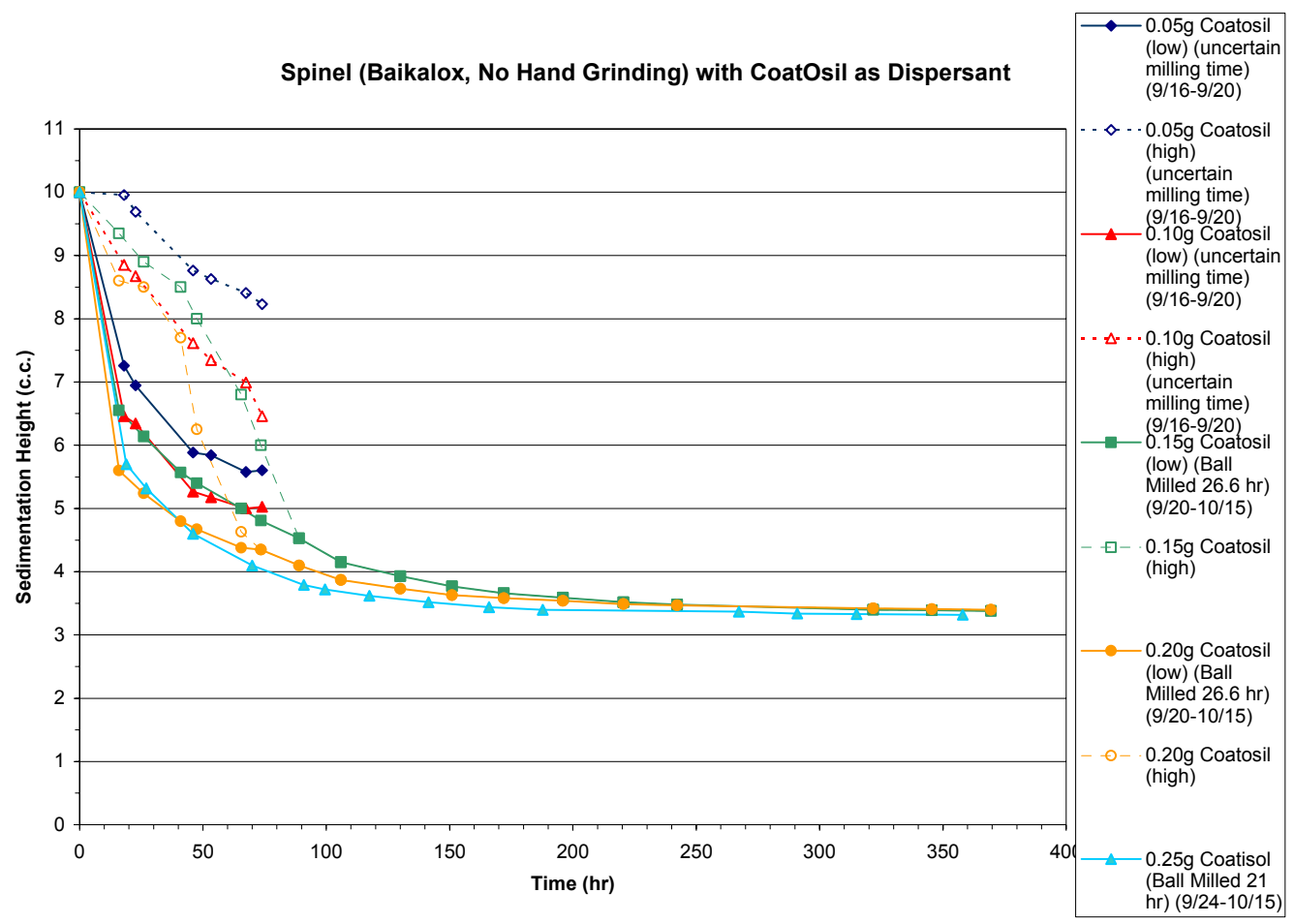

Figure 4-70. All five spinel samples dispersed with CoatOsil 1211. The full markers represent the sediment/solution interfaces and the hollow markers of the same colors the milky-solution/clearsolution interfaces in the samples. The CoatOsil amounts are $0.5,1.0,1.5,2.0$, and $2.5 \mathrm{w}$. \%. 


\subsubsection{Spinel Samples}

The experimental setup for two of the spinel batches with 0.15 and $0.20 \mathrm{w} . \%$ CoatOsil are shown in Figure 4-69. The samples have been tested for 16 hours. As shown in the figure, in contrast to most of the $\mathrm{MgO}$ samples, there exists another less well defined interface between the zone of milky solution and the zone of clear solution as marked by the green arrows. Also, the degree of cloudiness in the milky zone is greater than here compared to the $0 \mathrm{w} . \%$ CoatOsil $\mathrm{MgO}$ sample in Figure 4-67. These results imply that there is a larger amount of spinel fine particles suspended in the milky zone here. Also can be seen in the figure is that the milky zone in the $1.5 \mathrm{w} . \%$ sample is more cloudy than that of the $2.0 \mathrm{~W} . \%$ sample. This suggests that there are more fine particles suspended in the milky zone in the $1.5 \mathrm{w} . \%$ sample. The addition of more CoatOsil reduces the number of fine particles by agglomerating them together.

Both the levels of the sediment/solution interface and the milky-zone/clear-zone interface were recorded as a function of time. And the results are shown in Figure 4-70 for all five spinel samples. The CoatOsil amounts are 0.5, 1.0, 1.5, 2.0, and $2.5 \mathrm{w} . \%$. The filled markers represent the sediment/solution interfaces and the hallow markers of the same colors the milky-zone/clear-zone interfaces in the samples. The two interfaces in each sample merge at a certain time during the test. The rough general trends shown in Figure 4-70 are that the larger the amount of CoatOsil, the faster the settling rates of both the sediment zone (the filled markers) and the milky zone (the hollow markers). It is believed that CoatOsil also serves as flocculant in spinel solution. More CoatOsil renders larger flocs to be formed, and they settle with larger settling rates. In all samples the sediment settling rates are faster at the beginning of the test, and they get slower and slower as a function of time. On the contrary, the milky solution settling rates are slower at the beginning of the test and get faster later, resulting in curves concaving downwards. No explanation is available at this moment regarding these phenomena. For the three samples with the higher amounts of CoatOsil, 1.5, 2.0, and $2.5 \mathrm{w} . \%$, the final sediment heights are almost the same after 370 hours of test.

The agglomerate sizes of two samples, 0.5 and $2.0 \mathrm{w} . \%$, were calculated using the Stoke's Law. As shown in Figure 4-70, for the sample with 0.5 w. \% CoatOsil, the sediment settles for $2.74 \mathrm{~cm}$ in 18 hours, and the milky zone settles for only $1.24 \mathrm{~cm}$ in 46 hours. The particle sizes calculated using the Stoke's Law are $0.19 \mu \mathrm{m}$ for the sediment and $0.08 \mu \mathrm{m}$ for the milky solution. For the $2.0 \mathrm{w}$. \% CoatOsil sample, the sediment settles for $2.74 \mathrm{~cm}$ in 18 hours, and the milky zone settles for $1.24 \mathrm{~cm}$ in 46 hours. The calculated diameters using Stoke's Law are $0.25 \mu \mathrm{m}$ for the sediment and $0.11 \mu \mathrm{m}$ for the milky zone. These sizes are also smaller than the size estimated from optical microscopy, $0.5 \mu \mathrm{m}$ from Figure 4-66. Since CoatOsil was added to all samples as flocculant, the actual particle sizes of the spinel powders should be even smaller than the calculated values. It is believed that the Stoke's Law can not be applied to particle agglomerates due to the structure of the agglomerates. 


\subsubsection{Measurement of Numerical Aperture and Estimate of Mode Volume in Clad Sapphire Fiber}

In order to assess the effectiveness of the spinel cladding in reducing the number of guided modes in the sapphire fiber, an apparatus was assembled to measure the numerical aperture (NA) of a sapphire fiber before and after cladding. It is expected that the use of claddings (coatings) on sapphire fibers will reduce the number of electromagnetic modes that propagate in the fiber. Minimization of the number of propagating modes will permit the use of optical interferometry and fiber Bragg gratings with sapphire fibers, which in turn will facilitate the development of pressure sensors using sapphire fibers.

Optical fibers can be characterized by a parameter called the numerical aperture, which is a measure of the range of solid angles over which light incident on the input end of the fiber can be captured and transmitted by the fiber. As the modal volume (the number of propagating modes that the fiber can support) is decreased, the numerical aperture of the fiber decreases. Therefore, a change of numerical aperture can be correlated to a change in modal volume of a fiber.

For an ideal (no scattering at the core-cladding interface) step-index multimode fiber, the numerical aperture of the fiber depends only upon the refractive indices of the core and cladding:

$$
\mathrm{NA}=\sqrt{\mathrm{n}_{\mathrm{co}}^{2}-\mathrm{n}_{\mathrm{cl}}^{2}}[
$$

Equation 11

where $n_{c o}$ is the refractive index of the fiber core, and $n_{c l}$ is the refractive index of the cladding. Based on the c-axis refractive index of sapphire fiber (1.768) and the refractive index of magnesium aluminate spinel, a numerical aperture of 0.40 would be predicted for the spinel-clad sapphire fiber.

In the apparatus used to measure numerical aperture, the collimated light output by a helium neon laser is reflected by a mirror towards the end of a sapphire fiber at an angle $\theta$ with respect to the longitudinal axis of the fiber, as illustrated in Figure 4-71. Any light captured by the fiber is guided to the other end of the fiber, where an optical power meter measures the power output by the fiber. The mirror is adjustable so that the angle $\theta$ of light incident upon the fiber is variable. In this way, the light power captured by the fiber as a function of input angle of incidence may be measured, and from this, the numerical aperture may be calculated. Figure 4-72 is a photograph of the experimental set-up. 


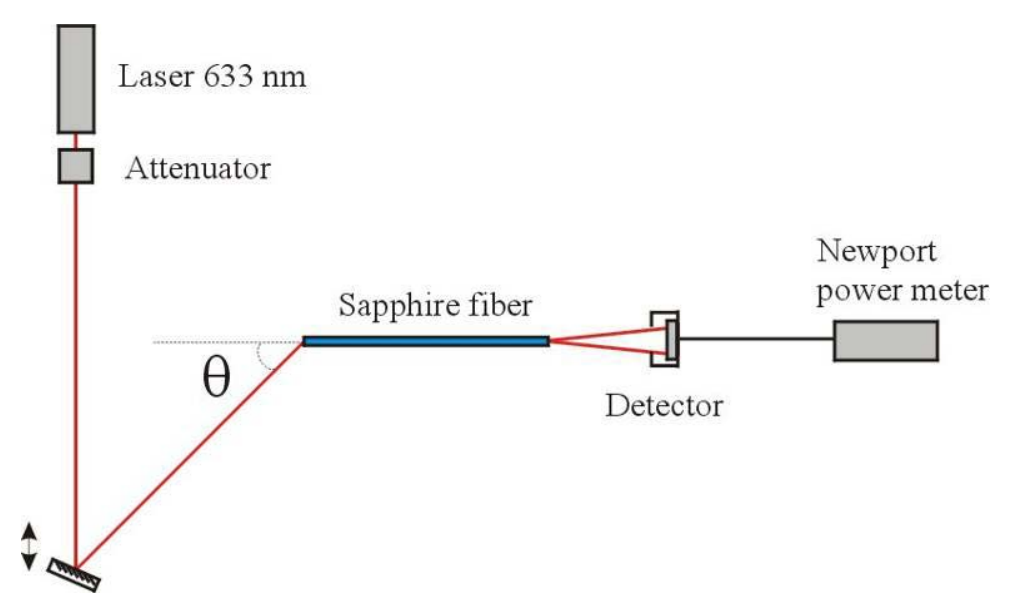

Figure 4-71. Schematic of Experimental Set-Up for Measurement of NA

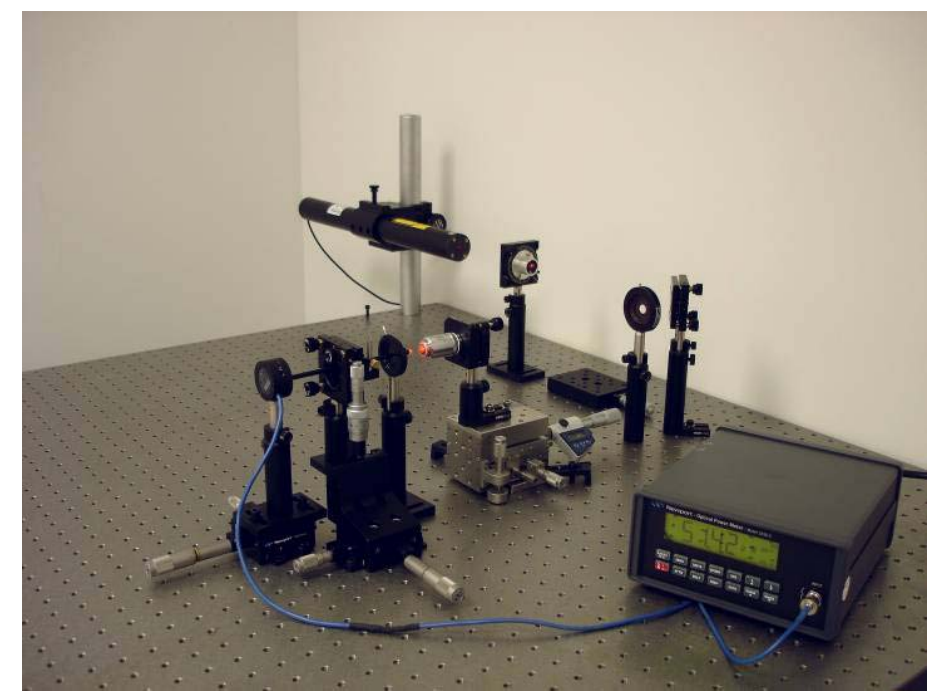

Figure 4-72. Photograph of Set-up for Measurement of NA

Two identical six-inch long samples of $125 \mu \mathrm{m}$ diameter sapphire fiber (obtained from Photran, Inc.) were used to evaluate the effect of the spinel cladding on the optical performance of the sapphire fiber. First, one of the sapphire fibers was polished before coating, and the numerical aperture of the fiber was measured using the set-up illustrated above. Using the Telecommunication Industry Association's Fiber Optic Test Procedure (FOTP) criteria for measurement of numerical aperture, the maximum angle acceptance for the fiber is chosen to be that angle at which the output power falls to $10 \%$ of the maximum power. ${ }^{27}$

\footnotetext{
27 "Optical Fibers -- Part 1-43: Measurement Methods and Test Procedures -- Numerical Aperture," (FOTP)-177, TIA-455-177-B, Telecommunication Industry Association (TIA), Arlington, VA.
} 
Figure 4-73 shows the variation in optical power accepted by the unclad sapphire fiber as a function of input angle. From the plot, the angle $\theta_{c}$ which the power falls to $10 \%$ is estimated to be $36^{\circ}$. By the definition of numerical aperture,

$$
N A=\sin \theta_{c}
$$

Equation 12

the NA of the unclad sapphire fiber was found to be approximately 0.59 .

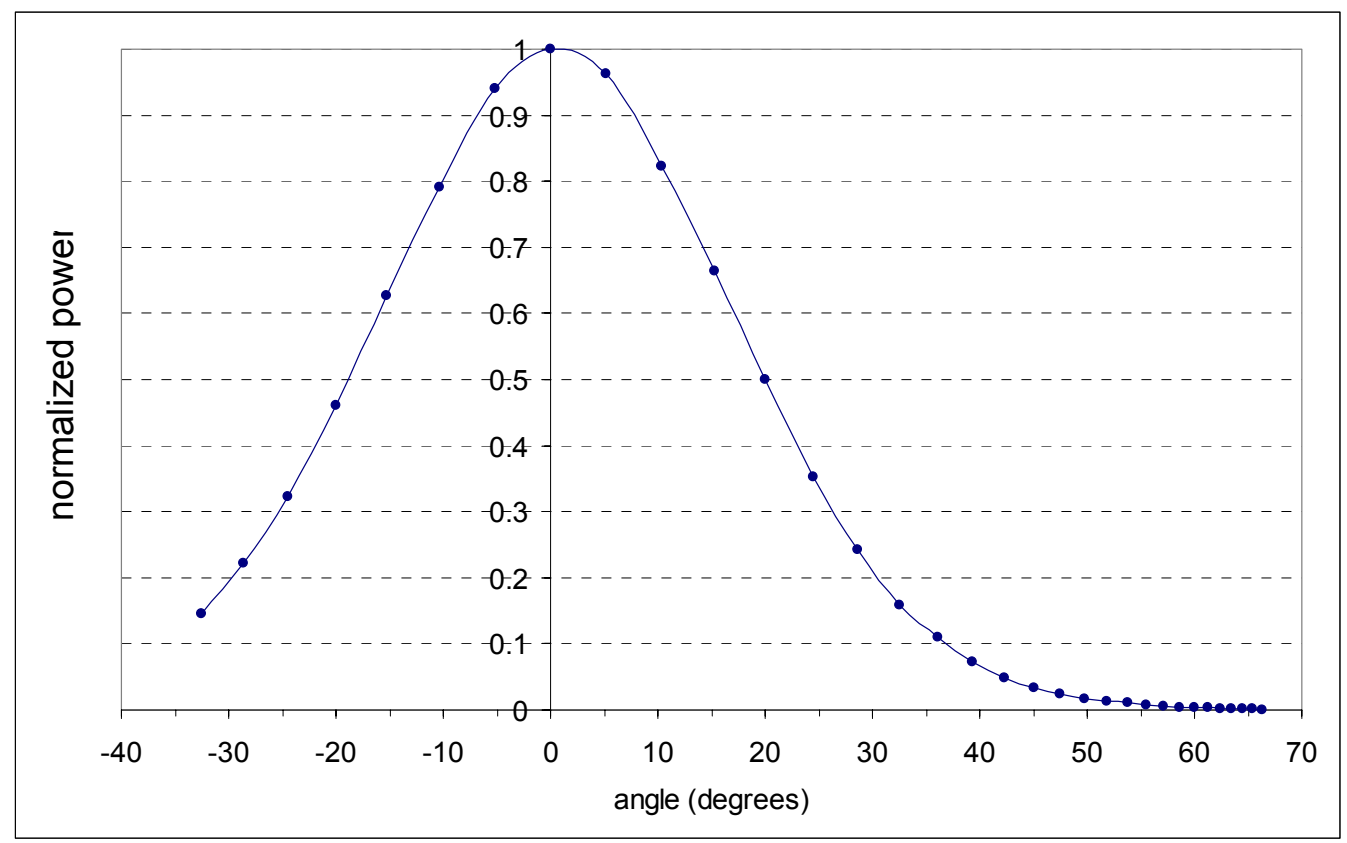

Figure 4-73. Plot of the normalized output power for unclad sapphire fiber as a function of angle of incidence of input light.

The second 6 inch sapphire fiber was then coated by dipping the sapphire fiber into the slurry consisting of $0.68 \mathrm{~g}$ of $\mathrm{MgO}$ powders from Aldrich, $1.21 \mathrm{~g}$ spinel powders from Baikalox, and $2.73 \mathrm{~g} \mathrm{MgO}$ powders from EM Science. All powders were dried at $500^{\circ} \mathrm{C}$ for 1 hour before slurry preparation. The slurry was prepared by mixing $20.9 \mathrm{~g}$ of 2 propanol with $150 \mu \mathrm{L}$ of menhaden fish oil (Aldrich) in a 4-oz polyethylene bottle filled with $133 \mathrm{~g} \mathrm{1/4-inch} \mathrm{grinding} \mathrm{media.} \mathrm{The} \mathrm{oxide} \mathrm{powders} \mathrm{were} \mathrm{added} \mathrm{to} \mathrm{the} \mathrm{slurry}$ immediately after being taken out from the oven. The slurry was then ball milled for 17.5 hours at $104 \mathrm{rpm}$. After ball milling, $2.2 \mathrm{~g}$ polyvinylpyrrolidone (PVP) and $2.1 \mathrm{~g} \mathrm{1-}$ methoxy-2-propanol were added to the slurry followed by 6 hours of ball milling.

Before dipping, the sapphire fiber was cleaned and soaked in 2-propanol for 15 minutes. Finally, the fiber was dipped in the slurry 100 times before firing, using a dipping speed of 0.11 inch per second. The heating profile of the dipped fiber is shown in Figure 4-74 below. The fiber was fired in air below $600^{\circ} \mathrm{C}\left(1,112^{\circ} \mathrm{F}\right)$ and in nitrogen above $600^{\circ} \mathrm{C}$ $\left(1,112^{\circ} \mathrm{F}\right)$. The nitrogen was admitted to the oven 15 minutes into the $600^{\circ} \mathrm{C}\left(1,112^{\circ} \mathrm{F}\right)$ dwell step. 


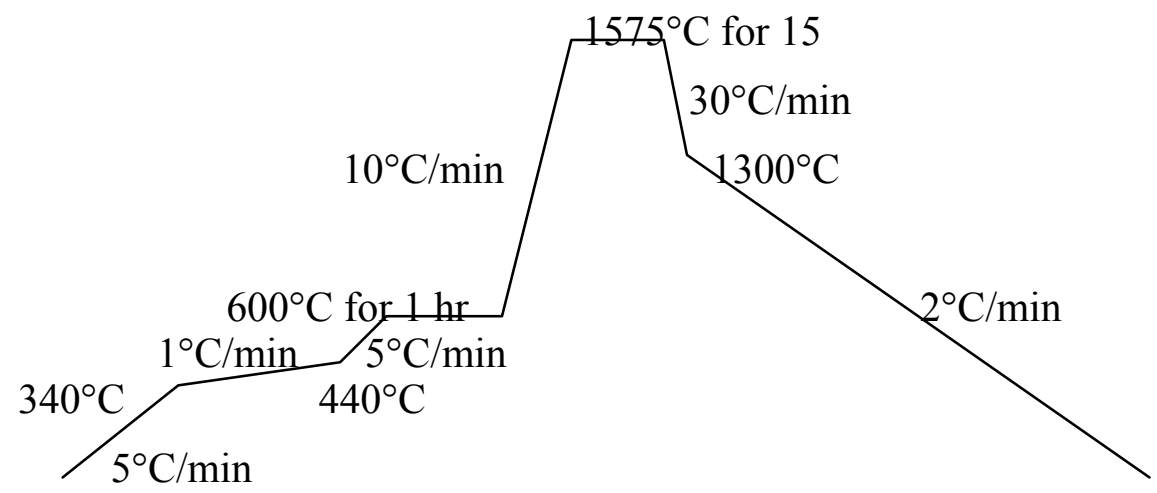

Figure 4-74. Heating profile used to process magnesium aluminate spinel coating on fiber for numerical aperture test.

The measurement setup was then used to measure the numerical aperture for this clad fiber. As Figure 4-75 shows, the acceptance angle was reduced to approximately $2^{\circ}$. Applying the definition above, the NA of the clad sapphire fiber is found to be approximately 0.3 .

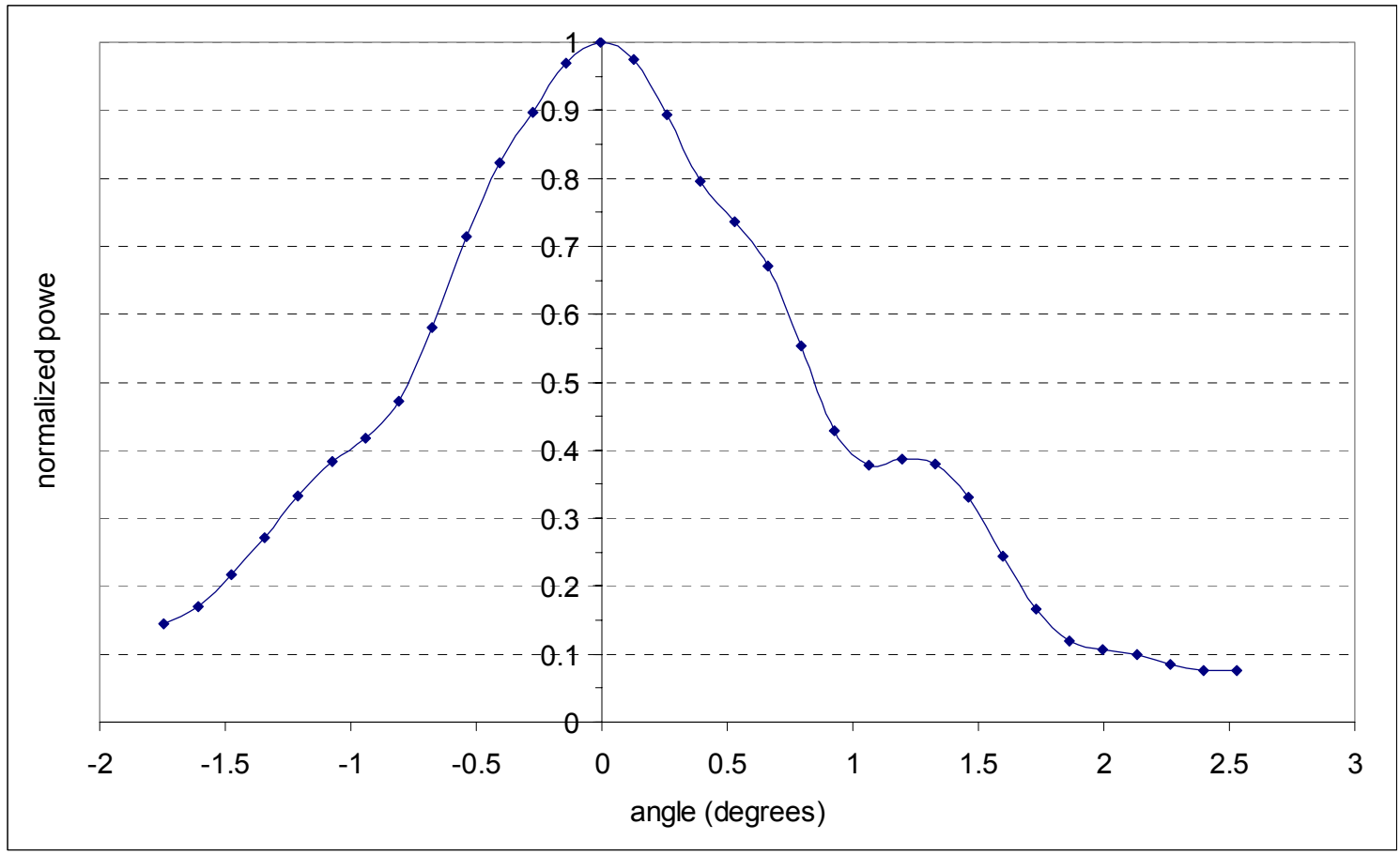

Figure 4-75. Plot of the normalized output power for clad sapphire fiber as a function of angle of incidence of input light. 
This value is in order of magnitude lower than that predicted by Equation. The reason for this is unknown and still under investigation, but a leading hypothesis is that a rough interface between the core and cladding in the coated fiber (seen in some of the photographs in the previous section) causes more scattering and stronger losses in higherorder modes rather than lower modes, thereby reducing the numerical aperture. Figure 4-76 combines the data from Figure 4-73 and Figure 4-75 onto a single semi-log plot. From the figure, it's clear that the transmission is strongly attenuated in the clad fiber, with the peak output $\left(\right.$ at $0^{\circ}$ ) about $15 \mathrm{~dB}$ down from that of the unclad fiber.

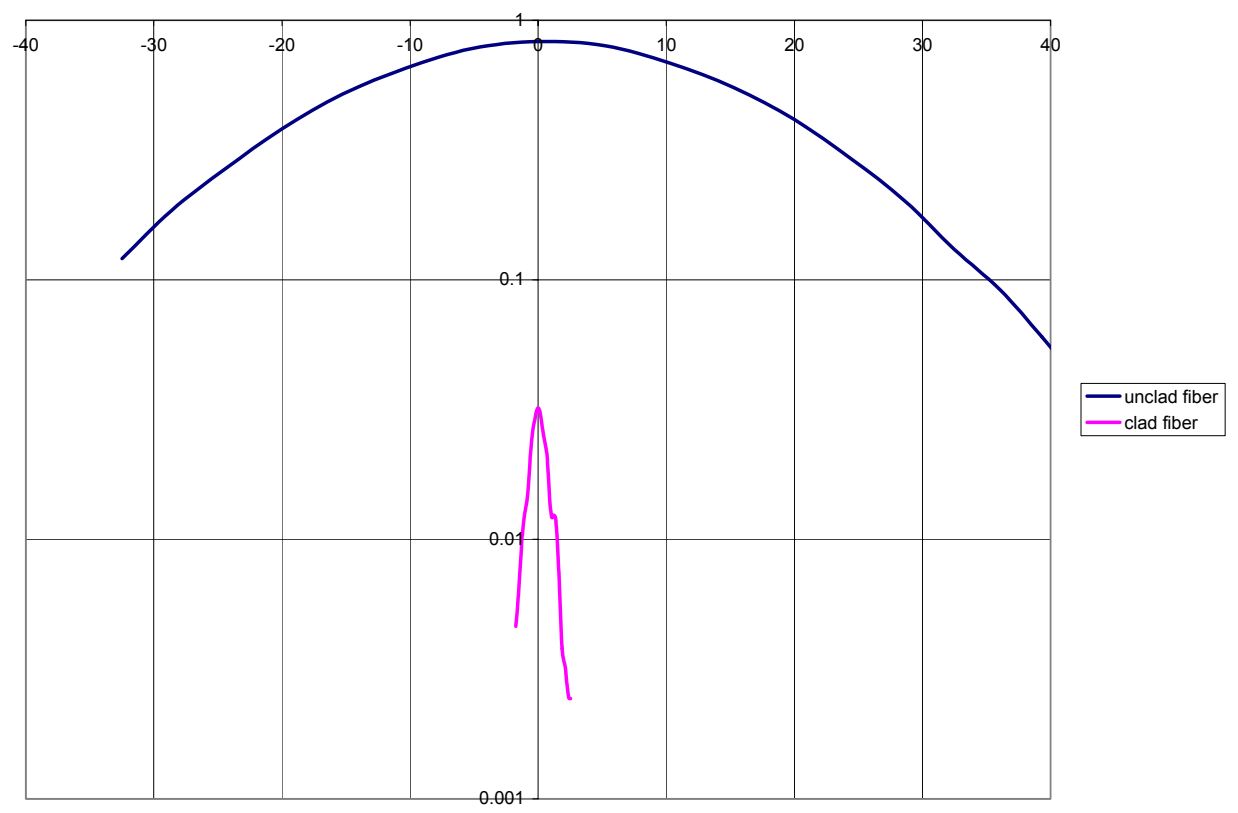

Figure 4-76. Comparison of far-field intensity distributions for unclad and clad sapphire fiber, on semi-log plot.

\subsubsection{Calculation of Mode Volume}

From electromagnetics theory, the number of electromagnetic modes guided by a step index multimode fiber may be estimated from the geometric and optical properties of the fiber. The number of guided modes $\mathrm{N}$ is given by

$$
\mathrm{N}=\frac{16 \cdot \mathrm{a}^{2}}{\lambda^{2}} \cdot \mathrm{NA}^{2}
$$

Equation 13

where $a$ is the radius of the core of the fiber, and $\lambda_{0}$ is a wavelength of light used to measure the numerical aperture. 


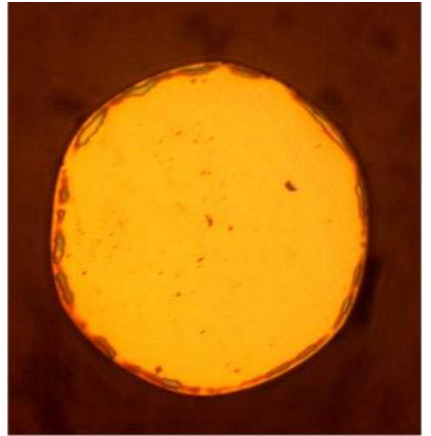

(a)

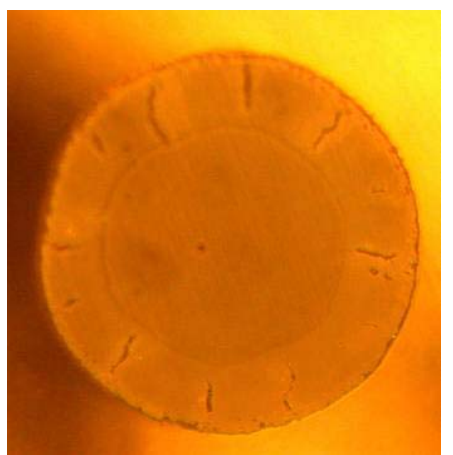

(b)

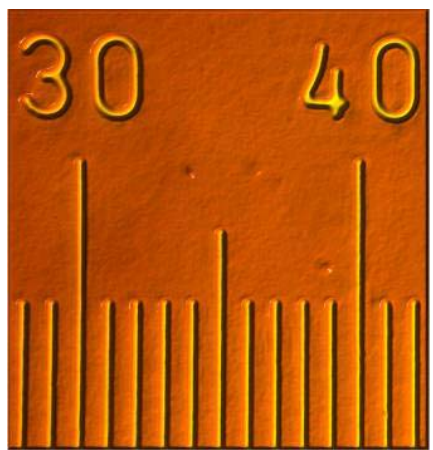

(c)

Figure 4-77. Photomicrographs of (a) unclad sapphire fiber, (the) clad sapphire fiber, and (see) graticule at same magnification as (a) and (b). Each division in (c) is ten micrometers.

From photomicrographs of the polished ends of the unclad and clad sapphire fibers (Figure 4-77), the core diameters of the fibers were measured. The diameter of the unclad fiber was measured to be $122 \mu \mathrm{m}$; the outside diameter of the clad fiber was found to be $137 \mu \mathrm{m}$, with a core diameter of $86 \mu \mathrm{m}$. Using these measurements for the unclad sapphire fiber, the number of guided modes propagating in the fiber is estimated to be approximately 52,000. In contrast, the number of guided modes for the clad sapphire fiber is estimated to be 66 .

The reduced mode volume in the clad fiber was also confirmed by examining the far-field intensity distribution of the clad sapphire fiber. Figure 4-79 shows the far-field radiation pattern output by the clad sapphire fiber. To obtain this image, the output of a helium neon laser was focused on to the end of the clad sapphire fiber using a 60x microscope objective, with a NA of 0.65, as shown in Figure 4-78. A CCD array, connected to a computer-based image acquisition system, was positioned approximately six inches from the output end of the fiber. The intensity distribution of the far-field pattern shows a few maxima consistent with interference from a few (less than 100) propagating modes. For comparison, Figure 4-80 shows the far-field pattern from a graded index multimode fiber with a $100 \mu \mathrm{m}$ core, which supports the propagation of thousands of modes.

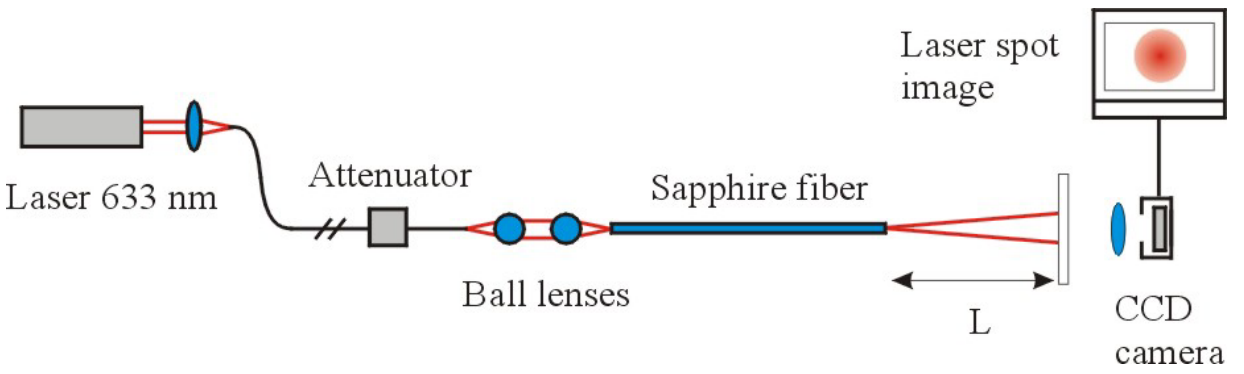

Figure 4-78. Set-up for imaging far-field intensity distribution from sapphire fiber. 


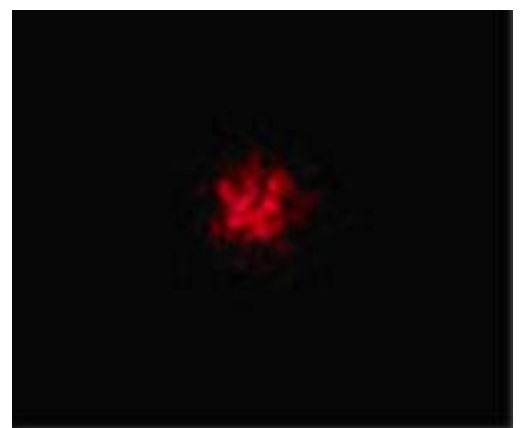

Figure 4-79. Far Field Radiation Pattern from Output of Clad Sapphire Fiber

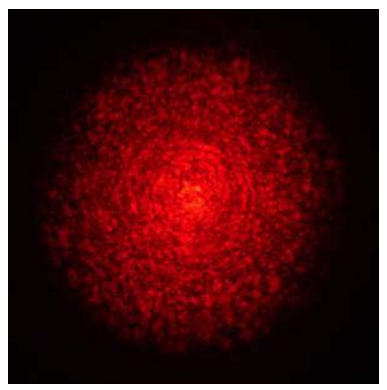

Figure 4-80. Far-Field Radiation Pattern Output by a $100 \mu \mathrm{m}$ Core, Graded Index Multimode Fiber

\subsubsection{Designed experiments for optimizing processing of clad sapphire fiber}

The Taguchi method is a technique for designing and performing experiments where the output depends on many factors, without tediously and uneconomically running the experiment using all possible combinations of values of those factors. By systematically choosing certain combinations of variables it is possible to separate their individual effects.

In the current project, a sapphire fiber is dip-coated in the slurry prepared using a variety of oxide powders and additives including dispersant and binder. In the high-temperature firing step, the magnesium oxide particles in the coating diffuse into the sapphire fiber and form the spinel cladding surrounding the fiber. A goal of this project is to fabricate mechanically strong spinel cladding with an optically smooth cladding/core interface. Many factors, including the type and amount of dispersant and binder, weight ratio of the magnesium oxide and spinel powders in the slurry, coating speed and number of dips, and the final firing profile, affect the outcome. It is difficult and tedious to sort out the individual effect of each factor without a systematically designed set of experiments. It is ideal in this situation to implement the Taguchi method to design the proper experimentation. Prof. Gary Pickrell at Virginia Tech guided the establishment of the Taguchi process for this portion of the program. 


\begin{tabular}{|c|c|c|c|c|c|}
\hline \multirow{2}{*}{$\begin{array}{l}\text { Factor } \\
\text { number }\end{array}$} & \multirow[t]{2}{*}{ Factor } & \multirow{2}{*}{$\begin{array}{l}\text { Number } \\
\text { of Levels }\end{array}$} & \multicolumn{3}{|c|}{ Level } \\
\hline & & & 1 & 2 & 3 \\
\hline 1 & Type of dispersant & 3 & Fish oil & $\begin{array}{l}\text { Darvan } \\
811\end{array}$ & PVP \\
\hline 2 & Amount of dispersant & 3 & $0.1 \mathrm{w} . \%$ & $0.5 \mathrm{w} . \%$ & $0.9 \mathrm{w} . \%$ \\
\hline 3 & Amount of binder (PVP) added & 3 & $3 \mathrm{w} . \%$ & 5 w. $\%$ & $10 \mathrm{w} . \%$ \\
\hline 4 & $\begin{array}{c}\text { Weight ratio of powders }(\mathrm{MgO} \\
\text { : spinel) }\end{array}$ & 3 & $3: 1$ & $4: 1$ & $5: 1$ \\
\hline 5 & Ultrasonic step & 3 & None & 30 seconds & $\begin{array}{c}60 \\
\text { seconds }\end{array}$ \\
\hline 6 & Dipping speed & 3 & $0.1 \mathrm{inch} / \mathrm{sec}$ & $\begin{array}{c}0.15 \\
\text { inch/sec }\end{array}$ & $\begin{array}{c}0.3 \\
\text { inch } / \mathrm{sec}\end{array}$ \\
\hline 7 & Numbers of dips & 3 & 50 & 100 & 150 \\
\hline 8 & The firing profile & 2 & Profile 1 & Profile 2 & \\
\hline
\end{tabular}

Table 4-17. Tentative factors and the levels of each factor

After careful review of the system of interest, it was decided to implement the "L18 Orthogonal Array" which consists of eighteen experiments with seven 3-level factors and one 2-level factor. The L18 array is shown in Table 4-18 below. Each of the eighteen rows represents one coated sapphire fiber sample prepared using the corresponding factor and level in each column. The eight factors and their designated levels are listed in Table 4-17 above.

\begin{tabular}{ccccccccc} 
& \multicolumn{1}{c}{ Columns } \\
EXPT NO & $\mathbf{1}$ & $\mathbf{2}$ & 3 & 4 & 5 & 6 & 7 & 8 \\
\hline 1 & $\mathbf{1}$ & $\mathbf{1}$ & 1 & 1 & 1 & 1 & 1 & 1 \\
2 & $\mathbf{1}$ & $\mathbf{1}$ & 2 & 2 & 2 & 2 & 2 & 2 \\
3 & $\mathbf{1}$ & $\mathbf{1}$ & 3 & 3 & 3 & 3 & 3 & 3 \\
4 & $\mathbf{1}$ & $\mathbf{2}$ & 1 & 1 & 2 & 2 & 3 & 3 \\
5 & $\mathbf{1}$ & $\mathbf{2}$ & 2 & 2 & 3 & 3 & 1 & 1 \\
6 & $\mathbf{1}$ & $\mathbf{2}$ & 3 & 3 & 1 & 1 & 2 & 2 \\
7 & $\mathbf{1}$ & $\mathbf{3}$ & 1 & 2 & 1 & 3 & 2 & 3 \\
8 & $\mathbf{1}$ & $\mathbf{3}$ & 2 & 3 & 2 & 1 & 3 & 1 \\
9 & $\mathbf{1}$ & $\mathbf{3}$ & 3 & 1 & 3 & 2 & 1 & 2 \\
& & & & & & & & \\
10 & $\mathbf{2}$ & $\mathbf{1}$ & 1 & 3 & 3 & 2 & 2 & 1 \\
11 & $\mathbf{2}$ & $\mathbf{1}$ & 2 & 1 & 1 & 3 & 3 & 2 \\
12 & $\mathbf{2}$ & $\mathbf{1}$ & 3 & 2 & 2 & 1 & 1 & 3 \\
13 & $\mathbf{2}$ & $\mathbf{2}$ & 1 & 2 & 3 & 1 & 3 & 2 \\
14 & $\mathbf{2}$ & $\mathbf{2}$ & 2 & 3 & 1 & 2 & 1 & 3 \\
15 & $\mathbf{2}$ & $\mathbf{2}$ & 3 & 1 & 2 & 3 & 2 & 1 \\
16 & $\mathbf{2}$ & $\mathbf{3}$ & 1 & 3 & 2 & 3 & 1 & 2 \\
17 & $\mathbf{2}$ & $\mathbf{3}$ & 2 & 1 & 3 & 1 & 2 & 3 \\
18 & $\mathbf{2}$ & $\mathbf{3}$ & 3 & 2 & 1 & 2 & 3 & 1 \\
& & & & & & & & \\
& & & & & & & &
\end{tabular}

Table 4-18. L18 array $\left(2^{1} \times 3^{7}\right)$ orthogonal array 
The performance of each clad fiber is judged using the following six criteria:

1. Optically smooth cladding/core interface;

2. Proper relative index of refractions between the sapphire core and the spinel cladding that can support light propagation;

3. A mechanically strong spinel cladding with the least amount of cracking;

4. Uniform cladding thickness along the whole length of the fiber;

5. Smooth and crack free surface of coated fibers after drying, binder burnout, and final firing.

Each criterion will be discussed in details in the following paragraphs.

\section{- Optically smooth cladding/core interface}

The quality of this response is to be judged by three criteria:

The concentricity of the cladding/core interface, that is, the degree of deviation of the interface from a perfect circle, is examined. First, the average distance between the interface and the center of the fiber is calculated, and a circle with a radius equal to the average distance is drawn overlapping the interface as shown in Figure 4-81. Finally, the root mean square difference between the circle and the interface in the radial direction at each unit segment of the interface is calculated.

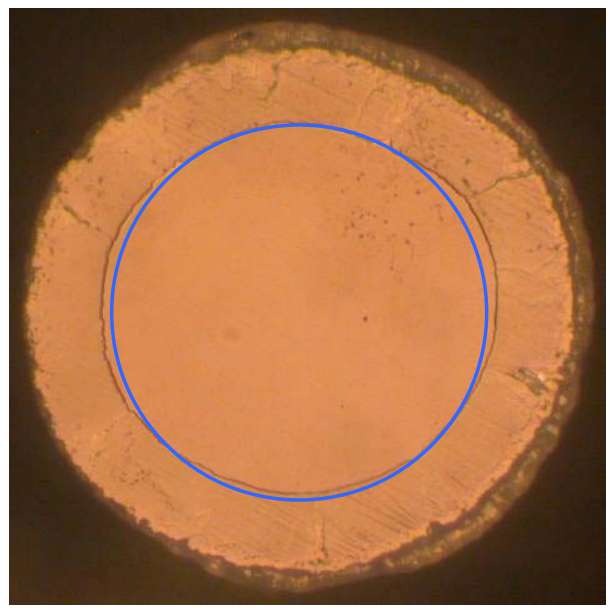

Figure 4-81. Determination of concentricity.

1.2 The second criterion is related to the smoothness of the interface itself. The procedures can best be illustrated by using a clad fiber with a typical rough interface as shown in Figure 4-82. In this figure, besides six large cracks with approximate hexagonal symmetry in the cladding, a zigzag core/cladding interface is clearly visible. The lower left corner of the core/cladding interface in Figure 4-82 is zoomed as shown in Figure 4-82. In order to measure the degree of 
smoothness of the interface, a running average of the interface is drawn along the interface as shown in Figure 4-82 as a, dotted blue curve, which can be drawn by naked eyes quite close to the accurate running average curve mathematically derived. The rigorous derivation of this curve will not be provided here as this moment. At each small unit segment on the curve, a line perpendicular to the curve shown as the dotted arrows in Figure 4-82 is drawn to intersect the core/cladding interface, and the root mean square difference between the curve and the interface is calculated as an indication of the degree of roughness of the interface.

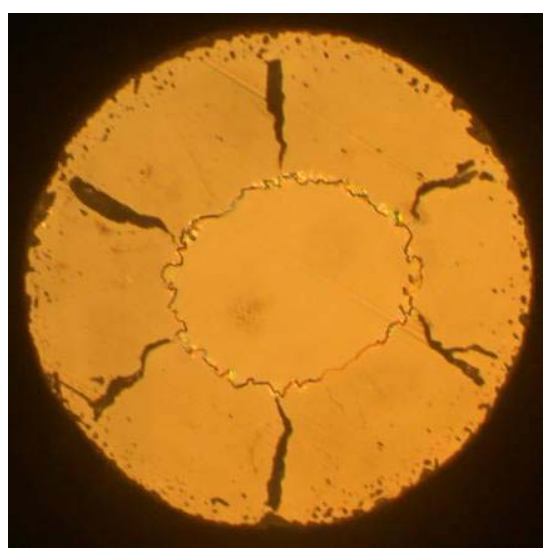

Figure 4-82

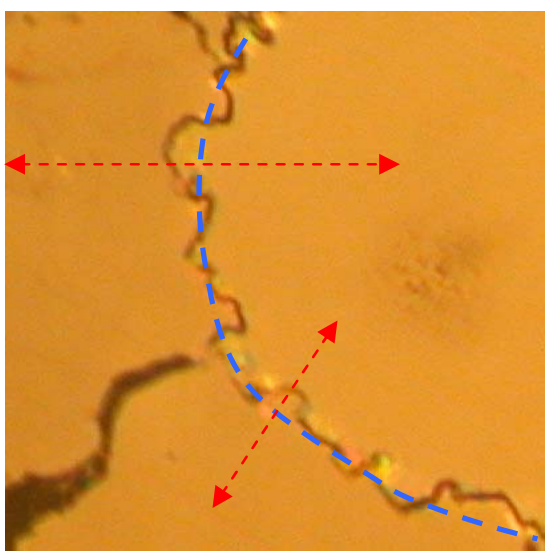

Figure 4-83

1.3 Another type of rough interface is shown in Figure 4-84, where granular, shiny, and thick sections of the interface occur in an otherwise smooth contour of the interface. Figure 4-84 is a local zoom at the upper left portion of the core/cladding interface in Figure 4-82. To evaluate the smoothness of the interface, the lengths of the widest sections are measured, and then the average width of the interface is calculated at each unit section of the interface. Both parameters are used to evaluate the smoothness. There are other occasions of the clad fibers where the entire interface is thick and granular; in these cases the same procedures described here can be implemented.

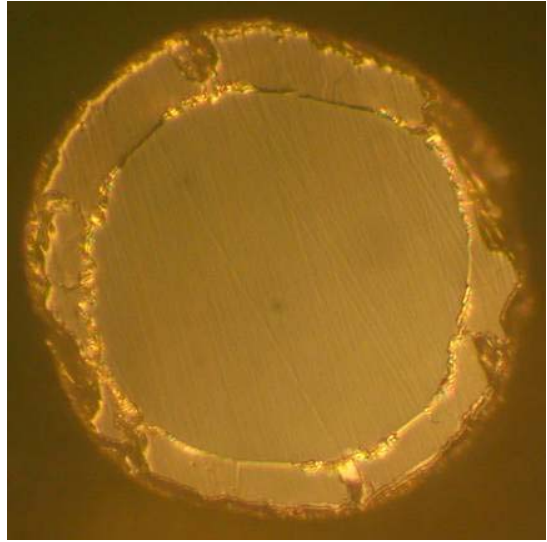

Figure 4-84

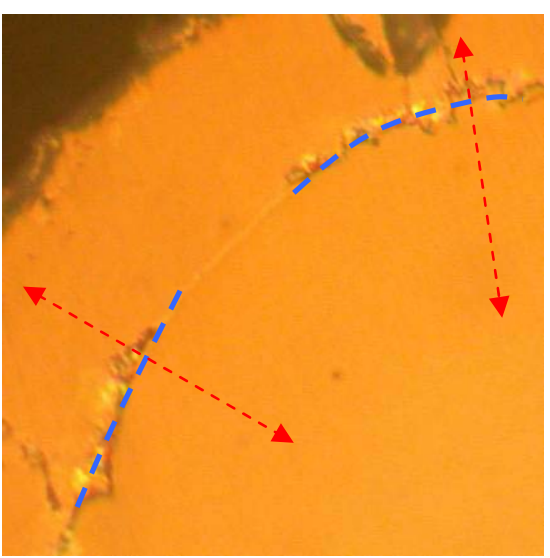

Figure 4-85 
- Proper relative index of refractions between the sapphire core and the spinal cladding that can support light propagation

Instead of directly measuring the indices of the spinel cladding and that of the sapphire core, the light guiding quality of clad fibers 6 inches long are examined by measuring the attenuation and the numerical aperture (NA) of the clad fibers. Both ends of the fibers are polished using diamond papers with abrasive sizes down to $0.5 \mu \mathrm{m}$. The attenuation and NA of the original sapphire fibers without cladding are measured as the reference of comparison.

- A mechanically strong spinel cladding with the least amount of cracking

Methods to quantitatively determine the mechanical strength of the clad fibers have not yet been implemented. However, the cracking in the cladding can be evaluated by both the lengths and the widths of the cracks. The methods are illustrated in Figure 4-86, in which the lower right crack in the clad fiber shown in Figure 4-82 is magnified. In this figure the unit length of the crack, $L$, is depicted a segment of blue line parallel to the crack, and the width of the crack, $W$, is depicted as the segment of red line perpendicular to the length of the crack. The degree of cracking of this segment of the crack is then designated as $L \times W$. All other segments of all cracks in the cladding are measured in the same way, and the sum of all $L_{i} \times W_{i}$ are calculated as the degree of cracking in the cladding for this sample.

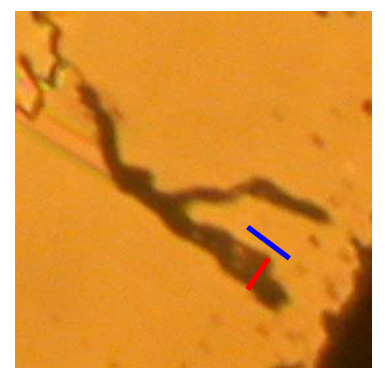

Figure 4-86

- Uniform cladding thickness along the whole length of the fiber

Transverse cross sections every 1 inch along the clad fiber are examined to determine the uniformity of the spinel cladding along the fiber. In each cross section, the thickness of cladding is determined by the average of the cladding thickness calculated in 8 locations as shown in Figure 4-87, where each pair of diagonally opposed directions are connected with a broken red line. 


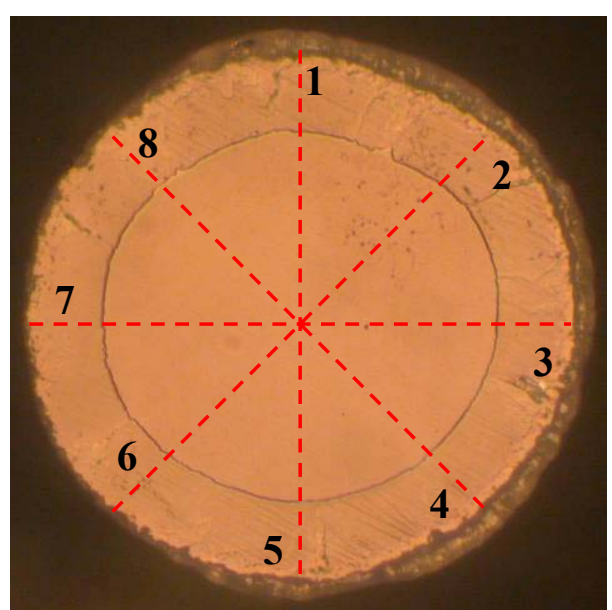

Figure 4-87

- A smooth and crack free surface of coated fibers after drying, binder burnout, and the final firing

The surface of the coated fiber is examined after each stage of drying, binder burnout, and the final high-temperature firing. The procedures of examining the cracking in the cladding from the transverse cross section of the fiber are used here to examine the surface cracking on the surface of clad fibers.

Each clad sapphire fiber is ranked as either "best", "good", "fair", "bad", or "worst" in each of the criteria stated above. The ranking is then substituted into the existing software program to calculate the response table in which the effect of each individual factor is plotted.

The Taguchi method is a critically important method to clarify the effect of each factor in preparing the spinel clad sapphire samples. By adjusting each factor according to the results from the Taguchi array, clad samples with optimal optical and mechanical properties can be fabricated.

\subsubsection{Application of designed experiments using the Taguchi method to optimize sapphire cladding}

Research directed towards development of the magnesium aluminate spinel cladding during the first year (Phase I) of this program showed that the mechanical and optical qualities of the cladding depends on several factors, some of which may be interdependent. In order to efficiently determine the slurry composition and processing procedure that leads to the optimal spinel cladding, we undertook a series of designed experiments using the Taguchi method.

The Taguchi method is a technique for designing and performing experiments. The goal is to investigate processes where the output depends on many factors without tediously 
and uneconomically running the process using all possible combinations of values of those factors. By systematically choosing certain combinations of variables, it is possible to separate their individual effects.

Successful implementation of designed experiments using the Taguchi Method requires that the objects of interest be carefully studied. Questions such as "What are the goals?", "What are the factors important in achieving the goals?", "How do you decide the levels in each important factor?", "What do you measure and how do you measure?" should be carefully answered before starting the experiments.

\subsubsection{Establishing goals for designed experiments}

The three major goals in implementing the designed experiments are (1) to create spinelclad sapphire fibers with optically smooth cladding/core interface, (2) to create mechanically strong spinel cladding without cracks, and (3) to achieve uniform cladding thickness along the whole length of the fiber.

\subsubsection{Determining factors for designed experiments}

Based on the experience obtained during cladding development during Phase I of the program, a list of factors that appeared to contribute to the quality of the sapphire cladding was tabulated. Potentially important factors include:

1. Smoothness of the sapphire fiber surface used for dipping

2. Procedures for cleaning of sapphire fibers before dipping

3. Type and amount of dispersant

4. Solid loading of ceramic powders

5. Weight ratio between $\mathrm{MgO}$ and spinel powders

6. Percentage of nano-MgO powders in all $\mathrm{MgO}$ powders

7. Types and amount of binder

8. Fiber dipping speed and the mechanical stability of the dipping apparatus

9. Number of dips

10. Dispersion milling session time and speed

11. Binder burnout heating profile and atmosphere

12. High temperature firing temperature, time, and atmosphere

The number of factors chosen for the Taguchi experiments also depends on the array selected. It was decided according to the time constrain of the project that an L18 array was most suitable. The L18 array is shown in Table 4-19 below. The L18 array consists of 7 three levels factors and 1 two level factor, and a total of 18 samples need to be fabricated. Each of the eighteen rows represents one coated sapphire fiber sample prepared using the corresponding factor and level in each column. 
Table 4-19: L18 array $\left(2^{1} \times 3^{7}\right)$ orthogonal array

\begin{tabular}{|c|c|c|c|c|c|c|c|c|}
\hline \multirow[b]{2}{*}{ Sample \# } & \multicolumn{8}{|c|}{ Columns (Factors) } \\
\hline & 1 & 2 & 3 & 4 & 5 & 6 & 7 & 8 \\
\hline 1 & 1 & 1 & 1 & 1 & 1 & 1 & 1 & 1 \\
\hline 2 & 1 & 1 & 2 & 2 & 2 & 2 & 2 & 2 \\
\hline 3 & 1 & 1 & 3 & 3 & 3 & 3 & 3 & 3 \\
\hline 4 & 1 & 2 & 1 & 1 & 2 & 2 & 3 & 3 \\
\hline 5 & 1 & 2 & 2 & 2 & 3 & 3 & 1 & 1 \\
\hline 6 & 1 & 2 & 3 & 3 & 1 & 1 & 2 & 2 \\
\hline 7 & 1 & 3 & 1 & 2 & 1 & 3 & 2 & 3 \\
\hline 8 & 1 & 3 & 2 & 3 & 2 & 1 & 3 & 1 \\
\hline 9 & 1 & 3 & 3 & 1 & 3 & 2 & 1 & 2 \\
\hline 10 & 2 & 1 & 1 & 3 & 3 & 2 & 2 & 1 \\
\hline 11 & 2 & 1 & 2 & 1 & 1 & 3 & 3 & 2 \\
\hline 12 & 2 & 1 & 3 & 2 & 2 & 1 & 1 & 3 \\
\hline 13 & 2 & 2 & 1 & 2 & 3 & 1 & 3 & 2 \\
\hline 14 & 2 & 2 & 2 & 3 & 1 & 2 & 1 & 3 \\
\hline 15 & 2 & 2 & 3 & 1 & 2 & 3 & 2 & 1 \\
\hline 16 & 2 & 3 & 1 & 3 & 2 & 3 & 1 & 2 \\
\hline 17 & 2 & 3 & 2 & 1 & 3 & 1 & 2 & 3 \\
\hline 18 & 2 & 3 & 3 & 2 & 1 & 2 & 3 & 1 \\
\hline
\end{tabular}

The task now is to pick the most important 8 factors out of the list above and determine their levels. Experience gained from previous experiments helped to narrow down the list of potentially important factors. For example, since a fiber cleaning procedure using 2-propanol has been proved effective in producing samples with smooth core/cladding interface, Factor 2 above was removed from the candidate list. As to the smoothness of the sapphire fiber surface, since no defects or scratches are discernable using optical microscopes, Factor 1 was also removed from the list. According to the results from previous experiments, it was decided that the solid loading, i.e., the weight ratio of ceramic powders, is to be fixed at $16 \%$ and the number of dips at 150 dips. Since the binder burnout profile seems to have less effect on the clad fiber properties than the high temperature firing profile, the former also will be removed from the list.

One of the most important factors is the type and amount of dispersant. Extensive research and study was performed during Phase I to determine the best dispersant for the current slurry system. The dispersants studied include Darvan 811 (dispersant agent from R.T. Vanderbilt Company, Inc), Triton QS-44 (surfactant from The Dow Chemical Company), Coatosil 1211 (coating additive from GE Silicones), and manhaden fish oil from (Sigma-Aldrich Inc.). The dispersants were studied using techniques such as particle size analysis and sedimentation tests. The most important observation on which the final decision was based was from the sedimentation tests. According to Stokes' Law, the terminal settling velocity of a smooth, rigid sphere in a viscous fluid is proportional to the square of the sphere radius. Therefore, in general, a fine ceramic particle would settle much slower than a course ceramic particle of the same kind. Figure 4-88 below shows the results of the sedimentation tests using either fish oil (Batch 1) or Darvan (Batch 3) as the dispersant, or without using any dispersant at all (Batch 2). As shown in the figure, the spinel powders settle the slowest in Batch 1, where fish oil was 
added as dispersant, with no clear-cut interface between sediment and the liquid above. In Batch 2, without any dispersant, the powders settle faster than in Batch 1 with a lower sediment/clear liquid interface. In Batch 3, where Darvan is added as the dispersant, the powders settle even faster. Most likely the powders agglomerate together in Batch 3 and form even larger agglomerates that increase the settling rate.

Other dispersant candidates such as Triton and Coatosil behave in a very similar manner as Darvan. It was therefore determined that fish oil is most capable of keeping the ceramic powders in a finely dispersed state. It seems that the dispersion capability of fish oil is enhanced by the presence of PVP. An observation supporting the beneficial role of PVP in dispersion is that the slurry viscosity always drops when PVP is added to the slurry containing fish oil as the dispersant.

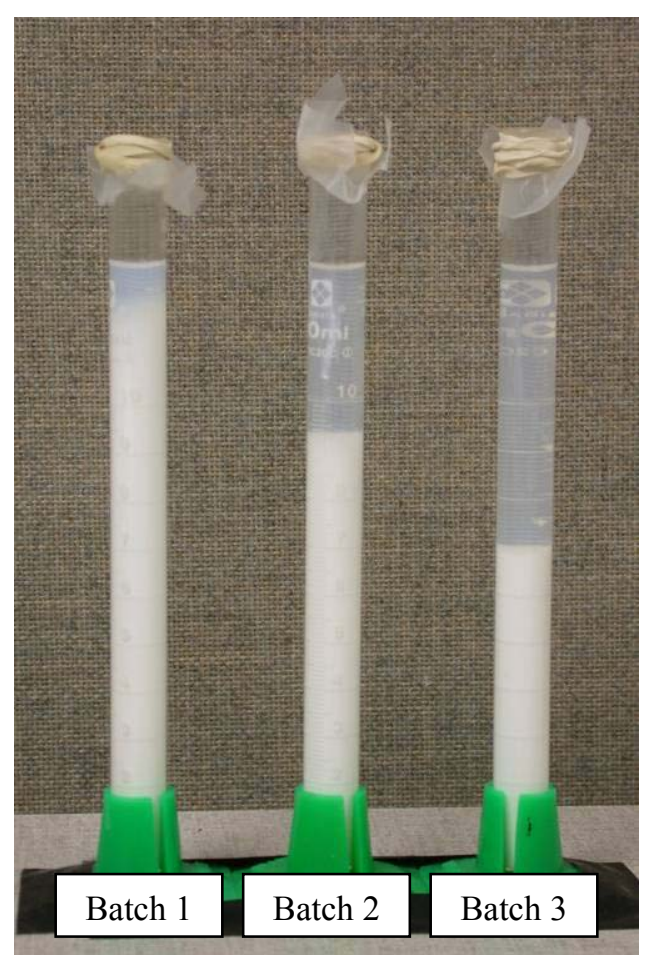

Figure 4-88: Sedimentation test results of spinel powders from Baikowski International after 106 days. The solid loading of each batch is 15.7 weight \% and the solvent are $18.66 \mathrm{~g}$ of 2-propanol and 2.12 g of 1-methoxy-2-propanol. In Batch 1, $50 \mu \mathrm{L}$ of fish oil and $0.2 \mathrm{~g}$ of PVP are added. In Batch 2, no dispersant is added. In batch 3, $50 \mu \mathrm{L}$ of Darvan 811 is added. All batches are ball milled for 21.5 hours.

The final 8 factors chosen are listed in Table 4-20 below. 
Table 4-20: Final chosen factors and their levels

\begin{tabular}{|l|l|l|l|l|}
\hline & Factors & Level 1 & Level 2 & Level 3 \\
\hline 1 & Nano MgO weight \% in all MgO powders & 10 & 20 & --------- \\
\hline 2 & Dipping speed $(\mathrm{mm} / \mathrm{sec})$ & 2.5 & 2.7 & 2.9 \\
\hline 3 & Maximum firing temperature $\left({ }^{\circ} \mathrm{C}\right)$ & 1550 & 1575 & 1600 \\
\hline 4 & MgO/Spinel ratio & $6: 1$ & $9: 1$ & $12: 1$ \\
\hline 5 & Dispersion milling session time $(\mathrm{hr})$ & 3 & 6 & 12 \\
\hline 6 & Fish oil amount $(\mu \mathrm{L})$ & 25 & 50 & 75 \\
\hline 7 & 10K PVP weight ratio $(\%)$ & 100 & 90 & 80 \\
\hline 8 & PVP amount (both $10 \mathrm{~K}$ and $55 \mathrm{~K})(\mathrm{g})$ & 0.4 & 0.5 & 0.6 \\
\hline
\end{tabular}

\subsection{Determining levels for factors}

The proper choice of levels for each factor is extremely important in the execution of the Taguchi Method. Usually it entails a series of trial-and-error experiments with some scientific judgment and luck. In practice, the determination of optimal levels starts with one set of current conditions showing the most promise. In this set of most promising conditions, only the levels of one factor are altered while keeping all other factors fixed. Then the resulting responses are analyzed to determine whether the levels just changed are feasible and practical for the experiment. For example, it was observed in previous Phase I experiments that the slurry viscosity increases dramatically when the nano- $\mathrm{MgO}$ weight \% (Factor 1) reaches about $25 \%$ in a certain set of experimental conditions. Since a set of full factorial experiments with the factors and levels shown in Table 1 consists of more than $4000\left(3^{7} \times 2\right)$ different sets of experiments, it is impossible to know for sure whether this huge increase in viscosity is also true in other sets of experimental conditions without extensive study, which is very time consuming. To keep it on the safe side, Level 2 in Factor 1 is set to $20 \%$ to prevent this huge increase in viscosity. On the other hand, if the levels are chosen too conservatively, it runs a risk that they make no difference at all to the results. So, some degree of scientific judgment is required in making the final decision. The levels for other factors are determined in a similar manner through a series of designed experiments.

\subsubsection{Measurement of experimental response}

To evaluate the design to experiment results, we measure responses appropriate for the goals we would like to achieve, namely, the smoothness of the cladding/core interface, the mechanical strength of the spinel cladding (and the presence or absence of cracks), and the uniformity of cladding thickness along the whole length of the fiber. There are in total 13 responses chosen, which may be grouped in three categories: geometrical responses, mechanical quality responses, and optical quality responses: 
Geometrical Response:

- G1: core diameter

- G2: overall cladding thickness

- G3: outer cladding thickness

- G4: skin thickness

- G5: overall fiber diameter

- G6: concentricity

Mechanical Quality Response:

- M1: flexibility from 3-point bending test

- Crack parameters:

○ M2: total area of cracks

- M3: length of the longest crack

- M4: number of cracks

Optical Quality Response:

- O1: core/cladding interface roughness

- O2: Numerical Aperture (NA) measurement

- O3: attenuation measurement

\subsection{Geometrical response}

The definitions used for the geometrical responses can be best illustrated by Figure 4-89 below. The figure shows a polished cross section of a clad fiber. The core diameter (G1) is defined as the distance between points A and B. The core diameters along three other diagonally symmetric directions depicted by three red dotted lines are also measured. The reported value of G1 is the average of all four measured core diameters. The overall cladding thickness (G2) is defined as the distance between points B and D. As seen in Figure 4-89 and other cross-sectional pictures to be shown later, the cladding in fact consists of two distinctive parts: the bulk part of cladding located between points $\mathrm{B}$ and $\mathrm{C}$, and a thinner layer of "outer cladding" between points $\mathrm{C}$ and $\mathrm{D}$, which is designated as the response G3, outer cladding thickness. Similarly, values for G2 and G3 at 7 other diagonally symmetric directions are also measured. The reported values of G2 and G3 are the average of eight "overall cladding thickness" and "outer cladding thickness" measurements, respectively, in all 8 locations. It is believed that both cladding parts are made of spinel. However, we believe that they are formed by different mechanisms. The bulk part of cladding, i.e., the spinel located between points $\mathrm{B}$ to $\mathrm{C}$, is formed by the reaction due to the $\mathrm{MgO}$ diffusing from the coating "into" the sapphire core, while the thin outer layer of spinel, i.e., the spinel located between points $\mathrm{C}$ to $\mathrm{D}$, is formed by the reaction between the aluminum oxide molecules diffusing "outward" from the core into the coating and the $\mathrm{MgO}$ molecules inside the coating. The region between points $\mathrm{D}$ and E in Figure 4-89 defines the "residual layer," G4. It is believed that this residual layer consists of materials not fully reacted in the coating after firing. The reported value of $\mathrm{G} 4$ is the average of all thickness measured at 8 symmetrical locations. The overall fiber 
diameter G5 is defined as the distance between points $\mathrm{E}$ and F. The last geometrical response is "concentricity", G6. It is defined as the distance between the center of the core/cladding interface, i.e., the interface containing points A and B in Figure 4-89, and the center of the cladding/skin interface, i.e., the interface containing point $\mathrm{D}$ in Figure 4-89.

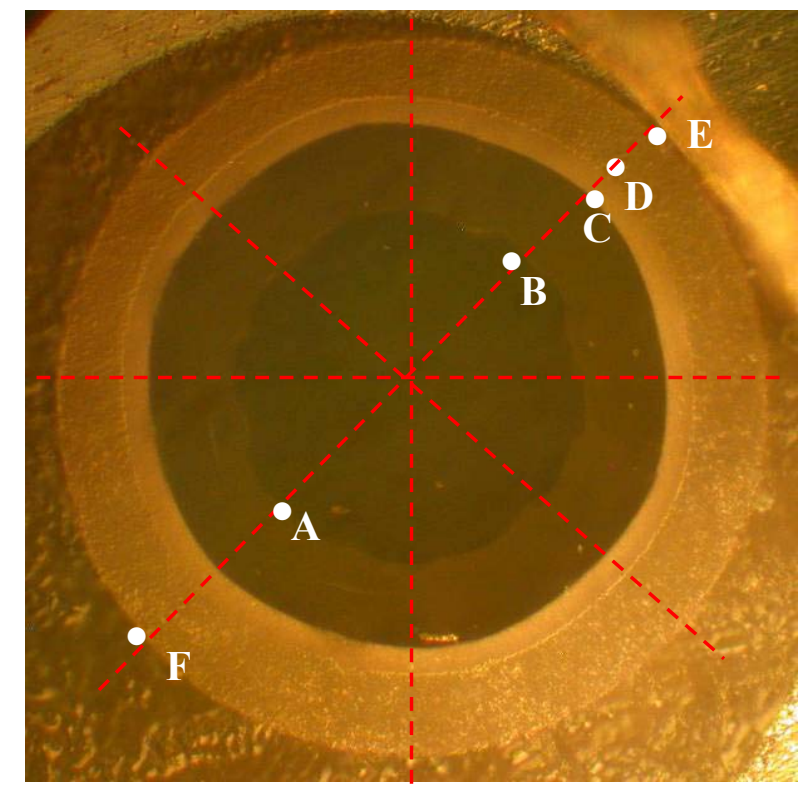

Figure 4-89: The polished cross section of a clad fiber.

\subsection{Mechanical quality response}

The first mechanical quality response is the "bending flexibility," M1, obtained from a 3-point bend test. The apparatus shown in Figure 4-90(a) was used to measure the radius of the induced bend in the clad fiber at the point at which the fiber fractured. As shown in the figure, the micrometer of an adjustable stage is coupled to a rotary motor which speed was controlled by a constant voltage source. A cylinder, i.e., cylinder C in Figure 4-90(b), is glued to the bottom of the adjustable stage. During the test, cylinder $\mathrm{C}$ descends in a constant rate of about $0.009 \mathrm{~mm} / \mathrm{sec}( \pm 0.001 \mathrm{~mm} / \mathrm{sec})$ until the test fiber breaks. Below the stage a cylinder, i.e., "cylinder A" in Figure 4-90(b), and a ceramic block, i.e., labeled "Stop" in the figure, are glued to a base plate. Another cylinder, cylinder B, positioned against the "stop" and is free to rotate leftward. The initial distance between cylinders A and B was $17 \mathrm{~mm}$. During measurement, the reading (H1) on the micrometer when Cylinder $\mathrm{C}$ in Figure 4-90(b) first touches the test fiber is recorded. Then a constant voltage is supplied to the motor causing cylinder $\mathrm{C}$ to descend at a predetermined rate. The reading on the micrometer $(\mathrm{H} 2)$ is once again recorded at the point of fiber breakage due to bending. The reported deflection is the difference between the two recorded readings, i.e., $\mathrm{H} 2-\mathrm{H} 1$, in millimeters, which in turn is reported as the value for response M1. 


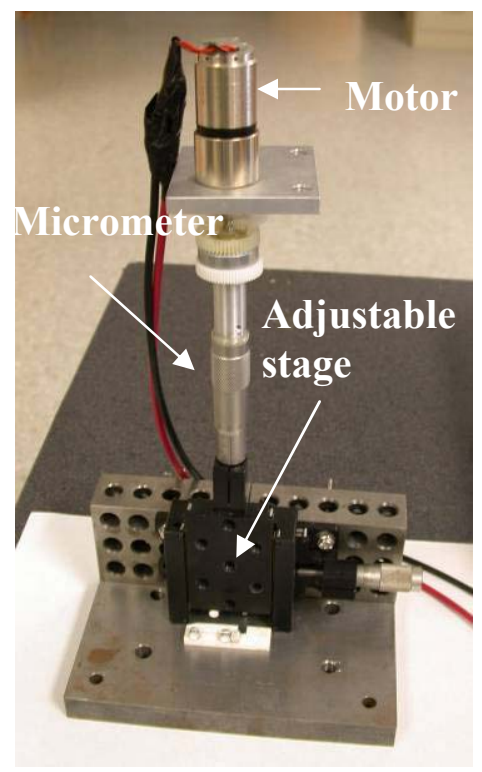

(a)

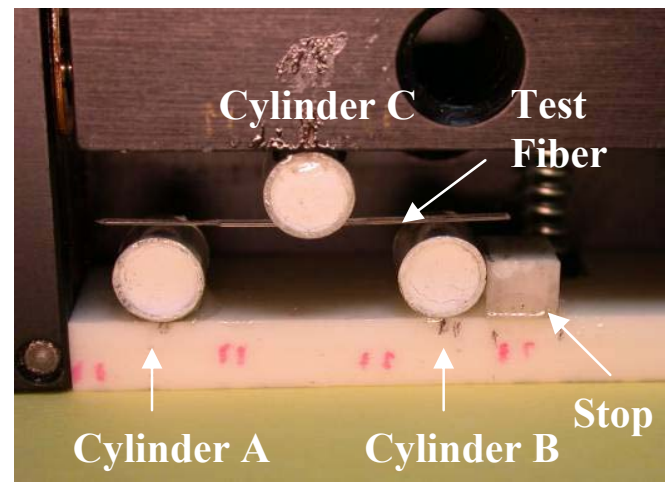

(b)

Figure 4-90 (a) and (b): the apparatus used to perform the bending test

The mechanical response M2 represents the surface area of cracks in $\mu \mathrm{m}^{2}$ in the cladding as depicted, for example, by the crack shown in Figure 4-88. The measure of the crack areas is obtained using a microscope with a video camera and image processing software. M3 is the length of the longest crack existing in the cladding, and M4 represents the number of individual cracks in the cladding.

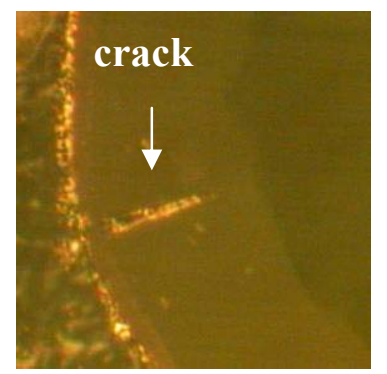

Figure 4-91: A portion of a polished cross section in the cladding of a clad fiber with large cracks.

\subsection{Optical quality response}

The procedures for obtaining the core/cladding interface roughness $(\mathrm{O} 1)$ can be illustrated by Figure 4-92. In this figure, a zig-zag core/cladding interface is clearly visible. In order to measure the degree of smoothness of the interface, a running average of the interface, as shown in the figure as a smooth blue dotted curve, is drawn along the interface. The sum of the areas enclosed between the core/cladding interface and the running average curve, i.e., the sum of areas A1, A2, A3, .... as shown in Figure 4-92, is calculated. This area is called the "roughness area (R.A.)". Furthermore, the area of the core enclosed by the running average curve is also calculated. This area is called the 
"core area (C.A.)". The core/cladding interface roughness is then defined as the ratio between the "roughness area" and the "core area", i.e., R.A/C.A.

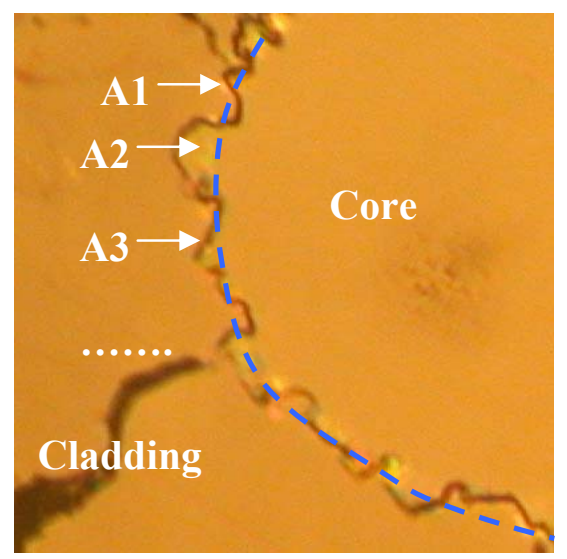

Figure 4-92: The calculation of core/cladding interface roughness

The second optical quality response $\mathrm{O} 2$ is the numerical aperture (NA) of the clad fiber. The NA is measured using the experimental setup depicted in Figure 4-93 below. The clad fiber sample, about 3.0 inches long, is packaged into a ceramic tube with

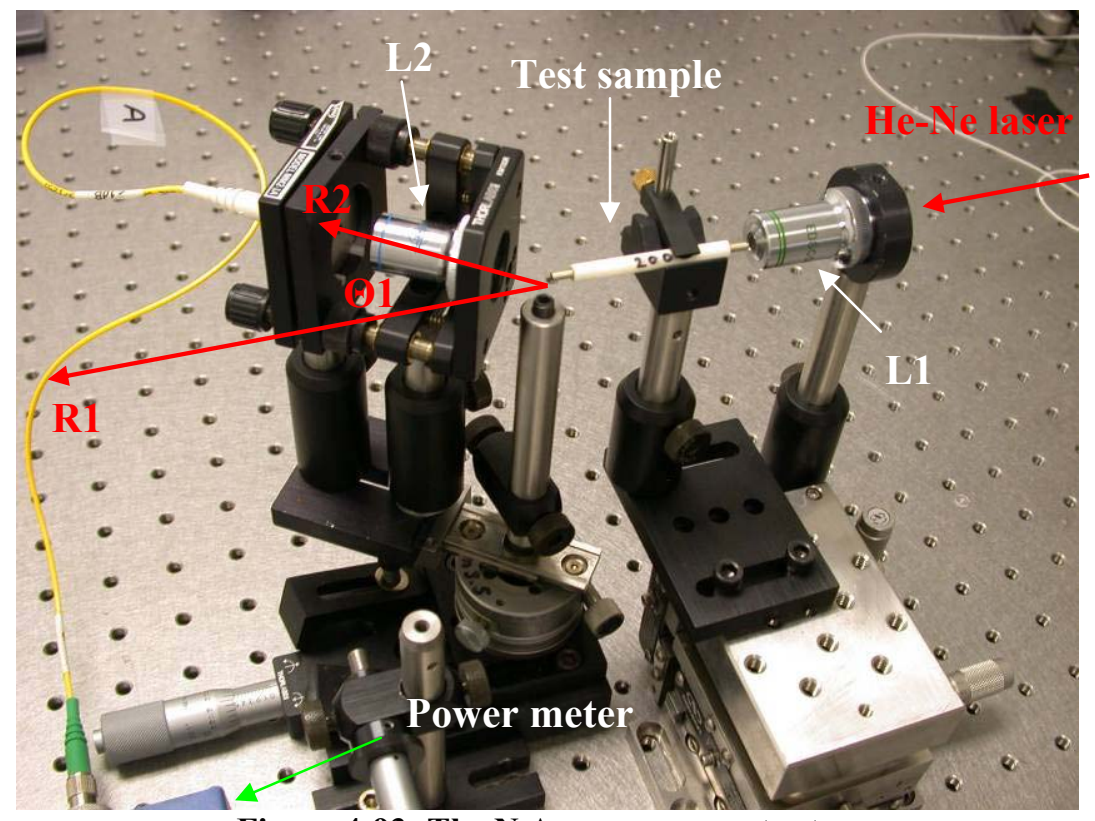

Figure 4-93: The N.A. measurement setup

stainless steel ferrules on both ends of the tube. The ends of the sapphire fiber are plotted into the ends of the steel ferrules using epoxy. The packaged fiber is polished on both ends using abrasive papers with sizes down to $0.5 \mu \mathrm{m}$.

An objective lens used to launch light from a helium neon laser $(633 \mathrm{~nm})$ into the test sample, as shown in Figure 4-93 as "L1", has a magnification of 10 and a NA of 0.4, and 
the lens to collect and focus light into one end of a single-mode patch cord for intensity measurement, as shown in the figure as "L2", has a magnification of 10 and a NA of 0.25 . The other end of the patch cord is connected to a handheld power meter.

At the beginning of measurement, the intensity of the light output by the test sample in the direction parallel to the axis of the sample, i.e., the direction depicted by ray " $\mathrm{R} 1$ " in Figure 4-93, is measured. Then the fixture holding both the objective lens "L2" and one end of the patch cord is swung counterclockwise along an arc which center is aligned with one end of the test sample until the power reading drops to $-13 \mathrm{~dB}$ of the value measured in the direction of ray "R1". The angle swung, i.e., $\theta 1$ in Figure 4-93, is recorded. The same procedure is repeated by swinging the fixture clockwise until the power drops to $-13 \mathrm{~dB}$, and the angle, i.e., $\theta 2$ where this occurs is recorded. The reported $\mathrm{NA}$ (the response $\mathrm{O} 2$ ) is then calculated using the equation

$$
N . A .=\operatorname{Sin}\left(\frac{\theta_{1}+\theta_{2}}{2}\right)
$$

Equation 14

The last optical quality response is the attenuation of clad fibers, O3. The experimental set-up used to measure attenuation is shown in Figure 4-94 below. The light output from a superluminescent diode (SLD) at $1550 \mathrm{~nm}$ is first launched into one end of a three inch long sapphire fiber without any cladding, and the power collected at the other end of the test sample, i.e., $\mathrm{P}_{0}$, is measured. Then the sample is replaced with a clad sapphire fiber of the same length and the same procedure is repeated. The measured power in this case is $\mathrm{P}$. The reported attenuation $\mathrm{O} 3$ is calculated as

$$
\text { Attenuation }=-10 \log \left(\frac{P_{0}}{P}\right) \text {. }
$$

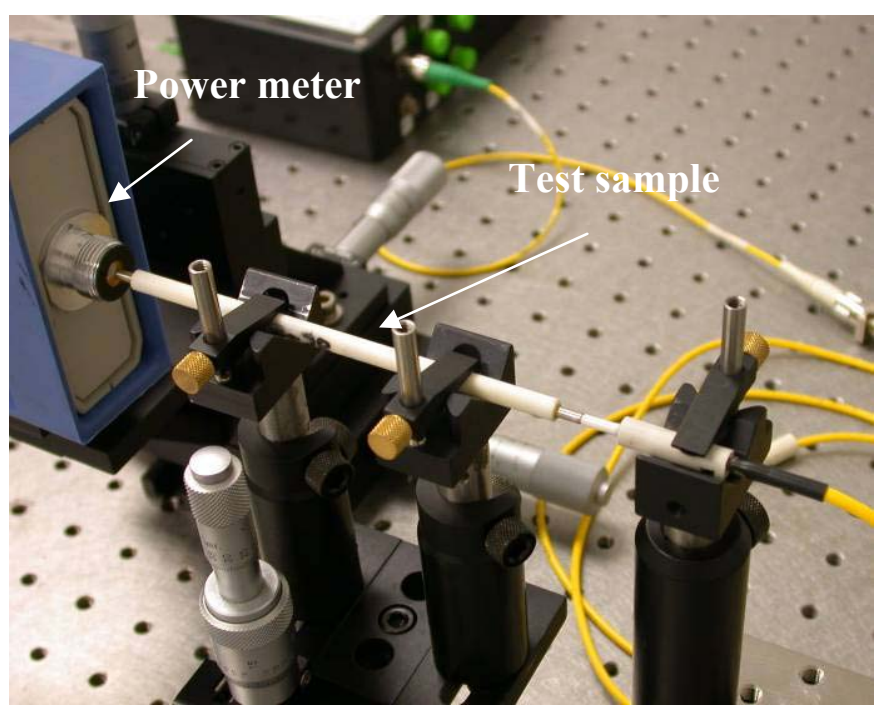

Figure 4-94: The attenuation measurement setup 


\subsubsection{Procedure for application of coatings}

The detailed steps for the preparation of clad fiber samples are listed below. The rationale or goal for each step is explained whenever possible. Some cautionary notes are also provided.

1. The ceramic powders are heated at $500{ }^{\circ} \mathrm{C}$ for 4 to 5 hours before slurry preparation. The goal is to remove adsorbed water moisture on the powder surface. This step is especially important for nano-powders due to their extremely large specific surface areas. Two types of magnesium oxide powders were used. One is a type of nanopowder, and the other has particle size in the micrometer range. A small amount of spinel powders is also added as nucleation agent for newly formed spinel crystallites.

2. At the same time, the grinding media used in slurry ball milling is also heated at $300{ }^{\circ} \mathrm{C}$ for 4 to 5 hours using a hot plate. The goal here is also to remove adsorbed water moisture. After the heating is completed, the grinding media is left to cool at room temperature for 15 to 20 minutes. A small fan is used to increase the cooling rate of the media.

3. The cooled grinding media is then put into a 4-oz wide-mouth polyethylene bottle with the desired amount of 2-propanol, the first of two solvents. Also added into the bottle is the dispersant, Menhaden fish oil. The bottle is then shaken by hand for 30 seconds. The choice of fish oil as the dispersant will be explained in one of the following sections.

4. Three types of ceramic powders, i.e., magnesium oxide nanopowders, magnesium aluminate spinel powders, and micron-size magnesium oxide powders, are added to the bottle sequentially. The bottle is shaken by hand for one minute after the addition of the first type of powder, another one minute after the addition of the second type of powder, and for two minutes after the last type of powder is added. The goal of hand shaking is to facilitate the dispersion of the powder in the slurry. Each type of powder is added to the bottle immediately after removal from the furnace at $500{ }^{\circ} \mathrm{C}\left(932{ }^{\circ} \mathrm{F}\right)$ to minimize the adsorption of moisture from room atmosphere.

5. The bottle is then ball milled for a specified number of hours. The speed of rotation of the bottle influences the degree of size reduction of powders. It should be kept constant in each ball milling session. This milling session is called the "dispersion milling session." Its goal is to disperse fully the powders without the possible interference of binder molecules in the dispersion performance.

6. After dispersion milling, two types of binder solutions are added to the slurry: both types are polyvinylpyrrolidone (PVP), one with $10 \mathrm{~K}$ molecular weight, and the other with $55 \mathrm{~K}$ molecular weight. The binder in powder form has been dissolved in 2propanol in advance. Since binder has tendency to stick together, the goal here is to 
ensure that the binder dissolves thoroughly in the slurry. After each step adding binder, the bottle is shaken by hand for 30 seconds.

7. The last chemical added to the bottle is 1-methoxy-2-propanol, the second solvent. Compared to 2-propanol, 1-methoxy-2-propanol has a smaller rate of evaporation. The proper amount of 1-methoxy-2-propanol, together with other favorable conditions, can effectively prevent the occurrence of "carrot" shape fiber caused by the "skinning effect" discussed in Section 4.1.15.7 below.

8. The bottle is then put back onto the ball miller for the final stage of milling, called "binder milling" session, for 3 to 4 hours. Binder milling longer than 3 to 4 hours may have a detrimental effect on the slurry property.

9. Now the slurry is ready for dipping. The sapphire fiber is first cleansed with Kimwipes soaked with 2-propanol. The entire fiber is then soaked in 2-propanol for about 1 hour. The exact time of soaking does not seem to matter; it could be as short as 15 minutes. However, the type of chemical used is important. The surface properties of fibers soaked with chemicals other than 2-propanol may be undesirably altered. It was observed that the ceramic slurry had difficulty sticking to the fibers soaked in ethanol in advance.

10. To prevent solvent evaporation, the top of the graduated cylinder containing the slurry is covered by a piece of Parafilm with a small hole at the center. The size of the hole is a little larger than that of the fiber. The hole should not be too small for the fiber to pass through comfortably. On the other hand, it should not be so large that it can not effectively reduce the rate of solvent evaporation. Up to two fibers can be dipped simultaneously using an automated dipping machine. The dipping speed is an important factor which influences coating thickness. In general, a faster dipping speed creates a thicker coating. The number of dips is another obvious factor affecting coating thickness that needs to be determined.

11. The dipped fibers are then transferred to an oven for binder burnout. The heating profile consists of four stages. In the first stage, the fiber is heated for a room temperature to $340^{\circ} \mathrm{C}\left(644^{\circ} \mathrm{F}\right)$ at a rate of $5^{\circ} \mathrm{C} / \mathrm{min}\left(9^{\circ} \mathrm{F} / \mathrm{min}\right)$. This is followed by a slow heating up from $340^{\circ} \mathrm{C}\left(644^{\circ} \mathrm{F}\right)$ to $440^{\circ} \mathrm{C}\left(824^{\circ} \mathrm{F}\right)$ at a rate of $1^{\circ} \mathrm{C}$ per minute $\left(1.8^{\circ} \mathrm{F} / \mathrm{min}\right)$. From a Differential Thermal Analysis (DTA) measurement performed at Virginia Tech, the PVP binder decomposes over a temperature range from $340{ }^{\circ} \mathrm{C}$ $\left(644^{\circ} \mathrm{F}\right)$ to $440^{\circ} \mathrm{C}\left(824^{\circ} \mathrm{F}\right)$. A slow heating is designed to reduce the decomposition rate of PVP to avoid the formation of large voids in the coating. Following a slow heating, the third stage consists of heating the fiber from $440{ }^{\circ} \mathrm{C}\left(824^{\circ} \mathrm{F}\right)$ to $600^{\circ} \mathrm{C}$ $\left(1,112^{\circ} \mathrm{F}\right)$ at a rate of $5^{\circ} \mathrm{C} / \mathrm{min}\left(9^{\circ} \mathrm{F} / \mathrm{min}\right)$. The fourth and final stage is a dwell period of one hour at $600{ }^{\circ} \mathrm{C}\left(1,112^{\circ} \mathrm{F}\right)$. The goal is to make sure that the PVP burns out completely.

12. After binder burnout, the fibers are transferred to another high temperature furnace for the final firing in argon. The maximum firing temperature and time are the most 
critical elements in the heating profile. A $50^{\circ} \mathrm{C}\left(90^{\circ} \mathrm{F}\right)$ increase in temperature may cause cracks to start forming in the cladding. A critical improvement regarding heating environment was made in the high temperature firing setup. It was realized that the samples need to be fired in very pure argon in order to have desirable overall properties. The argon gas was originally flowed into a 6 " $\times 6$ " $\times 6$ " heating chamber from a small hole on the top of chamber as shown in Figure 4-95(a). To increase argon concentration, the samples are now enclosed in a ceramic tube housing which argon gas enters directly from an opening at the top as shown in Figure 4-95(b). The properties of clad fiber, especially their bending strength and flexibility, improved significantly after this modification.

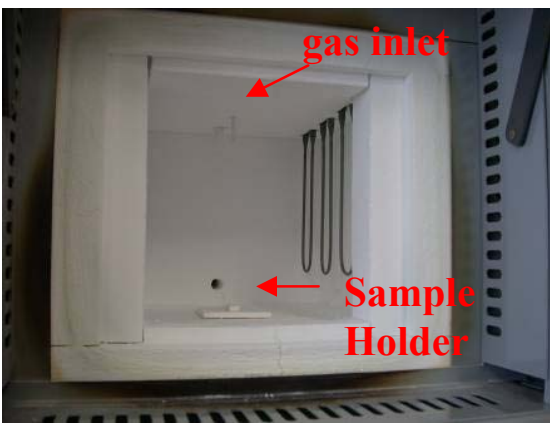

(a)

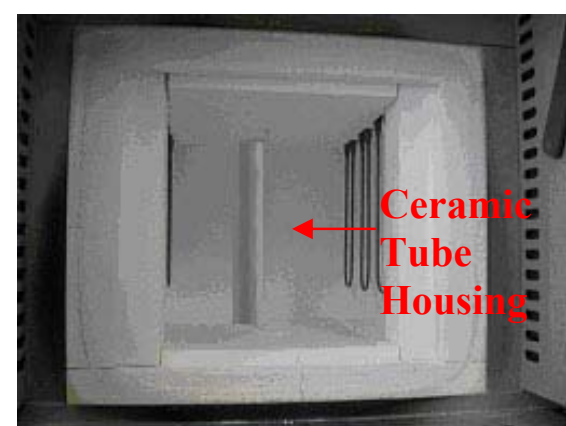

(b)

Figure 4-95: The samples in (b) are fired in much purer argon than in (a).

\subsubsection{Experimental results from designed experiments}

Eighteen samples were fabricated according to the L18 array listed in Table 4-19 and the factors and levels listed in Table 4-20. Each sample was prepared using to the steps described in Section 4.1.15.4. For each three-inch long clad samples, the cross-sections in three different locations, i.e., the top, the bottom, and the middle of the sample length, were polished and analyzed.

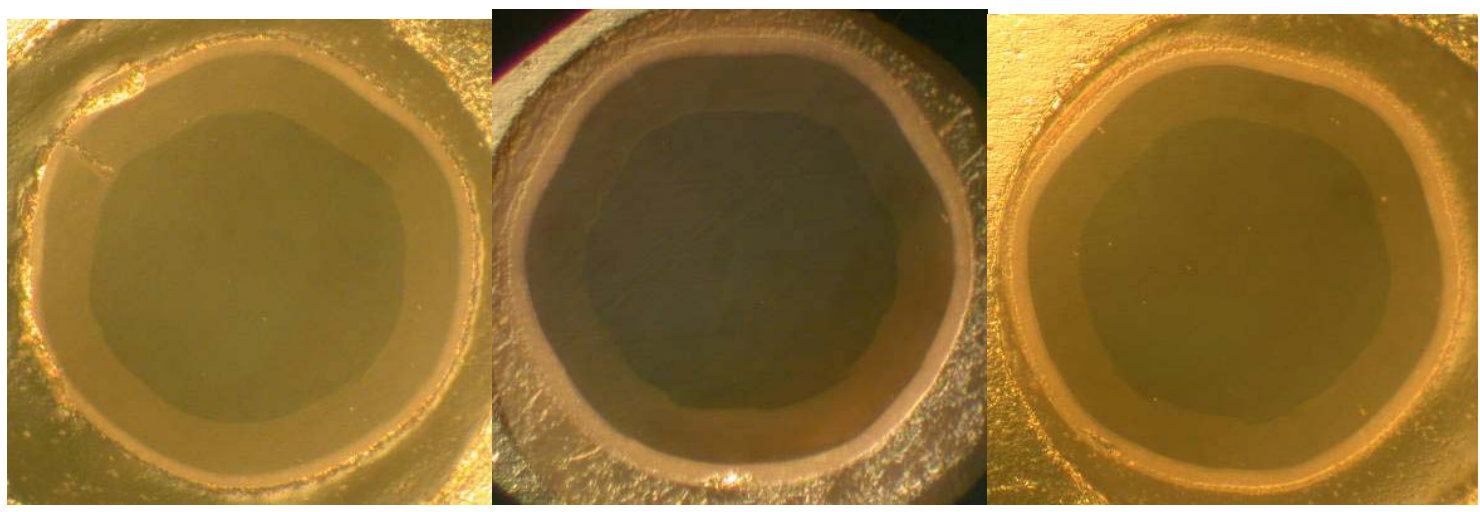

(a) cross-1 (top)

(b) cross-3 (middle)

(c) cross-2 (bottom)

Figure 4-96: the 3 cross sections polished and analyzed for sample 1

The three polished cross sections for Sample 1 are shown in Figure 4-96. In each cross section, 10 out of the 13 responses are analyzed. The results of the analysis for sample 1 are listed in Table 4-21, Table 4-22, and Table 4-23 for the geometrical, mechanical, and 
optical responses, respectively. The value of each of the 10 responses used for the following Taguchi analysis is the average value calculated from the responses measured at all three cross-sections. There is only one data point each measured for the responses M1 (bending deflection), O2 (N.A.) and O3 (attenuation), which are not listed in the tables, due to the nature of measurement. The results for all 13 responses of sample 1 are listed in Table 4-24.

Table 4-21: All 6 geometrical response data for sample 1

\begin{tabular}{|c|c|c|c|c|c|c|}
\hline $\begin{array}{c}\text { 6-1-05 } \\
\text { Sample 1- } \\
1\end{array}$ & $\begin{array}{c}\text { mean } \\
\text { core } \\
\text { diameter } \\
(\mu \mathrm{m}) \\
(\mathbf{G 1})\end{array}$ & $\begin{array}{c}\text { mean } \\
\text { overall } \\
\text { cladding } \\
\text { thickness } \\
(\mu \mathrm{m}) \\
(\mathbf{G} 2)\end{array}$ & $\begin{array}{c}\text { mean } \\
\text { outer } \\
\text { cladding } \\
\text { thickness } \\
(\mu \mathrm{m}) \\
(\mathrm{G})\end{array}$ & $\begin{array}{c}\text { mean skin } \\
\text { thickness } \\
(\mu \mathrm{m}) \\
(\mathbf{G 4})\end{array}$ & $\begin{array}{l}\text { mean } \\
\text { overall } \\
\text { fiber } \\
\text { diameter } \\
(\mu \mathrm{m}) \\
(\mathrm{G} 5)\end{array}$ & $\begin{array}{c}\text { interface } \\
\text { center } \\
\text { distance } \\
(\mu \mathrm{m}) \\
(\mathbf{G 6})\end{array}$ \\
\hline cross 1 & 95.2 & 20.6 & 4.4 & 2.10 & 136.3 & 0.2 \\
\hline cross 2 & 95.2 & 21.4 & 5.5 & 2.60 & 138.1 & 0.7 \\
\hline cross 3 & 92.4 & 22.3 & 5.4 & 1.00 & 137.5 & 1.1 \\
\hline Average & 94.3 & 21.4 & 5.1 & 1.9 & 137.3 & 0.67 \\
\hline
\end{tabular}

Table 4-22: The mechanical response data M2, M3, and M4 of sample 1

\begin{tabular}{|c|c|c|c|}
$\begin{array}{c}\text { Sample 1- } 1-05 \\
1\end{array}$ & $\begin{array}{c}\text { crack } \\
\text { parameter } \\
\left(\mu \mathrm{m}^{2}\right) \\
(\mathrm{M} 2)\end{array}$ & $\begin{array}{c}\text { crack } \\
\text { length } \\
(\mu \mathrm{m}) \\
(\mathrm{M} 3)\end{array}$ & $\begin{array}{c}\text { number } \\
\text { of } \\
\text { cracks } \\
(\mathrm{M} 4)\end{array}$ \\
\hline cross 1 & 19.7 & 19.5 & 1 \\
\hline cross 2 & 0.0 & 0.0 & 0 \\
\hline cross 3 & 3.5 & 17.0 & 1 \\
\hline Average & 7.7 & 12.2 & $\mathbf{0 . 7}$ \\
\hline
\end{tabular}

Table 4-23: The core/cladding interface roughness data, 01 , of sample 1

\begin{tabular}{|c|c|c|c|}
\hline Sample Name & $\begin{array}{c}\text { Core Area (C.A.) } \\
\text { (pixel) }\end{array}$ & $\begin{array}{c}\text { Roughness } \\
\text { Area (R.A.) } \\
\text { (pixel) }\end{array}$ & $\begin{array}{c}\text { Roughness } \\
\text { Factor } \\
\left(10{ }^{*} \text { R.A/C.A.) }\right. \\
\text { (O1) }\end{array}$ \\
\hline 6-1-05 sample 1-1 cross-1 & 651190 & 2563 & 0.39 \\
\hline 6-1-05 sample 1-1 cross-2 & 566041 & 2638 & 0.47 \\
\hline 6-22-05 sample 1-1 cross-3 & 557439 & 3011 & 0.54 \\
\hline Average & & & 0.47 \\
\hline
\end{tabular}

Table 4-24: The results of all 13 responses of sample 1

\begin{tabular}{|c|c|c|c|c|c|c|c|c|c|c|c|c|c|}
\hline & & & & & & & & & & & & & \\
\hline & $\begin{array}{c}\mathbf{G} 1 \\
(\mathrm{um})\end{array}$ & $\begin{array}{c}\text { G2 } \\
\text { (um) }\end{array}$ & $\begin{array}{c}\text { G3 } \\
\text { (um) }\end{array}$ & $\begin{array}{c}\text { G4 } \\
\text { (um) }\end{array}$ & $\begin{array}{c}\text { G5 } \\
\text { (um) }\end{array}$ & $\begin{array}{c}\mathbf{G 6} \\
(\mathrm{um})\end{array}$ & $\begin{array}{c}\text { M1 } \\
(\mathrm{mm})\end{array}$ & $\begin{array}{c}\text { M2 } \\
\left(u m^{\wedge} 2\right)\end{array}$ & $\begin{array}{c}\text { M3 } \\
\text { (um) }\end{array}$ & $\begin{array}{l}\text { M4 } \\
\text { (no } \\
\text { unit) }\end{array}$ & $\begin{array}{l}01 \\
\text { (no } \\
\text { unit) }\end{array}$ & $\begin{array}{l}\mathrm{O} 2 \\
\text { (no } \\
\text { unit) }\end{array}$ & $\begin{array}{c}\mathrm{O} 3 \\
(\mathrm{~dB})\end{array}$ \\
\hline 1 & 94.3 & 21.4 & 5.1 & 1.9 & 137.3 & 0.67 & 0.75 & 7.7 & 12.2 & 0.67 & 0.47 & 0.041 & 3.3 \\
\hline
\end{tabular}

(M1: bending deflection, O2: Numerical Aperture, and O3: attenuation) 
The optical microscopic pictures of all three polished cross sections for each of the 17 samples from sample 2 to sample 18 are shown in Appendix A. Also included in the figures are the results of all 13 responses used for Taguchi analysis for that specific sample.

All the data of all 18 samples used for the following Taguchi analysis are listed in Table 4-25 below.

Table 4-25. Data for all 18 samples

\begin{tabular}{|c|c|c|c|c|c|c|c|c|c|c|c|c|c|}
\hline & $\begin{array}{c}\mathbf{G 1} \\
(\mu \mathrm{m})\end{array}$ & $\begin{array}{c}\mathbf{G 2} \\
(\mu \mathrm{m})\end{array}$ & $\begin{array}{c}\text { G3 } \\
(\mu \mathrm{m})\end{array}$ & $\begin{array}{c}\mathbf{G 4} \\
(\mu \mathrm{m})\end{array}$ & $\begin{array}{c}\text { G5 } \\
(\mu \mathrm{m})\end{array}$ & $\begin{array}{c}\text { G6 } \\
(\mu \mathrm{m})\end{array}$ & $\begin{array}{c}\text { M1 } \\
(\mathrm{mm})\end{array}$ & $\begin{array}{c}\text { M2 } \\
\left(\mu \mathrm{m}^{\wedge} 2\right)\end{array}$ & $\begin{array}{c}\text { M3 } \\
(\mu \mathrm{m})\end{array}$ & M4 & 01 & $\mathrm{O2}$ & $\begin{array}{c}\text { O3 } \\
(\mathrm{dB})\end{array}$ \\
\hline 1 & 94.3 & 21.4 & 5.1 & 1.9 & 137.3 & 0.67 & 0.75 & 7.7 & 12.2 & 0.67 & 0.47 & 0.041 & 3.3 \\
\hline 2 & 89.9 & 22.1 & 3.9 & 1.6 & 134.3 & 0.81 & 0.71 & 0.0 & 0.0 & 0.00 & 0.40 & 0.046 & 8.4 \\
\hline 3 & 86.8 & 21.4 & 3.4 & 2.1 & 131.1 & 0.67 & 0.84 & 3.9 & 6.0 & 0.33 & 0.56 & 0.055 & 7.2 \\
\hline 4 & 94.4 & 20.1 & 4.6 & 1.5 & 134.4 & 1.22 & 0.88 & 0.0 & 0.0 & 0.00 & 0.24 & 0.068 & 3.1 \\
\hline $\begin{array}{c}5 \\
(\mathrm{R} 1) \\
\end{array}$ & 79.4 & 24.7 & 5.8 & 33.0 & 194.1 & 0.76 & 0.73 & 0.0 & 0.0 & 0.00 & 0.59 & 0.059 & 4.5 \\
\hline $\begin{array}{c}6 \\
\text { (R1) }\end{array}$ & 71.7 & 32.9 & 6.2 & 1.3 & 138.4 & 0.90 & 0.41 & 28.4 & 24.1 & 2.00 & 1.30 & 0.052 & 5.1 \\
\hline 7 & 92.8 & 21.6 & 4.9 & 2.5 & 138.9 & 0.82 & 0.83 & 0.0 & 0.0 & 0.00 & 0.02 & 0.063 & 4.9 \\
\hline $\begin{array}{c}8 \\
\text { (R1) } \\
\end{array}$ & 84.7 & 27.4 & 5.8 & 150.3 & 442.1 & 1.18 & 0.78 & 0.0 & 0.0 & 0.00 & 1.13 & 0.096 & 24 \\
\hline $\begin{array}{c}9 \\
(\mathrm{R} 1)\end{array}$ & 93.5 & 21.1 & 4.0 & 1.5 & 137.1 & 0.92 & 0.46 & 50.5 & 8.5 & 1.00 & 0.59 & 0.087 & 5.2 \\
\hline 10 & 90.4 & 22.5 & 4.4 & 5.5 & 140.2 & 0.60 & 0.67 & 0.0 & 0.0 & 0.00 & 0.41 & 0.052 & 2.5 \\
\hline $\begin{array}{c}11 \\
\text { (R1) }\end{array}$ & 89.3 & 25.2 & 5.5 & 2.1 & 141.1 & 0.50 & 0.59 & 17.6 & 6.7 & 0.67 & 0.44 & 0.061 & 7.2 \\
\hline 12 & 97.6 & 19.2 & 2.6 & 2.4 & 135.7 & 0.61 & 0.73 & 35.6 & 18.9 & 1.67 & 0.70 & 0.089 & 10.5 \\
\hline 13 & 92.4 & 22.6 & 4.9 & 2.6 & 137.8 & 0.93 & 1.08 & 9.3 & 7.1 & 0.33 & 0.65 & 0.152 & 5.3 \\
\hline 14 & 87.2 & 25.8 & 4.6 & 1.4 & 138.6 & 0.72 & 0.65 & 108.3 & 25.9 & 1.33 & 0.73 & 0.107 & 12 \\
\hline $\begin{array}{c}15 \\
(\mathrm{R} 1) \\
\end{array}$ & 80.4 & 33.4 & 8.3 & 10.5 & 165.5 & 0.93 & 0.54 & 0.0 & 0.0 & 0.00 & 0.99 & 0.052 & 3.9 \\
\hline 16 & 86.6 & 22.1 & 4.6 & 21.3 & 172.8 & 0.66 & 0.93 & 18.1 & 7.2 & 0.67 & 0.36 & 0.065 & 1.4 \\
\hline 17 & 88.5 & 23.0 & 4.5 & 1.5 & 134.2 & 0.77 & 0.81 & 12.7 & 7.3 & 0.33 & 1.06 & 0.107 & 11.3 \\
\hline $\begin{array}{c}18 \\
\text { (R1) }\end{array}$ & 67.5 & 31.1 & 6.3 & 37.8 & 206.1 & 0.59 & 0.64 & 0.0 & 0.0 & 0.00 & 1.88 & 0.044 & 5.3 \\
\hline
\end{tabular}

\subsubsection{Analysis of results}

The list of factors was examined to ensure that they were independent and that the samples could be made within the timeframe required. It was decided that the timeframe available for the experiments did not permit interactions to be studied. Also, the decision was made that a larger screening array would be used initially to determine the significant factors; subsequent arrays could be used if deemed necessary. Some preliminary experiments were required to ensure that all of the factors and levels in the array could 
actually be used to produce meaningful samples. In some cases, the levels had to be adjusted to allow this.

Overall, a large amount of variation was observed in the responses measured, and a significant portion of this variation for certain responses remained in the error term (unexplained variation). Analysis of the data provided a number of useful insights which were incorporated into subsequent fibers made according to the optimum recommendations from the experimental array design analysis. A rigorous experiment to confirm the resulsts was not performed. However, results from the additional fibers seemed to indicate marked improvement in many of the critical responses, indicating that the relations determined in the analysis of data may indeed be appropriate. For the analysis of each response presented, only the pertinent data is included. Taguchi methods of experimental design and the associated methodology for analysis were used for selection of the experimental design array and for interpretation of the data which resulted.

The L18 array presents a very large number of responses to collect and analyze. For each response, three measurements were taken and the averages and standard deviations calculated. The average values were used as the measured value for input into the ANOVA analysis. In some of the responses measured, a high standard deviation was encountered, indicating that the measurement process could be contributing significantly to some of the variation that was seen. Average values were used in the ANOVA input which should have mitigated this variation to some extent, although it was noted that the data indicated that the measurement process for many of the responses should be studied in more detail in the future. Below are listed some of the results of the analysis of the data for the responses measured.

\subsection{Sapphire core diameter (G1)}

The following is the analysis of the sapphire core diameter data generated in the 18 samples that were measured. The quality characteristic used was "smaller is better;" that is, it is desirable to have the core diameter as small as possible. The first column of Table 4-26 shows the factors considered, and the next three columns show the measured diameter response for the different levels of the factors. Since a total of 18 samples were prepared, each response value for factors 2 through 8 represents the average core diameter from six samples. As their only two levels for the first factor "Nano $\mathrm{MgO}$ amount", each value in the first 2 columns for that factor represents the average core diameter from the 9 samples.

Also listed in the last column of the Table is the difference between the averages of level 3 and level 1. For example, the average core diameter of the 6 samples with the factor "Dipping Speed" set to level 1 is $91.383 \mu \mathrm{m}$, and that to level 3 is $85.599 \mu \mathrm{m}$. So their difference, L3-L1, is -5.784 . This difference gives some indication of the relative influence of the factors to the variation of results. More rigorous measures of the influence of each factor will be decided by the F-Factor and Confidence Level shown in 
the Analysis of Variance (ANOVA) Table 4-27 below. It should be noted that all of the data generated will not be shown. For example, only L3-L1 is shown here. There is similarly data for level 2 minus level 1 and level 3 minus level 2 . These are not shown as information here because that they are already encoded into the other data presented.

Table 4-26

\begin{tabular}{|c|c|c|c|c|}
\hline Column \# / Factors & Level 1 & Leve1 2 & Leve1 3 & $\mathrm{~L} 3-\mathrm{L} 1$ \\
\hline $1 \mathrm{Nano} \mathrm{MgO}$ amount & 87.5 & 86.655 & & $\mathrm{n} / \mathrm{a}$ \\
\hline 2 Dipping Speed & 91.383 & 84.249 & 85.599 & -5.784 \\
\hline 3 Firing Profile & 91.816 & 86.5 & 82.916 & -8.901 \\
\hline $4 \mathrm{MgO} /$ Spinel ratio & 90.066 & 86.599 & 84.566 & -5.5 \\
\hline 5 First Stage Ball & 83.799 & 88.933 & 88.5 & 4.7 \\
\hline 6 Fish oil amount & 88.199 & 87.149 & 85.883 & -2.317 \\
\hline 7 PVP type (K) & 89.766 & 85.616 & 85.849 & -3.918 \\
\hline $8 \mathrm{PVP}$ amount & 82.783 & 87.233 & 91.216 & 8.432 \\
\hline
\end{tabular}

Table 4-27 shows the ANOVA table for the analysis of the factor influences. The Qualitek-4 software tool for automated design and analysis of Taguchi experiments, available from Nutek, Inc., was used in the preparation of these results. The next four tables show screen captures taken from the output of the software when applied to the analysis of our designed experiments.

The first column "DOF ( $f$ )" stands for "Degree of Freedom" and is equal to the number of levels minus 1. The second column "Sum of Sqrs $(S)$ " stands for "Sums of Squares," which are used to determine the significance of factor. For factors with an equivalent number of degrees of freedom, the larger the " $S$ " number, the more significant is the factor.

The value " $S$ " is calculated according to the equation,

$$
S=\sum_{i=1}^{L} \frac{A_{i}^{2}}{N_{A i}}-C . F .
$$

where

$A_{i}$ is the sum of results that include Factor $A$ at level $i$,

$N_{A i}$ is the total number of experiments in which level $i$ of factor $A$ is present,

$L$ the total number of levels, and

C.F. the Correction Factor, which is defined as $T^{2} / N$, in which $T$ is the sum of all results, and $N$ is the total number of results.

The column "Variance $(V)$ " is defined as $S / f$, and the column "F-Ratio $(F)$ " as $V / V_{e}$, where $V_{e}$ is the variance for the error terms (obtained by calculating the error sum of squares and dividing by the error degrees of freedom). In general, $V_{e}$ increases as the 
degree of data scattering increases. Therefore, a large "F-Ratio $(F)$ " number of a certain factor indicates that this factor possibly possesses a small degree of data scattering. This indicates that this factor is significant and has large influence on the results.

The column "Pure Sum $\left(S^{\prime}\right)$ " is defined as $S-\left(V_{e} \times f\right)$. In this column, when a factor is found to have little or no influence and is excluded from any statistical control, the number "0" would be entered. As shown in Table 4-27, all the factors, except for "Firing Profile" and "PVP amount," have little or no effect on the results since their entries are 0 's. The last column in the Table "Percent (P\%)" shows how sensitive the result is to the change of levels of the factors. Here, the most influential factor is the "Firing Profile", which has a relatively small influence of $6.3 \%$.

Table 4-27

\begin{tabular}{|l|c|r|r|r|r|r|}
\hline & $\begin{array}{c}\text { DOF } \\
\text { Col }\end{array}$ & $\begin{array}{r}\text { Sum of Sqrs. } \\
\text { (S) }\end{array}$ & $\begin{array}{c}\text { Variance } \\
\text { (V) }\end{array}$ & $\begin{array}{c}\text { F - Ratio } \\
\text { (F) }\end{array}$ & $\begin{array}{r}\text { Pure Sum } \\
\text { (S') }\end{array}$ & $\begin{array}{r}\text { Percent } \\
\text { P(\%) }\end{array}$ \\
\hline 1 Nano MgO amount & 1 & 3.207 & 3.207 & .037 & 0 & 0 \\
2 Dipping Speed & 2 & 172.294 & 86.147 & .996 & 0 & 0 \\
3 Firing Profile & 2 & 240.62 & 120.31 & 1.391 & 67.683 & 6.301 \\
4 MgO/Spinel ratio & 2 & 92.807 & 46.403 & .536 & 0 & 0 \\
5 First Stage Ball & 2 & 97.244 & 48.622 & .562 & 0 & 0 \\
6 Fish oil amount & 2 & 16.126 & 8.063 & .093 & 0 & 0 \\
7 PVP type (K) & 2 & 65.228 & 32.614 & .377 & 0 & 0 \\
8 PVP amount & 2 & 213.583 & 106.791 & 1.235 & 40.646 & 3.784 \\
\hline Other/Error & 2 & 172.936 & 86.468 & & & 89.915 \\
\hline Total: & 17 & 1074.051
\end{tabular}

The ANOVA analysis is applied to the results in Table 4-27, and calculates a "Confidence Level" for each factor. This Confidence Level is then displayed in the column titled "Pure Sum" in Table 4-28. The term "POOLED" is listed in the column "F-Ratio $(F)$ " for each factor in the table. When a factor is deemed unimportant, it can be factored out of the analysis by including (pooling) it into the error terms. The pooling is performed starting with the factors that show the least significance. In Table 4-28, all factors are pooled, which indicates that it was decided that none of the factors have significant influence on the results.

The "Confidence Level (C.L.)" is listed in the "Pure Sum $(S$ ')" column of the Table. The notation "NC" means "Not Calculated" for the factors with little or no influence. The value of "Confidence Level" is related directly to the magnitude of "F-Ratio $(F)$ " in Table 4-27; i.e., the larger the $F$ number, the higher would be the value of C.L. Here the confidence levels for the factors "Firing Profile" and "PVP amount" are $67.33 \%$ and $68.29 \%$ respectively. Therefore, based on the amount of scatter in the data, only "Firing Profile" and "PVP Amount" seem to have some degree of confident influence approaching $70 \%$ on the results. It is to be noted that $70 \%$ is a relatively small number for the Confidence Level. A desirable Confidence Level should approach about $95 \%$ to $100 \%$. More data would be needed to improve the estimates of these effects. 
Table 4-28

\begin{tabular}{|c|c|c|c|c|c|c|}
\hline Col\#/Factor & \begin{tabular}{|l} 
DOF \\
$(\mathrm{f})$
\end{tabular} & $\begin{array}{c}\text { Sum of Sqrs. } \\
\text { (S) }\end{array}$ & $\begin{array}{c}\text { Variance } \\
\text { (V) }\end{array}$ & $\begin{array}{l}\text { F - Ratio } \\
\text { (F) }\end{array}$ & $\begin{array}{c}\text { Pure Sum } \\
\left(S^{\prime}\right)\end{array}$ & $\begin{array}{r}\text { Percent } \\
\mathrm{P}(\%) \\
\end{array}$ \\
\hline $1 \mathrm{Nano} \mathrm{MgO}$ amount & (1) & (3.207) & & P O O L ED & $\left(\mathrm{CL}={ }^{*} \mathrm{NC}^{*}\right)$ & \\
\hline 2 Dipping Speed & (2) & $(172.294)$ & & P O OLED & $\left(\mathrm{CL}={ }^{*} \mathrm{NC}{ }^{*}\right)$ & \\
\hline 3 Firing Profile & (2) & $(240.62)$ & & P O OLED & $(C L=6733 \%$ & \\
\hline $4 \mathrm{MgO} / \mathrm{Sp}$ pinel ratio & (2) & $(92.807)$ & & P O OLED & $\left(\mathrm{CL}={ }^{*} \mathrm{NC}^{*}\right)$ & \\
\hline 5 First Stage Ball & (2) & (97.244) & & P O OLED & $\left(\mathrm{CL}={ }^{*} \mathrm{NC}^{*}\right)$ & \\
\hline 6 Fish oil amount & (2) & $(16.126)$ & & P O OLED & $\left(\mathrm{CL}={ }^{*} \mathrm{NC} *\right)$ & \\
\hline 7 PVP type (K) & (2) & $(65.228)$ & & P O OLED & $\left(\mathrm{CL}={ }^{*} \mathrm{NC}^{*}\right)$ & \\
\hline $8 \mathrm{PVP}$ amount & (2) & (213.583) & & P O OLED & $(\mathrm{CL}=68.29 \%$ & \\
\hline Other/Error & 17 & 1074.045 & 63.179 & & & 100 \\
\hline Total: & 17 & 1074.051 & & & & $100.00 \%$ \\
\hline
\end{tabular}

Figure 4-97 graphically illustrates the effect of each level of six of the factors on the measured response (in this case, core diameter). In each plot, the x-access refers to the level, and the y-axis refers to the measured core diameter in micrometers. In general, only six of the graphical representations are shown as the data for these graphs is encoded into the other data presented.

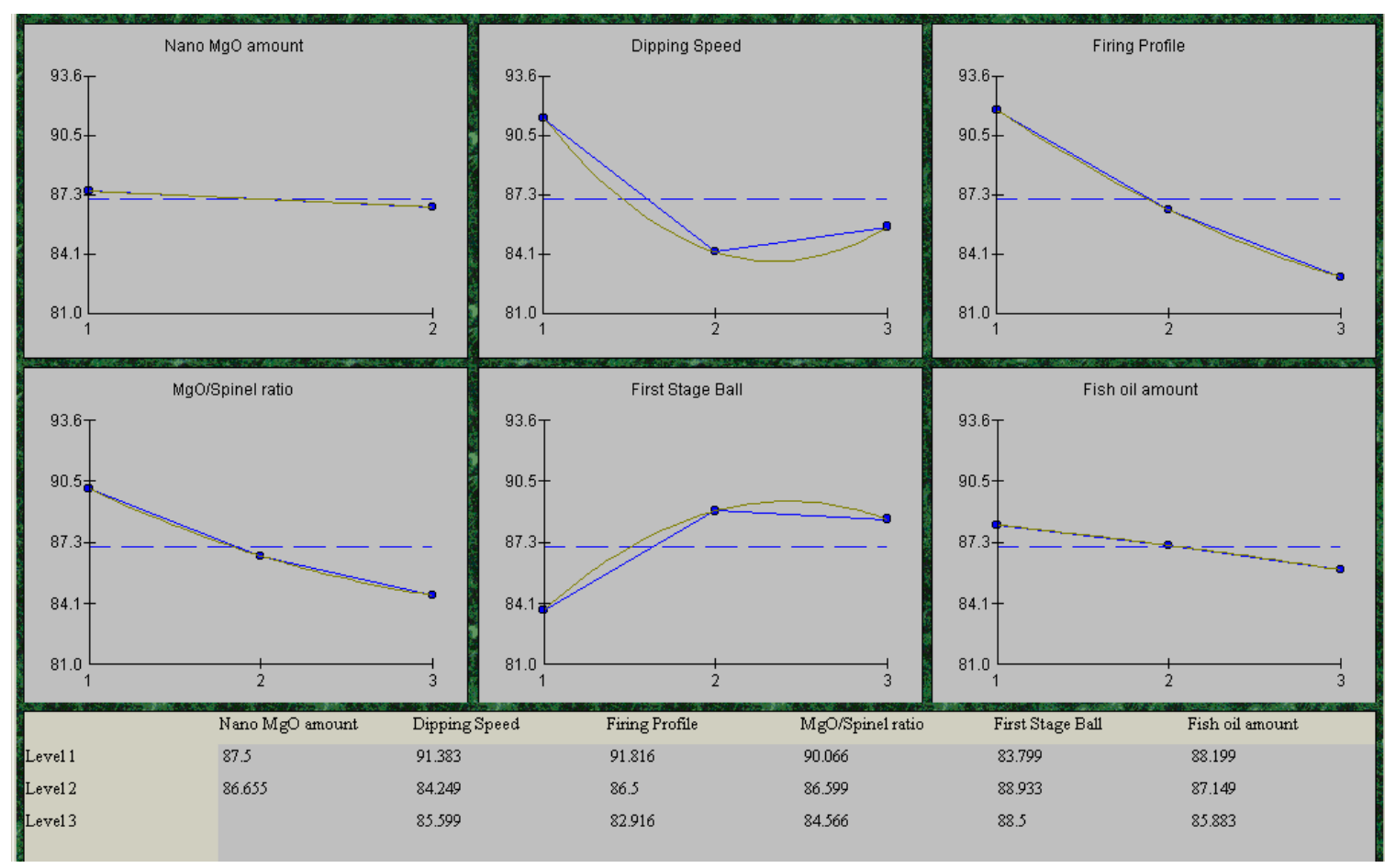

Figure 4-97. Effect of each level of six of the factors on the measured response

Table 4-29 shows the expected optimum performance for "Firing Profile" and "PVP amount" being determined as significant (with a confidence level less than $70 \%$ - so a very large amount of variation in the data relative to the factor level change). Based on 
the very large scatter in the data, the confidence interval around the predicted average (the "Expected Result at Optimum Condition" in Table 4-29) will be very large.

Table 4-29

\begin{tabular}{l|l|r|r|}
\hline Column \# / Factor & Level Description & Level & Contribution \\
\hline 3 Firing Profile & 1600 & 3 & -4.162 \\
8 PVP amount & 0.4 & 1 & -4.295 \\
& & & \\
\hline \\
Total Contribution From All Factors... & & -8.458 \\
Current Grand Average Of Performance... & \\
Expected Result At Optimum Condition... & & 78.077 \\
\end{tabular}

If we assumed that all the factors were significant the optimal levels would be as those listed in Table 4-30.

Table 4-30

\begin{tabular}{|l|l|l|r|}
\hline Column \# / F actor & Level Description & Level & Contribution \\
\hline 1 Nano MgO amount & 20 & 2 & -.423 \\
2 Dipping Speed & .105 & 2 & -2.828 \\
3 Firing Profile & 1600 & 3 & -4.162 \\
4 MgO/Spinel ratio & $12: 1$ & 3 & -2.512 \\
5 First Stage Ball & 3 & 1 & -3.278 \\
6 Fish oil amount & 75 & 3 & -1.195 \\
7 PVP type (K) & $90 \% 10 \mathrm{~K}$ & 2 & -1.462 \\
8 PVP amount & 0.4 & 1 & -4.295 \\
\hline
\end{tabular}

This gives the optimal levels as predicted by the data, but again due to the large scatter the factors mentioned above are not sufficiently high in significance to warrant inclusion in the optimal predictions.

The ANOVA results of the other 12 responses are listed in Appendix B.

\subsubsection{The determination of overall optimal levels from ANOVA analysis}

The factors determined to be significant and their optimal levels for each response are listed in Table 4-31 below. Unless otherwise noted, all levels in the table have confidence levels greater than $90 \%$. Levels with confidence levels less than $89 \%$ are not listed except for Factor 1 and 2. 
Table 4-31

\begin{tabular}{|c|c|c|c|c|c|c|c|c|}
\hline & F1 & $\mathrm{F} 2$ & F3 & F4 & F5 & F6 & F7 & F8 \\
\hline G1 & & & & & & & & \\
\hline $\mathrm{G} 2$ & & 1 & 1 & & 3 & & 1 & 3 \\
\hline G3 & & & & 2 & 3 & 1 & 1 & 3 \\
\hline G4 & $\begin{array}{c}2 \\
(71 \%)\end{array}$ & $\begin{array}{c}1 \\
(87 \%)\end{array}$ & & & & & 2 & 3 \\
\hline G5 & & & & & & & & 3 \\
\hline G6 & & & & & & & & \\
\hline M1 & & & & & & & & \\
\hline M2 & $\begin{array}{c}1 \\
(69 \%)\end{array}$ & & & & & & 3 & \\
\hline M3 & 1 & 3 & 1 & 2 & 2 & 3 & 3 & 1 \\
\hline M4 & & & & & & 3 & 3 & 1 \\
\hline $\mathrm{O} 1$ & $\begin{array}{c}1 \\
(75 \%)\end{array}$ & $\begin{array}{c}1 \\
(70 \%)\end{array}$ & 1 & & & & & \\
\hline $\mathrm{O} 2$ & $\begin{array}{c}1 \\
(68 \%)\end{array}$ & & & & & & & \\
\hline O3 & & & $\begin{array}{c}1 \\
(89 \%)\end{array}$ & & & & & \\
\hline
\end{tabular}

The responses are:

G1: core diameter,

G2: overall cladding thickness,

G3: outer cladding thickness,

G4: skin thickness, G5: overall fiber diameter,

G6: concentricity (inter-center distance),

M1: flexibility from 3-point bending test,

M2: total area of cracks,

M3: length of the longest crack,

M4: number of cracks,

O1: core/cladding interface roughness,

$\mathrm{O} 2$ : numerical aperture (N.A.) measurement,

O3: attenuation measurement,

F1: Nano $\mathrm{MgO}$ weight ratio,

F2: dipping speed,

F3: maximum firing temperature,

F4: $\mathrm{MgO} /$ Spinel ratio,

F5: dispersion milling time,

F6: fish oil amount,

F7: 10K PVP weight ratio,

F8: total PVP amount.

To determine the optimal overall levels, the relative importance of the responses measured is taken into account. A hierarchical structure was used for the optimal level selection, based on available time and the limited knowledge of the relative trade-offs between one response and the next.

The most important response was determined to be the "skin thickness" G4. One example of a "carrot shaped" dipped fiber with a thick and non-uniform skin is sample 
$18_{\mathrm{R} 1}$, shown in Figure 4-98 below. In the background of this picture are grid lines $1.4 \mathrm{~mm}$ apart. The top of dipped fiber is on the left hand side of this figure. As can be seen, the overall fiber diameter is quite large at the top of fiber, and the diameter gradually decreases towards the bottom of fiber.

This "carrot shape" indicates that the slurry has not been prepared using the optimized conditions. It may also create practical problems in fiber packaging in the future. So the first priority is to prevent this phenomenon from occurring. Therefore, according to row G4 in Table 4-31, Factor 7 and Factor 8 are set to be in Level 2 and Level 3 respectively. Factor 7 and 8 are related to the type and amount of PVP binder added to the slurry. Level 2 in Factor 7 represents a 10\% 55K PVP, i.e., 90\% 10K PVP, and Level 3 in Factor 8 represents a $0.6 \mathrm{~g}$ total amount of PVP. In fact, eventually, G4 turns out to be the only factor in the "Geometrical Response" category that has been taken into account in the determination of final optimum conditions.

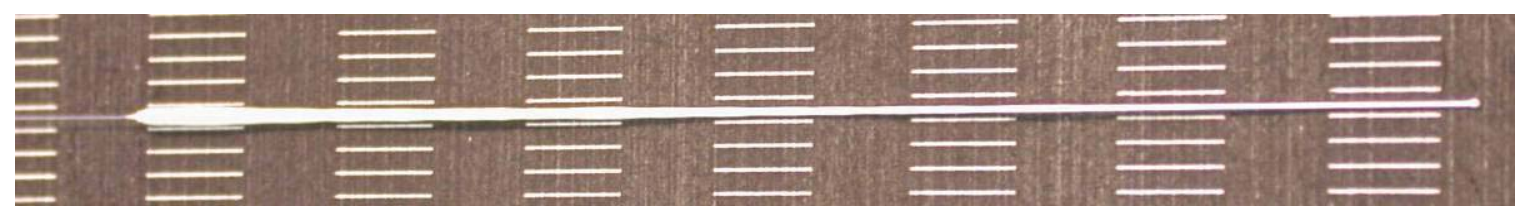

Figure 4-98: sample $18_{\mathrm{R} 1}$ after dipping

The second most important response was determined to be "core/cladding interface roughness", O1. According to row $\mathrm{O} 1$ in Table 4-31, Factor 3 (the maximum firing temperature) has to be set at Level $1\left(1550^{\circ} \mathrm{C}\right)$ in order to have smooth core/cladding interface. The results from "attenuation test", $\mathrm{O} 3$, as listed in row $\mathrm{O} 3$ in Table 4-31, also support the same conclusion.

The next most important response is the "length of the longest crack", M3. In fact, from Table 4-31, the mechanical responses including "total area of cracks (M2)" and "number of cracks (M4)" predict similar results regarding the optimum levels for Factors 6, 7 and 8. The reason for picking the "length of the longest crack", M3, as one of the most important factors is that all 8 factors are considered significant for this response. To achieve minimal cracking in cladding, Factor 7 needs to be set in Level 3 and Factor 8 in Level 1, indicating that the percentage of 55K PVP needs to be increased to $20 \%$ and the total amount of PVP decreased to $0.4 \mathrm{~g}$. However, since these levels are in conflict with the ones set to prevent "carrot" shape fibers, they are ignored.

Also from Row M3 in Table 4-31, Factor 3 (the maximum firing temperature) has to be set to Level $1\left(1550^{\circ} \mathrm{C}\right.$, or $\left.2,822^{\circ} \mathrm{F}\right)$ to prevent cracking. This level agrees with the one determined from the "core/cladding interface roughness" response, $\mathrm{O} 1$, to have a smooth interface. Finally, Factor 4 (MgO/Spinel ratio), Factor 5 (dispersion milling time), and Factor 6 (fish oil amount) are set to be in Level 2 (9:1), Level 2 (6 hours), and Level 3 $(75 \mu \mathrm{L})$ respectively according to Row M3 in Table 4-31.

The last two factors to be determined are Factor 1 (Nano $\mathrm{MgO}$ weight ratio) and Factor 2 (dipping speed). According to the results listed in column F1 and F2 in Table 4-31Table 
4-31, it was decided to set Factor 1 at Level 1 (10\% nano MgO powder) and Factor 2 at Level 2 (2.7 mm/second). The final optimal levels are listed in Table 4-32 below.

Table 4-32: Final recommendation from Taguchi experiments

\begin{tabular}{|c|l|c|c|}
\hline & Factors & Level & Value \\
\hline 1 & $\begin{array}{l}\text { Nano MgO weight ratio in all } \mathrm{MgO} \\
\text { powders }(\%)\end{array}$ & 1 & 10 \\
\hline 2 & Dipping speed $(\mathrm{mm} / \mathrm{sec})$ & 2 & 2.7 \\
\hline 3 & Maximum firing temperature $\left({ }^{\circ} \mathrm{C}\right)$ & 1 & 1550 \\
\hline 4 & MgO/Spinel ratio & 2 & $9: 1$ \\
\hline 5 & Dispersion milling session time $(\mathrm{hr})$ & 2 & 6 \\
\hline 6 & Fish oil amount $(\mu \mathrm{L})$ & 3 & 75 \\
\hline 7 & 10K PVP weight ratio $(\%)$ & 2 & 90 \\
\hline 8 & PVP amount $($ both $10 \mathrm{~K}$ and $55 \mathrm{~K})(\mathrm{g})$ & 3 & 0.6 \\
\hline
\end{tabular}

\subsubsection{Testing of optimized processing with $125 \mu \mathrm{m}$ sapphire fibers}

In order to assess the impact of the set of optimal processing parameters on the quality of the clad sapphire fiber, four pieces of clad fibers were fabricated using $125 \mu \mathrm{m}$ sapphire fibers and the final optimal conditions listed in Table 4-32. Each piece of clad fiber has a length of $6.3 \mathrm{~cm}$, except for the third sample, Sample 3, which is $4.9 \mathrm{~cm}$ long. Both ends of the clad fibers were polished and analyzed for geometrical, mechanical, and the core/cladding roughness (O1) responses. The optical microscopic pictures of each sample are shown in Figure 4-99 below. In general, all 4 samples have smooth core/cladding interfaces and claddings with very similar thicknesses.

The results of all the responses from the cross-sectional analysis are listed in Table 4-33 below. As can be seen in this table, the repeatability from sample to sample is quite good. For example, the average core diameters are 94.3, 95.5, 94.0, and $91.7 \mu \mathrm{m}$ for samples 1 , 2,3 , and 4 , respectively. The average of these 4 diameters is $93.9 \mu \mathrm{m}$ with a standard deviation of $1.6 \mu \mathrm{m}$. Other responses have very similar values among all 4 samples, except for a single crack occurring in the cross sections for sample 3 , while there are no cracks for samples 1, 2, and 4 . 

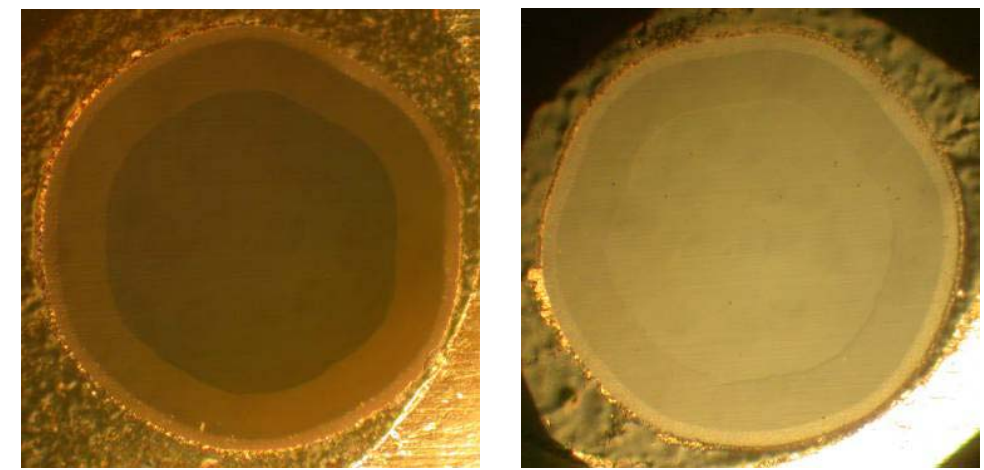

(a) Sample 1 - cross-section 1

(b) Sample 1 - cross-section 2
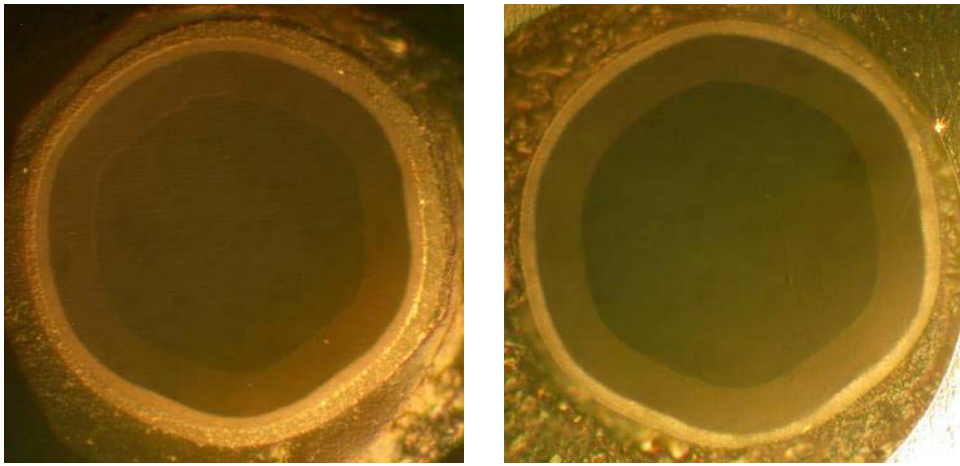

(c) Sample 2 - cross-section 1

(d) Sample 2 - cross-section 2
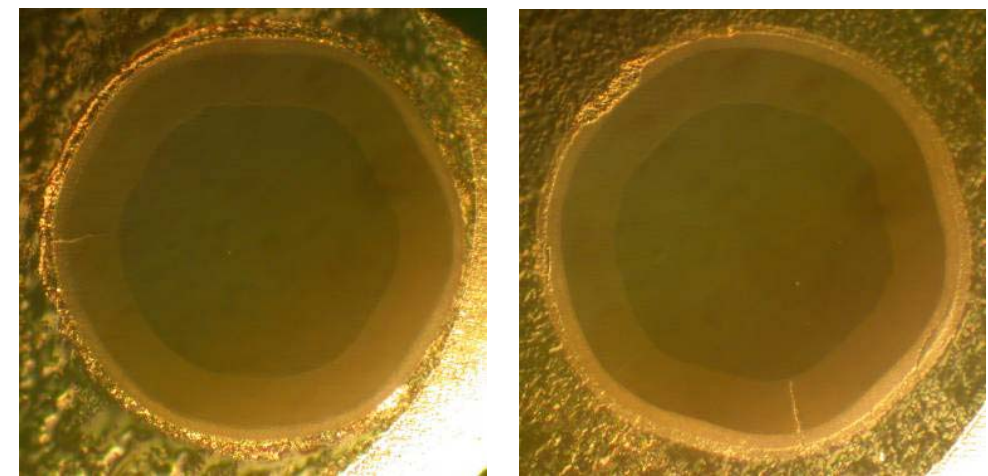

(e) Sample 3 - cross-section 1

(f) Sample 3 - cross-section 2
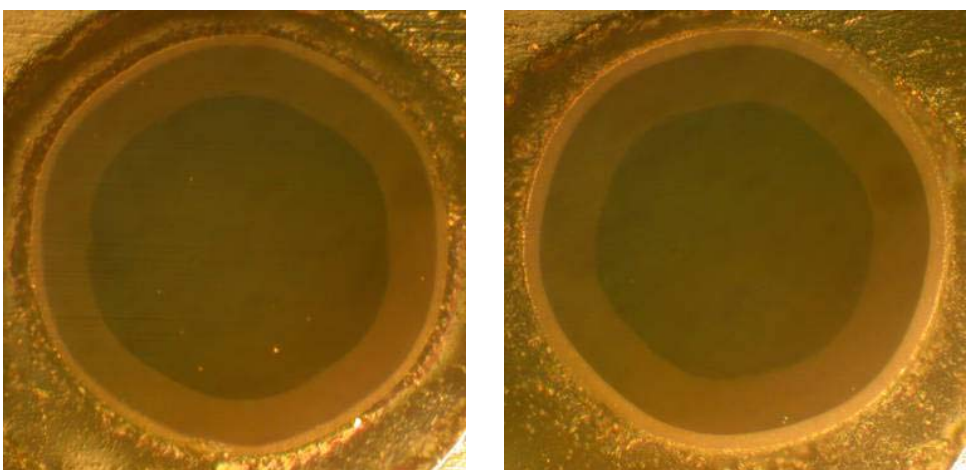

(g) Sample 4 - cross-section 1 (h) Sample 4 - cross-section 2 Figure 4-99: Cross sections of $125 \mu \mathrm{m}$ diameter confirmation clad fibers 
Table 4-33

\begin{tabular}{|c|c|c|c|c|c|c|c|c|c|c|c|}
\hline Sample & Cross & $\begin{array}{c}\mathbf{G 1} \\
(\mu \mathrm{m})\end{array}$ & $\begin{array}{c}\text { G2 } \\
(\mu \mathrm{m})\end{array}$ & $\begin{array}{c}\text { G3 } \\
(\mu \mathrm{m}) \\
\end{array}$ & $\begin{array}{c}\mathbf{G 4} \\
(\mu \mathrm{m})\end{array}$ & $\begin{array}{c}\text { G5 } \\
(\mu \mathrm{m})\end{array}$ & $\begin{array}{c}\text { G6 } \\
(\mu \mathrm{m})\end{array}$ & $\begin{array}{c}\mathrm{M} 2 \\
\left(\mu \mathrm{m}^{\wedge} 2\right)\end{array}$ & $\begin{array}{c}\text { M3 } \\
(\mu \mathrm{m})\end{array}$ & M4 & 01 \\
\hline 1 & 1 & 94.9 & 19.3 & 4.1 & 2.2 & 137.3 & 0.1 & 0 & 0 & 0 & 0.06 \\
\hline \multirow[t]{3}{*}{1} & 2 & 93.7 & 21.9 & 4.9 & 2.5 & 140.8 & 0.9 & 0 & 0 & 0 & 0.35 \\
\hline & Average & 94.3 & 20.6 & 4.5 & 2.35 & 139.05 & 0.46 & 0 & 0 & 0 & 0.21 \\
\hline & St Dev & 0.8 & 1.8 & 0.6 & 0.2 & 2.5 & 0.6 & 0 & 0 & 0 & 0.21 \\
\hline 2 & 1 & 94.1 & 20.7 & 4.6 & 5.6 & 147.5 & 0.4 & 0 & 0 & 0 & 0.46 \\
\hline \multirow[t]{3}{*}{2} & 2 & 97 & 19.2 & 4.3 & 1 & 136.2 & 0.5 & 0 & 0 & 0 & 0.03 \\
\hline & Average & 95.55 & 19.95 & 4.45 & 3.3 & 141.85 & 0.43 & 0 & 0 & 0 & 0.25 \\
\hline & St Dev & 2.1 & 1.1 & 0.2 & 3.3 & 8.0 & 0.0 & 0 & 0 & 0 & 0.30 \\
\hline 3 & 1 & 97.1 & 22.9 & 4.5 & 5.6 & 152.2 & 0.9 & 4.4 & 18.5 & 1 & 0.22 \\
\hline \multirow[t]{3}{*}{3} & 2 & 90.9 & 22.9 & 4.7 & 3.5 & 143.3 & 0.8 & 15.8 & 22.2 & 1 & 0.12 \\
\hline & Average & 94 & 22.9 & 4.605 & 4.55 & 147.75 & 0.83 & 10.1 & 20.4 & 1 & 0.17 \\
\hline & St Dev & 4.4 & 0.0 & 0.1 & 1.5 & 6.3 & 0.1 & 8.1 & 2.6 & 0 & 0.07 \\
\hline 4 & 1 & 95.2 & 18.3 & 3.3 & 4.1 & 138.3 & 0.6 & 0.0 & 0.0 & 0 & 0.06 \\
\hline \multirow[t]{3}{*}{4} & 2 & 88.3 & 22.9 & 5.0 & 2.5 & 138.3 & 0.5 & 0.0 & 0.0 & 0 & 0.07 \\
\hline & Average & 91.75 & 20.6 & 4.15 & 3.3 & 138.3 & 0.55 & 0 & 0 & 0 & 0.06 \\
\hline & St Dev & 4.9 & 3.3 & 1.2 & 1.1 & 0.0 & 0.1 & 0 & 0 & 0 & 0.01 \\
\hline
\end{tabular}

(G1: core diameter, G2: overall cladding thickness, G3: outer cladding thickness, G4: skin thickness, G5: overall fiber diameter, G6: concentricity (inter-center distance), M2: total area of cracks, M3: length of the longest crack, M4: number of cracks, O1: core/cladding interface roughness)

The 3-point bending test was performed on samples 1 and 3. For sample 1, three pieces of test samples, each of about $17 \mathrm{~mm}$ long, were cut from the original clad fiber. In the case of Sample 3, two pieces of test samples were prepared. The bending test results are listed in Table 4-34 below. As shown in the table, the average deflection for Sample 1 is 0.96 $\mathrm{mm}$ with a standard deviation of $0.05 \mathrm{~mm}$. Compared to the values of deflection of all 18 samples in the results of Taguchi experiments listed in Table 4-25, a deflection of $0.96 \mathrm{~mm}$ is the second largest deflection in the table. The average deflection of Sample 3 drops to $0.78 \mathrm{~mm}$, possibly due to the existence of a single crack in the cladding.

Table 4-34

\begin{tabular}{|l|c|c|c|c|c|}
\hline Sample & $\begin{array}{c}\text { Starting } \\
\text { Height (mm) } \\
(\mathrm{H} 1)\end{array}$ & $\begin{array}{c}\text { Ending Height } \\
(\mathrm{mm}) \\
(\mathrm{H} 2)\end{array}$ & $\begin{array}{c}\text { Time } \\
\text { Elapsed } \\
(\mathrm{s})\end{array}$ & $\begin{array}{c}\text { Change in } \\
\text { Length } \\
(\mathrm{H} 2-\mathrm{H} 1) \\
(\mathrm{mm})\end{array}$ & $\begin{array}{c}\text { Speed } \\
(\mathrm{mm} / \mathrm{s})\end{array}$ \\
\hline Sample 1-1 & 9.52 & 10.48 & 79 & 0.96 & 0.0122 \\
\hline Sample 1-2 & 9.56 & 10.57 & 87 & 1.01 & 0.0116 \\
\hline Sample 1-3 & 9.53 & 10.44 & 83 & 0.91 & 0.011 \\
\hline (Sample 1-No crack) & & & Average & 0.96 & \\
\hline & & & St Dev & 0.05 & \\
\hline & & & & & 0.94 \\
\hline Sample 3-1 & 9.58 & 10.52 & 85 & 0.0111 \\
\hline Sample 3-2 & 9.56 & 10.18 & 60 & 0.62 & 0.0103 \\
\hline (Sample 3-One crack) & & & Average & 0.78 & \\
\hline & & & St Dev & 0.23 & \\
\hline
\end{tabular}




\subsubsection{Testing of optimized processing with $75 \mu \mathrm{m}$ sapphire fibers}

The sample preparation conditions listed in Table 4-32 from the Taguchi analysis were also implemented on $75 \mu \mathrm{m}$ diameter sapphire fibers. The fibers were dipped for either 100 times, 150 times, or 200 times in the slurry, in order to assess the effect of the number of dips on the final cladding diameter. It is to be noted that all fibers in the Taguchi experiments are dipped for 150 times. Each $75 \mu \mathrm{m}$ diameter sapphire fiber is about $5.0 \mathrm{~cm}$ long. The polished cross sections of clad $75 \mu \mathrm{m}$ diameter sapphire fibers are shown in Figure 4-100. Only one cross section from each sample is shown in the figure. The figure reveals that the cladding thickness increases as the number of dips increases. In the case of 200 dips, there is one crack forming in the cladding and the core/cladding interface is very rough. The results of cross sectional analysis of Figure 4-100 are listed in Table 4-35. Compared with the cross sectional pictures of the $125 \mu \mathrm{m}$-diameter confirmation clad fibers illustrated in Figure 4-99, the core/cladding interface of the 150dip fiber, as shown in Figure 4-100(b), seems rougher in its details, although this is not reflected in the value of roughness "O1" measurement listed in Table 4-35. It is quite obvious that the 200-dip sample shown in Figure 4-100(c) has a very rough interface.

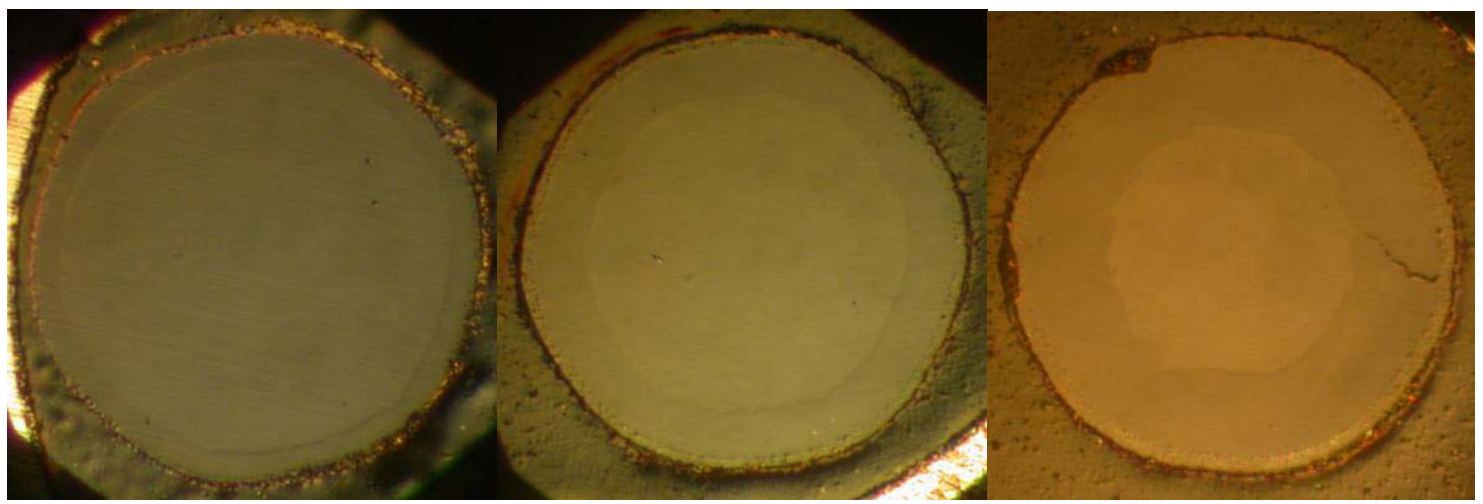

(a) 100 dips

(b) 150 dips

(c) 200 dips

Figure 4-100: Cross sections of $75 \mu \mathrm{m}$ diameter confirmation clad fibers

Table 4-35

\begin{tabular}{|c|c|c|c|c|c|c|c|c|c|c|}
\hline $\begin{array}{c}\text { \# of } \\
\text { dips }\end{array}$ & $\begin{array}{c}\text { G1 } \\
(\mu \mathrm{m})\end{array}$ & $\begin{array}{c}\text { G2 } \\
(\mu \mathrm{m})\end{array}$ & $\begin{array}{c}\text { G3 } \\
(\mu \mathrm{m})\end{array}$ & $\begin{array}{c}\mathbf{G 4} \\
(\mu \mathrm{m})\end{array}$ & $\begin{array}{c}\text { G5 } \\
(\mu \mathrm{m})\end{array}$ & $\begin{array}{c}\mathbf{G 6} \\
(\mu \mathrm{m})\end{array}$ & $\begin{array}{c}\text { M2 } \\
(\mu \mathrm{m} / 2)\end{array}$ & $\begin{array}{c}\text { M3 } \\
(\mu \mathrm{m})\end{array}$ & M4 & 01 \\
\hline 100 & 67.9 & 4.5 & 0 & 0 & 76.0 & 0.3 & 0 & 0 & 0 & 0.2 \\
\hline 150 & 64.5 & 12.4 & 2.3 & 0 & 86.3 & 0.8 & 0 & 0 & 0 & 0.15 \\
\hline 200 & 47.9 & 19.6 & 3.1 & 0 & 83.7 & 0.7 & 11.9 & 19.2 & 1 & 1.78 \\
\hline
\end{tabular}

The sample with 100 dips was cut into 3 pieces, each $17 \mathrm{~mm}$ long, for the 3-point bending test. The results are listed in Table 4-36 below. It can be seen in the table that the bending deflection of the $76 \mu \mathrm{m}$ sapphire fiber clad with a $4.5 \mu \mathrm{m}$ thick spinel cladding is very large $(1.71 \mathrm{~mm})$. The deflection here is about 1.7 times greater than that of a $125 \mu \mathrm{m}$ clad fiber as shown in Table 4-34 above. 
Table 4-36

\begin{tabular}{|l|c|c|c|c|c|}
\hline Sample (100 dips) & $\begin{array}{c}\text { Starting } \\
\text { Height (mm) } \\
(\mathrm{H} 1)\end{array}$ & $\begin{array}{c}\text { Ending Height } \\
(\mathrm{mm}) \\
(\mathrm{H} 2)\end{array}$ & $\begin{array}{c}\text { Time } \\
\text { Elapsed } \\
(\mathrm{s})\end{array}$ & $\begin{array}{c}\text { Change in } \\
\text { Length } \\
(\mathrm{H} 2-\mathrm{H} 1) \\
(\mathrm{mm})\end{array}$ & $\begin{array}{c}\text { Speed } \\
(\mathrm{mm} / \mathrm{s})\end{array}$ \\
\hline Conf.-75 micron 1-1 & 9.62 & 11.6 & 185 & 1.93 & 0.0104 \\
\hline Conf.-75 micron 1-2 & 9.63 & 11.28 & 141 & 1.65 & 0.0117 \\
\hline Conf.-75 micron 1-3 & 9.62 & 11.13 & 138 & 1.55 & 0.0112 \\
\hline & & & Average & 1.71 & \\
\hline & & & St Dev & 0.20 & \\
\hline
\end{tabular}

\subsubsection{High-temperature stability tests of sapphire fiber cladding}

Another important requirement for a practical high-temperature waveguide is its stability at high temperatures. From a microstructural point of view, it is important to understand, at elevated temperatures, whether further conversion from sapphire to spinel proceeds, or whether the core/cladding interface gets rougher due to spinel grain growth, and whether mechanical integrity degrades due to crack generation and propagation in the cladding.

4 pieces of clad fibers were fabricated according to the Taguchi recommendation as listed in Table 4-32 to study the high temperature stability from $1000{ }^{\circ} \mathrm{C}\left(1,832{ }^{\circ} \mathrm{F}\right)$ to $1500{ }^{\circ} \mathrm{C}$ $\left(2,732^{\circ} \mathrm{F}\right)$. One of the clad fibers was first heated to $100{ }^{\circ} \mathrm{C}\left(1,832^{\circ} \mathrm{F}\right)$ for 3 days, followed by each of the other 3 pieces heated to $1100^{\circ} \mathrm{C}\left(2,012^{\circ} \mathrm{F}\right), 1200^{\circ} \mathrm{C}\left(2,192{ }^{\circ} \mathrm{F}\right)$, and $1300{ }^{\circ} \mathrm{C}\left(2,372^{\circ} \mathrm{F}\right)$ sequentially. To study the stability at temperatures higher than $1300{ }^{\circ} \mathrm{C}\left(2,372{ }^{\circ} \mathrm{F}\right)$, the fiber heated at $1100{ }^{\circ} \mathrm{C}\left(2,012{ }^{\circ} \mathrm{F}\right)$ was once again put into the furnace and heated to $1400{ }^{\circ} \mathrm{C}\left(2,552{ }^{\circ} \mathrm{F}\right)$ for 3 days. Similarly, the fiber heated at $1200{ }^{\circ} \mathrm{C}\left(2,192{ }^{\circ} \mathrm{F}\right)$ was once again put into the furnace and heated to $1500{ }^{\circ} \mathrm{C}\left(2,732{ }^{\circ} \mathrm{F}\right)$ for 3 days. Each fiber was polished and its cross-section analyzed before and after the high temperature treatment.

The cross-sections of the clad fiber before and after the $1000{ }^{\circ} \mathrm{C}\left(1,832{ }^{\circ} \mathrm{F}\right)$ treatment are shown in Figure 4-101 below. Figure 4-101 (a) shows the cross section of the asfabricated clad fiber. Figure 4-101 (b) shows the same cross section after being heat treated at $1000{ }^{\circ} \mathrm{C}\left(1,832^{\circ} \mathrm{F}\right)$ for 3 days. Then the fiber was put into a mold and polished. Figure 4-101 (c) shows the cross section 1 to $2 \mathrm{~mm}$ below the surface shown in Figure 20 (b). As can be seen from these figures, there is little effect of the heat treatment on the fiber microstructure. 


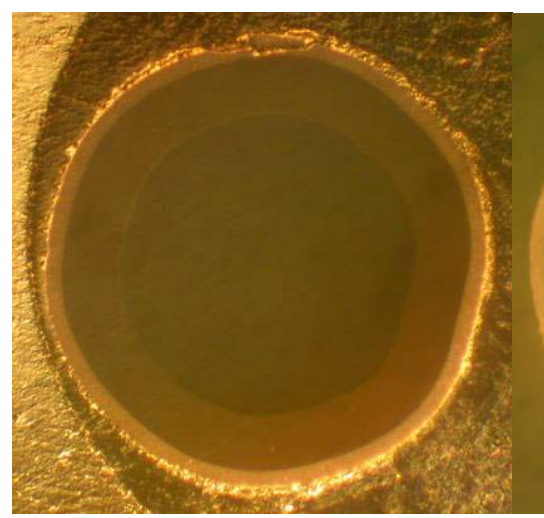

(a)

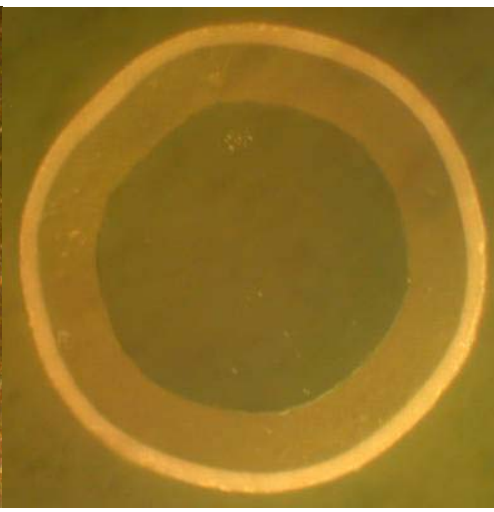

(b)

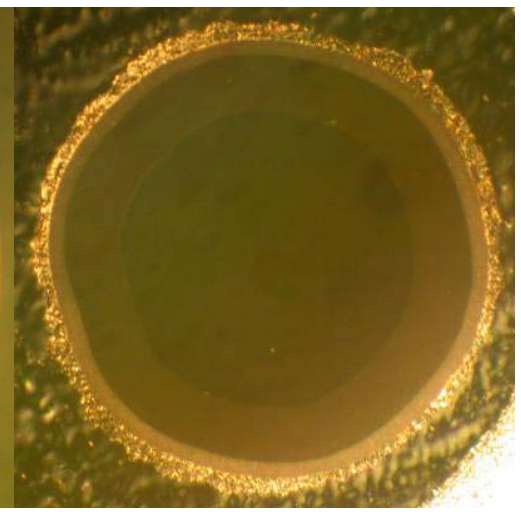

(c)

Figure 4-101: (a) The cross section of clad fiber before heat treatment, (b) the same cross section after heat treated at $1000{ }^{\circ} \mathrm{C}$ for 3 days, and (c) the polished cross section 1 to $2 \mathrm{~mm}$ below the surface shown in (b).

The cross-sectional pictures of the sample heat-treated at $1100^{\circ} \mathrm{C}\left(2,012^{\circ} \mathrm{F}\right)$ for 3 days are shown at Figure 4-102 below. In a similar fashion to the case of $1000{ }^{\circ} \mathrm{C}\left(1,832{ }^{\circ} \mathrm{F}\right)$ heat treatment, there is overall little observable difference caused by the $1100{ }^{\circ} \mathrm{C}$ $\left(2,012^{\circ} \mathrm{F}\right)$ treatment, except possibly a more distinctive core/cladding interface on the surface of the cross section as shown in Figure 4-102 (b).

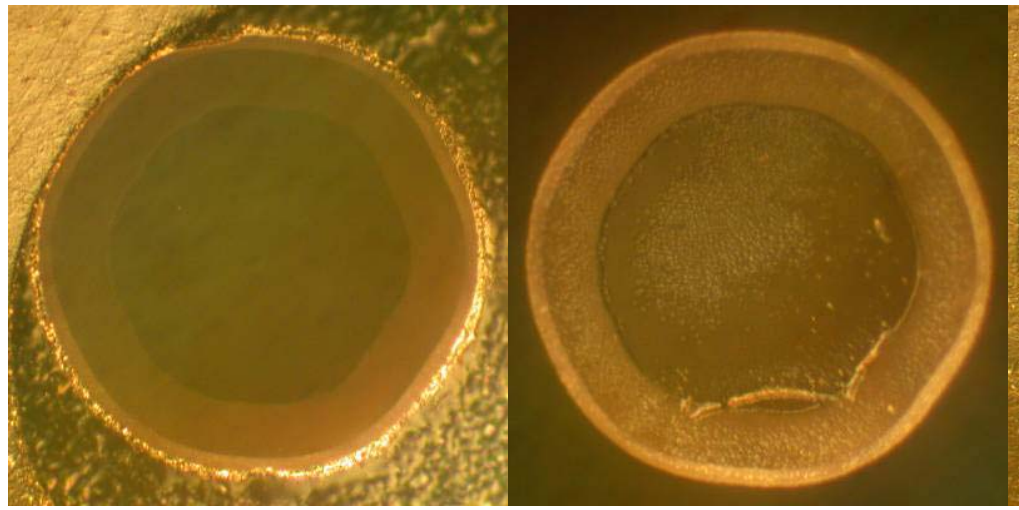

(a) (b)

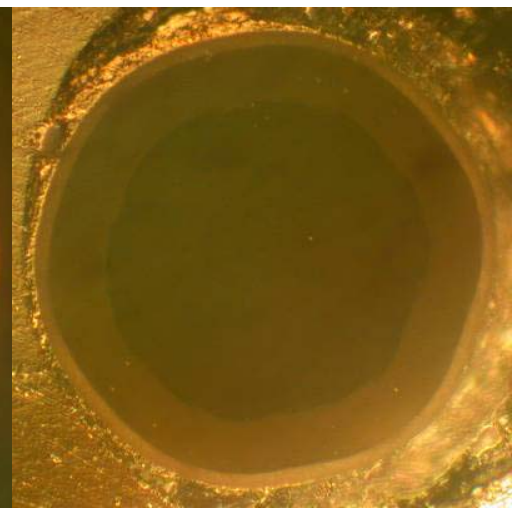

(c)

Figure 4-102: (a) The cross section of clad fiber before heat treatment, (b) the same cross section after heat treated at $1100^{\circ} \mathrm{C}$ for 3 days, and (c) the polished cross section 1 to $2 \mathrm{~mm}$ below the surface shown in (b).

The cross-sectional pictures of the sample heat treated at $1200{ }^{\circ} \mathrm{C}\left(2,192{ }^{\circ} \mathrm{F}\right)$ for 3 days are shown at Figure 4-103 below. Similarly, there is no significant difference except possible thickening of the core/cladding interface on the surface of the cross section as shown in Figure 4-103 (b). 


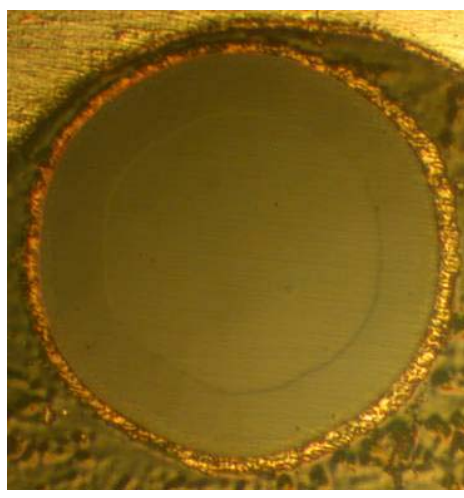

(a)

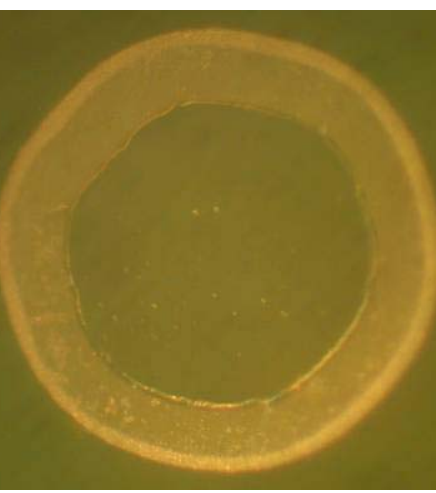

(b)

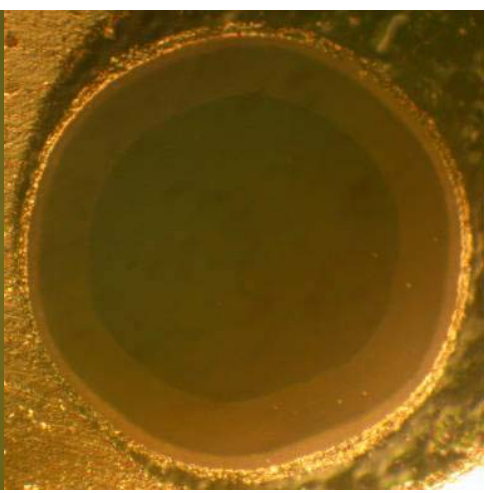

(c)

Figure 4-103: (a) The cross section of clad fiber before heat treatment, (b) the same cross section after heat treated at $1,200^{\circ} \mathrm{C}\left(2,192^{\circ} \mathrm{F}\right)$ for 3 days, and (c) the polished cross section 1 to $2 \mathrm{~mm}$ below the surface shown in (b).

The cross-sectional pictures of the sample heat-treated at $1300^{\circ} \mathrm{C}\left(2,372^{\circ} \mathrm{F}\right)$ for 3 days are shown at Figure 4-104 below. More microstructural changes are discernable after heat treatment at $1300{ }^{\circ} \mathrm{C}\left(2,372{ }^{\circ} \mathrm{F}\right)$ for 3 days. First, as shown in Figure 4-104 (b), the core/cladding interface at the surface after the heat treatment seems further thickening, and there seem to be surface cracks created in the cladding. However, as can be seen in Figure 4-104 (c), the polished cross section 1 to $2 \mathrm{~mm}$ below the surface shows no signs of cracks in the cladding. Yet again, as shown in Figure 4-104 (d), another polished cross section about $14 \mathrm{~mm}$ below the cross section in Figure 4-104 (c) does show signs of several cracks. Light was launched from the other end of the clad fiber and, as shown in Figure 4-104 (e), the light guiding property of the clad fiber does not seem to be greatly degraded due to the microstructural changes.

The sample heat treated at $1100{ }^{\circ} \mathrm{C}\left(2,012{ }^{\circ} \mathrm{F}\right)$ for 3 days was put back into the furnace and heated to $1400^{\circ} \mathrm{C}\left(2,552^{\circ} \mathrm{F}\right)$ for another 3 days. The cross-sectional pictures are shown at Figure 4-105 below. As shown in Figure 4-105 (b), at the core/cladding interface on the surface there is an even thicker layer of further converted material, presumably spinel. Also the cladding thickness in either Figure 4-105 (b) or (c) is visibly greater than that of Figure 4-105 (a).

The sample heat treated at $1,200^{\circ} \mathrm{C}\left(2,192^{\circ} \mathrm{F}\right)$ for 3 days was put back into the furnace and heated to $1,500^{\circ} \mathrm{C}\left(2,732^{\circ} \mathrm{F}\right)$ for another 3 days. The cross sectional pictures are shown at Figure 4-106 below. As shown in Figure 4-106 (b), the cladding thickness increases significantly at the expense of the sapphire core. There are also 4 cracks formed in the cladding, apparently due to this further conversion of sapphire into spinel. 


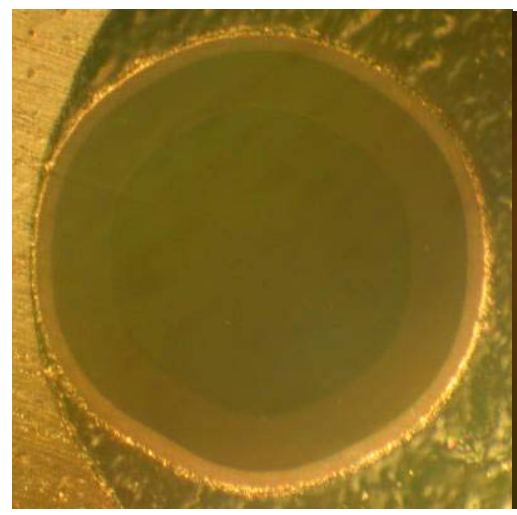

(a)

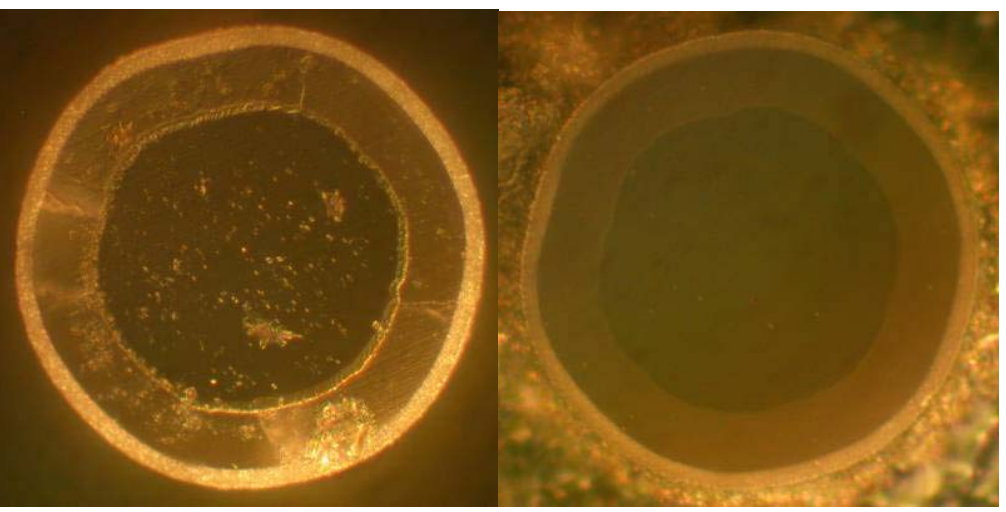

(b)

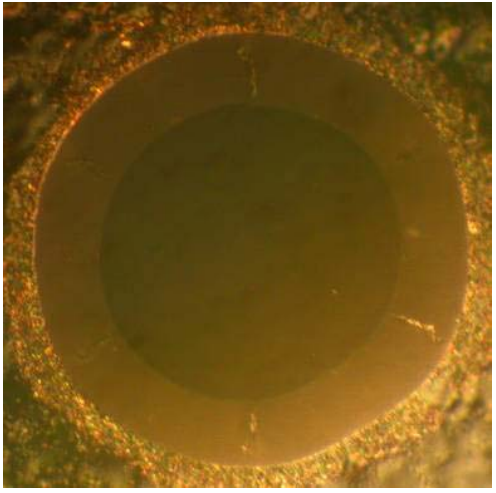

(d)

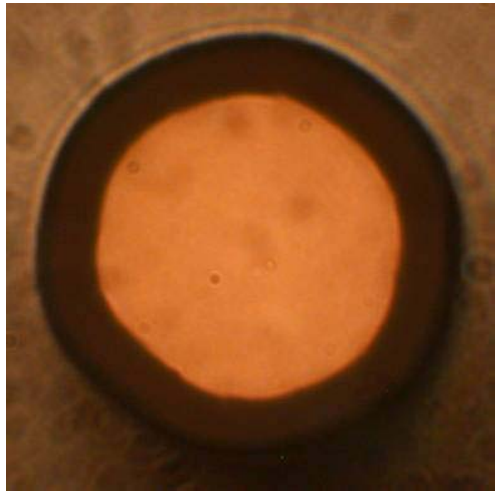

(e)

Figure 4-104: (a) The cross section of clad fiber before heat treatment, (b) the same cross section after heat treated at $1300^{\circ} \mathrm{C}$ for 3 days, (c) the polished cross section 1 to $2 \mathrm{~mm}$ below the surface shown in (b), (d) $14 \mathrm{~mm}$ below the surface shown in (c), and (e) the same surface as shown in (d) with light launched from the other end.

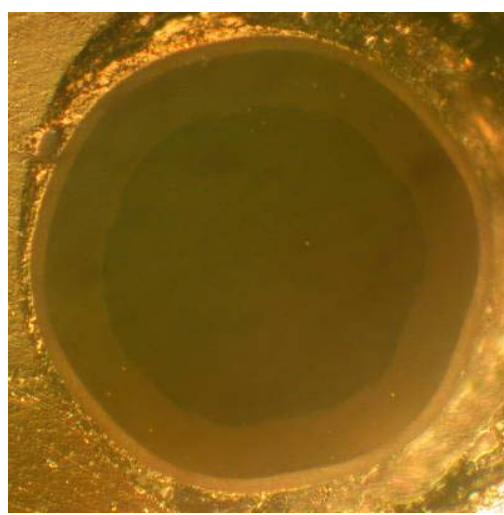

(a)

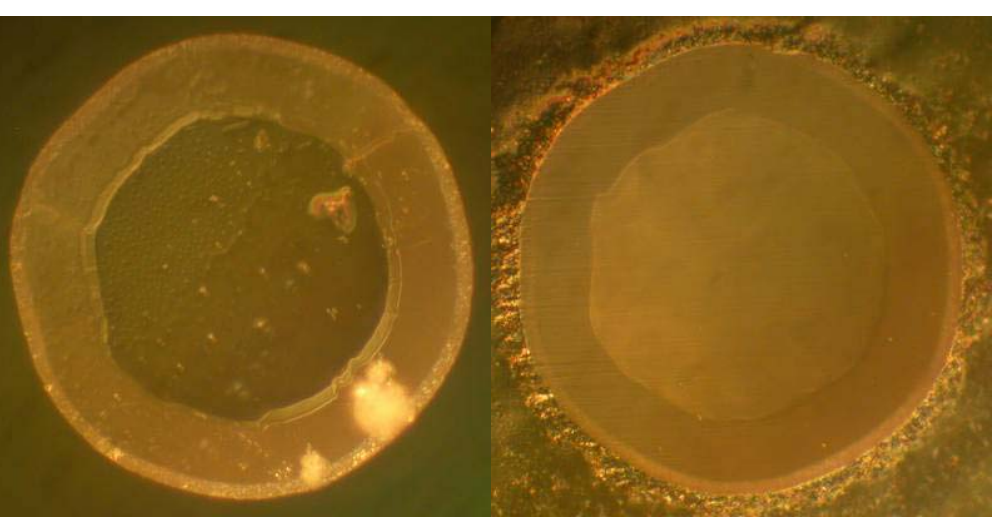

(b) (c)

Figure 4-105: (a) The cross section of clad fiber before heat treatment, (b) the same cross section after heat treated at $1,400{ }^{\circ} \mathrm{C}\left(2,552{ }^{\circ} \mathrm{F}\right)$ for 3 days, and (c) the polished cross section 1 to $2 \mathrm{~mm}$ below the surface shown in (b). 


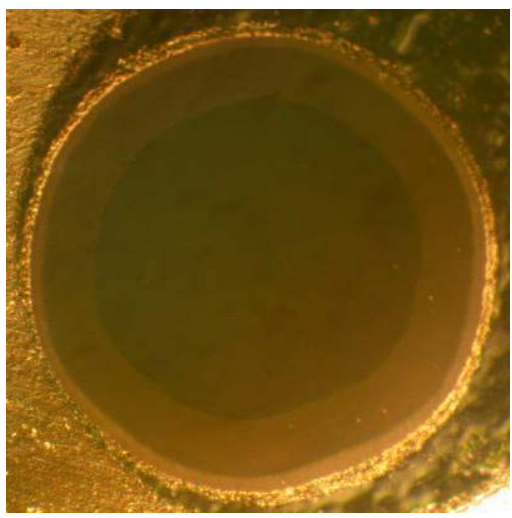

(a)

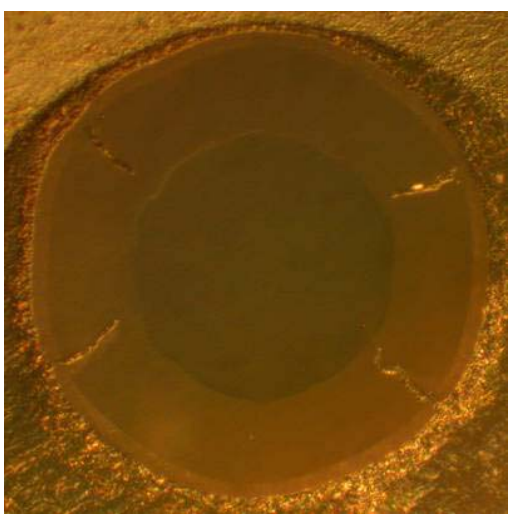

(b)

Figure 4-106: (a) The cross section of clad fiber before heat treatment, (b) the polished cross section 1 to $2 \mathrm{~mm}$ below the surface shown in (b) after heat treated at $1500{ }^{\circ} \mathrm{C}\left(2,732{ }^{\circ} \mathrm{F}\right)$ for 3 days.

The results of cross-sectional analysis, including core diameter, cladding thickness, ratio of cladding thickness and core diameter, overall fiber diameter, roughness, and crack parameter, for all the samples in the high temperature stability tests are listed in Table 4-37 below.

Instead of comparing the cladding thickness of each cross section directly, the ratio of cladding thickness and core diameter of each individual sample is used to determine whether there is further increase in cladding thickness. The results are outlined as follows:

- The structures of spinel clad fibers show no signs of change after heating at $1000{ }^{\circ} \mathrm{C}\left(1,832^{\circ} \mathrm{F}\right)$ for 3 days.

- The same is true after heating at $1100{ }^{\circ} \mathrm{C}\left(2,012^{\circ} \mathrm{F}\right)$ for 3 days and at $1200{ }^{\circ} \mathrm{C}$ $\left(2,192^{\circ} \mathrm{F}\right)$ for 3 days.

- After heating at $1300^{\circ} \mathrm{C}\left(2,372^{\circ} \mathrm{F}\right)$ for 3 days:

$\circ$ The spinel cladding thickness and the core/cladding interface roughness show little sign of change.

- Cracks may start to form in the cladding.

- After heating at $1400^{\circ} \mathrm{C}\left(2,552^{\circ} \mathrm{F}\right)$ for 3 days:

$\circ$ The spinel cladding appears to start growing in thickness.

- The core/cladding interface starts becoming rougher.

- After heating at $1500^{\circ} \mathrm{C}\left(2,732^{\circ} \mathrm{F}\right)$ for 3 days:

- The cladding starts to increase in thickness significantly at the expense of the sapphire core. The ratio between cladding thickness and core diameter increases $88 \%$ after firing.

- Large cracks form. 


\begin{tabular}{|c|c|c|c|c|c|c|c|}
\hline & & $\begin{array}{c}\text { Core } \\
\text { Diameter } \\
(\mathrm{A}) \\
(\mu \mathrm{m})\end{array}$ & $\begin{array}{c}\text { Cladding } \\
\text { Thickness } \\
(\mathrm{B}) \\
(\mu \mathrm{m})\end{array}$ & $\begin{array}{l}\text { B/A } \\
\text { (no } \\
\text { unit) }\end{array}$ & $\begin{array}{c}\text { Overall } \\
\text { Fiber } \\
\text { Diameter } \\
(\mu \mathrm{m})\end{array}$ & $\begin{array}{l}\text { Roughness } \\
\text { (no unit) }\end{array}$ & $\begin{array}{c}\text { Crack } \\
\text { Parameter } \\
\left(\mu \mathrm{m}^{2}\right)\end{array}$ \\
\hline \multirow[t]{2}{*}{$1000^{\circ} \mathrm{C}$} & Original & 89.9 & 21.7 & 0.241 & 132.3 & 1.03 & 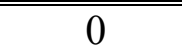 \\
\hline & 3 days & 90.8 & 22.2 & $\begin{array}{c}0.244 \\
(+1.2 \%)\end{array}$ & 135.8 & 0.68 & 0 \\
\hline \multirow[t]{2}{*}{$1100^{\circ} \mathrm{C}$} & Original & 94.4 & 21.9 & 0.232 & 136.8 & 0.92 & 0 \\
\hline & 3 days & 91.4 & 20.2 & $\begin{array}{c}0.221 \\
(-4.7 \%)\end{array}$ & 131.9 & 0.57 & 0 \\
\hline \multirow[t]{2}{*}{$1200^{\circ} \mathrm{C}$} & Original & 93.2 & 20.4 & 0.219 & 134.4 & $\bar{~} 0.96$ & 0 \\
\hline & 3 days & 95.5 & 19.4 & $\begin{array}{c}0.203 \\
(-7.3 \%)\end{array}$ & 134.2 & 0.99 & 0 \\
\hline \multirow{3}{*}{$1300^{\circ} \mathrm{C}$} & Original & 88.7 & 21.2 & 0.239 & 130.3 & 0.50 & 0 \\
\hline & 3 days & 90.5 & 22.7 & $\begin{array}{c}0.251 \\
(+5.0 \%)\end{array}$ & 135.9 & 0.76 & 0 \\
\hline & $\begin{array}{c}(14 \mathrm{~mm} \\
\text { below })\end{array}$ & 89.4 & 20.7 & 0.232 & 130.4 & 0.34 & 97.8 \\
\hline \multirow{2}{*}{$\begin{array}{c}1300^{\circ} \mathrm{C} \\
\left(1000^{\circ} \mathrm{C}\right)\end{array}$} & Original & 90.8 & 22.2 & 0.244 & 135.8 & 0.68 & 0 \\
\hline & 3 days & 88.5 & 22.8 & $\begin{array}{c}0.258 \\
(+5.7 \%) \\
\end{array}$ & 134.2 & 0.38 & 0 \\
\hline \multirow{2}{*}{$\begin{array}{c}1400^{\circ} \mathrm{C} \\
\left(1100^{\circ} \mathrm{C}\right)\end{array}$} & Original & 91.4 & 20.2 & 0.221 & 131.9 & 0.57 & 0 \\
\hline & 3 days & 88.6 & 21.5 & $\begin{array}{c}0.243 \\
(+10 \%) \\
\end{array}$ & 131.0 & 1.54 & 0 \\
\hline \multirow{2}{*}{$\begin{array}{c}1500^{\circ} \mathrm{C} \\
\left(1200^{\circ} \mathrm{C}\right)\end{array}$} & Original & 95.5 & 19.4 & 0.203 & 134.2 & 0.99 & 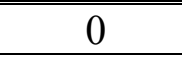 \\
\hline & 3 days & 80.1 & 30.6 & $\begin{array}{c}0.382 \\
(+88 \%)\end{array}$ & 140.9 & 0.89 & 128.8 \\
\hline
\end{tabular}

Table 4-37. High temperature stability results

\section{Interpretation of stability test results}

From the high temperature stability test results discussed in the previous section, there may be some concern regarding the operation in the desired industrial environments at temperatures at or above $1300^{\circ} \mathrm{C}$. These results suggest that they reaction that causes of sapphire to magnesium aluminate spinel has not reached an equilibrium when the final processing processing (sintering) of the cladding is concluded. Further reaction during inservice use is problematic: as the sapphire continues to dissolve, the core size will continue to shrink, and the refractive index of the cladding region will continue to change. Both of these changes will affect the mode volume of the sapphire fiber, which may change the sensor signal, depending upon the sensor interrogation scheme used.

The lack of equilibrium suggests the existence of a thermodynamic driving force for continued reaction of the alumina (sapphire) and magnesium oxide and spinel. One 
strategy for minimizing this effect is to adjust the store geometry of the slurry mixture so that the resulting coating composition is chosen to correspond to the tow phase region of spinel and alumina on the spinel solid solution boundary (on the alumina-magnesia binary phase diagram). Under this condition, the driving force for the alumina to dissolve in the spinel would be reduced. Furthermore, the dwell time for the high temperature firing (sintering) should be lengthened to ensure that the cladding has fully reacted during the process. However, possible detrimental effects, such as core-cladding roughness or cracks, that may result from a modified firing profile should be investigated as well.

\section{Development of Strain Gages for Solid Oxide Fuel Cells}

During this program, Prime Research investigated an optical strain sensor capable of operation at temperatures up to $800^{\circ} \mathrm{C}\left(1,472^{\circ} \mathrm{F}\right)$ for solid oxide fuel cell (SOFC) applications. The initial target application was a button cell with a diameter of $1.25 \mathrm{in}$ and thickness of $137 \mu \mathrm{m}$ Strain sensor technology in use by NETL for characterization of the button cell included an ITO based thin film strain gauge. This sensor provides a small footprint, low physical and thermal mass, and adequate sensitivity to detect small strain fluctuations. However, the installation process requires specialized equipment to deposit the thin film leads, sensor elements, and lead wire attachments. Prime identified ease of installation as a requirement for the optical strain sensor under development.

\subsection{Optical Sensor Technology Selection}

Building on the Fabry Perot strain gage design under development for the NETL power plant portion of this program, a similar design was initially investigated. Two optical fibers are to be aligned in parallel such that the two faces of the fiber ends are separated by an air gap (see the example in Figure 5-1). The fibers are bonded to a coupon or directly to the substrate (button fuel cell) such that strain is transferred directly to the sensor, yielding a direct measurement of the displacement.

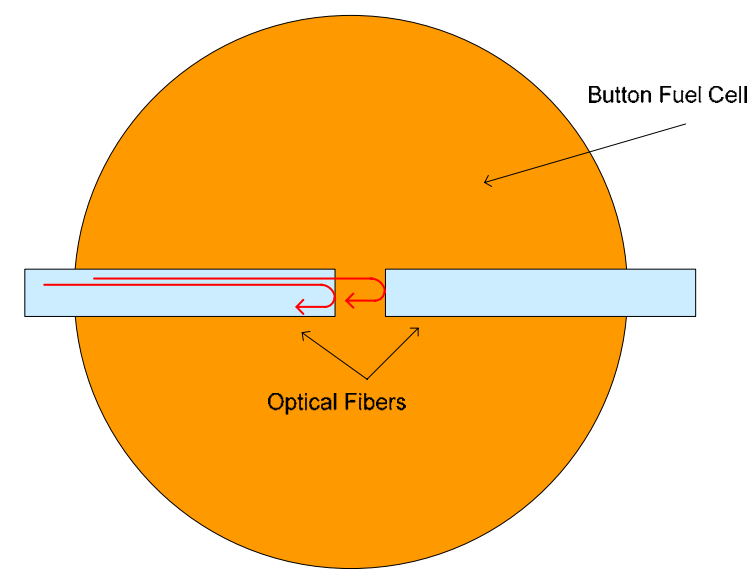

Figure 5-1. Fabry Perot reflections 
The power plant application requires multimode sapphire fiber for operation at temperatures up to $1,200^{\circ} \mathrm{C}\left(2,192^{\circ} \mathrm{F}\right)$. Installation in the power plant facility will be onsite, measurements taken with a multimode-compatible interrogator system, and sensor lifetime is expected to span over several months. In comparison, the fuel cell application requires a maximum operating temperature of $800^{\circ} \mathrm{C}\left(1,472^{\circ} \mathrm{F}\right)$, laboratory installation, and short lifetime (seconds). These differences have lead to the use of a different fiber material, minimization of packaging, and on-site build and installation of the sensor. It was determined by Prime Research that for the fuel cell gage feasibility study, multimode silica optical fiber would be used in place of sapphire, packaging would be developed specifically for the button cell testing application, and the design would allow NETL researchers to install sensors on-site without delay in testing schedules.

\subsubsection{Angular Sensitivity}

In order to evaluate the feasibility of external Fabry-Perot sensors for use in a fuel-cell bend test, a finite element model was developed. Evaluation of the angle between the fibers due to bending was inspired by a paper published by in the Center for Photonics Technology (CPT) at Virginia Tech in 2004. ${ }^{28}$ Their study modeled lightwave interference in low-finesse multimode Fabry Perot interferometers. The sensitivity of angular misalignment on fringe visibility was modeled. It was concluded that for two $100 \mu \mathrm{m}$ core diameter fibers with a $30 \mu \mathrm{m}$ gap between the fibers, there will be a drop in the fringe visibility to $4 \%$ with an angle of 0.5 degrees between the fibers as shown in Figure 5-2.

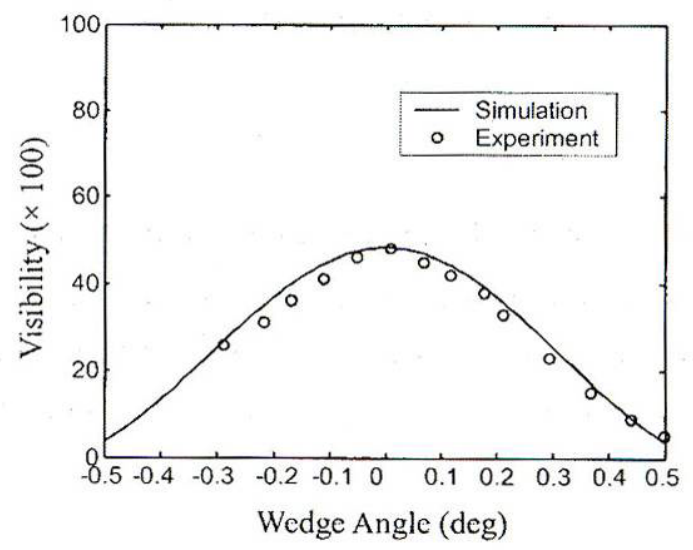

Figure 5-2. Fringe visibility versus wedge angle between the reflection surfaces ${ }^{2}$

The finite element model dimensions, applied load, and constraints were chosen to best represent the tests presented in the NETL presentation titled "Strain Gage

Characterization Test Rig (CTR)", by Rich Pineault, dated 2/2/2006. The model was for a 1.25 in diameter, $137 \mu \mathrm{m}$ thick wafer subject to a $1100 \mu \mathrm{m}$ center point displacement. The analysis was performed using Nastran for Windows version 2004r2. Due to the large displacements and rotations involved, a geometrically nonlinear static analysis was used.

\footnotetext{
${ }^{28}$ Ming Han and Anbo Wang. "Exact analysis of low-finesse multimode fiber extrinsic Fabry-Perot interferometers.” Applied Optics, Vol. 43, No. 24, pp4659-4666, 2004.
} 
The finite element model response to the bend test is shown in Figure 5-3. It can be seen that the maximum displacement occurs at the center of the wafer and is equal to 0.0433 in, or $1100 \mu \mathrm{m}$.

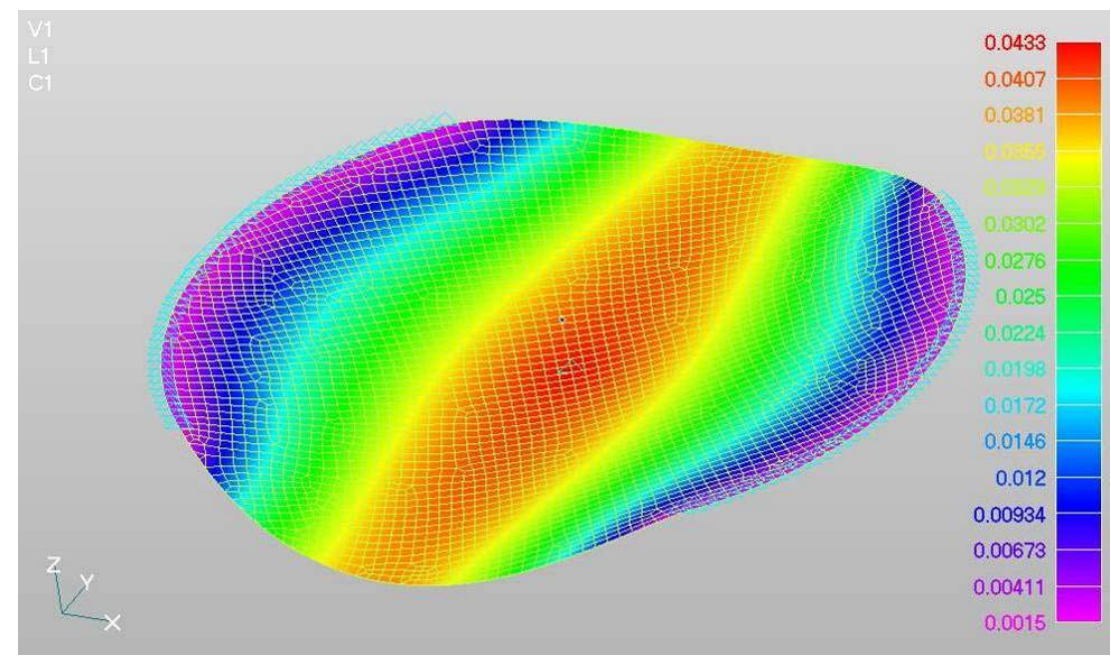

Figure 5-3. Finite element model of the fuel cell bend test. Contours of total displacement (in inches) are shown on a deformed mesh. Displacements are magnified for clarity. The light blue triangles at the edges represent the test fixture supports on the two 90 degree sectors.

In order to achieve adequate fringe visibility in an external Fabry-Perot sensor, it is important that the faces of the two reflectors remain as close to parallel as possible. However, in a bend test such as that performed on the fuel cell, there will be some inherent change in the angle between the reflector end-faces, as suggested by Figure 5-4. Based on the deformation results from the finite element analysis, rotations about the yaxis were calculated and used to predict the change in the angle between the Fabry-Perot reflectors. Figure 5-5 plots the rotation about the y-axis vs the distance from center line of the wafer.

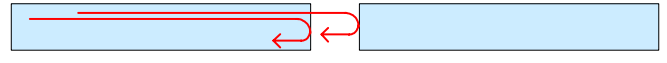

a. Fabry Perot reflections

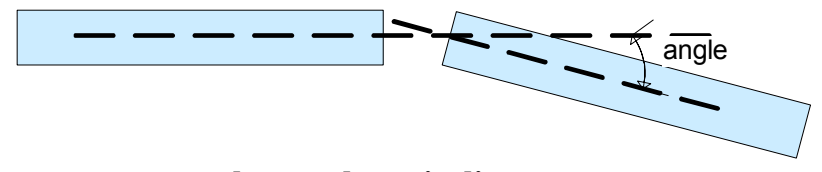

b. angular misalignment

Figure 5-4. External Fabry Perot

The Fabry-Perot gages under consideration for this work have a gap length of approximately $100 \mu \mathrm{m}$. If such a gage were to be positioned at the center of the fuel cell (just outside of the pushrod contact area), with one reflector face at distance $=0$ in and the other face at distance $=.00394 \mathrm{in}(100 \mu \mathrm{m})$, then the rotation between the reflectors would be approximately 0.2 degrees. According to the results shown in Figure 5-5, this angular misalignment could lower the fringe visibility to $\sim 30 \%$. This value was adjudged to be unacceptably low, so further development of Fabry-Perot strain gauges for SOFC instrumentation was discontinued, and alternate sensor designs were investigated, as described below. 


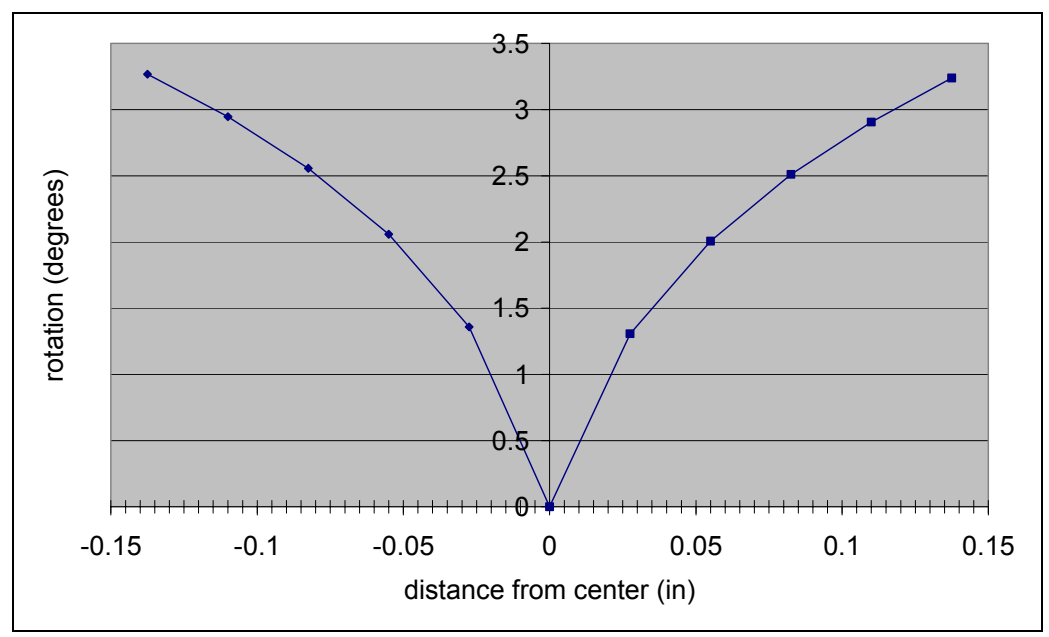

Figure 5-5. Rotation results from the finite element analysis shown in Figure 5-3. Rotations about the y-axis (see Figure 5-3) are plotted vs. the distance from the center of the wafer.

\subsection{Sensor Designs for Investigation}

\subsubsection{Double Angle Strain Sensor Design}

The Double Angle design is composed of two optical fibers with $45^{\circ}$ reflectors polished on the ends of the fibers and aligned in parallel such that light is transmitted between the fibers. Light is injected into Fiber A as shown in Figure 5-6 and transmitted to Fiber B after partial reflection off the $45^{\circ}$ angled fiber end faces. The fibers are mounted on a substrate so that strain developed in the substrate causes a change in the alignment of the sensor. This will cause a change in the transmitted power level. When the central points of the angles are aligned the power will be at the maximum.

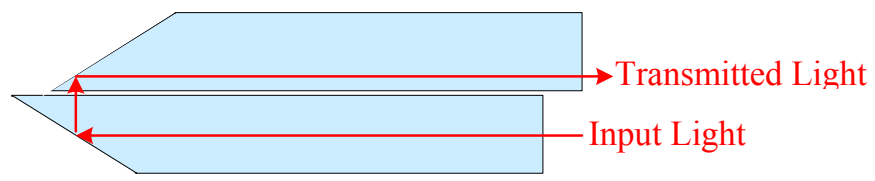

Figure 5-6. Double angle transmission sensor design

Feasibility studies were conducted for this design using silica multimode fiber $(62.5 / 125$ $\mu \mathrm{m})$, an $830 \mathrm{~nm}$ LED, and an Agilent 8163A Lightwave Multimeter. The fibers were aligned in parallel and held in alignment with a square glass ferrule as shown in Figure 5-7. The ferrule minimizes angular misalignment of the fibers as discussed above.

Each fiber could be moved independently with micrometer stages that include rotation in order to align the fiber angles as seen in Figure 5-7. The fibers could then be moved relative to each other to affect the transmitted power. As predicted, when the fiber angles were off center, the power levels decreased, as shown in Figure 5-8. 


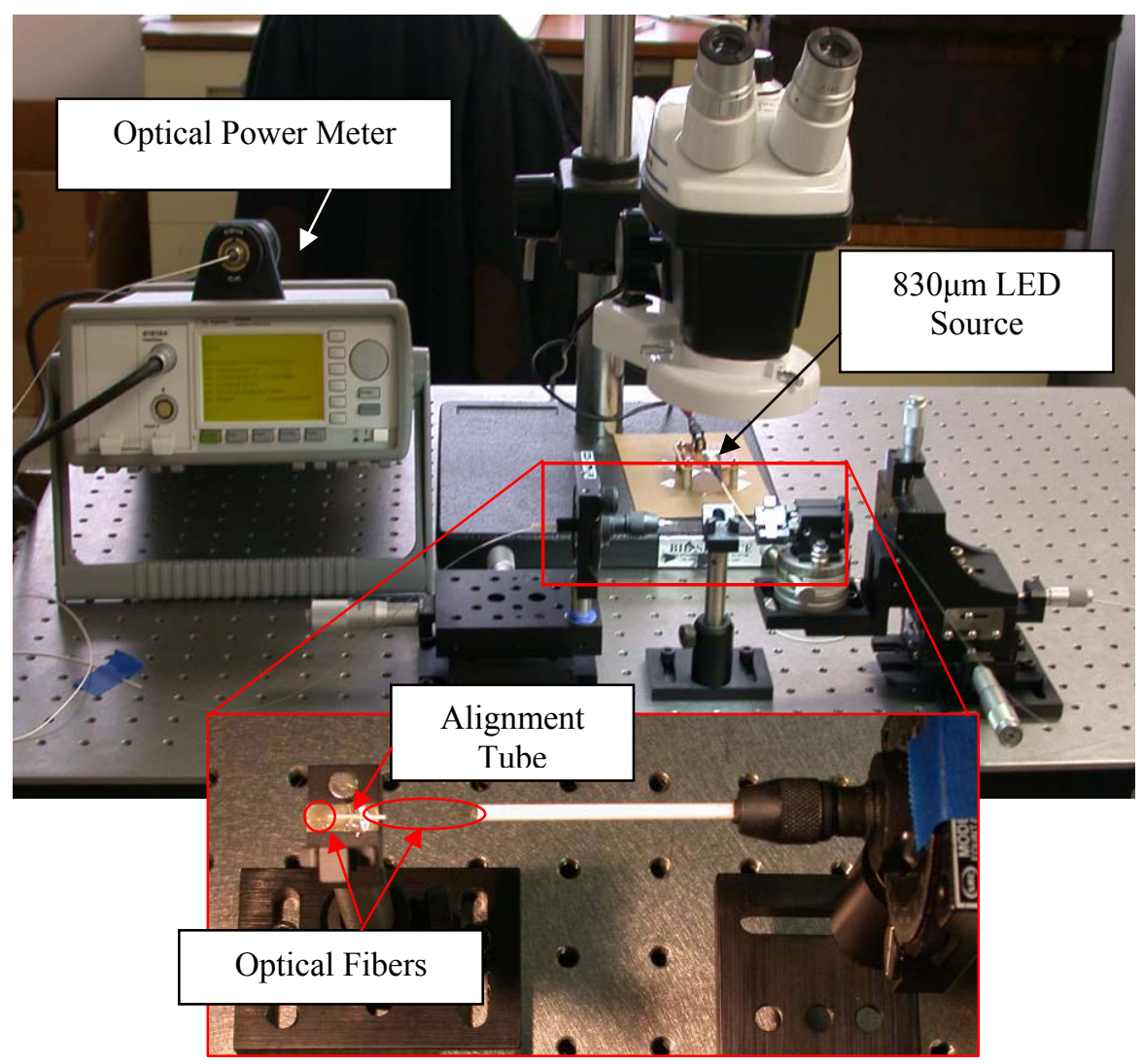

Figure 5-7. Experimental setup for feasibility testing

Continuation of this work will include the selection of an appropriate demodulation system. Work will also begin to focus on construction techniques for the sensor design. With the construction of initial prototype sensors, performance parameters such as resolution will be quantified.

\subsubsection{Resonant Strain Sensor Design}

Another approach evaluated for high temperature strain gage sensors for fuel-cell instrumentation employed the resonant sensing technology. For a general description of resonant sensing concepts, please refer to Section 3.7.1. The resonant strain gage is expected to be inexpensive, easy to install, and should lend itself well to high temperature applications.

A schematic of the resonant strain gage is shown in Figure 5-9. The structure to be instrumented is the ivory-colored block labeled "structure,", the resonant beam is shown in black, the driving fiber in blue, and the interrogation fiber in red. The resonant beam is bonded to the structure at the bond points indicated in yellow labeled "bond points.". The interrogation fiber end-face is polished to $45^{\circ}$ so that a Fabry-Perot interferometer is 


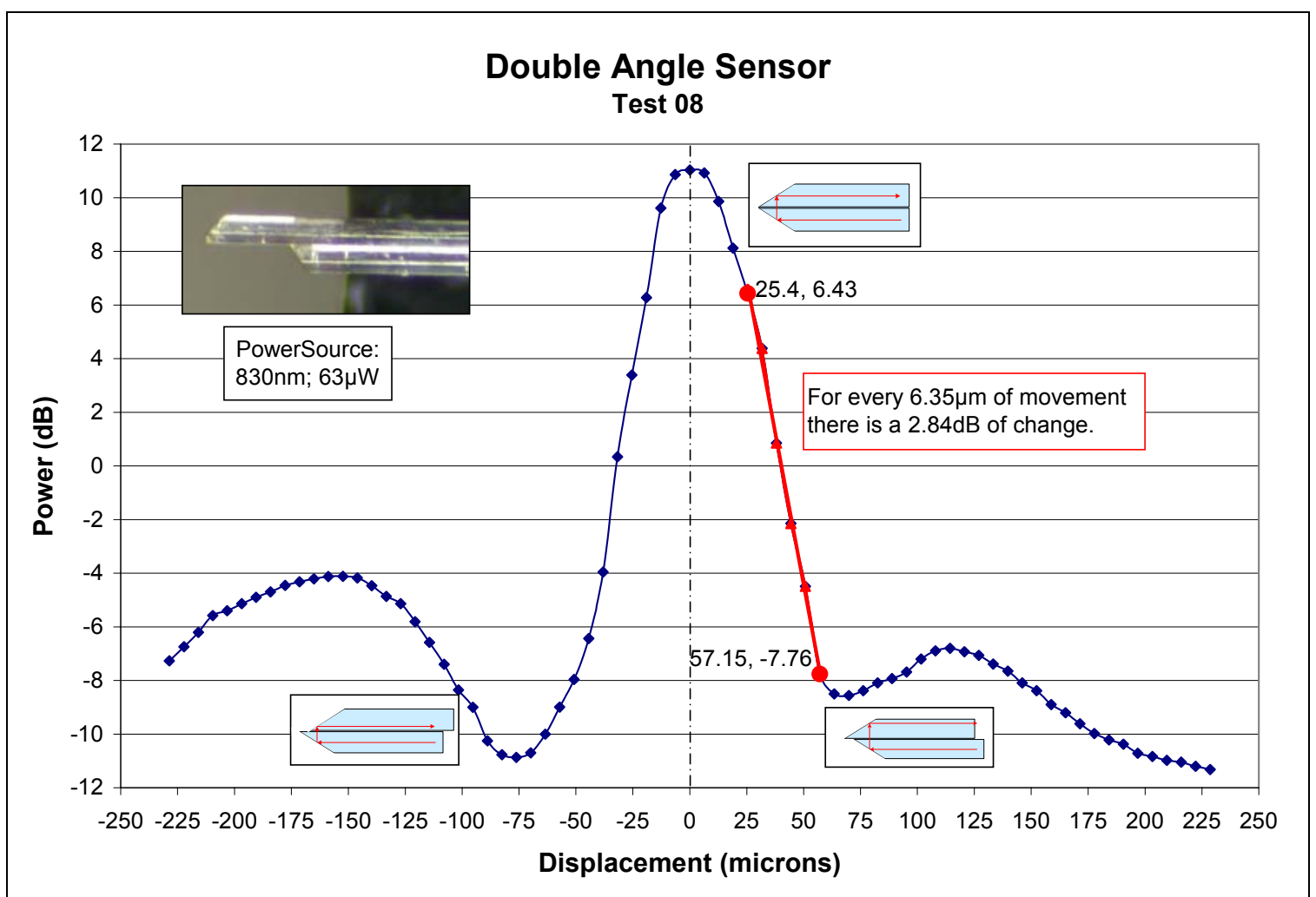

Figure 5-8. Power level versus displacement of the fiber angles

formed between the fiber end-face and the underside of the resonant beam. Strain in the instrumented structure causes a proportional change in the beam resonant frequency. A photograph of the prototype strain gage is shown in Figure 5-10. The prototype device differs from the schematic in Figure 5-9 only in the way the interrogation fiber is routed. For simplicity, the prototype device was fabricated with the interrogation fiber entering from the side of the beam, whereas a production quality device would allow the fiber to enter from the same direction as the driving fiber.

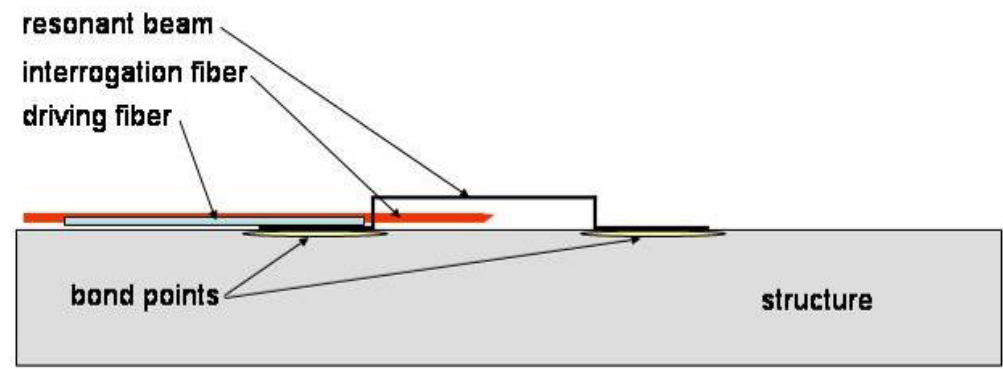

Figure 5-9. Schematic of a strain gage which utilizes the resonant sensing approach. 


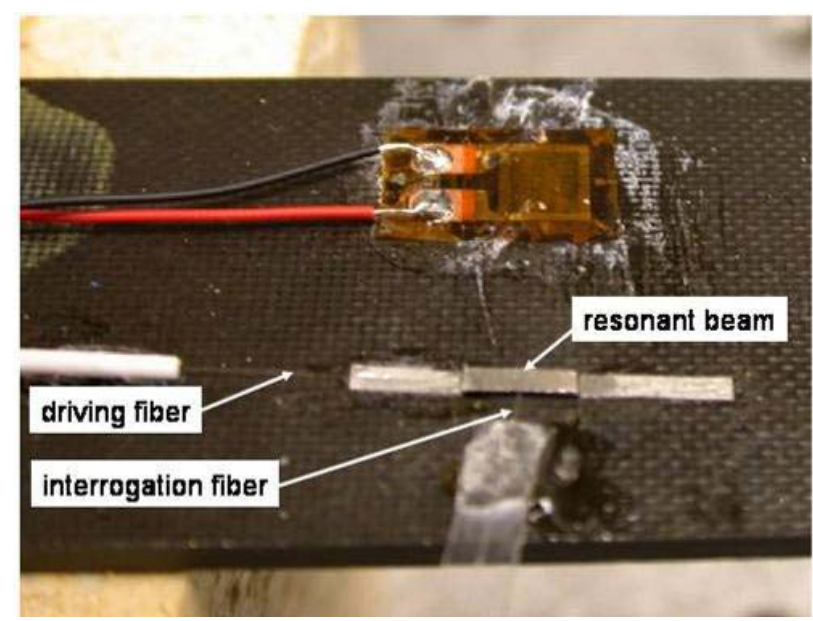

Figure 5-10. Photograph of carbon fiber beam instrumented with an optical resonator strain gage and a traditional thin film electrical gage.

\subsubsection{Miniature Fabry-Perot Strain Gages}

Also during the program, miniature strain gages consisting of Fabry-Perot cavities micromachined into optical fibers were investigated. The Miniature Fabry-Perot Sensor (MFPS) was invented at Virginia Tech; Prime Research licensed the background intellectual property and has been improving the sensor performance and manufacturing techniques. The MFPS strain gage is fabricated utilizing the etch/splice process show in Figure 5-11; a completed sensor is shown in Figure 5-12.

Pit-etched fiber

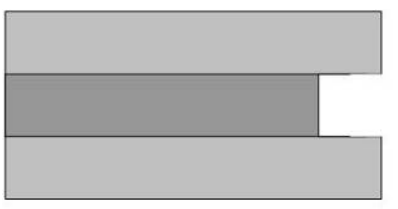

Reflector fiber

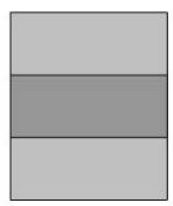

Completed strain gage
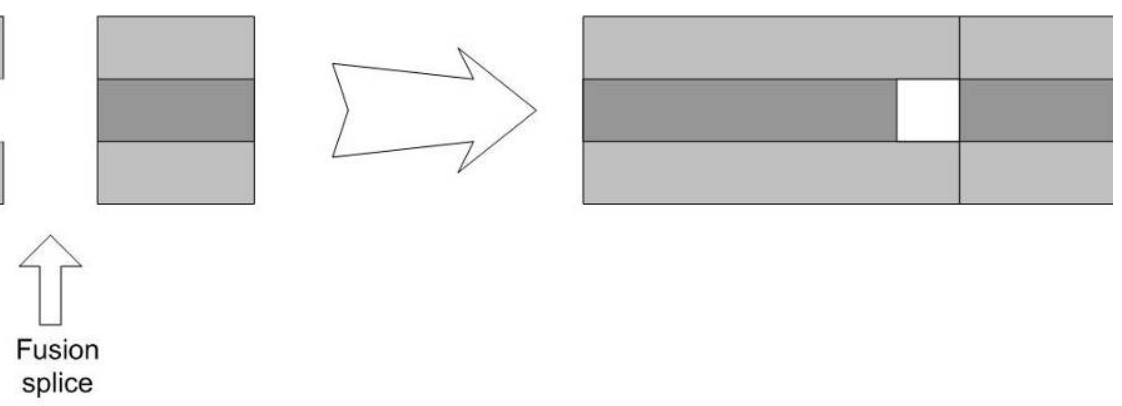

Figure 5-11. The MFPS strain gage is fabricated by pit-etching a cleaved fiber and then splicing to a reflector fiber.

In order to measure the strain of the fuel cell substrate, the MFPS sensor must be securely bonded to the surface such that any strain induced in the substrate is accurately transferred to the sensor. Initial bonding trials utilized an alumina based adhesive (Aremco 835M) that has a coefficient of thermal expansion (CTE) which approximates that of the fuel cell substrate. The Aremco 835M worked successfully at room temperature but was problematic at elevated temperatures. We suspect that since the 
CTE of the alumina adhesive is significantly greater than that of the silica fiber, that the mechanical adhesion provided at room temperature was lost at elevated temperatures. Tests showed that at only $100{ }^{\circ} \mathrm{C}\left(212^{\circ} \mathrm{F}\right)$, the fiber bonded with Aremco 835M did not accurately transfer strain from the substrate to the fiber. The solution to this problem was found by using a silica-based adhesive that has a CTE which approximates that of the silica strain gage. The silica-based adhesive utilized was Aremco 618N, and was shown to provide accurate transfer of strain over the full temperature range.

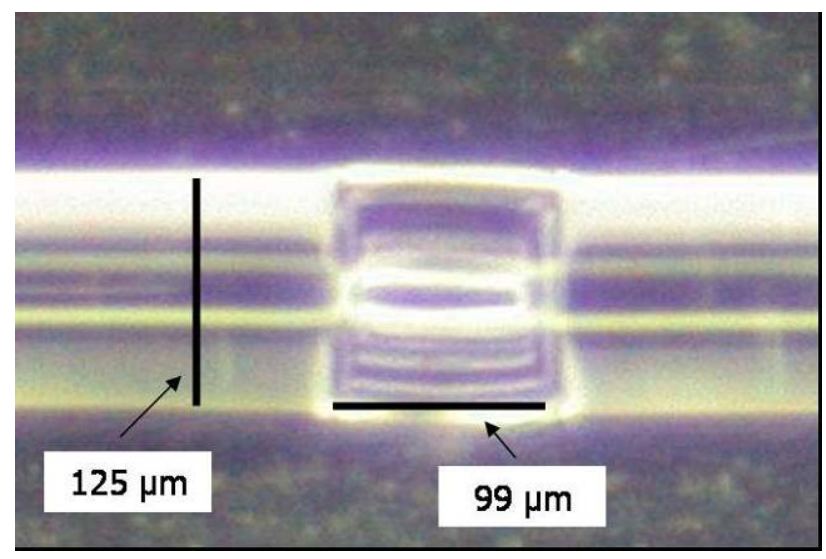

Figure 5-12. A magnified view of an MFPS strain gage. The $99 \mu \mathrm{m}$ Fabry-Perot air-gap is noted relative to the fiber diameter of $125 \mu \mathrm{m}$.

Evaluation of the MFPS strain gage was performed by instrumenting a thin alumina beam and subjecting it to a 3-point bend test. The alumina beams tested were $76 \mathrm{~mm}$ long $\mathrm{x} 13$ mm wide $\mathrm{x} 125 \mu \mathrm{m}$ thick ( 3 in $\mathrm{x} 0.5$ in $\mathrm{x} 0.005$ in). For room temperature testing, the alumina beam was suspended between glass rollers and was deflected using a free pivoting blade attached to a digital micrometer. In order to provide a source for comparison, a traditional thin-film electrical strain gage was bonded to the alumina beam at a location of symmetry to the optical strain gage. This room temperature 3-point bend test fixture is shown in Figure 5-13. A close-up of the alumina beam with electrical and optical gages is shown in Figure 5-14. Additional insight into the mechanics of the bend test was provided by the Finite Element Analysis (FEA) shown in Figure 5-15.

The comparison between the electrical and optical measurements, as well as the finite element analysis of the instrumented beam was very good. A plot of measured strain vs beam centerline displacement is shown in Figure 5-16. The analysis, using NASTRAN, predicted surface strains of $0.019 \%$ at the strain gauge location, for a $1.5 \mathrm{~mm}$ centerline displacement. 


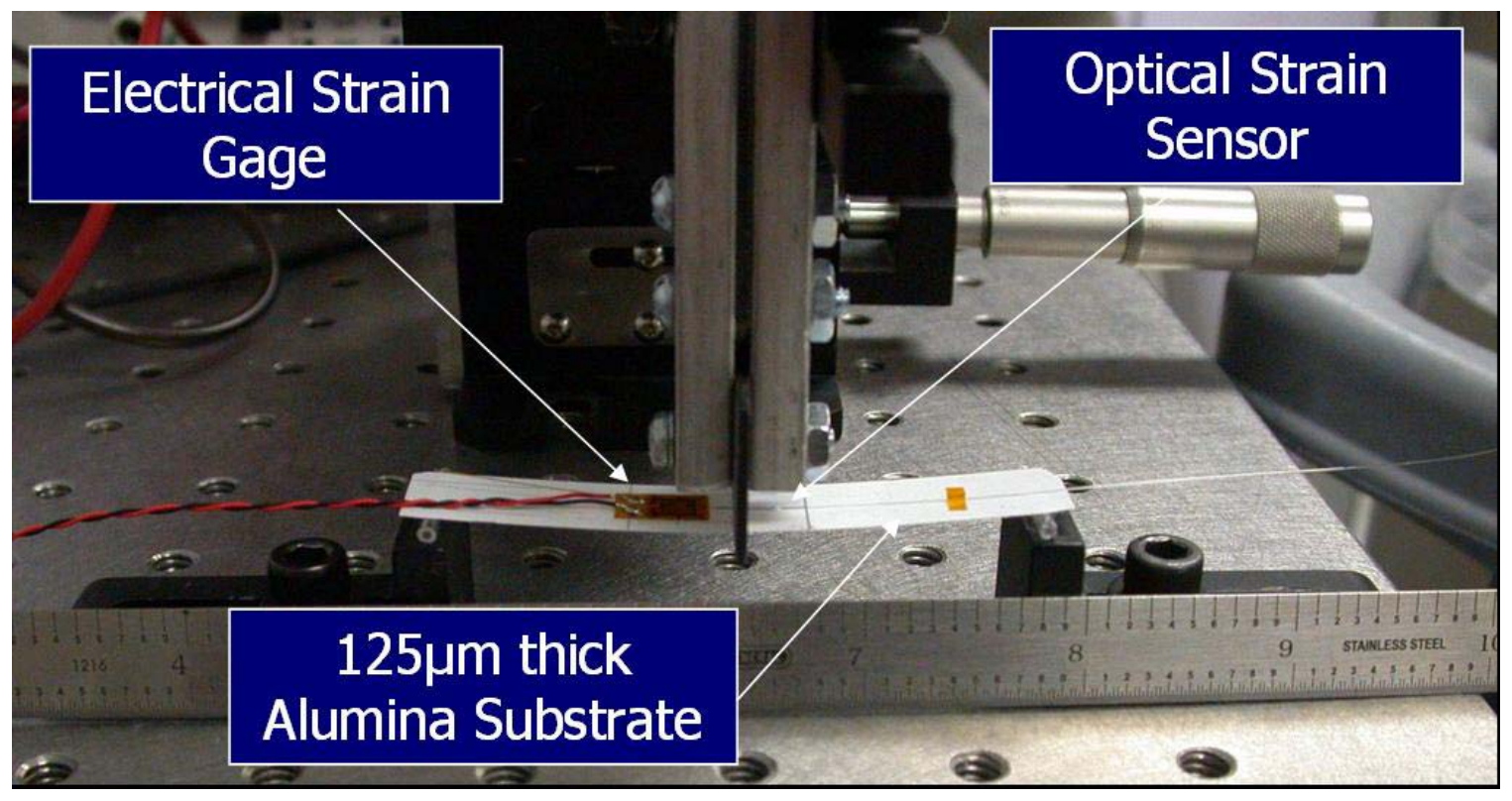

Figure 5-13. A picture of the 3-point bend test fixture utilized for room temperature evaluation of the MFPS strain gage. The 3" x 0.5 " $125 \mu \mathrm{m}$ alumina beam is instrumented with an electrical and an optical strain gage which are equidistant from the beam centerline.

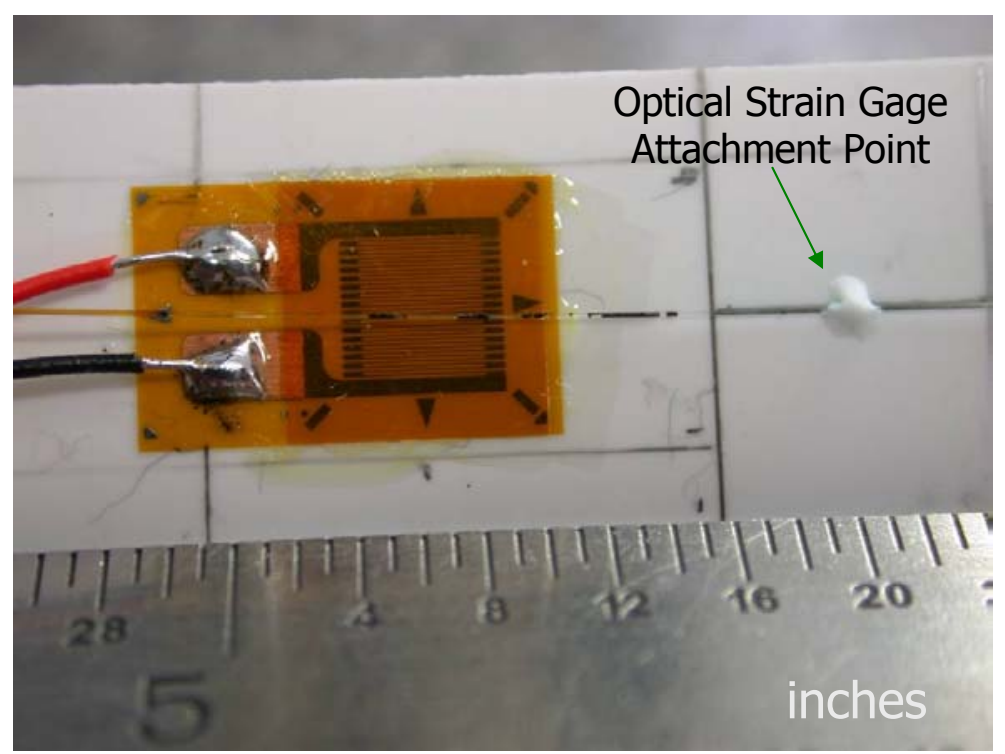

Figure 5-14. Close-up picture of the $125 \mu \mathrm{m}$ thick alumina beam instrumented with a traditional thin-film electrical strain gage and a Prime Research MFPS optical strain gage. 


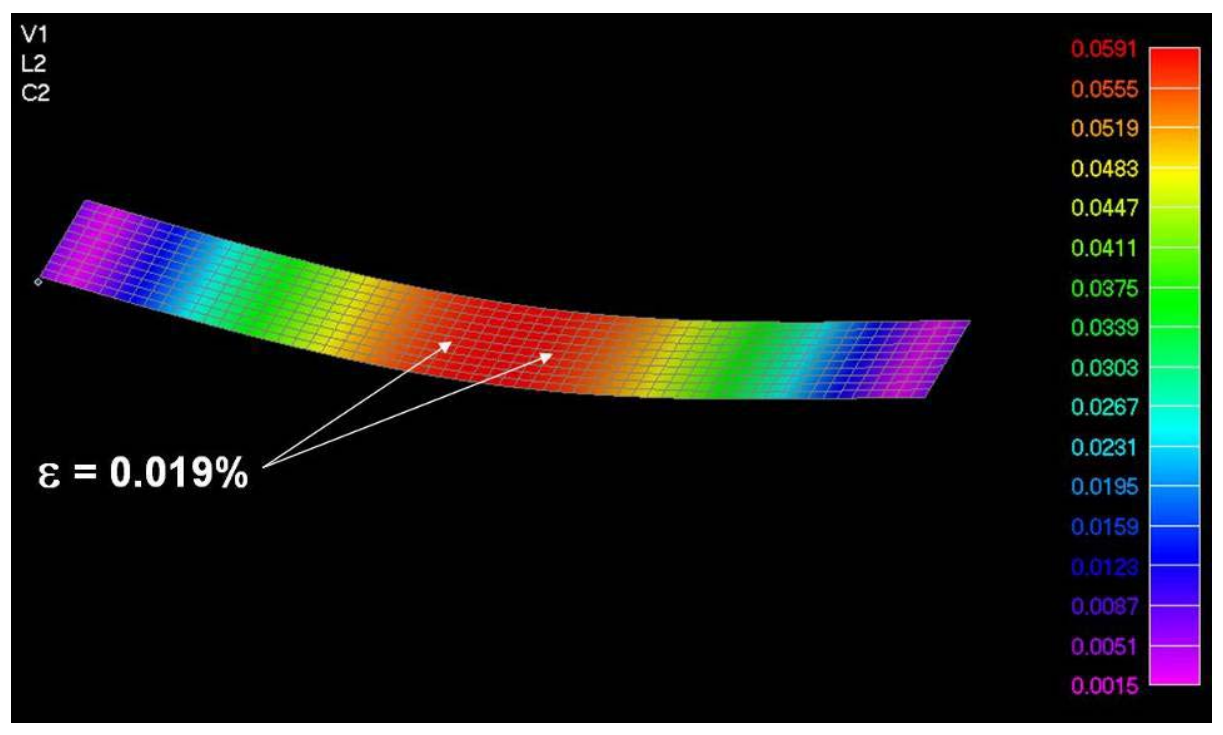

Figure 5-15. Finite element model of the 3" $\mathrm{x} 0.5$ " $\times 125 \mu \mathrm{m}$ alumina beam subject to a $1.5 \mathrm{~mm}$ centerline displacement. Contours of total displacement (in inches) are shown on a deformed mesh. Displacements are magnified for clarity. The calculated surface strain at the gage installation locations is $0.019 \%$.

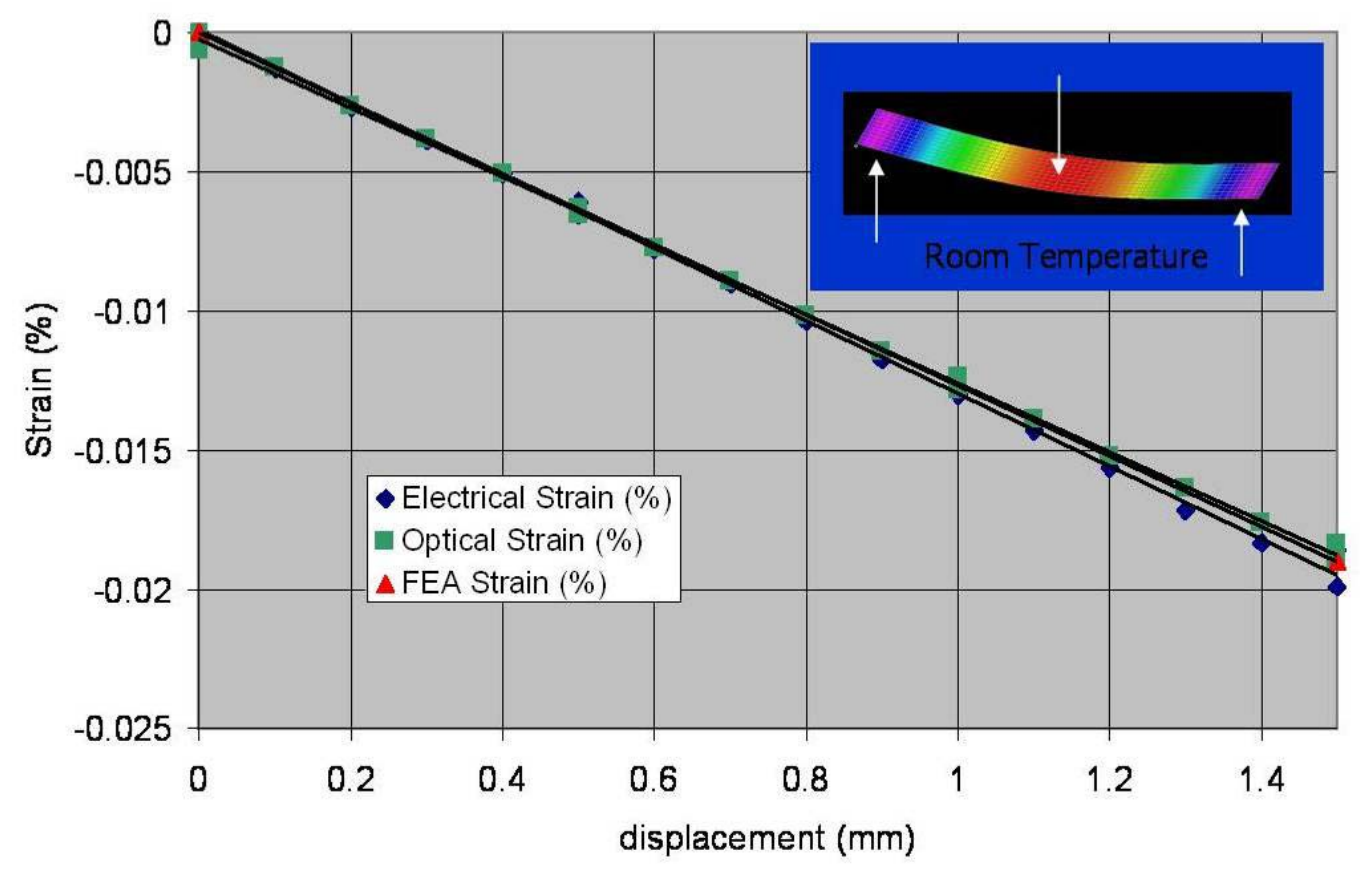

Figure 5-16. A plot showing the predicted and measured strain vs. beam centerline displacement. The optical and electrical strain measurements are seen to compare well with each other as well as the result predicted by finite element analysis. 
After successful demonstration of the MFPS strain gage at room temperature, a button cell test fixture based on NETL drawings was fabricated to evaluate performance at temperatures up to $800^{\circ} \mathrm{C}\left(1472^{\circ} \mathrm{F}\right)$. The button cell substrate holder was fabricated from Macor ceramic and was positioned inside a clam-shell furnace by an alumina tube. Displacements were applied to the test sample via an alumina push-rod/micrometer arrangement. This high temperature test apparatus is shown in Figure 5-17.

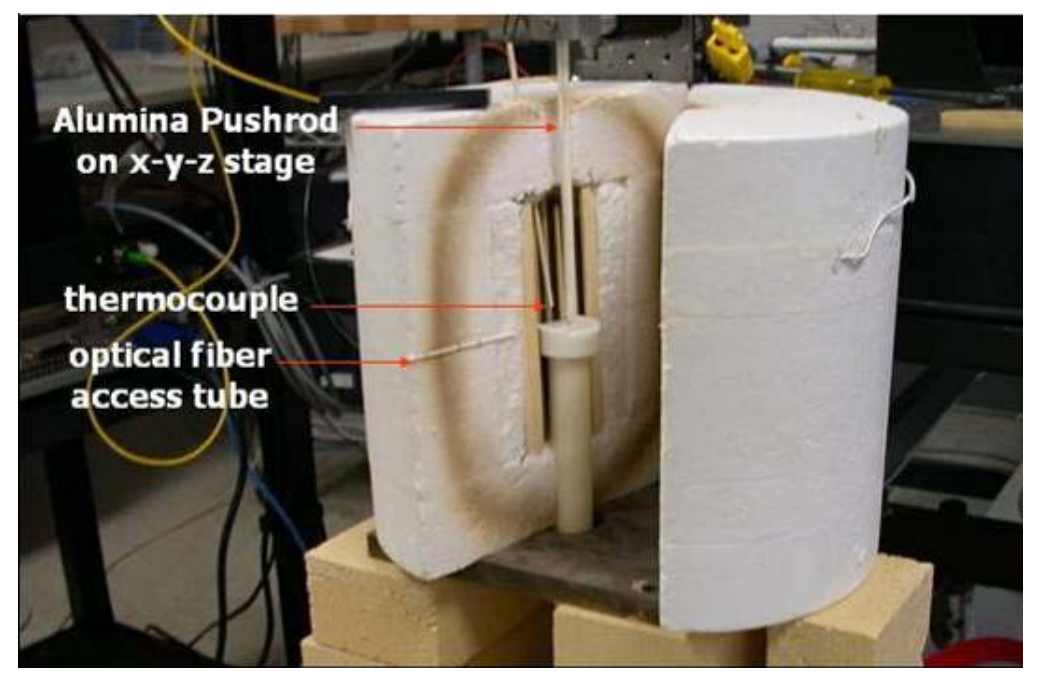

Figure 5-17. A picture showing the high temperature strain test fixture.

In order to debug the test rig before testing on expensive SOFC substrates, elevated temperature testing was performed on alumina substrates. MFPS optical strain gages were bonded to a 1 " long x 0.5 " wide x $125 \mu \mathrm{m}$ thick alumina beam with Aremco $618 \mathrm{~N}$. After performing the cure cycle required by the adhesive, the beam was heated to $800^{\circ} \mathrm{C}$ $\left(1472^{\circ} \mathrm{F}\right)$ and a mechanical load was applied using a pushrod to generate strain in the beam. The strain test involved using a micrometer to step the pushrod displacement from zero to $500 \mu \mathrm{m}$ and then back to zero in steps of $100 \mu \mathrm{m}$. A plot of the sensor response as a function of time for the entire test is shown in Figure 5-18, and an enlarged view in Figure 5-19.

After successful demonstration on alumina substrates at $800^{\circ} \mathrm{C}\left(1472^{\circ} \mathrm{F}\right)$, tests were performed on Notch Ionic substrates at $800^{\circ} \mathrm{C}$. The zirconia-based Hionic substrates were $28 \mathrm{~mm}$ in diameter and approximately $130 \mu \mathrm{m}$ thick. The fiber optic strain gage was bonded with Aremco $618 \mathrm{~N}$ ceramic adhesive to the Hionic substrate, and several strain tests were performed. The testing performed included two "step tests" and one "constant strain rate" test which loaded the substrate to its fracture limit. The entire test resulted in over 21000 data points taken once every 4 seconds, resulting in a total test duration of approximately 23 hours. The temperature and strain histories for the entire test are shown below in Figure 5-20. An enlarged view of one step test is shown in Figure 5-21. An enlarged view of the constant strain rate test is shown in Figure 5-22. 


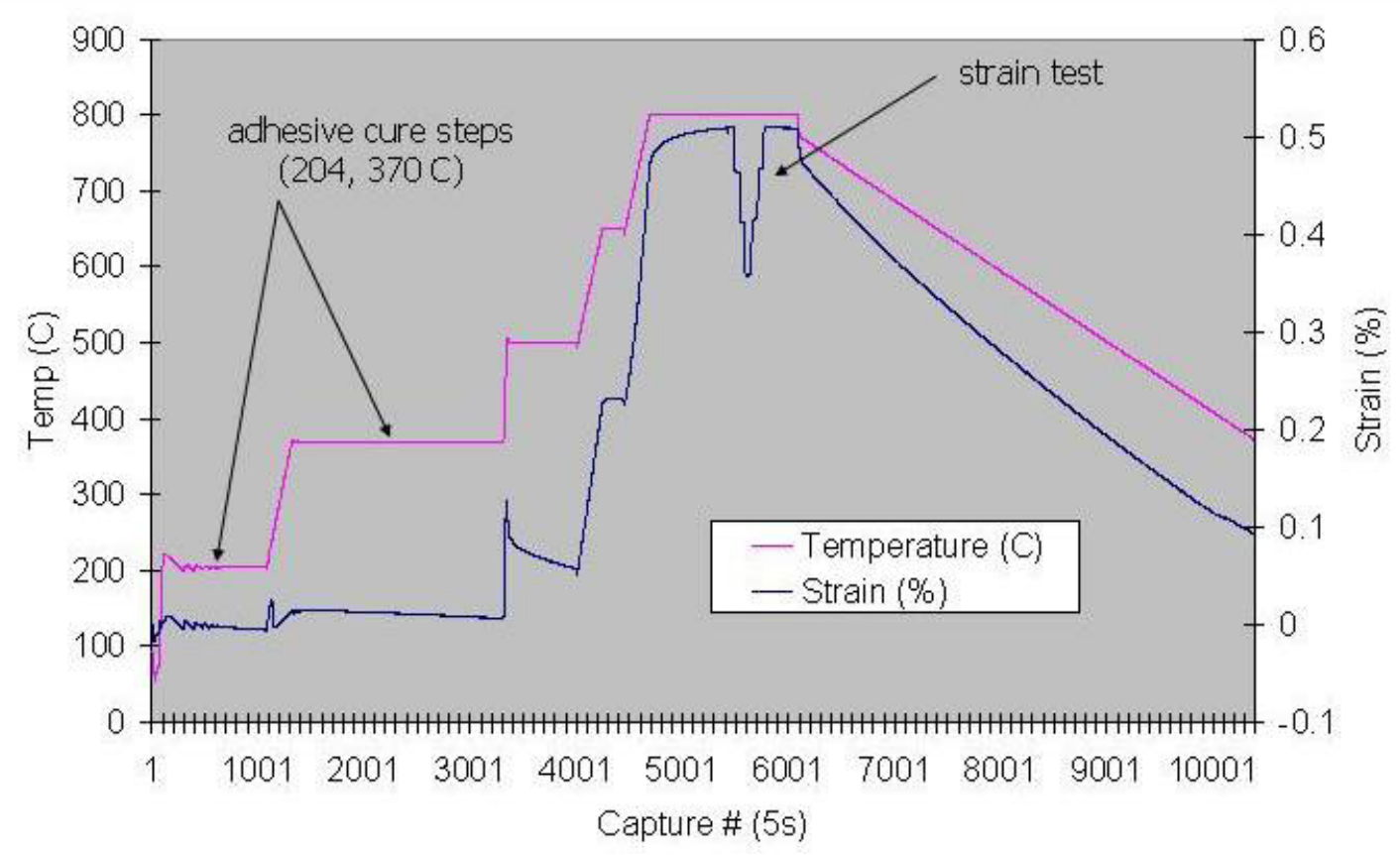

Figure 5-18. A plot showing the response of an MFPS optical strain gage to an elevated temperature test. The sensor response in \% strain is plotted as a function of time. Adhesive cure steps were followed by a strain test at $800^{\circ} \mathrm{C}\left(1472^{\circ} \mathrm{F}\right)$.

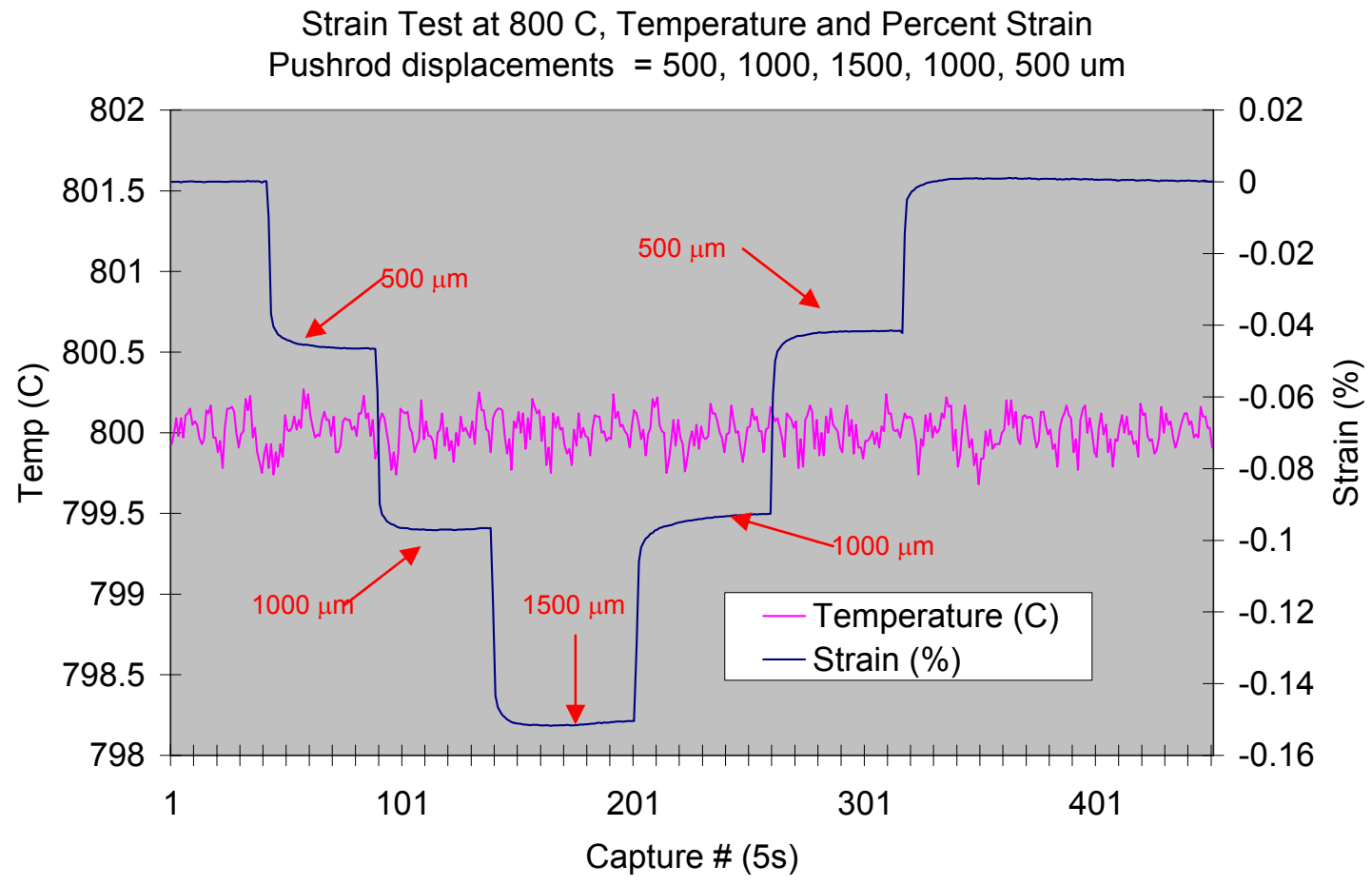

Figure 5-19. Close up view of the strain test shown in Figure 5-18. 


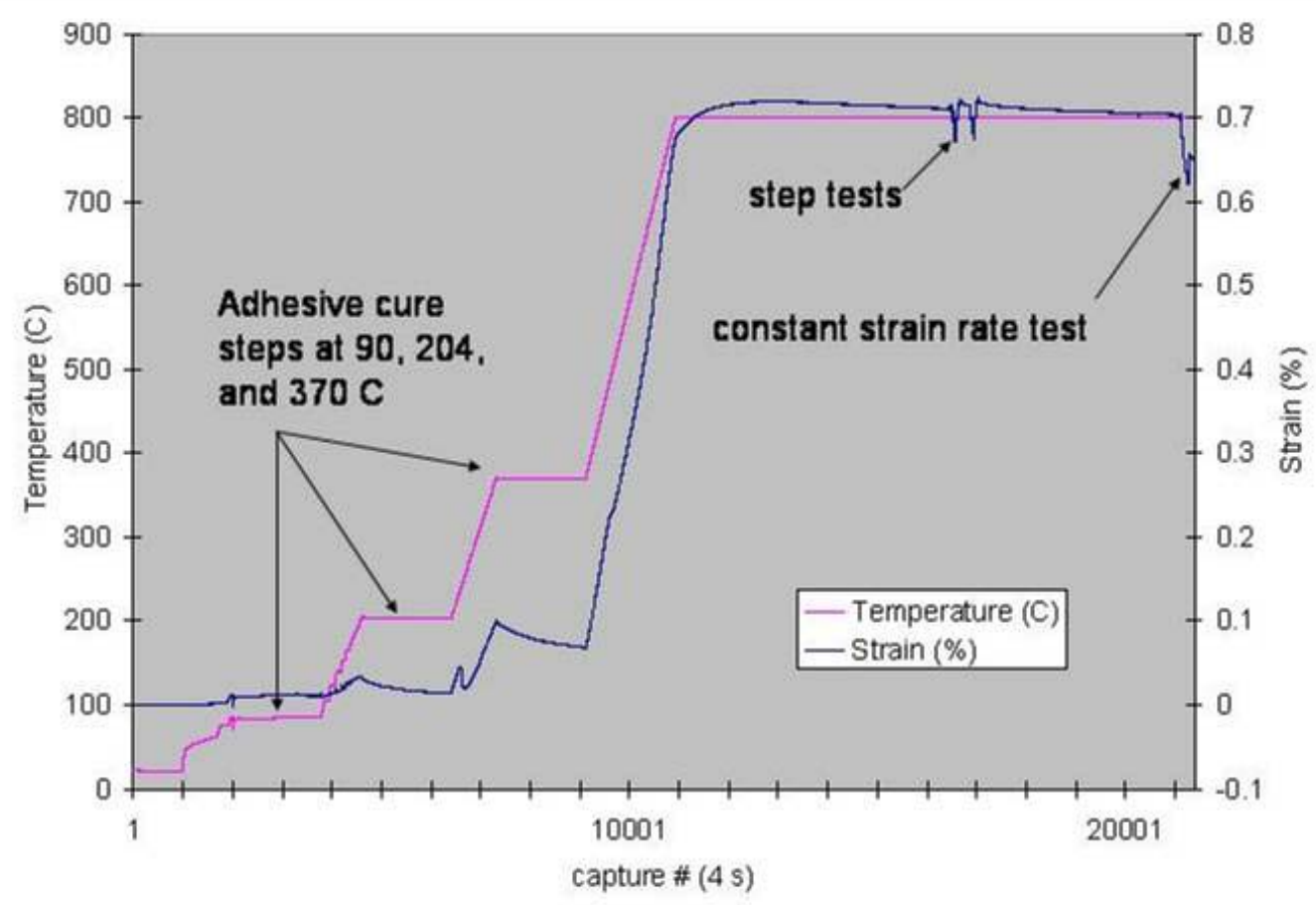

Figure 5-20. Temperature and strain histories from the evaluation of a fiber optic strain gage on a Hionic substrate. After the adhesive curing steps, several strain tests were conducted at $800^{\circ} \mathrm{C}$ $\left(1,472{ }^{\circ} \mathrm{F}\right)$.

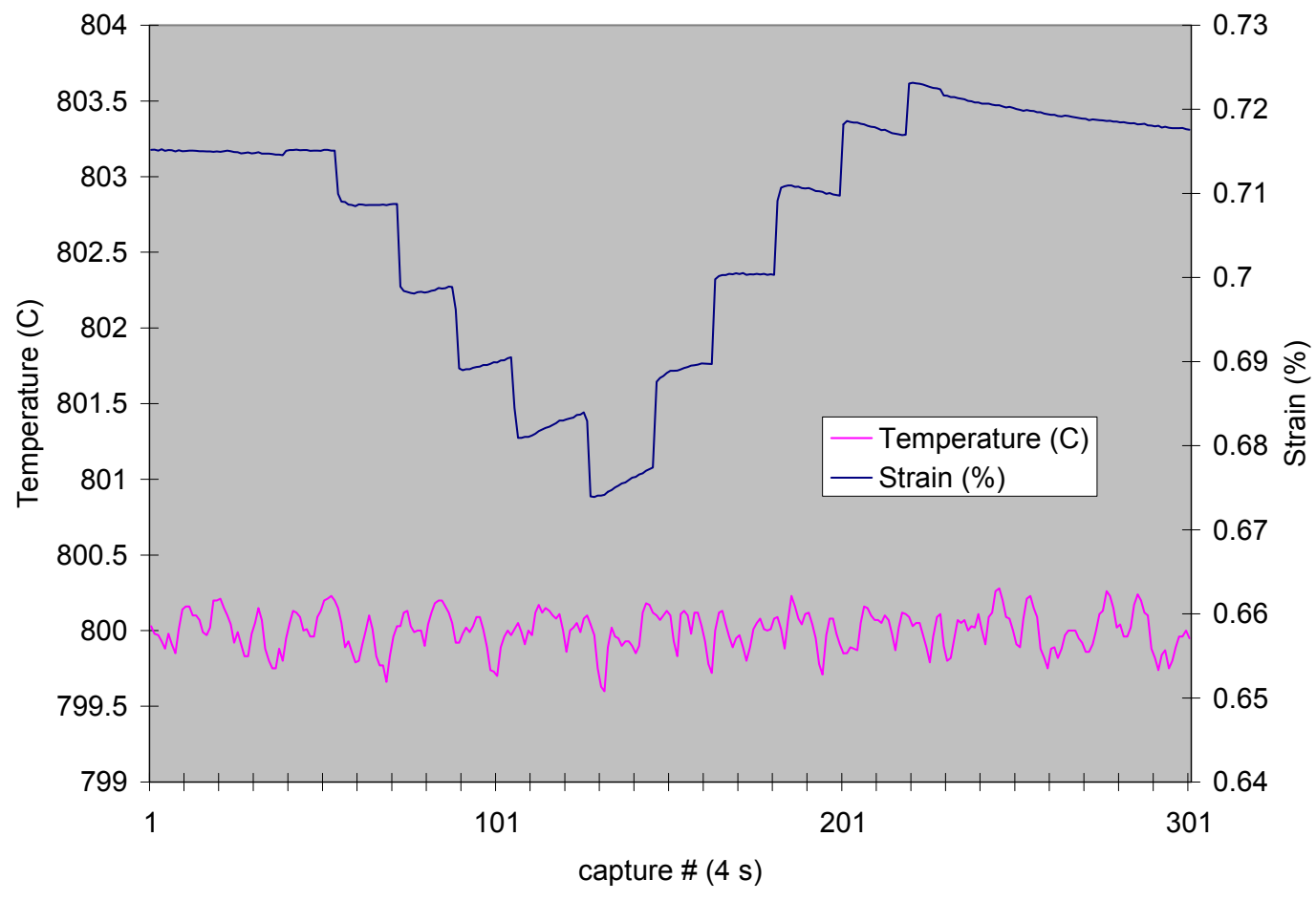

Figure 5-21. An enlarged view of one of the step tests seen in Figure 5-20. The test had a maximum pushrod displacement of $500 \mu \mathrm{m}$, with individual steps of $100 \mu \mathrm{m}$. 


\subsubsection{Test results}

Each step test involved discrete pushrod deflections of $100 \mu \mathrm{m}$ on the way up to, and back from, a maximum displacement of $500 \mu \mathrm{m}$. It can be seen in Figure 5-21 that there is some hysteresis in the response and a fairly significant drift, particularly at the larger deflections. Previous tests run on alumina substrates had occasionally exhibited this undesirable behavior, so it is believed to be unrelated to the Hionic substrate itself. Ordinarily, an intermittent problem usually indicates a quality control issue. In this case, the hysteresis and drift are believed to be due to slippage between the adhesive and the fiber, which is likely caused by inconsistencies in adhesive application. A close examination of and modification to the adhesive application procedure is likely to eliminate or greatly reduce the likelihood of slippage between the fiber and adhesive.

The constant strain rate test shown in Figure 5-22 was performed by controlling the pushrod micrometer with a voltage controlled motor. The pushrod displacement was increased at a rate of $165 \mu \mathrm{m} / \mathrm{min}$, and the test took almost exactly 10 minutes until the Hionic substrate failed. The center point deflection at failure was $1650 \mu \mathrm{m}$. The "bumps" in the response were due to drive belt slippage between the motor and micrometer movement that drove the pushrod. The curvature in the strain response is believed to be due, as with the step test data, to slippage between the adhesive and the fiber optic strain gage. As described above, this problem is likely to be eliminated with a careful inspection of the adhesive application procedure.

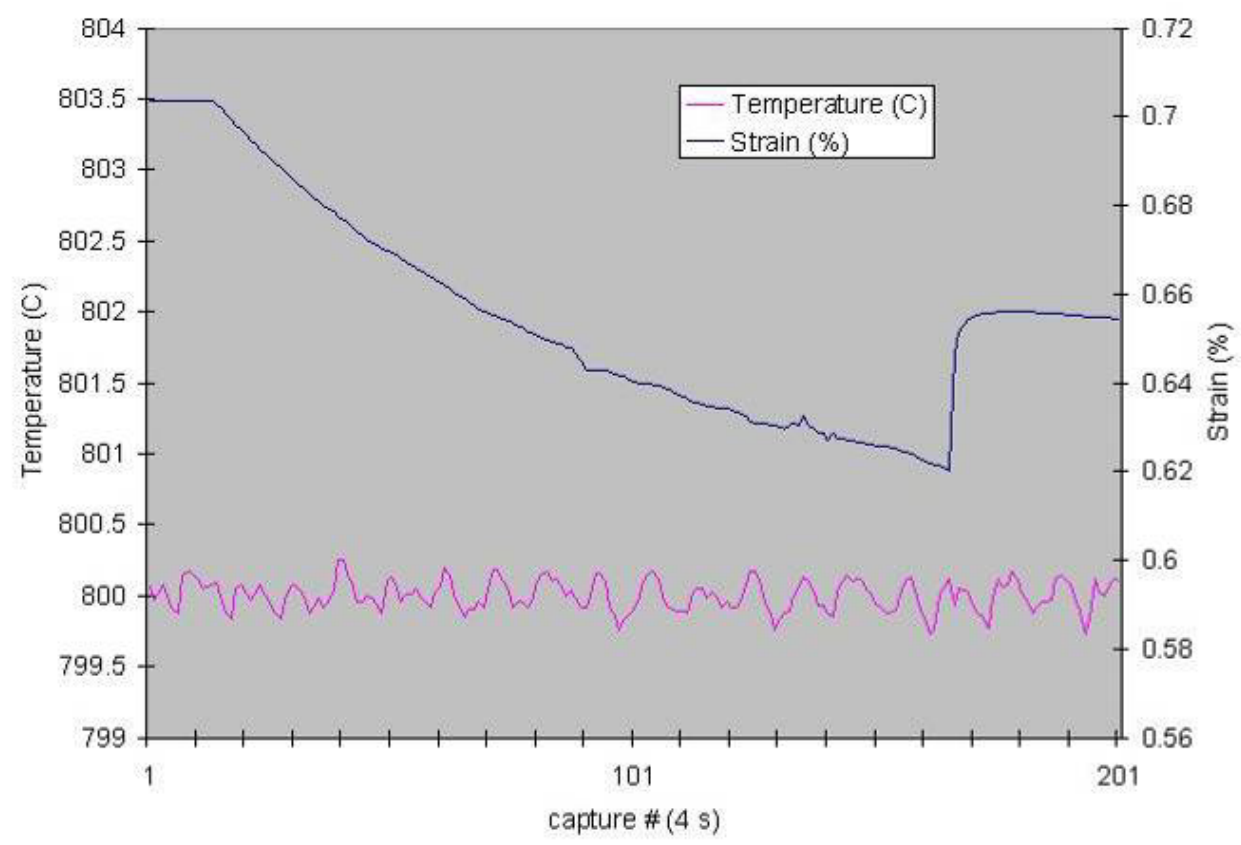

Figure 5-22. An enlarged view of the constant strain rate test shown in Figure 5-20. The entire plot covers a time of approximately 13 minutes. The pushrod was displaced at a rate of $165 \mu \mathrm{m} / \mathrm{min}$ and the Hionic substrate failed at a displacement of $1650 \mu \mathrm{m}$. 


\subsection{Distributed Sensors for SOFC Instrumentation}

In addition to the strain gage work intended for SOFC button cell applications, Prime Research researched the use of distributed sensors for instrumentation of full-size fuel cells. Current fuel cell technology utilizes stacks of ceramic plates that must withstand both the rigors of heating and the pressure of gaseous reactants, in addition to the forces used to compressively seal the fuel cell stack. Heating ceramics from room temperature to fuel cell working temperature causes thermally induced expansion that can easily crack the ceramic plates that must be also be clamped together to keep reactant gasses from escaping. When one plate in a stack fails, the entire stack becomes non-functional. Therefore, researchers have a strong need to understand both the mechanism of failure and the underlying reasons for it, with the ultimate goal of making more robust designs.

Once a fuel cell is at working temperature, fuel gas and compressed air are introduced to begin the generation of electricity. As these gasses flow, they are generally at a lower temperature than the fuel cell and cause a shift in the temperature profile across the cell, further complicating the issues of thermally induced stack failure. This change in temperature profile also affects the rate and efficiency of the cell reactions, which results in the generation of more or less additional reaction heat across the cell profile. The combination of these factors makes theoretical modeling a complex task. Without a way to measure the actual fuel cell cross-sectional strain and temperature profile, no real world validation of theoretical results is possible.

Much of the research and development in fuel cells up to now has been with stacks of modest dimensions, for example, a square approximately $17 \mathrm{~cm}$ on each side. However, as energy conversion plants scale up for bulk production, fuel cells with a larger cross sectional area are clearly needed. Knowledge about how temperature and strain profiles affect the failure rate of ceramic materials will become increasingly important in the future.

What is required is a large number of sensors in a tight distribution across the crosssectional area of a fuel cell, because it is the minute variations in cell temperature and strain that is important, not just a single bulk measurement. Any form of electrical type sensor technology would require a large number of wires to carry the profile information from the hot zone out to where the data can be used. With high temperatures, ceramic plates, and glass seals involved, this wiring represents a significant logistical challenge. For example, if the fuel cell stack working area is $17 \mathrm{~cm}$ by $17 \mathrm{~cm}$, and a spatial resolution of $1 \mathrm{~cm}$ is needed, then this represents a need for 289 sensors, which for electrical sensors would likely call for 578 wires.

In the case of fiber optic sensors, a large number of sensors can be built into a single fiber and read out using an instrument located far away from the hot zone. A sensor plate can be prepared in advance, and then installed in the stack during assembly, with only one or two optical fibers going to the outside. The result would be an easy-to-implement multisensor solution that will enable researchers to understand stack parameters better, even as stack cross sectional area increases. 
In principle, distributed strain and temperature measurements in SOFC fuel cells could be achieved by multiplexing a large number of ultrahigh temperature sensors located at discrete points along a single fiber. The fiber could then be attached to a fuel cell in a matter that profiles or images temperature in a cross section of the device under test, as illustrated in Figure 5-23. Multiplexing of the sensors and signal processing of the data will be performed in the interrogator and the resulting temperature profile will be displayed on a computer monitor, or the interrogator itself.

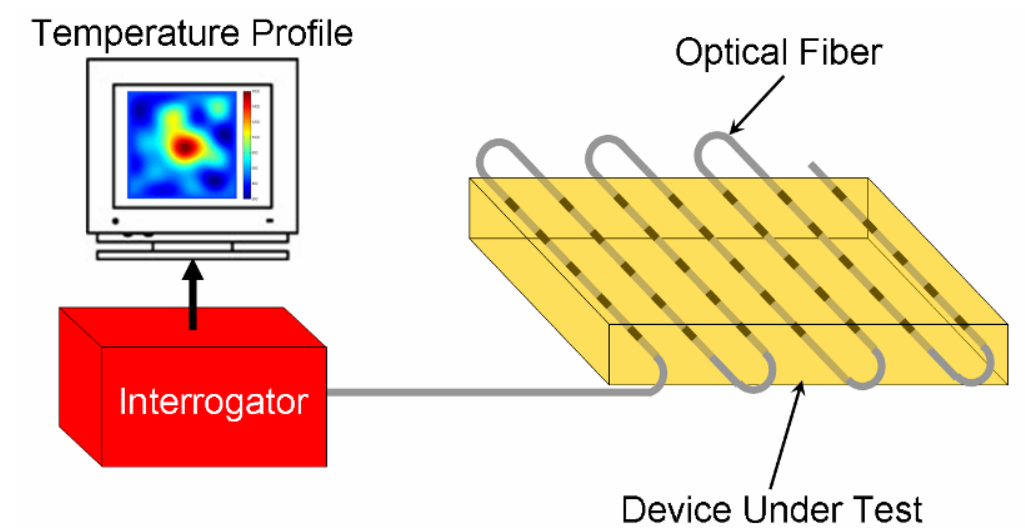

Figure 5-23. System concept for fiber optic ultrahigh temperature profiler

\subsubsection{Distributed sensor technology}

With the clear need for many sensors on a single fiber, Prime Research has examined how this has been done in the past and how a fresh approach might advance the state of the art in this area. First, a survey of existing technologies was undertaken, including both single sensor approaches and multiple sensor approaches. Table 5-1 below indicates the results of that survey so far.

When multiple sensors are placed on one fiber, the mechanism for determining one sensor response from another is called the discrimination method.

Fiber Bragg Gratings, as shown in the table, are easy to manufacture, widely available and well understood, but they suffer from several limitations. When wavelength division multiplexing is used as the multi-sensor discrimination method, the conveniently available spectral width (provided by telecommunication components) limits the number of sensors that can be put in one fiber to the order of tens of sensors. In addition, most gratings are fabricated in photosensitive germanosilicate fiber. Such Type-I gratings are manufactured using either continuous wave or pulsed UV laser irradiation, resulting in color centers that change the index of refraction of the core. Because of this method, Type-I gratings suffer from signal fading effects and are essentially erased at approximately $200^{\circ} \mathrm{C}$. This is a moderately high temperature for a telecommunications environment, but a relatively low temperature compared to the solid oxide fuel cell environment. 
Optical Time Domain Reflectometry (OTDR) methods work well when the structures are large because the discrimination method is the time of flight for an individual pulse of light. Any change of index of refraction encountered along the fiber causes a light reflection that returns to the instrument. The exact time of the return of the reflection depends upon how far down the fiber the index of refraction change is from the instrument. Light travels very fast, so even fast electrical timing methods translate into long physical distances, much larger than the dimensional scale of solid oxide fuel cells.

Table 5-1. Survey of Sensor Applicability

\begin{tabular}{|c|c|c|c|c|c|c|c|c|}
\hline & $\begin{array}{l}\text { Ease of } \\
\text { Manufacturing }\end{array}$ & $\begin{array}{l}\text { Applicability } \\
\text { for Pressure } \\
\text { Measurement }\end{array}$ & $\begin{array}{l}\text { Applicability for } \\
\text { Temperature } \\
\text { Measurement }\end{array}$ & $\begin{array}{l}\text { Applicability for } \\
\text { Strain } \\
\text { Measurement }\end{array}$ & $\begin{array}{l}\text { Cost of } \\
\text { Development }\end{array}$ & Multiplexibility & Resolution & Limitations \\
\hline FBG & high & poor & good & very good & moderate & very good & very good & $\begin{array}{l}\text { Requires IP } \\
\text { licenses }\end{array}$ \\
\hline LPG & high & moderate & good & moderate & moderate & moderate & moderate & $\begin{array}{l}\text { Requires IP } \\
\text { licenses }\end{array}$ \\
\hline $\begin{array}{l}\text { IFPI + } \\
\text { OTDR }\end{array}$ & high & poor & good & good & moderate & good & poor & $\begin{array}{l}\text { Slow } \\
\text { response, } \\
\text { poor } \\
\text { resolution, } \\
\text { poor stability, } \\
\text { only tested to } \\
300 \text { C, }\end{array}$ \\
\hline $\begin{array}{l}\text { IFPI + } \\
\text { SLI }\end{array}$ & high & poor & very good & very good & moderate & moderate & very good & $\begin{array}{l}\text { Maximum of } \\
10 \text { sensors, } \\
\text { only tested to } \\
300 \text { C }\end{array}$ \\
\hline EFPI & moderate & good & good & very good & low & moderate & very good & $\begin{array}{l}\text { Maximum of } \\
10 \text { sensors }\end{array}$ \\
\hline Microbend & high & good & moderate & moderate & low & poor & poor & \\
\hline OFDR & low & poor & good & good & high & very good & good & $\begin{array}{l}\text { limited to } \\
\text { short } \\
\text { distances }\end{array}$ \\
\hline DTS & moderate & $\begin{array}{l}\text { not } \\
\text { applicable }\end{array}$ & very good & not applicable & high & very good & very good & $\begin{array}{l}\text { limited to } \\
<250^{\circ} \mathrm{C}\end{array}$ \\
\hline Polarimetric & low & moderate & good & good & low & poor & moderate & $\begin{array}{l}\text { Requires } \\
\text { special fibers }\end{array}$ \\
\hline $\begin{array}{l}\text { Black Body } \\
\text { Radiation }\end{array}$ & moderate & $\begin{array}{l}\text { not } \\
\text { applicable }\end{array}$ & very good & not applicable & moderate & poor & moderate & \\
\hline
\end{tabular}

IFPI = intrinsic Fabry-Perot interferometer

FBG $=$ fiber Bragg grating

OFDR = optical frequency domain reflectometer

DTS $=$ distributed temperature sensor

EFPI = extrinsic Fabry-Perot interferometer

$\mathrm{SLI}=$ swept laser interrogation

Extrinsic Fabry-Perot Interferometric have been the subject of extensive development, but the extrinsic nature of their construction makes multiplexing difficult due to the high optical losses of each sensor element.

Intrinsic Fabry-Perot interferometry does not suffer so much from the light loss issue, because the light is confined to the optical fiber. However, IFPI cavities written using 
methods similar to Type-I Fiber Bragg Gratings also suffer from signal fading above $200{ }^{\circ} \mathrm{C}\left(392^{\circ} \mathrm{F}\right)$. Due to the similarity in fabrication methods for FBG sensors and IFPI sensors, it is likely that persistent IFPI sensors suitable for use at the higher temperatures can be fabricated through the use of a high-power excimer laser.

\subsubsection{Extending the Temperature Range of IFPIs}

When Fiber Bragg gratings are written into photosensitive fibers using a UV laser, minute structural changes in the fiber are created. These structural changes cause a strong reflection when made in the form of a Bragg grating. However, these effects are significantly reduced if the fiber is exposed to temperatures above $200{ }^{\circ} \mathrm{C}\left(392{ }^{\circ} \mathrm{F}\right)$.

Instead, when gratings are formed using a very intense, single pulse of light $\left(>500 \mathrm{~mJ} / \mathrm{cm}^{2}\right)$, such as with a high-power excimer laser, Type-II gratings are obtained. Type-II photosensitivity is caused by the resulting damage at the core-cladding interface. This fusion of the glass matrix at the writing point is a highly nonlinear dynamic process. Type-II photosensitivity can lead to a refractive change as high as $1 \%$. Gratings made a using a Type-II process show great stability at high temperature, withstanding temperatures as high as $800^{\circ} \mathrm{C}\left(1,472{ }^{\circ} \mathrm{F}\right)$ without erasure.

There is a threshold of pulse energy density of about $650 \mathrm{~mJ} / \mathrm{cm}^{2}$ where the Type-II effect begins. When the pulse energy is below this threshold, refractive index changes linearly with energy density. Above this threshold, the refractive index change increases dramatically with increasing energy density. When the pulse energy is higher than $1000 \mathrm{~mJ} / \mathrm{cm}^{2}$, the refractive index change effect begins to saturate.

Because of this damage at the interface of fiber core and cladding, the fiber becomes somewhat fragile at the writing point. This fragility may limit the applicability of a TypeII sensor as a tensile strain gage.

By changing the mechanism of the reflection from color centers as in Type-I gratings to structural decomposition as in Type-II gratings, we expect to be able to extend the working reflection temperature to $800^{\circ} \mathrm{C}\left(1,472{ }^{\circ} \mathrm{F}\right)$, where solid oxide fuel cells operate.

\subsubsection{Fabrication of High-Temperature IFPI Sensors}

The sensor design best suited for implementation of a distributed sensor system based on multiplexed interferometry is the intrinsic Fabry-Perot interferometer, or IFPI. In this approach, a high-power ultraviolet laser is used to increase the refractive index of the germania-doped silica core of an optical fiber. The modified section of the fiber core acts as a Fabry-Perot cavity with low finesse, as shown in Figure 5-24. . Such intrinsic Fabry-Perot sensors have two attributes that make them well suited for the multiplexed sensor system. First, IFPI sensors are fabricated as an integral part of the interrogation fiber. This greatly simplifies the sensor system and allows for a minimally intrusive measurement. Second, IFPIs typically have a low reflectance, which means more sensors 
can be interrogated for a given optical source power. The technique for fabrication of IFPI sensors though the use of an excimer laser was invented at Virginia Tech; Prime Research has licensed the intellectual property and further developed the technology as explained below.

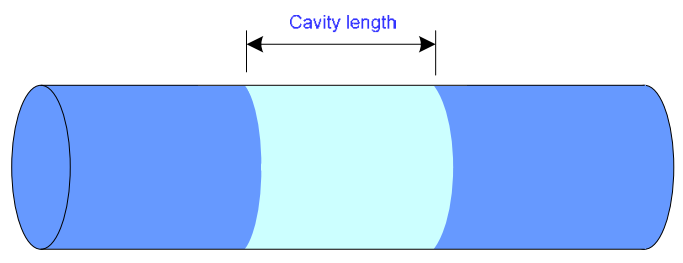

Figure 5-24. IFPI cavity written by a single UV exposure (light blue represents exposed glass).

Fabrication of IFPI sensors requires intense ultraviolet light from a krypton fluoride excimer laser, which irradiates a short length (a few hundred microns) of a photosensitive optical fiber. The irradiation causes a small increase in the refractive index in the exposed section of the fiber. The discontinuities in refractive index between the exposed glass and the unexposed glass at the two ends of the exposed section form two weak reflectors, which define an optical cavity. Changes in length of this optical cavity can be detected and used to transduce temperature or strain.

Previous research of IFPI sensors at Prime Research show that limitations of the sensor fabrication are (1) fabrication of a given IFPI cavity length was difficult to reproduce, (2) damage to the fiber due to UV exposure resulted in a very low yield rate of usable sensors, due to the resulting fragility of the exposed glass, and (3) the visibility (a measure of the interferometric fringe contrast which is related to the sensor signal-tonoise ratio) of the cavities was small (2-3 dB). All of these limitations have been eliminated or minimized with the current procedure for fabrication.

Microscopic examination of exposed fiber sections revealed that the high laser power was causing ablation of some of the glass around the circumference of the fiber, leading to stress concentration sites in the fiber. Physical damage to the fiber has been greatly reduced by careful manipulation of laser energy, beam focusing optics, and fixturing of the fiber. The recent cavities have shown very little damage and can be easily handled for further processing/packaging without fear of breaking the fiber.

Previous IFPI fringe visibility of 2-3 dB has been increased to $10-15 \mathrm{~dB}$. It is believed that this improvement was achieved by changing the material used to construct the shutter blades which define the exposure aperture (Figure 5-25 and Figure 5-26). The material was changed from stainless steel to silicon. This change resulted in sharper cavity definition and eliminated the oxidation and ablation problems associated with the steel blades. 


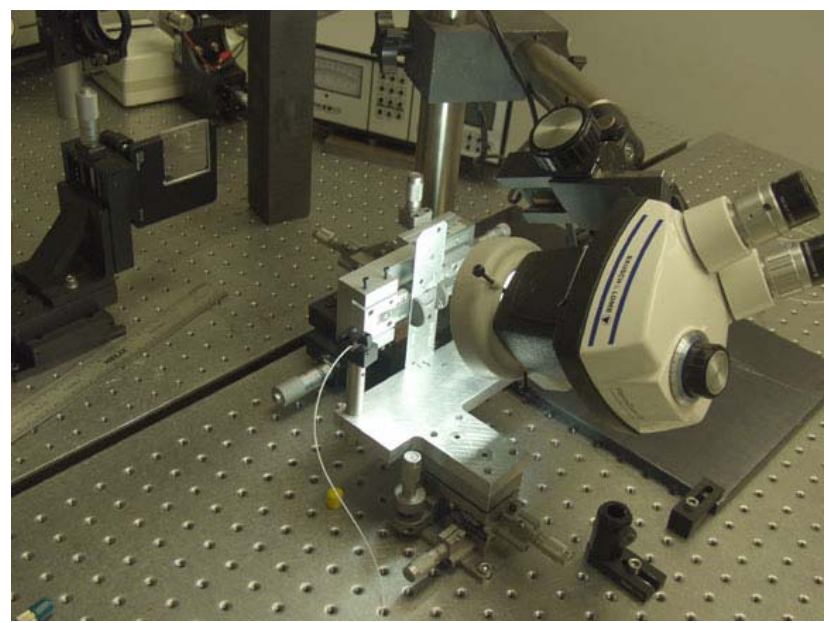

Figure 5-25. Set-up for exposing fiber to excimer laser output.

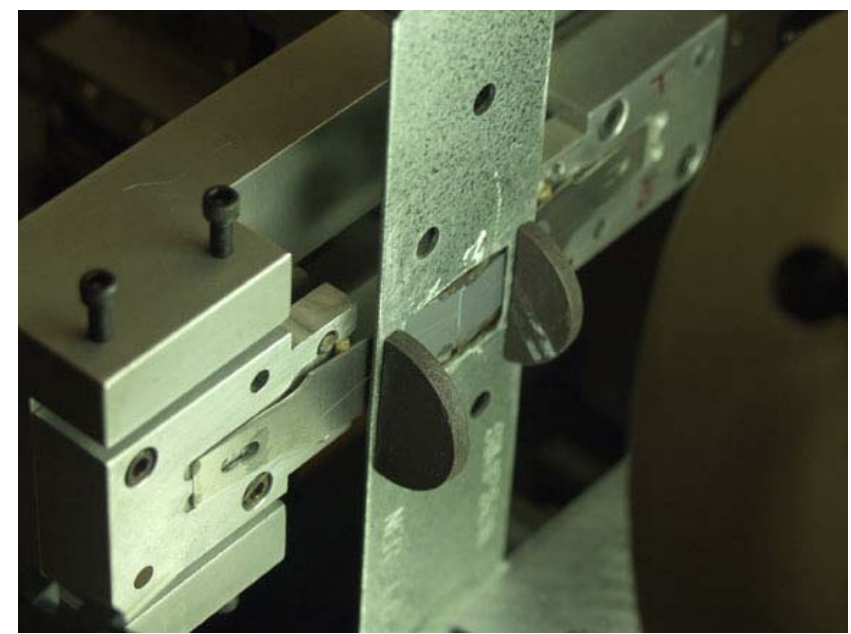

Figure 5-26. Close-up of set-up, showing silicon shutters used to define exposure aperture.

Additional improvements to the fabrication process were obtained by changing the lens used to focus the UV energy onto the aperture. Previous efforts utilized a cylindrical lens which is only capable of focusing the laser energy on one axis. When viewed at the focal plane, the cylindrical lens produces a beam profile resembling a narrow line. Current efforts utilize a spherical lens; which focuses the laser on both axis and results in a small spot at the focal plane. Reduction in the spot size allows the laser to be run at lower power; and condenses any irregularities evident in the un-collapsed beam profile. To date, many IFPI sensors have been fabricated for use in demonstration of the multiplexed sensor system. These sensors have been fabricated with various cavity lengths and sensor spacing along the fiber. One such sensor string consists of four IFPI sensors with cavity lengths of approximately $100 \mu \mathrm{m}$, located at $1 \mathrm{~cm}$ intervals. 


\subsubsection{Improvements in IFPI Fabrication Methods}

The principal mechanism for fabrication of an IFPI is through the creation of two photoinduced features within the core of the optical fiber. A schematic of the system used to create these features is shown in Figure 5-27. Ultraviolet light is generated by an excimer laser (GSI Lumonics Pulsemaster ${ }^{\mathrm{TM}}$ ), which is shown in the back of Figure 5-28. The pulse then passes through an attenuator to achieve the desired power throughput. After attenuation, the pulse passes through a pair of spatial filters, the first of which is cylindrical. In addition to providing spatial filtering, these systems also act as demagnifiers, condensing the optical power into a smaller beam. Finally the beam passes through the focusing optics, which results in an extremely small, nearly diffraction limited focal point within the target optical fiber.

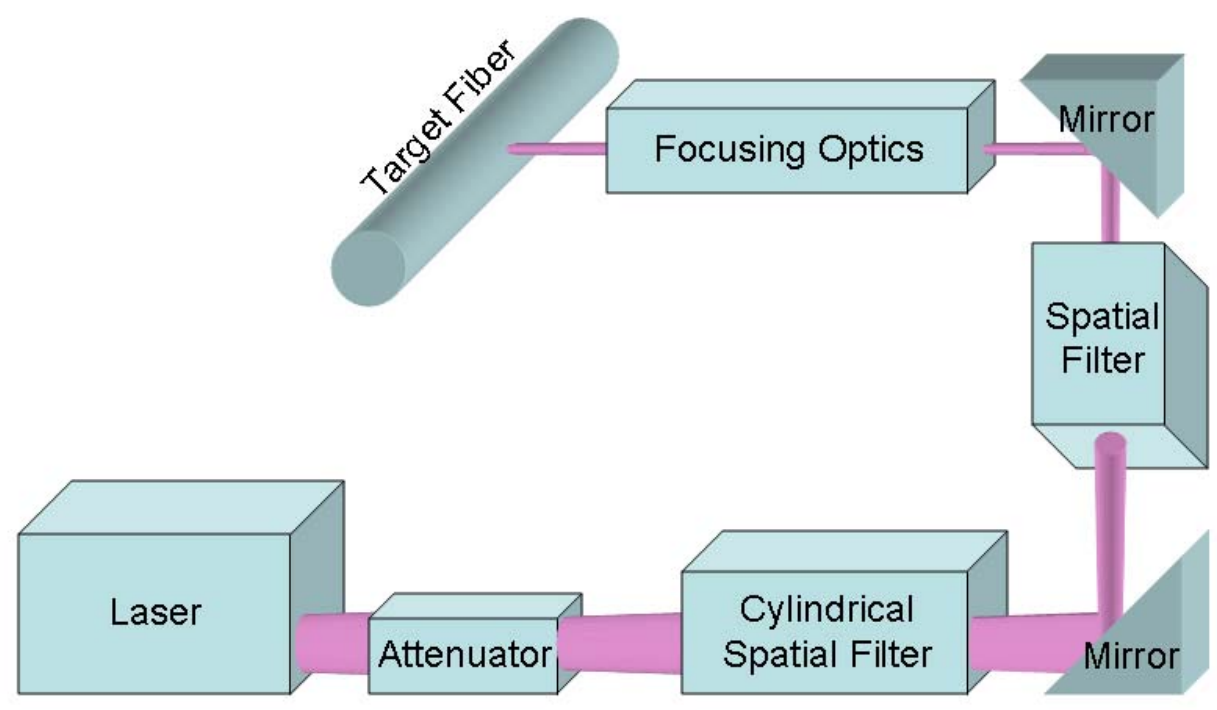

Figure 5-27: Schematic of the IFPI writing system

Several different fibers were tested for use as the target of the IFPI fabrication process. A listing of those fibers is given in Table 5-2. All of these fibers were commercially available singlemode fibers. One of them (GF1AA) is a specially designed fiber which is engineered to have an uncommonly high photosensitivity (a strong response to irradiation with ultraviolet light.) Although the details of the fiber composition are not available, it is likely that both the Nufern and the OFS fiber contain significantly higher concentrations of germanium oxide in the core, which is the primary required precursor for the dominant photosensitive process. 


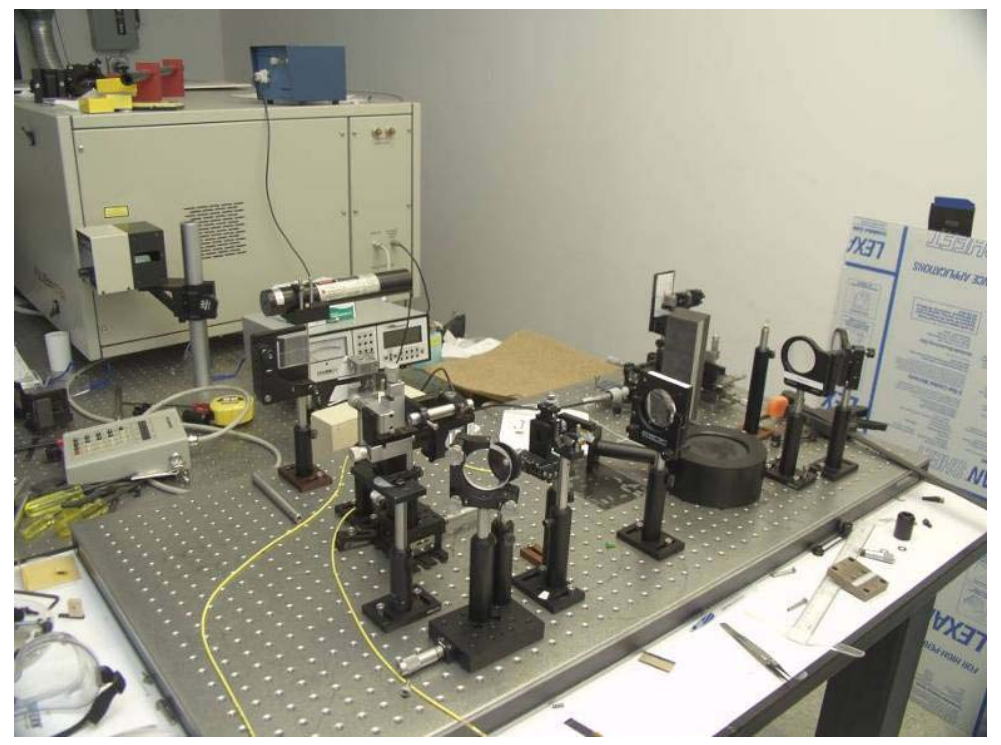

Figure 5-28. Optical set-up for writing IFPI sensors with excimer laser.

A number of IFPI sensors were fabricated during this study. Efforts were made to make the fabrication process as uniform as possible, but there were significant differences between the particular sensors. The likely cause of this difference is the well known inconsistency of power and mode pattern associated with excimer lasers. This inconsistency is especially relevant in the case of the IFPI fabrication, because each reflector was written with a single pulse from the laser, rather than multiple pulses which would have the effect of averaging out any variations in the pulse profile. The pulses which were incident on the fiber had a total power of approximately $2.5 \mathrm{~mJ}$. A key requirement in the fabrication of a successful IFPI is that the longitudinal spatial extent of the feature written into the fiber must be as narrow as possible. This is to enhance the strength of the reflection from each interface.

Table 5-2: IFPI Target Fibers

\begin{tabular}{|l|l|l|l|}
\hline Fiber Name & Manufacturer & IFPI capable & Number of Sensors Produced \\
\hline GF1AA & Nufern & Yes & 6 \\
\hline SMB-E1550J & OFS & Yes & 1 \\
\hline SMF-28 & Corning & No & 0 \\
\hline
\end{tabular}

Figure 5-29 shows a micrograph of one of the IFPIs (serial number 071001-1). This image was taken by focusing a microscope on the midplane of the optical fiber while illuminating from both above and below. One immediately apparent feature is the dissimilarity between the two reflectors shown in the fiber. The one on the left is significantly clearer, with highly parallel interfaces. The one on the right, however, shows a more inconsistent pattern of damage, possibly indicating an excessive pulse power delivered to the target fiber site. Other micrographs are shown below. 


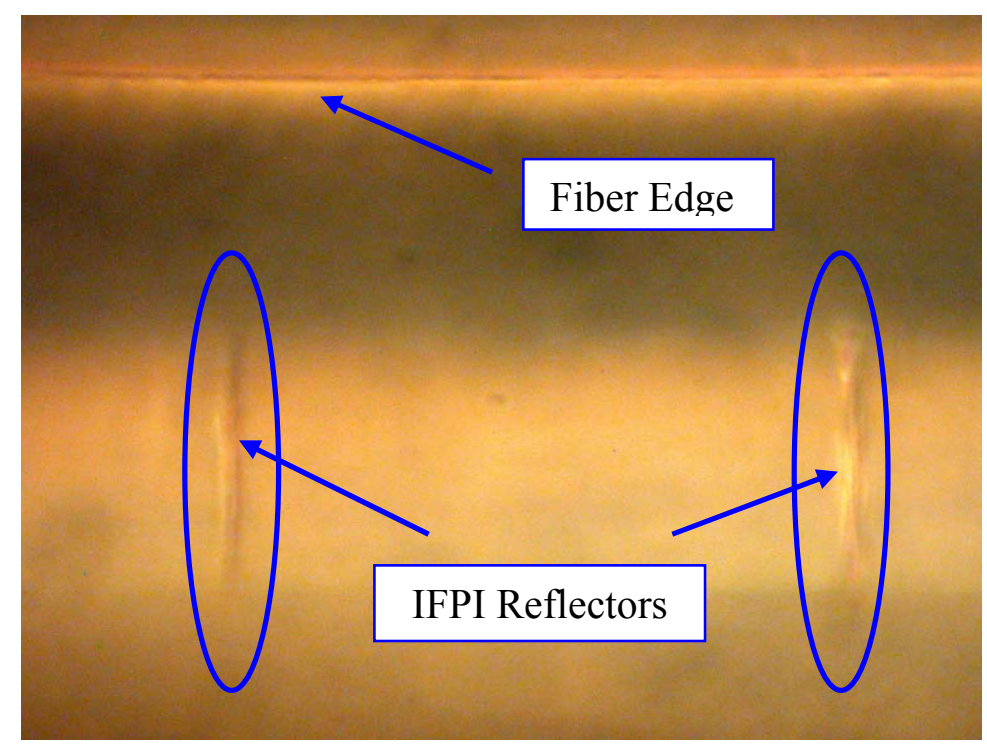

Figure 5-29: Microphotograph of an IFPI

One fiber had a feature which became evident under microscopy, but which cannot be represented in a single image. In the case of this IFPI, one of the reflection features possessed a corkscrew pattern in which the plane of the reflector rotated as it passes through the fiber. This is an indication of a highly asymmetric beam profile which would result from astigmatism in the focusing optics, in which case the different portions of the beam focus at different depths within the fiber. An exaggerated schematic of the approximate behavior is shown in Figure 5-30.

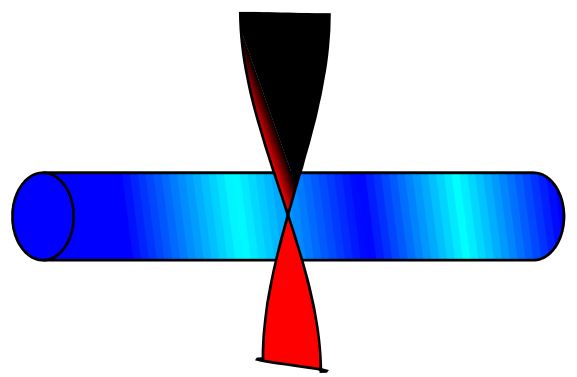

Figure 5-30: Schematic of fiber showing the corkscrew IFPI feature

A summary of the key characteristics of several IFPIs is provided in Table 5-3. Listed are the optical gap length, the peak power, the fringe visibility, and the power throughput. Optical gap length is defined as the equivalent distance which light would have to travel in vacuum to accumulate the measured amount of phase change. Peak power, reported in $\mathrm{dB}$, is a measure of the strength of a single reflector. A peak power of $0 \mathrm{~dB}$ would indicate $100 \%$ of the power was returned at that wavelength. The fringe visibility is the difference between the maximum and minimum power returned as the wavelength illuminating the IFPI is scanned. The power throughput, expressed as a percentage of the power injected, is the amount of power which successfully travels through the sensor in one direction. 
Table 5-3: Summary of IFPI properties. Entries in bold are shown below.

\begin{tabular}{|c|c|c|r|l|}
\hline Serial \# & $\begin{array}{c}\text { Optical Gap } \\
\text { Length }(\mu \mathrm{m})\end{array}$ & $\begin{array}{c}\text { Peak Power } \\
(\mathrm{dB})\end{array}$ & $\begin{array}{c}\text { Fringe } \\
\text { Visibility } \\
(\mathrm{dB})\end{array}$ & Throughput (\%) \\
\hline $070921-1$ & 221.5 & -24.0 & 1.5 & not measured \\
\hline $070921-2$ & 144.7 & -35.0 & 5.0 & not measured \\
\hline $071001-1$ & 142.2 & -37.0 & 11.0 & not measured \\
\hline $\mathbf{0 7 1 0 0 1 - 2}$ & 189.9 & -35.8 & 10.2 & 37 \\
\hline $071001-3$ & 251.0 & -43.0 & 8.0 & not measured \\
\hline $\mathbf{0 7 1 0 0 1 - 4}$ & 299.7 & -25.6 & 22.4 & 14 \\
\hline
\end{tabular}

There is a significant amount of variation in the peak power returned fringe visibility of the various sensors. This is an indication that the repeatability of the laser exposure used to generate the IFPIs is inconsistent. Throughput percentage is a key characteristic in determining how many sensors can be assembled into a single chain. As the throughput drops more light is being lost from the fiber, preventing detection of subsequent sensors. In the case of a perfectly formed sensor, the peak power combined with the throughput should represent all of the light launched into the sensor. In the case of sensor 071001-4, this sum is approximately $14.5 \%$, indicating that the vast majority of the light is being lost out the sides of the fiber. A probable reason for this can be seen by examining the micrographs shown in Figure 5-31.

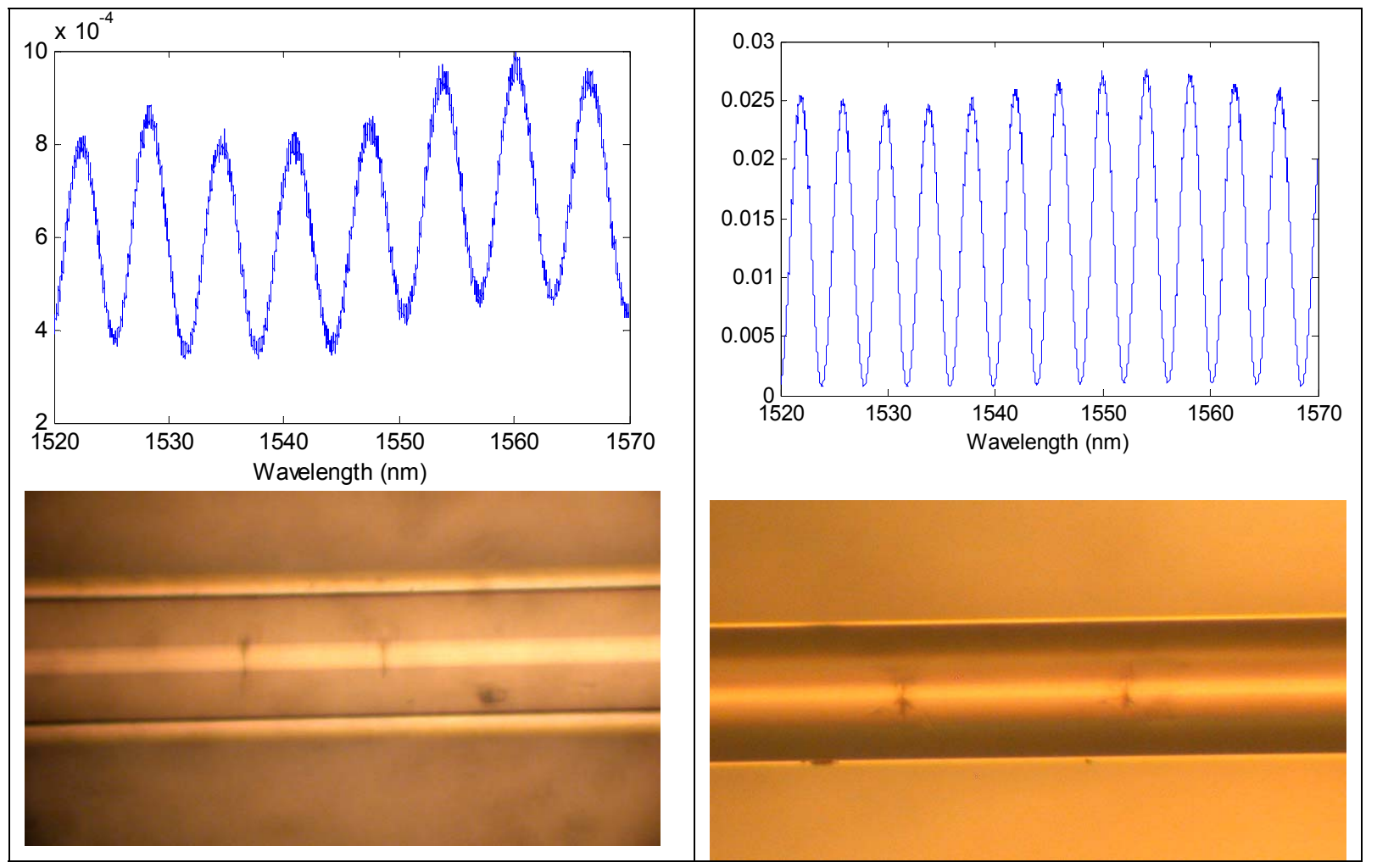

Figure 5-31: Interference spectra and microphotographs of IFPIs 071001-2 (left) and 071001-4 (right) 
A closer view of the left hand reflector from 071001-4 is shown in Figure 5-32. The fiber structure has suffered significant damage as a result of the laser pulse. It is not clear exactly what the visible features are, although they may be small voids or stress fractures embedded within the glass. Ideal fabrication will require fine tuning the level of irradiation to reduce the amount of excess damage caused to the fiber in feature formation.

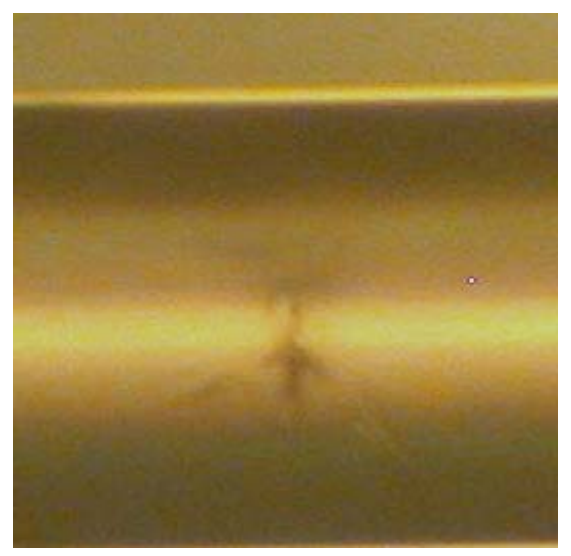

Figure 5-32: Close image of the left reflector from IFPI 071001-4

\subsubsection{Alternative methods for fabricating IFPI sensors}

In order to expedite development of the signal processing for concatenated IFPI sensors, it was necessary to use other Fabry-Perot sensors to substitute for IFPI sensors while the techniques for writing IFPI sensors with an excimer laser were being developed. Another approach to fabricating IFPI sensors that was attempted involved splicing a section of microstructured holey fiber between two standard singlemode fibers. For this experiment, LMA-10 Large Mode Area Fiber with a $10 \mu \mathrm{m}$ core from Crystal Fibre was used. This fiber has a quartz core and a honey comb of glass tubes with holes for cladding. The LMA-10 fiber can be spliced to standard silica optical fiber using an arc fusion splicer if reduced power is used and the arc moved off center away from the holey fiber. If too much heat is used for the splice, the holes in the holey fiber can melt together.

Figure 5-34 below shows the typical results that were obtained for IFPI cavities fabricated using the LMA-10 fiber as the cavity. The two traces correspond to the interference signal that resulted from interrogating the IFPI from each of the two ends of the IFPI cavity. The numbers on the y-axis represent reflectance, where the maximum is $100 \%$. As can be seen, the best reflection achieved was about $0.002 \%(-47 \mathrm{~dB})$ which is far lower than a standard 3.5\% reflection for one air/glass interface. This low reflectance is due to the very small difference in refractive indices of the cores of the singlemode fiber and the LMA-10 fiber. Generally, for the interrogation electronics to achieve a usable signal-to-noise ration, the reflectance should be on the order of $0.05 \%$ or more. 


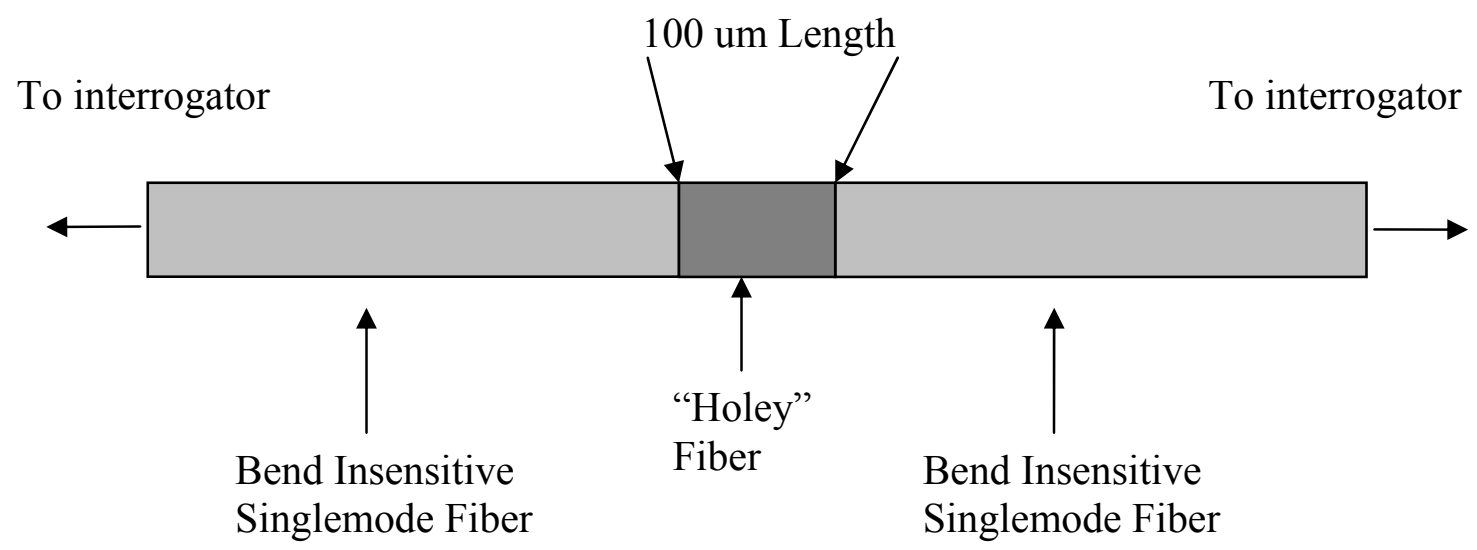

Figure 5-33. Fabrication of IFPI sensor using microstructured "holey" fiber

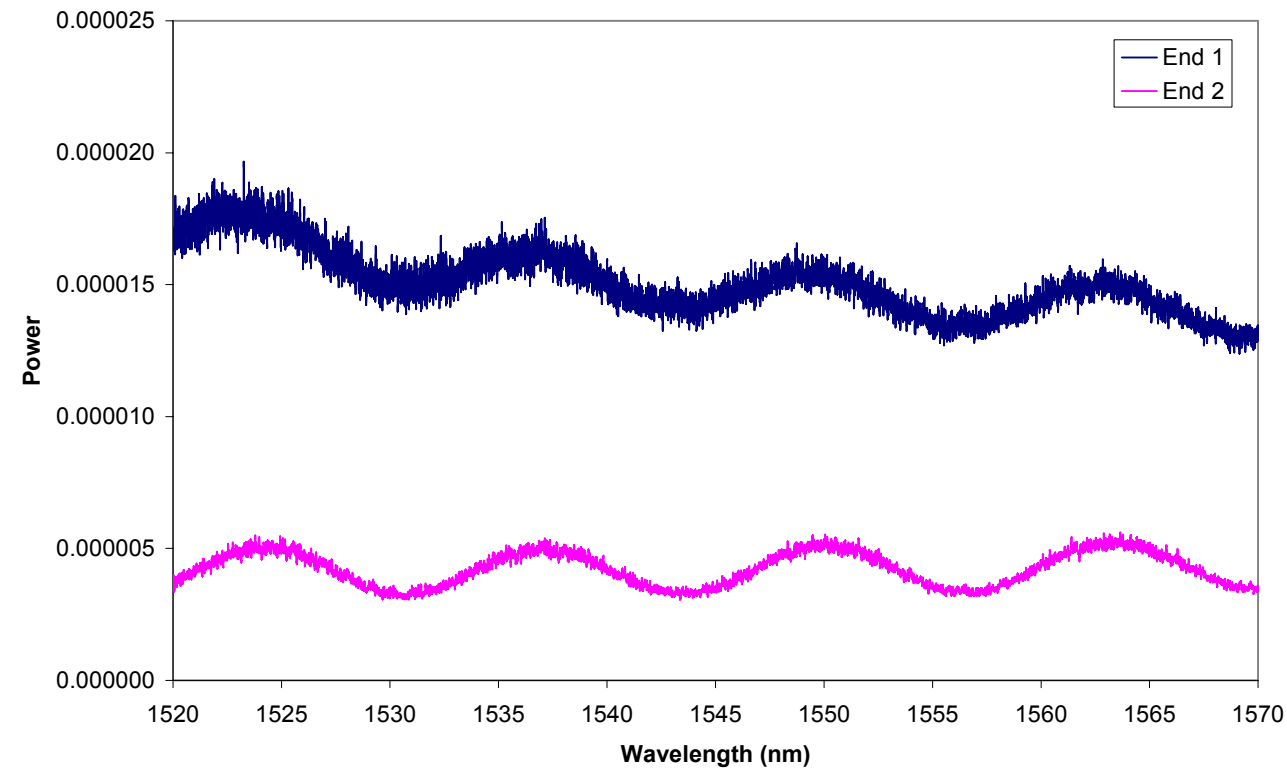

Figure 5-34. Interference fringes obtained from IFPI fabricated with holey fiber. The two traces correspond to signals obtained by interrogating the cavity from each end of the IFPI.

\subsubsection{Methods for Interrogation of Concatenated IFPI Sensors}

Many methods exist to multiplex a number of sensors along a single fiber including optical frequency-domain reflectometry (OFDR), multiplexed Michelson interferometry (MMI), and fiber Bragg grating sensors. 


\subsection{Optical Frequency Domain Reflectometry}

The principle employed in Optical Frequency Domain Reflectometry (OFDR) is to decode the signals returned from multiple sensors based on the varying response of the system to different optical frequencies, or wavelengths. There are many varieties of OFDR sensors and methods of decoding the signal. For this application, we adopted a goal of a distributed sensing system containing 300 temperature or strain sensors spaced at $1 \mathrm{~cm}$ physical separation, with a target temperature resolution of 1 degree centigrade. Therefore, the overall length of the sensing string is 3 meters. Furthermore, the analysis assumed the use of standard telecommunications fiber.

The most common class of OFDR measurement is based on the use of fiber Bragg gratings (FBGs). In this instance, the measurand is encoded directly in the wavelength of the FBGs reflection peak. This mechanism is effective as long as the system can satisfy the conditions of mutually non-interfering sensors. That is, the spectral peak of any given Bragg grating must be uniquely identifiable as having come from that particular sensor. Another method of decoding an OFDR system is to Fourier transform the frequency response of a system into the spatial domain. If one considers the hypothetical situation of a string of broadband partial reflectors in a fiber, each contributes a back reflection to an overall signal. For simplicity, we will consider the case with no occurrence of multiple reflections. Each of these reflections will interfere with all of the others. In order to discriminate between the various reflections, one needs to add an additional reference reflection which will interfere with each sensor reflection to produce a unique spectral response. One method for achieving this is to have a reference reflector which is further from one end of the sensor string than the total length of the string of concatenated sensors. Note that the reference reflector does not have to be located in the same physical fiber, as long as the distance from the separation point satisfies the condition described above. Based on the design assumptions listed above, we can then ascertain that the reference reflector can be no less than 3 meters in physical length (or 4.5 meters of optical path length) from one end of the sensor string. Therefore, the longest cavity required for a three meter sensor string must be no less than six meters of physical length, or nine meters of optical length.

For any low finesse interferometer, the fringe spacing is described by

$$
I \propto \cos \left(\frac{4 \pi O P L v}{c}\right),
$$

where $I$ is the intensity profile, $O P L$ is the optical path length (index of refraction $n$ multiplied by length $L$ ) of the cavity in one direction, $v$ is the optical frequency, and $c$ is the speed of light. Because this has a maximum whenever the argument is equal to $m 2 \pi$ where $m$ is any integer, the spacing of any two adjacent peaks in frequency domain is given by 


$$
\Delta v_{p k-p k}=\frac{c}{2 O P L} H z
$$

Equation 18

or conversely, the frequency of fringes is the reciprocal of this relation. In the case of a cavity with a nine meter optical path length, this yields a fringe frequency of

$$
f_{\max }=\frac{1}{\Delta v_{p k=p k}}=\frac{2 O P L}{c}=\frac{2 * 9 m}{3 * 10^{8} \mathrm{~m} / \mathrm{s}}=6 * 10^{-8} \text { fringes } / \mathrm{Hz}=60 \text { fringes } / \mathrm{GHz} \text {. }
$$

Equation 19

Expressed another way, there will be a peak in the interference pattern every $16.67 \mathrm{MHz}$. There are two characteristics which are derived based on the maximum anticipated frequency. The first is the sampling frequency. The Nyquist criterion indicates that in order to detect the presence of a signal at frequency $f$, the data must be sampled at no less than twice that frequency: $f_{S} \geq 2 f_{\max }$. The other related characteristic is that the resolution of a Fourier transform is determined by the number of data points $N_{\text {samples }}$ relative to the sampling frequency $f_{s}$ :

$$
\Delta f_{\text {res }}=\frac{f_{S}}{N_{\text {samples }}}
$$

In this application, the next question becomes what resolution of the measurement in frequency space is required. Considering the case of a temperature sensor fabricated from silica fiber, we will assume the best case scenario: that each sensing region occupies the largest possible space (that is, we are measuring the change in optical path length for the entire centimeter spacing between sensors). The change in fringe frequency as a function of temperature is given by:

$$
\frac{d f}{d T}=\frac{d}{d T}\left(\frac{2 n L}{c}\right)=\frac{2}{c}\left[n \frac{d L}{d T}+L \frac{d n}{d T}\right]
$$

Equation 21

The first derivative $d L / d T$ is the coefficient of thermal expansion, which has a value of $5.5^{*} 10^{-7}\left({ }^{\circ} \mathrm{C}\right)^{-1}$. The second derivative measures the change in refractive index as a function of temperature, with a value of approximately $10^{-5}\left({ }^{\circ} \mathrm{C}\right)^{-1}$. Thus, if we consider the case of a one centimeter sensor made of silica, we find that the change in fringe frequency per degree Celsius is:

$$
\Delta f=\frac{2}{3 * 10^{8} \mathrm{~m} / \mathrm{s}}\left[1.45\left(5.5 * 10^{-7}\right) 1 \mathrm{~cm}+1 \mathrm{~cm}\left(10^{-5}\right)\right] \Delta T \approx 7.2 * 10^{-7} \frac{\text { fringes }}{\mathrm{GHz}} /{ }^{\circ} \mathrm{C} \quad \text { Equation } 22
$$


Therefore, in order to detect a change in temperature of 1 degree Celsius, the number of samples required would be:

$$
N_{\text {Samples }} \geq \frac{f_{S}}{\Delta f}=\frac{2 * 60 \text { fringes } / \mathrm{GHz}}{7.2 * 10^{-7} \text { fringes } / \mathrm{GHz}}=166,666,667 \text { Samples! } \quad \text { Equation } 23
$$

Even were it reasonable to collect over 166 million data points, the computational time required to perform the FFT on that amount of data would be prohibitive. As a result, direct measurement of the distributed sensor string, via Fourier Transform OFDR was deemed impracticable for instrumentation of the SOFC fuel cell, and was not further considered.

\subsection{Multiplexed Michelson Interferometry}

Another concept evaluated for application to distributed sensing of strain and temperature in SOFC fuel cells was multiplexed Michelson interferometry. The method chosen here may be best described as simply multiplexed interferometry (MI) and a system schematic is illustrated in Figure 5-35.

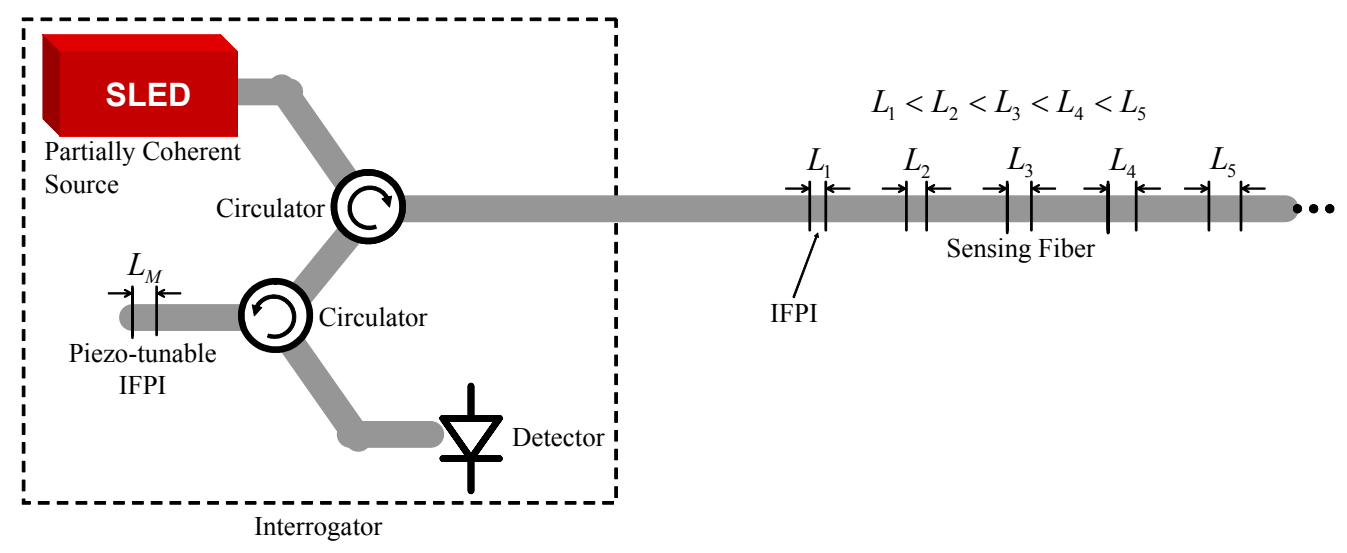

Figure 5-35. Fiber optic multiplexed interferometer

A series of reflection points will be written into the fiber core along the length of an optical fiber using an excimer laser, using a process similar to that described in Section 3.2.1.2. This will form what could be described as a series of intrinsic Fabry-Perot interferometers (IFPI) with varying cavity lengths, but the interrogation method will not utilize interference inside the cavities. A three-port optical circulator will route light from a broadband LED source to the sensing fiber, and the same circulator will route reflected light from the sensors to a second circulator. The second circulator will route this reflected light to a tunable Fabry-Perot interferometer (TFPI); when this light is then reflected from the TFPI, the second circulator then directs it to a photodetector. As the tunable interferometer is adjusted, bright fringes will be observed when the cavity length 
of the TFPI equals the cavity length of a sensing IFPI. As long as the cavity lengths of the sensing IFPIs are sufficiently different, they may be distinguished and small changes in length can be detected in each IFPI separately from which temperature will be calculated.

Assuming the reflection points have low reflectance (i.e. less than 5\%), Fabry-Perot type resonances between reflection points may be ignored. Partial coherence of the source will also work to reduce these effects.

For the $i^{\text {th }}$ sensing IFPI, light reflects from two points that differ in path length by $2 L_{i}$. Upon reaching the TFPI, light again reflects from two points that differ in path length by $2 L_{M}$. Given this, there are four paths the light will traverse. These four paths are illustrated in Figure 5-36. In this figure, the superscripts indicate which of the reflection points is being referenced.

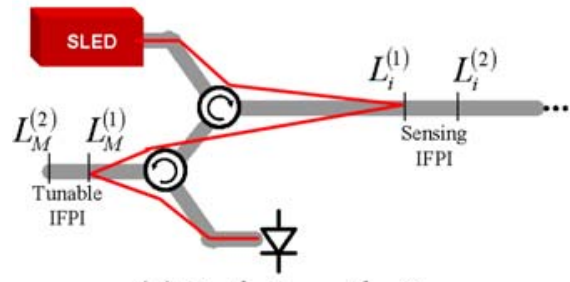

(a) Path Length: 0

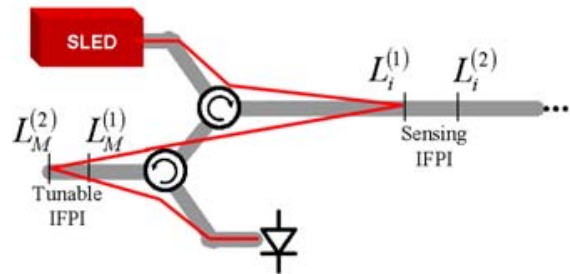

(c) Path Length: $2 L_{M}$

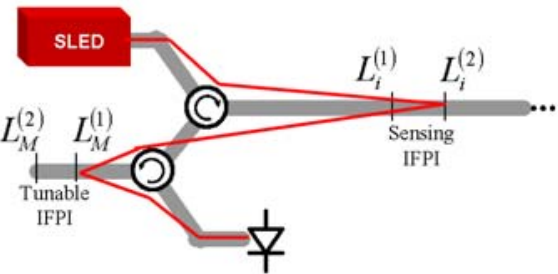

(b) Path Length: $2 L_{i}$

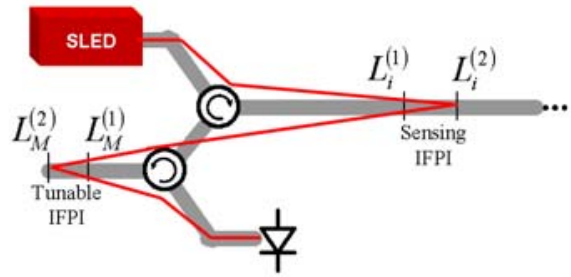

(d) Path Length: $2 L_{i}+2 L_{M}$

Figure 5-36. Four possible paths light may traverse for each sensing IFPI

Given an appropriate partially coherent source, the only two light paths that can be close enough in length to produce visible interference are (b) and (c) when $L_{M} \approx L_{i}$. Using a high precision piezoelectric micropositioner to adjust the tunable IFPI, and hence $L_{M}$, subtle changes in the cavity length of the sensing IFPI will be detected from which temperature will be calculated through knowledge of the coefficient of thermal expansion of the sensor. As long as the cavity lengths of all sensing IFPIs are different by a length greater than temperature induced changes, they will be easily distinguished by the interrogator.

The principle which LCPMI operates on is the limited coherence length of optical sources. The coherence length is defined in Equation 24, where $\Delta v$ and $\Delta \lambda$ are measures of optical bandwidth. The implication of the limited coherence length is that when a 
given optical signal is split, and later recombined, if the cumulative optical path difference (OPD) is less than the coherence length, the signals with add interferometrically (the detected power is highly dependent on the value of the OPD). When the OPD is greater than the coherence length, the signals will add in the intensity domain (the detected power is strictly the sum of the power from each of the two paths).

$$
L_{c o h}=\frac{c}{\Delta v} \approx c \frac{\Delta \lambda}{\lambda^{2}}
$$

Equation 24

In order to utilize this phenomenon as a mechanism for decoding many sensors which are multiplexed onto a single optical fiber, we simply need to apply a few assumptions about the nature of the sensors which we intend to deploy. In order to uniquely identify each sensor, we impose a requirement that the cavity length of each sensor is unique from all other sensors in the chain. To be more explicit, the length of each sensor should be at least one coherence length away from the length of any other sensor in the chain. This ensures that at no time is more than one cavity responsible for returning an interferometric signal. Then by sweeping a set of optics which is part of the interrogator through the range of cavity lengths, one can sequentially determine the actual cavity size of each sensor by locating the path length at which the interferometric signal achieves a peak.

A schematic of the interrogation system used to decode the IFPI is shown in Figure 5-37. A broad-band light source is coupled into a modulator, which is used to create a regularly oscillating signal, at a frequency determined by a lock in amplifier. The combination of the lock in amplifier with the modulator creates a tremendous amount of amplification, which is helpful in the detection of the signals which are quite small due to the necessarily small reflected power from each sensor. The modulated light then travels through a circulator, and into the fiber containing the string of IFPI sensors. The light which is reflected off of the IFPI sensors returns to the circulator and is then directed through the path matching optics, and to a detector. The path matching optics were formed by attaching an fiber optic extrinsic Fabry-Perot interferometer to a servocontrolled piezopositioner (Physik Instrumente E-660). By varying the voltage applied to the positioner's controller, the cavity length of the extrinsic Fabry-Perot interferometer could be changed with nanometer precision.

The detector was coupled into the lock-in amplifier, with the result being passed on to an oscilloscope. The length of the extrinsic Fabry-Perot interferometer was controlled by a slowly varying voltage from a function generator, which is also coupled into the oscilloscope to act as the reference for the amplified optical data. 


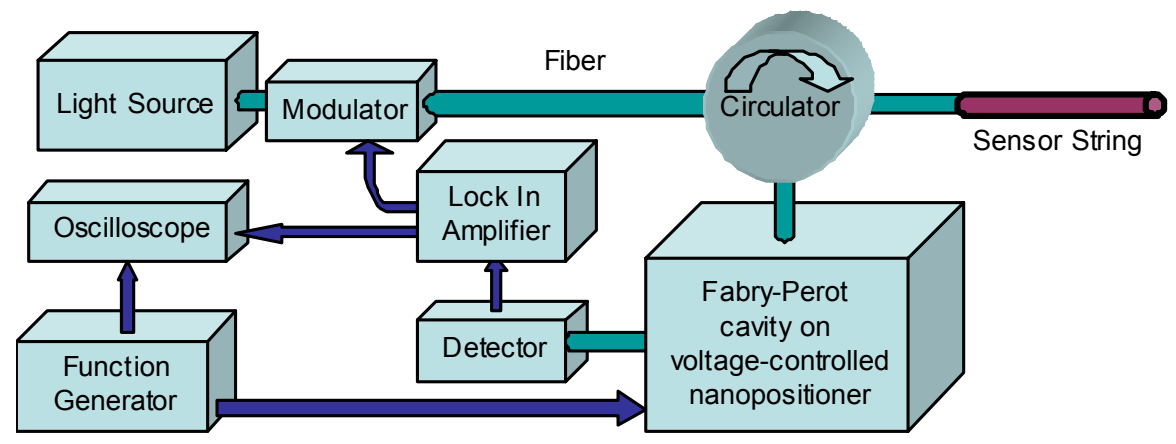

Figure 5-37: Schematic of the IFPI interrogation system

A sample of the output from the interrogation system is shown in Figure 5-38. As the path matching optics are swept back and forth, there are bursts of output which occur at times when the path length comes within one coherence length of one of the three sensors which are in the sensor string. In the arrangement which was tested, a lower voltage from the function generator (shown in green) corresponds to a larger sensor size. The different magnitude of the envelopes is a result of the relative amount of power which is reaching the detector from each of the three sensors. In this instance, the largest envelope (which occurs around sample number $1.2 * 10^{4}$ ) is the signal from the second sensor in the chain.

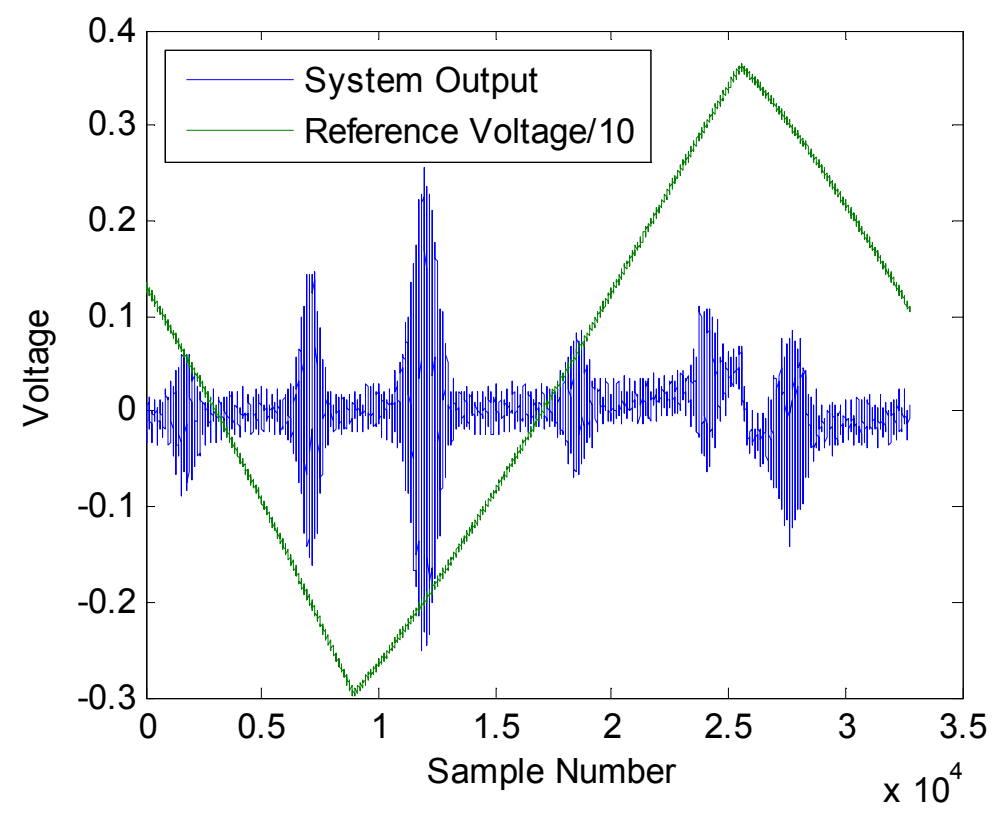

Figure 5-38: Sample traces of the outputs from the lock in amplifier (blue) and the function generator (green). In order to display both traces effectively the green trace has been scaled and centered to match the output from the lock in amplifier. 


\subsubsection{Test and Evaluation of Distributed IFPI Sensors Interrogated Using Multiplexed Michelson Interferometry}

Testing was conducted on the combined sensing system to determine whether multiple sensors could be discriminated. The principal sensor chain which was tested is shown as photographed in Figure 5-39. Each of the sensing elements is contained within a Petri dish with the connection to the interrogation system being on the left hand end of the chain.

A schematic representation of the sensors which were tested is seen below in Figure 5-40. An item of note in the sequencing of the sensors is that they are arranged not by the length of the cavities, bur rather by a dual selection of increasing reflected power and decreasing throughput. This is important because the further down the sensing chain a particular transducer is, the more attenuation will occur to the light reaching it and returning from it. Ideally one would be able to select the sensor order such that, for every sensor, the following product remains constant:

$$
P_{n}=\text { Reflection }_{n} \prod_{i=1: n-1} \text { Throughput }_{i}^{2} .
$$

Equation 25

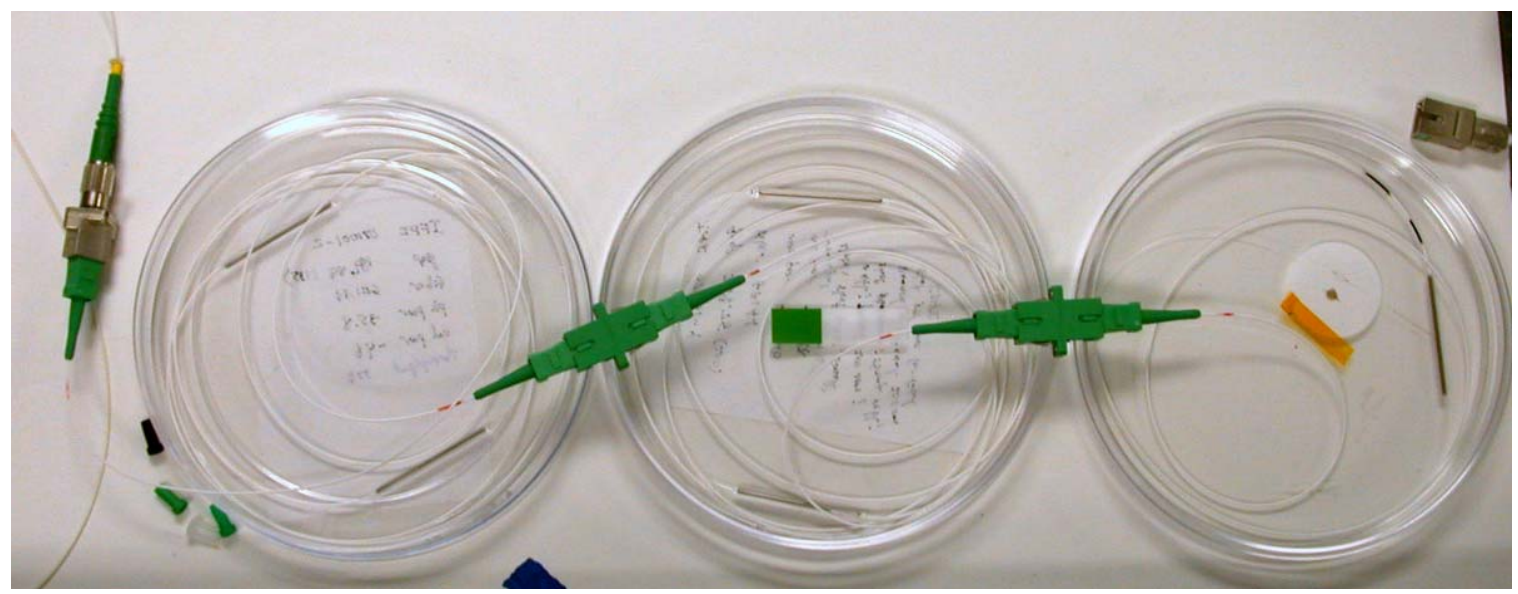

Figure 5-39: Photograph showing three sensors connected in series. The cavity sizes, from left to right are: 189.9, 299.8, and 87 microns

This equation indicates that the power detected from the $\mathrm{n}^{\text {th }}$ sensor in the chain is the product of the square of the throughputs of each sensor before it, times the reflection strength of the particular sensor. The throughputs must be squared to account for the losses in both directions. In the arrangement shown here, the values for $\mathrm{P}_{\mathrm{n}}$ from left to right are $0.026 \%, 0.038 \%$, and $0.055 \%$. This results in a maximum difference ratio of 2.1. For comparison, if the first two sensors were reversed the values would be: $0.28 \%$, $0.00051 \%$, and $0.055 \%$ with a maximum difference ratio of 549.0 !

It should be noted, however, that this limitation is a function of the very low throughputs which were obtained with these particular sensors. If the sensors were fabricated in a 
lossless fashion, the throughputs would be on the order of $99.75 \%$, in which case the sequencing of the sensors becomes far less important for power considerations. The regard in which the sequence does remain important is in the ability to correlate the sensor response with a particular location on the fiber chain, as the data returned from the interrogator is presented in order of the cavity size, rather than a spatial arrangement of the sensors.

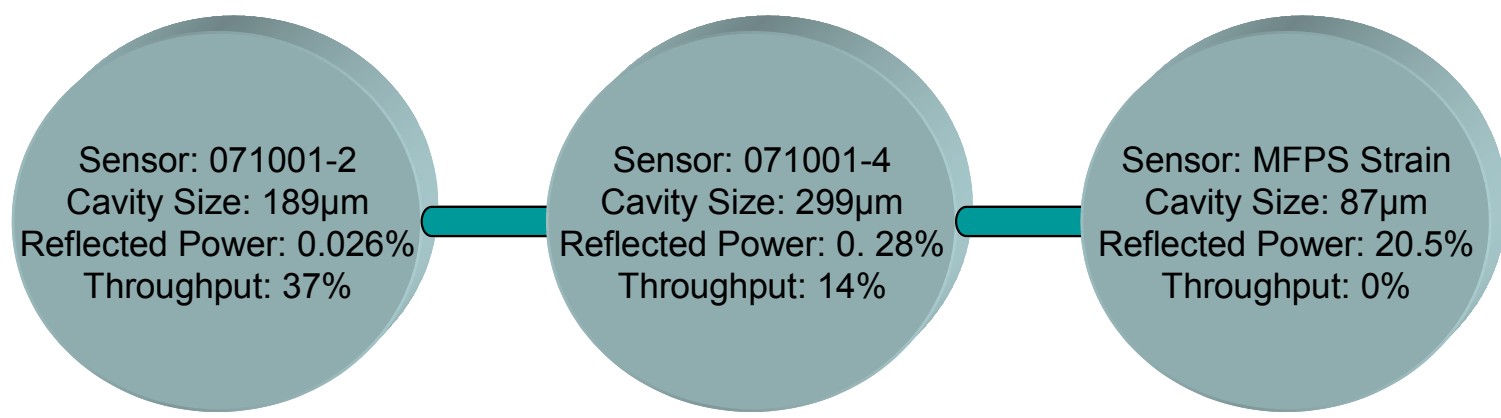

Figure 5-40: Schematic representation of sensor chain. The interrogation system is on the left hand end of the chain.

A sample of the raw data collected can be seen in Figure 5-41. The left hand side contains an overview of a full, bi-directional sweep of the path matching optics. The green line shows the voltage applied to the drive electronics of the nanopositioner used to adjuste the length of the receiver Fabry-Perot cavity; this voltage identifies the exact length of the optical path match, while the blue line represents the time based interferogram of the LCPMI. Each region in which the blue trace undergoes large scale oscillation is indicative of an optical path match, which means the path matching optics are matched to one of the sensors in the chain. On the right hand side of Figure 5-41 is a close view of the oscillations which occur when the system passes through a matched path.
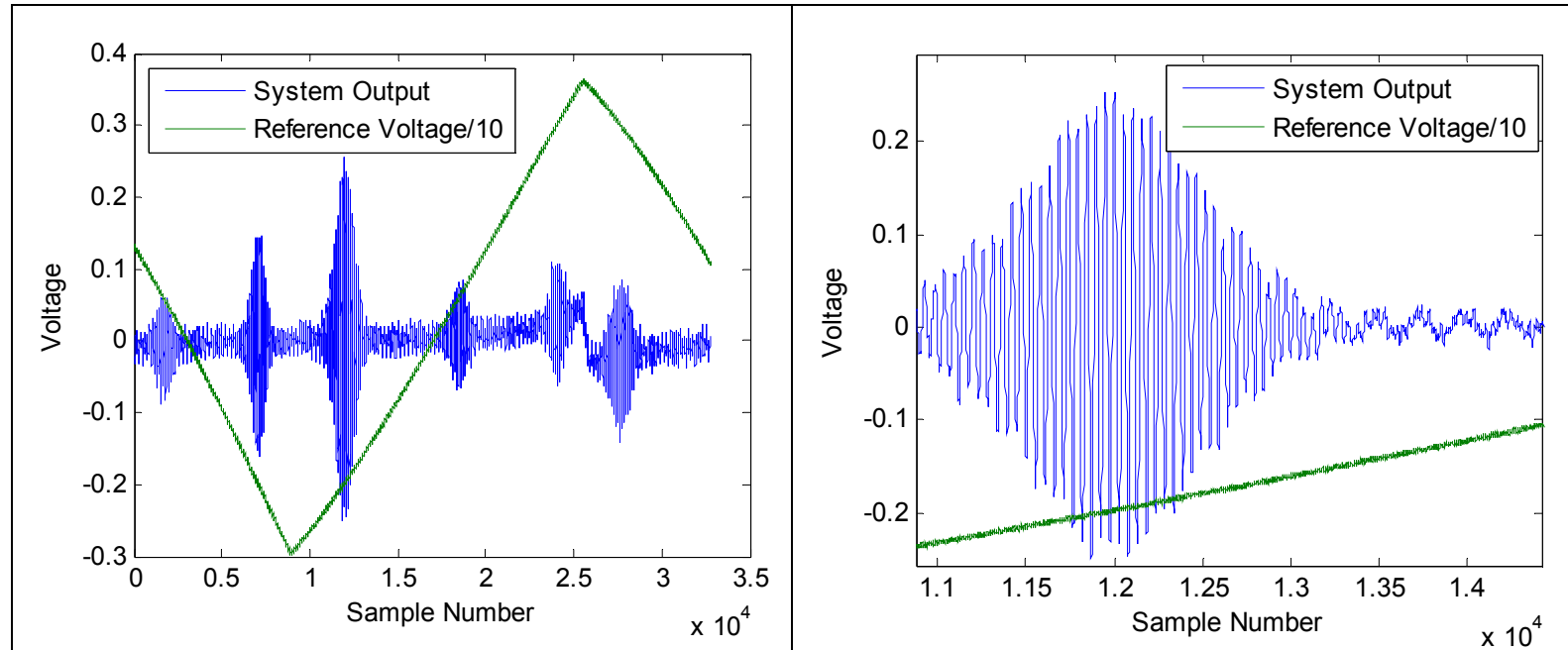

Figure 5-41 Output from the sensing system. Left: A full sweep of the sensor chain in both directions. Right: A close view of the response due to a single IFPI. In both cases the blue trace represents the output of the IFPI system, and the green line is the reference voltage which identifies the state of the path matching optics. 
The interpretation of the data is based on determining the value of the reference voltage which corresponds to the peak of the resonant condition (the largest point in the amplitude oscillation). The first step in the process is to convert the data reference from the time based domain in which it is actually gathered into one which is based on the reference voltage. Equivalently, we are converting it into a format which is plotted against optical cavity size. This conversion is shown in Figure 5-42.

Once the data is being manipulated in terms of the reference voltage, the next task in the signal processing is to determine the location of the peak of each of the resonances. The steps of this process are shown in Figure 5-43. For clarity the plot has been zoomed in to show only the response from a single IFPI. The base data from above is shown in blue. The next step is to eliminate the oscillations from the overall signal, which is done through application of the Hilbert transform. The output of this step is shown in green, but still contains some oscillations, as well as a fair amount of noise. Following envelope extraction, a zero phase offset filter is applied to create a smoothly varying representation of the envelope (red).
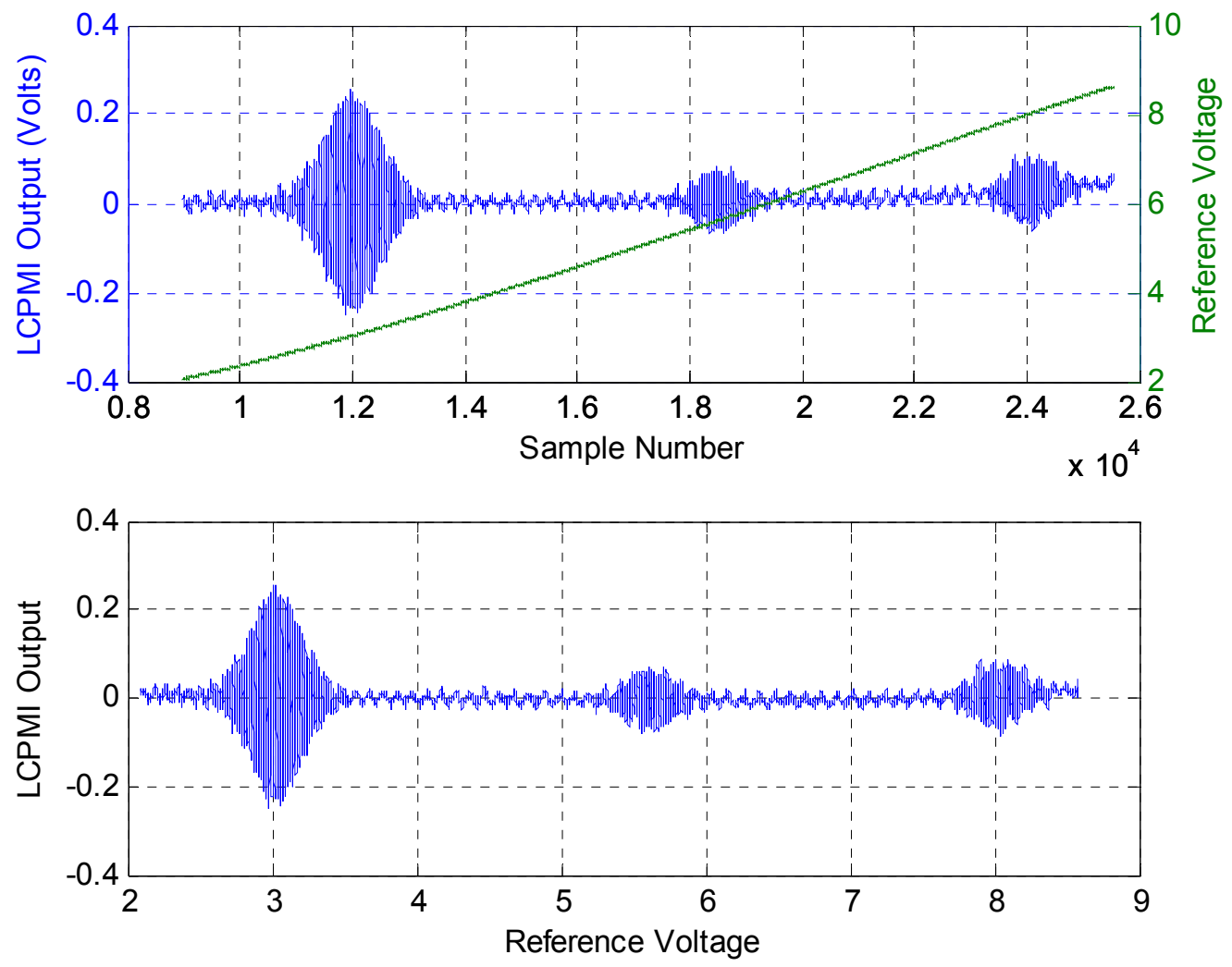

Figure 5-42: Adaptation of the raw, time-based data (top) into data plotted against the reference voltage (bottom)

Showing an overview of multiple readings of the entire sensor chain are the plots in Figure 5-44. In the top plot of the figure is an overlay of ten sweeps of the system after it has been plotted against the reference voltage. The multiple sweeps were chosen to reveal the occurrence of any non-repeatable phenomena in the data. In the lower plot is 
the filtered envelope extracted from each of these ten sweeps of data. At the top of each of the grid lines in the lower figure are the values of optical cavity size which correlate with the reference voltages plotted on the abscissa. Note that in the configuration used, an increasing reference voltage corresponds to a decreasing optical cavity size.

Therefore, the longest optical cavity in the system (from IFPI 071001-4, with a length of approximately $299 \mu \mathrm{m}$ ) is shown at the left end of the plot.

Once this has been done, the remaining steps are to determine the true peak of the envelope, and to apply the calibration which relates the reference voltage at which the peak occurs to the physical cavity size of the IFPI sensor in question. The first step is done by determining the full-width half-max centroid of each peak in the sequence. The algorithm seeks out as many peaks as are present with significant power. For each of these peaks the half-maximum crossings are identified, and a weighted average of the peak is determined to identify the true center of mass for the peak.

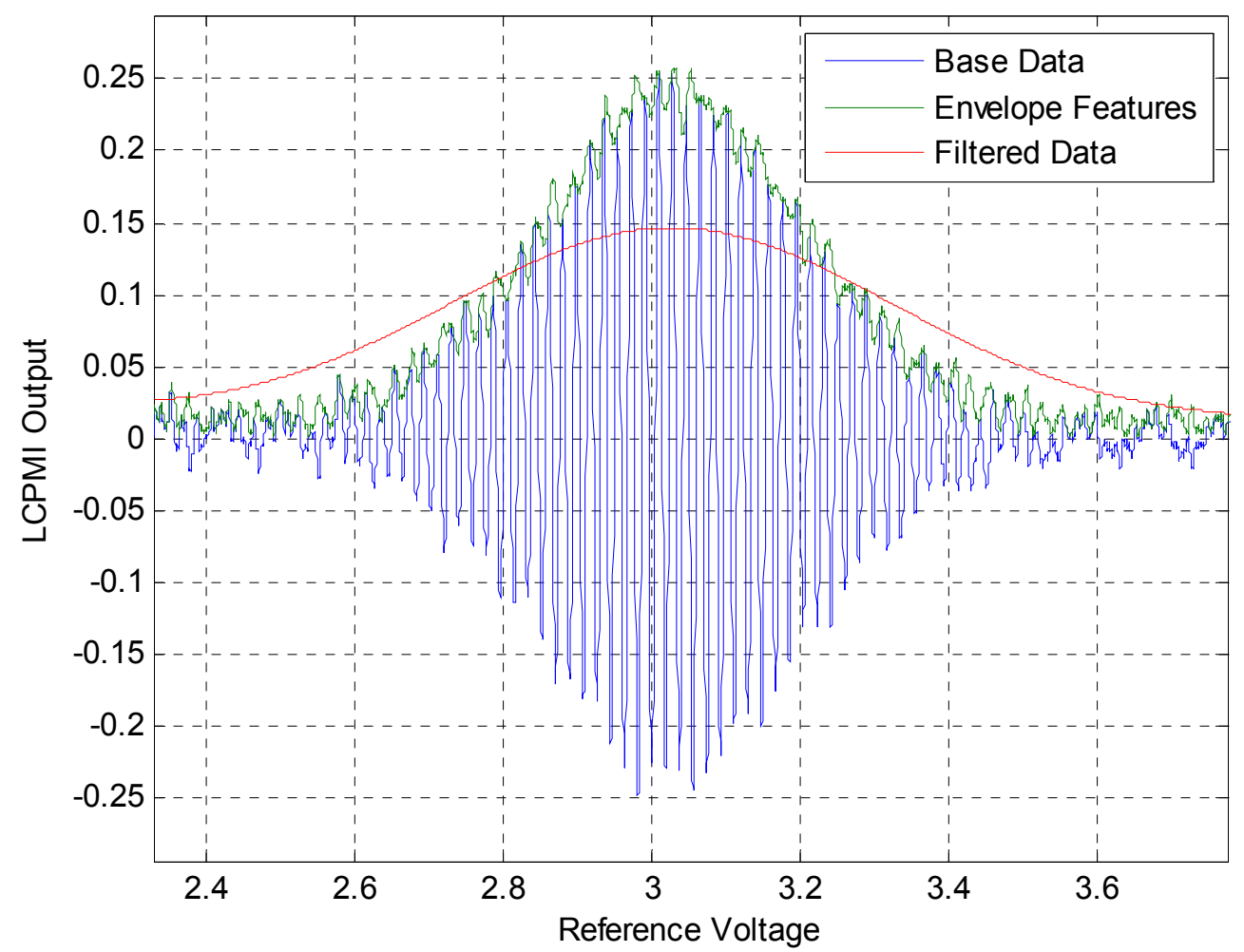

Figure 5-43: Stages of preparing data from a single sensor's response for peak detection. The traces are the base data (blue), the envelope of the oscillation (green), and a filtered copy of the envelope (red)

The results of this processing for the ten sweeps shown above are displayed in Figure 5-45. In each of the three graphs of the figure are the measured cavity sizes for one of the three sensors. The order of the plots is determined by the relative strength of the signals returned. In each case the standard deviation in the optical cavity calculation is reported. 
To view these results in terms of the temperature resolution which the sensors can report, we need to determine what the change in optical path length of the sensor per degree is. This is given by the relation:

$$
\frac{d O P L}{d T}=n \frac{d L}{d T}+L \frac{d n}{d T}=L n C T E+L \frac{d n}{d T}=L\left(n C T E+\frac{d n}{d T}\right) \quad \text { Equation } 26
$$

In this relation, $\mathrm{n}$ is the refractive index of the optical fiber (approximately 1.5), $\mathrm{L}$ is the physical length of the cavity, and CTE is the coefficient of thermal expansion $\left(0.55 * 10^{-6} C^{-1}\right.$ for silica). The change in refractive index with respect to temperature, $\frac{d n}{d T}$, is approximately $10^{-5} C^{-1}$. This indicates that for a physical cavity length of 200 microns (the length of 071001-4) the optical path length changes by $2.17 \mathrm{~nm}$ per degree C. Therefore, as tested here, the measurements of 071001-4 have a standard deviation of approximately $70^{\circ} \mathrm{C}$. It is worthy of note, however, that no efforts were made to utilize signal processing tools, such as averaging of samples or high resolution data collection, to improve these results.

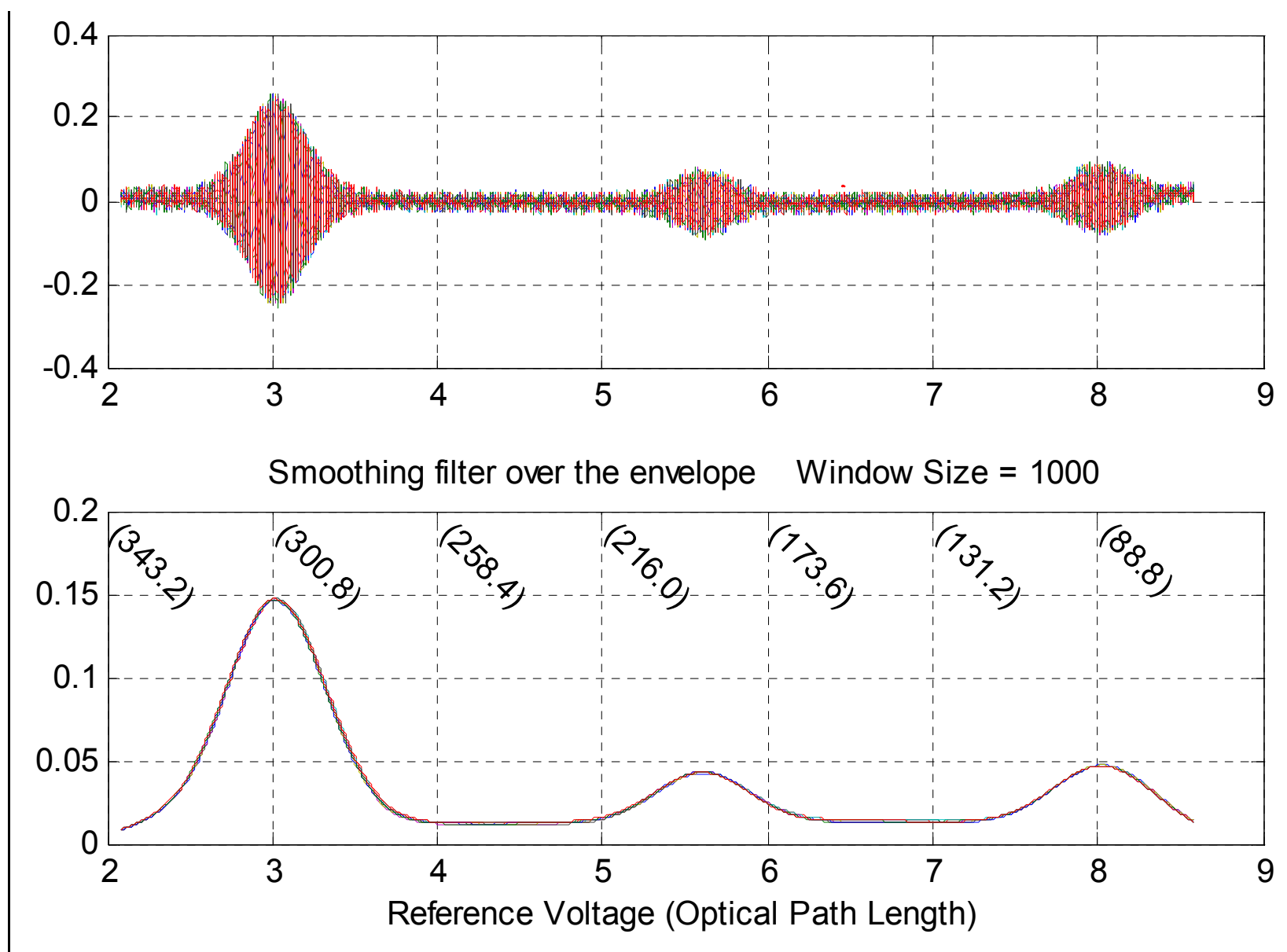

Figure 5-44: Full scale view of ten sweeps of the full sensor chain. Top: Raw baseline data as a function of reference voltage. Bottom: Filtered envelope data. The numbers in parenthesis indicate the optical cavity sizes which correlate with each voltage. 


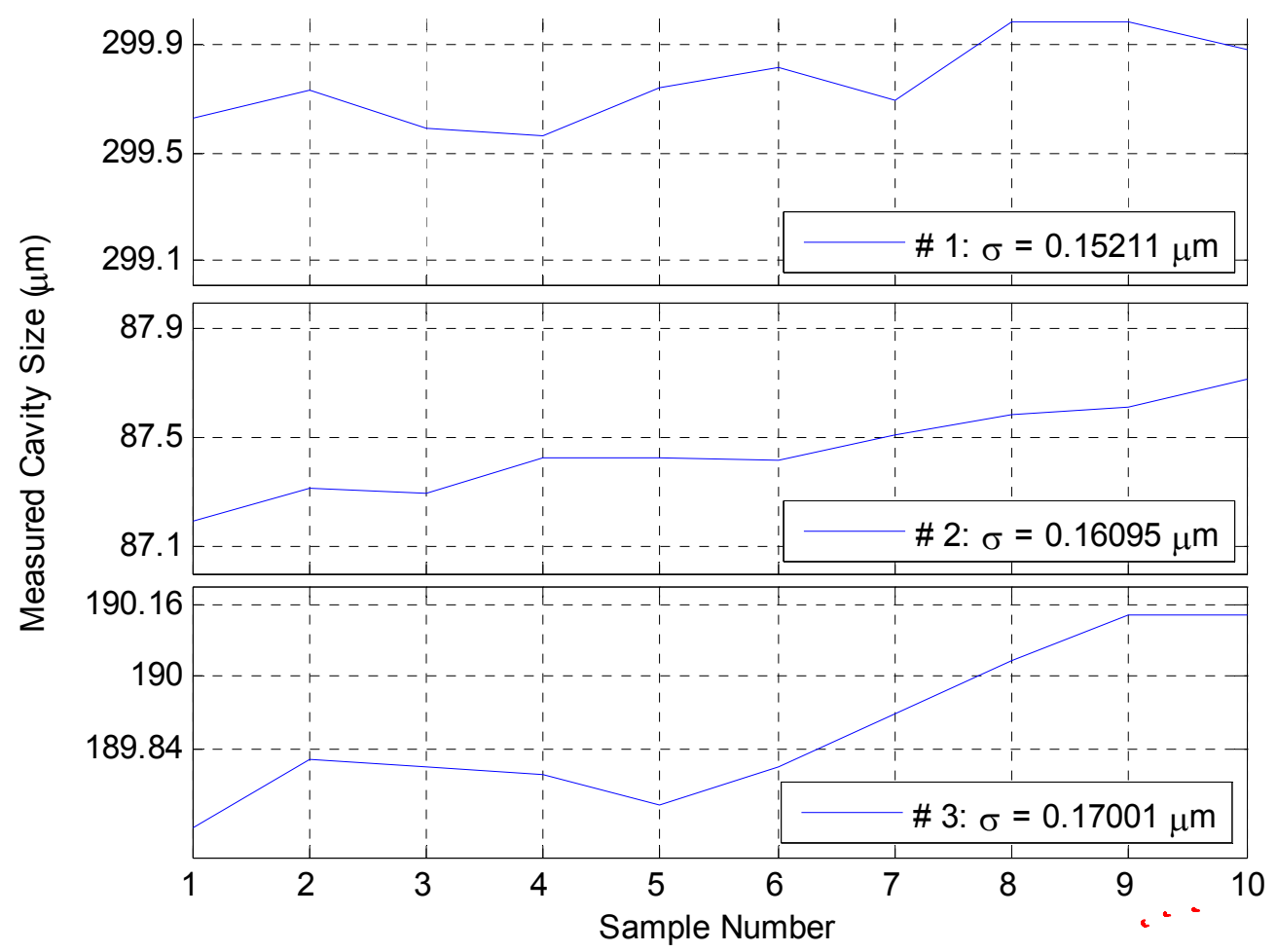

Figure 5-45: Distributed Sensor system performance over ten sweeps for three sensors. The sensors shown are 071001-4 (top), MFPS strain (center), and 071001-2 (bottom).

\subsection{Conclusions Regarding Distributed Sensing}

We have demonstrated that use of the IFPI sensor technology in combination with LCPMI can successfully be used to achieve a quasi-distributed optical measurement system for temperature and strain. As implemented to date, there are a few specific limiting factors which are resulting in reduced performance. The primary of these factors is the relatively low quality IFPI sensors which were fabricated, due to imperfect control of the laser system. The impacts of this issue are the limited number of IFPIs which can be multiplexed onto a chain. An additional impact is that the high throughput losses dramatically increase the overall noise for all subsequent sensors in the chain. The overall power level which was measured returning from the sensors in the chain is reduced to approximately $2 \%$ of what could be attained with higher quality IFPIs.

The second limiting factor of the system as implemented is the optical source which was available to drive the interrogation system. The source had a much narrower spectrum than desired, approximately 50 nanometers at full-width half-max, resulting in a much longer coherence length. The impact of this is to make it much more difficult to localize the peaks of the resonance conditions which are indicative of having achieved the optical path match. Neither of these factors represents any limitation on the fundamental capabilities of the technology, or its applicability to high temperature use, such as in solid oxide fuel cells. It is clear, however, that further development is needed to render the technology into a field usable tool. 


\section{Conclusions}

The goal of this program was to explore the potential of optical sensors based on modified sapphire fibers for instrumentation of advanced fossil fuel power generation systems and solid oxide fuel cells. Specific objectives of this program were:

- to enable revolutionary improvements in the efficiency and output of fossil fueled power plants through the development of new, robust photonic sensors based on improved high-temperature sapphire fibers,

- to demonstrate the application of those sensor systems to monitoring of the powerplants, and

- to investigate and demonstrate the use of high-temperature optical fiber sensors for characterization of the mechanical properties of developmental solid oxide fuel cells.

Achievements of the program included the development of new processing methods to produce ultrahigh-temperature clad sapphire fiber, identification of applications within advanced boiler plants for new ultrahigh-temperature photonic sensors, the development and demonstration of prototype sensors for fossil fuel power plants, the development and demonstration of high-temperature strain gages for SOFCs, and the development of technology for making distributed measurements along a single optical fiber.

\subsection{Determination of Requirements}

Through a subcontract, Babcock \& Wilcox Research Center (B\&W) investigated possible applications of photonic sensors in existing and future boiler plants. Four classes of measurements within supercritical boiler plants were identified as the most desirable for monitoring and control of the plants. These include temperature measurements for thermal barrier coatings, in-situ strain measurements on superheater tubes, measurement of steam flow in membrane walls and tube banks, and dynamic pressure measurements for monitoring of combustion dynamics. Three specific locations were identified as primary candidates for sensor development in this program: the furnace water wall (tube temperature, heat flux, water flow), secondary superheater tubes (tube temperature, steam temperature, strain), and burners (temperature of burner components). Required sensor measurands for these locations include temperature, pressure, and strain.

Issues that were identified as potential barriers to increased use of instrumentation in advanced plants included limited availability of materials capable of surviving under typical power plant conditions (which include corrosive and reducing atmospheres), very high temperatures due to oxygen firing, a need for improved models, difficulty in interfacing with existing control systems, and resistance of customers to pay for sensors and controls. 
Based on the input from Babcock \& Wilcox, a matrix of sensing techniques and a list of requirements for high-temperature fiber and cladding were developed. Based on the results of this comparative study, the requirements determined by Babcock \& Wilcox, and the experience and expertise of Prime Research engineers, sapphire sensors based on extrinsic Fabry-Perot interferometry were chosen for development as sensors for power plant applications.

\subsection{Development of High-Temperature Optical Cladding for Sapphire Fiber}

During the program, methods for coating sapphire fibers with magnesium aluminate spinel which function as optical claddings were researched. Claddings are required on optical fibers for optimal operation of several classes of optical fiber sensors, including fiber Bragg gratings (FBGs) and interferometric sensors. While it is possible to minimize the problems resulting from the use of multimode fibers such as sapphire fibers by exciting only a small number of modes in the multimode fiber, this approach is successful only with straight, inflexible rods. Bends, splices and connectors in the fiber (which are difficult to avoid in real-world applications) cause a redistribution of power into a larger number of modes, causing intermodal interference and reducing the practicality of such multimode sensors.

Magnesium aluminate spinel is well-suited for cladding of sapphire fibers due to its high melting temperature, refractive index slightly less than that of sapphire, and coefficient of thermal expansion closely matched to that of sapphire. Application of spinel coatings involves dipping the sapphire fiber in a slurry of spinel and magnesium oxide powders, firing the slurry on the fiber, and then sintering the coating to densify it on the fiber.

Research conducted during the program on solid-state methods for the coating of magnesium aluminate spinel claddings onto sapphire fibers demonstrated that there are several variables that influence the optical and mechanical quality of the resulting clad fibers. An investigation was undertaken in which certain parameters such as the firing profiles, the slurry dipping procedures, the firing atmosphere, the particle sizes of the powders, and slurry compositions were systematically varied. Preliminary results indicated that a smooth interface between the sapphire core and spinel cladding, which reduces scattering and mode conversion in the fiber, can be achieved through a carefully tailored firing procedure in an argon atmosphere. In addition, it was found that the core diameter of the sapphire fiber can be reduced by a specified amount through the addition of a specified proportion of magnesium oxide in the slurry.

These results also indicated that the fabrication variables, which include chemical composition of the coating, processing steps for preparation of the coating slurry, and firing profiles for binder burn-out and sintering of the coatings, often interact, making determination of an optimal set of variables difficult. Therefore, a set of experiments designed using Taguchi methodology was undertaken to efficiently determine the optimal set of processing variables to yield clad fibers with good optical and mechanical 
properties. Specific fabrication parameters studied included incorporation of nanopowders into the slurry, improved coating density through optimized particle packing, separation of powder milling into dispersion milling and binder milling to prevent agglomeration of powders, testing of several dispersants to control agglomeration, and determination of proper binder burnout temperature. The desired responses included good optical quality, good mechanical quality, and acceptable fiber geometry.

The measured results of the designed experiments were statistically analyzed to determine the significance of each factor in contributing to the desired responses. The output of these analyses were then used to predict the set of factors that would result in a spinel cladding with the optimal geometrical, mechanical, and optical properties. To confirm the predictions of the designed experiments, a set of four sapphire fibers were clad with the magnesium aluminate spinel coating using the predicted optimal set of factors. In general, the clad fibers demonstrated high quality, exceeding the best results previously obtained.

Tests of the high-temperature stability of the clad fibers were also conducted. Four samples of clad fibers were fabricated using the optimal factors and were then heated in a furnace at temperatures of $1000^{\circ} \mathrm{C}, 1100{ }^{\circ} \mathrm{C}, 1200{ }^{\circ} \mathrm{C}, 1300{ }^{\circ} \mathrm{C}, 1400{ }^{\circ} \mathrm{C}$, and $1500{ }^{\circ} \mathrm{C}$, for three days at each temperature. Microscopic cross-sectional analysis of the heated fibers indicated that the clad fibers were stable at temperatures up to $1300{ }^{\circ} \mathrm{C}$ for three days. At the higher temperatures, some changes in the geometry of the fibers were observed, with more dramatic changes occurring upon exposure to $1500{ }^{\circ} \mathrm{C}$ for three days, including the formation of cracks, the growth of the cladding thickness, and a reduction in the fiber core diameter. A strategy for stabilizing the cladding, involving an improved composition of the spinel slurry during coating, was proposed but not tested during this program.

In order to assess the effectiveness of the spinel cladding in reducing the number of guided modes in the sapphire fiber, an apparatus was assembled to measure the numerical aperture (NA) of a sapphire fiber before and after cladding. As the number of propagating modes in a fiber is decreased, the numerical aperture of the fiber decreases. Therefore, a change of numerical aperture can be correlated to a change in modal volume of a fiber. Using the measurement apparatus, the NA of a six-inch long unclad sapphire fiber was found to be approximately 0.59. An identical sapphire fiber was coated with a magnesium aluminate spinel cladding, and after measurement was found to have an NA of approximately 0.3 , demonstrating the efficacy of the cladding in reducing the modal volume in the sapphire fiber.

\subsection{Development of Sapphire Sensors and Interrogators for Instrumentation of Coal-Fired Power Plants}

A key objective for the program was the demonstration of new, ultrahigh-temperature optical sensors for specific measurements required for advanced powerplant control 
systems, as identified by powerplant manufacturers/integrators/operators. Towards this end, new techniques to produce sensor elements within sapphire fibers were explored and evaluated. Tests were performed to evaluate the performance of three sensor classes: interferometric (extrinsic and intrinsic Fabry-Perot interferometer), gratings (fiber Bragg gratings and long period gratings), and microbend sensors. Of the sensor configurations evaluated, sapphire sensors based on extrinsic Fabry-Perot interferometers were determined to be the most promising approach for coal-fired power plant instrumentation.

The design and fabrication of sapphire sensors based on Fabry Perot interferometers for measurement of temperature were then researched. The specific sensor configuration uses a polished sapphire wafer as the temperature-sensitive element. The wafer is attached to a sapphire fiber (clad or unclad), and is interrogated as a Fabry-Perot cavity. Changes in the temperature of the wafer cause a change in the optical path length of the Fabry-Perot cavity, which can be related to the change in temperature through the known coefficient of thermal expansion. The high degree of parallelism of the two sides of the wafer (and thus the Fabry-Perot cavity) permits the achievement of reasonable fringe visibility, even with multimode fibers. Methods for assembling and packaging the sensor were investigated. The use of materials that can withstand the harsh environments of boiler plants was a priority in determining the assembly process.

Several prototype sapphire temperature sensors were fabricated, tested, and evaluated over the course of the program. The most significant engineering challenge was to design the sensor to eliminate the formation of a gap between the sapphire wafer and the end of the sapphire fiber, which forms a small spurious Fabry-Perot cavity that adds undesired fringes to the sensor output.

Methods for fabrication of high-temperature strain sensors using sapphire fibers configured as Fabry-Perot interferometers were also investigated for applications in power generating facilities. In general, assembly of sapphire strain gages based on Fabry-Perot interferometric cavities is difficult due to the tight alignment tolerances required for multimode waveguide (sapphire fiber) components. Several designs and fabrication methods were attempted and evaluated during the program. Best results were found to result with the use of a zirconia sleeve to align the two fiber ends forming the Fabry-Perot cavity. Both sapphire fibers (input and reflector) were potted in zirconia ferrules commonly used for fiber optic connectors, using a ceramic adhesive to pot the fibers in the ferrules. After curing the adhesive, the fiber and ferrule ends were polished on diamond polishing media using a polishing puck. When the polished fibers were aligned using a zirconia sleeve, interference fringes were obtained. Due to the relative simplicity of fabrication of this technique compared to the others evaluated, this fabrication method was used to fabricate the sapphire strain gages assembled for the field test.

Methods for joining, or splicing, the sapphire fiber to a fused silica communications fiber, were investigated and developed. Experiments showed that the most reliable splices were obtaind by using fused silica capillary tubing to capture the two fiber ends, with borosilicate glass between them to bond the fiber ends. 
Efforts to develop signal processing techniques to convert the optical signals from the sapphire Fabry-Perot cavities to engineering values of temperature or strain concentrated on methods to facilitate the processing of Fabry-Perot spectra, specifically concentrating on techniques to reduce the effects of intermodal interference resulting from the use of multimode sapphire fibers.

Investigation of methods for interrogation of the sapphire Fabry-Perot interferometer sensors concentrated on the use of optical spectrum analyzers to determine the interference spectra of the sensors over the telecommunications C-band (roughly from $1520 \mathrm{~nm}$ to $1570 \mathrm{~nm}$ ). In early tests, the presence of additional undesired fringes in the output spectra suggested that modal noise was responsible for an undesired modulation of the sensor output. If the output of a multimode fiber (such as a sapphire fiber) is restricted by an aperture of smaller spatial area than the core of the multimode fiber, the aperture will act as a spatial filter, passing a limited subset of the modes that propagate in the multimode fiber. When the number of propagating modes is filtered, intermodal interference will result. This spurious modal noise is particularly sensitive to the movement of the multimode fiber. Experiments indicated that the influence of the modal noise can be greatly minimized, if not completely eliminated, through the use of multimode fibers exclusively in the sensor system, in addition to the use of a spectrometer designed for use with multimode fibers.

Algorithms were investigated for processing the spectral responses of the sapphire FabryPerot sensors to yield absolute measurements (in which no data regarding the sensor history is required to obtain an accurate measurement of the environment), as opposed to relative measurements (which give only the change from a previous measurement). Digital spectral filters were designed and implemented to eliminate undesired frequency components. In addition, work was directed to develop methods for uniquely determining the phase of the output signal, even when the total phase excursion of the signal exceeds $2 \pi$. The objective of those efforts was to eliminate the ambiguous output when the phase excursion exceeds $2 \pi$. Best results were realized by first calculating a coarse measure of the optical cavity length by using a measurement of the period of the spectral response of the cavity. This coarse measurement can then be used to detect and eliminate $2 \pi$ phase jumps in the calculation of cavity length from the phase of the spectral response. The phase yields a measurement of higher resolution, but is prone to modulo $2 \pi$ phase jumps if the coarse measurement of cavity length is not used to unwrap the phase jumps.

\subsection{Field Tests of Sapphire Sensors}

Prime Research worked with engineers at the Babcock \& Wilcox Research Center to identify a test site for field tests of the prototype sapphire strain and temperature sensors. Two facilities were considered: a municipal power plant in Hamilton, Ohio with B\&W boilers, and a combustion research facility operated by B\&W in Alliance, Ohio. Plans were first made for tests in the Hamilton facility, and procedures for sensor installation 
were developed, along designs for mounting adapters for specific locations in the plant. When the City of Hamilton, OH decided not to commit to field tests, Babcock \& Wilcox (B\&W) offered an opportunity to install and test the sensors in the B\&W Clean Environment Development Facility (CEDF) in Alliance, Ohio. This plant replicates key operating conditions of steam generating systems, including burners for pulverized coal, a convection pass with simulated superheater tubes, and sootblowers for cleaning the tubes.

B\&W engineers suggested that the prototype sensors could be mounted to these simulated superheater tubes, and that the conditions presented to the sensors would be representative of those that would be seen in a convection pass in a conventional coalfired supercritical boiler plant. Prime engineers visited the CEDF facility to examine the proposed installation site, and after taking measurements of critical dimensions (tube diameter, wall thickness), began designing packaging and planning for installation of the sapphire sensors for the CEDF facility.

Two sapphire temperature sensors were assembled and packaged for installation in the Babcock \& Wilcox Clean Environment Development Facility (CEDF) combustion test facility in Alliance, $\mathrm{OH}$ for field tests. The temperature sensors and their packaging were designed for instrumentation of two locations in the $2^{\text {nd }}$ tube bank of the CEDF.

In addition, two sapphire strain gages were assembled and packaged for installation in the B\&W CEDF plant. The sensors used an extrinsic Fabry-Perot interferometer design; that is, an air gap was formed between two sapphire fibers, so that when light is injected into one of the sapphire fibers, the reflections generated by the sapphire fiber ends that form the air gap will interfere. When the two fibers are attached to a tube in the CEDF plant, then stresses applied to the tube will result in strains that will correspondingly cause a change in the sensor cavity length, which can be measured interferometrically. The sensors were packaged in Inconel housings, in order to protect the sapphire fibers from mechanical damage during handling, installation, and in service. During the final packaging of the second strain gage, the sapphire fiber was broken during insertion of the fiber into the Inconel tube, rendering that sensor unusable.

On June 21, 2007, the two sapphire temperature sensors, two thermocouples, and one sapphire strain gage were installed in the B\&W CEDF plant by Prime Research engineers. Prior to the installation date, B\&W personnel drilled through the CEDF wall and installed a port with a gas shield, so that the sensors could penetrate the plant wall. One sapphire temperature sensor, the sapphire strain gage, and one Type K thermocouple were attached to a CEDF simulated superheater tube using resistance welding. Due to the high temperatures anticipated by B\&W engineers (up to $2000{ }^{\circ} \mathrm{F}\left(1093{ }^{\circ} \mathrm{C}\right.$ )), it was not possible to mount a conventional electrical strain gage on the tube to serve as a reference for the sapphire sensor. The sapphire strain gage was mounted on a tube that was suspended from the ceiling of the superheater tube pass. Since the bottom of the tube was freely hanging, any strain resulting in the tube during the testing would result solely from the thermal expansion of the tube. Therefore, for the purpose of testing the 
sapphire strain gage on the CEDF tube, the measured strain was correlated with temperature.

In addition to the sapphire temperature sensor and strain gage mounted to the CEDF tube, an additional sapphire temperature sensor and thermocouple were installed. These were mounted so that the sensors sampled the temperature of the air approximately two inches from the interior wall of the CEDF.

A dustproof enclosure was used to hold two optical interrogators and a data acquisition computer. The interrogators used were a Micron Optics CTS scanning laser spectrometer (modified with a large area photodetector) for the sapphire strain gage, and an Ocean Optics USB4000 spectrometer for the two sapphire temperature sensors. The data acquisition computer was used to $\log$ the outputs from the two interrogators.

Data acquisition from the sapphire strain gage, one sapphire temperature sensor, and one thermocouple began on 21 August 2007. The sapphire temperature sensor was set up to record full interference spectra every 30 seconds. Over a duration of forty-five days, a total of 130,411 spectra were recorded, ending on 5 October, 2007. On 5 October, the data collection system ceased acquisition due to a glitch in the commercial software used for recording the data. Full spectra of the output of the sapphire strain gage were recorded at one minute intervals. The sensor operated from the installation on 21 August, 2007 through 28 September, 2007. On September 28, the sensor suffered a failure at which time discernable interferometric fringes were no longer present in the captured signal. Approximately 55,000 usable spectra were logged from the strain gage.

Data obtained from the thermocouple logs indicate that the CEDF plant was not fired until 5 September 2007, so that data logged previous to that date were not used in sensor evaluation. On 18 December 2007, the sapphire sensors in the thermocouples were removed from the CEDF during a plant shutdown, for further analysis at Prime Research laboratories. At the same time, the logged data and spectra were retrieved from the data acquisition computer for further analysis.

In order to process the temperature sensor data, a series of pre-processing steps were first applied. The data were trimmed to the region of interest, and then flattened to remove the envelope of the light source used. The data were then filtered and normalized in order to yield an optimum interferogram which was then be processed in order to extract the key parameters which represent the measurement, specifically the frequency and phase of the signal.

An algorithm was then applied to extract data from the pre-processed spectra in order to determine the changes in the fundamental Fabry-Perot cavities. When the interference fringes acquired by the interrogator are expressed as a function of wavenumber (the reciprocal of wavelength), the frequency of the fringes is a direct measure of the FabryPerot cavity length. In the processed data from the field tests, most sections of the temperature sensor data were smoothly varying and corresponded well with the temperature changes of the system. However, other sections displayed excessive noise; it 
is suspected that the appearance of a second optical cavity (in addition to the sapphire wafer which serves as the measurement element and primary optical cavity) created a low-frequency interference signal in addition to the desired measurement signal. Since the frequency of the signal from the second cavity is inversely proportional to the optical length of the cavity, a small (less than one micron) cavity can generate a signal of very low frequency. This low frequency signal would heterodyne (mix) with the measurement signal, shifting the frequency of the measurement signal by an amount equal to the frequency of the second cavity. While the source of this secondary cavity has not been definitively determined, possible causes include the development of a gap between the sapphire fiber and silica fiber in the splice region, or the development of a gap between the sapphire wafer and the sapphire fiber.

In sections where high noise levels were observed, "phase jumps" resulted in offsets in the reported output, compromising the system accuracy. The phase jumps result from the periodic nature of any calculation of phase. Every $2 \pi(\sim 6.28)$ radians is a "phase jump", and one of the requirements of an interferometric sensing system is to correctly identify whether a phase jump has occurred, and unwrap it in the event that one has. In the case of excessive noise, a situation can arise in which such phase jumps are either missed, or falsely identified.

To process the data logged from the sapphire strain gage, the data was first scaled and low pass filtered to extract the highest frequency noise content. Next, the data was decimated (or downsampled) to reduce the data volume in order to maintain a reasonably short processing time. A proprietary algorithm was then used to extract the frequency, amplitude, and phase content of the signals from the decimated data.

As explained above, it was expected that the thermally induced strain in the CEDF tube would correlate well with the temperature of the tube. When the phase of the sensor output (which is proportional to the strain measured by the sensor) was plotted against the thermocouple output, a correlation coefficient of 0.7955 was obtained (with 1.0 indicating perfect correlation.)

A close inspection of the strain gage data showed that the low data sampling rate used (one sample per minute) resulted in phase jumps that reduced the accuracy of the system. When the sensor system was installed in the CEDF, it was not known definitively when the plant would begin operation. Therefore, a sampling interval of one minute was selected in order that the data acquisition computer's storage medium not be filled before the first firing of the plant. The actual rate at which the temperature within the environment changed was much higher than anticipated, and as a result the data collection at one minute intervals resulted in a number of phase jumps being missed. When the sensor response was successfully decoded, the slope of the phase versus temperature plot was uniform. However, when the rate of temperature change was large, the software at times failed to correctly unwrap the phase, resulting in a temporary reduction in sensitivity and a consequent reduction in accuracy. To avoid this problem in future uses of the sensor, the data acquisition rate for the strain gage interrogator should be carefully set to ensure that sufficient data is obtained to avoid phase jumps. 


\subsection{Development of High-Temperature Strain Gages for Solid Oxide Fuel Celss}

Strain sensors for the mechanical testing of solid oxide fuel cell (SOFC) components were developed and evaluated during the program. The target application was a button cell with a diameter of $1.25 \mathrm{in}$ and thickness of $137 \mu \mathrm{m}$ and operated at a temperature of approximately $800^{\circ} \mathrm{C}\left(1,472^{\circ} \mathrm{F}\right)$. Miniature Fabry-Perot strain gages based on micromachined fused silica optical fibers were evaluated.

Several adhesives for bonding the optical strain gauge to the solid oxide fuel cell substrate were investigated, and a ceramic adhesive with silica filler was found to provide the best strain transfer fidelity, and to give the most stable measurements at temperatures up to $800^{\circ} \mathrm{C}\left(1,472{ }^{\circ} \mathrm{F}\right)$. Development of signal processing techniques to convert the optical signals from the sapphire Fabry-Perot cavities to engineering values of temperature or strain was also addressed during the program.

Tests of the Fabry-Perot strain gage, mounted on an alumina beam next to an electrical strain gage, showed a close match between the optical and electrical strain gages when the beam was loaded in a three point configuration at room temperature. In addition, both gages showed good agreement with strains predicted by finite element analysis.

Further tests of the strain gage were performed on zirconia-based Hionic substrates at $800^{\circ} \mathrm{C}\left(1472^{\circ} \mathrm{F}\right)$. A Fabry-Perot strain gage was attached to a Hionic substrate using a ceramic adhesive, and the substrate was subjected to increasing deflection using a threepoint bend set-up. The sensor output showed changes in cavity length that were clearly correlated with the beam deflection (and hence the strain), although some hysteresis and drift was present and apparently due to slippage between the fiber sensor and the ceramic adhesive. In a final test of the sensor, the Hionic substrate was gradually loaded until failure. In this test, the increasing strain was monitored by the fiber sensor, and again some evidence of poor adhesion of the ceramic to the fiber was seen.

\subsection{Development of Technology for Distributed Measurement of Strain in SOFCs}

Technologies to measure the distribution of temperature and strain across the surface of solid oxide fuel cell components were investigated and developed during the program. By concatenating multiple sensor elements along a single optical fiber, and applying optical signal processing methods to separate the responses of the individual sensors, the temperature and/or strain distribution across the fuel cell component may be mapped.

After considering various candidate sensor designs, a weakly-reflecting intrinsic FabryPerot interferometer (IFPI) was chosen as the most promising sensor type for the proposed application. Since temperatures are not expected to exceed $800{ }^{\circ} \mathrm{C}\left(1,472{ }^{\circ} \mathrm{F}\right)$ 
in the solid oxide fuel cells, optical fiber sensors based on doped fused silica fiber can be applied.

Techniques to write IFPI cavities in germania-doped fused silica fiber using a krypton fluoride excimer laser were developed. Efforts were made to make the fabrication process as uniform as possible, but there were significant differences observed between the particular sensors. The likely cause of this difference is the inconsistency of power and mode pattern associated with excimer lasers.

Two methods for demultiplexing serially concatenated IFPIs sensors were explored: multiplexed Michelson interferometry and optical frequency domain reflectometry. A theoretical analysis was used to determine that the number of data samples required to demultiplex a $3 \mathrm{~m}$ long string of IFPI temperature sensors using optical frequency domain reflectometry (OFDR) with $1^{\circ} \mathrm{C}\left(1.8^{\circ} \mathrm{F}\right)$ resolution was prohibitively high $\left(>10^{8}\right.$ samples). An alternate approach, multiplexed Michelson interferometry (MMI), was chosen as the best alternative for further development.

The principle of operation of MMI (also called low-coherence path matching interferometry) is to use a reference cavity which is maintained within the interrogation system to sequentially match the optical cavities of each of the transducers. By accurately monitoring output signal as a function of the size of the reference cavity, one can determine the precise size of each of the transducer cavities. A prototype interrogator was assembled, and was used to successfully interrogate three concatenated Fabry-Perot sensors on a single fiber.

The number of IFPI sensors that were multiplexed was limited by high throughput losses in the sensors, which increased the overall noise for all subsequent sensors in the chain. The overall power level which was measured returning from the sensors in the chain was approximately $2 \%$ of what could be attained with higher quality IFPIs.

A second limiting factor of the system as implemented was the optical source which was available to drive the interrogation system. The source had a much narrower spectrum than desired, resulting in a much longer coherence length. The impact of this is to make it much more difficult to localize the peaks of the resonance conditions which are indicative of having achieved the optical path match.

\section{Suggestions for Future Work}

The achievements of the program demonstrated the potential for the use of hightemperature sensors for instrumentation of coal-fired power plants and solid oxide fuel cells. However, considerable work remains to make the technology practical for these applications. Specific recommendations for future work are proposed below: 


\subsection{High-temperature optical cladding for sapphire fiber}

The results of the development of the solid-state processing of magnesium aluminate spinel for coatings on sapphire fibers to function as optical cladding demonstrated that although the claddings reduce the modal volume of the sapphire fibers, additional development is required to improve the process and the coated fiber.

The spinel coated fibers produced using the optimized processing identified through the designed experiments were dramatically stronger than earlier spinel coated fibers. However, the fragility of the fibers still necessitates some care in handling. Photomicrographs of cross-sections of coated fibers revealed small cracks in the surface of the spinel coating. When the fiber is submitted to tensile stresses, these cracks can propagate and result in fracture of the fiber. The number and concentration of surface cracks in the spinel coating were greatly reduced when optimized processing parameters were applied, but some cracks remained. Strategies for either eliminating the cracks during application and sintering of the coating, or healing the cracks after sintering, should be researched to improve the mechanical strength of the clad sapphire fibers.

One possibility for achieving high-strength spinel clad sapphire fiber may be the application of spinel coatings through vapor phase processing. During the conduct of the research on the solid-state processing of the spinel cladding, it was discovered through a review of the technical literature that the coating of sapphire lenses with magnesium aluminate spinel had been demonstrated using a vapor phase technique. No strength measurements had been conducted, but photographs of the coated lens suggested that the coatings may have been crack free, implying a high tensile strength for the coated fibers.

In this program, the length of sapphire fiber that could be coated with magnesium aluminate spinel was limited by the size of the box furnace that was used to fire the green coating on the fiber. The largest furnace available to Prime Research for this effort limited the coated fiber length to approximately six inches. To make the spinel coated sapphire fiber practical for use in coal-fired power plants, the length of coated fiber should be scaled up, ideally to one meter, through the use of larger or custom furnaces.

Optical tests of the spinel clad sapphire fiber demonstrated that the cladding was effective in reducing the numerical aperture of the fiber, and hence the modal volume, which was a desirable result. In addition, the tests showed that the attenuation of the sapphire fiber increased with the application of the cladding. The mechanism for this is unclear, but could be related to scattering losses at the interface between the sapphire core and the spinel cladding. The causes for this increased loss should be investigated with a goal of reducing the attenuation of the clad sapphire fiber.

Cross-sectional measurement of sapphire fiber dimensions before and after application of the spinel cladding demonstrated that the cladding process reduced the diameter of the pure sapphire core of the fiber, even though the coating increased the outside diameter of the coated fiber.. This appears to be the result of the diffusion of magnesium oxide from the coating into the sapphire fiber, converting some of the outer circumference of the 
sapphire to magnesium aluminate spinel. Consideration of equilibrium phase diagrams for the magnesium aluminate spinel system indicates that the conversion of sapphire to spinel will progress until excess magnesium oxide is consumed. This suggests that it may be possible to carefully choose the proportions of magnesium oxide and magnesium aluminate spinel in the slurry applied to the sapphire fiber to convert all but a few microns of the sapphire diameter to spinel. Under this scenario, the sapphire fiber would be limited to single mode propagation when the light source emits in the near infrared spectrum. This possibility should be explored, as the achievement of single moded sapphire fibers would facilitate development and application of interferometric sapphire sensors, as well as sapphire fiber Bragg gratings.

\subsection{Sapphire sensor systems}

Laboratory tests of the final version of the sapphire temperature sensors were successfully tested in the laboratory at elevated temperatures. Although the fibers connected to the Fabry-Perot cavity were multimode, the effects of intermodal interference were minimized by using a wafer with parallel faces for the Fabry-Perot cavity. Analysis has shown that, for a Fabry-Perot cavity with parallel face, intermodal interference effects are minimized. Under those conditions, the reflectance spectrum of the sensor can be successfully processed to yield a measurement of the optical path length of the Fabry-Perot cavity.

During the field test of the sapphire temperature sensor at the Babcock \& Wilcox Clean Environment Development Facility, the quality of the reflectance spectrum degraded, making the processing and demodulation of the spectrum more difficult. The spectral data revealed spurious interference fringes that appeared to be consistent with the appearance of a short undesired cavity in the sensor. The most likely source for the extra fringes is the development of a gap between the wafer and the sapphire fiber connected to the wafer. One priority for continued development of the sapphire temperature sensor should be an improved package design that will ensure that the sapphire fiber end be fixed to the sapphire wafer.

Similarly, the B\&W CEDF field test indicated that improvements are required for the sapphire strain gage. Since the Fabry-Perot cavity for that sensor is formed by an air gap between the polished ends of two sapphire fibers, parallelism of the reflectors in the Fabry-Perot cavity is not guaranteed, and is more difficult to achieve than in the sapphire temperature sensor. By potting the sapphire fiber ends in commercially available zirconia ferrules, and then polishing the ferrules like standard zirconia optical connectors, the polishing fixture ensured that the polished ends were perpendicular to the axis of the fiber within a tight tolerance. Then, the use of a split zirconia sleeve to align the two ferrules resulted in an air gap between the fibers with good parallelism between the fiber ends. However, installation of the strain gage on the CEDF simulated superheater tube required that two inconel straps be resistance welded to the tube, each to hold one of the two sapphire fibers. One of these welds failed midway during the field test, rendering the sensor inoperable for the remainder of the test. An improved weld, or an improved 
mount for the strain gage, should be a priority for continued development of the sapphire strain gage.

Due to the lengths of high-temperature fiber required for the field test, and the limited lengths of spinel-clad fiber that could be produced ( $<6$ inches), the sapphire fibers used in the tests at the CEDF were unclad and highly multimoded. Considerable emphasis was directed towards developing signal processing algorithms to make determining the cavity length from the Fabry-Perot spectrum relatively insensitive to intermodal interference. Despite this, however, noise in the sensor output often confounded the signal processing algorithm. A major step in the algorithm is the determination of the phase of a sinusoidal signal. The resulting phase can then be used to determine the cavity length through calibration or physical principles. Since an infinite number of phase values (each separated by $2 \pi$ ) yield the same sinusoid, a critical step is to determine which of those phase values resulted in the sinusoid measured by the interrogator. In general, if the physical measurand can be expected to be smoothly varying, then the algorithm can be designed to choose the phase value which closest to the previous value. The presence of noise, however, can result in undesired phase jumps or missed phase jumps, resulting in erroneous sensor outputs.

The presence of noise in the temperature sensor output, apparently resulting from an extraneous cavity, resulted in just such undesired and/or missed phase jumps. Continued development of the signal processing algorithm, to improve its immunity to noise, is required to make the interrogators for the sapphire sensors practical for use in power plants.

In the case of the sapphire strain gage, undesired and/or missed phase jumps also contributed to inaccuracies in the sensor system output, although for that sensor the cause appeared to be due to the low sampling rate of the data collection. The rate of temperature changes of the simulated superheater tube to which the sensor was mounted was much higher than anticipated. As a result, jumps in phase that occurred between data samples were missed, leading to inaccuracies in the sensor output. This highlights the need to carefully choose the sampling rate to ensure that sufficient data is obtained to detect all phase jumps.

The commercially available data acquisition software used to record the output spectra of the temperature sensor failed during the field test. Clearly, for critical applications such as power plant monitoring, more robust data acquisition software is required.

Due to the complexity of the signal processing employed, the algorithms used for processing the data ran on a personal computer separate from the interrogator hardware. While implementing the algorithm on the internal processor in the interrogator could be achieved, it was beyond the scope of this program. However, for industrial applications, the algorithm should run on the interrogator processor, to eliminate the need for a separate computer. 


\subsection{High-temperature strain gages for solid oxide fuel cells}

The research into distributed temperature sensors for solid oxide fuel cells (SOFCs) demonstrated the feasibility of intrinsic Fabry-Perot interferometer (IFPI) strain gages written in germanium doped silica fiber with an excimer laser, and of the use of multiplexed Michelson interferometry to interrogate serially concatenated IFPI sensors. However, the total number of IFPI sensors that could successfully be interrogated was limted to three by the high losses in the IFPI sensors. Further development of this concept will require additional work to improve sensor fabrication and reduce the sensor insertion losses.

In addition, the measurement resolution achievable with the multiplexed Michelson interferometry system depends on the coherence length of the optical source used. Achieving a high resolution requires a source with low coherence. In general, though, low cost, low coherence sources, such as white light sources, are not well suited for injecting high power into optical fibers. Despite an extensive search, an affordable high power, low coherence source was not found for this effort. Further development of this multiplexed Michelson interferometry system with IFPI sensors will require the identification and use of a better source. 
Appendices 


\section{Appendix A}

In this Appendix, the original cross-sectional pictures and the average responses of 17 samples coated with magnesium aluminate spinel are listed from Figure A-2 to Figure A18 follow..

\section{A-2: Sample 2}

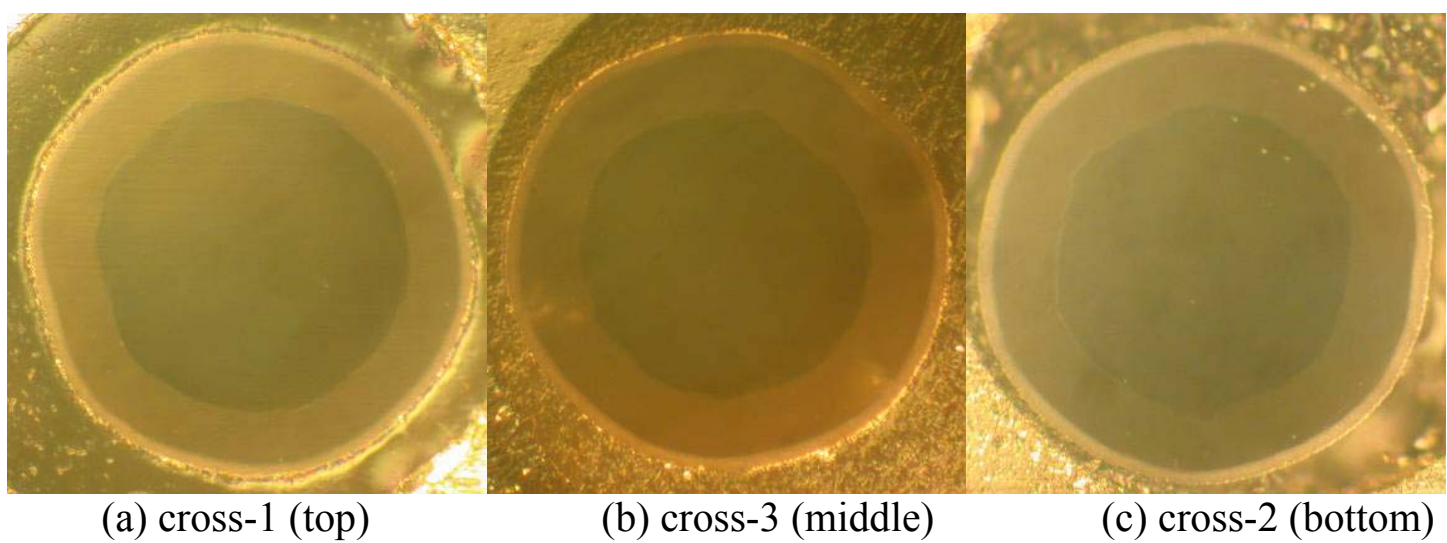

Figure A-2 (a) to (c): the 3 cross sections polished and analyzed for sample 2, and (d): the results of all 13 responses for sample 2.

\begin{tabular}{|c|c|c|c|c|c|c|c|c|c|c|c|c|c|}
\hline & $\begin{array}{c}\mathbf{G 1} \\
\text { (um) }\end{array}$ & $\begin{array}{c}\mathbf{G} 2 \\
\text { (um) }\end{array}$ & $\begin{array}{c}\text { G3 } \\
\text { (um) }\end{array}$ & $\begin{array}{c}\mathbf{G} 4 \\
\text { (um) }\end{array}$ & $\begin{array}{c}\text { G5 } \\
\text { (um) }\end{array}$ & $\begin{array}{c}\mathbf{G 6} \\
(\mathrm{um})\end{array}$ & $\begin{array}{c}\text { M1 } \\
(\mathrm{mm})\end{array}$ & $\begin{array}{c}\text { M2 } \\
\left(\mathrm{um}^{\wedge} 2\right)\end{array}$ & $\begin{array}{c}\text { M3 } \\
\text { (um) }\end{array}$ & $\begin{array}{c}\text { M4 } \\
\text { (no } \\
\text { unit) }\end{array}$ & $\begin{array}{c}01 \\
\text { (no } \\
\text { unit) }\end{array}$ & $\begin{array}{c}\mathrm{O} 2 \\
\text { (no } \\
\text { unit) }\end{array}$ & $\begin{array}{c}\mathrm{O} 3 \\
(\mathrm{~dB})\end{array}$ \\
\hline 2 & 89.9 & 22.1 & 3.9 & 1.6 & 134.3 & 0.81 & 0.71 & 0.0 & 0.0 & 0.00 & 0.40 & 0.046 & 8.4 \\
\hline
\end{tabular}

Table A-2: The results of all 13 responses for sample 2

\section{$\underline{\text { A-3: Sample } 3}$}

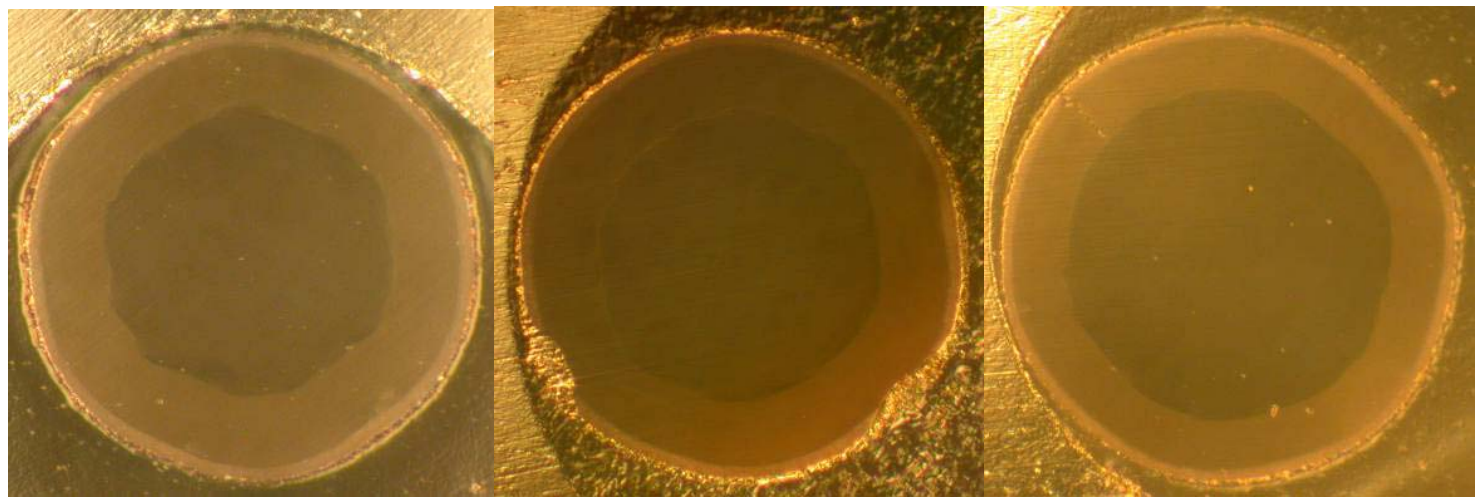

(a) cross-1 (top)

(b) cross-3 (middle)

(c) cross-2 (bottom)

Figure A-3 (a) to (c): the 3 cross sections polished and analyzed for sample 3, and (d): the results of all 13 responses for sample 3 . 


\begin{tabular}{|c|c|c|c|c|c|c|c|c|c|c|c|c|c|}
\hline & $\begin{array}{c}\mathbf{G} 1 \\
\text { (um) }\end{array}$ & $\begin{array}{c}\mathbf{G 2} \\
\text { (um) }\end{array}$ & $\begin{array}{c}\text { G3 } \\
\text { (um) }\end{array}$ & $\begin{array}{c}\mathbf{G 4} \\
\text { (um) }\end{array}$ & $\begin{array}{c}\text { G5 } \\
\text { (um) }\end{array}$ & $\begin{array}{c}\text { G6 } \\
\text { (um) }\end{array}$ & $\begin{array}{c}\text { M1 } \\
(\mathrm{mm})\end{array}$ & $\begin{array}{c}\text { M2 } \\
\left(u^{\wedge} 2\right)\end{array}$ & $\begin{array}{l}\text { M3 } \\
\text { (um) }\end{array}$ & $\begin{array}{l}\text { M4 } \\
\text { (no } \\
\text { unit) }\end{array}$ & $\begin{array}{l}01 \\
\text { (no } \\
\text { unit) }\end{array}$ & $\begin{array}{c}\mathrm{O} 2 \\
\text { (no } \\
\text { unit) }\end{array}$ & $\begin{array}{c}\mathrm{O} 3 \\
(\mathrm{~dB})\end{array}$ \\
\hline 3 & 86.8 & 21.4 & 3.4 & 2.1 & 131.1 & 0.67 & 0.84 & 3.9 & 6.0 & 0.33 & 0.56 & 0.055 & 7.2 \\
\hline
\end{tabular}

Table A-3: The results of all 13 responses for sample 3

\section{A-4: Sample 4}

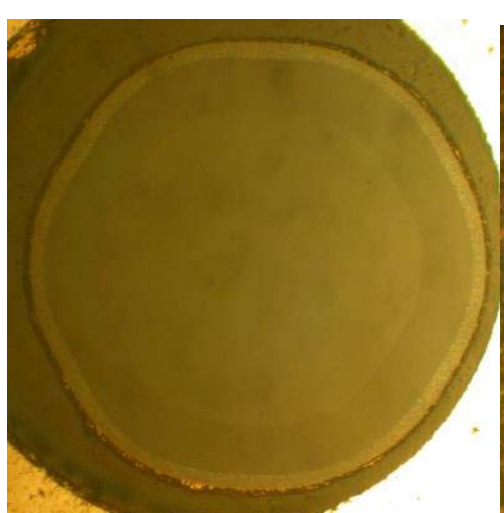

(a) cross-1 (top)

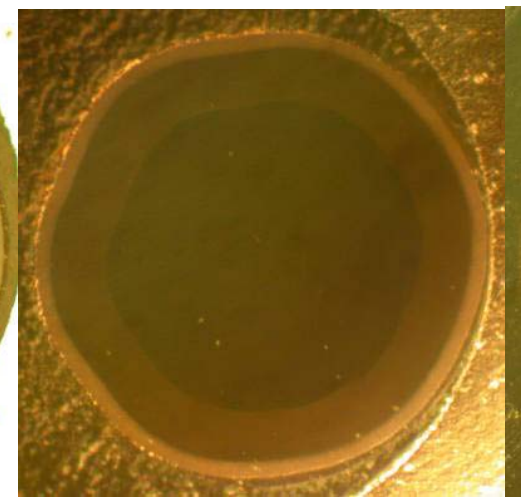

(b) cross-3 (middle)

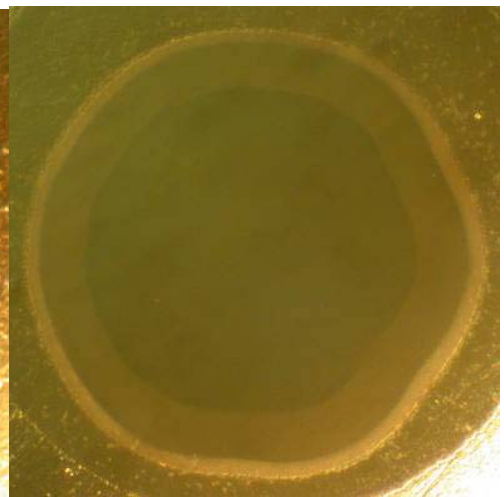

(c) cross-2 (bottom)

Figure A-4 (a) to (c): the 3 cross sections polished and analyzed for sample 4, and (d): the results of all 13 responses for sample 4.

\begin{tabular}{|c|c|c|c|c|c|c|c|c|c|c|c|c|c|}
\hline & $\begin{array}{c}\mathbf{G 1} \\
\text { (um) }\end{array}$ & $\begin{array}{c}\mathbf{G 2} \\
\text { (um) }\end{array}$ & $\begin{array}{c}\text { G3 } \\
\text { (um) }\end{array}$ & $\begin{array}{c}\mathbf{G 4} \\
\text { (um) }\end{array}$ & $\begin{array}{c}\text { G5 } \\
\text { (um) }\end{array}$ & $\begin{array}{c}\mathbf{G 6} \\
\text { (um) }\end{array}$ & $\begin{array}{c}\text { M1 } \\
(\mathrm{mm})\end{array}$ & $\begin{array}{c}\text { M2 } \\
\left(u^{\wedge} 2\right)\end{array}$ & $\begin{array}{c}\text { M3 } \\
\text { (um) }\end{array}$ & $\begin{array}{l}\text { M4 } \\
\text { (no } \\
\text { unit) }\end{array}$ & $\begin{array}{l}\text { O1 } \\
\text { (no } \\
\text { unit) }\end{array}$ & $\begin{array}{c}\mathrm{O} 2 \\
\text { (no } \\
\text { unit) }\end{array}$ & $\begin{array}{c}\mathrm{O} 3 \\
\text { (dB) }\end{array}$ \\
\hline 4 & 94.4 & 20.1 & 4.6 & 1.5 & 134.4 & 1.22 & 0.88 & 0.0 & 0.0 & 0.00 & 0.24 & 0.068 & 3.1 \\
\hline
\end{tabular}

Table A-4: The results of all 13 responses for sample 4

\section{$\underline{\text { A-5: Sample } 5}$}

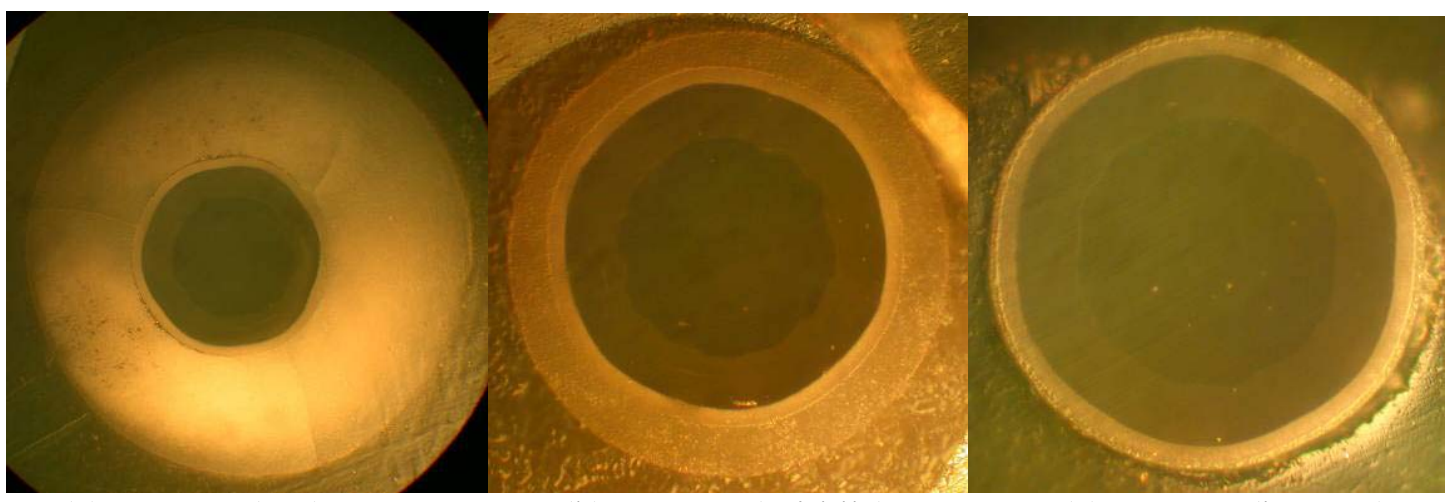
(a) cross-1 (top)
(b) cross-3 (middle)
(c) cross-2 (bottom)

Figure A-5 (a) to (c): the 3 cross sections polished and analyzed for sample $5_{\mathrm{R} 1}$ 


\begin{tabular}{|c|c|c|c|c|c|c|c|c|c|c|c|c|c|}
\hline & $\begin{array}{c}\mathbf{G 1} \\
\text { (um) }\end{array}$ & $\begin{array}{c}\mathbf{G 2} \\
\text { (um) }\end{array}$ & $\begin{array}{c}\text { G3 } \\
\text { (um) }\end{array}$ & $\begin{array}{c}\mathbf{G 4} \\
\text { (um) }\end{array}$ & $\begin{array}{c}\text { G5 } \\
\text { (um) }\end{array}$ & $\begin{array}{c}\mathbf{G 6} \\
\text { (um) }\end{array}$ & $\begin{array}{c}\text { M1 } \\
\text { (mm) }\end{array}$ & $\begin{array}{c}\text { M2 } \\
\text { (um^2) }\end{array}$ & $\begin{array}{c}\text { M3 } \\
\text { (um) }\end{array}$ & $\begin{array}{c}\text { M4 } \\
\text { (no } \\
\text { unit) }\end{array}$ & $\begin{array}{c}\text { O1 } \\
\text { (no } \\
\text { unit) }\end{array}$ & $\begin{array}{c}\mathrm{O} 2 \\
\text { (no } \\
\text { unit) }\end{array}$ & $\begin{array}{c}\mathrm{O} 3 \\
(\mathrm{~dB})\end{array}$ \\
\hline $\begin{array}{c}5 \\
\text { (R1) }\end{array}$ & 79.4 & 24.7 & 5.8 & 33.0 & 194.1 & 0.76 & 0.73 & 0.0 & 0.0 & 0.00 & 0.59 & 0.059 & 4.5 \\
\hline
\end{tabular}

Table A-5: The results of all 13 responses for sample $5_{R 1}(R 1$ indicates that the results are from a repeated sample prepared using the same conditions)

It is to be noted that sample $5_{\mathrm{R} 1}$ is the first sample showing a significant "carrot" shape. The skin thickness, G4, of the cross section at the top of the fiber is $82.5 \mu \mathrm{m}$. The thickness decreases to $13.9 \mu \mathrm{m}$ at the middle of fiber, and to $2.5 \mu \mathrm{m}$ at the bottom of fiber

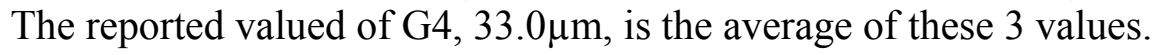

\section{A-6: Sample 6}

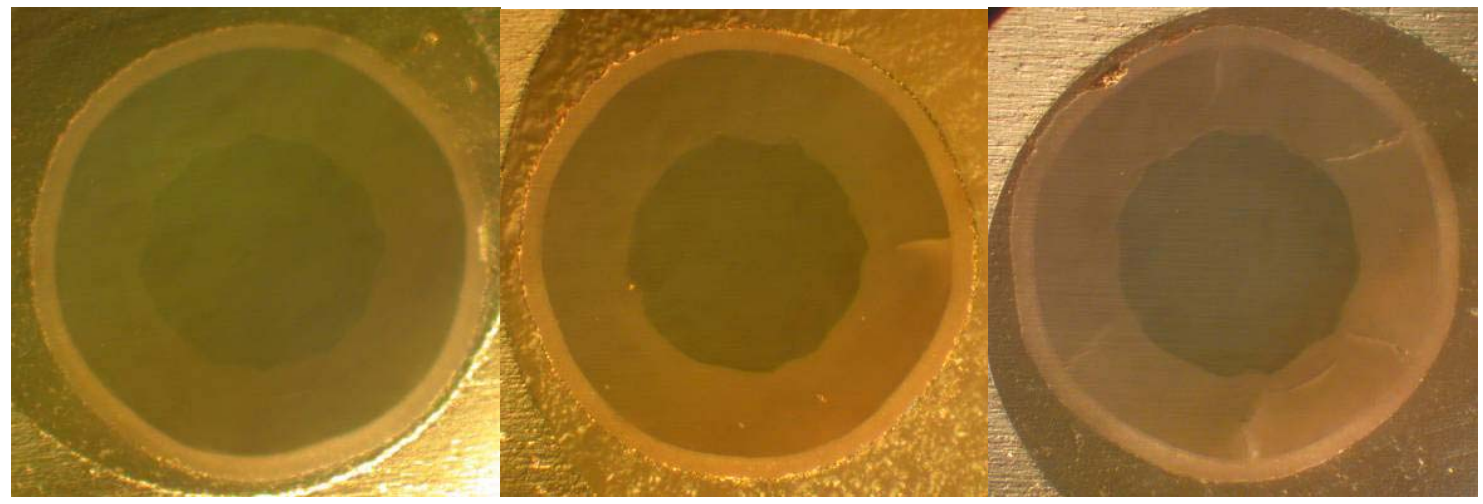
(a) cross-1 (top)
(b) cross-3 (middle)
(c) cross-2 (bottom)

Figure A-6 (a) to (c): the 3 cross sections polished and analyzed for sample $6_{\mathrm{R} 1}$

\begin{tabular}{|c|c|c|c|c|c|c|c|c|c|c|c|c|c|}
\hline & $\begin{array}{c}\text { G1 } \\
(\mathrm{um})\end{array}$ & $\begin{array}{c}\text { G2 } \\
(\mathrm{um})\end{array}$ & $\begin{array}{c}\text { G3 } \\
(\mathrm{um})\end{array}$ & $\begin{array}{c}\text { G4 } \\
(\mathrm{um})\end{array}$ & $\begin{array}{c}\text { G5 } \\
(\mathrm{um})\end{array}$ & $\begin{array}{c}\text { G6 } \\
(\mathrm{um})\end{array}$ & $\begin{array}{c}\text { M1 } \\
(\mathrm{mm})\end{array}$ & $\begin{array}{c}\text { M2 } \\
\left(\mathrm{um} \mathrm{m}^{\wedge}\right)\end{array}$ & $\begin{array}{c}\text { M3 } \\
(\mathrm{um})\end{array}$ & $\mathbf{M} 4$ & 01 & O2 & $\begin{array}{c}\text { O3 } \\
(\mathrm{dB})\end{array}$ \\
\hline $\begin{array}{c}6 \\
\text { (R1) }\end{array}$ & 71.7 & 32.9 & 6.2 & 1.3 & 138.4 & 0.90 & 0.41 & 28.4 & 24.1 & 2.00 & 1.30 & 0.052 & 5.1 \\
\hline
\end{tabular}

Table A-6: The results of all 13 responses for sample $6_{R 1}(R 1$ indicates that the results are from a repeated sample prepared using the same conditions) 


\section{A-7: Sample 7}

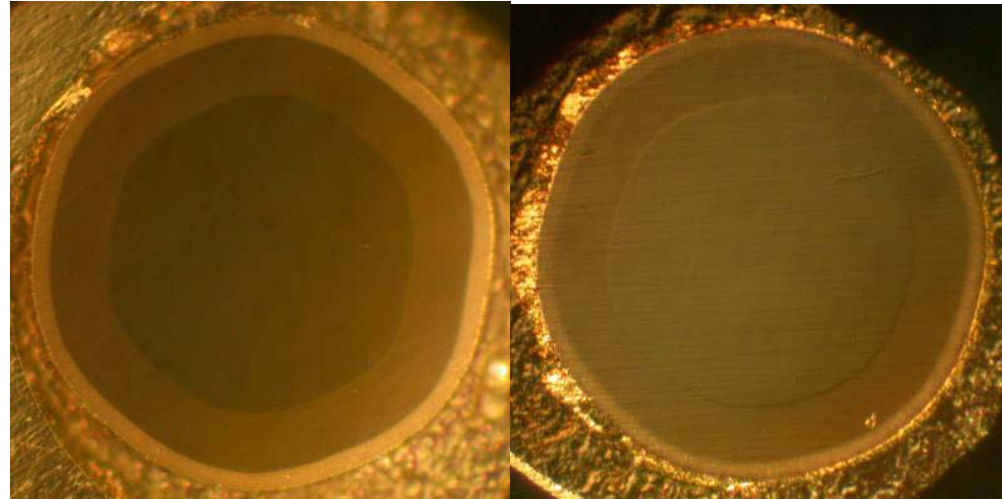

(a) cross-1 (top) (b) cross-3 (middle)

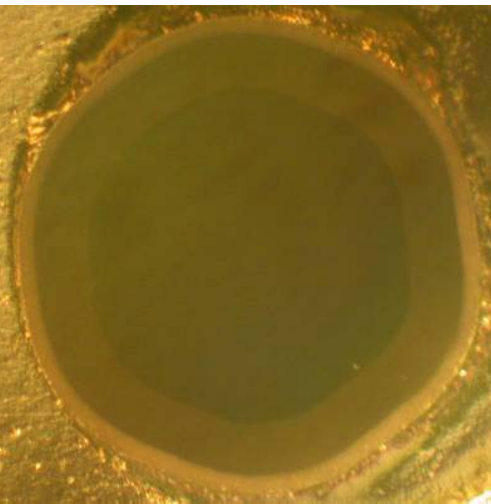

(c) cross-2 (bottom)

Figure A-7 (a) to (c): the 3 cross sections polished and analyzed for sample 7

\begin{tabular}{|c|c|c|c|c|c|c|c|c|c|c|c|c|c|}
\hline & $\begin{array}{c}\mathbf{G} 1 \\
\text { (um) }\end{array}$ & $\begin{array}{c}\mathbf{G} 2 \\
\text { (um) }\end{array}$ & $\begin{array}{c}\text { G3 } \\
\text { (um) }\end{array}$ & $\begin{array}{c}\mathbf{G 4} \\
\text { (um) }\end{array}$ & $\begin{array}{c}\mathbf{G 5} \\
\text { (um) }\end{array}$ & $\begin{array}{c}\mathbf{G 6} \\
\text { (um) }\end{array}$ & $\begin{array}{c}\text { M1 } \\
(\mathrm{mm})\end{array}$ & $\begin{array}{c}\text { M2 } \\
\left(\mathrm{um}^{\wedge} 2\right)\end{array}$ & $\begin{array}{c}\text { M3 } \\
\text { (um) }\end{array}$ & M4 & 01 & $\mathrm{O} 2$ & $\begin{array}{c}\mathrm{O} 3 \\
(\mathrm{~dB})\end{array}$ \\
\hline 7 & 92.8 & 21.6 & 4.9 & 2.5 & 138.9 & 0.82 & 0.83 & 0.0 & 0.0 & 0.00 & 0.02 & 0.063 & 4.9 \\
\hline
\end{tabular}

Table A-7: The results of all 13 responses for sample 7

\section{A-8: Sample 8}

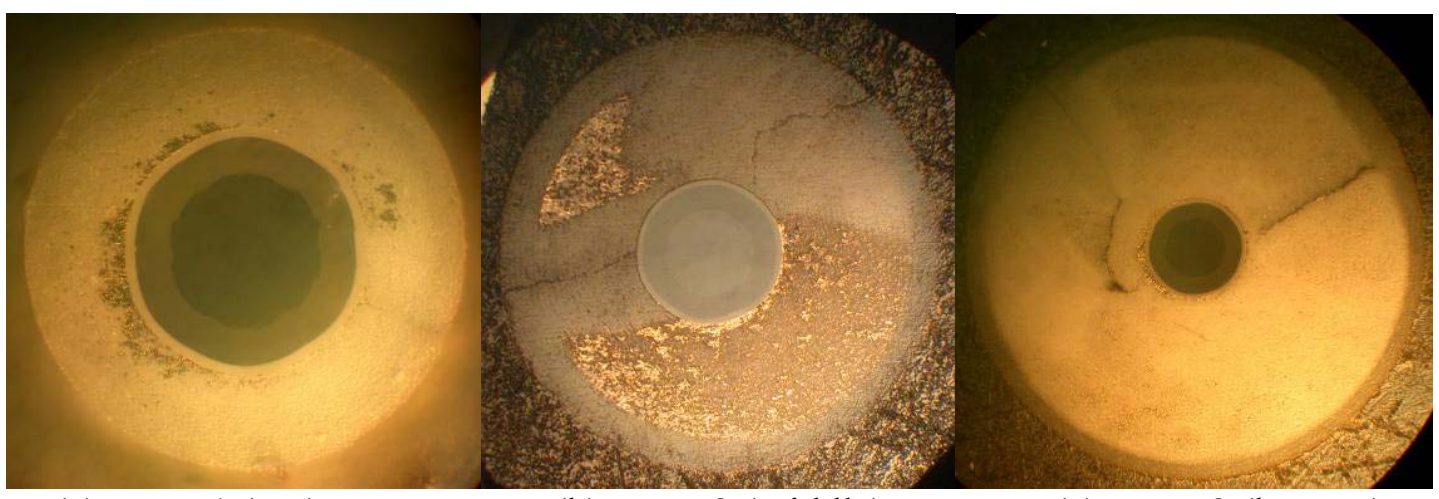
(a) cross-1 (top)
(b) cross-3 (middle)
(c) cross-2 (bottom)

Figure A-8 (a) to (c): the 3 cross sections polished and analyzed for sample $8_{\mathrm{R} 1}$

\begin{tabular}{|c|c|c|c|c|c|c|c|c|c|c|c|c|c|}
\hline & $\begin{array}{c}\text { G1 } \\
(\mathrm{um})\end{array}$ & $\begin{array}{c}\text { G2 } \\
(\mathrm{um})\end{array}$ & $\begin{array}{c}\text { G3 } \\
(\mathrm{um})\end{array}$ & $\begin{array}{c}\text { G4 } \\
(\mathrm{um})\end{array}$ & $\begin{array}{c}\text { G5 } \\
(\mathrm{um})\end{array}$ & $\begin{array}{c}\text { G6 } \\
(\mathrm{um})\end{array}$ & $\begin{array}{c}\text { M1 } \\
(\mathrm{mm})\end{array}$ & $\begin{array}{c}\text { M2 } \\
\left(\mathrm{um} \mathrm{m}^{\wedge}\right)\end{array}$ & $\begin{array}{c}\text { M3 } \\
(\mathrm{um})\end{array}$ & M4 & 01 & O2 & $\begin{array}{c}\text { O3 } \\
(\mathrm{dB})\end{array}$ \\
\hline $\begin{array}{c}8 \\
\text { (R1) }\end{array}$ & 84.7 & 27.4 & 5.8 & 150.3 & 442.1 & 1.18 & 0.78 & 0.0 & 0.0 & 0.00 & 1.13 & 0.096 & 24 \\
\hline
\end{tabular}

Table A-8: The results of all 13 responses for sample $8_{\mathrm{R} 1}$ 


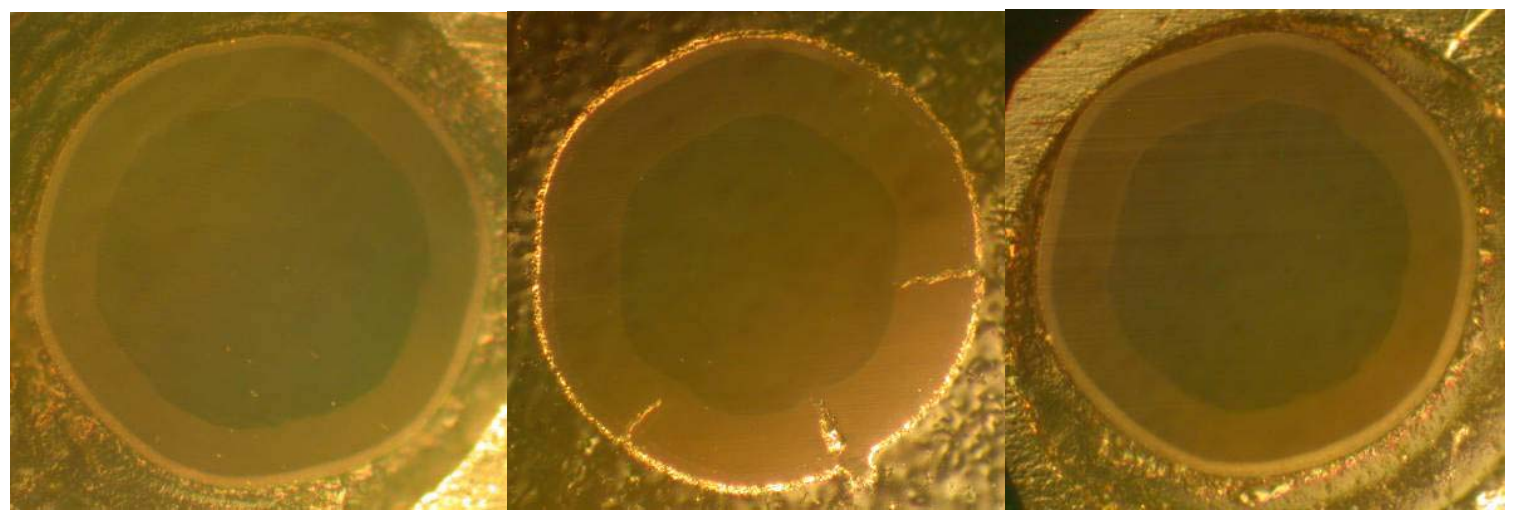
(a) cross-1 (top)
(b) cross-3 (middle)
(c) cross-2 (bottom)

Figure A-9 (a) to (c): the 3 cross sections polished and analyzed for sample $9_{\mathrm{R} 1}$

\begin{tabular}{|c|c|c|c|c|c|c|c|c|c|c|c|c|c|}
\hline & $\begin{array}{c}\mathbf{G 1} \\
\text { (um) }\end{array}$ & $\begin{array}{c}\mathbf{G} 2 \\
\text { (um) }\end{array}$ & $\begin{array}{c}\text { G3 } \\
\text { (um) }\end{array}$ & $\begin{array}{c}\mathbf{G} 4 \\
\text { (um) }\end{array}$ & $\begin{array}{c}\text { G5 } \\
\text { (um) }\end{array}$ & $\begin{array}{c}\mathbf{G 6} \\
\text { (um) }\end{array}$ & $\begin{array}{c}\text { M1 } \\
(\mathrm{mm})\end{array}$ & $\begin{array}{c}\text { M2 } \\
\left(u m^{\wedge} 2\right)\end{array}$ & $\begin{array}{c}\text { M3 } \\
\text { (um) }\end{array}$ & M4 & 01 & $\mathrm{O} 2$ & $\begin{array}{c}\mathrm{O} 3 \\
(\mathrm{~dB})\end{array}$ \\
\hline $\begin{array}{c}9 \\
\text { (R1) }\end{array}$ & 93.5 & 21.1 & 4.0 & 1.5 & 137.1 & 0.92 & 0.46 & 50.5 & 8.5 & 1.00 & 0.59 & 0.087 & 5.2 \\
\hline
\end{tabular}

Table A-9: The results of all 13 responses for sample $9_{\mathrm{R} 1}$

\section{A-10: Sample 10}

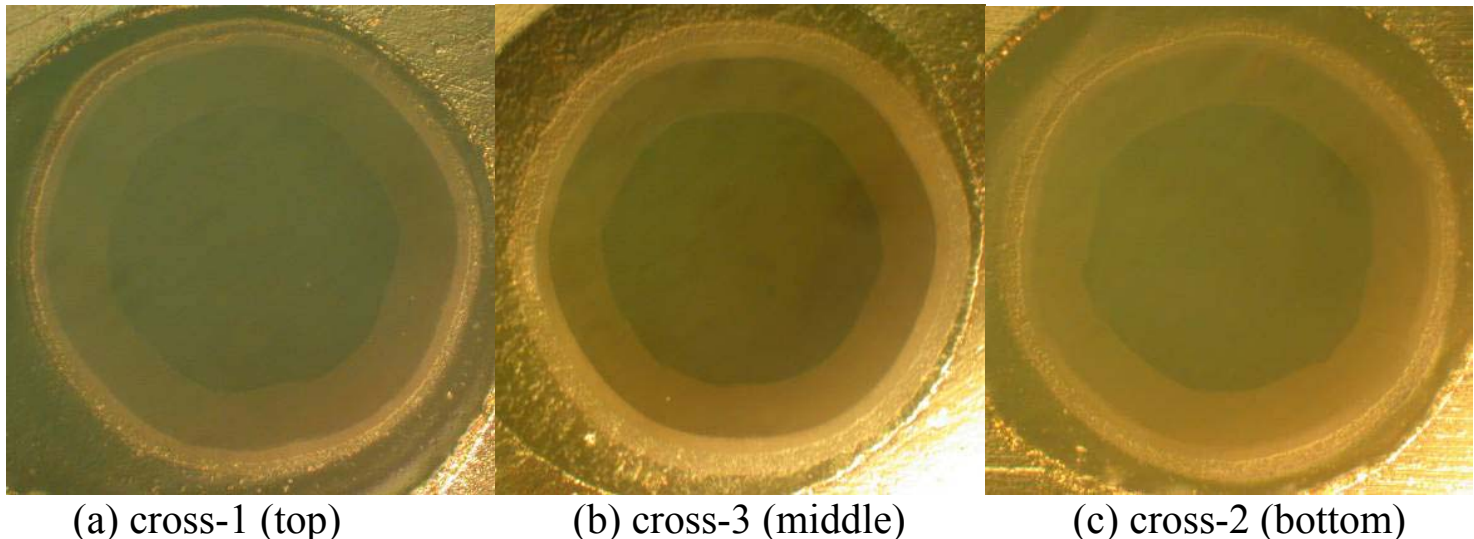

Figure A-10 (a) to (c): the 3 cross sections polished and analyzed for sample 10

\begin{tabular}{|c|c|c|c|c|c|c|c|c|c|c|c|c|c|}
\hline & $\begin{array}{c}\text { G1 } \\
(\mathrm{um})\end{array}$ & $\begin{array}{c}\mathbf{G} 2 \\
(\mathrm{um})\end{array}$ & $\begin{array}{c}\mathbf{G} \text { (um) } \\
(\mathrm{um})\end{array}$ & $\begin{array}{c}\mathbf{G} 4 \\
(\mathrm{um})\end{array}$ & $\begin{array}{c}\text { G5 } \\
(\mathrm{um})\end{array}$ & $\begin{array}{c}\text { G6 } \\
(\mathrm{um})\end{array}$ & $\begin{array}{c}\text { M1 } \\
(\mathrm{mm})\end{array}$ & $\begin{array}{c}\text { M2 } \\
\left(\mathrm{um} \mathrm{m}^{\wedge}\right)\end{array}$ & $\begin{array}{c}\text { M3 } \\
(\mathrm{um})\end{array}$ & M4 & 01 & O2 & $\begin{array}{c}\text { O3 } \\
(\mathrm{dB})\end{array}$ \\
\hline 10 & 90.4 & 22.5 & 4.4 & 5.5 & 140.2 & 0.60 & 0.67 & 0.0 & 0.0 & 0.00 & 0.41 & 0.052 & 2.5 \\
\hline
\end{tabular}

Table 10: The results of all 13 responses for sample 10 


\section{A-11: Sample 11}

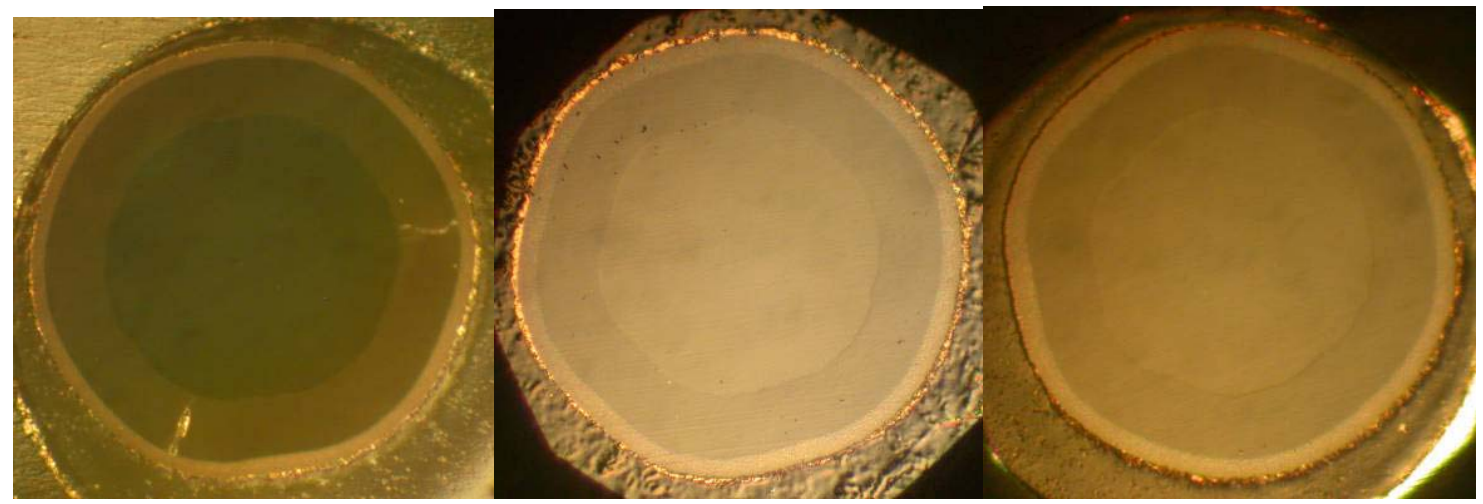
(a) cross-1 (top)
(b) cross-3 (middle)
(c) cross-2 (bottom)

Figure A-11 (a) to (c): the 3 cross sections polished and analyzed for sample $11_{\mathrm{R} 1}$

\begin{tabular}{|c|c|c|c|c|c|c|c|c|c|c|c|c|c|}
\hline & $\begin{array}{c}\text { G1 } \\
\text { (um) }\end{array}$ & $\begin{array}{c}\text { G2 } \\
\text { (um) }\end{array}$ & $\begin{array}{c}\text { G3 } \\
\text { (um) }\end{array}$ & $\begin{array}{c}\text { G4 } \\
\text { (um) }\end{array}$ & $\begin{array}{c}\text { G5 } \\
\text { (um) }\end{array}$ & $\begin{array}{c}\text { G6 } \\
\text { (um) }\end{array}$ & $\begin{array}{c}\text { M1 } \\
\text { (mm) }\end{array}$ & $\begin{array}{c}\text { M2 } \\
\text { (um²) }\end{array}$ & $\begin{array}{c}\text { M3 } \\
\text { (um) }\end{array}$ & M4 & O1 & O2 & $\begin{array}{c}\text { O3 } \\
\text { (dB) }\end{array}$ \\
\hline $\begin{array}{c}11 \\
\text { (R1) }\end{array}$ & 89.3 & 25.2 & 5.5 & 2.1 & 141.1 & 0.50 & 0.59 & 17.6 & 6.7 & 0.67 & 0.44 & 0.061 & 7.2 \\
\hline
\end{tabular}

Table A-11. The results of all 13 responses for sample $11_{R 1}$

\section{A-12: Sample 12}

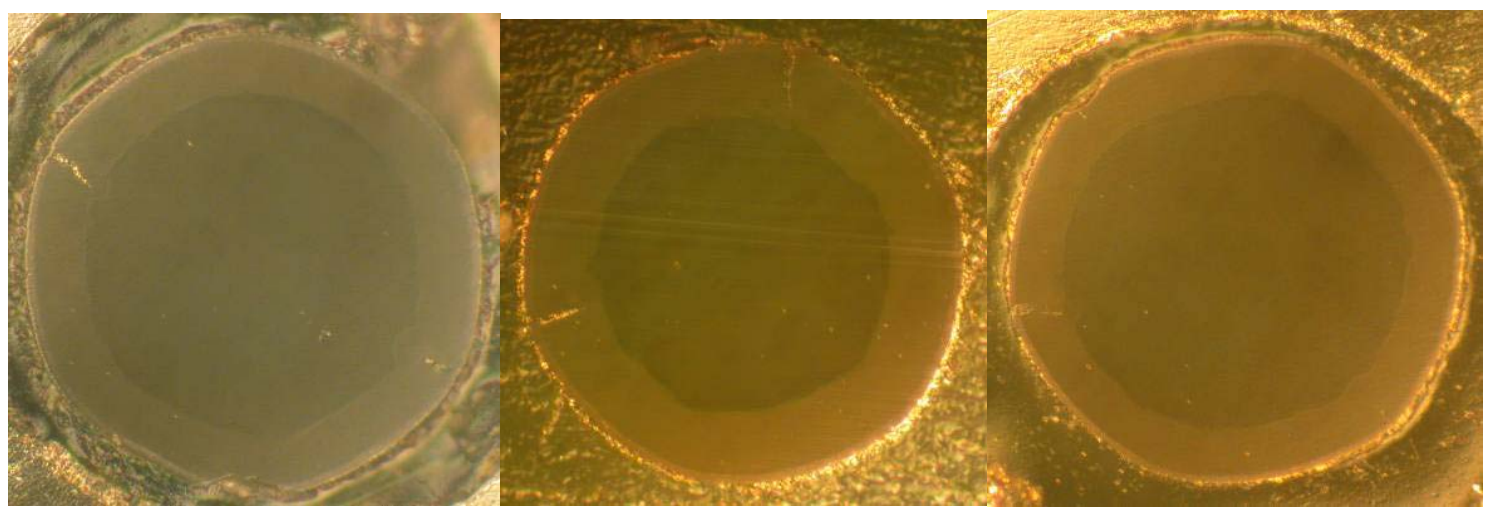

(a) cross-1 (top)

(b) cross-3 (middle)

(c) cross-2 (bottom)

Figure A-12 (a) to (c): the 3 cross sections polished and analyzed for sample 12

\begin{tabular}{|c|c|c|c|c|c|c|c|c|c|c|c|c|c|}
\hline & $\begin{array}{c}\text { G1 } \\
\text { (um) }\end{array}$ & $\begin{array}{c}\text { G2 } \\
\text { (um) }\end{array}$ & $\begin{array}{c}\text { G3 } \\
\text { (um) }\end{array}$ & $\begin{array}{c}\text { G4 } \\
\text { (um) }\end{array}$ & $\begin{array}{c}\text { G5 } \\
\text { (um) }\end{array}$ & $\begin{array}{c}\text { G6 } \\
\text { (um) }\end{array}$ & $\begin{array}{c}\text { M1 } \\
\text { (mm) }\end{array}$ & $\begin{array}{c}\text { M2 } \\
\text { (um²) }\end{array}$ & $\begin{array}{c}\text { M3 } \\
\text { (um) }\end{array}$ & M4 & O1 & O2 & $\begin{array}{c}\text { O3 } \\
\text { (dB) }\end{array}$ \\
\hline 12 & 97.6 & 19.2 & 2.6 & 2.4 & 135.7 & 0.61 & 0.73 & 35.6 & 18.9 & 1.67 & 0.70 & 0.089 & 10.5 \\
\hline
\end{tabular}

Table A-12: The results of all 13 responses for sample 12 


\section{A-13: Sample 13}

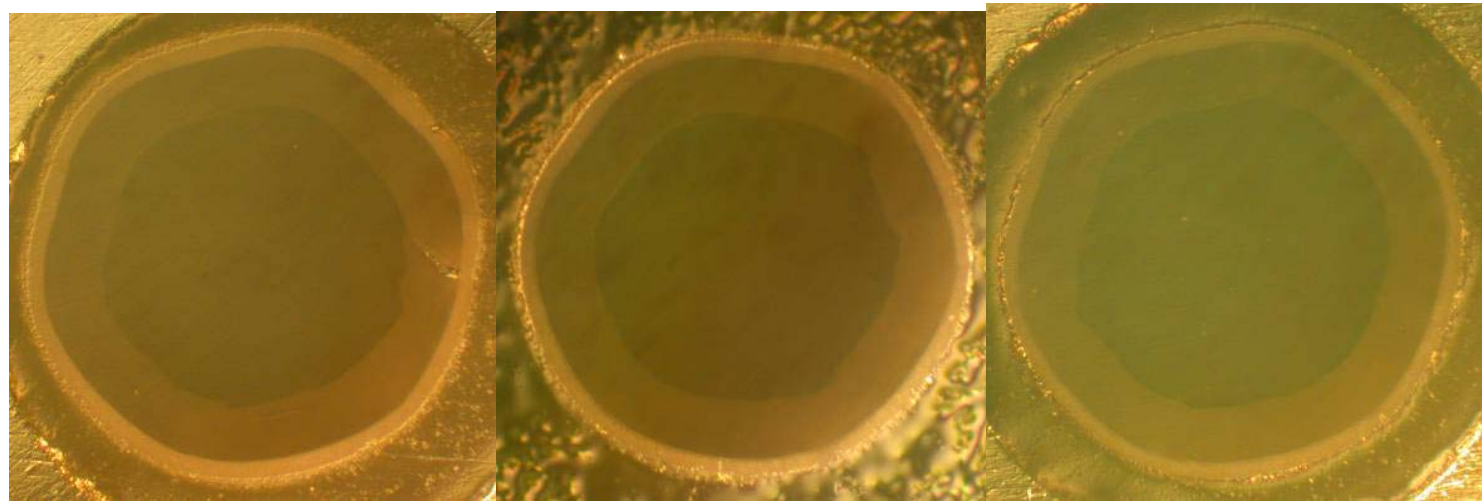
(a) cross-1 (top)
(b) cross-3 (middle)
(c) cross-2 (bottom)

Figure A-13 (a) to (c): the 3 cross sections polished and analyzed for sample 13

\begin{tabular}{|c|c|c|c|c|c|c|c|c|c|c|c|c|c|}
\hline & $\begin{array}{c}\text { G1 } \\
(\mathrm{um})\end{array}$ & $\begin{array}{c}\mathbf{G} 2 \\
(\mathrm{um})\end{array}$ & $\begin{array}{c}\mathbf{G} \text { (um) } \\
(\mathrm{um})\end{array}$ & $\begin{array}{c}\text { G4 } \\
(\mathrm{um})\end{array}$ & $\begin{array}{c}\text { G5 } \\
(\mathrm{um})\end{array}$ & $\begin{array}{c}\text { G6 } \\
(\mathrm{um})\end{array}$ & $\begin{array}{c}\text { M1 } \\
(\mathrm{mm})\end{array}$ & $\begin{array}{c}\text { M2 } \\
\left(\mathrm{um} \mathrm{m}^{\wedge}\right)\end{array}$ & $\begin{array}{c}\text { M3 } \\
(\mathrm{um})\end{array}$ & M4 & 01 & O2 & $\begin{array}{c}\text { O3 } \\
(\mathrm{dB})\end{array}$ \\
\hline 13 & 92.4 & 22.6 & 4.9 & 2.6 & 137.8 & 0.93 & 1.08 & 9.3 & 7.1 & 0.33 & 0.65 & 0.152 & 5.3 \\
\hline
\end{tabular}

Table A-13: The results of all 13 responses for sample 13

\section{A-14: Sample 14}

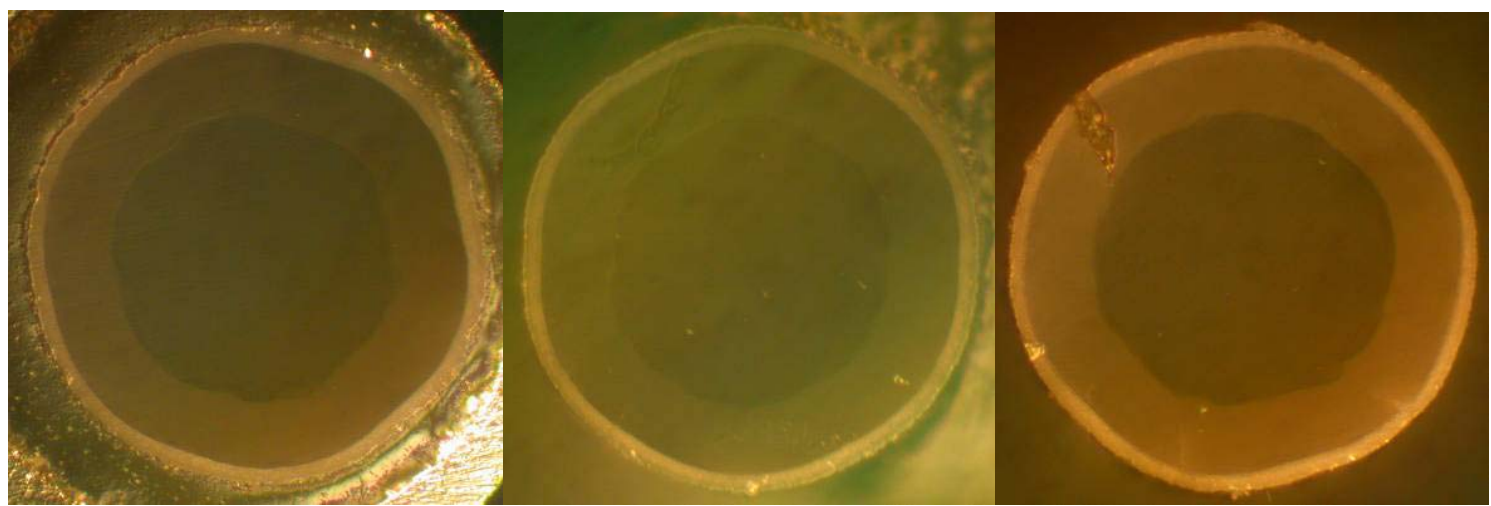

(a) cross-1 (top)

(b) cross-3 (middle)

(c) cross-2 (bottom)

Figure A-14 (a) to (c): the 3 cross sections polished and analyzed for sample 14

\begin{tabular}{|c|c|c|c|c|c|c|c|c|c|c|c|c|c|}
\hline & $\begin{array}{c}\text { G1 } \\
(\mathrm{um})\end{array}$ & $\begin{array}{c}\mathbf{G} 2 \\
(\mathrm{um})\end{array}$ & $\begin{array}{c}\text { G3 } \\
(\mathrm{um})\end{array}$ & $\begin{array}{c}\text { G4 } \\
(\mathrm{um})\end{array}$ & $\begin{array}{c}\text { G5 } \\
(\mathrm{um})\end{array}$ & $\begin{array}{c}\text { G6 } \\
(\mathrm{um})\end{array}$ & $\begin{array}{c}\text { M1 } \\
(\mathrm{mm})\end{array}$ & $\begin{array}{c}\text { M2 } \\
\left(\mathrm{um} \mathrm{m}^{\wedge} 2\right)\end{array}$ & $\begin{array}{c}\text { M3 } \\
(\mathrm{um})\end{array}$ & M4 & O1 & O2 & $\begin{array}{c}\text { O3 } \\
(\mathrm{dB})\end{array}$ \\
\hline 14 & 87.2 & 25.8 & 4.6 & 1.4 & 138.6 & 0.72 & 0.65 & 108.3 & 25.9 & 1.33 & 0.73 & 0.107 & 12 \\
\hline
\end{tabular}

Table A-14: The results of all 13 responses for sample 14 


\section{A-15: Sample 15}

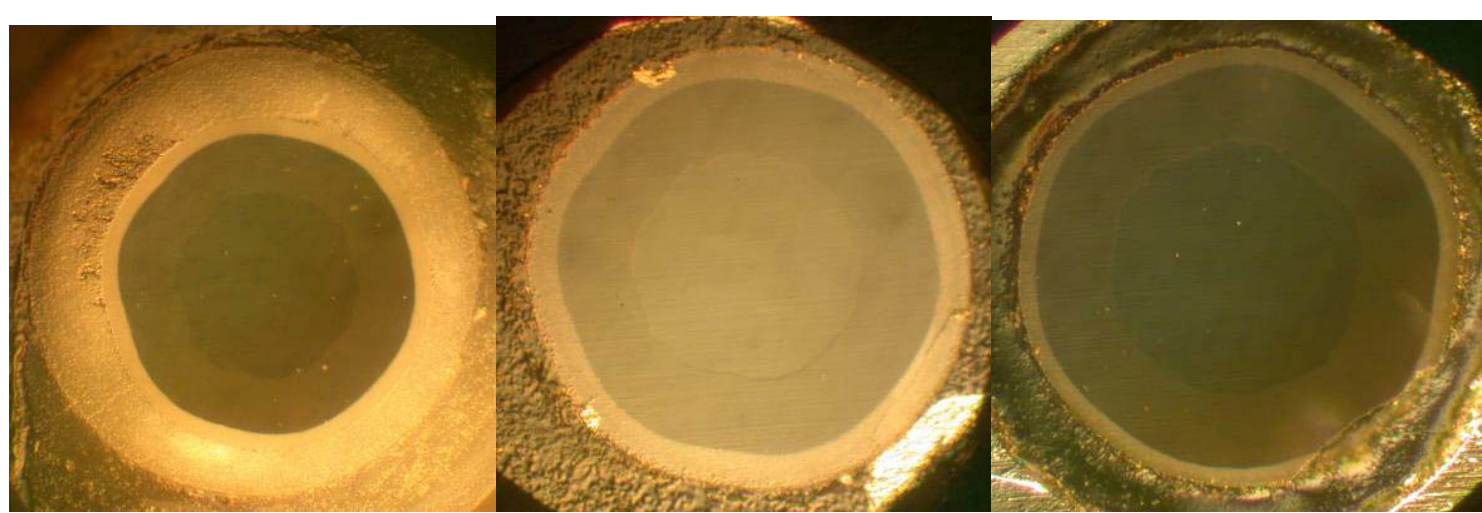

(a) cross-1 (top)

(b) cross-3 (middle)

(c) cross-2 (bottom)

Figure A-15 (a) to (c): the 3 cross sections polished and analyzed for sample $1_{\mathrm{R} 1}$

\begin{tabular}{|c|c|c|c|c|c|c|c|c|c|c|c|c|c|}
\hline & $\begin{array}{c}\text { G1 } \\
(\mathrm{um})\end{array}$ & $\begin{array}{c}\text { G2 } \\
(\mathrm{um})\end{array}$ & $\begin{array}{c}\text { G3 } \\
(\mathrm{um})\end{array}$ & $\begin{array}{c}\text { G4 } \\
(\mathrm{um})\end{array}$ & $\begin{array}{c}\text { G5 } \\
(\mathrm{um})\end{array}$ & $\begin{array}{c}\text { G6 } \\
(\mathrm{um})\end{array}$ & $\begin{array}{c}\text { M1 } \\
(\mathrm{mm})\end{array}$ & $\begin{array}{c}\text { M2 } \\
\left(\mathrm{um}^{\wedge} 2\right)\end{array}$ & $\begin{array}{c}\text { M3 } \\
(\mathrm{um})\end{array}$ & M4 & 01 & O2 & $\begin{array}{c}\text { O3 } \\
(\mathrm{dB})\end{array}$ \\
\hline $\begin{array}{c}15 \\
\text { (R1) }\end{array}$ & 80.4 & 33.4 & 8.3 & 10.5 & 165.5 & 0.93 & 0.54 & 0.0 & 0.0 & 0.00 & 0.99 & 0.052 & 3.9 \\
\hline
\end{tabular}

Table A-15: The results of all 13 responses for sample $15_{\mathrm{R} 1}$

\section{A-16: Sample 16}

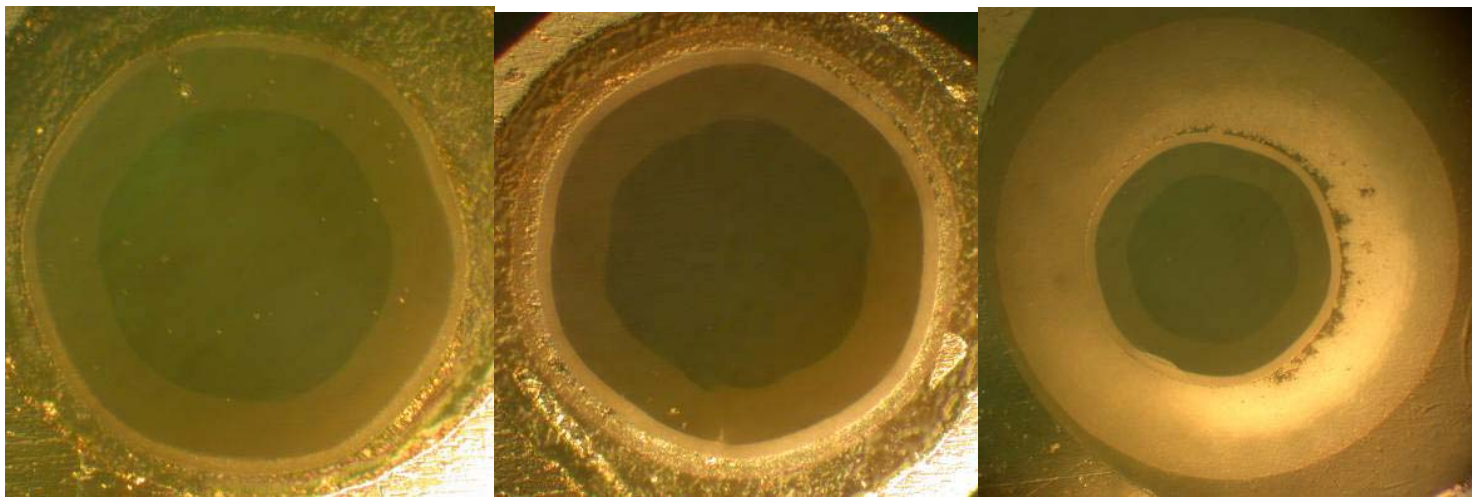
(a) cross-1 (top)
(b) cross-3 (middle)
(c) cross-2 (bottom)

Figure A-16 (a) to (c): the 3 cross sections polished and analyzed for sample 16

\begin{tabular}{|c|c|c|c|c|c|c|c|c|c|c|c|c|c|}
\hline & $\begin{array}{c}\text { G1 } \\
(\mathrm{um})\end{array}$ & $\begin{array}{c}\mathbf{G} 2 \\
(\mathrm{um})\end{array}$ & $\begin{array}{c}\mathbf{G} \text { (um) } \\
(\mathrm{um})\end{array}$ & $\begin{array}{c}\mathbf{G} 4 \\
(\mathrm{um})\end{array}$ & $\begin{array}{c}\text { G5 } \\
(\mathrm{um})\end{array}$ & $\begin{array}{c}\text { G6 } \\
(\mathrm{um})\end{array}$ & $\begin{array}{c}\text { M1 } \\
(\mathrm{mm})\end{array}$ & $\begin{array}{c}\text { M2 } \\
(\mathrm{um} / 2)\end{array}$ & $\begin{array}{c}\text { M3 } \\
(\mathrm{um})\end{array}$ & M4 & 01 & O2 & $\begin{array}{c}\text { O3 } \\
(\mathrm{dB})\end{array}$ \\
\hline 16 & 86.6 & 22.1 & 4.6 & 21.3 & 172.8 & 0.66 & 0.93 & 18.1 & 7.2 & 0.67 & 0.36 & 0.065 & 1.4 \\
\hline
\end{tabular}

Table A-16: The results of all 13 responses for sample 16 


\section{A-17: Sample 17}

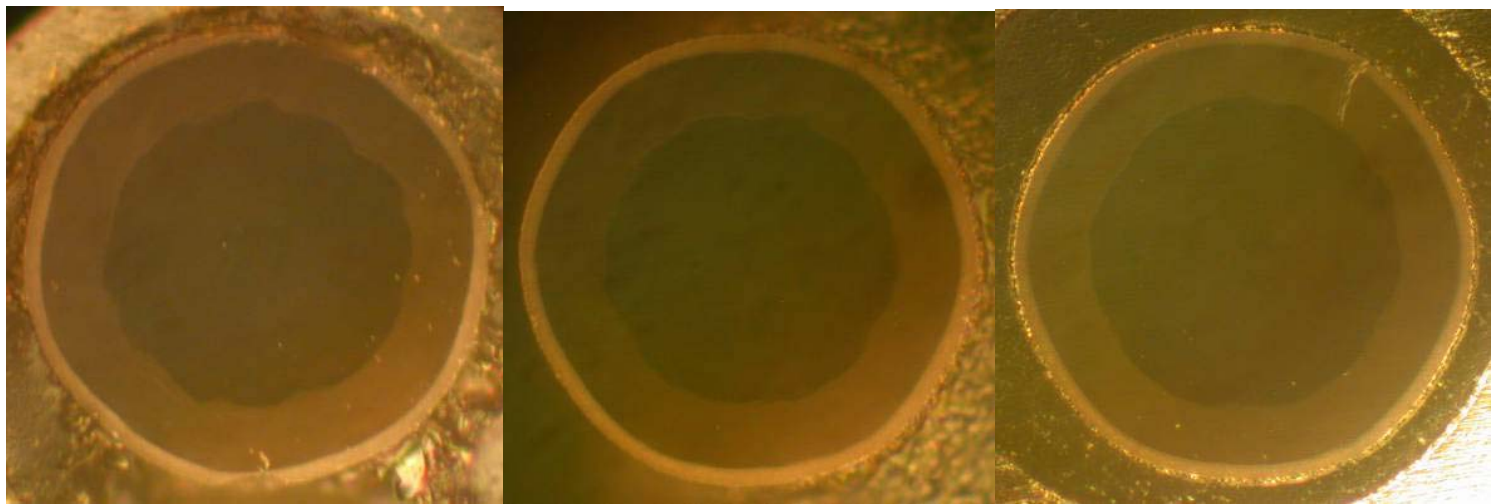
(a) cross-1 (top)
(b) cross-3 (middle)
(c) cross-2 (bottom)

Figure A-17 (a) to (c): the 3 cross sections polished and analyzed for sample 17

\begin{tabular}{|c|c|c|c|c|c|c|c|c|c|c|c|c|c|}
\hline & $\begin{array}{c}\text { G1 } \\
(\mathrm{um})\end{array}$ & $\begin{array}{c}\mathbf{G} 2 \\
(\mathrm{um})\end{array}$ & $\begin{array}{c}\mathbf{G} \text { (um) } \\
(\mathrm{um})\end{array}$ & $\begin{array}{c}\mathbf{G} 4 \\
(\mathrm{um})\end{array}$ & $\begin{array}{c}\text { G5 } \\
(\mathrm{um})\end{array}$ & $\begin{array}{c}\text { G6 } \\
(\mathrm{um})\end{array}$ & $\begin{array}{c}\text { M1 } \\
(\mathrm{mm})\end{array}$ & $\begin{array}{c}\text { M2 } \\
(\mathrm{um} / 2)\end{array}$ & $\begin{array}{c}\text { M3 } \\
(\mathrm{um})\end{array}$ & M4 & 01 & O2 & $\begin{array}{c}\text { O3 } \\
(\mathrm{dB})\end{array}$ \\
\hline 17 & 88.5 & 23.0 & 4.5 & 1.5 & 134.2 & 0.77 & 0.81 & 12.7 & 7.3 & 0.33 & 1.06 & 0.107 & 11.3 \\
\hline
\end{tabular}

Table A-17: The results of all 13 responses for sample 17

\section{A-18: Sample 18}

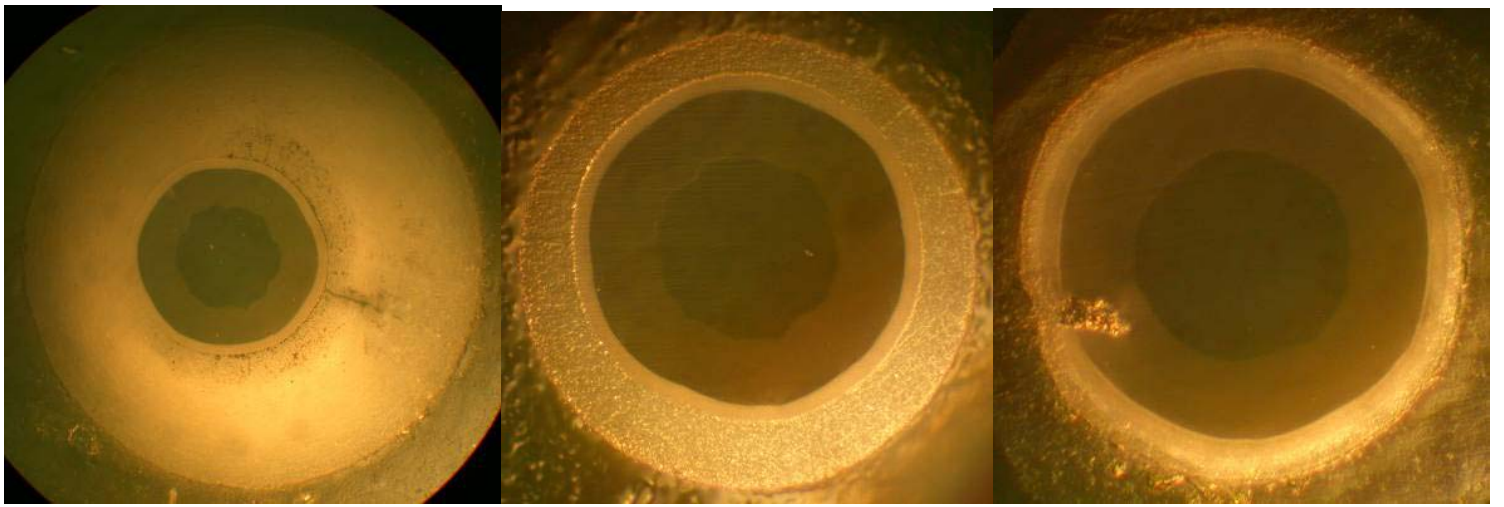

(a) cross-1 (top)

(b) cross-3 (middle)

(c) cross-2 (bottom)

Figure A-18 (a) to (c): the 3 cross sections polished and analyzed for sample $18_{\mathrm{R} 1}$

\begin{tabular}{|c|c|c|c|c|c|c|c|c|c|c|c|c|c|}
\hline & $\begin{array}{c}\mathbf{G 1} \\
\text { (um) }\end{array}$ & $\begin{array}{c}\text { G2 } \\
\text { (um) }\end{array}$ & $\begin{array}{c}\text { G3 } \\
\text { (um) }\end{array}$ & $\begin{array}{c}\mathbf{G 4} \\
\text { (um) }\end{array}$ & $\begin{array}{c}\mathbf{G 5} \\
\text { (um) }\end{array}$ & $\begin{array}{c}\mathbf{G 6} \\
\text { (um) }\end{array}$ & $\begin{array}{c}\text { M1 } \\
(\mathrm{mm})\end{array}$ & $\begin{array}{c}\text { M2 } \\
\left(u^{\wedge} 2\right)\end{array}$ & $\begin{array}{c}\text { M3 } \\
\text { (um) }\end{array}$ & M4 & 01 & $\mathrm{O} 2$ & $\begin{array}{c}\mathrm{O} 3 \\
(\mathrm{~dB})\end{array}$ \\
\hline $\begin{array}{c}18 \\
\text { (R1) }\end{array}$ & 67.5 & 31.1 & 6.3 & 37.8 & 206.1 & 0.59 & 0.64 & 0.0 & 0.0 & 0.00 & 1.88 & 0.044 & 5.3 \\
\hline
\end{tabular}

Table A-18: The results of all 13 responses for sample $18_{\mathrm{R} 1}$ 


\section{Appendix B}

In this Appendix, the results of the Taguchi analysis of eleven of the twelve responses are presented. The results of the analysis of response G1, Core Diameter, are presented in Section 4.1.15.

\section{G2 - Overall Cladding Thickness}

The following is the analysis of the sapphire fiber overall cladding thickness data generated in the 18 samples that were measured. The quality characteristic used was "smaller is better". Table G2-1 shows the factors and levels and the averages of level 3 minus level 1.

\section{Table G2-1}

\begin{tabular}{|l|r|r|r||r|}
\multicolumn{1}{|c|}{ Column \# / Factors } & Leve1 1 & \multicolumn{1}{c|}{ Leve1 2 } & \multicolumn{1}{c|}{ Leve1 3 } & L3 - L1 \\
\hline 1 Nano MgO amount & 23.633 & 24.988 & & N/a \\
2 Dipping Speed & 21.966 & 26.583 & 24.383 & 2.416 \\
3 Firing Profile & 21.716 & 24.7 & 26.516 & 4.799 \\
4 MgO/Spinel ratio & 24.033 & 23.55 & 25.35 & 1.317 \\
5 First Stage Ball & 26.333 & 24.05 & 22.549 & -3.784 \\
6 Fish oil amount & 24.416 & 23.783 & 24.733 & .317 \\
7 PVP type (K) & 22.383 & 25.916 & 24.633 & 2.25 \\
8 PVP amount & 26.75 & 24.333 & 21.85 & -4.9 \\
\hline
\end{tabular}

Table G2-2 shows the ANOVA table for the analysis of the factor influences. It appears that almost all the factors are significant at the $99 \%$ level.

Table G2-2

\begin{tabular}{|l|l|r|r|r|r|r|}
\hline & $\begin{array}{c}\text { DOF } \\
\text { Col }\end{array}$ & $\begin{array}{r}\text { Sum of Sqrs. } \\
\text { (S) }\end{array}$ & $\begin{array}{c}\text { Variance } \\
\text { (V) }\end{array}$ & $\begin{array}{c}\text { F - Ratio } \\
\text { (F) }\end{array}$ & $\begin{array}{c}\text { Pure Sum } \\
\text { (S') }\end{array}$ & $\begin{array}{r}\text { Percent } \\
\text { P(\%) }\end{array}$ \\
\hline 1 Nano MgO amount & 1 & 8.27 & 8.27 & 11.825 & 7.571 & 2.431 \\
2 Dipping Speed & 2 & 63.987 & 31.993 & 45.744 & 62.588 & 20.103 \\
3 Firing Profile & 2 & 70.482 & 35.241 & 50.387 & 69.083 & 22.189 \\
4 MgO/Spinel ratio & 2 & 10.414 & 5.207 & 7.445 & 9.016 & 2.895 \\
5 First Stage Ball & 2 & 43.554 & 21.777 & 31.136 & 42.155 & 13.54 \\
6 Fish oil amount & 2 & 2.807 & 1.403 & 2.006 & 1.408 & .452 \\
7 PVP type (K) & 2 & 38.387 & 19.193 & 27.442 & 36.988 & 11.88 \\
8 PVP amount & 2 & 72.034 & 36.017 & 51.497 & 70.635 & 22.687 \\
\hline OtherEmor & 2 & 1.397 & .698 & & & 3.823 \\
\hline
\end{tabular}


Table G2-3 shows the results of the ANOVA analysis. As seen in this table, the confidence levels of all the factors except for three are greater than $99 \%$.

Table G2-3

\begin{tabular}{|c|c|c|c|c|c|c|}
\hline $\mathrm{Col} \# /$ Factor & \begin{tabular}{|l|}
$\mathrm{DOF}$ \\
$(\mathrm{f})$
\end{tabular} & $\begin{array}{c}\text { Surn of Squrs. } \\
\text { (S) }\end{array}$ & $\begin{array}{l}\text { Variance } \\
\text { (V) }\end{array}$ & $\begin{array}{c}\text { F - Ratio } \\
\text { (F) }\end{array}$ & $\begin{array}{c}\text { Pure Sum } \\
\left(S^{\prime}\right)\end{array}$ & $\begin{array}{c}\text { Percent } \\
\mathrm{P}(\%) \\
\end{array}$ \\
\hline $1 \mathrm{Nano} \mathrm{MgO}$ amount & (1) & $(8.27)$ & & P O OLED & $(\mathrm{CL}=98.98 \%$ & \\
\hline 2 Dipping Speed & 2 & 63.987 & 31.993 & 45.744 & 62.588 & 20.103 \\
\hline 3 Firing Profile & 2 & 70.482 & 35.241 & 50.387 & 69.083 & 22.189 \\
\hline $4 \mathrm{MgO} /$ Spinel ratio & (2) & (10.414) & & P O OLED & $(\mathrm{CL}=88.43 \%$ & \\
\hline 5 First Stage Ball & 2 & 43.554 & 21.777 & 31.136 & 42.155 & 13.54 \\
\hline 6 Fish oil amount & (2) & $(2.807)$ & & P O OLED & $(\mathrm{CL}=7551 \%$ & \\
\hline 7 PVP type $(\mathrm{K})$ & 2 & 38.387 & 19.193 & 27.442 & 36.988 & 11.88 \\
\hline $8 \mathrm{PVP}$ amount & 2 & 72.034 & 36.017 & 51.497 & 70.635 & 22.687 \\
\hline Other/Error & 7 & 22.888 & 3.27 & & & 9.601 \\
\hline Total: & 17 & 311.337 & & & & $100.00 \%$ \\
\hline
\end{tabular}

Figure $\mathrm{G} 2$ shows the plots of the effects of each of the factors on the measured response.

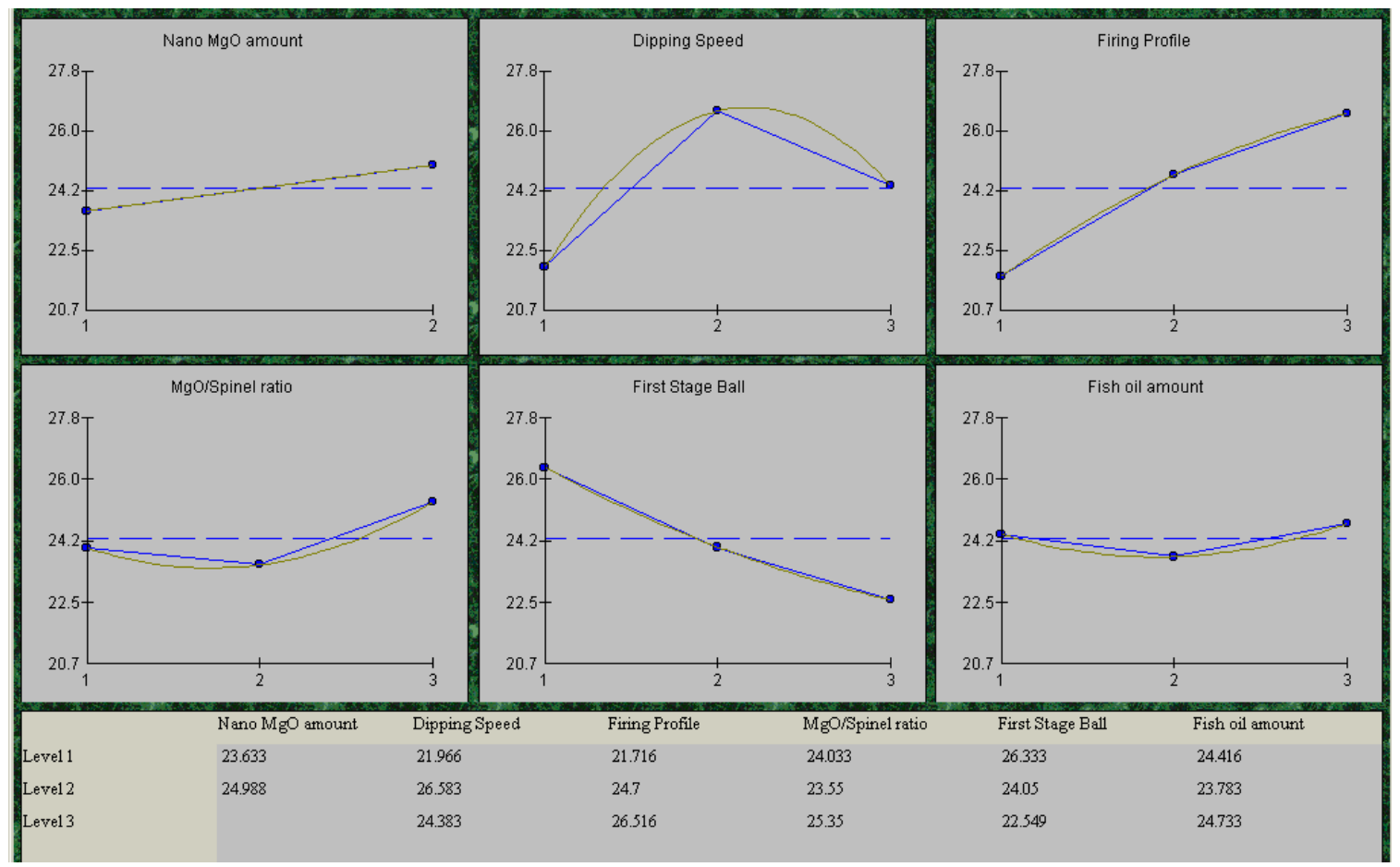

Figure G2

Table G2-4 shows the expected optimal performance for the factors determined as significant (with a confidence level greater than 99\%). 
Table G2-4

\begin{tabular}{|l|l|c|r|}
\hline Column \# / Factor & Level Description & Level & Contribution \\
\hline 2 Dipping Speed & .09 & 1 & -2.345 \\
3 Firing Profile & 1550 & 1 & -2.595 \\
5 First Stage Ball & 12 & 3 & -1.762 \\
7 PVP type (K) & 10 & 1 & -1.928 \\
8 PVP amount & 0.6 & 3 & -2.462 \\
\hline \\
Total Contribution From All Factors... \\
Current Grand Average Of Performance... \\
Expected Result At Optimum Condition...
\end{tabular}

If we assumed that all the factors were significant the optimal levels would be as those listed in Table G2-5 below.

Table G2-5

\begin{tabular}{|l|l|c|r|}
\hline Column \# / F actor & Level Description & Level & Contribution \\
\hline 1 Nano MgO amount & 10 & 1 & -.678 \\
2 Dipping Speed & .09 & 1 & -2.345 \\
3 Firing Profile & 1550 & 1 & -2.595 \\
4 MgO/Spinel ratio & $9: 1$ & 2 & -.762 \\
5 First Stage Ball & 12 & 3 & -1.762 \\
6 Fish oil amount & 50 & 2 & -.528 \\
7 PVP type (K) & 10 & 1 & -1.928 \\
8 PVP amount & 0.6 & 3 & -2.462 \\
\hline
\end{tabular}

This gives the optimal levels as predicted by the data, but as stated before most of the factors are significant at the $99 \%$ level.

\section{G3 - Outer Cladding Thickness}

The following is the analysis of the sapphire fiber outer cladding thickness data generated in the 18 samples that were measured. The quality characteristic used was "smaller is better". Table G3-1 shows the factors and levels and the averages of level 3 minus level 1. 
Table G3-1

\begin{tabular}{|l|r|r|r||r|}
\multicolumn{1}{|c|}{ Column \#/Factors } & Leve1 1 & Leve1 2 & Leve1 3 & L3 - L1 i \\
\hline 1 Nano MgO amount & 4.856 & 4.822 & & $\mathrm{n} / \mathrm{a}$ \\
2 Dipping Speed & 4.151 & 5.349 & 5.016 & .865 \\
3 Firing Profile & 4.366 & 5.016 & 5.134 & .768 \\
4 MgO/Spinel ratio & 5.333 & 4.35 & 4.835 & -.499 \\
5 First Stage Ball & 5.433 & 4.966 & 4.118 & -1.315 \\
6 Fish oil amount & 4.466 & 4.633 & 5.418 & .951 \\
7 PVP type (K) & 4.45 & 5.366 & 4.701 & .25 \\
8 PVP amount & 5.95 & 4.466 & 4.101 & -1.85 \\
\hline
\end{tabular}

Table G3-2 shows the ANOVA table for the analysis of the factor influences. It appears that almost all the factors are significant at the $90 \%$ level.

Table G3-2

\begin{tabular}{|c|c|c|c|c|c|c|}
\hline Col\#/Factor & \begin{tabular}{|l|}
$D O F$ \\
(f)
\end{tabular} & $\begin{array}{c}\text { Sum of Squrs. } \\
\text { (S) }\end{array}$ & $\begin{array}{l}\text { Variance } \\
\text { (V) }\end{array}$ & $\begin{array}{c}\text { F - Ratio } \\
\text { (F) }\end{array}$ & $\begin{array}{c}\text { Pure Sum } \\
\left(S^{\prime}\right)\end{array}$ & $\begin{array}{r}\text { Percent } \\
\mathrm{P}(\%) \\
\end{array}$ \\
\hline $1 \mathrm{Nano} \mathrm{MgO}$ amount & 1 & .005 & .005 & .038 & 0 & 0 \\
\hline 2 Dipping Speed & 2 & 4.59 & 2.295 & 16.436 & 4.311 & 13.284 \\
\hline 3 Firing Profile & 2 & 2.053 & 1.026 & 7.353 & 1.774 & 5.467 \\
\hline $4 \mathrm{MgO} /$ Spinel ratio & 2 & 2.901 & 1.45 & 10.387 & 2.621 & 8.078 \\
\hline 5 First Stage Ball & 2 & 5.333 & 2.666 & 19.095 & 5.054 & 15.572 \\
\hline 6 Fish oil amount & 2 & 3.099 & 1.549 & 11.096 & 2.82 & 8.689 \\
\hline 7 PVP type (K) & 2 & 2.691 & 1.345 & 9.637 & 2.412 & 7.433 \\
\hline $8 \mathrm{PVP}$ amount & 2 & 11.499 & 5.749 & 41.174 & 11.22 & 34.573 \\
\hline OtheriError & 2 & .279 & .139 & & & 6.904 \\
\hline Total: & 17 & 32.454 & & & & $100.00 \%$ \\
\hline
\end{tabular}

Table G3-3 shows the results of the ANOVA analysis. As seen in this table, the confidence levels of all the factors except the first three are greater than $99 \%$. 
Table G3-3

\begin{tabular}{|c|c|c|c|c|c|c|}
\hline Col\#/Factor & \begin{tabular}{|l|}
$\mathrm{DOF}$ \\
$(\mathrm{f})$
\end{tabular} & $\begin{array}{c}\text { Sum of Sqrs. } \\
\text { (S) }\end{array}$ & $\begin{array}{c}\text { Variance } \\
\text { (V) }\end{array}$ & $\begin{array}{c}\text { F - Ratio } \\
\text { (F) }\end{array}$ & $\begin{array}{l}\text { Pure Sum } \\
\text { (S') }\end{array}$ & $\begin{array}{c}\text { Percent } \\
\mathrm{P}(\%) \\
\end{array}$ \\
\hline $1 \mathrm{Nano} \mathrm{MgO}$ amount & (1) & $(.005)$ & & P O O L ED & $\left(\mathrm{CL}={ }^{*} \mathrm{NC}^{*}\right)$ & \\
\hline 2 Dipping Speed & (2) & $(4.59)$ & & P O OL ED & $(\mathrm{CL}=9758 \%$ & \\
\hline 3 Firing Profile & (2) & (2.053) & & P O OLED & $(\mathrm{CL}=96.86 \%$ & \\
\hline $4 \mathrm{MgO} / \mathrm{Sp}$ pinel ratio & 2 & 2.901 & 1.45 & 10.387 & 2.621 & 8.078 \\
\hline 5 First Stage Ball & 2 & 5.333 & 2.666 & 19.095 & 5.054 & 15.572 \\
\hline 6 Fish oil amount & 2 & 3.099 & 1.549 & 11.096 & 2.82 & 8.689 \\
\hline 7 PVP type (K) & 2 & 2.691 & 1.345 & 9.637 & 2.412 & 7.433 \\
\hline $8 \mathrm{PVP}$ amount & 2 & 11.499 & 5.749 & 41.174 & 11.22 & 34.573 \\
\hline OtheriEnor & 7 & 6.927 & .989 & & & 25.655 \\
\hline Total: & 17 & 32.454 & & & & $100.00 \%$ \\
\hline
\end{tabular}

Figure G3 shows the plots of the effects of each of the factors on the measured response.

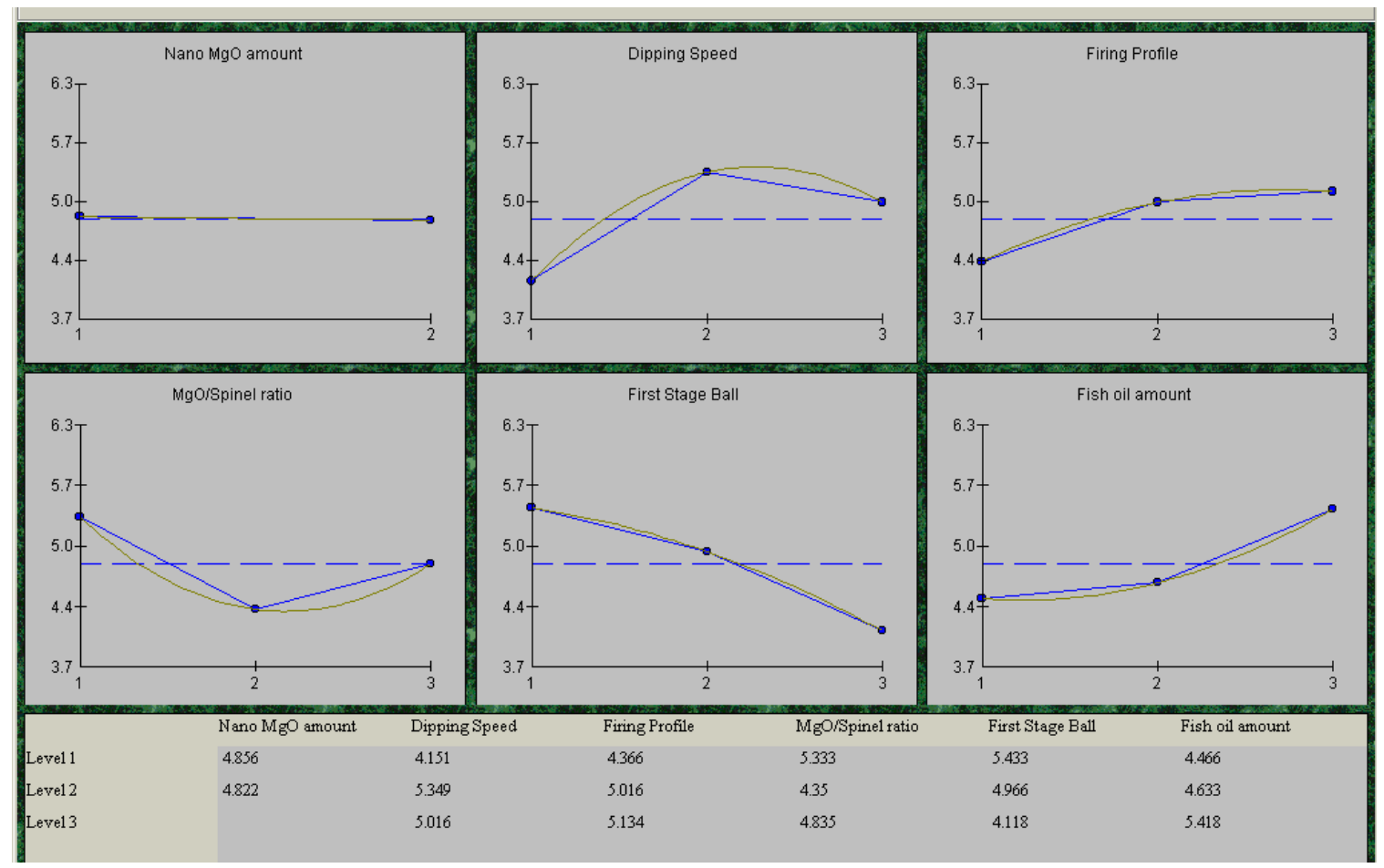

Figure G3

Table G3-4 shows the expected optimal performance for the factors determined as significant (with a confidence level greater than 99\%). 
Table G3-4

\begin{tabular}{|l|l|r|r|}
\hline Column \# / Factor & Level Description & Level & Contribution \\
\hline 4 MgO/Spinel ratio & $9: 1$ & 2 & -.49 \\
5 First Stage Ball & 12 & 3 & -.722 \\
6 Fish oil amount & 25 & 1 & -.373 \\
7 PVP type (K) & 10 & 1 & -.39 \\
8 PVP amount & 0.6 & 3 & -.738 \\
\hline \\
Total Contribution From All Factors... \\
Current Grand Average Of Performance... \\
Expected Result At Optimum Condition...
\end{tabular}

If we assumed that all the factors were significant the optimal levels would be those listed in Table G3-5.

Table G3-5

\begin{tabular}{|l|l|r|r|}
\hline Column \# / Factor & Level Description & Leve1 & Contribution \\
\hline 1 Nano MgO amount & 20 & 2 & -.018 \\
2 Dipping Speed & .09 & 1 & -.688 \\
3 Firing Profile & 1550 & 1 & -.473 \\
4 MgO/Spinel ratio & $9: 1$ & 2 & -.49 \\
5 First Stage Ball & 12 & 3 & -.722 \\
6 Fish oil amount & 25 & 1 & -.373 \\
7 PVP type (K) & 10 & 1 & -.39 \\
8 PVP amount & 0.6 & 3 & -.738 \\
\hline
\end{tabular}

This gives the optimal levels as predicted by the data, but as stated before most of the factors are significant at the $99 \%$ level.

\section{G4 - Skin Thickness}

The following is the analysis of the skin thickness data generated in the 18 samples that were measured. The quality characteristic used was "smaller is better". Table G4-1 shows the factors and levels and the averages of level 3 minus level 1. 
Table G4-1

\begin{tabular}{|l|r|r|r||r|}
\multicolumn{1}{|c|}{ Column \#/Factors } & Leve1 1 & Leve1 2 & Leve1 3 & L3 - L1 i \\
\hline 1 Nano MgO amount & 21.742 & 9.455 & & $\mathrm{n} / \mathrm{a}$ \\
2 Dipping Speed & 2.6 & 8.383 & 35.813 & 33.213 \\
3 Firing Profile & 5.883 & 31.649 & 9.263 & 3.379 \\
4 MgO/Spinel ratio & 3.163 & 13.316 & 30.316 & 27.152 \\
5 First Stage Ball & 7.833 & 31.266 & 7.696 & -.138 \\
6 Fish oil amount & 26.666 & 8.213 & 11.916 & -14.75 \\
7 PVP type (K) & 10.246 & 3.816 & 32.733 & 22.486 \\
8 PVP amount & 39.833 & 5.063 & 1.899 & -37.934 \\
\hline
\end{tabular}

Table G4-2 shows the ANOVA table for the analysis of the factor influences. It appears that two of the factors are significant at the $90 \%$ level.

Table G4-2

\begin{tabular}{|l|l|r|r|r|r|r|}
\hline & $\begin{array}{l}\text { DOF } \\
\text { (f) }\end{array}$ & $\begin{array}{r}\text { Sum of Sqrs. } \\
\text { (S) }\end{array}$ & $\begin{array}{c}\text { Variance } \\
\text { (V) }\end{array}$ & $\begin{array}{c}\text { F - Ratio } \\
\text { (F) }\end{array}$ & $\begin{array}{r}\text { Pure Sum } \\
\text { (S') }\end{array}$ & $\begin{array}{r}\text { Percent } \\
\text { P(\%) }\end{array}$ \\
\hline 1 Nano MgO amount & 1 & 679.329 & 679.329 & 1.575 & 248.246 & 1.161 \\
2 Dipping Speed & 2 & 3777.955 & 1888.977 & 4.381 & 2915.788 & 13.646 \\
3 Firing Profile & 2 & 2353.016 & 1176.508 & 2.729 & 1490.849 & 6.977 \\
4 MgO/Spinel ratio & 2 & 2258.786 & 1129.393 & 2.619 & 1396.619 & 6.536 \\
5 First Stage Ball & 2 & 2209.369 & 1104.684 & 2.562 & 1347.203 & 6.305 \\
6 Fish oil amount & 2 & 1143.605 & 571.802 & 1.326 & 281.438 & 1.317 \\
7 PVP type (K) & 2 & 2766.338 & 1383.169 & 3.208 & 1904.172 & 8.912 \\
8 PVP amount & 2 & 5315.795 & 2657.897 & 6.165 & 4453.629 & 20.844 \\
\hline Other/Error & 2 & 862.166 & 431.083 & & 34.302 \\
\hline Total: & 17 & 21366.365 \\
\hline
\end{tabular}

Table G4-3 shows the results of the ANOVA analysis. As seen in this table, the confidence levels of all the factors except for two are less than $90 \%$. 
Table G4-3

\begin{tabular}{|c|c|c|c|c|c|c|}
\hline Col\#/Factor & \begin{tabular}{|l|}
$\mathrm{DOF}$ \\
$(\mathrm{f})$
\end{tabular} & $\begin{array}{c}\text { Sum of Squrs. } \\
\text { (S) }\end{array}$ & $\begin{array}{l}\text { Variance } \\
\text { (V) }\end{array}$ & $\begin{array}{l}\text { F - Ratio } \\
\text { (F) }\end{array}$ & $\begin{array}{l}\text { Pure Sum } \\
\text { (S') }\end{array}$ & $\begin{array}{r}\text { Percent } \\
\mathrm{P}(\%) \\
\end{array}$ \\
\hline $1 \mathrm{Nano} \mathrm{MgO}$ amount & (1) & $(679.329)$ & & P O OLED & $(\mathrm{CL}=71.15 \%$ & \\
\hline 2 Dipping Speed & (2) & $(3777.955)$ & & P O OLED & $(\mathrm{CL}=87.42 \%$ & \\
\hline 3 Firing Profile & (2) & $(2353.016)$ & & P O OLED & $(\mathrm{CL}=8456 \%$ & \\
\hline $4 \mathrm{MgO} / \mathrm{Sp}$ pinel ratio & (2) & $(2258.786)$ & & P O OL ED & $(\mathrm{CL}=86.19 \%$ & \\
\hline 5 First Stage Ball & (2) & $(2209.369)$ & & P O OLED & $(\mathrm{CL}=87.16 \%$ & \\
\hline 6 Fish oil amount & (2) & $(1143.605)$ & & P O OLED & $(\mathrm{CL}=69.92 \%$ & \\
\hline 7 PVP type (K) & 2 & 2766.338 & 1383.169 & 3.208 & 1904.172 & 8.912 \\
\hline $8 \mathrm{PVP}$ amount & 2 & 5315.795 & 2657.897 & 6.165 & 4453.629 & 20.844 \\
\hline OtherrError & 13 & 13284.226 & 1021.863 & & & 70.244 \\
\hline Total: & 17 & 21366.365 & & & & $100.00 \%$ \\
\hline
\end{tabular}

Figure G4 shows the plots of the effects of each of the factors on the measured response.

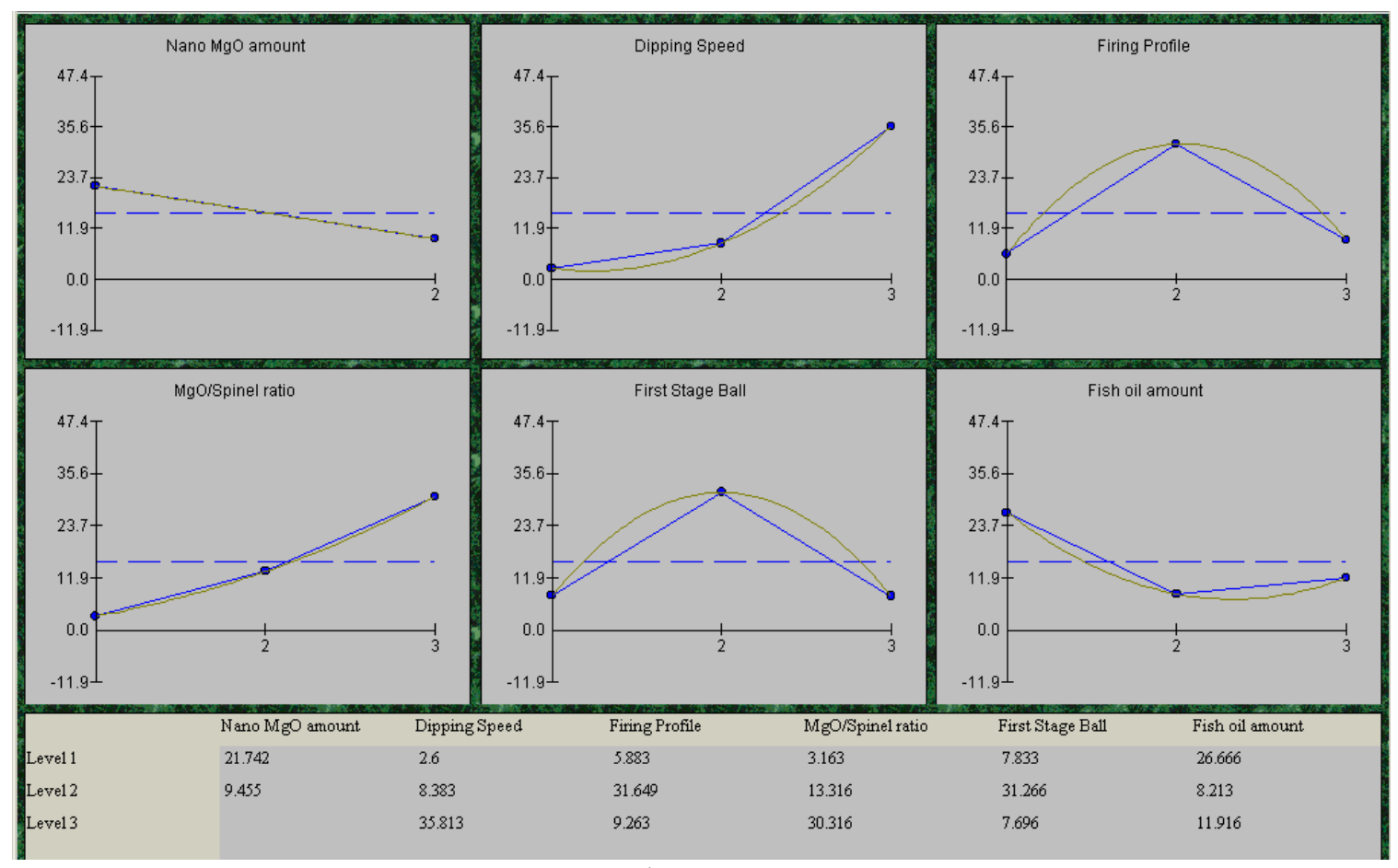

Figure G4

Table G4-4 shows the expected optimal performance for the factors determined as significant (with a confidence level greater than 90\%). 
Table G4-4

\begin{tabular}{l|l|l|r|}
\hline Column \# / Factor & Leve1 Description & Level & Contribution \\
\hline 7 PVP type (K) & $90 \% 10 \mathrm{~K}$ & 2 & -11.783 \\
8 PVP amount & 0.6 & 3 & -13.699 \\
& & & \\
\hline \\
Total Contribution From All Factors... & & -25.482 \\
Current Grand Average Of Performance... & 15.598 \\
Expected Result At Optimum Condition... & -9.884
\end{tabular}

If we assumed that all the factors were significant the optimal levels would be as those listed in Table G4-5.

\section{Table G4-5}

\begin{tabular}{|l|l|l|r|}
\hline Column \# / Factor & Level Description & Level & Contribution \\
\hline 1 Nano MgO amount & 20 & 2 & -6.144 \\
2 Dipping Speed & .09 & 1 & -12.999 \\
3 Firing Profile & 1550 & 1 & -9.716 \\
4 MgO/Spinel ratio & $6: 1$ & 1 & -12.436 \\
5 First Stage Ball & 12 & 3 & -7.903 \\
6 Fish oil amount & 50 & 2 & -7.386 \\
7 PVP type (K) & $90 \% 10 \mathrm{~K}$ & 2 & -11.783 \\
8 PVP amount & 0.6 & 3 & -13.699 \\
\hline
\end{tabular}

This gives the optimal levels as predicted by the data, but as stated before all but two of the factors are not significant at the $90 \%$ level.

\section{G5 - Overall fiber diameter}

The following is the analysis of the sapphire fiber overall fiber diameter data generated in the 18 samples that were measured. The quality characteristic used was "smaller is better". Table G5-1 shows the factors and levels and the averages of level 3 minus level 1 . 
Table G5-1

\begin{tabular}{|l|r|r|r||r|}
\hline \multicolumn{1}{|c|}{ Column \# / Factors } & Leve1 1 & Leve1 2 & Leve1 3 & L3 - L1 i \\
\hline 1 Nano MgO amount & 176.411 & 152.444 & & n/a \\
2 Dipping Speed & 136.616 & 151.466 & 205.199 & 68.582 \\
3 Firing Profile & 143.566 & 197.399 & 152.316 & 8.75 \\
4 MgO/Spinel ratio & 141.6 & 157.816 & 193.866 & 52.266 \\
5 First Stage Ball & 150.066 & 197.466 & 145.75 & -4.317 \\
6 Fish oil amount & 187.583 & 148.449 & 157.25 & -30.333 \\
7 PVP type (K) & 152.6 & 141.916 & 198.766 & 46.165 \\
8 PVP amount & 214.216 & 143.583 & 135.483 & -78.734 \\
\hline
\end{tabular}

Table G5-2 shows the ANOVA table for the analysis of the factor influences. It appears that almost all the factors are not significant at the $90 \%$ level.

Table G5-2

\begin{tabular}{|c|c|c|c|c|c|c|}
\hline Col\#/Factor & $\begin{array}{l}\mathrm{DOF} \\
\text { (f) }\end{array}$ & $\begin{array}{c}\text { Sum of Squrs. } \\
\text { (S) }\end{array}$ & $\begin{array}{l}\text { Variance } \\
\text { (V) }\end{array}$ & $\begin{array}{l}\text { F - Ratio } \\
\text { (F) }\end{array}$ & $\begin{array}{c}\text { Pure Sum } \\
\left(\mathrm{S}^{\prime}\right)\end{array}$ & $\begin{array}{c}\text { Percent } \\
\mathrm{P}(\%) \\
\end{array}$ \\
\hline $1 \mathrm{Nano} \mathrm{MgO}$ amount & 1 & 2584.763 & 2584.763 & 1.134 & 305.889 & .34 \\
\hline 2 Dipping Speed & 2 & 15622.936 & 7811.468 & 3.427 & 11065.187 & 12.326 \\
\hline 3 Firing Profile & 2 & 10014.131 & 5007.065 & 2.197 & 5456.382 & 6.078 \\
\hline $4 \mathrm{MgO} / \mathrm{Sp}$ pinel ratio & 2 & 8588.814 & 4294.407 & 1.884 & 4031.065 & 4.49 \\
\hline 5 First Stage Ball & 2 & 9880.022 & 4940.011 & 2.167 & 5322.274 & 5.928 \\
\hline 6 Fish oil amount & 2 & 5057.911 & 2528.955 & 1.109 & 500.163 & .557 \\
\hline 7 PVP type (K) & 2 & 10954.835 & 5477.417 & 2.403 & 6397.087 & 7.126 \\
\hline 8 PVP amount & 2 & 22507.176 & 11253.588 & 4.938 & 17949.428 & 19.995 \\
\hline Other/Error & 2 & 4557.748 & 2278.874 & & & 43.16 \\
\hline Total: & 17 & 89768.34 & & & & $100.00 \%$ \\
\hline
\end{tabular}

Table G5-3 shows the results of the ANOVA analysis. As seen in this table, the confidence levels of all the factors except PVP amount are less than $90 \%$. 
Table G5-3

\begin{tabular}{|c|c|c|c|c|c|c|}
\hline Col\#/Factor & $\begin{array}{l}\mathrm{DOF} \\
\text { (f) }\end{array}$ & $\begin{array}{c}\text { Sum of Sqrs. } \\
\text { (S) }\end{array}$ & $\begin{array}{l}\text { Variance } \\
\text { (V) }\end{array}$ & $\begin{array}{l}\text { F - Ratio } \\
\text { (F) }\end{array}$ & $\begin{array}{c}\text { Pure Sum } \\
\left(\mathrm{S}^{\prime}\right)\end{array}$ & $\begin{array}{r}\text { Percent } \\
\mathrm{P}(\%) \\
\end{array}$ \\
\hline $1 \mathrm{Nano} \mathrm{MgO}$ amount & (1) & $(2584.763)$ & & POOLED & $(\mathrm{CL}=6436 \%$ & \\
\hline 2 Dipping Speed & (2) & (15622.930) & & P O OLED & $(C L=8356 \%$ & \\
\hline 3 Firing Profile & (2) & (10014.131) & & P O OLED & $(\mathrm{CL}=79.74 \%$ & \\
\hline $4 \mathrm{MgO} /$ Spinel ratio & (2) & $(8588.814)$ & & P O OLED & $(\mathrm{CL}=78.26 \%$ & \\
\hline 5 First Stage Ball & (2) & $(9880.022)$ & & P O OLED & $(\mathrm{CL}=83.31 \%$ & \\
\hline 6 Fish oil amount & (2) & $(5057.911)$ & & P O OLED & $(\mathrm{CL}=63.77 \%$ & \\
\hline 7 PVP type (K) & (2) & $(10954.835)$ & & POOLED & $(\mathrm{CL}=8736 \%$ & \\
\hline $8 \mathrm{PVP}$ amount & 2 & 22507.176 & 11253.588 & 4.938 & 17949.428 & 19.995 \\
\hline Other/Enror & 15 & 67261.16 & 4484.077 & & & 80.005 \\
\hline Total: & 17 & 89768.34 & & & & $100.00 \%$ \\
\hline
\end{tabular}

Figure G5 shows the plots of the effects of each of the factors on the measured response.

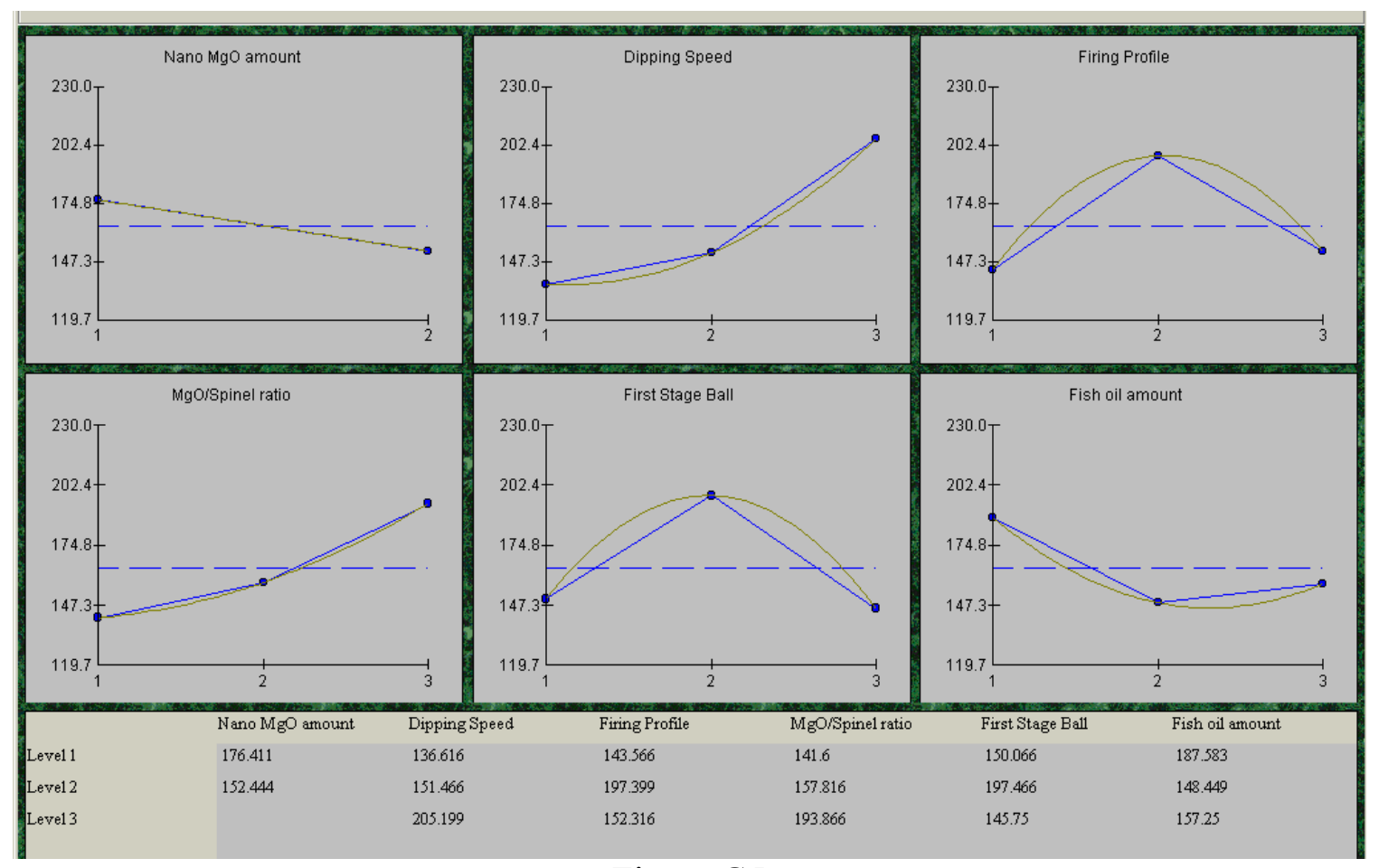

Figure G5

Table G5-4 shows the expected optimal performance for the factors determined as significant (with a confidence level greater than $90 \%$ ). 
Table G5-4

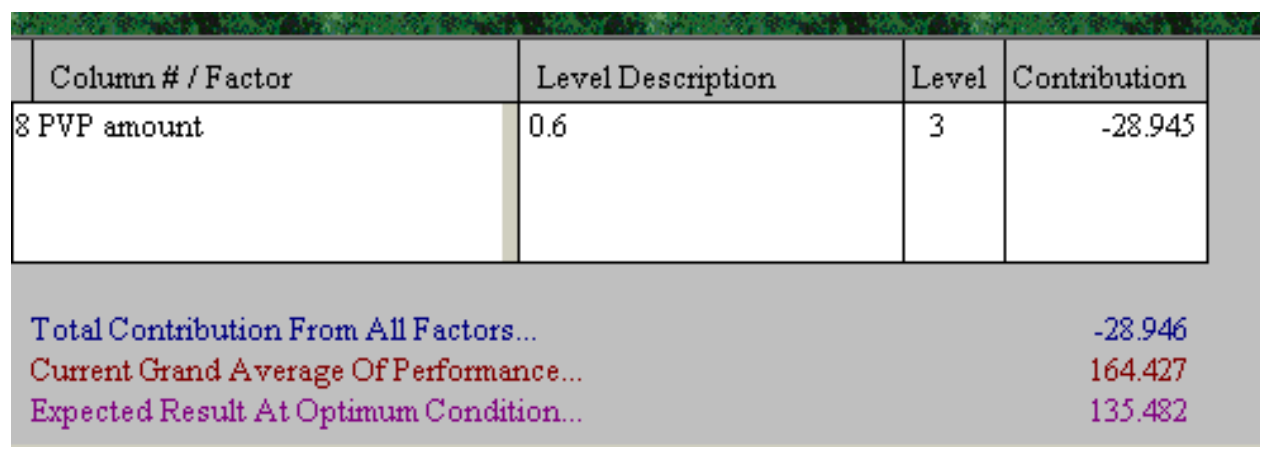

If we assumed that all the factors were significant the optimal levels would be as shown in Table G5-5.

Table G5-5

\begin{tabular}{|l|l|c|r|}
\hline Column \# / Factor & Level Description & Level & Contribution \\
\hline 1 Nano MgO amount & 20 & 2 & -11.984 \\
2 Dipping Speed & .09 & 1 & -27.812 \\
3 Firing Profile & 1550 & 1 & -20.862 \\
4 MgO/Spinel ratio & $6: 1$ & 1 & -22.828 \\
5 First Stage Ball & 12 & 3 & -18.678 \\
6 Fish oil amount & 50 & 2 & -15.978 \\
7 PVP type (K) & $90 \% 10 \mathrm{~K}$ & 2 & -22.512 \\
8 PVP amount & 0.6 & 3 & -28.945 \\
\hline
\end{tabular}

This gives the optimal levels as predicted by the data, but as stated before all of the factors except one are not significant at the $90 \%$ level.

\section{G6 - Concentricity - Inter Center Distance}

The following is the analysis of the sapphire interface center distance data generated in the 18 samples that were measured. The quality characteristic used was "smaller is better". Table G6-1 shows the factors and levels and the averages of level 3 minus level 1 . 
Table G6-1

\begin{tabular}{|c|c|c|c|c|}
\hline Column\# / Factors & Level 1 & Level 2 & Level 3 & $\mathrm{~L} 3-\mathrm{L1}$ \\
\hline $1 \mathrm{Nano} \mathrm{MgO}$ amount & 11.003 & .701 & & $\mathrm{n} / \mathrm{a}$ \\
\hline 2 Dipping Speed & .643 & .91 & 16.003 & 15.36 \\
\hline 3 Firing Profile & .816 & .789 & 15.949 & 15.132 \\
\hline $4 \mathrm{MgO/Spinel} \mathrm{ratio}$ & 16.014 & .753 & .788 & -15.226 \\
\hline 5 First Stage Ball & 699 & .901 & 15.954 & 15.255 \\
\hline 6 Fish oil amount & .843 & 15.989 & .723 & -.12 \\
\hline 7 PVP type (K) & 15.903 & .805 & .848 & -15.056 \\
\hline 8 PVP amount & .788 & 15.966 & .801 & .013 \\
\hline
\end{tabular}

Table G6-2 shows the ANOVA table for the analysis of the factor influences. It appears that all the factors are not significant at the $70 \%$ level.

Table G6-2

\begin{tabular}{|c|c|c|c|c|c|c|}
\hline Col\#/Factor & $\begin{array}{l}\mathrm{DOF} \\
\text { (f) }\end{array}$ & $\begin{array}{c}\text { Sum of Sqrs. } \\
\text { (S) }\end{array}$ & $\begin{array}{l}\text { Variance } \\
\text { (V) }\end{array}$ & $\begin{array}{l}\text { F - Ratio } \\
\text { (F) }\end{array}$ & $\begin{array}{l}\text { Pure Sum } \\
\text { (S') }\end{array}$ & $\begin{array}{c}\text { Percent } \\
\mathrm{P}(\%)\end{array}$ \\
\hline $1 \mathrm{Nano} \mathrm{MgO}$ amount & 1 & 477.61 & 477.61 & 1.024 & 11.365 & .144 \\
\hline 2 Dipping Speed & 2 & 927.618 & 463.809 & .994 & 0 & 0 \\
\hline 3 Firing Profile & 2 & 917.688 & 458.844 & .984 & 0 & 0 \\
\hline $4 \mathrm{MgO} / \mathrm{Spinel}$ ratio & 2 & 929.542 & 464.771 & .996 & 0 & 0 \\
\hline 5 First Stage Ball & 2 & 918.716 & 459.358 & .985 & 0 & 0 \\
\hline 6 Fish oil amount & 2 & 925.013 & 462.506 & .991 & 0 & 0 \\
\hline 7 PVP type (K) & 2 & 909.229 & 454.614 & .975 & 0 & 0 \\
\hline $8 \mathrm{PVP}$ amount & 2 & 920.718 & 460.359 & .987 & 0 & 0 \\
\hline Other/Error & 2 & 932.489 & 466.244 & & & 99.856 \\
\hline Total: & 17 & 7858.628 & & & & $100.00 \%$ \\
\hline
\end{tabular}

Table G6-3 shows the results of the ANOVA analysis. As seen in this table, the confidence levels of all the factors except for one are less than $60 \%$. 
Table G6-3

\begin{tabular}{|c|c|c|c|c|c|c|}
\hline Col\#/Factor & \begin{tabular}{|l|}
$\mathrm{DOF}$ \\
$(\mathrm{f})$
\end{tabular} & $\begin{array}{c}\text { Sum of Sqrs. } \\
\text { (S) }\end{array}$ & $\begin{array}{c}\text { Variance } \\
\text { (V) }\end{array}$ & $\begin{array}{l}\text { F - Ratio } \\
\text { (F) }\end{array}$ & $\begin{array}{c}\text { Pure Sum } \\
\text { (S') }\end{array}$ & $\begin{array}{r}\text { Percent } \\
\mathrm{P}(\%) \\
\end{array}$ \\
\hline $1 \mathrm{Nano} \mathrm{MgO}$ amount & (1) & (477.61) & & POOLED & $(\mathrm{CL}=62.1 \%)$ & \\
\hline 2 Dipping Speed & (2) & $(927.618)$ & & P O OLED & $\left(\mathrm{CL}={ }^{*} \mathrm{NC}^{*}\right)$ & \\
\hline 3 Firing Profile & (2) & (917.688) & & P O OLED & $\left(\mathrm{CL}={ }^{*} \mathrm{NC}^{*}\right)$ & \\
\hline $4 \mathrm{MgO} / \mathrm{Sp}$ pinel ratio & (2) & $(929.542)$ & & P O OLED & $\left(\mathrm{CL}={ }^{*} \mathrm{NC}{ }^{*}\right)$ & \\
\hline 5 First Stage Ball & (2) & (918.716) & & P O OLED & $\left(\mathrm{CL}={ }^{*} \mathrm{NC}{ }^{*}\right)$ & \\
\hline 6 Fish oil amount & (2) & (925.013) & & P O OLED & $\left(\mathrm{CL}={ }^{*} \mathrm{NC}^{*}\right)$ & \\
\hline 7 PVP type (K) & (2) & $(909.229)$ & & P O OLED & $\left(\mathrm{CL}={ }^{*} \mathrm{NC}^{*}\right)$ & \\
\hline 8 PVP amount & (2) & $(920.718)$ & & P O OLED & $\left(\mathrm{CL}={ }^{*} \mathrm{NC}^{*}\right)$ & \\
\hline Other/Error & 17 & 7858.623 & 462.272 & & & 100 \\
\hline Total: & 17 & 7858.628 & & & & $100.00 \%$ \\
\hline
\end{tabular}

Figure G6 shows the plots of the effects of each of the factors on the measured response.

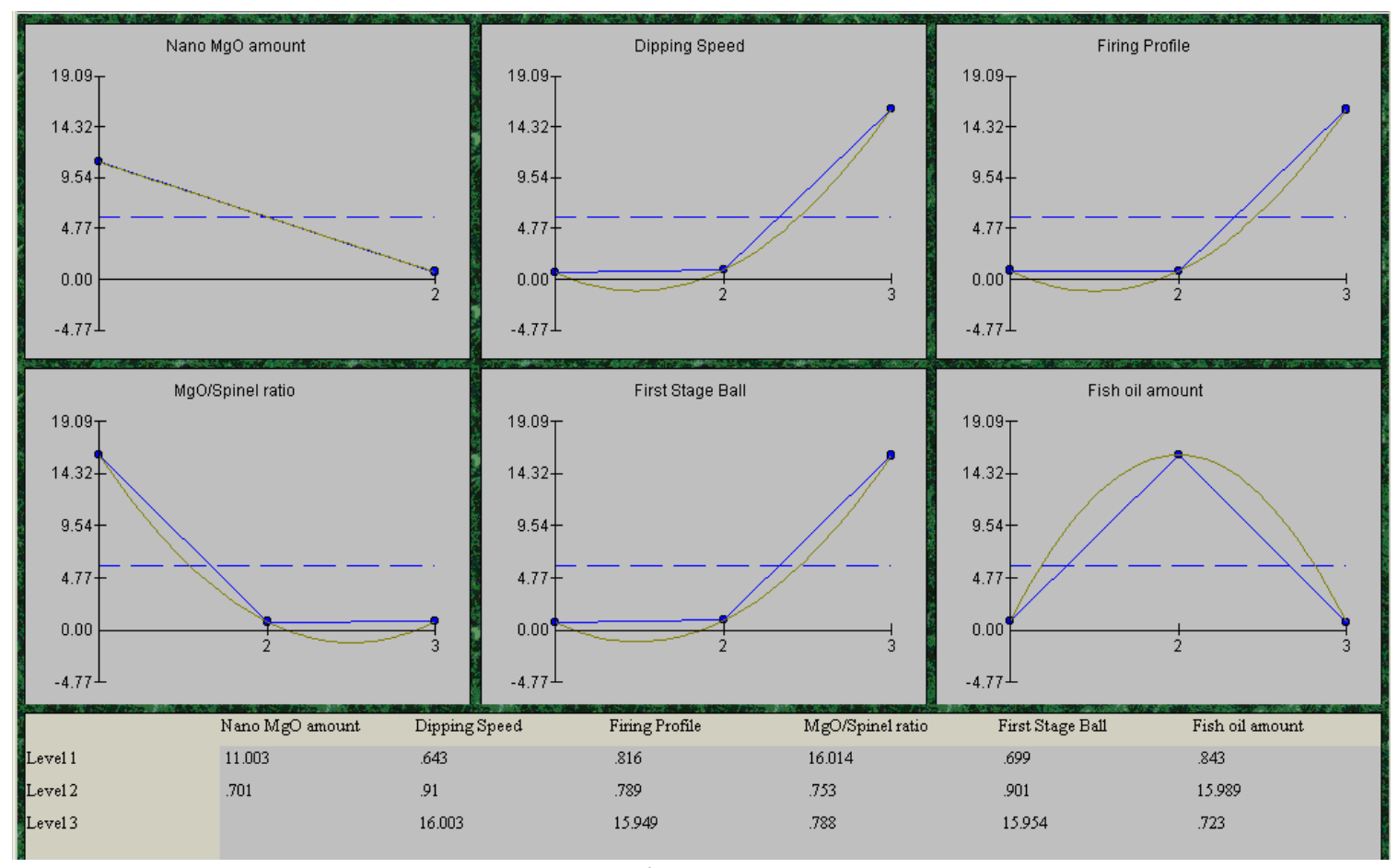

Figure G6

Table G6-4 shows the expected optimal performance for the factors determined as significant (with a confidence level greater than $60 \%$ ). 
Table G6-4

\begin{tabular}{|l|l|r|r|}
\hline Column \# / Factor & Level Description & Level & Contribution \\
\hline 1 Nano MgO amount & 20 & 2 & -5.152 \\
& & & \\
\hline & & \\
Total Contribution From All Factors... & -5.153 \\
Current Grand Average Of Performance... & 5.852 \\
Expected Result At Optimum Condition... & 7 \\
\hline
\end{tabular}

If we assumed that all the factors were significant the optimal levels should be as those shown in Table G6-5.

Table G6-5

\begin{tabular}{|l|l|l|r|}
\hline Column \# / Factor & Level Description & Level & Contribution \\
\hline 1 Nano MgO amount & 20 & 2 & -5.152 \\
2 Dipping Speed & .09 & 1 & -5.209 \\
3 Firing Profile & 1575 & 2 & -5.063 \\
4 MgO/Spinel ratio & $9: 1$ & 2 & -5.099 \\
5 First Stage Ball & 3 & 1 & -5.153 \\
6 Fish oil amount & 75 & 3 & -5.129 \\
7 PVP type (K) & $90 \% 10 \mathrm{~K}$ & 2 & -5.048 \\
8 PVP amount & 0.4 & 1 & -5.064 \\
\hline
\end{tabular}

This gives the optimal levels as predicted by the data, but as stated before most of the factors are not significant at the $70 \%$ level.

\section{M1: Bend Analysis}

The following is the analysis of bend data generated in the 18 samples that were measured. The quality characteristic used was "bigger is better".

Table M1-1 shows the results of the anova analysis. As seen in this table, the confidence levels of all the factors but one - firing profile - are less than $90 \%$. Therefore, based on the amount of scatter in the data, only the effect of the firing profile seems significant when approaching 90\%. More data would be needed to improve the estimates of these effects. 
Table M1-1

\begin{tabular}{|c|c|c|c|c|c|c|}
\hline Col\#/Factor & $\begin{array}{l}\text { DUF } \\
\text { (f) }\end{array}$ & $\begin{array}{c}\text { sum or sqrs. } \\
\text { (S) }\end{array}$ & $\begin{array}{c}\text { Variance } \\
\text { (V) }\end{array}$ & $\begin{array}{c}\text { F- Ratio } \\
\text { (F) }\end{array}$ & $\begin{array}{c}\text { rure sum } \\
\text { (S') }\end{array}$ & $\begin{array}{r}\text { Percent } \\
\mathrm{P}(\%) \\
\end{array}$ \\
\hline $1 \mathrm{Nano} \mathrm{MgO}$ amount & (1) & $(.003)$ & & P O OLED & $\left(\mathrm{CL}={ }^{*} \mathrm{NC}^{*}\right)$ & \\
\hline 2 Dipping Speed & (2) & $(.002)$ & & P O OLED & $\left(\mathrm{CL}={ }^{*} \mathrm{NC}^{*}\right)$ & \\
\hline 3 Firing Profile & 2 & .193 & .096 & 4.973 & .154 & 33.294 \\
\hline $4 \mathrm{MgO} /$ Spinel ratio & (2) & (.04) & & P O OLED & $(\mathrm{CL}=5854 \%$ & \\
\hline 5 First Stage Ball & (2) & $(.056)$ & & P O OLED & $(\mathrm{CL}=70.42 \%$ & \\
\hline 6 Fish oil amount & (2) & $(.028)$ & & P O OLED & $\left(\mathrm{CL}={ }^{*} \mathrm{NC} *\right)$ & \\
\hline 7 PVP type (K) & (2) & (.06) & & P O OLED & $(C L=75.14 \%$ & \\
\hline 8 PVP amount & (2) & $(.039)$ & & P O OLED & $(\mathrm{CL}=61.47 \%$ & \\
\hline Other/Error & 15 & .266 & .018 & & & 66.706 \\
\hline Total: & 17 & .465 & & & & 100.00 \\
\hline
\end{tabular}

The optimal level for firing profile is level 1.

Table M1-2

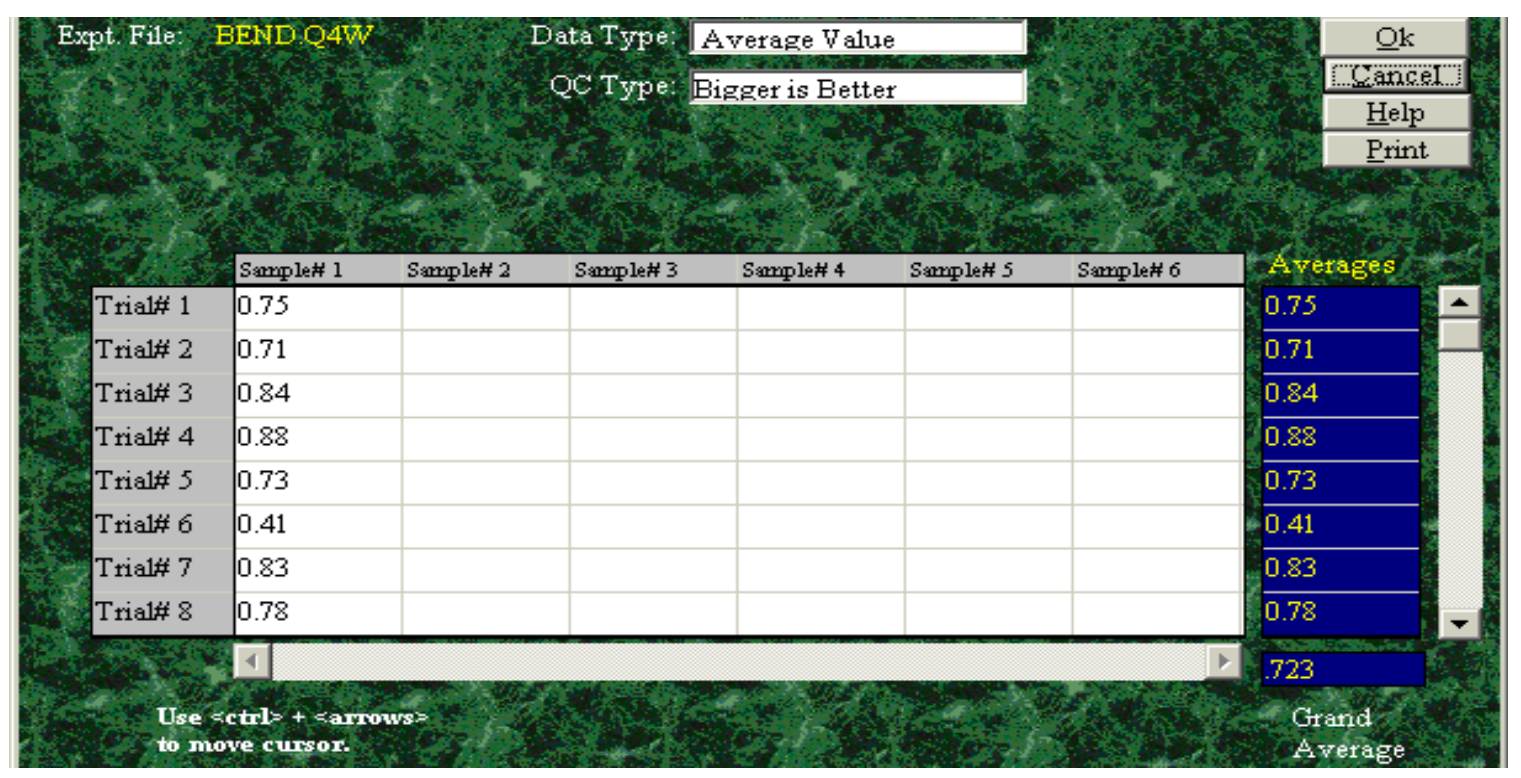


Table M1-3

\begin{tabular}{|l|l|r|r|}
\hline Column \#/Factor & Level Description & Level & Contribution \\
\hline 3 Firing Profile & 1550 & 1 & .132 \\
& & & \\
& & & \\
& & .132 \\
Total Contribution From All Factors... & .723 \\
Current Grand Average Of Performance... & .855 \\
Expected Result At Optimum Condition... & \\
\hline
\end{tabular}

Table M1-4

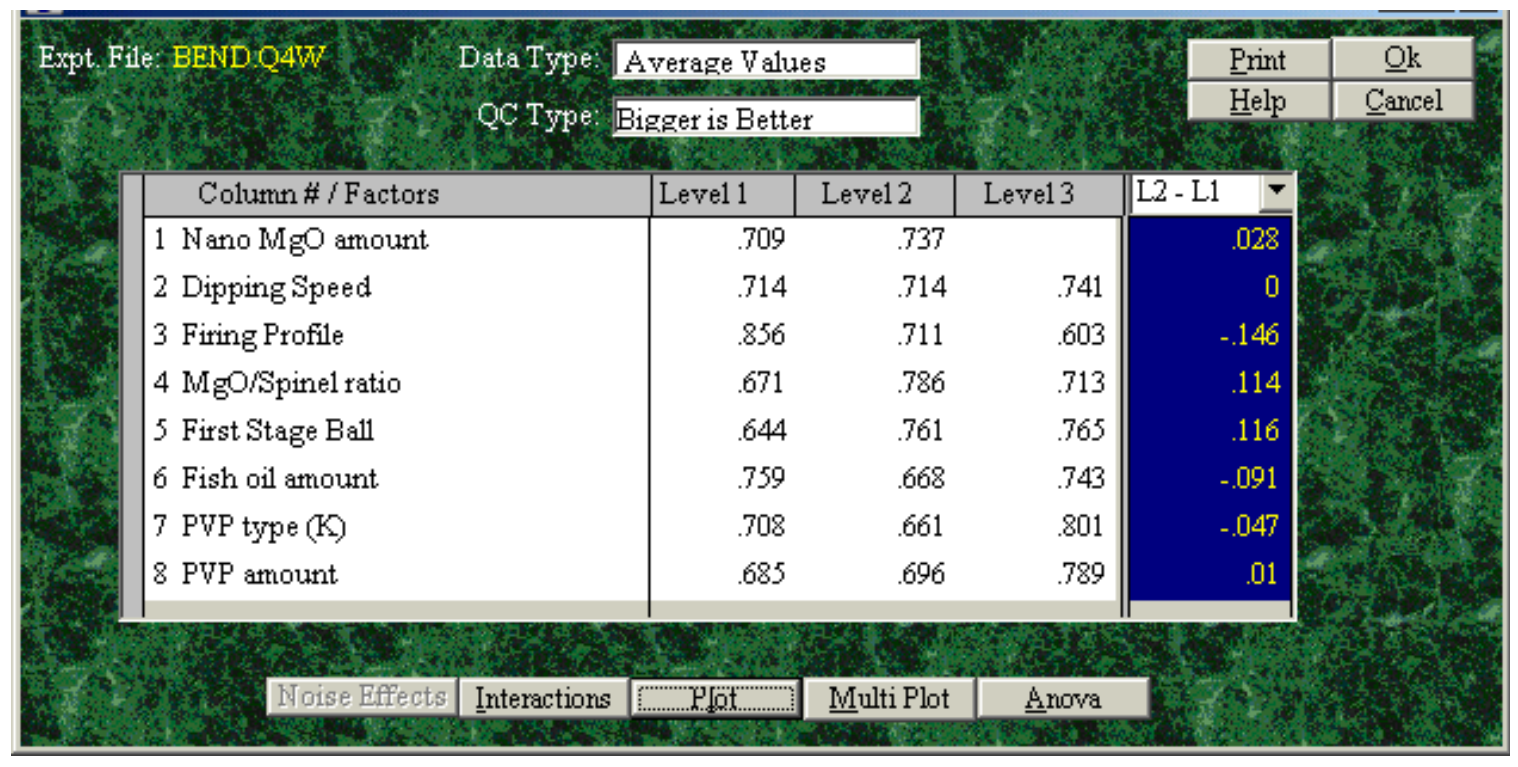




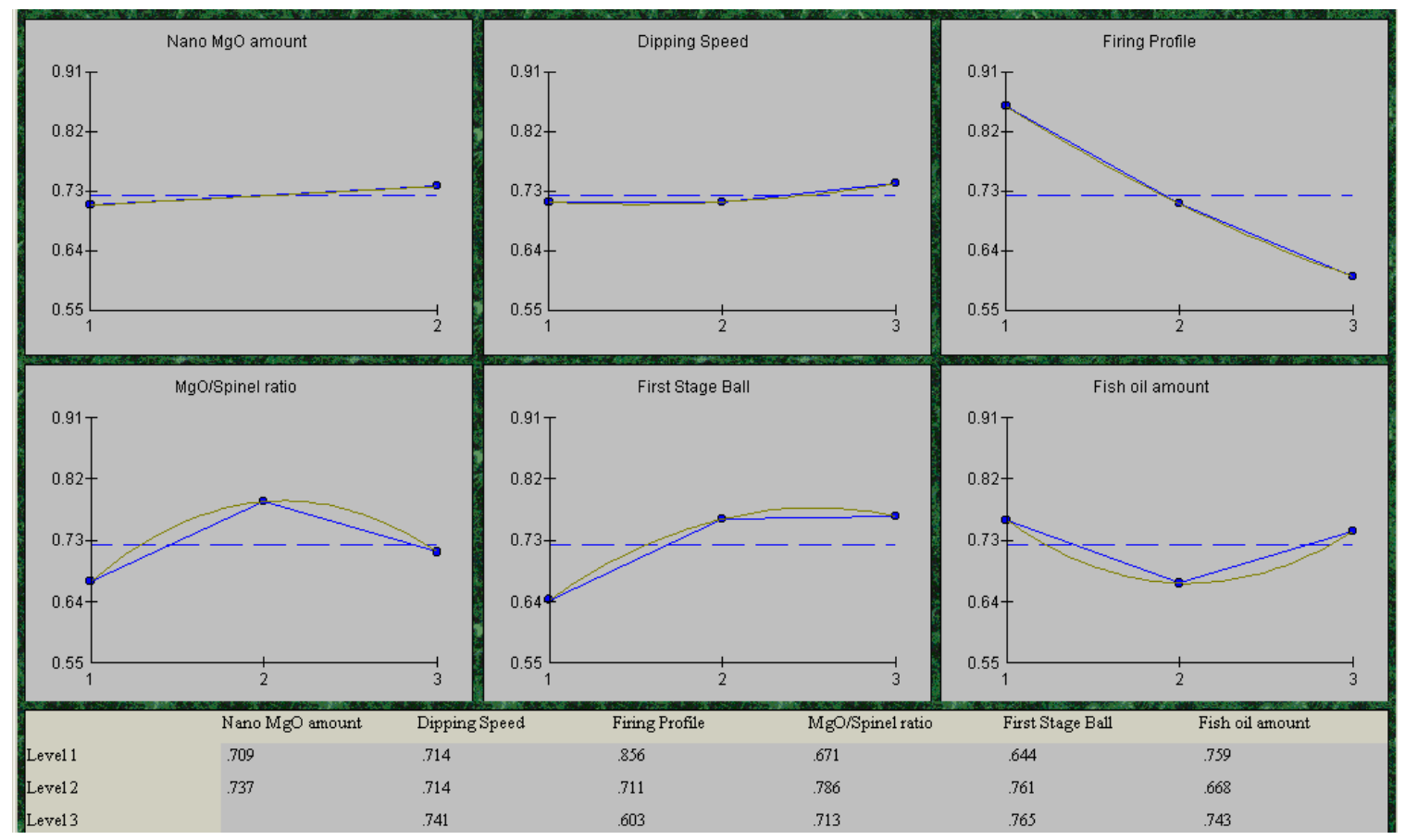

Figure M1-1

\section{M2 - Total Area of Crack}

The following is the analysis of the sapphire crack parameter (total area of crack) data generated in the 18 samples that were measured. The quality characteristic used was "smaller is better". Table M2-1 shows the factors and levels and the averages of level 3 minus level 1.

Table M2-1

\begin{tabular}{|l|r|r|r|r|}
\hline \multicolumn{1}{|c|}{ Column \# / Factors } & Leve1 1 & Leve1 2 & Leve1 3 & L3 - L1 i \\
\hline 1 Nano MgO amount & 10.055 & 22.4 & & n/a \\
2 Dipping Speed & 10.8 & 24.333 & 13.55 & 2.75 \\
3 Firing Profile & 5.85 & 23.1 & 19.733 & 13.883 \\
4 MgO/Spinel ratio & 14.75 & 7.483 & 26.45 & 11.699 \\
5 First Stage Ball & 27 & 8.95 & 12.733 & -14.267 \\
6 Fish oil amount & 15.616 & 26.466 & 6.6 & -9.017 \\
7 PVP type (K) & 36.7 & 6.85 & 5.133 & -31.568 \\
8 PVP amount & 1.283 & 20.649 & 26.75 & 25.467 \\
\hline
\end{tabular}

Table M2-2 shows the ANOVA table for the analysis of the factor influences. It appears that almost all the factors are not significant at the $90 \%$ level. 
Table M2-2

\begin{tabular}{|c|c|c|c|c|c|c|}
\hline Col\#/Factor & $\begin{array}{l}\mathrm{DOF} \\
\text { (f) }\end{array}$ & $\begin{array}{c}\text { Sum of Sqrs. } \\
\text { (S) }\end{array}$ & $\begin{array}{l}\text { Variance } \\
\text { (V) }\end{array}$ & $\begin{array}{c}\text { F - Ratio } \\
\text { (F) }\end{array}$ & $\begin{array}{c}\text { Pure Sum } \\
\left(S^{\prime}\right)\end{array}$ & $\begin{array}{r}\text { Percent } \\
\mathrm{P}(\%) \\
\end{array}$ \\
\hline $1 \mathrm{Nano} \mathrm{MgO}$ amount & 1 & 685.734 & 685.734 & 1.38 & 188.993 & 1.503 \\
\hline 2 Dipping Speed & 2 & 613.987 & 306.993 & .618 & 0 & 0 \\
\hline 3 Firing Profile & 2 & 1003.288 & 501.644 & 1.009 & 9.806 & .078 \\
\hline $4 \mathrm{MgO} / \mathrm{Sp}$ pinel ratio & 2 & 1098.857 & 549.428 & 1.106 & 105.376 & .838 \\
\hline 5 First Stage Ball & 2 & 1087.307 & 543.653 & 1.094 & 93.825 & .746 \\
\hline 6 Fish oil amount & 2 & 1187.414 & 593.707 & 1.195 & 193.933 & 1.542 \\
\hline 7 PVP type (K) & 2 & 3780.846 & 1890.423 & 3.805 & 2787.365 & 22.17 \\
\hline $8 \mathrm{PVP}$ amount & 2 & 2121.656 & 1060.828 & 2.135 & 1128.174 & 8.973 \\
\hline Other/Error & 2 & 993.481 & 496.74 & & & 64.15 \\
\hline Total: & 17 & 12572.574 & & & & $100.00 \%$ \\
\hline
\end{tabular}

Table M2-3 shows the results of the ANOVA analysis. As seen in this table, the confidence levels of all the factors except PVP type are less than $90 \%$.

Table M2-3

\begin{tabular}{|c|c|c|c|c|c|c|}
\hline Col\#/Factor & $\begin{array}{l}\text { DOF } \\
\text { (f) }\end{array}$ & $\begin{array}{c}\text { Sum of Sqrs. } \\
\text { (S) }\end{array}$ & $\begin{array}{l}\text { Variance } \\
\text { (V) }\end{array}$ & $\begin{array}{l}\text { F - Ratio } \\
\text { (F) }\end{array}$ & $\begin{array}{l}\text { Pure Sum } \\
\left(S^{\prime}\right)\end{array}$ & $\begin{array}{c}\text { Percent } \\
\mathrm{P}(\%)\end{array}$ \\
\hline $1 \mathrm{Nano} \mathrm{MgO}$ amount & (1) & $(685.734)$ & & P O OLED & $(\mathrm{CL}=6851 \%$ & \\
\hline 2 Dipping Sppeed & (2) & $(613.987)$ & & P O O L ED & $\left(\mathrm{CL}={ }^{*} \mathrm{NC}^{*}\right)$ & \\
\hline 3 Firing Profile & (2) & $(1003.288)$ & & P O OLED & $(\mathrm{CL}=5752 \%$ & \\
\hline $4 \mathrm{MgO/Spinel} \mathrm{ratio}$ & (2) & $(1098.857)$ & & P O O L ED & $(\mathrm{CL}=62.13 \%$ & \\
\hline 5 First Stage Ball & (2) & $(1087.307)$ & & P O OLED & $(\mathrm{CL}=62.84 \%$ & \\
\hline 6 Fish oil amount & (2) & $(1187.414)$ & & P O OL ED & $(\mathrm{CL}=66.48 \%$ & \\
\hline 7 PVP type (K) & 2 & 3780.846 & 1890.423 & 3.805 & 2787.365 & 22.17 \\
\hline $8 \mathrm{PVP}$ amount & (2) & $(2121.656)$ & & P O O L ED & $(\mathrm{CL}=8455 \%)$ & \\
\hline OtherrEnor & 15 & 8791.724 & 586.115 & & & 77.83 \\
\hline Total: & 17 & 12572.574 & & & & $100.00 \%$ \\
\hline
\end{tabular}

Figure M2 shows the plots of the effects of each of the factors on the measured response. 


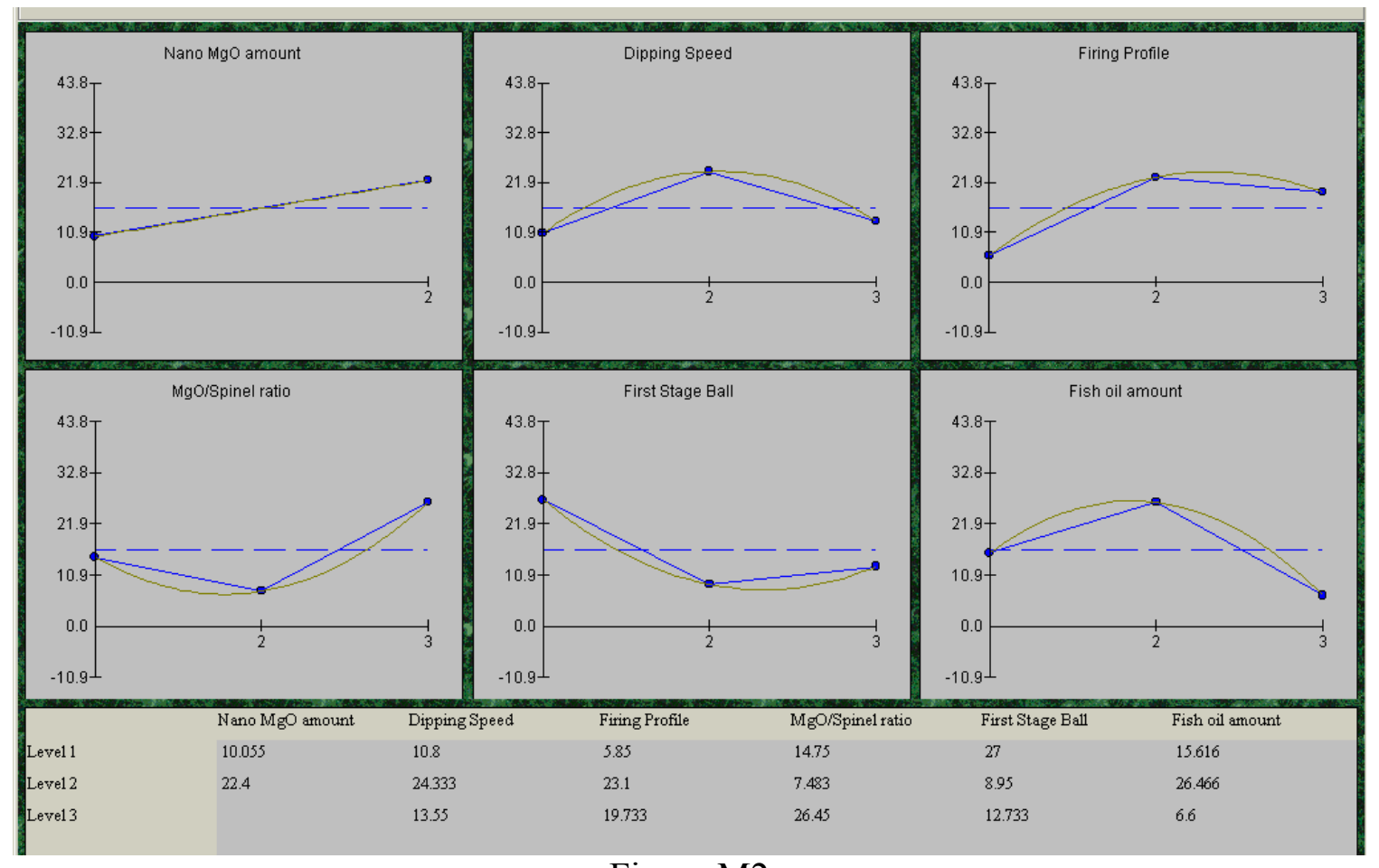

Figure M2

Table M2-4 shows the expected optimal performance for the factors determined as significant (with a confidence level greater than 90\%).

\section{Table M2-4}

\begin{tabular}{|l|l|l|l|}
\hline Column \# / Factor & Leve1 Description & Level & Contribution \\
\hline 7 PVP type (K) & $80 \% 10 \mathrm{~K}$ & 3 & -11.095 \\
& & & \\
\hline
\end{tabular}

If we assumed that all the factors were significant the optimal levels would be as those shown in Table M2-5. 
Table M2-5

\begin{tabular}{|l|l|c|r|}
\hline Column \# / Factor & Level Description & Level & Contribution \\
\hline 1 Nano MgO amount & 10 & 1 & -6.173 \\
2 Dipping Speed & .09 & 1 & -5.428 \\
3 Firing Profile & 1550 & 1 & -10.378 \\
4 MgO/Spinel ratio & $9: 1$ & 2 & -8.745 \\
5 First Stage Ball & 6 & 2 & -7.278 \\
6 Fish oil amount & 75 & 3 & -9.628 \\
7 PVP type (K) & $80 \% 10 \mathrm{~K}$ & 3 & -11.095 \\
8 PVP amount & 0.4 & 1 & -14.945 \\
\hline
\end{tabular}

This gives the optimal levels as predicted by the data, but as stated before most of the factors are not significant at the $90 \%$ level.

\section{M3 - Length of the Longest Crack}

The following is the analysis of the length of the longest crack data generated in the 18 samples that were measured. The quality characteristic used was "smaller is better". Table M3-1 shows the factors and levels and the averages of level 3 minus level 1.

\section{Table M3-1}

\begin{tabular}{|l|rrr|r||r|}
\hline \multicolumn{1}{|c|}{ Column \# / Factors } & Leve1 1 & Leve1 2 & Leve1 3 & L3 - L1 & v \\
\hline 1 Nano MgO amount & 5.644 & 8.122 & & n/a \\
2 Dipping Speed & 7.3 & 9.516 & 3.833 & -3.467 \\
3 Firing Profile & 4.416 & 6.65 & 9.583 & 5.166 \\
4 MgO/Spinel ratio & 5.783 & 4.333 & 10.533 & 4.749 \\
5 First Stage Ball & 11.483 & 4.35 & 4.816 & -6.668 \\
6 Fish oil amount & 11.6 & 5.733 & 3.316 & -8.284 \\
7 PVP type (K) & 12.116 & 5.233 & 3.3 & -8.816 \\
8 PVP amount & 2.033 & 8.933 & 9.683 & 7.649 \\
\hline
\end{tabular}

Table M3-2 shows the ANOVA table for the analysis of the factor influences. It appears that almost all the factors are significant at the $90 \%$ level. 
Table M3-2

\begin{tabular}{|l|c|r|r|r|r|r|}
\hline \hline Col\#/Factor & $\begin{array}{c}\text { DOF } \\
\text { (f) }\end{array}$ & $\begin{array}{r}\text { Sum of Sqrs. } \\
\text { (S) }\end{array}$ & $\begin{array}{c}\text { Variance } \\
\text { (V) }\end{array}$ & $\begin{array}{c}\text { F - Ratio } \\
\text { (F) }\end{array}$ & $\begin{array}{r}\text { Pure Sum } \\
\text { (S') }\end{array}$ & $\begin{array}{r}\text { Percent } \\
\text { P(\%) }\end{array}$ \\
\hline 1 Nano MgO amount & 1 & 27.627 & 27.627 & 78.805 & 27.276 & 2.247 \\
2 Dipping Speed & 2 & 98.463 & 49.231 & 140.43 & 97.762 & 8.056 \\
3 Firing Profile & 2 & 80.573 & 40.286 & 114.915 & 79.872 & 6.581 \\
4 MgO/Spinel ratio & 2 & 126.209 & 63.104 & 180.003 & 125.508 & 10.342 \\
5 First Stage Ball & 2 & 191.093 & 95.546 & 272.542 & 190.392 & 15.689 \\
6 Fish oil amount & 2 & 217.743 & 108.871 & 310.551 & 217.042 & 17.885 \\
7 PVP type (K) & 2 & 257.703 & 128.851 & 367.542 & 257.001 & 21.178 \\
8 PVP amount & 2 & 213.389 & 106.694 & 304.341 & 212.688 & 17.526 \\
\hline OtheriEror & 2 & .701 & .35 & & .496 \\
\hline Total: & 17 & 1213.504 & & & & \\
\hline
\end{tabular}

Table M3-3 shows the results of the ANOVA analysis. As seen in this table, the confidence levels of all the factors are greater than $90 \%$.

Table M3-3

\begin{tabular}{|c|c|c|c|c|c|c|}
\hline Col\#/Factor & \begin{tabular}{|l|} 
DOF \\
(f)
\end{tabular} & $\begin{array}{c}\text { Sum of Sqrs. } \\
\text { (S) }\end{array}$ & $\begin{array}{l}\text { Variance } \\
\text { (V) }\end{array}$ & $\begin{array}{l}\text { F - Ratio } \\
\text { (F) }\end{array}$ & $\begin{array}{c}\text { Pure Sum } \\
\left(S^{\prime}\right)\end{array}$ & $\begin{array}{r}\text { Percent } \\
\mathrm{P}(\%)\end{array}$ \\
\hline $1 \mathrm{Nano} \mathrm{MgO}$ amount & (1) & $(27.627)$ & & POOLED & $(\mathrm{CL}=98.9 \%)$ & \\
\hline 2 Dipping Speed & (2) & $(98.463)$ & & P O OLED & $(C L=99.76 \%$ & \\
\hline 3 Firing Profile & 2 & 80.573 & 40.286 & 114.915 & 79.872 & 6.581 \\
\hline $4 \mathrm{MgO} /$ Spinel ratio & 2 & 126.209 & 63.104 & 180.003 & 125.508 & 10.342 \\
\hline 5 First Stage Ball & 2 & 191.093 & 95.546 & 272.542 & 190.392 & 15.689 \\
\hline 6 Fish oil amount & 2 & 217.743 & 108.871 & 310.551 & 217.042 & 17.885 \\
\hline 7 PVP type (K) & 2 & 257.703 & 128.851 & 367.542 & 257.001 & 21.178 \\
\hline $8 \mathrm{PVP}$ amount & 2 & 213.389 & 106.694 & 304.341 & 212.688 & 17.526 \\
\hline Other:Error & 5 & 126.791 & 25.358 & & & 10.799 \\
\hline Total: & 17 & 1213.504 & & & & $100.00 \%$ \\
\hline
\end{tabular}

Figure M3 shows the plots of the effects of each of the factors on the measured response. 


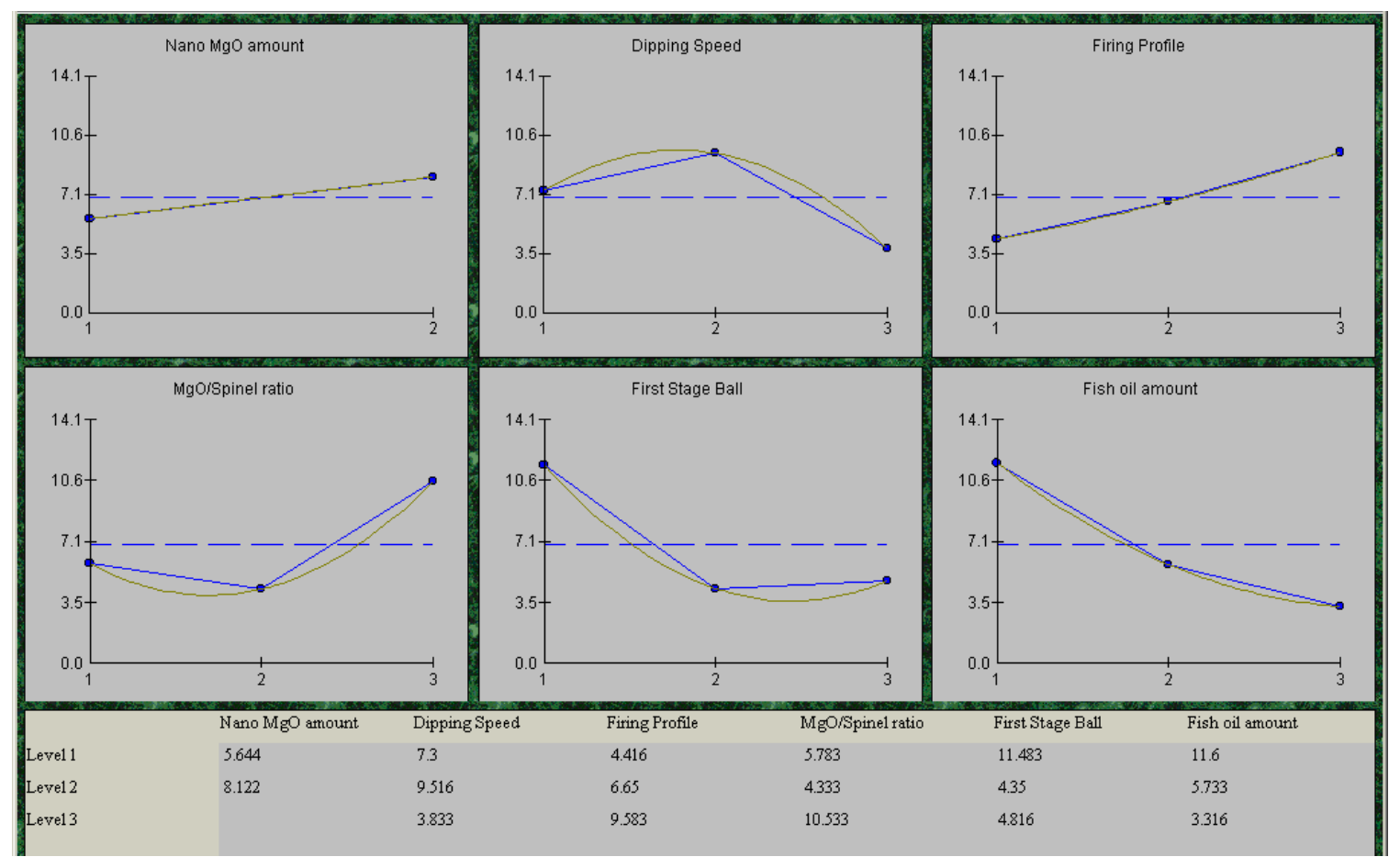

Figure M3

Table M3-4 shows the expected optimal performance for the factors determined as significant (with a confidence level greater than $99.5 \%$.

Table M3-4

\begin{tabular}{|l|l|r|r|}
\hline Column \# / Factor & Level Description & Leve1 & Contribution \\
\hline 3 Firing Profile & 1550 & 1 & -2.467 \\
4 MgO/Spinel ratio & $9: 1$ & 2 & -2.55 \\
5 First Stage Ball & 6 & 2 & -2.534 \\
6 Fish oil amount & 75 & 3 & -3.567 \\
7 PVP type (K) & $80 \% 10 \mathrm{~K}$ & 3 & -3.584 \\
8 PVP amount & 0.4 & 1 & -4.85 \\
\hline \\
Total Contribution From All Factors... & & \\
Current Grand Average Of Performance... & & -19.552 \\
Expected Result At Optimum Condition... & -12.669 \\
\hline
\end{tabular}

If we assumed that all the factors were significant the optimal levels would be as those shown in Table M3-5. 
Table M3-5

\begin{tabular}{|l|l|l|r|}
\hline Column \# / Factor & Level Description & Level & Contribution \\
\hline 1 Nano MgO amount & 10 & 1 & -1.239 \\
2 Dipping Speed & .120 & 3 & -3.05 \\
3 Firing Profile & 1550 & 1 & -2.467 \\
$4 \mathrm{MgO} /$ Spinel ratio & $9: 1$ & 2 & -2.55 \\
5 First Stage Ball & 6 & 2 & -2.534 \\
6 Fish oil amount & 75 & 3 & -3.567 \\
7 PVP type (K) & $80 \% 10 \mathrm{~K}$ & 3 & -3.584 \\
8 PVP amount & 0.4 & 1 & -4.85 \\
\hline
\end{tabular}

This gives the optimal levels as predicted by the data.

\section{M4 - Number of Cracks}

The following is the analysis of the sapphire number of cracks data generated in the 18 samples that were measured. The quality characteristic used was "smaller is better". Table M4-1 shows the factors and levels and the averages of level 3 minus level 1.

Table M4-1

\begin{tabular}{|l|r|r|r|r|}
\hline \multicolumn{1}{|c|}{ Column \# / Factors } & Leve1 1 & Leve1 2 & Leve1 3 & L3 - L1 i \\
\hline 1 Nano MgO amount & .444 & .555 & \\
2 Dipping Speed & .566 & .6 & .333 & $\mathrm{n} / \mathrm{a}$ \\
3 Firing Profile & .283 & .383 & .833 & -.233 \\
4 MgO/Spinel ratio & .45 & .333 & .716 & .549 \\
5 First Stage Ball & .783 & .4 & .316 & -.468 \\
6 Fish oil amount & .833 & .383 & .283 & -.55 \\
7 PVP type (K) & .9 & .383 & .216 & -.685 \\
8 PVP amount & .116 & .783 & .6 & .483 \\
\hline
\end{tabular}

Table M4-2 shows the ANOVA table for the analysis of the factor influences. It appears that the firing profile and fish oil amount are the only significant factors affecting the attenuation from the data generated. 
Table M4-2

\begin{tabular}{|l|c|r|r|r|r|r|}
\hline Col \# / Factor & $\begin{array}{l}\text { DOF } \\
\text { (f) }\end{array}$ & $\begin{array}{r}\text { Sum of Sqrs. } \\
\text { (S) }\end{array}$ & $\begin{array}{c}\text { Variance } \\
\text { (V) }\end{array}$ & $\begin{array}{c}\text { F - Ratio } \\
\text { (F) }\end{array}$ & $\begin{array}{r}\text { Pure Sum } \\
\text { (S') }\end{array}$ & $\begin{array}{r}\text { Percent } \\
\text { P(\%) }\end{array}$ \\
\hline 1 Nano MgO amount & 1 & .055 & .055 & .373 & 0 & 0 \\
2 Dipping Speed & 2 & .253 & .126 & .85 & 0 & 0 \\
3 Firing Profile & 2 & 1.03 & .515 & 3.458 & .732 & 10.736 \\
4 MgO/Spinel ratio & 2 & .463 & .231 & 1.555 & .165 & 2.427 \\
5 First Stage Ball & 2 & .743 & .371 & 2.496 & .445 & 6.533 \\
6 Fish oil amount & 2 & 1.029 & .514 & 3.458 & .732 & 10.736 \\
7 PVP type (K) & 2 & 1.523 & .761 & 5.115 & 1.225 & 17.969 \\
8 PVP amount & 2 & 1.423 & .711 & 4.779 & 1.125 & 16.503 \\
\hline Other/Error & 2 & .296 & .148 & & & 35.096 \\
\hline Total: & 17 & 6.819 & & & & \\
\hline
\end{tabular}

Table M4-3 shows the results of the ANOVA analysis. As seen in this table, the confidence levels of all of the last three factors are greater than $90 \%$.

Table M4-3

\begin{tabular}{|c|c|c|c|c|c|c|}
\hline Col\#/Factor & \begin{tabular}{|l} 
DOF \\
$(\mathrm{f})$
\end{tabular} & $\begin{array}{c}\text { Sum of Sqrs. } \\
\text { (S) }\end{array}$ & $\begin{array}{l}\text { Variance } \\
\text { (V) }\end{array}$ & $\begin{array}{l}\text { F - Ratio } \\
\text { (F) }\end{array}$ & $\begin{array}{c}\text { Pure Sum } \\
\text { (S') }\end{array}$ & $\begin{array}{r}\text { Percent } \\
\mathrm{P}(\%) \\
\end{array}$ \\
\hline $1 \mathrm{Nano} \mathrm{MgO}$ amount & (1) & $(.055)$ & & P O OLED & $\left(\mathrm{CL}={ }^{*} \mathrm{NC}^{*}\right)$ & \\
\hline 2 Dipping Speed & (2) & $(.253)$ & & P O OL ED & $\left(\mathrm{CL}={ }^{*} \mathrm{NC}^{*}\right)$ & \\
\hline 3 Firing Profile & (2) & (1.03) & & P O OLED & $(\mathrm{CL}=88.91 \%$ & \\
\hline $4 \mathrm{MgO/Spinel} \mathrm{ratio}$ & (2) & $(.463)$ & & P O OL ED & $(\mathrm{CL}=72.81 \%$ & \\
\hline 5 First Stage Ball & (2) & $(.743)$ & & P O OLED & $(\mathrm{CL}=86.6 \%)$ & \\
\hline 6 Fish oil amount & 2 & 1.029 & .514 & 3.458 & .732 & 10.736 \\
\hline 7 PVP type (K) & 2 & 1.523 & .761 & 5.115 & 1.225 & 17.969 \\
\hline $8 \mathrm{PVP}$ amount & 2 & 1.423 & .711 & 4.779 & 1.125 & 16.503 \\
\hline Other/Error & 11 & 2.84 & .258 & & & 54.792 \\
\hline Total: & 17 & 6.819 & & & & $100.00 \%$ \\
\hline
\end{tabular}

Figure M4 shows the plots of the effects of each of the factors on the measured response. 


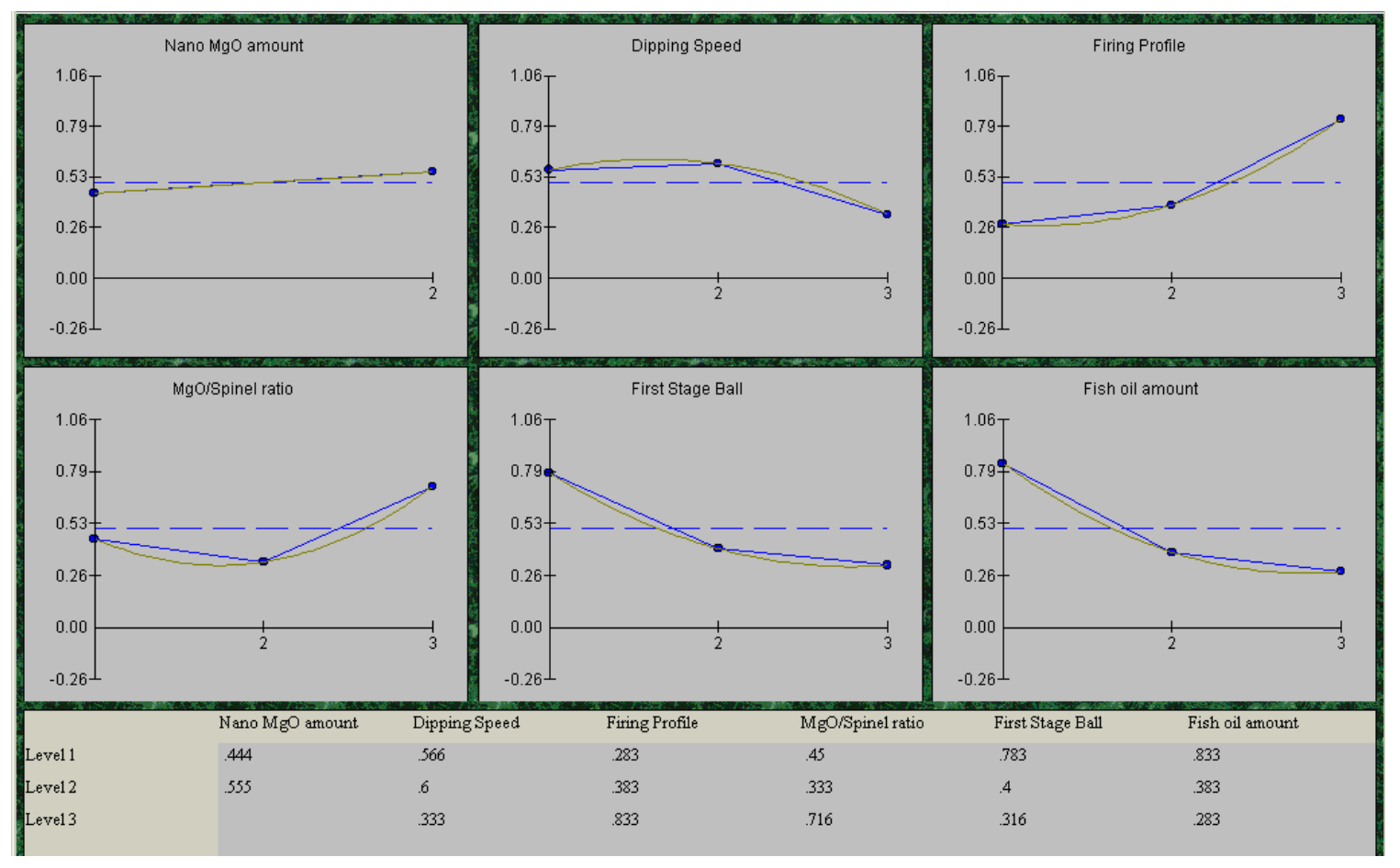

Figure M-4

Table M4-4 shows the expected optimal performance for PVP type, PVP amount and fish oil amount being determined as significant (with a confidence level less than 90).

Table M4-4

\begin{tabular}{l|l|l|r|}
\hline Column \# / Factor & Level Description & Level & Contribution \\
\hline 6 Fish oil amount & 75 & 3 & -.217 \\
7 PVP type (K) & $80 \% 10 \mathrm{~K}$ & 3 & -.284 \\
8 PVP amount & 0.4 & 1 & -.384 \\
\hline \\
Total Contribution From All Factors... \\
Current Grand Average Of Performance... \\
Expected Result At Optimum Condition... \\
\hline
\end{tabular}

If we assumed that all the factors were significant the optimal levels would be as those shown in Table M4-5. 
Table M4-5

\begin{tabular}{|l|l|l|r|}
\hline Column \# / Factor & Level Description & Level & Contribution \\
\hline 1 Nano MgO amount & 10 & 1 & -.056 \\
2 Dipping Speed & .120 & 3 & -.167 \\
3 Firing Profile & 1550 & 1 & -.217 \\
4 MgO/Spinel ratio & $9: 1$ & 2 & -.167 \\
5 First Stage Ball & 12 & 3 & -.184 \\
6 Fish oil amount & 75 & 3 & -.217 \\
7 PVP type (K) & $80 \% 10 \mathrm{~K}$ & 3 & -.284 \\
8 PVP amount & 0.4 & 1 & -.384 \\
\hline
\end{tabular}

\section{O1: Core/Cladding Interface Roughness Analysis}

The following is the analysis of the interface roughness data generated in the 18 samples that were measured. The quality characteristic used was "smaller is better".

Table O1-1 shows the results of the Anova analysis. As seen in this table, the confidence levels of all the factors but one - firing profile - are less than $90 \%$. Therefore, based on the amount of scatter in the data, only the effect of the firing profile seems significant when approaching 90\%. More data would be needed to improve the estimates of these effects.

Table O1-1

\begin{tabular}{|c|c|c|c|c|c|c|}
\hline Col\#/Factor & $\begin{array}{l}\text { DUF } \\
\text { (f) }\end{array}$ & $\begin{array}{c}\text { sum or squs. } \\
\text { (S) }\end{array}$ & $\begin{array}{l}\text { Variance } \\
\text { (V) }\end{array}$ & $\begin{array}{l}\mathrm{F} \text { - Ratio } \\
\text { (F) }\end{array}$ & $\begin{array}{l}\text { Pure sum } \\
\text { (S') }\end{array}$ & $\begin{array}{r}\text { rercent } \\
\mathrm{P}(\%)\end{array}$ \\
\hline $1 \mathrm{Nano} \mathrm{MgO}$ amount & (1) & (.204) & & P O OLED & $(\mathrm{CL}=74.88 \%$ & \\
\hline 2 Dipping Speed & (2) & $(.38)$ & & P O OL ED & $(\mathrm{CL}=6957 \%$ & \\
\hline 3 Firing Profile & 2 & 1.255 & .627 & 5.901 & 1.043 & 31.781 \\
\hline $4 \mathrm{MgO} / \mathrm{Sp}$ inel ratio & (2) & $(.041)$ & & P O OLED & $\left(\mathrm{CL}={ }^{*} \mathrm{NC} *\right)$ & \\
\hline 5 First Stage Ball & (2) & $(.111)$ & & P O OLED & $\left(\mathrm{CL}={ }^{*} \mathrm{NC}{ }^{*}\right)$ & \\
\hline 6 Fish oil amount & (2) & $(461)$ & & P O OLED & $(C L=83.33 \%$ & \\
\hline 7 PVP type (K) & (2) & $(.177)$ & & P O OLED & $\left(\mathrm{CL}={ }^{*} \mathrm{NC}^{*}\right)$ & \\
\hline 8 PVP amount & (2) & $(435)$ & & P O OLED & $(\mathrm{CL}=83.47 \%$ & \\
\hline OtherrError & 15 & 2.021 & .135 & & & 68.219 \\
\hline
\end{tabular}

The optimal level for firing profile is level 1. 
Table O1-2

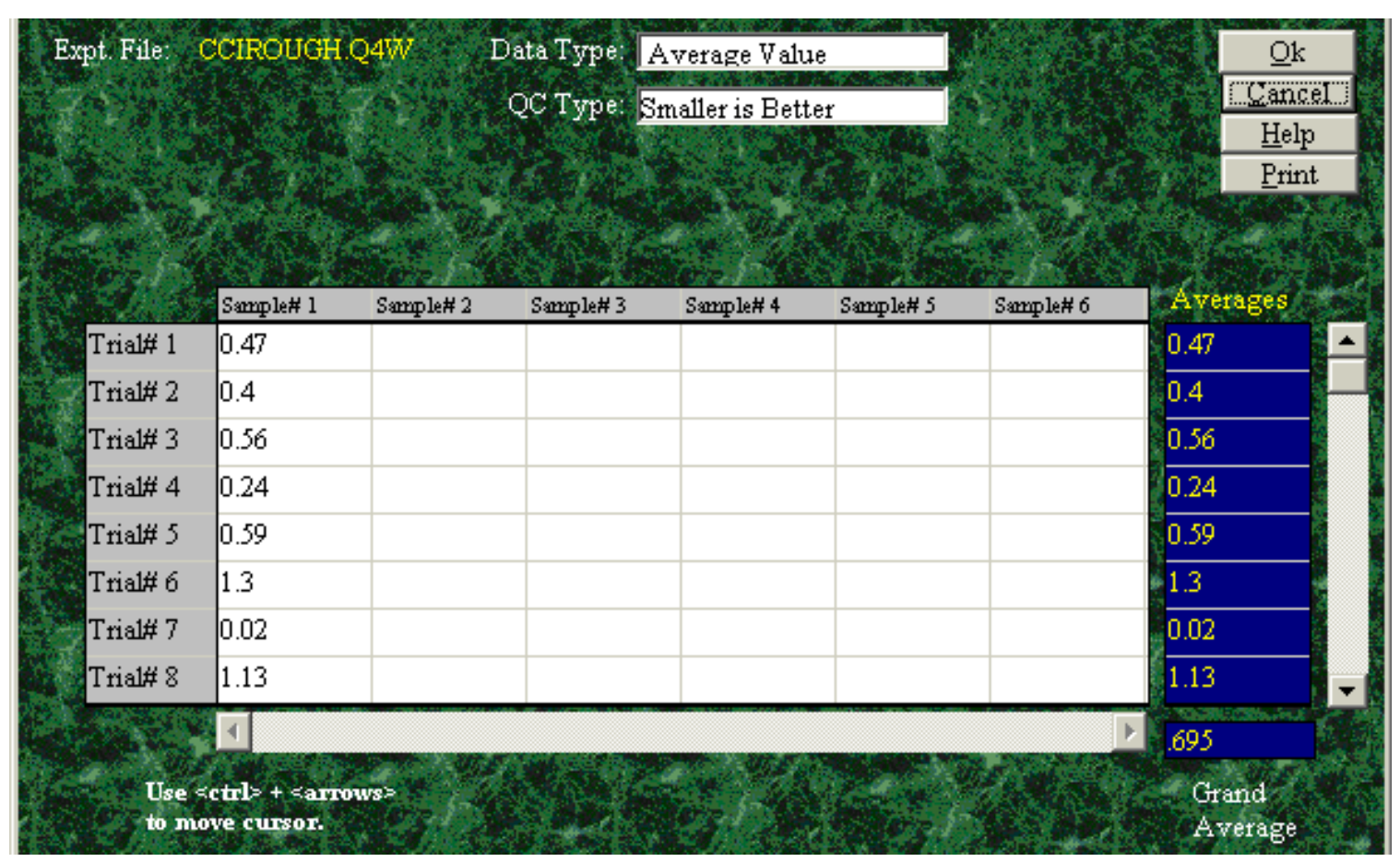

Table O1-3

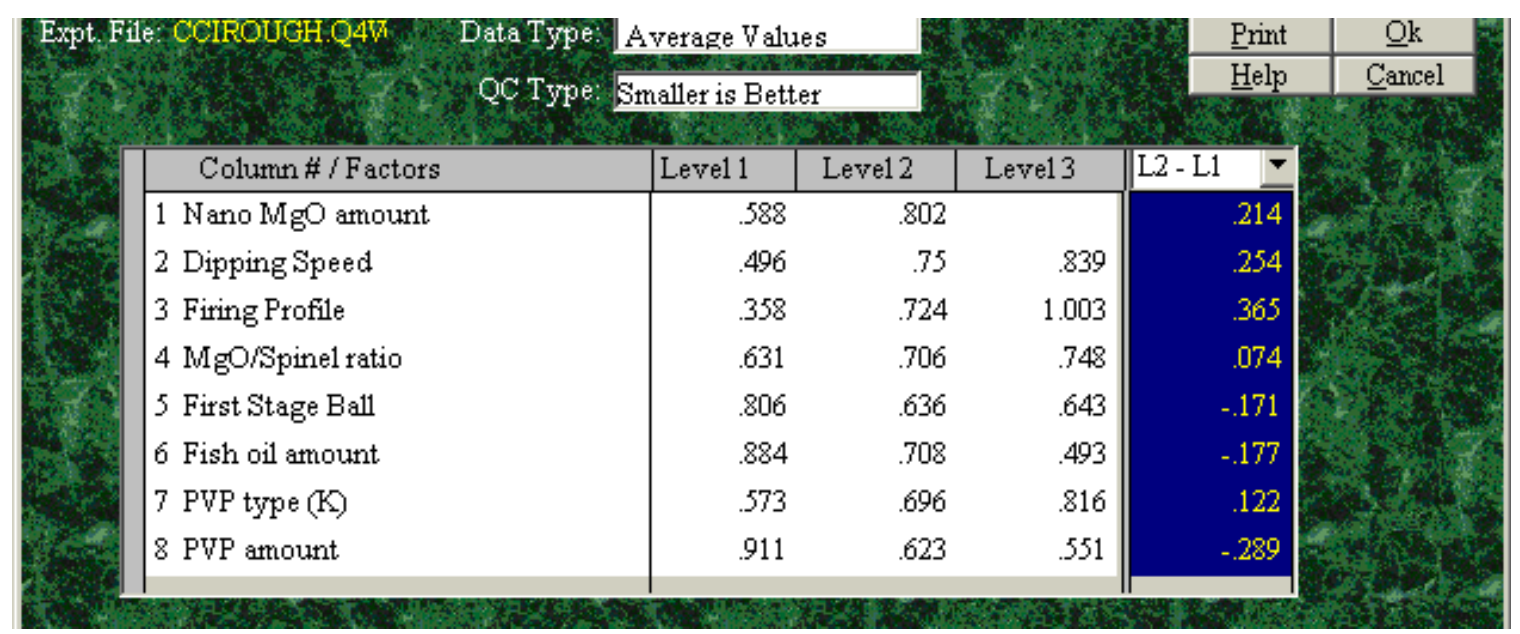


Table O1-4

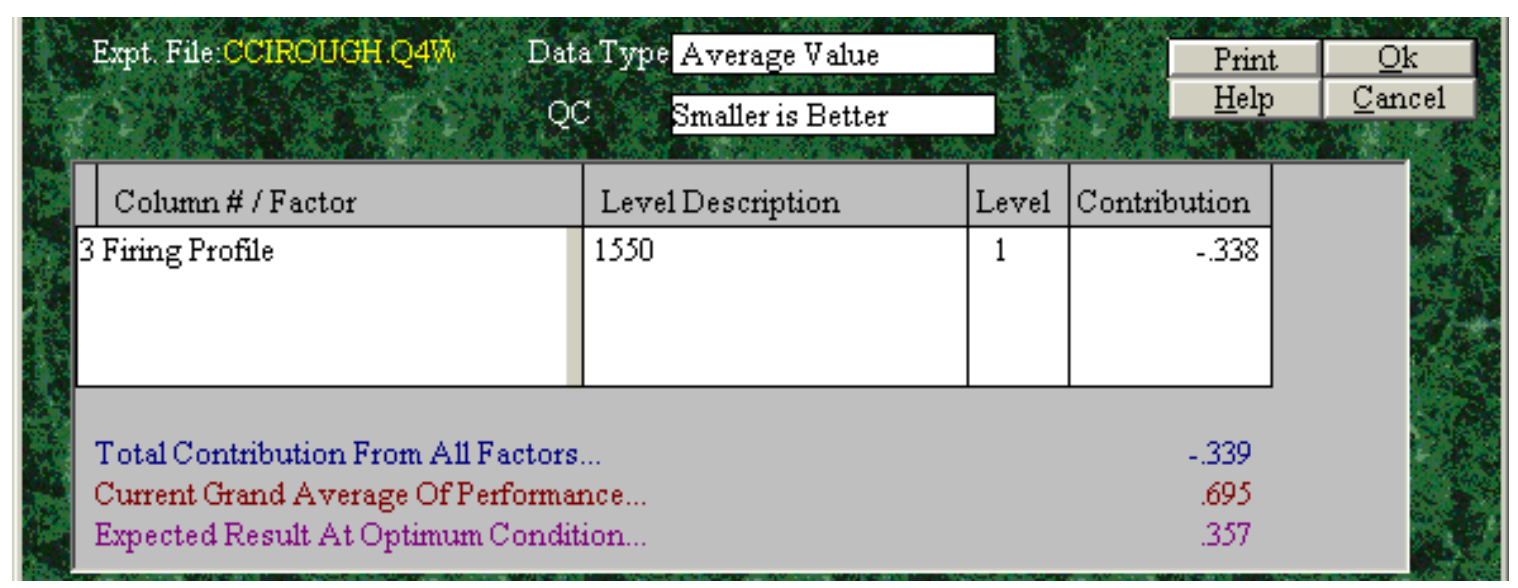

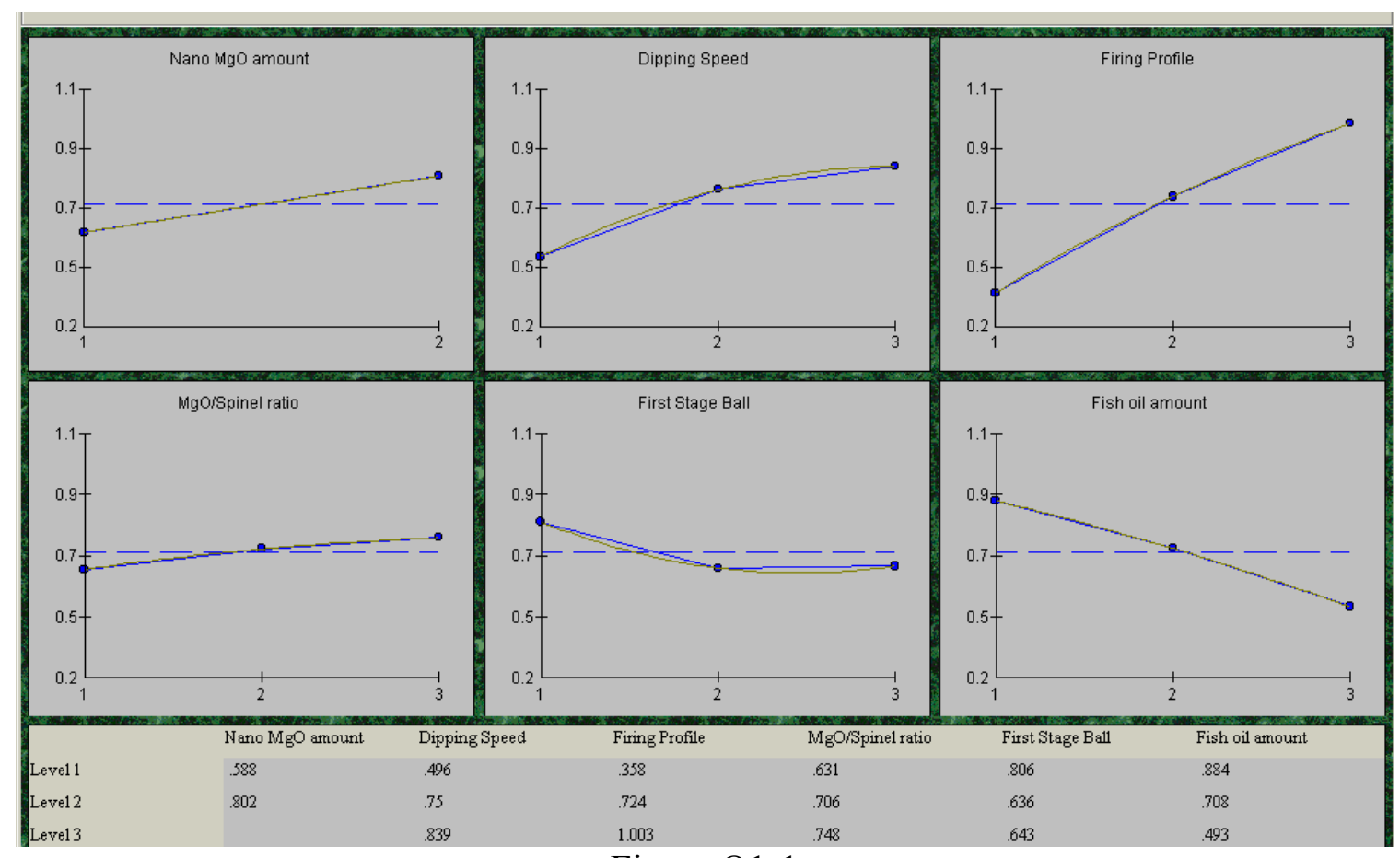

Figure 01-1

\section{2 - Numerical Aperture Measurement}

The following is the analysis of the sapphire numerical aperture data generated in the 18 samples that were measured. The quality characteristic used was "smaller is better". Table O2-1 shows the factors and levels and the averages of level 3 minus level 1. 
Table O2-1

\begin{tabular}{|c|c|c|c|c|}
\hline Column \# / Factors & Level 1 & Leve1 2 & Leve1 3 & L3 - L1 \\
\hline $1 \mathrm{Nano} \mathrm{MgO}$ amount & .062 & .081 & & $\mathrm{n} / \mathrm{a}$ \\
\hline 2 Dipping Speed & .057 & .081 & .076 & .018 \\
\hline 3 Firing Profile & .073 & .079 & .063 & -.01 \\
\hline $4 \mathrm{MgO} / \mathrm{Sp}$ inel ratio & .069 & .075 & .071 & .001 \\
\hline 5 First Stage Ball & .061 & .069 & .085 & .024 \\
\hline 6 Fish oil amount & .089 & .067 & .059 & -.03 \\
\hline 7 PVP type (K) & .074 & .062 & .079 & .005 \\
\hline 8 PVP amount & .057 & .077 & .081 & .024 \\
\hline
\end{tabular}

Table O2-2 shows the ANOVA table for the analysis of the factor influences. It appears that none of the factors has a very high percent contribution.

Table O2-2

\begin{tabular}{|c|c|c|c|c|c|c|}
\hline Col\#/Factor & $\begin{array}{l}\mathrm{DOF} \\
\text { (f) }\end{array}$ & $\begin{array}{c}\text { Sum of Sqrs. } \\
\text { (S) }\end{array}$ & $\begin{array}{l}\text { Variance } \\
\text { (V) }\end{array}$ & $\begin{array}{c}\text { F - Ratio } \\
\text { (F) }\end{array}$ & $\begin{array}{c}\text { Pure Sum } \\
\text { (S') }\end{array}$ & $\begin{array}{r}\text { Percent } \\
\mathrm{P}(\%) \\
\end{array}$ \\
\hline $1 \mathrm{Nano} \mathrm{MgO}$ amount & 1 & .001 & .001 & 1.327 & 0 & 2.519 \\
\hline 2 Dipping Speed & 2 & .002 & .001 & .911 & 0 & 0 \\
\hline 3 Firing Profile & 2 & 0 & 0 & .366 & 0 & 0 \\
\hline $4 \mathrm{MgO} / \mathrm{Spinel}$ ratio & 2 & 0 & 0 & .054 & 0 & 0 \\
\hline 5 First Stage Ball & 2 & .001 & 0 & .816 & 0 & 0 \\
\hline 6 Fish oil amount & 2 & .002 & .001 & 1.346 & 0 & 5.322 \\
\hline 7 PVP type (K) & 2 & 0 & 0 & .439 & 0 & 0 \\
\hline $8 \mathrm{PVP}$ amount & 2 & .001 & 0 & .907 & 0 & 0 \\
\hline OtheriError & 2 & .001 & 0 & & & 92.159 \\
\hline Total: & 17 & .014 & & & & $100.00 \%$ \\
\hline
\end{tabular}

Table O2-3 shows the results of the ANOVA analysis. As seen in this table, the confidence levels of all the factors are less than $71 \%$. 
Table O2-3

\begin{tabular}{|c|c|c|c|c|c|c|}
\hline Col\#/Factor & $\begin{array}{l}\text { DOF } \\
\text { (f) }\end{array}$ & $\begin{array}{c}\text { Sum of Sqrs. } \\
\text { (S) }\end{array}$ & $\begin{array}{l}\text { Variance } \\
\text { (V) }\end{array}$ & $\begin{array}{l}\text { F - Ratio } \\
\text { (F) }\end{array}$ & $\begin{array}{c}\text { Pure Sum } \\
\left(S^{\prime}\right)\end{array}$ & $\begin{array}{r}\text { Percent } \\
\mathrm{P}(\%) \\
\end{array}$ \\
\hline $1 \mathrm{Nano} \mathrm{MgO}$ amount & (1) & $(.001)$ & & POOLED & $(\mathrm{CL}=67.71 \%$ & \\
\hline 2 Dipping Speed & (2) & $(.002)$ & & P O O L ED & $\left(\mathrm{CL}={ }^{*} \mathrm{NC}^{*}\right)$ & \\
\hline 3 Firing Profile & (2) & (0) & & P O O L ED & $\left(\mathrm{CL}={ }^{\prime} \mathrm{NC} *\right)$ & \\
\hline $4 \mathrm{MgO} /$ Spinel ratio & (2) & (D) & & P O O L E D & $\left(\mathrm{CL}={ }^{*} \mathrm{NC}^{*}\right)$ & \\
\hline 5 First Stage Ball & (2) & $(.001)$ & & P O OLED & $\left(\mathrm{CL}={ }^{*} \mathrm{NC}^{*}\right)$ & \\
\hline 6 Fish oil amount & (2) & $(.002)$ & & P O OLED & $(\mathrm{CL}=70.4 \%)$ & \\
\hline 7 PVP type (K) & (2) & (D) & & P O O L ED & $\left(\mathrm{CL}={ }^{*} \mathrm{NC}^{*}\right)$ & \\
\hline $8 \mathrm{PVP}$ amount & (2) & $(001)$ & & P O O L ED & $\left(\mathrm{CL}={ }^{*} \mathrm{NC}^{*}\right)$ & \\
\hline OtheriEnor & 17 & .008 & 0 & & & 100 \\
\hline Total: & 17 & .014 & & & & $100.00 \%$ \\
\hline
\end{tabular}

Figure $\mathrm{O} 2$ shows the plots of the effects of each of the factors on the measured response.

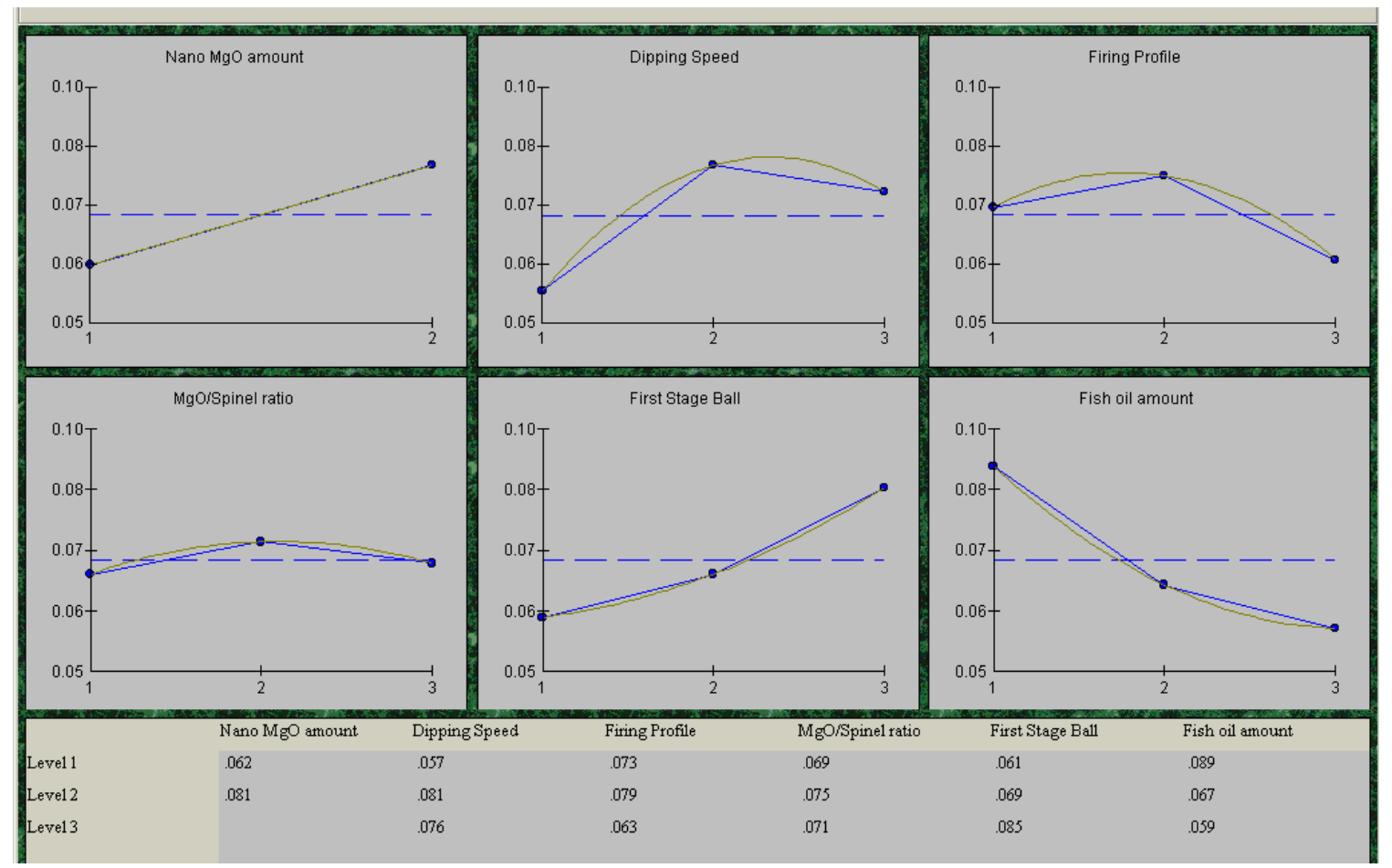

Figure $\mathrm{O} 2$

Table O2-4 shows the expected optimal performance for Nano MgO amount, PVP amount and fish oil amount being determined as significant (with a confidence level less than $70 \%$ - so a very large amount of variation in the data relative to the factor level change. 
Table O2-4

\begin{tabular}{|l|l|r|r|}
\hline Column \# / Factor & Level Description & Level & Contribution \\
\hline 1 Nano MgO amount & 10 & 1 & -.01 \\
6 Fish oil amount & 75 & 3 & -.013 \\
8 PVP amount & 0.4 & 1 & -.015 \\
\hline \\
Total Contribution From All Factors... \\
Current Grand Average Of Performance... \\
Expected Result At Optimum Condition... \\
\hline
\end{tabular}

Based on the very large scatter in the data, the confidence interval around the predicted average will be very large.

If we assumed that all the factors were significant the optimal levels would be as those shown in Table O2-5.

Table O2-5

\begin{tabular}{|l|l|l|r|}
\hline Column \# / Factor & Leve1 Description & Leve1 & Contribution \\
\hline 1 Nano MgO amount & 10 & 1 & -.01 \\
2 Dipping Speed & .09 & 1 & -.015 \\
3 Firing Profile & 1600 & 3 & -.009 \\
$4 \mathrm{MgO}$ Spinel ratio & $6: 1$ & 1 & -.003 \\
5 First Stage Ball & 3 & 1 & -.011 \\
6 Fish oil amount & 75 & 3 & -.013 \\
7 PVP type (K) & $90 \% 10 \mathrm{~K}$ & 2 & -.01 \\
8 PVP amount & 0.4 & 1 & -.015 \\
\hline
\end{tabular}

This gives the optimal levels as predicted by the data, but again due to the large scatter the factors mentioned above are not sufficiently high in significance to warrant inclusion in the optimal predictions.

\section{3 - Attenuation Measurement}

The following is the analysis of the sapphire attenuation data generated in the 18 samples that were measured. The quality characteristic used was "smaller is better".

Table O3-1 shows the factors and levels and the averages of level 3 minus level 1. 
Table O3-1

\begin{tabular}{|l|r|r|r||r|}
\hline Column \# / Factors & Leve1 1 & Leve1 2 & Leve1 3 & L3 - L1 \\
\hline 1 Nano MgO amount & 7.299 & 6.6 & & $\mathrm{n} / \mathrm{a}$ \\
2 Dipping Speed & 6.516 & 5.65 & 8.683 & 2.166 \\
3 Firing Profile & 3.416 & 11.233 & 6.2 & 2.784 \\
4 MgO/Spinel ratio & 5.666 & 6.483 & 8.699 & 3.032 \\
5 First Stage Ball & 6.299 & 8.55 & 6 & -.3 \\
6 Fish oil amount & 9.916 & 6.083 & 4.849 & -5.068 \\
7 PVP type (K) & 6.15 & 6.016 & 8.683 & 2.532 \\
8 PVP amount & 7.25 & 5.433 & 8.166 & .916 \\
\hline
\end{tabular}

Table O3-2 shows the ANOVA table for the analysis of the factor influences. It appears that the firing profile and fish oil amount are the only significant factors affecting the attenuation from the data generated.

Table O3-2

\begin{tabular}{|c|c|c|c|c|c|c|}
\hline $\mathrm{Col} \# /$ Factor & $\begin{array}{l}\text { DOF } \\
\text { (f) }\end{array}$ & $\begin{array}{c}\text { Sum of Sqrs. } \\
\text { (S) }\end{array}$ & $\begin{array}{c}\text { Variance } \\
\text { (V) }\end{array}$ & $\begin{array}{c}\text { F - Ratio } \\
\text { (F) }\end{array}$ & $\begin{array}{l}\text { Pure Sum } \\
\left(S^{\prime}\right)\end{array}$ & $\begin{array}{c}\text { Percent } \\
\mathrm{P}(\%)\end{array}$ \\
\hline $1 \mathrm{Nano} \mathrm{MgO}$ amount & 1 & 2.205 & 2.205 & .082 & 0 & 0 \\
\hline 2 Dipping Speed & 2 & 29.293 & 14.646 & .549 & 0 & 0 \\
\hline 3 Firing Profile & 2 & 188.363 & 94.181 & 3.532 & 135.043 & 29.348 \\
\hline $4 \mathrm{MgO} / \mathrm{Sp}$ pinel ratio & 2 & 29.563 & 14.781 & .554 & 0 & 0 \\
\hline 5 First Stage Ball & 2 & 23.31 & 11.655 & .437 & 0 & 0 \\
\hline 6 Fish oil amount & 2 & 83.773 & 41.886 & 1.571 & 30.453 & 6.618 \\
\hline 7 PVP type $(\mathrm{K})$ & 2 & 27.093 & 13.546 & .508 & 0 & 0 \\
\hline $8 \mathrm{PVP}$ amount & 2 & 23.223 & 11.611 & .435 & 0 & 0 \\
\hline Other/Error & 2 & 53.319 & 26.659 & & & 64.034 \\
\hline Total: & 17 & 460.145 & & & & $100.00 \%$ \\
\hline
\end{tabular}

Table O3-3 shows the results of the ANOVA analysis. As seen in this table, the confidence levels of all the factors are less than $90 \%$. Therefore, based on the amount of scatter in the data, only the effect of the firing profile seems significant when approaching $90 \%$. More data would be needed to improve the estimates of these effects. 
Table O3-3

\begin{tabular}{|c|c|c|c|c|c|c|}
\hline Col\#/Factor & \begin{tabular}{|l|}
$\begin{array}{l}D O F \\
\text { (f) }\end{array}$ \\
\end{tabular} & $\begin{array}{l}\text { Sum of Sqrs. } \\
\text { (S) }\end{array}$ & $\begin{array}{l}\text { Variance } \\
\text { (V) }\end{array}$ & $\begin{array}{l}\text { F - Ratio } \\
\text { (F) }\end{array}$ & $\begin{array}{l}\text { Pure Sum } \\
\text { (S') }\end{array}$ & $\begin{array}{r}\text { Percent } \\
\mathrm{P}(\%) \\
\end{array}$ \\
\hline $1 \mathrm{Nano} \mathrm{MgO}$ amount & (1) & (2.205) & & POOLED & $\left(\mathrm{CL}={ }^{*} \mathrm{NC} *\right)$ & \\
\hline 2 Dipping Speed & (2) & $(29.293)$ & & P O OLED & $\left(\mathrm{CL}={ }^{\prime} \mathrm{NC}^{\star}\right)$ & \\
\hline 3 Firing Profile & (2) & $(188.363)$ & & P O OLED & $(\mathrm{CL}=89.24 \%$ & \\
\hline $4 \mathrm{MgO} /$ spinel ratio & (2) & $(29.563)$ & & P O OLED & $\left(\mathrm{CL}={ }^{\prime} \mathrm{NC} *\right)$ & \\
\hline 5 First Stage Ball & (2) & (23.31) & & P O O L ED & $\left(\mathrm{CL}={ }^{*} \mathrm{NC}^{*}\right)$ & \\
\hline 6 Fish oil amount & (2) & $(83.773)$ & & P O OLED & $(\mathrm{CL}=75.28 \%$ & \\
\hline 7 PVP type (K) & (2) & $(27.093)$ & & P O OLED & $\left(\mathrm{CL}={ }^{\prime} \mathrm{NC}{ }^{\star}\right)$ & \\
\hline $8 \mathrm{PVP}$ amount & (2) & (23.223) & & P O OLED & $\left(\mathrm{CL}={ }^{2} \mathrm{NC} *\right)$ & \\
\hline Other/Error & 17 & 460.142 & 27.067 & & & 100 \\
\hline Total: & 17 & 460.145 & & & & $100.00 \%$ \\
\hline
\end{tabular}

It should be noted that the attenuation measurement was developed quickly during the period where samples were being prepared. A rigorous analysis of the repeatability and reproducibility of the measurement procedure was decided against due to the extremely tight time constraints under which the project operated. The data indicate that there is a large amount of unexplained error present in the system and this may be due in part to the variation of the measurement process. Future work should focus on characterization of the variation from the measurement of the attenuation.

Figure $\mathrm{O} 3$ shows the plots of the effects of each of the factors on the measured attenuation.

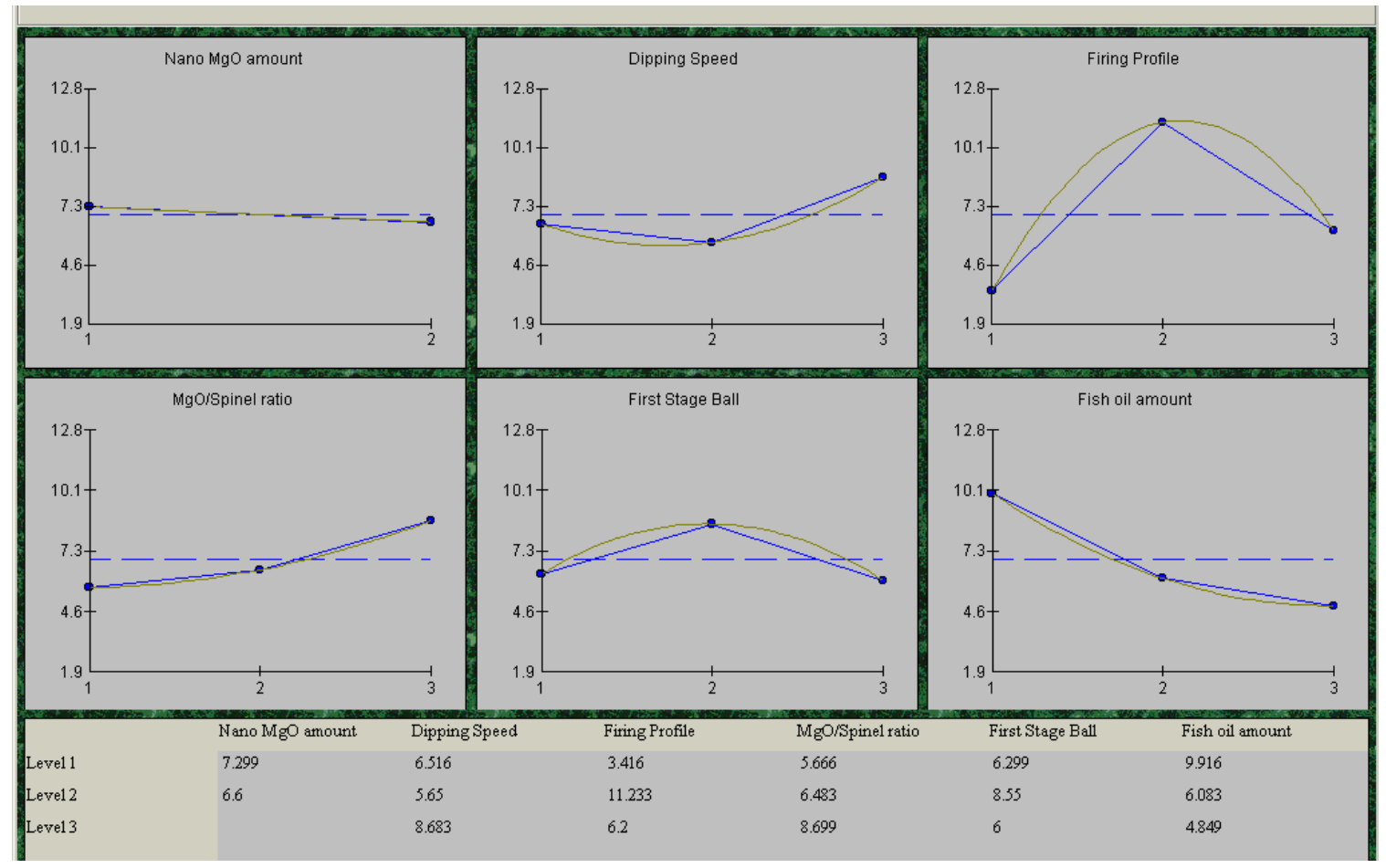

Figure $\mathrm{O} 3$ 
Table O3-4 shows the expected optimal performance for firing profile and fish oil amount being determined as significant.

Table O3-4

\begin{tabular}{|l|l|r|r|}
\hline Column \# / Factor & Level Description & Level & Contribution \\
\hline 3 Firing Profile & 1550 & 1 & -3.534 \\
6 Fish oil amount & 75 & 3 & -2.101 \\
& & & \\
\hline \\
Total Contribution From All Factors... & \\
Current Grand Average Of Performance... & -5.635 \\
Expected Result At Optimum Condition... & 6.95 \\
\hline
\end{tabular}

Based on the very large scatter in the data, the confidence interval around the predicted average will be very large.

If we assumed that all the factors were significant the optimal levels would be as those shown in Table O3-5.

Table O3-5

\begin{tabular}{|l|l|c|}
\hline Column \# / Factor & Level Description & Leve1 \\
\hline 1 Nano MgO amount & 20 & 2 \\
2 Dipping Speed & .105 & 2 \\
3 Firing Profile & 1550 & 1 \\
4 MgO/Spinel ratio & $6: 1$ & 1 \\
5 First Stage Ball & 12 & 3 \\
6 Fish oil amount & 75 & 3 \\
7 PVP type (K) & $90 \% 10 \mathrm{~K}$ & 2 \\
8 PVP amount & 0.5 & 2 \\
\hline
\end{tabular}

This gives the optimal levels as predicted by the data, but again due to the large scatter the factors other than the two mentioned above are not sufficiently high in significance to warrant inclusion in the optimal predictions. 


\section{Appendix C: Slurry preparation and fiber dipping procedures}

1. Powder Preparation:

1.1. $\mathrm{MgO}$ powders are hand-ground in a mortar using a pestle for 1 to 2 hours. During the grinding isopropyl alcohol is added to the mortar to form a paste. After grinding the powder slurry is poured into a polypropylene bottle (250c.c.) with alumina grinding medium filled to about $50 \%$ in volume of the bottle. More alcohol is added to cover the grinding media if needed. The jar is rotated using a ball mill at a speed of 60 to 70 turns per minute for a few days to further break down the particles.

1.2. $\mathrm{MgAl}_{2} \mathrm{O}_{4}$ powders are also ground using the same steps described in step 1.1.

1.3. $4.45 \mathrm{~g}$ of $\mathrm{MgO}$ powders ( $75 \mathrm{wt}$. $\%$ ), and $1.49 \mathrm{~g}$ of $\mathrm{MgAl}_{2} \mathrm{O}_{4}$ powders $(25 \mathrm{wt} . \%)$ are mixed in a beaker.

2. Slurry Preparation:

2.1. A bath of $24.97 \mathrm{~g}$ of isopropyl alcohol (90.2 wt. \%), $2.04 \mathrm{~g}$ of propylene glycol methyl ether (8.2 wt. \%, Fluka 1-methoxy-2-propanol), $0.3 \mathrm{~g}$ of polyvinylpyrrolidone (1.2 wt. $\%)$ and $0.12 \mathrm{~g}$ of polyethylene glycol $(0.4 \mathrm{wt} . \%)$ is prepared in a beaker on a hot plate. The bath in a beaker is covered with parafilm and is stirred using a magnetic stir bar on a hot plate for about 10 minutes. The polyvinylpyrrolidone crystals are fully dissolved at the end of stirring.

2.2. The powder prepared in step 1 is added to the solution bath, one small scoop at a time. The spinning magnetic bar is also used to enhance the dissolution of the powders.

2.3. The slurry is poured from the beaker into a polypropylene bottle (250c.c.) with alumina grinding medium filled to about $50 \%$ in volume of the bottle. More alcohol is added to cover the grinding media if needed. The jar is rotated using a ball mill at a speed of 60 to 70 turns per minute for a few days to mix the slurry thoroughly and to further break down the particles. After ball milling, the slurry is stirred in a beaker using a magnetic spin bar until the desired viscosity is reached.

3. Application of slurry (dipping processes):

3.1. The slurry was transferred to a graduated cylinder or a small test tube for dipping. To prevent the slurry from drying, the cylinder or the test tube is capped, and a small hole is drilled in the cap to let the fiber pass through. The cylinder or the test tube sits on a small lab jack.

3.2. Before dipping, the fibers are first cleaned with isopropyl alcohol using Kimwipe, and then the fibers are immersed in $\mathrm{HCl}$ for 1 minute, followed by in deionized water for 1 minute and finally in isopropyl alcohol for another 1 minute. The fiber is then clamped vertically to a sample holder.

3.3. The fiber is dipped by slowly raising the graduated cylinder containing the slurry with a lab jack until the desired length of the fiber is immersed in the slurry. Then the cylinder is slowly lowered to complete the dipping. This procedure is repeated for multiple dipping. There is a 10 to 15 seconds interval between each dipping for the slurry coating on the fiber to dry. 


\section{Appendix D: The firing profile of sample 14(17)s}

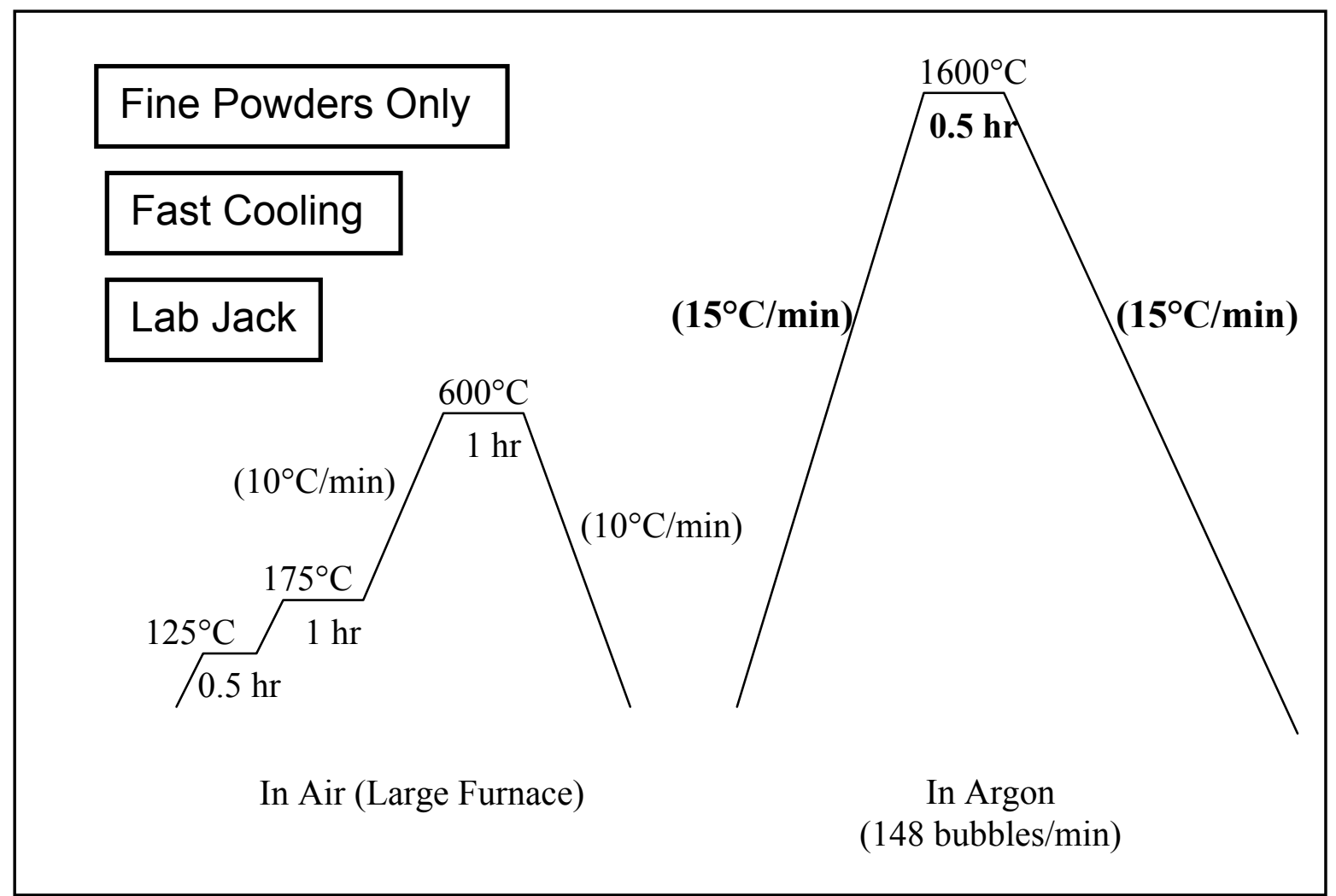

(Note: The first firing profile in air is designed to remove water and the binder in the spinel slurry coating.) 


\section{Appendix E: Preparation conditions of cladding samplea}

\begin{tabular}{|c|c|c|c|c|c|c|}
\hline $\begin{array}{l}\text { Sample } \\
\text { I.D. }\end{array}$ & $\begin{array}{c}\text { Date } \\
\text { Dipped }\end{array}$ & $\begin{array}{l}\text { Date } \\
\text { Fired }\end{array}$ & $\begin{array}{c}\text { Atmos- } \\
\text { phere }\end{array}$ & Firing Profiles** & $\begin{array}{l}\text { Powder } \\
\text { type*** }\end{array}$ & $\begin{array}{c}\# \text { of } \\
\text { dips } * * * * *\end{array}$ \\
\hline Sp-1 & $11 / 26 / 03$ & $12 / 4 / 03$ & Air & $0.5 \mathrm{~h}$ in at $1750^{\circ} \mathrm{C}$ & Coarse & 1 \\
\hline Sp-2 & $11 / 26 / 03$ & $12 / 4 / 03$ & Air & $0.5 \mathrm{~h}$ at $1750^{\circ} \mathrm{C}$ & Coarse & 2 \\
\hline Sp-3 & $11 / 26 / 03$ & $12 / 4 / 03$ & Air & $0.5 \mathrm{~h}$ at $1750^{\circ} \mathrm{C}$ & Coarse & 3 \\
\hline $1 \mathrm{a}$ & $1 / 23 / 04$ & $1 / 23 / 04$ & Air & $\begin{array}{c}2.5 \text { days at } 1650^{\circ} \mathrm{C} \text { (Furnace } \\
\text { cooled) }\end{array}$ & Coarse & 1 \\
\hline $2 a$ & $1 / 23 / 04$ & $1 / 23 / 04$ & Air & $\begin{array}{c}2.5 \text { days at } 1650^{\circ} \mathrm{C} \text { (Furnace } \\
\text { cooled) }\end{array}$ & Coarse & 2 \\
\hline $4 a$ & $1 / 23 / 04$ & $1 / 23 / 04$ & Air & $\begin{array}{c}2.5 \text { days at } 1650^{\circ} \mathrm{C} \text { (Furnace } \\
\text { cooled) }\end{array}$ & Coarse & 4 \\
\hline $1 b$ & $1 / 26 / 04$ & $1 / 26 / 04$ & Air & $\begin{array}{c}16 \mathrm{hr} \text { at } 1650^{\circ} \mathrm{C}\left(10^{\circ} \mathrm{C} / \mathrm{min} .\right. \\
\text { cooling rate })\end{array}$ & Coarse & 1 \\
\hline $1 b-1^{*}$ & $1 / 26 / 04$ & $1 / 26 / 04$ & Air & $\begin{array}{c}16 \mathrm{hr} \text { at } 1650^{\circ} \mathrm{C}\left(10^{\circ} \mathrm{C} / \mathrm{min} .\right. \\
\text { cooling rate })\end{array}$ & Coarse & 1 \\
\hline $2 b$ & $1 / 26 / 04$ & $1 / 26 / 04$ & Air & $\begin{array}{c}16 \mathrm{hr} \text { at } 1650^{\circ} \mathrm{C}\left(10^{\circ} \mathrm{C} / \mathrm{min} .\right. \\
\text { cooling rate })\end{array}$ & Coarse & 2 \\
\hline $3 b$ & $1 / 27 / 04$ & $1 / 27 / 04$ & Air & $\begin{array}{c}16 \mathrm{hr} \text { at } 1650^{\circ} \mathrm{C} \text { (Furnace } \\
\text { cooled) }\end{array}$ & Coarse & 3 \\
\hline $4 \mathrm{~b}$ & $1 / 27 / 04$ & $1 / 27 / 04$ & Air & $\begin{array}{c}16 \mathrm{hr} \text { at } 1650^{\circ} \mathrm{C} \text { (Furnace } \\
\text { cooled) }\end{array}$ & Coarse & 4 \\
\hline 8 & $1 / 20 / 04$ & $1 / 20 / 04$ & Air & $16 \mathrm{hr}$ at $1650^{\circ} \mathrm{C}$ & Coarse & 8 \\
\hline 12 & $1 / 20 / 04$ & $1 / 20 / 04$ & Air & $16 \mathrm{hr}$ at $1650^{\circ} \mathrm{C}$ & Coarse & 12 \\
\hline $2 \mathrm{c}$ & $2 / 20 / 04$ & $2 / 24 / 04$ & Air & $\begin{array}{l}16 \mathrm{hr} \text { at } 1650^{\circ} \mathrm{C}\left(10^{\circ} \mathrm{C} / \mathrm{min} .\right. \\
\text { heating and cooling rate })\end{array}$ & Fine & 2 \\
\hline $3 \mathrm{c}$ & $2 / 20 / 04$ & $2 / 24 / 04$ & Air & $\begin{array}{l}16 \mathrm{hr} \text { at } 1650^{\circ} \mathrm{C}\left(10^{\circ} \mathrm{C} / \mathrm{min} .\right. \\
\text { heating and cooling rate })\end{array}$ & Fine & 3 \\
\hline $4 \mathrm{c}$ & $2 / 20 / 04$ & $2 / 24 / 04$ & Air & $\begin{array}{l}16 \mathrm{hr} \text { at } 1650^{\circ} \mathrm{C}\left(10^{\circ} \mathrm{C} / \mathrm{min} .\right. \\
\text { heating and cooling rate })\end{array}$ & Fine & 4 \\
\hline $6 e$ & $3 / 3 / 04$ & $3 / 3 / 04$ & Air & $\begin{array}{l}16 \mathrm{hr} \text { at } 1650^{\circ} \mathrm{C}\left(10^{\circ} \mathrm{C} / \mathrm{min} .\right. \\
\text { heating and cooling rate })\end{array}$ & Fine & 6 \\
\hline $10 \mathrm{f}$ & 3/9/04 & $3 / 9 / 04$ & Air & $\begin{array}{l}4 \mathrm{hr} \text { at } 1700^{\circ} \mathrm{C}\left(10^{\circ} \mathrm{C} / \mathrm{min} .\right. \\
\text { heating and cooling rate })\end{array}$ & Fine & 10 \\
\hline $4(8) g-1$ & $3 / 10 / 04$ & $3 / 10 / 04$ & Air & $\begin{array}{c}2 \mathrm{hr} \text { at } 1700^{\circ} \mathrm{C}\left(10^{\circ} \mathrm{C} / \mathrm{min} .\right. \\
\text { heating and } 5^{\circ} \mathrm{C} / \mathrm{min} \text {. } \\
\text { cooling rate })\end{array}$ & $\begin{array}{l}\text { Coarse }+ \\
\text { Fine }\end{array}$ & 4 \\
\hline $4(8) g-2$ & $3 / 10 / 04$ & $3 / 10 / 04$ & Air & $\begin{array}{c}2 \mathrm{hr} \text { at } 1700^{\circ} \mathrm{C}\left(10^{\circ} \mathrm{C} / \mathrm{min} \text {. }\right. \\
\text { heating and } 5^{\circ} \mathrm{C} / \mathrm{min} \text {. } \\
\text { cooling rate })\end{array}$ & $\begin{array}{l}\text { Coarse }+ \\
\text { Fine }\end{array}$ & 8 \\
\hline $12(17) \mathrm{h}-1$ & $3 / 11 / 04$ & $3 / 11 / 04$ & Air & $\begin{array}{c}2 \mathrm{hr} \text { at } 1700^{\circ} \mathrm{C}\left(10^{\circ} \mathrm{C} / \mathrm{min} .\right. \\
\text { heating and } 5^{\circ} \mathrm{C} / \mathrm{min} \text {. } \\
\text { cooling rate })\end{array}$ & $\begin{array}{l}\text { Coarse }+ \\
\text { Fine }\end{array}$ & 17 \\
\hline $12(17) \mathrm{h}-2$ & $3 / 11 / 04$ & $3 / 11 / 04$ & Air & $\begin{array}{c}2 \mathrm{hr} \text { at } 1700^{\circ} \mathrm{C}\left(10^{\circ} \mathrm{C} / \mathrm{min} .\right. \\
\text { heating and } 5^{\circ} \mathrm{C} / \mathrm{min} \text {. } \\
\text { cooling rate })\end{array}$ & $\begin{array}{l}\text { Coarse }+ \\
\text { Fine }\end{array}$ & 12 \\
\hline $20(24) \mathrm{i}-1$ & $3 / 12 / 04$ & $3 / 12 / 04$ & Air & $\begin{array}{c}1 \mathrm{hr} \text { at } 1700^{\circ} \mathrm{C}\left(10^{\circ} \mathrm{C} / \mathrm{min} .\right. \\
\text { heating and } 5^{\circ} \mathrm{C} / \mathrm{min} \text {. } \\
\text { cooling rate })\end{array}$ & $\begin{array}{l}\text { Coarse }+ \\
\text { Fine }\end{array}$ & 24 \\
\hline $20(24) \mathrm{i}-2$ & $3 / 12 / 04$ & $3 / 12 / 04$ & Air & $\begin{array}{l}1 \mathrm{hr} \text { at } 1700^{\circ} \mathrm{C}\left(10^{\circ} \mathrm{C} / \mathrm{min} \text {. }\right. \\
\text { heating and } 5^{\circ} \mathrm{C} / \mathrm{min} \text {. }\end{array}$ & $\begin{array}{l}\text { Coarse }+ \\
\text { Fine }\end{array}$ & 20 \\
\hline
\end{tabular}




\begin{tabular}{|c|c|c|c|c|c|c|}
\hline & & & & cooling rate) & & \\
\hline $6(10) 1$ & $3 / 28 / 04$ & $3 / 28 / 04$ & Argon & $\begin{array}{c}1 \mathrm{hr} \text { at } 1600^{\circ} \mathrm{C}\left(10^{\circ} \mathrm{C} / \mathrm{min} .\right. \\
\text { heating and } 5^{\circ} \mathrm{C} / \mathrm{min} . \\
\text { cooling rate })\end{array}$ & $\begin{array}{c}\text { Coarse }+ \\
\text { Fine }\end{array}$ & $6 \& 10$ \\
\hline $20(26) \mathrm{m}$ & $3 / 29 / 04$ & $3 / 29 / 04$ & Argon & $\begin{array}{l}4 \mathrm{hr} \text { at } 1600^{\circ} \mathrm{C}\left(10^{\circ} \mathrm{C} / \mathrm{min} .\right. \\
\text { heating and } 5^{\circ} \mathrm{C} / \mathrm{min} \text {. } \\
\text { cooling rate })\end{array}$ & $\begin{array}{l}\text { Coarse }+ \\
\text { Fine }\end{array}$ & $20 \& 26$ \\
\hline 12(17)n & $3 / 30 / 04$ & $3 / 30 / 04$ & Argon & $\begin{array}{c}1 \mathrm{hr} \text { at } 1600^{\circ} \mathrm{C}\left(10^{\circ} \mathrm{C} / \mathrm{min} .\right. \\
\text { heating and } 5^{\circ} \mathrm{C} / \mathrm{min} \text {. } \\
\text { cooling rate })\end{array}$ & $\begin{array}{l}\text { Coarse }+ \\
\text { Fine }\end{array}$ & $12 \& 17$ \\
\hline$(20-35) \mathrm{o}$ & $3 / 31 / 04$ & $3 / 31 / 04$ & Argon & $\begin{array}{l}1 \mathrm{hr} \text { at } 1600^{\circ} \mathrm{C}\left(10^{\circ} \mathrm{C} / \mathrm{min} .\right. \\
\text { heating and } 5^{\circ} \mathrm{C} / \mathrm{min} \text {. } \\
\text { cooling rate })\end{array}$ & $\begin{array}{c}\text { Coarse }+ \\
\text { Fine }\end{array}$ & 20 to 35 \\
\hline $20(25) \mathrm{p}$ & $4 / 1 / 04$ & $4 / 2 / 04$ & Argon & $\begin{array}{c}1 \mathrm{hr} \text { at } 1600^{\circ} \mathrm{C}\left(5^{\circ} \mathrm{C} / \mathrm{min} .\right. \\
\text { heating and } 1^{\circ} \mathrm{C} / \mathrm{min} . \\
\text { cooling rate })\end{array}$ & $\begin{array}{c}\text { Coarse }+ \\
\text { Fine }\end{array}$ & $20 \& 25$ \\
\hline $40(50) \mathrm{q}$ & $4 / 3 / 04$ & $4 / 3 / 04$ & Argon & $\begin{array}{l}1 \mathrm{hr} \text { at } 1600^{\circ} \mathrm{C}\left(5^{\circ} \mathrm{C} / \mathrm{min} .\right. \\
\text { heating and } 1^{\circ} \mathrm{C} / \mathrm{min} . \\
\text { cooling rate })\end{array}$ & Fine & $40 \& 50$ \\
\hline $19(25) \mathrm{r}$ & $4 / 6 / 04$ & $4 / 6 / 04$ & Argon & $\begin{array}{c}1 \mathrm{hr} \text { at } 1600^{\circ} \mathrm{C}\left(10^{\circ} \mathrm{C} / \mathrm{min} .\right. \\
\text { heating and } 5^{\circ} \mathrm{C} / \mathrm{min} \text {. } \\
\text { cooling rate })\end{array}$ & Fine & $19 \& 25$ \\
\hline 14(17)s & $4 / 12 / 04$ & $4 / 12 / 04$ & Argon & $\begin{array}{c}0.5 \mathrm{hr} \text { at } 1600^{\circ} \mathrm{C}\left(15^{\circ} \mathrm{C} / \mathrm{min} .\right. \\
\text { heating and } 15^{\circ} \mathrm{C} / \mathrm{min} . \\
\text { cooling rate })\end{array}$ & Fine & $14 \& 17$ \\
\hline $30 t$ & $4 / 15 / 04$ & $4 / 15 / 04$ & Argon & $\begin{array}{c}0.5 \mathrm{hr} \text { at } 1600^{\circ} \mathrm{C}\left(15^{\circ} \mathrm{C} / \mathrm{min} .\right. \\
\text { heating and } 15^{\circ} \mathrm{C} / \mathrm{min} . \\
\text { cooling rate })\end{array}$ & Fine & 30 \\
\hline $50 \mathrm{u}$ & $4 / 20 / 04$ & $4 / 20 / 04$ & Argon & $\begin{array}{c}10 \mathrm{~min} . \text { at } 1600^{\circ} \mathrm{C} \\
\left(15^{\circ} \mathrm{C} / \mathrm{min} \text {. heating and }\right. \\
\left.15^{\circ} \mathrm{C} / \mathrm{min} \text {. cooling rate }\right)\end{array}$ & Fine & 50 \\
\hline $35 v$ & $4 / 21 / 04$ & $4 / 21 / 04$ & Argon & $\begin{array}{c}10 \mathrm{~min} . \text { at } 1600^{\circ} \mathrm{C} \\
\left(15^{\circ} \mathrm{C} / \mathrm{min} \text {. heating and }\right. \\
\left.15^{\circ} \mathrm{C} / \mathrm{min} \text {. cooling rate }\right)\end{array}$ & Fine & 35 \\
\hline $70 w$ & $4 / 21 / 04$ & $4 / 21 / 04$ & Argon & $\begin{array}{c}2 \mathrm{~min} \text {. at } 1600^{\circ} \mathrm{C} \\
\left(15^{\circ} \mathrm{C} / \mathrm{min} \text {. heating and }\right. \\
\left.15^{\circ} \mathrm{C} / \mathrm{min} \text {. cooling rate }\right)\end{array}$ & Nano & 70 \\
\hline $34 x$ & $4 / 22 / 04$ & $4 / 22 / 04$ & Argon & $\begin{array}{c}2 \mathrm{~min} . \text { at } 1600^{\circ} \mathrm{C} \\
\left(15^{\circ} \mathrm{C} / \mathrm{min} \text {. heating and }\right. \\
\left.15^{\circ} \mathrm{C} / \mathrm{min} \text {. cooling rate }\right)\end{array}$ & Nano & 34 \\
\hline
\end{tabular}

* Sample 1b-1 and Sample 1b are sections broken from the same dipped and fired sapphire fiber.

** Each fiber is dried at $80^{\circ} \mathrm{C}$ for 15 minutes and at $130^{\circ} \mathrm{C}$ for another 15 minutes in air. For samples treated in argon, another heating step at $600^{\circ} \mathrm{C}$ for 1 hour in air was added to remove the binder.

*** Coarse: $\mathrm{MgO}(15 \mu \mathrm{m})$ and $\mathrm{MaAl}_{2} \mathrm{O}_{4}(3 \mu \mathrm{m})$; Fine: after hand grinding for a few hours and ball milling for a few days; Nano: $\mathrm{MgO}\left(130 \mathrm{~m}^{2} / \mathrm{g}, 13 \mathrm{~nm}\right.$ diameter $)$ and $\mathrm{MgAl}_{2} \mathrm{O}_{4}\left(10 \mathrm{~m}^{2} / \mathrm{g}\right.$, submicron diameter).

**** If there are two numbers for one sample, such as ( $\mathrm{x} \& \mathrm{y}), \mathrm{x}<\mathrm{y}$, it means that the bottom part of the fiber is dipped $y$ times and the upper part of the fiber is dipped $x$ times. 


\section{Appendix F: Heating profiles of sample (20-35)o and 19(25)r}
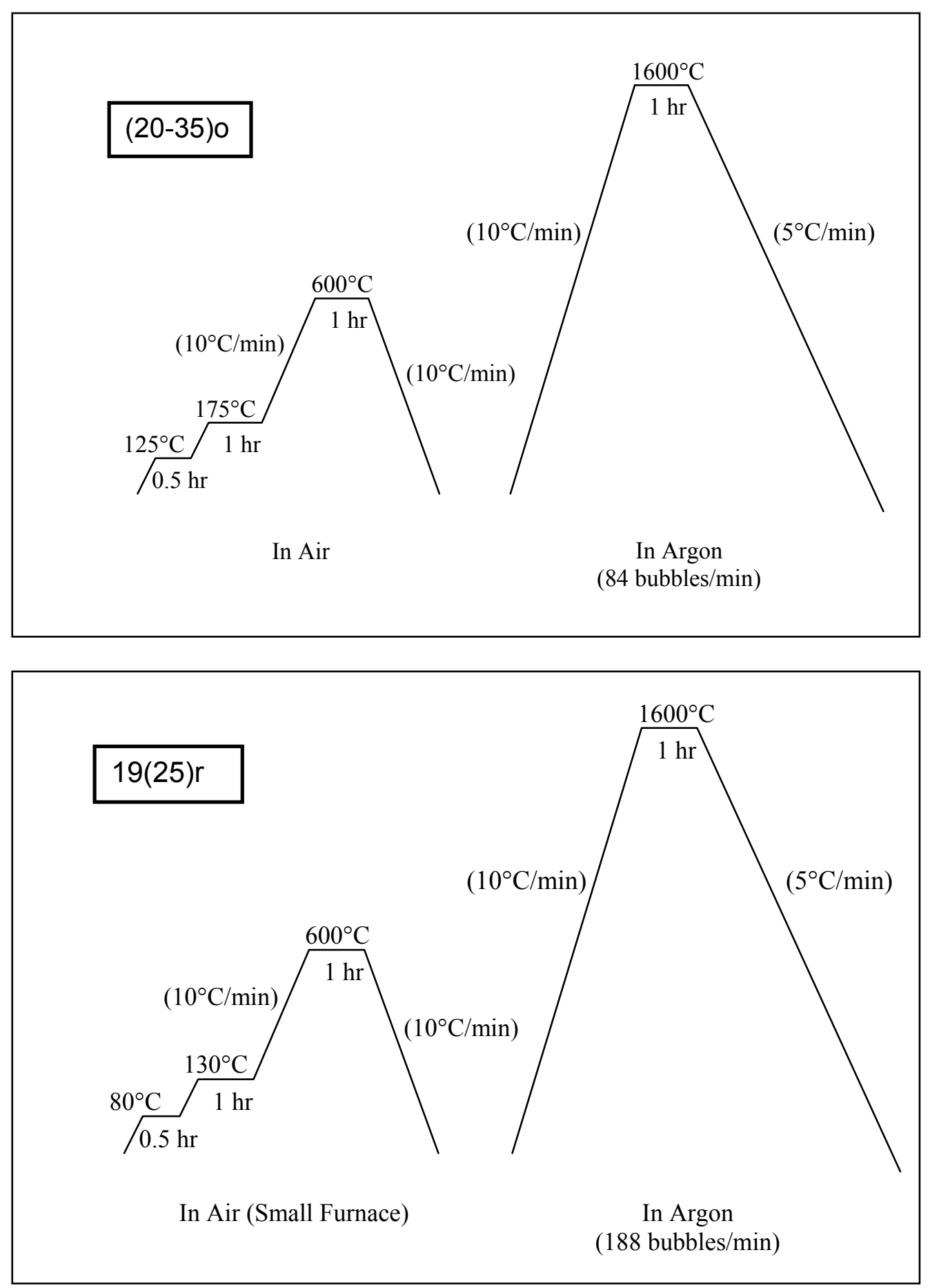

(Note: There exists slight difference in the above two firing profiles in the first heating stage to remove the water and the binder. However, the high temperature sintering steps, which are more influential to the results, are exactly the same in both firing profiles.) 


\section{Appendix G: Results of EDS chemical analysis for 9 samples}

\begin{tabular}{|c|c|c|c|c|c|c|}
\hline \multirow[t]{2}{*}{$\begin{array}{l}\text { Sample } \\
\text { Name }\end{array}$} & \multicolumn{2}{|c|}{ Surface } & \multicolumn{2}{|c|}{$\begin{array}{c}\text { Fractured cross section } \\
\text { (Core) }\end{array}$} & \multicolumn{2}{|c|}{$\begin{array}{c}\text { Fractured cross section } \\
\text { (Cladding) }\end{array}$} \\
\hline & $\mathrm{Mg}($ at $\%)$ & $\mathrm{Al}($ at $\%)$ & $\mathrm{Mg}($ at $\%)$ & $\mathrm{Al}($ at $\%)$ & $\mathrm{Mg}($ at $\%)$ & $\mathrm{Al}($ at $\%)$ \\
\hline $4 a$ & 15 & 85 & 4 & 96 & 15 & 85 \\
\hline $2 \mathrm{a}$ & 22 & 78 & 1 & 99 & 23 & 77 \\
\hline $1 \mathrm{a}$ & 22 & 78 & & & & \\
\hline $4 \mathrm{~b}$ & 15 & 85 & 1 & 99 & 15 & 85 \\
\hline $3 b$ & 15 & 85 & & & & \\
\hline $2 b$ & 16 & 84 & 1 & 99 & 16 & 84 \\
\hline $1 \mathrm{~b}$ & 24 & 76 & & & & \\
\hline $8 * *$ & 23 & 77 & & & 22 & 78 \\
\hline $12 * *$ & 53 & 47 & & & 31 & 69* \\
\hline
\end{tabular}

* A large amount of suspected phosphor contamination exists in both surface and cross section EDS data.

** In samples 8 and 12, the sapphire fibers are fully converted to spinel before the reaction between the slurry coating and the sapphire fiber is completed. This causes the $\mathrm{Mg}$ at $\%$ of these samples in the cladding parts significantly higher than those of the other samples. 